\title{
Regierung und Verwaltung auf einen Blick 2013
}

\section{(1)}
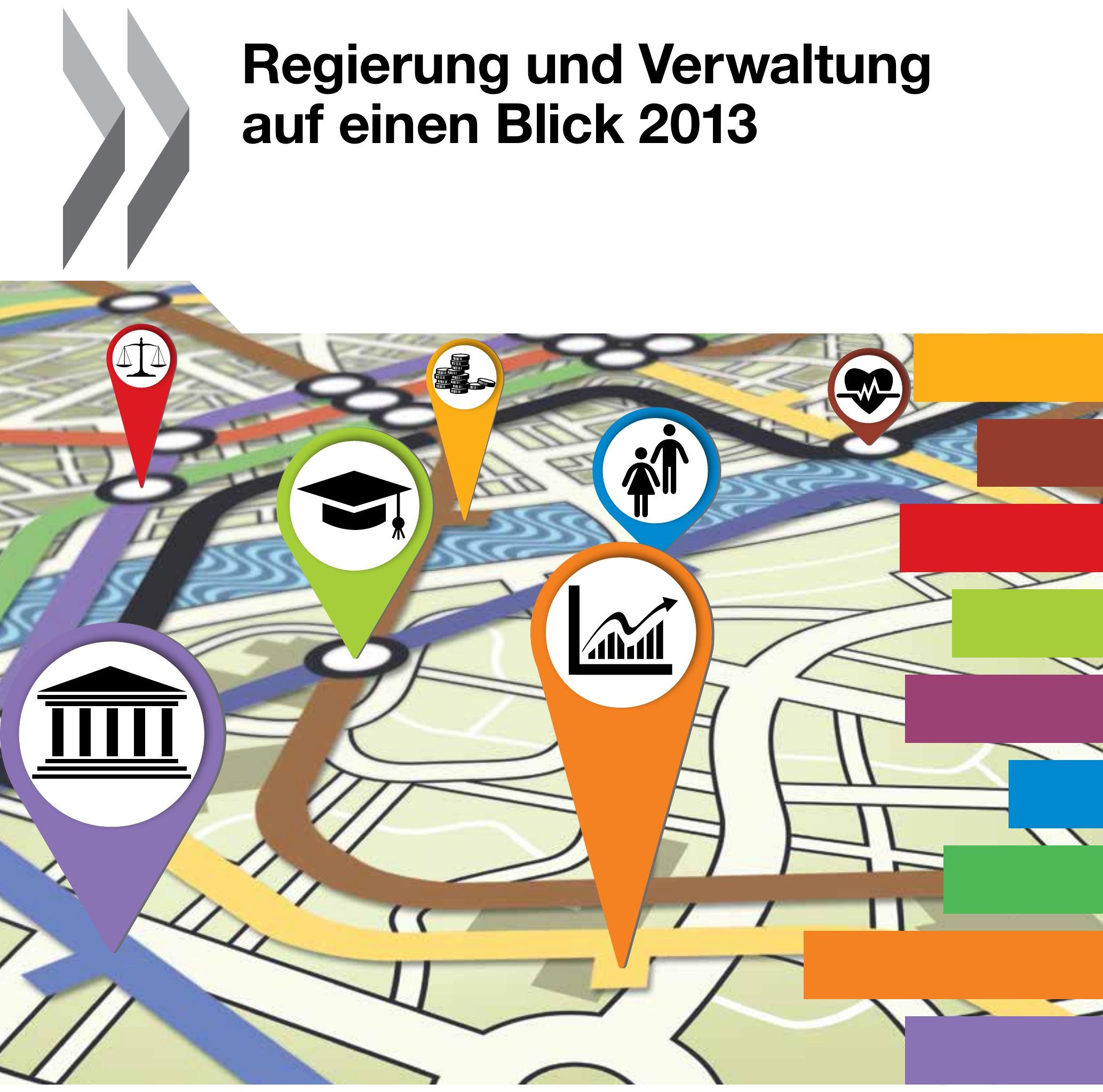



\section{Regierung und Verwaltung auf einen Blick 2013}

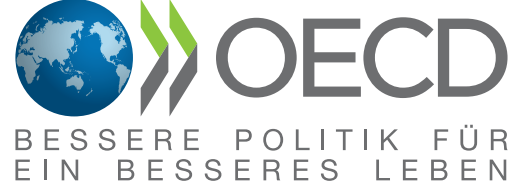


Das vorliegende Dokument wird unter der Verantwortung des Generalsekretärs der OECD veröffentlicht. Die darin zum Ausdruck gebrachten Meinungen und Argumente spiegeln nicht zwangsläufig die offizielle Einstellung der OECD-Mitgliedstaaten wider.

Dieses Dokument und die darin enthaltenen Karten berühren nicht den völkerrechtlichen Status und die Souveränität über Territorien, den Verlauf der internationalen Grenzen und Grenzlinien sowie den Namen von Territorien, Städten und Gebieten.

Bitte zitieren Sie diese Publikation wie folgt:

OECD (2014), Regierung und Verwaltung auf einen Blick 2013, OECD Publishing.

http://dx.doi.org/10.1787/9789264209541-de

ISBN 978-92-64-20109-5 (Print)

ISBN 978-92-64-20650-2 (PDF)

ISBN 978-92-64-20142-2 (HTML)

Die statistischen Daten für Israel wurden von den zuständigen israelischen Stellen bereitgestellt, die für sie verantwortlich zeichnen. Die Verwendung dieser Daten durch die OECD erfolgt unbeschadet des völkerrechtlichen Status der Golanhöhen, von Ost-Jerusalem und der israelischen Siedlungen im Westjordanland.

Originaltitel: Government at a Glance 2013 - Panorama des administrations publiques 2013

Übersetzung durch den Deutschen Übersetzungsdienst der OECD.

Foto(s): Deckblatt @ Robert Adrian Hillman/Shutterstock.com, @ tovovan/Shutterstock.com.

Kapitel 2 to $9 \odot$ Shutterstock/kentoh

Korrigenda zu OECD-Veröffentlichungen sind verfügbar unter: www.oecd.org/about/publishing/corrigenda.htm.

(C) OECD 2014

Die OECD gestattet das Kopieren, Herunterladen und Abdrucken von OECD-Inhalten für den eigenen Gebrauch sowie das Einfügen von Auszügen aus OECD-Veröffentlichungen, -Datenbanken und -Multimediaprodukten in eigene Dokumente, Präsentationen, Blogs, Websites und Lehrmaterialien, vorausgesetzt die Quelle und der Urheberrechtsinhaber werden in geeigneter Weise genannt. Sämtliche Anfragen bezüglich Verwendung für öffentliche oder kommerzielle Zwecke bzw. Übersetzungsrechte sind zu richten an: rights@oecd.org. Die Genehmigung zur Kopie von Teilen dieser Publikation für den öffentlichen oder kommerziellen Gebrauch ist direkt einzuholen beim Copyright Clearance Center (CCC) unter info@copyright.com oder beim Centre français d'exploitation du droit de copie (CFC) unter contact@cfcopies.com. 


\section{Vorwort}

$D_{\text {i }}$ ie Finanz- und Wirtschaftskrise, die 2008 begann und die Mehrzahl der OECD-Länder erfasste, hat die Debatte über die Rolle des Staates bzw. darüber, wie und wo und mit welchen Zielen er in das wirtschaftliche Geschehen eingreifen sollte, neu entfacht. Regierung und Verwaltung auf einen Blick 2013 liefert wichtige quantitative und qualitative Daten, die eine evidenzbasierte Entscheidungsfindung ermöglichen und den Ländern dabei helfen, für die Zukunft zu planen. Die Publikation gestattet einen Vergleich staatlicher Aktivitäten, Vorgehensweisen und Ergebniskennzahlen in einer Reihe wichtiger Bereiche und weist auf Aspekte hin, die eingehender untersucht werden sollten. Das einleitende Politikkapitel befasst sich mit den Zusammenhängen zwischen dem Vertrauen in den Staat und den Politiken bzw. Institutionen der Staats- und Verwaltungsführung.

Die Arbeiten an dieser Publikation wurden von Zsuzsanna Lonti unter der Aufsicht von Rolf Alter und Edwin Lau geleitet und von Natalia Nolan-Flecha, Santiago González, Jean-François Leruste und Alessandro Lupi vorbereitet. Wichtige Textbeiträge kamen von Mario Marcel und Stéphane Jacobzone (Kapitel 1 „Vertrauen in den Staat, Wirksamkeit des staatlichen Handelns und die Agenda für die Staatsund Verwaltungsführung"), Catherine Gamper und Alice Lazzati (Kapitel 2 „Strategische Governance"), Monica Brezzi, Arthur Mickoleit und Camila Vammalle (Kapitel 3 „Öffentliche Finanzen und wirtschaftliches Handeln des Staates"), Ronnie Downes, Ian Hawkesworth, Joung Jin Jang, Knut Klepvisk und Lisa Von Trapp (Kapitel 4 „Haushaltspraxis und -verfahren“), Robert Ball und Maya Beauvallet (Kapitel 5 „Beschäftigung und Bezahlung im öffentlichen Sektor"), Robert Ball, Michelle Marshalian und Tatyana Teplova (Kapitel 6 „Frauen in Regierung und Verwaltung"), Elodie Beth, María-Emma Cantera und Ulrika Kilnes (Kapitel 7 „Öffentliches Beschaffungswesen“), Julio Bacio Terracino, Janos Bertok, Maria-Emma Cantera, Ronnie Downes, Ulrika Kilnes, Knut Klepvisk, Arthur Mickoleit, Adam Mollerup und Barbara Ubaldi (Kapitel 8 „Offene und inklusive Regierung"); Filippo Cavassini, Alice Lazzati and Adam Mollerup (Kapitel 9 „Sonderbeitrag - Im Dienste der Bürger: Zugänglichkeit und Qualität öffentlicher Dienstleistungen"). Unser besonderer Dank gilt Lia Beyeler, Laura Boutin, Kate Lancaster, Natasha Lawrance, Sophie Limoges, Jennifer Stein und Deirdre Wolfender, die den Text redaktionell für die Publikation aufbereitet haben.

In diese Publikation sind Beiträge aus verschiedensten Quellen zu einem breiten Spektrum von Fachbereichen eingeflossen. Wertvolle Vorarbeit leisteten insbesondere der OECD-Ausschuss für öffentliche Governance und der Lenkungsausschuss Regierung und Verwaltung auf einen Blick (Einzelheiten hierzu in Anhang F), der OECD-Statistikausschuss, die Arbeitsgruppe Beschäftigung und Verwaltung im öffentlichen Sektor, die Arbeitsgruppe hochrangiger Verantwortlicher für die öffentliche Haushaltspolitik, die OECD-Sachverständigengruppe Interessenkonflikte, das Netzwerk hochrangiger Verantwortlicher für E-Government, die Gruppe der führenden Fachleute des öffentlichen Beschaffungswesens und die Sachverständigengruppe Innovative und Offene Regierung. Wertvolle Kommentare kamen zudem von Peter Van de Ven und Catherine La Rosa-Elkaim (OECD-Direktion Statistik), Richard Highfield, Mehmet Ceylan und Devi Thani (Zentrum für Steuerpolitik), Gaetan Lafortune, Nicolaas Sieds Klazinga und Valerie Paris (OECD-Direktion Beschäftigung, Arbeit und Sozialfragen), Corine Heckmann und Joris Ranchin (OECD-Direktion Bildung), Peter Hoeller und Giussepe Nicoletti (OECD-Hauptabteilung Wirtschaft), Messaoud Hammouya (Internationale Arbeitsorganisation, Genf) sowie Zoltan Mikolas (Consultant). 



\section{Inhaltsverzeichnis}

Geleitwort - Auf die Governance kommt es an!.................... 9

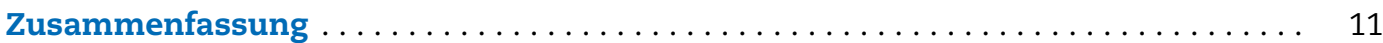

Kapitel 1 Vertrauen in den Staat, Wirksamkeit des staatlichen Handelns

und die Governance-Agenda . . . . . . . . . . . . . . . . . . 25

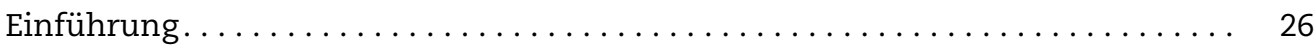

Was ist mit Vertrauen in den Staat gemeint? .................... 27

Weshalb ist Vertrauen in den Staat wichtig? ..................... 28

Messung des Vertrauens in den Staat .................... 30

Muster und Trends im Hinblick auf das Vertrauen in den Staat

in den OECD-Ländern. . . . . . . . . 32

Bestimmungsfaktoren für das Vertrauen in den Staat $\ldots \ldots \ldots \ldots \ldots \ldots \ldots .36$

Schlussbetrachtungen ................................ 44

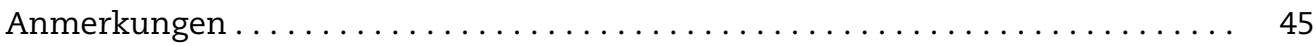

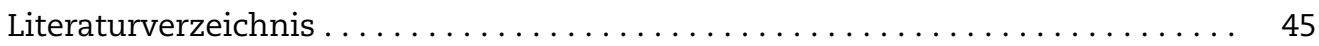

Kapitel 2 Strategische Governance. ......................... 47

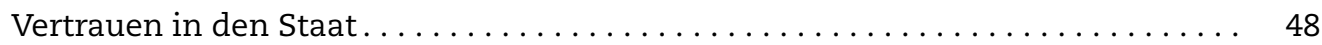

Ausgewählte Politikmaßnahmen zur Verbesserung der Gerechtigkeit . . . . . . 50

Rechtsstaatlichkeit.................................... 52

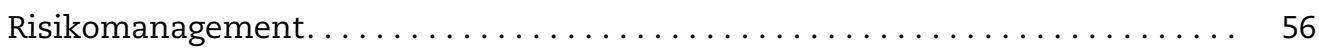

Tragfähigkeit der öffentlichen Finanzen .................... 58

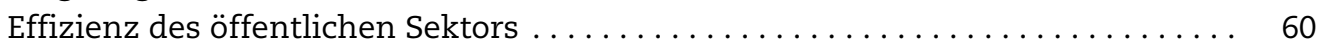

Kosteneffektivität des öffentlichen Sektors ..................... 64

Kapitel 3 Öffentliche Finanzen und wirtschaftliches Handeln des Staates . . . . . . . 69

Gesamtstaatlicher Finanzierungssaldo ....................... 70

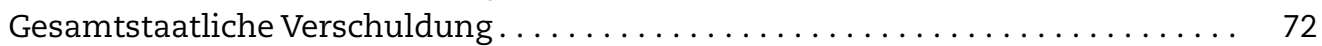

Finanzierungssaldo und Verschuldung nach staatlichen Ebenen .......... 74

Gesamtstaatliche Einnahmen. ........................ 76

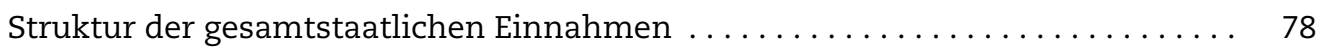

Einnahmestruktur nach staatlichen Ebenen $\ldots \ldots \ldots \ldots \ldots \ldots \ldots \ldots . \ldots \ldots$

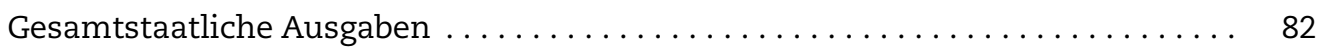

Struktur der gesamtstaatlichen Ausgaben nach Verwendungszweck (COFOG) . . 84

Ausgabenstruktur nach staatlichen Ebenen.................... 86

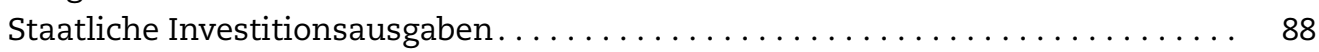

Produktionskosten des Staates und Outsourcing staatlicher Aufgaben. . . . . . . . 90

Sonderthema: Gesamtstaatliche IKT-Ausgaben. . . . . . . . . . . . . . 92

Kapitel 4 Haushaltspraxis und -verfahren ........................ 95

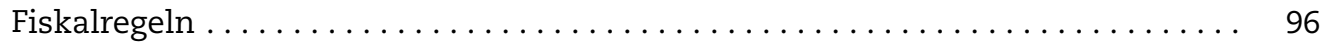

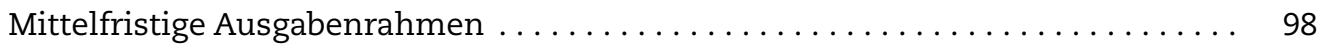

Haushaltsflexibilität der Exekutive . . . . . . . . . . . . . . . . . . . 100 
Ergebnisorientierte Budgetierung. ........................ 102

Öffentlich-private Partnerschaften . . . . . . . . . . . . . . . . . . . 104

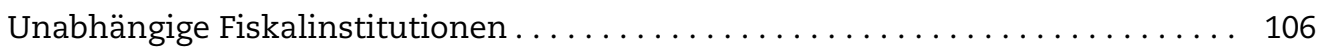

Kapitel 5 Beschäftigung und Bezahlung im öffentlichen Sektor .............. 109

Beschäftigung im Sektor Staat und in öffentlichen Unternehmen.......... . 110

Beschäftigung nach staatlichen Ebenen ..................... 112

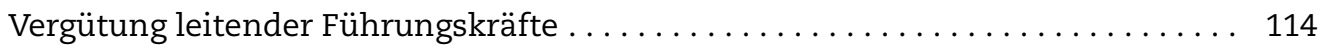

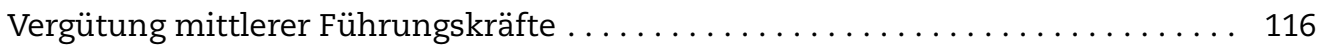

Vergütung von akademischen Fachkräften auf Ebene der Zentralregierung. . . . . 118

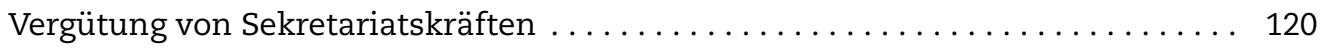

Vergütung in ausgewählten Dienstleistungsberufen $\ldots \ldots \ldots \ldots \ldots \ldots \ldots \ldots . \ldots \ldots$

Gehälter von Lehrkräften . . . . . . . . . . . . . . . . . . . . . . . . 124

Kapitel 6 Frauen in Regierung und Verwaltung. . . . . . . . . . . . . . . . . . 127

Die Beschäftigung von Frauen im Staatssektor .................... 128

Beschäftigung von Frauen auf Ebene der Zentralregierung . . . . . . . . . . 130

Frauen in leitenden Verwaltungspositionen auf Ebene der Zentralregierung . . . . . 132

Frauen in der Politik . . . . . . . . . . . . . . . . . . . . . . 134

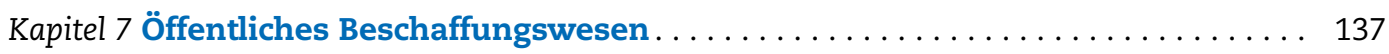

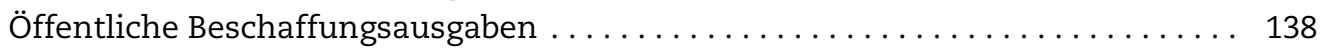

Innovative Instrumente im öffentlichen Beschaffungswesen . . . . . . . . 140

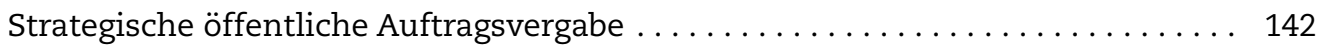

Fairer Wettbewerb im öffentlichen Beschaffungswesen und unter KMU. . . . . . . 144

Kapitel 8 Offene und inklusive Regierung $\ldots \ldots \ldots \ldots \ldots \ldots \ldots \ldots \ldots \ldots \ldots \ldots \ldots$

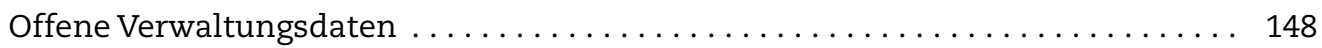

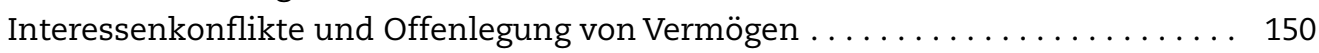

Haushaltstransparenz . . . . . . . . . . . . . . . . . . . . . 152

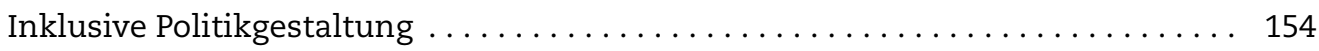

Kapitel 9 Sonderbeitrag - Im Dienste der Bürger: Zugänglichkeit und Qualität

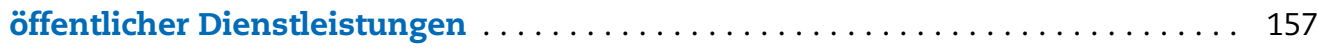

Zugang zu öffentlichen Dienstleistungen: Erschwinglichkeit ........... 158

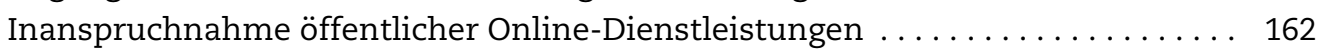

Bürgerorientierung öffentlicher Dienstleistungen: Zeitgerechtigkeit. . . . . . . 166

Verlässlichkeit öffentlicher Dienstleistungen: Sicherung der Rechte der Bürger . . 170

Zufriedenheit der Bürger mit öffentlichen Dienstleistungen. . . . . . . . . . . 174

Anhang A Methodik bezüglich der Einnahmeaggregate .................. 177

Anhang B Klassifikation der Aufgabenbereiche des Staats (COFOG) . . . . . . . . . 178

Anhang C Zusammengesetzte Indizes zur Haushaltspraxis ................ 180

Anhang D Methodik und weitere Anmerkungen zur Vergütung

der Beschäftigten im öffentlichen Dienst. . . . . . . . . . . . . . . . 184

Anhang E Detaillierte Daten über die Offenlegung von Interessenkonflikten . . . . 195

Anhang F Mitglieder des Lenkungsausschusses .................. 200

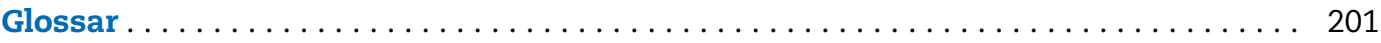




\section{Folgen Sie OECD-Veröffentlichungen auf:}

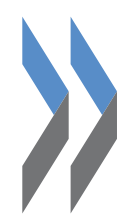

http://twitter.com/OECD_Pubs

$f$ http://www.facebook.com/OECDPublications

in. http://www.linkedin.com/groups/OECD-Publications-4645871

Youl he http://www.youtube.com/oecdilibrary

OECD

http://www.oecd.org/oecddirect/

Dieser Bericht enthält...

Suchen Sie die StatLinks rechts unter den in diesem Bericht wiedergegebenen Tabellen oder Abbildungen. Um die entsprechende Datei im Excel-Format herunterzuladen, genügt es, den jeweiligen Link, beginnend mit http://dx.doi.org, in den Internetbrowser einzugeben. 



\section{Geleitwort - Auf die Governance kommt es an!}

$\mathrm{D}$

ie Aussichten für die Weltwirtschaft verbessern sich zwar allmählich, weltweit haben die Länder jedoch weiterhin mit den Konsequenzen der globalen Finanz-, Wirtschafts- und Sozialkrise zu kämpfen. Schwaches Wachstum, hohe Staatsverschuldung, anhaltende Arbeitslosigkeit und zunehmende Ungleichheiten machen energische Korrekturmaßnahmen erforderlich. Von den Regierungen wird erwartet, dass sie unsere Volkswirtschaften wieder auf Kurs bringen, um ein stärkeres, umweltfreundlicheres und inklusiveres Wachstum zu erzielen.

Die Bürger richten den Blick auf die Regierungen, damit sie den Weg weisen. Fehlt es an einer starken, auf eine wirkungsvolle Politik gestützten Führung kann das Vertrauen der Bürger rasch schwinden. Und in der Tat wurde das Vertrauen in den Staat durch die Krise stark beeinträchtigt. Im gesamten OECD-Raum haben Bürgerinnen und Bürger das Vertrauen in die Fähigkeit der politisch Verantwortlichen verloren, wirtschaftliche Probleme zu lösen und ihren Bedürfnissen und Anforderungen gerecht zu werden. Die Regierungen müssen daher unbedingt das Vertrauen ihrer Bürger zurückgewinnen, um notwendige Reformen durchführen zu können.

Ein entscheidendes Instrument, um Vertrauen zu schaffen, sind gute Leistungen. Regierung und Verwaltung auf einen Blick 2013 liefert vor diesem Hintergrund wichtige Informationen, die es politischen Entscheidungsträgern und Bürgern gestatten, die Leistung ihrer Regierungen zu analysieren und zu vergleichen und Bereiche zu identifizieren, in denen die Effektivität und Effizienz des öffentlichen Sektors gesteigert werden können. Die fünfzig Indikatoren erstrecken sich über die gesamte Produktionskette öffentlicher Güter und Dienstleistungen (Vorleistungen, Verfahren, Ergebnisse und Wirkungen) und betreffen entscheidende Aspekte der öffentlichen Verwaltung und Governance, z.B. Haushaltspraxis, Integrität und offene Regierung, E-Government und IKT-Strategien.

Regierung und Verwaltung auf einen Blick 2013 zeigt, dass die staatlichen Instanzen zwar Schritte unternommen haben, um die Institutionen zu stärken und das Preis-LeistungsVerhältnis ihrer Arbeit zu verbessern, dass diesbezüglich aber noch immer viel zu tun bleibt. Beispielsweise muss die Lage der öffentlichen Finanzen trotz der beträchtlichen Konsolidierungsanstrengungen, die in vielen Ländern unternommen wurden, weiter verbessert werden. Zudem bestehen immer noch erhebliche Ungleichgewichte zwischen Männern und Frauen. So sind z.B. nur 40\% der Posten der mittleren Führungsebene und nur 29\% der Posten der oberen Führungsebene mit Frauen besetzt. Und obwohl offene Verwaltungsdaten (Open Government Data - OGD) als Governance-Instrument zunehmend an Bedeutung gewinnen - 56\% der OECD-Länder haben nationale Strategien für die Offenlegung von Verwaltungsdaten eingerichtet -, muss noch deutlich mehr unternommen werden, um sicherzustellen, dass die Bürger die verfügbaren Informationen auch effektiv nutzen können. Im Hinblick auf diesen und andere Bereiche müssen Reformen des öffentlichen Sektors weiterhin weit oben auf der Prioritätenliste stehen, um die Verwirklichung unserer wirtschaftlichen und sozialen Ziele zu unterstützen. 
Indem wir den Erfassungsbereich und die Aktualität unserer Governance-Indikatoren und -Analysen kontinuierlich verbessern und sie im Interesse eines einfacheren Zugangs in verschiedenen elektronischen Formaten zur Verfügung stellen, hoffen wir, dass Regierung und Verwaltung auf einen Blick 2013 einen entscheidenden Beitrag zu den Überlegungen von politisch Verantwortlichen, Bürgern und Wissenschaftlern darüber, wie eine bessere Politik für ein besseres Leben aussehen sollte, leisten kann.

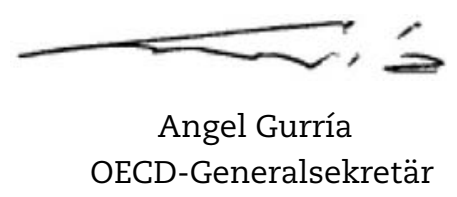




\section{Zusammenfassung}

$\mathrm{D}$ ie Finanz- und Wirtschaftskrise sowie ihre Folgen veranlassten viele Regierungen im OECD-Raum, zur Wiederherstellung solider öffentlicher Finanzen strukturelle Anpassungsprogramme umzusetzen. Das Vertrauen in den Staat hat dadurch jedoch deutlich abgenommen, da es mit den begrenzten staatlichen Mitteln schwierig wurde, den wachsenden Erwartungen der Bürgerinnen und Bürger gerecht zu werden. Zwischen 2007 und 2012 sank das den nationalen Regierungen entgegengebrachte Vertrauen im Schnitt von $45 \%$ auf $40 \%$, was die Bemühungen der nationalen staatlichen Stellen, Unterstützung für notwendige Reformen zu finden, erschwerte.

Damit die Regierungen den Erwartungen der Bürgerinnen und Bürger mit den begrenzten, ihnen zur Verfügung stehenden Mitteln gerecht werden können, bedarf es im Bereich der öffentlichen Governance eines neuen Ansatzes. Dieser sollte auf der Schaffung strategischer Kapazitäten, starker Institutionen und wirksamer Instrumente und Prozesse sowie auf eindeutig messbaren Ergebnissen aufbauen. Die in Regierung und Verwaltung auf einen Blick 2013 vorgestellten Indikatoren veranschaulichen, welche Fortschritte die OECDLänder in dieser Hinsicht erzielt haben.

Wichtigste Ergebnisse

- Trotz beträchtlicher Konsolidierungsbemühungen der Länder bleiben die Herausforderungen im Bereich der öffentlichen Finanzen bestehen. Die OECD hat Schätzungen des Umfangs der Verbesserung des strukturellen Primärsaldos vorgelegt, die erforderlich wäre, um die Bruttostaatsverschuldung bis 2030 auf 60\% des BIP zu senken. Im OECDDurchschnitt müsste eine Verbesserung um rd. 3\% des potenziellen BIP gegenüber der Haushaltsposition von 2012 erzielt werden. Mehrere OECD-Länder sind jedoch nach wie vor mit steigenden Staatsschuldenquoten konfrontiert, und im Schnitt lagen die Staatsausgaben im Jahr 2011 über den Staatseinnahmen. Dies war u.a. den Kosten der Konjunkturpakete und den stagnierenden staatlichen Einnahmen infolge der Wirtschaftskrise sowie dem Anstieg der alterungsbedingten Ausgaben zuzuschreiben.

- Die Länder haben neue Haushaltsverfahren eingeführt und neue GovernanceInstitutionen geschaffen. Veränderungen des Rahmens für die globale Wirtschaftsgovernance, die erforderlich wurden, als sich die vorhandenen Mechanismen zur Wahrung der Haushaltsdisziplin als unwirksam erwiesen, veranlassen die Länder, die gegenwärtig genutzten Instrumente zu optimieren und neue Strategien umzusetzen. So wurden inzwischen beispielsweise in 97\% der OECD-Länder Haushaltsregeln eingeführt, und die durchschnittliche Zahl dieser Regeln pro Land hat sich erhöht. Zwischen 2009 und 2013 wurden in acht Ländern unabhängige fiskalische Institutionen eingerichtet, um die Haushaltsdisziplin zu stärken, Wirtschaftsdaten bereitzustellen und eine Ressourcenallokation nach dem Prinzip der Nutzenmaximierung sicherzustellen. 
- Das öffentliche Beschäftigungsniveau ist in der Tendenz langfristig stabil. Die Beschäftigung im Staatssektor blieb mit einem Anteil von knapp unter 16\% der Gesamterwerbsbevölkerung zwischen 2001 und 2011 weitgehend konstant. Gemessen an den durchschnittlichen Ausgaben des öffentlichen Sektors, die sich im Jahr 2011 auf $45,4 \%$ des BIP beliefen, ist dies vergleichsweise wenig und veranschaulicht die zentrale Rolle des Outsourcing. Zwar haben mehrere OECD-Länder im Rahmen ihrer Haushaltskonsolidierungspläne Einstellungsstopps bzw. Stellenkürzungen angekündigt, ein deutlicher Rückgang der Beschäftigung im öffentlichen Sektor ist auf lange Sicht jedoch nur schwer zu erreichen, da die Forderungen der Bürgerinnen und Bürger laufend zunehmen.

- Es bedarf zusätzlicher Mechanismen, um die Gleichstellung der Geschlechter im öffentlichen Sektor zu gewährleisten. Die Regierungen haben eine Reihe von Maßnahmen ergriffen, um die Chancengleichheit von Arbeitnehmerinnen und Arbeitnehmern im öffentlichen Dienst sicherzustellen; beispielsweise haben sie Einstellungsund Beförderungsziele sowie Maßnahmen zur besseren Vereinbarkeit von Berufs- und Privatleben eingeführt. Die Daten zeigen jedoch, dass mehr als 50\% (in manchen Fällen fast 90\%) der Sekretariatstätigkeiten von Frauen ausgeübt werden, während Frauen in gehobeneren Positionen wesentlich weniger gut repräsentiert sind. Die Korrekturmaßnahmen zum Ausgleich solcher Disparitäten umfassen u.a. ein Gender Responsive Budgeting (GRB), also eine Haushaltsplanung unter geschlechtsspezifischen Gesichtspunkten, durch die der Frage der Gleichstellung von Mann und Frau in allen Etappen des Haushaltszyklus Rechnung getragen wird. Dadurch sollen „geschlechtsblinde Ausgaben" vermieden und die Wirksamkeit staatlicher Programme durch die Identifizierung von in geschlechtsspezifischer Hinsicht unangemessenen Folgen der Mittelverwendung erhöht werden. Eine solche Haushaltsplanung wurde jedoch in weniger als der Hälfte der OECD-Länder eingeführt.

- Das öffentliche Auftragswesen wird von den Ländern strategischer eingesetzt. Viele OECD-Länder nutzen innovative Vergabeinstrumente, um Skaleneffekte zu erzielen $(94 \%$ etwa nutzen Rahmenvereinbarungen), strukturieren die Beschaffungsabteilungen neu, konsolidieren die Beschaffung und machen im Beschaffungsprozess von Informationsund Kommunikationstechnologien (IKT) Gebrauch (97\% nutzen nationale E-Procurement-Systeme für öffentliche Ausschreibungen). Außerdem setzen zahlreiche OECD-Länder die öffentliche Beschaffungspolitik nicht nur zur Optimierung des KostenNutzen-Verhältnisses ein, sondern verfolgen damit auch andere Zielsetzungen wie Innovation, nachhaltiges Wirtschaftswachstum (73\% fördern ein umweltfreundliches Beschaffungswesen), KMU-Förderung (70\% fördern die Auftragsvergabe an KMU) und faire Rahmenbedingungen beim Zugang zu wirtschaftlichen Möglichkeiten.

- Die Offenlegung der Vermögensverhältnisse und Privatinteressen von Entscheidungsträgern bleibt ein wichtiges Instrument bei der Behandlung von Interessenkonflikten. In fast allen Ländern sind die Entscheidungsträger verpflichtet, ihre Vermögensverhältnisse und Einkommensquellen offenzulegen. Eine Offenlegung von vorherigen Beschäftigungsverhältnissen und Verbindlichkeiten ist indes nur in wenigen Ländern erforderlich.

- Offene Verwaltungsdaten (Open Government Data - OGD) entwickeln sich zunehmend zu einem wichtigen Instrument im Bereich der Governance. Knapp über die Hälfte der OECD-Länder verfügt über eine nationale Strategie zur Bereitstellung offener Verwaltungsdaten für Bürgerinnen und Bürger. 12\% der Länder gaben an, dass in den einzelnen Fachministerien gesonderte OGD-Strategien verfolgt werden, und $28 \%$ haben 
sowohl auf nationaler als auch auf regionaler Ebene diesbezügliche Strategien entwickelt. Als wichtigste Prioritäten im Zusammenhang mit offenen Verwaltungsdaten werden Transparenz, Offenheit, Steigerungen des Geschäftsvolumens im privaten Sektor sowie Unternehmensneugründungen genannt. Darüber hinaus sind sich die Länder des Potenzials offener Verwaltungsdaten im Hinblick auf eine Verbesserung der Dienstleistungserbringung bewusst. Die potenziellen Auswirkungen auf die Beteiligung der Bürger an öffentlichen Debatten und am Entscheidungsprozess gehören allerdings nicht zu den wichtigsten Prioritäten.

- Die Bürgerinnen und Bürger haben größeres Vertrauen in die öffentlichen Dienstleistungen, die sie in Anspruch nehmen, als in das abstrakte Konzept Staat. Obwohl das Vertrauen in den „Staat" sinkt, sind die Bürgerinnen und Bürger eigenen Angaben zufolge mit den öffentlichen Dienstleistungen zufrieden. So gaben etwa $72 \%$ an, Vertrauen in die örtliche Polizei zu haben. Fast der gleiche Prozentsatz betrachtete das Gesundheitsversorgungsangebot als zufriedenstellend, und $66 \%$ äußerten sich zufrieden mit dem Bildungssystem und den Schulen in ihrer Stadt oder Gegend. Im Schnitt blieb der Grad der Zufriedenheit während und unmittelbar nach der weltweiten Finanz- und Wirtschaftskrise weitgehend unverändert.

- Den Regierungen der OECD-Länder ist es in zunehmendem Maße ein Anliegen, hochwertige öffentliche Güter und Dienstleistungen für ein breites Spektrum der Bevölkerung bereitzustellen. Viele Länder führen Leistungsstandards für die Erbringung von Dienstleistungen ein und greifen dabei auf Mechanismen zurück, durch die von der Bevölkerung Feedback eingeholt und in den Prozess integriert werden kann. In dieser Ausgabe von Regierung und Verwaltung auf einen Blick werden erstmals vier Aspekte der Dienstleistungsqualität - Erschwinglichkeit, Bürgerorientierung, Zuverlässigkeit und Bürgerzufriedenheit - verglichen, und zwar nicht nur zwischen den Ländern, sondern auch zwischen den wichtigen öffentlichen Dienstleistungsbereichen Bildung, Gesundheitswesen, Justiz und Steuerverwaltung. 



\section{Hinweise für den Leser}

$\mathrm{U}_{\mathrm{m}}$ $m$ die in Regierung und Verwaltung auf einen Blick enthaltenen Informationen richtig interpretieren zu können, müssen die Leser mit einigen methodischen Konzepten vertraut sein, die für eine ganze Reihe von Indikatoren Anwendung finden. Wie in den vergangenen Ausgaben werden diese Indikatoren jeweils auf zwei Seiten präsentiert. Ein paar Indikatoren - z.B. zur Rechtsstaatlichkeit und zur Qualität öffentlicher Dienstleistungen werden allerdings auf vier Seiten dargelegt. Bei den auf zwei Seiten dargestellten Indikatoren wird auf der ersten Seite die Bedeutung des Themas erläutert und auf die wichtigsten Unterschiede hingewiesen, die im OECD-Vergleich festzustellen sind. Im Anschluss daran werden im Abschnitt „Methodik und Definitionen“ die Datenquellen genannt und wichtige Informationen für die Interpretation der Daten gegeben. Am Ende der ersten Seite finden sich nützliche Hinweise auf weiterführende Literatur mit Hintergrundinformationen zu den vorgestellten Daten. Auf der zweiten Seite sind die Daten in Schaubildern dargestellt. Dabei handelt es sich um Abbildungen zum derzeitigen Stand sowie zu den Trends im Zeitverlauf, falls entsprechende Daten vorliegen. Am Ende der Publikation befindet sich ein Glossar zu wichtigen im Text verwendeten Definitionen.

\section{Kalenderjahr/Finanzjahr in den Daten aus den Volkswirtschaftlichen Gesamtrechnungen}

Sofern nicht anders angegeben, beziehen sich die Angaben aus den Volkswirtschaftlichen Gesamtrechnungen der OECD-Länder auf Kalenderjahre.

Die Daten für Australien und Neuseeland beziehen sich auf Finanzjahre. In Australien beginnt das Finanzjahr am 1. Juli des genannten Jahres und endet am 30. Juni, in Neuseeland beginnt es am 1. April des genannten Jahres und endet am 31. März. Für Japan beziehen sich die Daten zu den Unterbereichen des Staatssektors und den Ausgaben nach Verwendungszweck (COFOG - Classification of the Functions of Government) auf Finanzjahre.

Die auf dem System der Volkwirtschaftlichen Gesamtrechnungen (SNA) beruhenden Daten stammen aus der OECD-Datenbank OECD National Accounts Statistics, Stand 12. August 2013.

\section{Berücksichtigte Länder}

Regierung und Verwaltung auf einen Blick 2013 enthält je nach Verfügbarkeit Daten zu allen 34 OECD-Ländern. Die statistischen Daten für Israel wurden von den zuständigen israelischen Stellen bereitgestellt, die für sie verantwortlich zeichnen. Die Verwendung dieser Daten durch die OECD erfolgt unbeschadet des völkerrechtlichen Status der Golanhöhen, von Ost-Jerusalem und der israelischen Siedlungen im Westjordanland.

Einige weitere Länder, z.B. die Russische Föderation (die derzeit in Beitrittsverhandlungen mit der OECD steht), sowie Länder, die im Ausschuss für öffentliche Governance der OECD 
einen Teilnehmerstatus haben (Brasilien, Ägypten, Südafrika, Ukraine), steuerten zu einigen Indikatoren ebenfalls Daten bei. Die Daten für Nichtmitgliedsländer sind gesondert am Ende der Tabellen und Abbildungen aufgeführt.

\section{Ländercodes (ISO-Codes)}

\begin{tabular}{|c|c|c|c|}
\hline OECD-Mitgliedsländer & & Polen & POL \\
\hline Australien & AUS & Portugal & PRT \\
\hline Österreich & AUT & Slowakische Republik & SVK \\
\hline Belgien & BEL & Slowenien & SVN \\
\hline Kanada & CAN & Spanien & ESP \\
\hline Chile & $\mathrm{CHL}$ & Schweden & SWE \\
\hline Tschechische Republik & CZE & Schweiz & $\mathrm{CHE}$ \\
\hline Dänemark & DNK & Türkei & TUR \\
\hline Estland & EST & Vereinigtes Königreich & GBR \\
\hline Finnland & FIN & Vereinigte Staaten & USA \\
\hline Frankreich & FRA & & \\
\hline Deutschland & DEU & OECD-Beitrittsland & \\
\hline Griechenland & GRC & Russische Föderation & RUS \\
\hline Ungarn & HUN & & \\
\hline Island & ISL & Sonstige große Volkswirtschaften & \\
\hline Irland & IRL & Brasilien (nimmt am OECD-Ausschuss für öffentliche Governance teil) & BRA \\
\hline Israel & ISR & China & $\mathrm{CHN}$ \\
\hline Italien & ITA & Indien & IND \\
\hline Japan & JPN & Indonesien & IDN \\
\hline Korea & KOR & Südafrika (nimmt am OECD-Ausschuss für öffentliche Governance teil) & ZAF \\
\hline Luxemburg & LUX & & \\
\hline Mexiko & MEX & Weitere Teilnehmerländer des OECD-Ausschusses für öffentliche Governance & \\
\hline Niederlande & NLD & Ägypten & EGY \\
\hline Neuseeland & NZL & Ukraine & UKR \\
\hline Norwegen & NOR & & \\
\hline
\end{tabular}

\section{OECD-Durchschnittswerte und OECD-Gesamtwerte}

\section{Durchschnittswerte}

Die Durchschnittswerte in den Abbildungen und im Text beziehen sich auf das ungewichtete arithmetische Mittel der OECD-Länder, für die Daten verfügbar sind. Daten für Nichtmitgliedsländer sind nicht berücksichtigt. In den Anmerkungen sind die OECDLänder genannt, für die jeweils keine Daten vorliegen.

Wenn in einer Abbildung Daten zu einem oder mehreren Jahren dargestellt sind, umfasst der OECD-Durchschnitt alle Mitgliedsländer, für die Daten verfügbar sind. Ein OECD-Durchschnittswert für das Jahr 2009 beinhaltet beispielsweise alle derzeitigen OECDLänder, für die Daten aus diesem Jahr vorliegen, selbst wenn einige dieser Länder 2009 noch nicht Mitglied der OECD waren.

\section{Gesamtwerte}

OECD-Gesamtwerte sind vor allem in Tabellen zu finden und entsprechen der Summe der Werte in der jeweiligen Spalte für die Länder, für die Daten verfügbar sind. Daten für Nichtmitgliedsländer sind nicht berücksichtigt. In den Anmerkungen sind die OECDLänder genannt, für die jeweils keine Daten vorliegen. 


\section{Online-Ergänzungen}

$\mathrm{Zu}$ einigen Indikatoren sind online zusätzliche Tabellen und Abbildungen mit länderspezifischen Daten verfügbar. Auf diese Tabellen und Abbildungen wird im Abschnitt „Methodik und Definitionen“ des jeweiligen Indikators hingewiesen. Regierung und Verwaltung auf einen Blick 2013 gestattet auch den Zugriff auf StatLinks, über die die Leser die entsprechenden Excel-Dateien herunterladen können. Die StatLinks befinden sich unten rechts von den Tabellen oder Abbildungen und können in einen Web-Browser eingegeben werden. In elektronischen Ausgaben können sie einfach angeklickt werden.

Außerdem sind folgende zusätzliche Unterlagen online verfügbar unter www.oecd.org/ gov/govataglance.htm:

- Kurzdarstellungen zu den einzelnen Ländern mit wichtigen Daten, die mit OECDDurchschnittswerten verglichen werden (Country Fact Sheets).

- Statistische Datenbank zu Regierung und Verwaltung auf einen Blick mit regelmäßig aktualisierten Daten zu einer Auswahl von Indikatoren (über OECD.Stat).

- Länderspezifische Kontextinformationen zu wichtigen Merkmalen der politischen und administrativen Struktur der verschiedenen Mitgliedsländer (Contextual Factors).

\section{Pro-Kopf-Werte}

Einige Indikatoren (z.B. zu den Ausgaben und Einnahmen und zur Staatsverschuldung) sind auf Pro-Kopf-Basis, d.h. im Verhältnis zur Einwohnerzahl angegeben. Die zu Grunde liegenden Bevölkerungsschätzungen basieren auf dem im System der Volkswirtschaftlichen Gesamtrechnungen verwendeten Wohnsitz-Konzept. Sie umfassen - unabhängig von ihrer Staatsangehörigkeit - Personen, die mindestens ein Jahr im betreffenden Land ansässig sind. Erfasst werden dabei auch Bedienstete ausländischer diplomatischer Vertretungen und Mitglieder der Streitkräfte und deren Familien sowie Studierende, die im Ausland studieren, und Patienten, die sich im Ausland behandeln lassen, selbst wenn ihr Auslandsaufenthalt länger als ein Jahr dauert. Die Einjahresregel bedeutet, dass Gebietsansässige, die weniger als ein Jahr in einem anderen Land leben, in der Einwohnerzahl erfasst werden, während ausländische Besucher (z.B. Urlauber), die sich weniger als ein Jahr im betreffenden Land aufhalten, nicht berücksichtigt werden. In diesem Zusammenhang gilt es darauf hinzuweisen, dass ein und dieselben Personen in einem Land als abhängig Beschäftigte (die über die Produktion zum Bruttoinlandsprodukt dieses Landes beitragen), in einem anderen Land jedoch als Gebietsansässige (deren Löhne und Gehälter in das Bruttonationaleinkommen ihres Wohnsitzlandes eingehen) erfasst sein können.

\section{Kaufkraftparitäten}

Kaufkraftparitäten (KKP) sind Umrechnungssätze, die eine Äquivalisierung der Kaufkraft der verschiedenen Länder ermöglichen, indem zwischen den Ländern bestehende Unterschiede beim Preisniveau herausgerechnet werden. Wenn Ausgaben anhand von Kaufkraftparitäten umgerechnet werden, können sie in verschiedenen Ländern bezogen auf einen einheitlichen Katalog von Preisen ausgedrückt werden, was bedeutet, dass ein vergleichbarer Korb von Waren und Dienstleistungen in beiden Ländern gleich viel kostet. So wird es möglich, Vergleiche zwischen den Ländern anzustellen, in denen nur die Unterschiede in der Menge der eingekauften Waren und Dienstleistungen zum Ausdruck kommen. 
2001 und 2009: Bei den KKP für alle europäischen Länder handelt es sich um jährliche, von Eurostat zur Verfügung gestellte Benchmark-Ergebnisse. Bei den KKP für die nichteuropäischen Länder und die Russische Föderation handelt es sich um OECDSchätzungen.

2011: Bei den KKP für alle Mitgliedsländer sowie die Russische Föderation handelt es sich um vorläufige Benchmark-Ergebnisse, die von der OECD errechnet wurden. Bei der Interpretation von Schätzungen und vorläufigen Ergebnissen ist Vorsicht geboten, da sie u.U. noch revidiert werden müssen.

Weitere Informationen sind auf der OECD-Internetseite zu den Kaufkraftparitäten zu finden: www.oecd.org/std/prices-ppp.

\section{Zusammengesetzte Indikatoren}

Diese Publikation enthält mehrere deskriptive, zusammengesetzte Indizes für eng definierte Bereiche im Zusammenhang mit Interessenkonflikten sowie Haushaltspraxis und -verfahren. Solche zusammengesetzten Indizes sind ein zweckmäßiges Instrument zur Zusammenfassung diskreter qualitativer Informationen. Die in dieser Publikation vorgestellten zusammengesetzten Indizes wurden gemäß den Anleitungen im Handbook on Constructing Composite Indicators (Nardo et al., 2008) erstellt.

Einzelheiten zu den Variablen und Gewichtungen, die zur Erstellung der zusammengesetzten Indizes zu den Themen Haushaltspraxis und -verfahren sowie Interessenkonflikte verwendet wurden, können Anhang C und Anhang E entnommen werden. Während die zusammengesetzten Indikatoren in Kooperation mit Mitgliedsländern entwickelt wurden und auf theoretischen Erkenntnissen und/oder besten Verfahrensweisen beruhen, basieren die Variablen, aus denen sich die Indizes zusammensetzen, sowie deren Gewichtungen auf Expertenbeurteilungen und können sich daher im Zeitverlauf ändern. Die zusammengesetzten Indizes zu Haushaltspraxis und -verfahren sind nicht mit denen der Ausgabe 2009 von Regierung und Verwaltung auf einen Blick vergleichbar, da die Fragen in der letzten Erhebungsrunde des Survey of Budget Practices and Procedures und des Survey on Performance-Budgeting (2012) teilweise etwas anders formuliert waren als 2007. Außerdem wurden zusätzliche Fragen aufgenommen, und einige der Gewichtungen wurden neu definiert.

\section{Zeichen und Abkürzungen}

.. Fehlende Daten oder kein Nachweis vorhanden

$\mathrm{x} \quad$ Nicht anwendbar

EUR Euro

US-\$ US-Dollar

p.p. Prozentpunkte 


\section{Einführung}

Ziele

Die Wirtschaftskrise der jüngsten Zeit hat deutlich gemacht, welche Bedeutung dem Staat als wichtigem Akteur moderner Gesellschaften zukommt. Vom Staat wird erwartet, dass er die erforderlichen Rahmenbedingungen für ein Wirtschaftswachstum schafft, das die Lebensqualität seiner Bürger erhöht; außerdem muss er im Interesse des Gemeinwohls Verhaltensregeln für Unternehmen und Bürger aufstellen, eine Einkommensumverteilung zur Verbesserung der sozialen Gerechtigkeit vornehmen, der Bevölkerung öffentliche Güter und Dienstleistungen zur Verfügung stellen und dabei zugleich Haushaltszwängen und demografischen Belastungen Rechnung tragen. Die Fähigkeit des Staats, effektiv und effizient zu handeln, hängt u.a. von seiner Politik und seinen Verfahrensweisen in so verschiedenen Bereichen wie Budgetierung, Personalverwaltung und Beschaffungswesen ab.

Das Hauptziel der Publikationsreihe Regierung und Verwaltung auf einen Blick besteht darin, verlässliche und international vergleichbare Daten zu den staatlichen Aktivitäten und ihren Ergebnissen in den OECD-Ländern sowie einigen anderen Ländern zu liefern. Diese Daten können dann von den einzelnen Ländern genutzt werden, um die Leistung ihrer staatlichen Stellen mit der anderer Länder zu vergleichen, die Entwicklungen im eigenen Land sowie auf internationaler Ebene im Zeitverlauf zu betrachten und Entscheidungen zur Politikgestaltung ausgehend von einer soliden Faktengrundlage treffen zu können.

Die Indikatoren von Regierung auf einen Blick werden zunehmend zum Standard für die Leistungsmessung in vielen Bereichen der Staats- und Verwaltungsführung. Zusätzlich zu den Kernindikatoren, die das Markenzeichen dieser Publikation sind, enthält diese dritte Ausgabe eine Auswahl neuer Indikatoren mit zusätzlichen Datenquellen, die es ermöglichen, ein umfassenderes Bild der öffentlichen Verwaltung in den OECDMitgliedsländern zu zeichnen.

\section{Was ist neu in Regierung und Verwaltung 2013?}

Im Vergleich zu Regierung und Verwaltung auf einen Blick 2011 bringt die Ausgabe 2013 mehrere neue Elemente. Zunächst einmal enthält sie drei neue Kapitel: Kapitel 2 "Strategische Governance“, das Elemente der vorangegangenen Ausgaben zusammenbringt, aber auch neue Indikatoren zum Vertrauen in die Institutionen, zum Risikomanagement und zur Rechtsstaatlichkeit umfasst, Kapitel 6 „Frauen in Regierung und Verwaltung“, wo auf die Rolle der Frauen in allen staatlichen Bereichen, in der öffentlichen Verwaltung, der Justiz und im Parlament eingegangen wird, und Kapitel 9 „Sonderbeitrag - Im Dienste der Bürger: Zugänglichkeit und Qualität öffentlicher Dienstleistungen“, das sich auf ein neues Rahmenkonzept zur Beurteilung der Dienstleistungsqualität stützt (Zugänglichkeit, Bürgerorientierung, Zuverlässigkeit und Bürgerzufriedenheit). Diese Qualitätsaspekte werden ausgehend von einer Reihe entscheidender Merkmale für die Bereiche Bildung, Gesundheitsversorgung, Justiz und Steuerverwaltung gemessen. 
Die Daten zu den öffentlichen Finanzen sind für 2001, 2009 und 2011 angegeben, stellvertretend für die Zeit vor, während und nach der Wirtschaftskrise. Die neuen Indikatoren zu Schuldenstand, Finanzierungssalden und Investitionen sind nach staatlichen Ebenen aufgeschlüsselt, um einen genaueren Eindruck von der Fiskalstruktur der verschiedenen Mitgliedsländer und der Verteilung der Zuständigkeiten auf Gliedstaaten und Kommunen zu vermitteln.

Die Umfragen zu Haushaltspraxis und -verfahren, ergebnisorientierter Budgetierung, öffentlicher Beschaffung und Personalvergütungen, die 2012 durchgeführt wurden, gestatteten die Aufnahme neuer Indikatorenreihen zu diesen Themen. Mit den neuen Fassungen dieser Fragebogen konnten genauere und zuverlässigere Informationen gesammelt werden. In der Erhebung zu den Personalvergütungen wurden z.B. zum ersten Mal Daten zu wichtigen Dienstleistungsberufen gesammelt, die einen Vergleich von Vergütungsniveau und -strukturen für Polizeikommissare und Kriminalbeamte, Polizisten, Beamte der Grenzpolizei, Zollbeamte und Steuerprüfer in den verschiedenen OECDLändern gestatten. Als Sonderbeitrag enthält diese Ausgabe zudem einen Indikator zu Ausgaben für Informations- und Kommunikationstechnologien (IKT).

\section{Definition des Sektors Staat}

Die Daten zu den öffentlichen Finanzen basieren auf der Definition des Sektors „Staat “ gemäß dem System der Volkswirtschaftlichen Gesamtrechnungen (SNA). Dieser Definition zufolge setzt sich der Sektor Staat zusammen aus den Ministerien, Behörden und Ämtern (sowie teilweise auch Organisationen ohne Erwerbszweck) auf Ebene der Zentralregierung, der Gliedstaaten und der Gemeinden sowie den Sozialversicherungsträgern. Die Daten zu Einnahmen und Ausgaben werden für die zentrale Ebene und die nachgeordneten Ebenen, d.h. Gliedstaaten und Gemeinden, sowie gegebenenfalls auch für die Sozialversicherungsträger präsentiert. Die Daten zur Beschäftigung beziehen sich allerdings auf den öffentlichen Sektor, d.h. den Sektor Staat zuzüglich der öffentlichen Unternehmen, z.B. staatseigene Banken, Häfen und Flughäfen. Die Daten zu den Regeln und Verfahren der öffentlichen Verwaltung beziehen sich ausschließlich auf die zentrale Ebene.

\section{Rahmenkonzept}

Regierung und Verwaltung auf einen Blick enthält Angaben zu den 34 Mitgliedsländern sowie - falls entsprechende Daten verfügbar sind - zu Beitrittsländern, z.B. der Russischen Föderation, und anderen großen Volkswirtschaften wie China, Indien, Indonesien und Südafrika. Für einige Indikatoren wurden auch Daten aus Teilnehmerländern des Ausschusses für öffentliche Verwaltung aufgenommen (Brasilien, Ägypten, Südafrika und Ukraine). Diese verschiedenen Länder spielen eine große und zunehmend wichtige Rolle in der Weltwirtschaft und den internationalen Politikstrukturen.

Diese dritte Ausgabe von Regierung und Verwaltung auf einen Blick enthält Kontext-, Input-, Verfahrens- sowie Ergebnis- und Wirkungsindikatoren. Sie enthält einen umfassenderen Katalog von Indikatoren $\mathrm{zu}$ wichtigen Aspekten der Leistungsfähigkeit des Staats in ausgewählten Sektoren, die an Ergebnissen (Outputs) und Wirkungen (Outcomes) gemessen wird; dabei wird erstmalig auch auf das Justizsystem sowie auf die Qualitätskomponente öffentlicher Dienstleistungen - in den Bereichen Gesundheitsversorgung, Bildung, Justiz und Steuerverwaltung - eingegangen. Abbildung 0.1 liefert einen Überblick über das Rahmenkonzept von Regierung und Verwaltung auf einen Blick. 
Abbildung 0.1. Rahmenkonzept von Regierung und Verwaltung auf einen Blick 2013

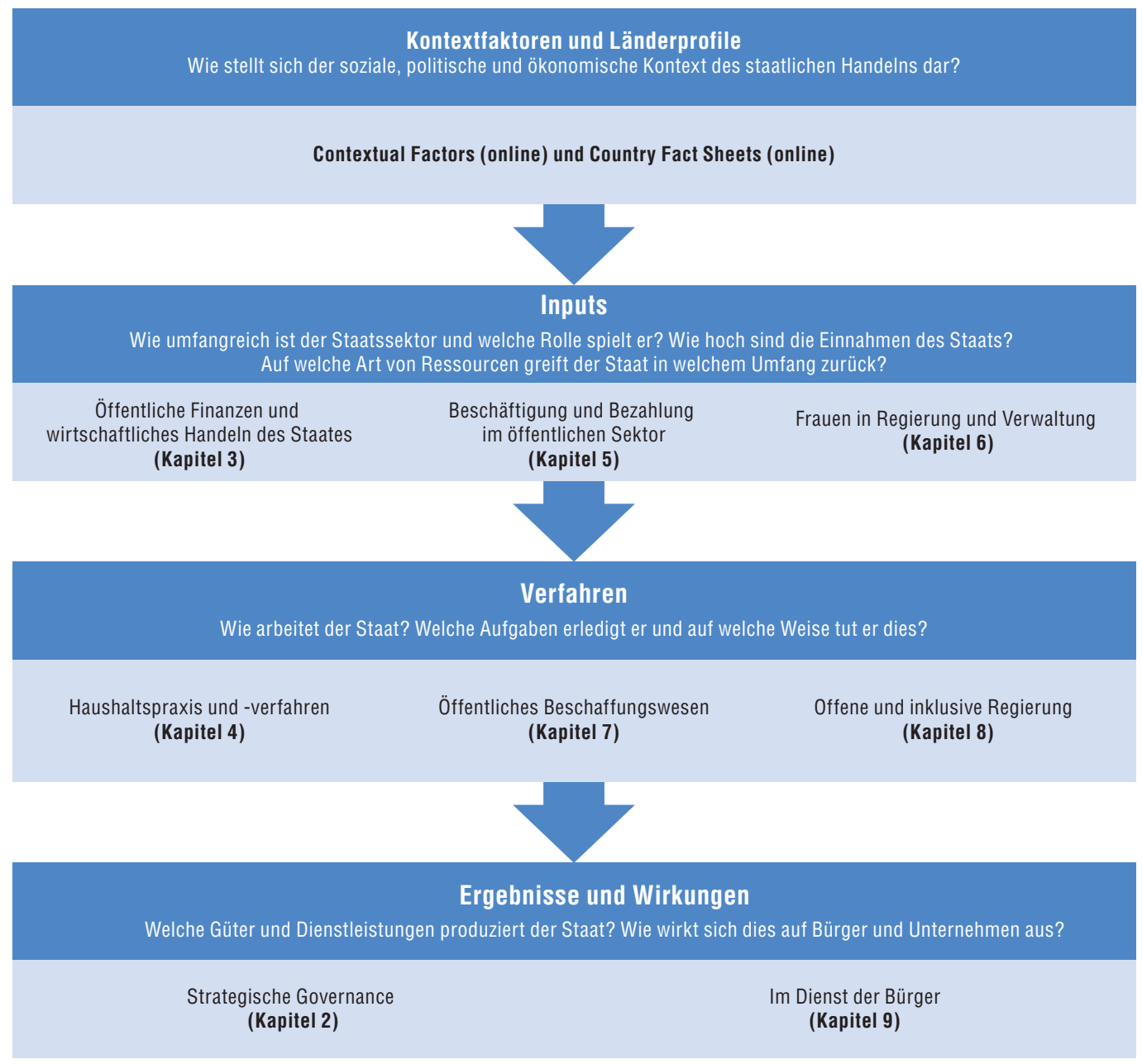

Inputs

Das Konzept der „Inputs“ bezieht sich auf die Ressourcen, die der Staat zur Ausübung seiner Produktionsfunktion einsetzt, sowie auf die Art und Weise, wie diese Ressourcen bzw. Produktionsfaktoren - es handelt sich um Arbeit und Kapital - kombiniert werden. Die Kapitel, die sich mit diesen Inputs befassen, sind „Öffentliche Finanzen und wirtschaftliches Handeln des Staates“, „Beschäftigung und Bezahlung im öffentlichen Sektor" sowie „Frauen in Regierung und Verwaltung“. Diese Kapitel enthalten Indikatoren zu Staatsausgaben, Produktionskosten, Beschäftigung und Beschäftigten. Durch die Differenzierung dieser Indikatoren lassen sich Unterschiede bei der Kapazität staatlicher Stellen zur Produktion und Bereitstellung öffentlicher Güter für die Bevölkerung leichter analysieren.

\section{Verfahren}

Bei den „Verfahren“ handelt es sich um die öffentlichen Verwaltungsverfahren sowie die von den staatlichen Stellen gewählten Vorgehensweisen zur Politikumsetzung. Dabei geht es unmittelbar um die Mittel, die öffentliche Verwaltungen einsetzen, um ihre Aufgaben zu erfüllen und ihre Ziele zu erreichen. Daher sind diese Verfahren oft von entscheidender Bedeutung, um Rechtsstaatlichkeit und Fairness zu gewährleisten, Rechenschaft zu legen und dem Grundsatz der Offenheit staatlichen Handelns zu 
genügen. Reformen des öffentlichen Sektors zielen üblicherweise auf eine Verbesserung der Verfahren ab, weshalb sie in der Öffentlichkeit besonders große Aufmerksamkeit finden. Diese Ausgabe enthält diesbezügliche Informationen zu Haushaltspraxis und -verfahren, öffentlicher Beschaffung sowie zum Thema offene und inklusive Regierung.

\section{Ergebnisse und Wirkungen}

Die begriffliche Abgrenzung zwischen „Ergebnissen“ - Outputs - und „Wirkungen“ Outcomes - ist nicht immer ganz eindeutig; die Ergebnisse beziehen sich auf die Menge der Güter und Dienstleistungen, die vom Staat produziert werden, während es bei den Wirkungen um die Effekte geht, die staatliche Maßnahmen und Vorgehensweisen für Bürger und Unternehmen haben. Der Erfolg einer Politikmaßnahme sollte zunächst anhand der Ergebnisse gemessen werden, die mit ihr erzielt werden; um ein abschließendes Urteil über ihren Erfolg zu fällen, müssen jedoch die Wirkungen untersucht werden. In dieser Ausgabe wurde versucht, mehr Indikatoren zu Ergebnissen und Wirkungen aufzunehmen. Angesichts der Schwierigkeiten, die bei der Messung von Wirkungen auftreten, wurde als Instrument hierfür das vorstehend erwähnte Rahmenkonzept zur Messung der Qualität entwickelt, mit dem die verschiedenen Aspekte evaluiert werden sollen, die bei der Erbringung staatlicher Dienstleistungen für die Bürger ins Spiel kommen. Beispiele der entsprechenden Indikatoren sind im „Sonderbeitrag - Im Dienste der Bürger: Zugänglichkeit und Qualität öffentlicher Dienstleistungen“ (Kapitel 9) zu finden.

\section{Aufbau der Publikation}

Regierung und Verwaltung auf einen Blick 2013 ist wie folgt aufgebaut: Die Publikation beginnt mit einem einführenden Politikkapitel, das sich mit dem Thema Vertrauen in Staat und Regierung befasst, wobei auf die derzeitige Situation sowie die Herausforderungen eingegangen wird, die auf die OECD-Länder in diesem Bereich zukommen werden.

Die Kapitel 2 bis 8 setzen sich mit den folgenden Aspekten öffentlicher Verwaltung auseinander: „Strategische Governance“, „Öffentliche Finanzen und wirtschaftliches Handeln des Staates“, „Haushaltspraxis und -verfahren“, „Beschäftigung und Bezahlung im öffentlichen Sektor“, „Frauen in Regierung und Verwaltung“, „Öffentliches Beschaffungswesen" und „Offene und inklusive Regierung“; zusätzlich gibt es ein Sonderkapitel (Kapitel 9) mit dem Titel „Sonderbeitrag - Im Dienste der Bürger: Zugänglichkeit und Qualität öffentlicher Dienstleistungen“. Diese verschiedenen Kapitel machen zudem deutlich, dass wir die Evidenzgrundlage zur Beurteilung von Wirkung und Nutzen der verschiedenen gewählten Instrumente der öffentlichen Verwaltung weiter verbessern müssen. Die Publikation schließt mit einem Glossar sowie mehreren Anhängen zu Fragen der Methodik.

\section{Künftige Herausforderungen}

Für die Publikation Regierung und Verwaltung auf einen Blick arbeitet die OECD eng mit anderen Organisationen zusammen - darunter die Internationale Arbeitsorganisation (ILO), das World Justice Project, die Europäische Kommission für die Wirksamkeit der Justiz (European Commission for the Efficiency of Justice - CEPEJ), Gallup sowie die Europäische Kommission -, um so einen umfassenden Überblick über das staatliche Handeln und die Methoden staatlichen Handelns bieten zu können und die Doppelerfassung von Daten zu vermeiden. Diese Zusammenarbeit gewährleistet, dass die Daten zwischen den in der Publikation behandelten Ländern vergleichbar sind. 
Für künftige Ausgaben der Publikation plant das Team von Regierung und Verwaltung auf einen Blick Arbeiten in folgenden Bereichen:

- Kartierung der verschiedenen staatlichen Stellen und ihrer Merkmale.

- Erfassung neuer Daten zu Methoden des Regulierungsmanagements und ihrer Leistungsfähigkeit.

- Indikatoren zum Aufbau, zu den Aufgaben, Befugnissen, Zuständigkeiten und Prioritäten der Regierungszentralen (Centres of Government), d.h. der Stellen, die die gemeinsame Arbeit der Exekutive und des Premierministers bzw. Staatspräsidenten unterstützen.

- Vergleich von Vergütungsniveau und -strukturen im öffentlichen und privaten Sektor (steht noch nicht fest).

- Neue Indikatoren zu Lobbying und Parteienfinanzierung.

- Neuauflage der Umfrage zum strategischen Personalmanagement. 



\section{Vertrauen in den Staat, Wirksamkeit des staatlichen Handelns und die Governance-Agenda}

Die statistischen Daten für Israel wurden von den zuständigen israelischen Stellen bereitgestellt, die für sie verantwortlich zeichnen. Die Verwendung dieser Daten durch die OECD erfolgt unbeschadet des völkerrechtlichen Status der Golanhöhen, von Ost-Jerusalem und der israelischen Siedlungen im Westjordanland. 


\section{Einführung}

Die Finanz- und Wirtschaftskrise, die 2008 begann, führte zu einem erheblichen Verlust an Vertrauen in den Staat. 2012 sahen im Durchschnitt nur vier von zehn Personen in den OECD-Mitgliedsländern ihre Regierungen als vertrauenswürdig an. Auf der Suche nach einem Weg zur wirtschaftlichen Erholung besteht die Herausforderung, der sich die Regierungen gegenübersehen, nicht nur in der Auswahl geeigneter Politikmaßnahmen, sondern auch in der Umsetzung dieser Maßnahmen. Die Fähigkeit zur Umsetzung ist indessen in entscheidendem Maße vom Vertrauen abhängig. Ohne Vertrauen in den Staat, die Märkte und die Institutionen ist es schwierig, Unterstützung für die notwendigen Reformen zu mobilisieren, insbesondere dann, wenn diese kurzfristig mit Opfern verbunden sind und der langfristige Nutzen weniger greifbar ist. Der starke Verlust des Vertrauens in den Staat macht deutlich, dass Vertrauen ein entscheidendes, aber oftmals vernachlässigtes Element erfolgreicher Politikgestaltung ist.

Sinkt das Vertrauen, so führt dies u.U. dazu, dass Regeln und Rechtsvorschriften seltener eingehalten werden. Die Risikoaversion von Bürgern und Unternehmen kann darüber hinaus zunehmen, was zu Verzögerungen bei Investitionen, Innovation und Beschäftigungsentscheidungen führt, die von entscheidender Bedeutung sind, um die Wettbewerbsfähigkeit wiederherzustellen und das Wachstum wieder in Schwung zu bringen. Vertrauensfördernde Maßnahmen stellen daher eine Investition in die wirtschaftliche Erholung und das soziale Wohlergehen in der Zukunft dar. Vertrauen ist sowohl Input für Reformen des öffentlichen Sektors, der für die Umsetzung entsprechender Reformen unerlässlich ist, als auch Ergebnis solcher Reformen, da diese die für das wirtschaftliche und soziale Wohlergehen relevanten Einstellungen und Entscheidungen der Bürger und Unternehmen beeinflussen. Infolgedessen ist das Vertrauen der Bürger und Unternehmen in den Staat für eine effektive und effiziente Politikgestaltung von grundlegender Bedeutung - sowohl in guten als auch in schlechten Zeiten. Vertrauensbildende Maßnahmen sollten als neuer und zentraler Ansatz zur Wiederherstellung des Wirtschaftswachstums und zur Stärkung des sozialen Zusammenhalts betrachtet werden und das Signal aussenden, dass die Regierungen Lehren aus der Krise ziehen.

Die Herausforderung, das Vertrauen zu bewahren, wird durch den rascheren und vielfältigeren Informationsfluss in der Gesellschaft verkompliziert, wie er z.B. durch die Zivilgesellschaft, das Internet und die sozialen Netzwerke erfolgt. Diese Umstände lassen darauf schließen, dass das Umfeld komplexer geworden ist, in dem der Staat das Vertrauen der Beteiligten aufrechterhalten muss. In diesem Umfeld sind eine gute Politikgestaltung und eine wirtschaftliche Erholung u.U. nicht ausreichend, um das Vertrauen wiederherzustellen, wenn die Bürger dem Politikgestaltungsprozess skeptisch gegenüberstehen und die Verteilung von Kosten und Nutzen als ungerecht wahrnehmen. Es ist von entscheidender Bedeutung, ein Verständnis dafür zu entwickeln, welche Faktoren das Vertrauen in den Staat fördern, um einen positiven Kreislauf zu schaffen, der mittelfristig Wirtschaftswachstum und Wohlergehen sichern kann. 
Im vorliegenden Kapitel sollen die Zusammenhänge zwischen dem Vertrauen in den Staat und den Politiken bzw. Institutionen der Staats- und Verwaltungsführung untersucht werden. Es wird darauf eingegangen, wie Vertrauen, und insbesondere Vertrauen in den Staat, definiert werden kann, wie es gemessen wird und auf welche Art und Weise es die Einstellungen und Reaktionen der Bürger auf staatliche Maßnahmen beeinflussen kann. Auf der Grundlage der verfügbaren Befunde wird in dem Kapitel erörtert, wie sich Vertrauen fördern lässt; ferner werden darin Möglichkeiten identifiziert, um Politikmaßnahmen verlässlicher, flexibler, offener, inklusiver und gerechter zu gestalten. Bei der Analyse handelt es sich um eine erste Untersuchung eines Themas, das in der Vergangenheit von Experten der Staats- und Verwaltungsführung, Volkswirten und Soziologen aus relativ unterschiedlichen Blickwinkeln betrachtet wurde. Auch wenn u.U. weitere Forschungsarbeiten erforderlich sein werden, um eine einheitliche Perspektive und belastbarere Schlüsse für die Politik zu ziehen, bietet das Vertrauen in den Staat bereits einen anderen Blickwinkel, aus dem die Staats- und Verwaltungsführung betrachtet werden kann - ein Blickwinkel, der den Wahrnehmungen der Menschen sowie der Art und Weise, wie diese ihre Reaktion auf Politikmaßnahmen und Reformen beeinflussen, weitaus größere Aufmerksamkeit zuteil werden lässt. Wenn sie die verschiedenen Dimensionen des Vertrauens verstehen, könnte dies die Politikverantwortlichen und die Analysten der Staats- und Verwaltungsführung daher stärker für die Erwartungen der Bürger sensibilisieren, damit sie diese flexibler berücksichtigen können.

\section{Was ist mit Vertrauen in den Staat gemeint?}

Vertrauen bedeutet, eine positive Wahrnehmung der Handlungen einer Person oder einer Organisation zu haben. Es handelt sich dabei um ein subjektives Phänomen, das im Ermessen des Betrachters liegt und dessen Bedeutung sich insbesondere danach richtet, in welchem Maße es das Verhalten beeinflusst. Vertrauen in den Staat bezieht sich auf die Zuversicht, mit der die Bürger davon ausgehen, dass „die staatlichen Stellen das tun, was richtig ist und als gerecht wahrgenommen wird“ (Easton, 1965). Es hängt von der Übereinstimmung zwischen den Präferenzen der Bürger - ihrer Interpretation dessen, was richtig und gerecht und was ungerecht ist - und dem wahrgenommenen tatsächlichen Funktionieren des Staats ab (Bouckaert und van de Walle, 2003). Da die Präferenzen der Bürger vielfältig sind, verwenden sie eine Vielzahl unterschiedlicher Kriterien, um die Maßnahmen/Ergebnisse des Staats zu bewerten. Was von einer Person als richtig und gerecht betrachtet wird, wird von einer anderen möglicherweise anders eingeschätzt. Um zu analysieren, was das Vertrauen in den Staat beeinflusst, müssen die Präferenzen der Bürger mit ihren Wahrnehmungen des Funktionierens des Staats verglichen werden. Da es nicht die tatsächlichen Ergebnisse des Staats sind, sondern die wahrgenommenen Ergebnisse, die für das Vertrauen in den Staat bedeutsam sind, müssen darüber hinaus auch die Faktoren identifiziert werden, die die Wahrnehmungen neben den staatlichen Ergebnissen beeinflussen.

Ganz allgemein stützt sich das Vertrauen in den Staat auf zwei wesentliche Komponenten: 1. soziales Vertrauen, das das Vertrauen der Bürger in ihre soziale Gemeinschaft widerspiegelt, und 2. politisches Vertrauen, bei dem die Bürger den Staat und seine Institutionen beurteilen. Politisches Vertrauen umfasst sowohl das Vertrauen auf der Makroebene, das diffus und systembasiert ist, als auch das institutionsbasierte Vertrauen. Die Teilhabe am Leben der Gesellschaft und das zwischenmenschliche Vertrauen tragen nachweislich zum allgemeinen sozialen Vertrauen bei (Putnam, 2000). Dieser Zusammenhang ist indessen nicht automatisch und wird u.U. durch eine Reihe von Kontextfaktoren beeinflusst. So gibt es beispielsweise Länder, in denen sich die Menschen 
gegenseitig misstrauen - und das soziale Vertrauen somit gering ist - und sich dann für die Wahrnehmung ihrer Interessen auf die Institutionen verlassen (Aghion et al., 2010).

Die Erwartungen der Bürger sind für ihr Vertrauen in den Staat von entscheidender Bedeutung. Mit zunehmender Bildung der Bürger steigen ihre Erwartungen an die Leistungsfähigkeit des Staats. Wenn die Erwartungen der Bürger rascher steigen als die tatsächlichen Ergebnisse des Staats, könnten ihr Vertrauen und ihre Zufriedenheit sinken. Solche Veränderungen der Erwartungen erklären möglicherweise einen größeren Teil der Erosion der politischen Unterstützung als die realen Ergebnisse des Staats (Dalton, 2005) und könnten Politikverantwortliche überraschen, die sich an den Gegebenheiten der Vergangenheit orientieren.

Darüber hinaus wird das Vertrauen der Bürger gegenüber dem Staat unterschiedlich beeinflusst, je nachdem ob sie positive oder negative Erfahrungen mit der Erbringung der öffentlichen Dienstleistungen machen. Negative Erfahrungen haben weitaus stärkere Auswirkungen auf das Vertrauen in den Staat als positive. Die Ausrichtung von Politikmaßnahmen auf unzufriedene Bürger wird daher größere Auswirkungen auf das Vertrauen in den Staat haben (Kampen et al., 2006).

Ein Großteil der Analyse über das Vertrauen der Bürger in den Staat gilt auch für Unternehmen und sogar für die Beschäftigten des öffentlichen Sektors, die Art und Weise, wie Wahrnehmungen entstehen, und die Faktoren, die diese beeinflussen, unterscheiden sich jedoch möglicherweise.

\section{Weshalb ist Vertrauen in den Staat wichtig?}

Vertrauen in den Staat ist als eine der wichtigsten Grundlagen identifiziert worden, auf der Legitimität und Nachhaltigkeit politischer Systeme beruhen. Vertrauen ist von entscheidender Bedeutung für den sozialen Zusammenhalt und das gesellschaftliche Wohlergehen, da es Auswirkungen auf die Regierungsfähigkeit der Staaten hat und diese in die Lage versetzt, ohne Zwangsausübung zu agieren. Folglich handelt es sich um ein effizientes Mittel, um die Transaktionskosten in allen sozialen, wirtschaftlichen und politischen Zusammenhängen zu senken (Fukuyama, 1995). Ein hohes Maß an Vertrauen in den Staat könnte die Effizienz und die Wirksamkeit der Verwaltung steigern.

Ein Mindestma $\beta$ an Vertrauen in den Staat ist für das gerechte und effektive Funktionieren der staatlichen Institutionen notwendig - z.B. die Einhaltung des Prinzips der Rechtsstaatlichkeit oder die Sicherung der öffentlichen Daseinsvorsorge sowie die Bereitstellung von Infrastruktur. Rechtsstaatlichkeit und eine unabhängige Justiz sind besonders wichtig, da ihre reibungslose Funktionsweise ein wichtiger Bestimmungsfaktor für das Vertrauen in den Staat ist, wie in verschiedenen Studien herausgefunden wurde (Knack und Zak, 2003; Johnston, Krahn und Harrison, 2006; Blind, 2007). Da gut funktionierende staatliche Institutionen für die Investitionsentscheidungen der Unternehmen von Bedeutung sind, ist Vertrauen in diese ein notwendiges Element zur Ankurbelung des Wirtschaftswachstums (Dasgupta, 2009; Algan und Cuha, 2013).

Das Vertrauen in die staatlichen Institutionen beeinflusst zugleich das Verhalten des Einzelnen auf eine Art und Weise, die die gewünschten Politikergebnisse unterstützen könnte. Dies kann sich sowohl auf eher eng definierte Politikmaßnahmen und Programme beziehen (z.B. die Teilnahme an Impfkampagnen) als auch auf breiter angelegte Reformen (z.B. Umweltschutzbestimmungen oder eine Rentenreform). Vertrauen ist wichtig, weil zahlreiche öffentliche Programme die Möglichkeit zum Trittbrettfahren bzw. für opportunistisches Verhalten schaffen. Vertrauen könnte das Risiko eines solchen Verhaltens in dem Maße verringern, wie die Menschen bereit sind, auf bestimmte 
unmittelbare Vorteile zu verzichten, wenn sie positive Erwartungen bezüglich des längerfristigen Ergebnisses staatlicher Politikmaßnahmen haben, entweder auf persönlicher Ebene (Renten) oder durch Leistung eines Beitrags zum Gemeinwohl (Umverteilung des Einkommens über das Steuersystem).

Vertrauen in den Staat kann den Regierungen bei der Umsetzung von Strukturreformen helfen, die mit langfristigen Vorteilen verbunden sind. Viele Reformen sind mit der Zurückstellung kurzfristiger Zufriedenheit zu Gunsten längerfristigen Nutzens verbunden und werden einen breiteren sozialen und politischen Konsens erfordern, um wirkungsvoll und nachhaltig zu sein. In einem sehr vertrauensvollen Umfeld können solche Reformen nicht nur angemessen verabschiedet und umgesetzt, sondern auch lange genug aufrechterhalten werden, um Früchte zu tragen. Dies vergrößert den zeitlichen Horizont politischer Entscheidungen. In einem wenig vertrauensvollen Klima werden die Bürger dem unmittelbar verwendbaren Teilnutzen den Vorzug geben und Politiker dazu verleiten, durch Trittbrettfahren und populistisches Verhalten kurzfristige bzw. opportunistische Gewinne anzustreben (Gyorffy, 2013).

Vertrauen in den Staat könnte die Einhaltung von Regeln und Rechtsvorschriften verbessern und die Umsetzungskosten verringern. Regeln und Rechtsvorschriften sind nie vollkommen und vollständig genug, um Missbrauch zu unterbinden. Ihre Wirksamkeit hängt von dem Maße $\mathrm{ab}$, wie die Menschen sie als gerecht und legitim genug ansehen, um den Vorteil der Zuwiderhandlung wettzumachen. Dies ist insbesondere für Rechtsvorschriften wichtig, bei denen der Abstand zwischen den Befolgungskosten und dem persönlichen Nutzen groß ist und die Überwachung schwieriger ist. Besteuerung ist ein Beispiel für ersteres, wohingegen Verkehrsregeln ein Beispiel für das Zweitgenannte sind. Vertrauen in die für die Regulierung zuständige Stelle kann zu höherer freiwilliger Befolgung führen (Murphy, 2004).

Vertrauen in die staatlichen Institutionen könnte dazu beitragen, das Vertrauen in die Wirtschaft zu stärken, indem wirtschaftliche Entscheidungen erleichtert werden, z.B. in den Bereichen Investitionen und Verbrauch, die das Wirtschaftswachstum fördern. Vertrauen in die Institutionen sowie zwischenmenschliches Vertrauen senken u.U. die wahrgenommenen Risiken im Zusammenhang mit Entscheidungen, die von der Anschaffung von Gebrauchsgütern bis zur Arbeitsplatzmobilität, zur Einstellung von Arbeitskräften und zu Investitionen reichen. Die Stärkung des Vertrauens zwischen den Menschen steigert die Gesamtfaktorproduktivität und fördert damit den wirtschaftlichen Fortschritt (Dasgupta, 2009). Dies wiederum stützt das Wirtschaftswachstum und erweitert den Planungshorizont der Wirtschaftsakteure, was die wirtschaftliche Dynamik erhöht.

Vertrauen in den Staat scheint in Krisensituationen besonders entscheidend zu sein, etwa im Fall von Naturkatastrophen, Wirtschaftskrisen oder politischen Unruhen, was das Augenmerk auf die Kernfunktionen der Staats- und Verwaltungsführung richtet. Die Fähigkeit der Regierungen zum Krisenmanagement und zur Umsetzung erfolgreicher Ausstiegsstrategien ist oft eine Voraussetzung für ihr Fortbestehen und ihre Wiederwahl. In der Zeit nach einer größeren Katastrophe kann mangelndes Vertrauen die Krisen- und Wiederaufbaumaßnahmen behindern, was der Gesellschaft großen Schaden zufügt und die Handlungsfähigkeit der Regierung beeinträchtigt. Ebenso zeigt die gegenwärtige Wirtschaftskrise möglicherweise Dimensionen des Vertrauens auf, die in den Vorjahren angesichts der schrittweisen Entwicklung der Länder nicht offen zu Tage getreten waren.

Vertrauen kann in verschiedene Richtungen gehen. Nicht nur das Vertrauen der Bürger und Unternehmen in den Staat ist für die Wirksamkeit des staatlichen Handelns wichtig; das Vertrauen des Staats in die Bürger und Organisationen sowie das Vertrauen innerhalb des Staats ist für die Politikgestaltung und ihre Ergebnisse u.U. ebenfalls von 
Bedeutung (Bouckaert, 2012). Wie groß das Vertrauen des Staats in Bürger und Unternehmen ist, spiegelt sich in der Art und Weise wider, wie der Staat arbeitet und wie die öffentlichen Dienstleistungen organisiert sind, ebenso wie in ihrer Effizienz und Effektivität - z.B. im Hinblick auf das Steuersystem sowie die Möglichkeit der Selbstregulierung und der Selbstüberwachung. Darüber hinaus beeinflussen sich das Vertrauen der Bürger und Unternehmen in den Staat und das Vertrauen des Staats in die Bürger und Unternehmen gegenseitig. Ein offener und bürgerorientierter Staat bietet ein günstiges Umfeld, um das Vertrauen zwischen Staat und Bürgern in beide Richtungen zu stärken. Leider ist das Vertrauen seitens und innerhalb des Staats in der Fachliteratur deutlich weniger gut dokumentiert als das Vertrauen in den Staat.

Vertrauen benötigt zwar Zeit, um sich zu bilden, kann jedoch rasch verspielt werden. Es reicht nicht aus, den Effekt von Vertrauen in den Staat auf die Ergebnisse des Staats, der Wirtschaft und der Gesellschaft zu erörtern, notwendig ist darüber hinaus eine Beschreibung dessen, was im Falle zunehmenden Misstrauens gegenüber dem Staat geschehen könnte. Dies könnte zu sinkender Bereitschaft seitens der Bürger (und Unternehmen) führen, die Gesetze einzuhalten, in Krisenzeiten Opfer zu bringen oder Steuern zu zahlen. Dies könnte die Kosten für den Staat erhöhen, was zu sinkender Effizienz führen würde, oder die Einnahmen beeinträchtigen. Sinkt das Vertrauen in den Staat, könnte es auch schwieriger werden, kompetente Arbeitskräfte für staatliche Institutionen anzuwerben und zu binden.

\section{Messung des Vertrauens in den Staat}

Vertrauen beruht auf Wahrnehmungen, und für seine Messung müssen zahlreiche Herausforderungen bewältigt werden. Dies trifft auf nationaler Ebene zu und noch viel mehr auf internationaler Ebene. Da Vertrauen für eine positive Wahrnehmung des Staats steht, wird es durch Meinungsumfragen gemessen, bei denen Bürger, Unternehmen oder Experten gefragt werden, ob sie dem Staat, der politischen Führung und/oder den konkreten staatlichen Institutionen (z.B. den Kommunalbehörden oder der Justiz) Vertrauen entgegenbringen. Darüber hinaus werden oftmals Fragen zu ihrer Zufriedenheit mit den öffentlichen Dienstleistungen gestellt, z.B. der örtlichen Polizei, dem Bildungsoder Gesundheitswesen, auch wenn diesen Fragen ein etwas anderes Konzept zu Grunde liegt als Vertrauen.

Mehrere internationale Erhebungen sammeln Daten über das Vertrauen der Bürger in den Staat (vgl. Tabelle 1.1). Der Gallup World Poll erhebt in allen 34 OECD-Mitgliedsländern in ausreichender Regelmäßigkeit Daten, um den Effekt der weltweiten Finanz- und Wirtschaftskrise auf das Vertrauen in den Staat zu erfassen. Der World Values Survey hat das Vertrauen in den Staat über den längsten Zeitraum gemessen, die Datensätze sind jedoch bruchstückhaft, und Daten liegen lediglich für Mehrjahreszeiträume vor, wobei die letzte Welle im Zeitraum 2005-07 erfolgte. Das Eurobarometer der Europäischen Union bietet die konsistentesten Datensätze (u.a. werden zweimal im Jahr Daten erhoben), deckt jedoch leider nur 23 OECD-Mitgliedsländer ab. Das Edelman-Trust-Barometer bietet Zeitreihen lediglich für eine begrenzte Stichprobe der Bevölkerung (die Kriterien für die Stichprobenauswahl umfassen einen Hochschulabschluss und ein Haushaltseinkommen im obersten Quartil).

Da internationale Erhebungen konzipiert wurden, um länderübergreifende Vergleiche zu ermöglichen, können die Fragen, anhand derer das Vertrauen in den Staat gemessen wird, unklar formuliert sein und unterliegen oftmals der Interpretation der Umfrageteilnehmer, da der mehrdeutige englische Begriff government in der Regel nicht näher definiert ist (auch wenn er in deutschsprachigen Fragebogen oftmals mit dem enger gefassten Begriff Regierung 
Tabelle 1.1. Internationale Erhebungen, in denen das Vertrauen in den Staat gemessen wird

\begin{tabular}{|c|c|c|c|c|}
\hline Name der Erhebung & $\begin{array}{l}\text { Zahl der erfassten } \\
\text { OECD-Länder }\end{array}$ & $\begin{array}{l}\text { Erfasste Jahre } \\
\text { und Häufigkeit }\end{array}$ & Messgröße & Antwortskala \\
\hline Gallup World Poll & 34 & $\begin{array}{l}\text { 2005-12 } \\
\text { (jährlich) }\end{array}$ & $\begin{array}{l}\text { Vertrauen in die } \\
\text { nationale Regierung }\end{array}$ & 2: ja/nein \\
\hline World Values Survey & 25 & $\begin{array}{l}4 \text { Wellen: 1989-93, } \\
\text { 1994-98, 1999-2004, } \\
2005-08\end{array}$ & $\begin{array}{c}\text { Vertrauen } \\
\text { in die Regierung }\end{array}$ & $\begin{array}{l}\text { 4: groß/ziemlich groß/ } \\
\text { nicht besonders groß/ } \\
\text { gar nicht groß }\end{array}$ \\
\hline Eurobarometer & 23 & $\begin{array}{c}\text { 2003-13 } \\
\text { (zweimal jährlich) }\end{array}$ & $\begin{array}{l}\text { Vertrauen } \\
\text { in die Regierung }\end{array}$ & $\begin{array}{l}\text { 2: vertraue eher/ } \\
\text { vertraue eher nicht }\end{array}$ \\
\hline Edelman-Trust-Barometer & 15 & $\begin{array}{l}2001-13 \\
\text { (jährlich) }\end{array}$ & $\begin{array}{l}\text { Vertrauen } \\
\text { in die Regierung }\end{array}$ & $\begin{array}{l}\text { 9-Punkte-Skala: } 1 \text { bedeutet „vertraue } \\
\text { überhaupt nicht“ } \\
\text { und } 9 \text { bedeutet „vertraue sehr“ }\end{array}$ \\
\hline Latinobarómetro & 3 & $\begin{array}{c}\text { 1995-2012 } \\
\text { (jährlich) }\end{array}$ & $\begin{array}{c}\text { Vertrauen } \\
\text { in die Regierung }\end{array}$ & $\begin{array}{c}\text { 4: viel/einiges/ein wenig/kein } \\
\text { Vertrauen }\end{array}$ \\
\hline
\end{tabular}

wiedergegeben wird - A.d.Ü). Die internationalen Erhebungen wenden in Bezug auf die Stichprobenziehung ähnliche Methoden an, unterscheiden sich jedoch in Bezug auf die Formulierung der Fragen in den Fragebogen (z.B. wird zwischen einer Frage zur Zuversicht und einer Frage zum Vertrauen in die Regierung nuanciert und werden unterschiedliche Antwortskalen verwendet) sowie in Bezug auf andere Messgrößen des Vertrauens, die Vergleichsgrößen liefern könnten (z.B. Vertrauen in nationale Parlamente, Finanzinstitute, Politiker, Beamte, internationale Organisationen, öffentliche Dienstleistungen etwa im Gesundheits- und Bildungssektor, Unternehmen, religiöse Einrichtungen).

Auf Grund der Einschränkungen internationaler Erhebungen ist es schwierig, eingehend zu verstehen, wie sich das Vertrauen der Bürger in den Staat im Lauf der Zeit entwickelt und wodurch das Vertrauen in den Staat in den OECD-Ländern und darüber hinaus beeinflusst wird. Durch das Vorliegen kultureller Faktoren in Bezug auf die Art und Weise, wie die Menschen öffentlichen Institutionen begegnen, sind länderübergreifende Vergleiche des Vertrauens in den Staat eine besondere Herausforderung. Für die Zwecke der vorliegenden Analyse vielleicht am wichtigsten ist, dass mit den bestehenden Erhebungen nicht beabsichtigt wurde, Politikanalysen zu unterstützen oder Politikempfehlungen zu ermöglichen.

Auch wenn nationale Erhebungen, die das Vertrauen in den Staat messen, nicht für länderübergreifende Vergleichszwecke verwendet werden können, bieten sie aus vielen Gründen eine bessere Grundlage für Politikanalysen. Im Vergleich zu internationalen Erhebungen bieten sie größere Erkenntnisse in Bezug auf die Faktoren, die Vertrauen begünstigen, und können um Wahlzyklen bereinigt werden. So werden etwa im Rahmen der von Metroscopia in Spanien durchgeführten Erhebung Barómetro de Confianza Ciudadana Monatsdaten veröffentlicht, anhand derer die Zufriedenheit mit der Regierung mit der Wahrnehmung der Wirtschaftslage verglichen werden kann. In nationalen Erhebungen wird darüber hinaus das Vertrauen im Bereich des ganzen Spektrums des öffentlichen Lebens eingehender untersucht. So veröffentlicht etwa IPSOS Mori im Vereinigten Königreich zweimal jährlich die trendmäßige Entwicklung des Vertrauens in verschiedene öffentliche Institutionen (z.B. unterschiedliche staatliche Ebenen, das Parlament), öffentliche Dienstleistungen, wirtschaftspolitische Maßnahmen (z.B. bezogen auf das Wirtschaftswachstum, die Arbeitslosigkeit, die Inflation, die Kaufkraft), politische Parteien und Vertreter der Politik (Führungskräfte in der Exekutive, Politiker, Mitglieder des Parlaments) sowie der Wahrnehmung der Korruption in der Regierung. Nationale Erhebungen können darüber hinaus Messgrößen des Vertrauens in bestehende Politikmaßnahmen bieten. Beispielsweise werden die Bürger in Frankreich von IFOP nach 
ihrem Vertrauen in die Umsetzung der Ziele der Regierung befragt, die bei Amtsantritt angekündigt worden waren. Die Zeitreihen nationaler Erhebungen sind überdies oftmals wesentlich länger, beispielsweise erhebt das PEW Research Center in den Vereinigten Staaten seit Ende der 1950er Jahre Daten zum Vertrauen in den Staat.

Die vorstehende Diskussion lässt darauf schließen, dass mehr getan werden könnte, um die Vergleichbarkeit der durch Meinungsumfragen gewonnenen Daten über das Vertrauen der Bürger zu erhöhen und die Politikdiskussion zu fördern. Erstens könnte die Repräsentativität der Erhebungen verbessert werden. Die derzeitigen Erhebungen arbeiten mit kleinen Stichproben und sind in geografischer Hinsicht innerhalb eines Landes selten repräsentativ. Zusätzliche Merkmale der Befragten - z.B. Alter, Geschlecht, ethnische Zugehörigkeit, Bildungsstand, Personenstand, Einkommensniveau, ob sie eine staatliche Dienstleistung in Anspruch genommen haben oder nicht usw. - beeinflussen ihre Wahrnehmung der Regierung, weshalb es sinnvoll wäre, wenn die Stichprobe diesen Merkmalen ebenfalls Rechnung tragen würde. Zweitens könnten die Erhebungsfragen verbessert werden. Die Schlüsselbegriffe müssen präzise definiert werden: So wurden die Befragten etwa im Gallup World Poll gefragt, wie groß ihr Vertrauen in die nationale Regierung (national government) ist, ohne nähere Erläuterung, was damit gemeint ist. Die Befragten könnten die Regierung mit dem Kabinett oder dem Verwaltungsapparat gleichsetzen (wobei dieses Problem auf Grund der Mehrdeutigkeit von government im Englischen gravierender als im Deutschen sein könnte - A.d.Ü). Die Erhebungsfragen und die dazugehörigen Antwortkategorien müssen darüber hinaus so formuliert sein, dass die Regierungen auf der Grundlage der gewonnenen Informationen handeln können, etwa durch eine Verhaltensänderung. Und schließlich wird die Erhebung von Daten in regelmäßigen Abständen zusätzlich zu länderübergreifenden Vergleichen zu einem bestimmten Zeitpunkt auch die Feststellung von Veränderungen im Zeitverlauf und Trends sowohl in den einzelnen Ländern als auch im Ländervergleich ermöglichen.

\section{Muster und Trends im Hinblick auf das Vertrauen in den Staat in den OECD-Ländern}

Trotz der methodischen Schwierigkeiten bei der Messung des Vertrauens in den Staat zeigen die verfügbaren Daten verschiedene Muster, Trends und Korrelationen auf, die einiges über den Zustand des Vertrauens in den Staat in den OECD-Ländern aussagen und den Politikverantwortlichen dabei helfen könnten, sich tiefer mit der Materie auseinanderzusetzen.

Erstens lassen die jüngsten verfügbaren Daten für die OECD-Länder darauf schließen, dass die Antworten der Bürger von einem Land zum anderen erheblich auseinandergehen, wenn sie über ihr Vertrauen in die Regierung befragt werden, wobei die positiven Antworten im Durchschnitt zuletzt bei deutlich unter 50\% lagen (Abbildung 1.1). Mit anderen Worten gab weniger als die Hälfte der Bürger der OECD-Länder im Rahmen von Umfragen an, dass sie ihrer jeweiligen Regierung Vertrauen entgegenbringen. Die nationalen Durchschnitte lagen zwischen nahezu $80 \%$ in der Schweiz und $12 \%$ in Griechenland. Die Verteilung innerhalb dieses Spektrums scheint weder auf den Lebensstandard noch auf das Pro-Kopf-BIP oder die Höhe des Wirtschaftswachstums zurückzuführen zu sein. Während die Vertrauenswerte in Japan - einem Land der oberen Einkommensgruppe - und Korea - einem wachstumsstarken Land - jeweils unter dem OECD-Durchschnitt lagen, lag dieser Wert in der Türkei, die ein geringeres Pro-Kopf-BIP aufweist, deutlich darüber. Dies lässt darauf schließen, dass das Vertrauen in den Staat u.U. weniger auf die langfristige Wirtschaftsentwicklung bzw. absolute Lebensstandards reagiert als vielmehr auf kulturelle Faktoren, sich verändernde Erwartungen und politische 


\section{Abbildung 1.1. Vertrauen in die nationale Regierung im Jahr 2012 und Veränderung seit 2007}

Angeordnet in absteigender Reihenfolge nach der Veränderung in Prozentpunkten zwischen 2007 und 2012

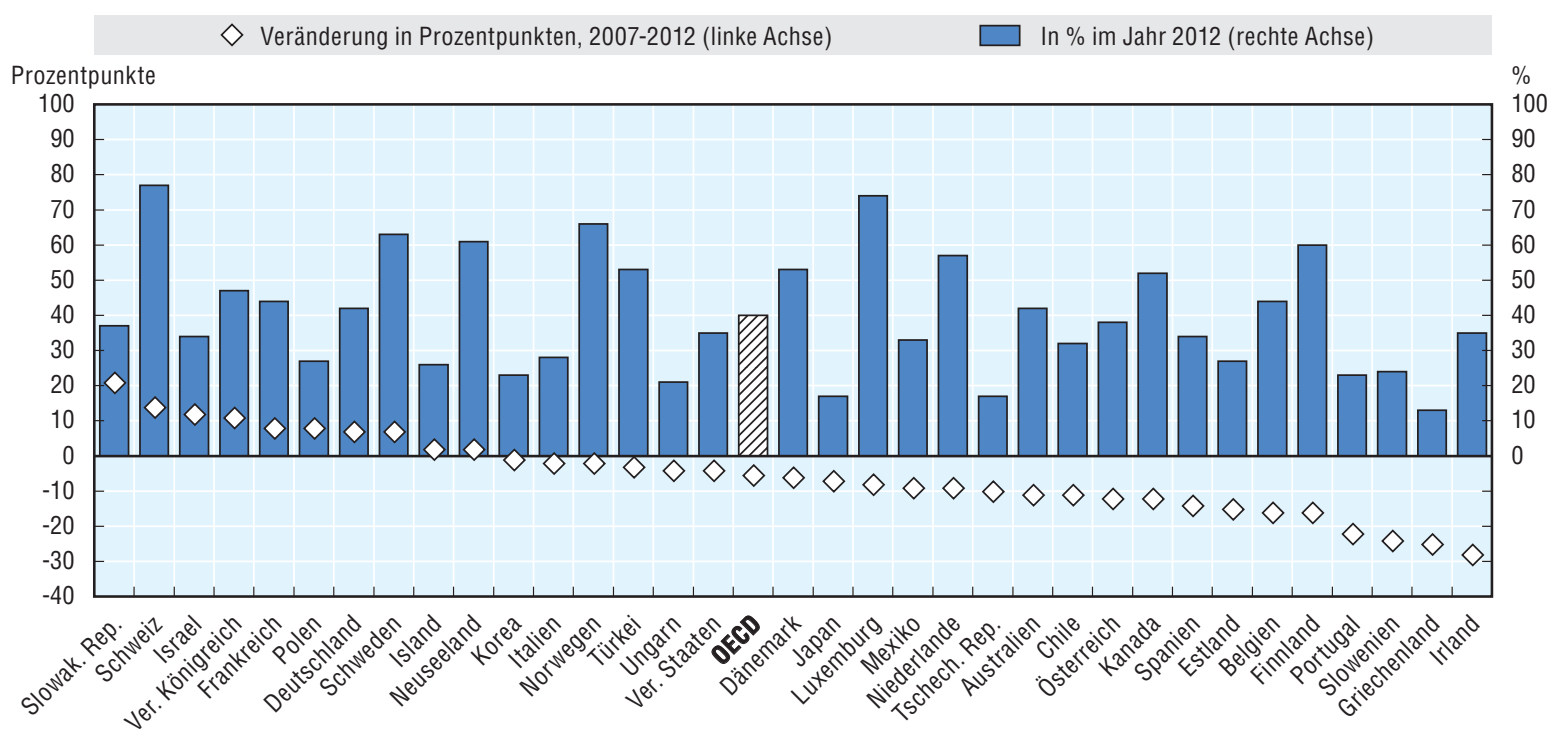

Anmerkung: Die Daten beziehen sich auf den Prozentsatz der Befragten, die mit „Ja“ auf die folgende Frage antworteten: „Haben Sie in diesem Land Vertrauen in die folgenden Einrichtungen/Institutionen oder nicht? Wie sieht es aus mit der nationalen Regierung?" Die Daten für Chile, Deutschland und das Vereinigte Königreich beziehen sich auf 2011 anstelle von 2012. Die Daten für Island und Luxemburg beziehen sich auf 2008 anstelle von 2007. Die Daten für Österreich, Finnland, Irland, Norwegen, Portugal, die Slowakische Republik, Slowenien und die Schweiz beziehen sich auf 2006 anstelle von 2007.

Quelle: Gallup World Poll.

Entwicklungen. Diese Schlussfolgerung wird durch die vorhandenen Daten für manche aufstrebenden Länder erhärtet, was den Einfluss der Erwartungen der Bürger gegenüber dem staatlichen Handeln auf den Vertrauensgrad der Bürger unterstreicht (Kasten 1.1).

Zweitens zeigen die Daten, dass das Vertrauen in den Staat $2012 \mathrm{im}$ Durchschnitt unter dem Vorkrisenniveau des Jahres 2007 lag (unterer Teil der Abbildung 1.1). Der Anteil der Befragten, die im Jahr 2012 Vertrauen in den Staat äußerten, hat sich gegenüber $2007 \mathrm{im}$ Durchschnitt um 5 Prozentpunkte verringert (von 45\% auf 40\%). Hinter diesem Vergleich verbergen sich weitaus größere Unterschiede zwischen den einzelnen Ländern, da über zwei Drittel der OECD-Länder im Zeitraum 2007-12 einen Verlust des Vertrauens in den Staat verzeichneten. Zu stärkeren Vertrauensverlusten kam es in Ländern, in denen entweder eine politische, fiskalpolitische oder Wirtschaftskrise ausbrach, z.B. in Griechenland, Slowenien, Irland, Spanien, Belgien und Portugal. In anderen Ländern stieg das Vertrauen in den Staat hingegen, insbesondere in der Slowakischen Republik, in Israel, dem Vereinigten Königreich, Polen, Frankreich, der Schweiz, Deutschland und Schweden.

Drittens ist das Vertrauen in die Regierung im Durchschnitt ebenso hoch wie das Vertrauen in zwei wichtige Institutionen des privaten Sektors: a) Finanzinstitute und Banken und b) die Medien, wobei jedoch auch hier erhebliche Unterschiede zwischen den einzelnen OECD-Ländern bestehen. Insgesamt ist das Vertrauen in Finanzinstitute und Banken in den OECD-Ländern mit 43\% etwas höher als das Vertrauen in die Regierung (40\%) (Abbildung 1.2). In einigen Ländern, die von der Finanzkrise von 2008 am wenigsten betroffen waren, genießen Finanzinstitute und Banken hohes Vertrauen, so z.B. in Kanada, Polen, Finnland, Norwegen, Mexiko, Australien und Japan. Hingegen ist das Vertrauen in die Regierung in Ländern, die am stärksten in Mitleidenschaft gezogen wurden, verhältnismäßig höher als in Finanzinstitute, so z.B. in Irland, Spanien und Italien. 


\section{Kasten 1.1. Das Vertrauen in den Staat in den BRIICS (2012)}

Im Durchschnitt der BRIICS brachte die Mehrheit der Bürger (54\%) im Jahr 2012 ihr Vertrauen in den Staat zum Ausdruck. Das Vertrauen in den Staat war in Indonesien und China am höchsten (zwei Drittel der Bürger) und in Südafrika, Brasilien und der Russischen Föderation am niedrigsten (in all diesen Ländern lag der Wert bei 45-47\%). Das Vertrauen in den Staat lag in allen BRIICS über dem OECD-Durchschnitt von 40\%. Im Zeitraum 2007-12 sank das Vertrauen in den Staat im Durchschnitt der BRIICS (außer China) um 3 Prozentpunkte, was ein geringerer Rückgang als in den OECD-Mitgliedsländern war, wo das Vertrauen im Durchschnitt um 5 Prozentpunkte abnahm. Der größte Vertrauenszuwachs wurde in Indonesien verzeichnet (15 Prozentpunkte), der größte Vertrauensverlust hingegen in Indien (27 Prozentpunkte). Der höhere Vertrauensgrad in den BRIICS im Vergleich zu den meisten OECD-Mitgliedsländern kann auf kulturelle und kontextspezifische Faktoren zurückzuführen sein, er kann jedoch auch durch unterschiedliche Erwartungen bedingt sein, die die Bürger gegenüber den staatlichen Dienstleistungen und Ergebnissen hegen, wenn sie sich in unterschiedlichen Phasen der sozioökonomischen Entwicklung befinden. Dennoch herrscht Konsens zwischen den Forschern, dass es wichtiger ist, den optimalen Vertrauensgrad zu erreichen als den maximalen Vertrauensgrad (Knack und Keefer, 1997; Nooteboom, 2006; Dasgupta, 2009; Bouckeart, 2012).

\section{Das Vertrauen in den Staat ist in den BRIICS größer als im OECD-Raum}

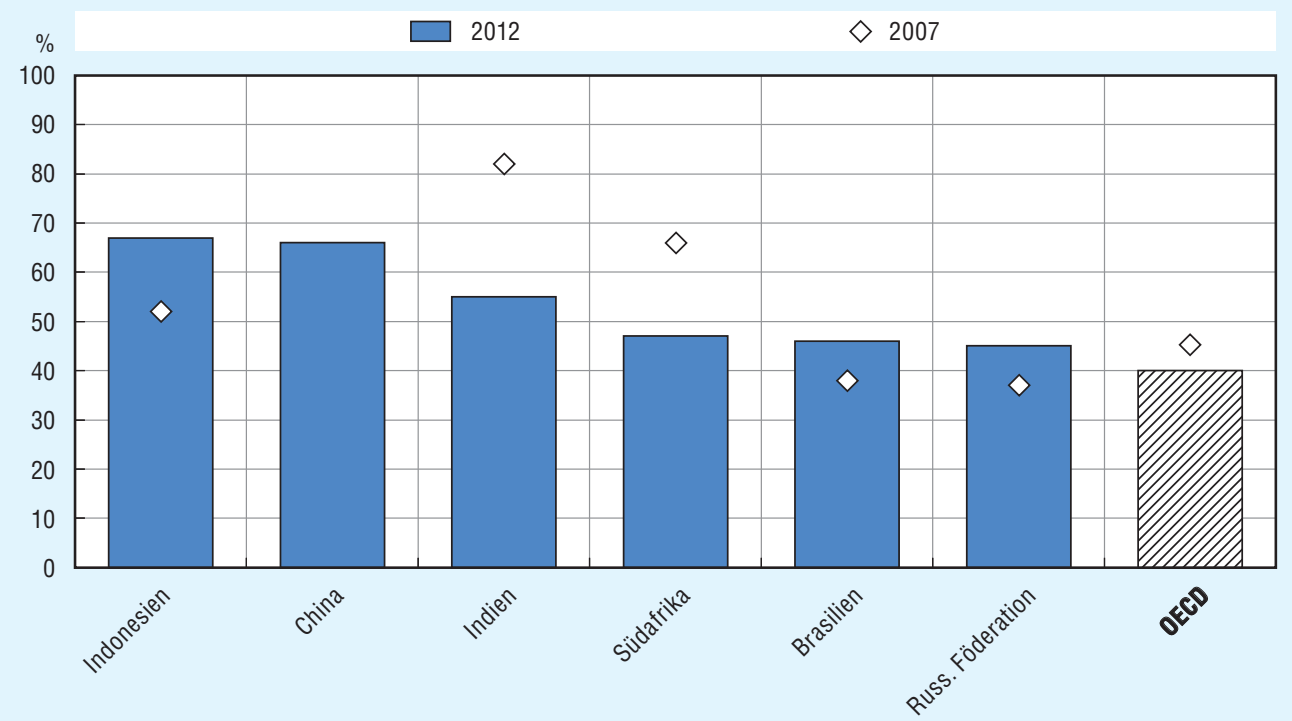

Anmerkung: Die Daten beziehen sich auf den Prozentsatz der Befragten, die mit „Ja“ auf die folgende Frage antworteten: „Haben Sie in diesem Land Vertrauen in die folgenden Einrichtungen/Institutionen oder nicht? Wie sieht es aus mit der nationalen Regierung?" Quelle: Gallup World Poll.

StatLink तilsts $h$ ttp://dx.doi.org/10.1787/888932940740

Das Vertrauen in die Medien war in Irland, Spanien und Portugal im Jahr 2010 - dem Jahr, für das Daten vorliegen - deutlich höher als das Vertrauen in die Regierung, wohingegen es in der Türkei, in Schweden, den Niederlanden und Luxemburg deutlich niedriger war (Abbildung 1.3). Da die Länder in der ersten Gruppe zu denen zählen, in denen im Lauf der Krise der größte Verlust an Vertrauen in die Regierung verzeichnet wurde, die Länder in der zweiten Gruppe wiederum zu denen, in denen das Vertrauen am größten und stabilsten ist, sagt der Vergleich möglicherweise mehr über die Entwicklung des Vertrauens in die Regierung aus als über das Vertrauen in die Medien. Beim Vergleich 


\section{Abbildung 1.2. Vertrauen in Finanzinstitute im Vergleich zur Regierung}

Vergleich des Vertrauens in Finanzinstitute/Banken und in die Regierung (2012)

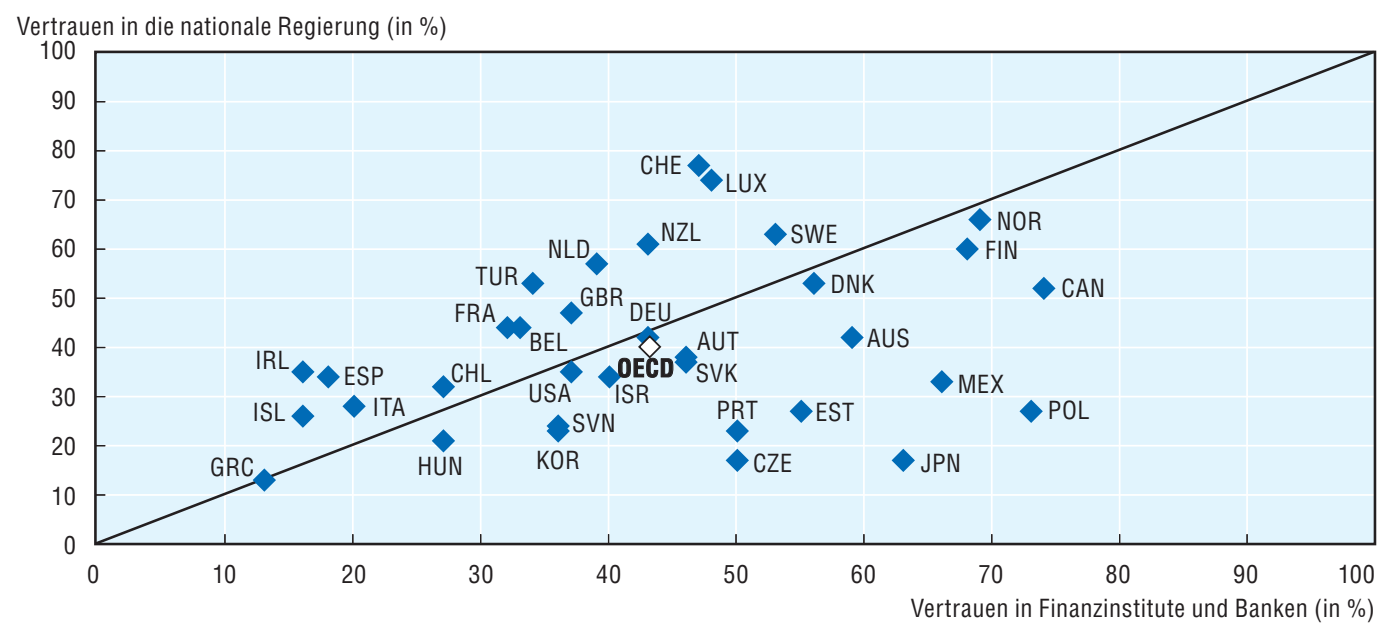

Anmerkung: Die Daten über das Vertrauen in die nationale Regierung beziehen sich auf den Prozentsatz der Befragten, die mit „Ja“ auf die folgende Frage antworteten: „Haben Sie in diesem Land Vertrauen in die folgenden Einrichtungen/Institutionen oder nicht? Wie sieht es aus mit der nationalen Regierung?" Die Daten über das Vertrauen in Finanzinstitute und Banken beziehen sich auf den Prozentsatz der Befragten, die mit „Ja“ auf die folgende Frage antworteten: „Haben Sie in diesem Land Vertrauen in die folgenden Einrichtungen/Institutionen oder nicht? Wie sieht es aus mit Finanzinstituten oder Banken?" Die Daten für Chile, Deutschland und das Vereinigte Königreich beziehen sich auf 2010 anstelle von 2011. In den Ländern unterhalb der Linie ist das Vertrauen in Finanzinstitute und Banken höher als das Vertrauen in die Regierung.

Quelle: Gallup World Poll.

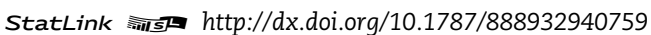

\section{Abbildung 1.3. Vertrauen in die Medien und in die Regierung}

Vergleich des Vertrauens in die nationale Regierung bzw. in die Medien (2010)

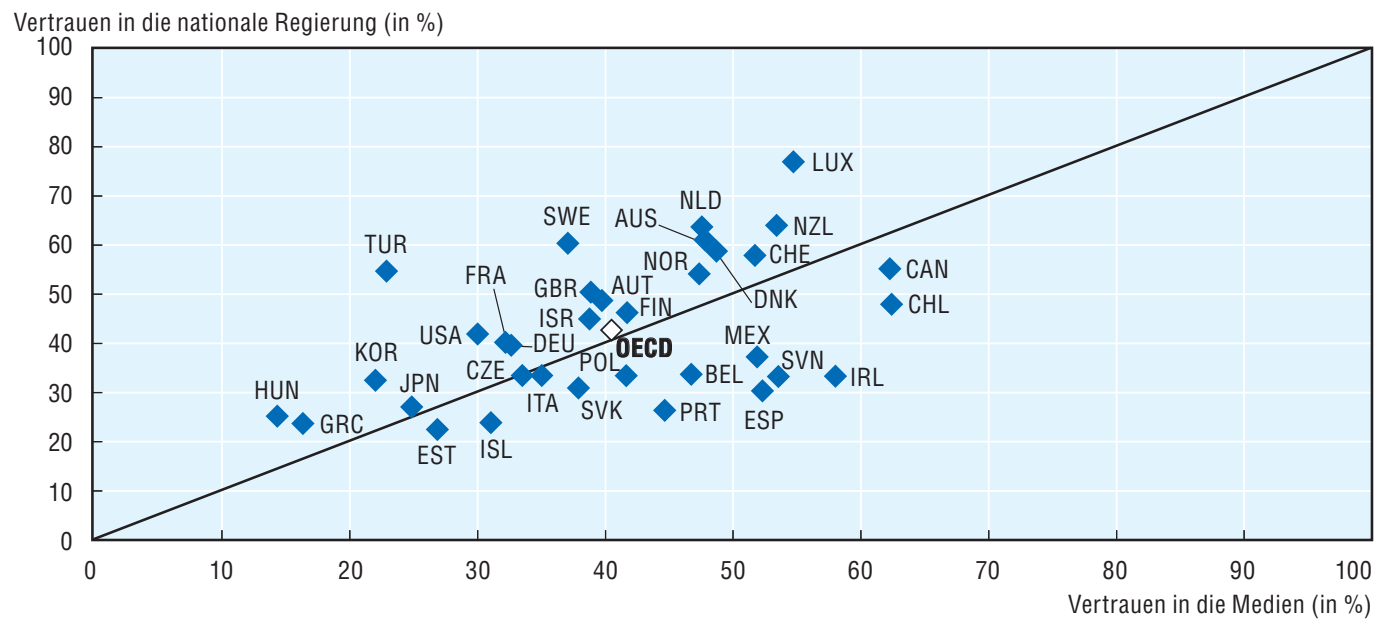

Anmerkung: Die Daten über das Vertrauen in die nationale Regierung beziehen sich auf den Prozentsatz der Befragten, die mit „Ja“ auf die folgende Frage antworteten: „Haben Sie in diesem Land Vertrauen in die folgenden Einrichtungen/Institutionen oder nicht? Wie sieht es aus mit der nationalen Regierung?“ Die Daten über das Vertrauen in die Medien beziehen sich auf den Prozentsatz der Befragten, die mit "Ja“ auf die folgende Frage antworteten: „Haben Sie in diesem Land Vertrauen in die folgenden Einrichtungen/Institutionen oder nicht? Wie sieht es aus mit der Qualität und der Integrität der Medien?" Die Daten für Island und Norwegen beziehen sich auf 2008 anstelle von 2010. Die Daten für die Schweiz und Estland beziehen sich auf 2009 anstelle von 2010. In den Ländern unterhalb der Linie ist das Vertrauen in die Medien höher als das Vertrauen in die Regierung. Quelle: Gallup World Poll.

StatLink त्ञाजी http://dx.doi.org/10.1787/888932940778

zwischen dem Vertrauen in die Regierung und dem Vertrauen in Finanzinstitute ist u.U. das Gegenteil zu beobachten, wobei die Entwicklung des Vertrauens in die Finanzinstitute in diesem Zusammenhang ausschlaggebend ist. 


\section{Bestimmungsfaktoren für das Vertrauen in den Staat}

Das Vertrauen in den Staat hat zahlreiche Facetten und beruht auf einem Mix aus wirtschaftlichen, sozialen und politischen Wechselwirkungen zwischen den Bürgern und dem Staat. In der umfangreichen empirischen Fachliteratur ${ }^{1}$ werden die Zusammenhänge zwischen dem Vertrauen in den Staat sowie wirtschaftlichen, sozialen und Governancebezogenen Parametern erörtert. Vier breite Gruppen von Bestimmungsfaktoren für das Vertrauen in den Staat werden darin identifiziert: 1. Kultur, 2. institutionelle Rahmenbedingungen, 3. wirtschaftliche und soziale Ergebnisse und 4. Ergebnisse der Institutionen. Es besteht zwar mehr oder weniger Konsens über das Spektrum dieser Bestimmungsfaktoren, die Befunde widersprechen sich jedoch in Bezug auf die Stärke ihres Einflusses sowie der Wechselwirkungen zwischen diesen Faktoren und dem Vertrauen. Eine allgemeine Erkenntnis besteht darin, dass Vertrauen und die meisten seiner Bestimmungsfaktoren miteinander verbunden und selbstverstärkend sind und daher ergänzende Wirkung im Zusammenhang mit der Staats- und Verwaltungsführung sowie der wirtschaftlichen Entwicklung haben.

Bouckaert (2012) zufolge kann das Vertrauen in den Staat auf drei Ebenen analysiert werden. Auf der Makroebene bezieht sich Vertrauen auf politische Institutionen und das Funktionieren der Demokratie. Auf Mesoebene bezieht sich Vertrauen auf die Politikgestaltung die Fähigkeit der Regierungen, Maßnahmen zur Bewältigung wirtschaftlicher und sozialer Probleme zu ergreifen und positive Erwartungen in Bezug auf das künftige Wohlergehen zu schaffen. Und schließlich bezieht sich Vertrauen auf der Mikroebene auf den Effekt, den staatliches Handeln mittels der Erbringung öffentlicher Dienstleistungen auf das Alltagsleben der Menschen hat. Auch wenn sich diese drei Ebenen voneinander unterscheiden, bestehen Wechselwirkungen zwischen ihnen, und ein deutlicher Vertrauensmangel auf einer Ebene kann das Vertrauen auf den anderen Ebenen beeinträchtigen und die Politikergebnisse beeinflussen. Bemühungen zur Stärkung des Vertrauens müssen daher Synergien zwischen all diesen verschiedenen Sphären verstärken.

Bouckaerts Systematik ist aus zwei Gründen besonders nützlich. Erstens lässt sie den Schluss zu, dass Vertrauen nicht einfach etwas ist, das Regierungen zufällig entgegengebracht wird, sondern etwas, das die Regierungen durch ihre Handlungen und Politikmaßnahmen beeinflussen können. Zweitens legt sie nahe, dass es bei der Beeinflussung des Vertrauens nicht nur auf das was der öffentlichen Politikmaßnahmen ankommt, sondern auch auf das wie, für wen und mit wem. Folglich sind nicht nur die Endergebnisse für Bürger und Unternehmen wichtig, sondern auch die Prozesse, die zu diesen Ergebnissen führen.

Der Aspekt der Staats- und Verwaltungsführung - die institutionellen Rahmenbedingungen und die durch diese erzielten Ergebnisse - in Bezug auf das Vertrauen lässt sich u.U. besser verstehen, wenn dieses Konzept in eine Reihe miteinander zusammenhängender Prozess-Komponenten aufgeschlüsselt wird, die die Erwartungen der Bürger gegenüber dem Staat zusammenfassen. Die OECD hat folgende Komponenten vorgeschlagen:

- Verlässlichkeit: die Fähigkeit der Regierungen, die Unsicherheit des wirtschaftlichen, sozialen und politischen Umfelds für ihre Bürger auf ein Mindestmaß zu begrenzen und konsequent und vorhersehbar zu agieren.

- Bürgerorientierung: die Bereitstellung gut zugänglicher, effizienter und bürgerorientierter öffentlicher Dienstleistungen, die dem Bedarf und den Erwartungen der Öffentlichkeit effektiv Rechnung tragen. 
- Offenheit und Inklusivität: ein systematischer, umfassender Ansatz zur Institutionalisierung einer beidseitigen Kommunikation mit den beteiligten Parteien, in deren Rahmen relevante, verwertbare Informationen geliefert und Interaktionen gefördert werden, um Transparenz, Rechenschaftspflicht und Engagement zu verbessern.

- Integrität: die Ausrichtung der Regierung und der öffentlichen Institutionen an allgemeineren Prinzipien und Verhaltensmaßstäben, die zur Wahrung des öffentlichen Interesses beitragen und zugleich Korruption verhindern.

- Gerechtigkeit: im Verfahrenssinne die einheitliche Behandlung von Bürgern (und Unternehmen) im Rahmen der Politikgestaltungs- und -umsetzungsprozesse.

Im Folgenden verwenden wir Bouckaerts Dreiebenenmodell, um die potenziellen Bestimmungsfaktoren des Vertrauens im Bereich der Staats- und Verwaltungsführung zu identifizieren und auf Befunde aus internationalen Erhebungen hinzuweisen, die auf eine statistische Korrelation schließen lassen. Hierbei handelt es sich indessen um eine vorläufige Untersuchung, die weit davon entfernt ist, Kausalzusammenhänge abschließend festzustellen, die jedoch als Orientierungspunkt für weitere Forschungsarbeiten und Erörterungen dienen könnte.

\section{Makroebene}

Auf der Makroebene wichtig für das Vertrauen in den Staat sind politische Institutionen und das Funktionieren der Demokratie. Eine entscheidende Voraussetzung, um der OECD beizutreten, besteht darin, dass es sich bei dem betreffenden Staat um eine Demokratie mit gut entwickelten politischen Institutionen handeln muss ${ }^{2}$.

Im Hinblick auf die politischen Institutionen sprechen die Bürger - zumindest in den europäischen Ländern, für die Daten verfügbar sind - der Regierung durchgängig größeres Vertrauen aus als den politischen Parteien (Abbildung 1.4 und Abbildung 1.5). 2013 war das Vertrauen der Bürger in die Regierung lediglich in einem europäischen

\section{Abbildung 1.4. Das Vertrauen in die politischen Parteien ist in Europa im Zeitverlauf (2005-13) deutlich geringer als das Vertrauen in die Regierung}

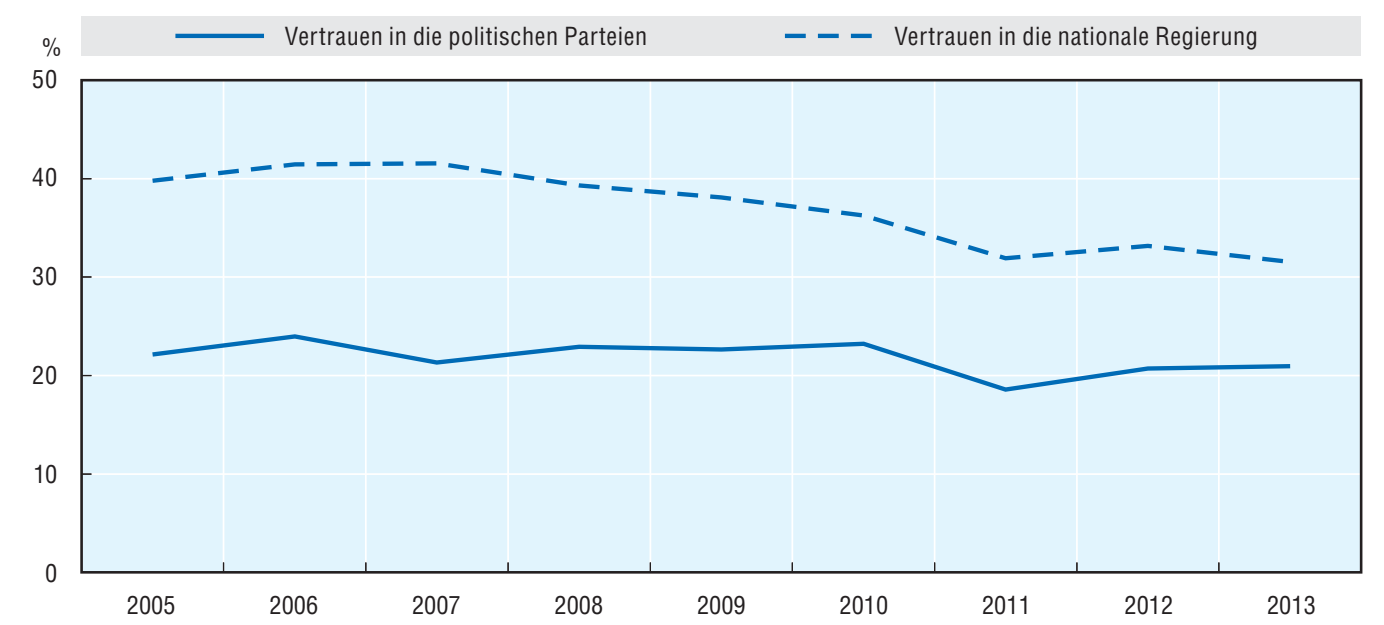

Anmerkung: Die Daten beziehen sich auf den Prozentsatz der Befragten, die mit „eher vertrauen“ auf die folgenden Fragen antworteten: „Sagen Sie mir bitte für jede der folgenden Institutionen, ob Sie ihr eher vertrauen oder eher nicht vertrauen: der (nationalen) Regierung; den politischen Parteien." Die Daten beziehen sich auf die Jahresdurchschnittswerte für 23 OECD-Mitgliedsländer: Für Australien, Kanada, Chile, Israel, Japan, Korea, Mexiko, Neuseeland, Norwegen, die Schweiz und die Vereinigten Staaten liegen keine Daten vor. Quelle: Eurobarometer (Datenbank), OECD-Berechnungen. 


\section{Abbildung 1.5. Vertrauen in die Regierung und in die politischen Parteien in den europäischen OECD-Mitgliedsländern (2013)}

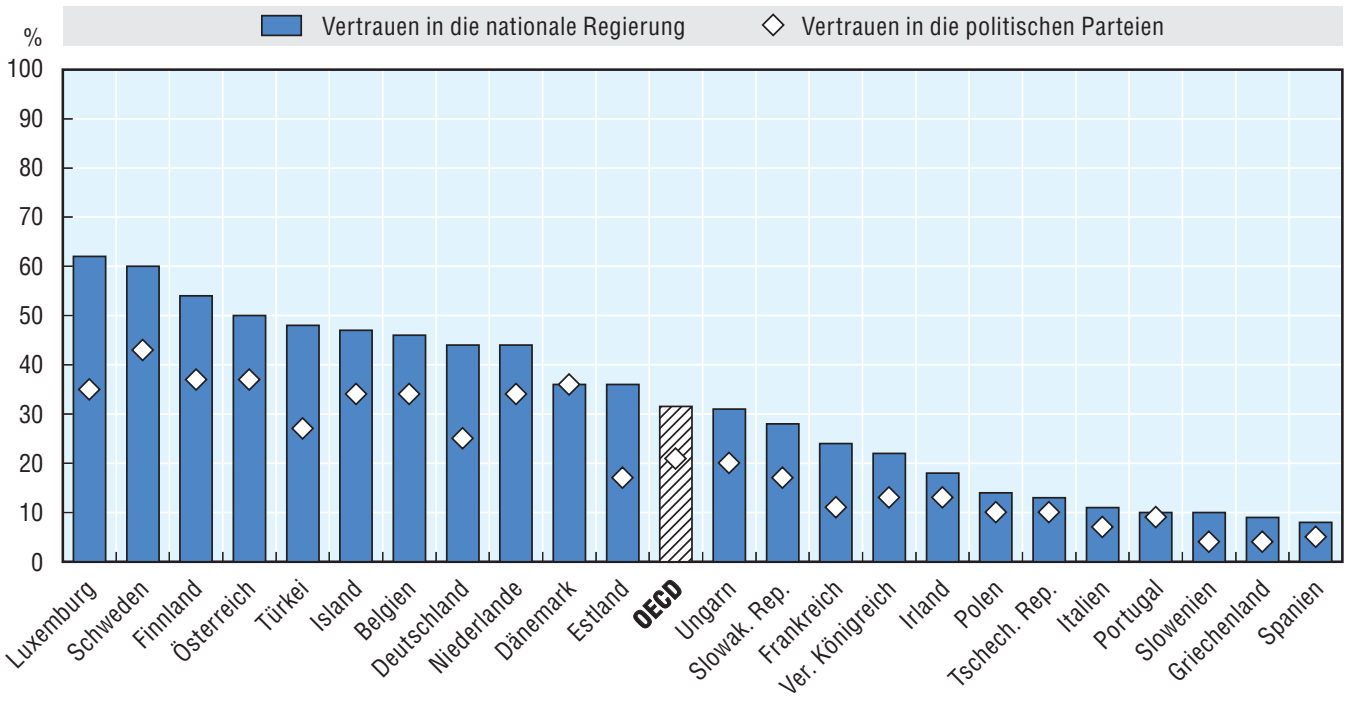

Anmerkung: Die Daten beziehen sich auf den Prozentsatz der Befragten, die mit „eher vertrauen“ auf die folgenden Fragen antworteten: „Sagen Sie mir bitte für jede der folgenden Institutionen, ob Sie ihr eher vertrauen oder eher nicht vertrauen: der (nationalen) Regierung; den politischen Parteien." Die Daten beziehen sich auf die Jahresdurchschnittswerte für 23 OECD-Mitgliedsländer: Für Australien, Kanada, Chile, Israel, Japan, Korea, Mexiko, Neuseeland, Norwegen, die Schweiz und die Vereinigten Staaten liegen keine Daten vor. Quelle: Eurobarometer (Datenbank).

OECD-Mitgliedsland, nämlich in Dänemark, ebenso hoch wie in die politischen Parteien; in allen anderen Ländern genießen die politischen Parteien ein geringeres Vertrauen. Das Vertrauen in die politischen Parteien ist mit unter $10 \%$ der Befragten in den Ländern am niedrigsten, die am stärksten von den Krisen der öffentlichen Haushalte in Mitleidenschaft gezogen wurden, z.B. Slowenien, Griechenland, Spanien, Italien und Portugal. Dies sind zugleich die Länder, in denen das Vertrauen in die Regierung ebenfalls am niedrigsten ist.

Ein Grundsatz der Demokratie - neben freien und fairen Wahlen - ist die Einhaltung des Prinzips der Rechtsstaatlichkeit, die sowohl eine Ergebnis- als auch eine Prozessmessgröße ist. Dies bedeutet, dass niemand - auch nicht der Staat - über dem Gesetz steht, die Gesetze die Grundrechte schützen und die Gerichtsbarkeit für alle zugänglich ist. Dies spiegelt sich in der starken Korrelation zwischen dem Vertrauen wider, das die Menschen in ihre nationale Regierung und in das Justizsystem setzen (Abbildung 1.6). Das Vertrauen in das Justizsystem stellt sowohl ein Ergebnis als auch einen wichtigen Aspekt der Staats- und Verwaltungsführung dar und steht mit der Integrität in engem Zusammenhang.

Ein anderer üblicherweise herangezogener Hilfsindikator für die Messung des Vertrauens in das politische System ist die Wahlbeteiligung. Es gibt indessen zwei konkurrierende Hypothesen bezüglich des Zusammenhangs zwischen der Wahlbeteiligung und dem Vertrauen: Die erste lautet, dass eine höhere Wahlbeteiligung ein größeres Vertrauen in das politische System widerspiegeln könnte, die zweite hingegen, dass ein geringeres Vertrauen in die amtierende Regierung zu einer höheren Wahlbereitschaft führen könnte, um diese abzuwählen. Der Korrelationskoeffizient zwischen dem Vertrauen in die Regierung und der Wahlbeteiligung ist indessen unerheblich.

Wenn sie ihrem Vertrauen in die nationale Regierung Ausdruck verleihen, urteilen die Bürger auch über die politische Führung ihres Landes ( $\left.R^{2}: 0.9\right)$ (Abbildung 1.7). Es bleibt dahingestellt, ob sich diese politische Führung nur auf die Regierungspolitiker oder auch auf 


\section{Abbildung 1.6. Das Vertrauen in das Justizsystem ist wichtig für das Vertrauen in die nationale Regierung}

Korrelation zwischen dem Vertrauen in die nationale Regierung und dem Vertrauen in das Justizsystem (2012)

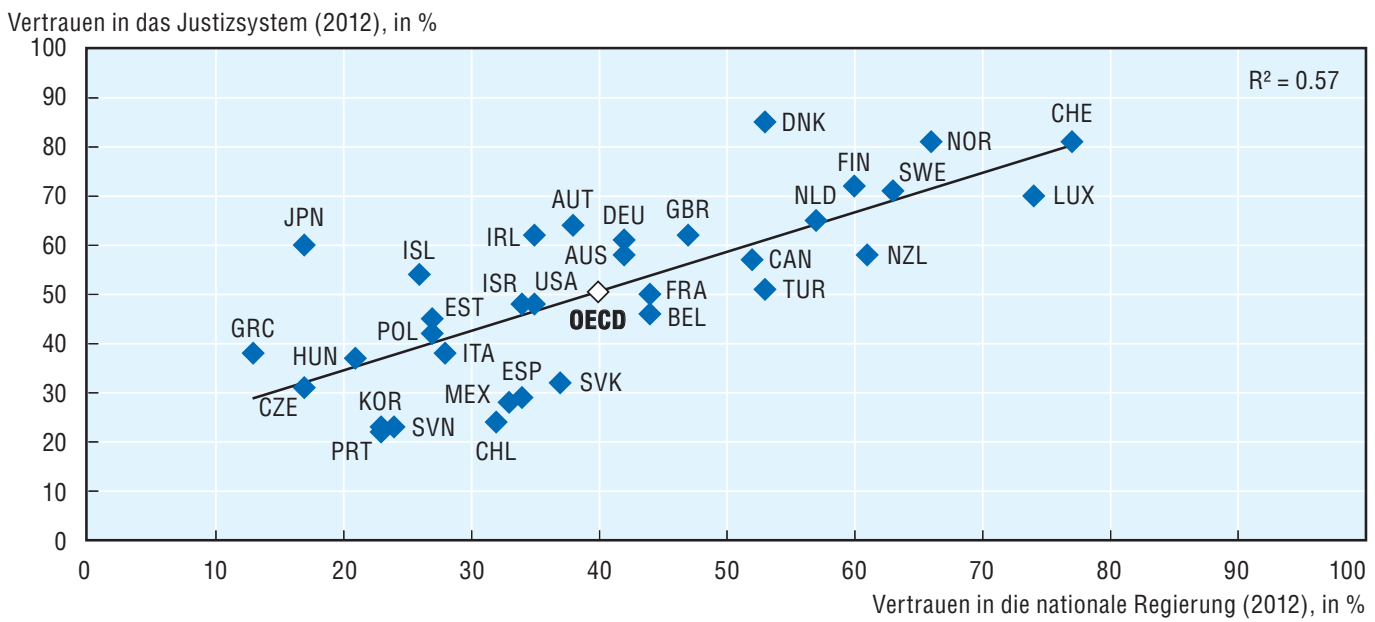

Anmerkung: Die Daten über das Vertrauen in die nationale Regierung beziehen sich auf den Prozentsatz der Befragten, die mit „Ja“ auf die folgende Frage antworteten: „Haben Sie in diesem Land Vertrauen in die folgenden Einrichtungen/Institutionen oder nicht? Wie sieht es aus mit der nationalen Regierung?“ Die Daten über das Vertrauen in das Justizsystem beziehen sich auf den Prozentsatz der Befragten, die mit "Ja" auf die folgende Frage antworteten: „Haben Sie in diesem Land Vertrauen in die folgenden Einrichtungen/Institutionen oder nicht? Wie sieht es aus mit dem Justizsystem und den Gerichtshöfen?" Die Daten für Chile, Deutschland und das Vereinigte Königreich beziehen sich auf 2011 anstelle von 2012.

Quelle: Gallup World Poll.

\section{Abbildung 1.7. Führungskompetenz ist entscheidend für das Vertrauen in die nationale Regierung}

Korrelation zwischen dem Vertrauen in die nationale Regierung und dem Vertrauen in die politische Führung eines Landes (2012)

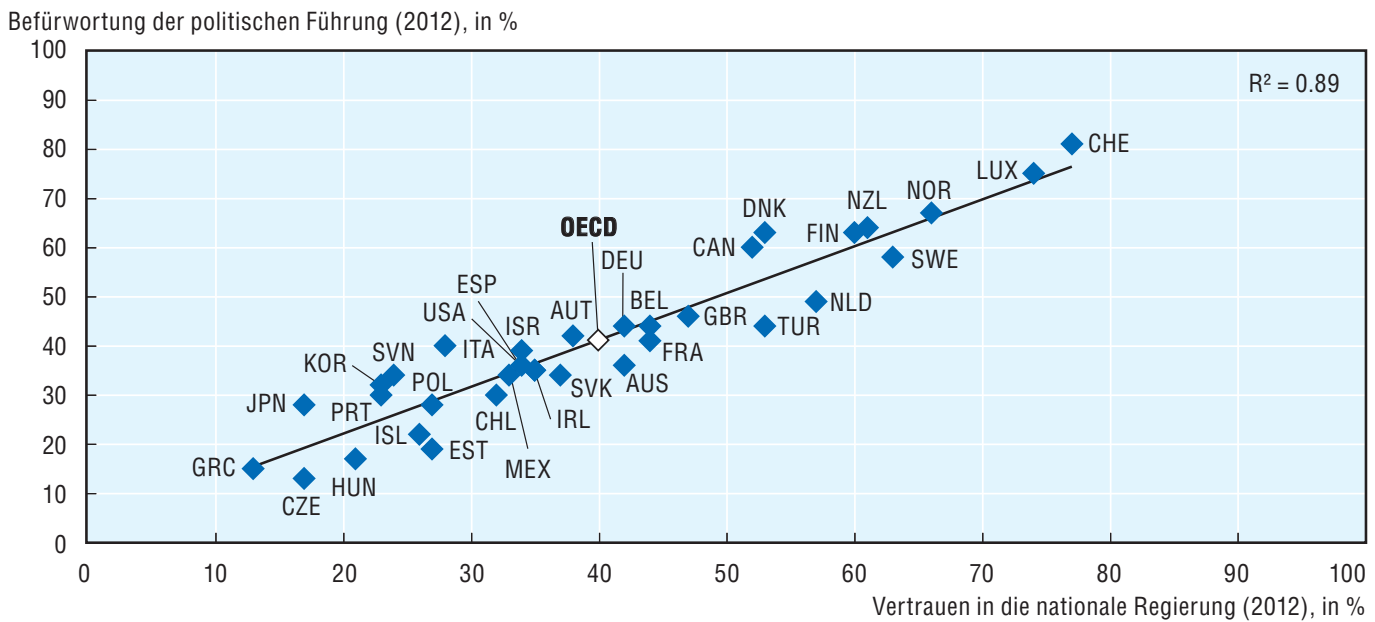

Anmerkung: Die Daten zum Vertrauen in die nationale Regierung beziehen sich auf den Prozentsatz der Befragten, die mit „Ja“ auf die folgende Frage antworteten: „Haben Sie in diesem Land Vertrauen in die folgenden Einrichtungen/ Institutionen oder nicht? Wie sieht es aus mit der nationalen Regierung?“ Die Daten zur Befürwortung der politischen Führung beziehen sich auf den Prozentsatz der Befragten, die mit „Befürworten“ auf die folgende Frage antworteten: „Befürworten Sie die Arbeit der politischen Führung oder nicht?“ Die Daten für Chile, Deutschland und das Vereinigte Königreich beziehen sich auf 2011 anstelle von 2012. Quelle: Gallup World Poll.

die Verwaltungsspitzen bezieht. Dies zeigt jedoch, wie wichtig Führungskompetenz im Rahmen der Staats- und Verwaltungsführung ist und dass eine gut funktionierende politisch-administrative Schnittstelle notwendig ist, die die Vision, Ergebnisse und Integrität der Regierung unterstützt. 


\section{Mesoebene}

Auf Mesoebene kann sich Vertrauen auf die strategische Politikgestaltung beziehen - die Fähigkeit der Regierungen, wirtschaftliche und soziale Probleme zu bewältigen sowie positive Erwartungen für das künftige Wohlergehen zu wecken. Regierung und Verwaltung auf einen Blick 2013 enthält mehrere Indikatoren, die die Komponenten und Ergebnisse der strategischen Politikgestaltung untersuchen, z.B. Gerechtigkeit (Kapitel 2), Risikomanagement (Kapitel 2), Tragfähigkeit der öffentlichen Finanzen (Kapitel 2), Finanzierungssalden (Kapitel 3), Verschuldungsniveau (Kapitel 3) und Haushaltspraxis (Kapitel 4). Werden diese Indikatoren jedoch auf den Grad und die Veränderung des Vertrauens in den Staat bezogen, besteht in keinem dieser Fälle eine starke Korrelation.

Die Höhe der Ausgaben für die soziale Sicherung (einschließlich Arbeitslosigkeit, Versicherung, Renten und Sozialleistungen) wies eine geringe Korrelation mit dem Grad des Vertrauens in den Staat auf $\left(R^{2}: 0.44\right)$. Da die Sozialprogramme in einer Reihe von Ländern in den Fokus der Haushaltskonsolidierung geraten sind, könnte das Vertrauen in den Staat durch Veränderungen der Komponenten und Zugangsregelungen solcher Programme eine zusätzliche Eintrübung erfahren, da dies als Änderung des Gesellschaftsvertrags zwischen dem Staat und seinen Bürgern aufgefasst wird. Der Effekt auf das Vertrauen der Öffentlichkeit ließe sich indessen durch die Verfahren abschwächen, mittels derer die Reformen durchgeführt werden. Dies zeigt, wie wichtig Gerechtigkeit ist, sowohl in Bezug auf die Ergebnisse - wobei das Augenmerk darauf liegt, wer in welchem Umfang betroffen ist und wie gerecht die Last verteilt ist - als auch auf die Prozesse, durch die Entscheidungen getroffen werden, wobei es darauf ankommt, wie transparent der Entscheidungsprozess und die diesem zu Grunde liegenden Erkenntnisse sind und welche Möglichkeiten zur Mitwirkung den von den Entscheidungen Betroffenen offenstehen. Auf diese Weise lässt sich das Vertrauen in den Staat weiter stützen, indem Mitwirkung gefördert und Vertrauen in die von den Entscheidungsträgern verwendeten Erkenntnisse und Kriterien aufgebaut und damit die Legitimität ihrer Entscheidungen erhöht wird.

Es ist nicht unbedingt ein klarer Zusammenhang zwischen einer umsichtigen Finanzpolitik und dem Vertrauen in den Staat festzustellen. Offenbar besteht im Falle eines geordneten Staatshaushalts überhaupt kein großer Zusammenhang. Wenn Länder jedoch ernsthafte Schwierigkeiten mit ihrem Staatshaushalt haben, wird dieser zu einem vorrangigen Problem. Dies ist in Abbildung 1.8 gut dokumentiert, in der die starke negative Korrelation $\left(R^{2}: 0,81\right)$ für die fünf europäischen Länder mit gravierendem Staatsschuldenproblem (vgl. den Teil „Im Fokus“) dargestellt ist. Eine hohe Verschuldung im Verhältnis zum BIP stellt daher u.U. die Verlässlichkeit der Regierung sowie ihre Fähigkeit zur Begrenzung der Unsicherheit auf ein Mindestmaß in Frage.

\section{Mikroebene}

Auf der Mikroebene liegt das Augenmerk auf den Erfahrungen, die der Bürger mit dem Staat macht, wenn dieser öffentliche Dienstleistungen erbringt. Die Zufriedenheit mit den öffentlichen Dienstleistungen ist deutlich höher als das Vertrauen in den Staat, eine höhere Zufriedenheit mit den Dienstleistungen schlägt sich jedoch nicht unbedingt in höherem Vertrauen in den Staat nieder.

Die Umfrageergebnisse lassen darauf schließen, dass die Bürger zwischen den verschiedenen Bereichen und Stellen unterscheiden können, die den öffentlichen Sektor ausmachen, wenn sie konkret danach befragt werden (Abbildung 1.9). 2012 war das Vertrauen in die örtliche Polizei sowie das Gesundheitswesen im Durchschnitt der OECDMitgliedsländer mit 72\% bzw. 71\% am höchsten, gefolgt vom Bildungswesen (66\%) und dem 


\section{Abbildung 1.8. Die Rolle der Staatsverschuldung spielt nur in Ländern mit Haushaltskrise eine Rolle}

Korrelation zwischen dem Vertrauen in die nationale Regierung (2012) und dem öffentlichen Schuldenstand (2011)

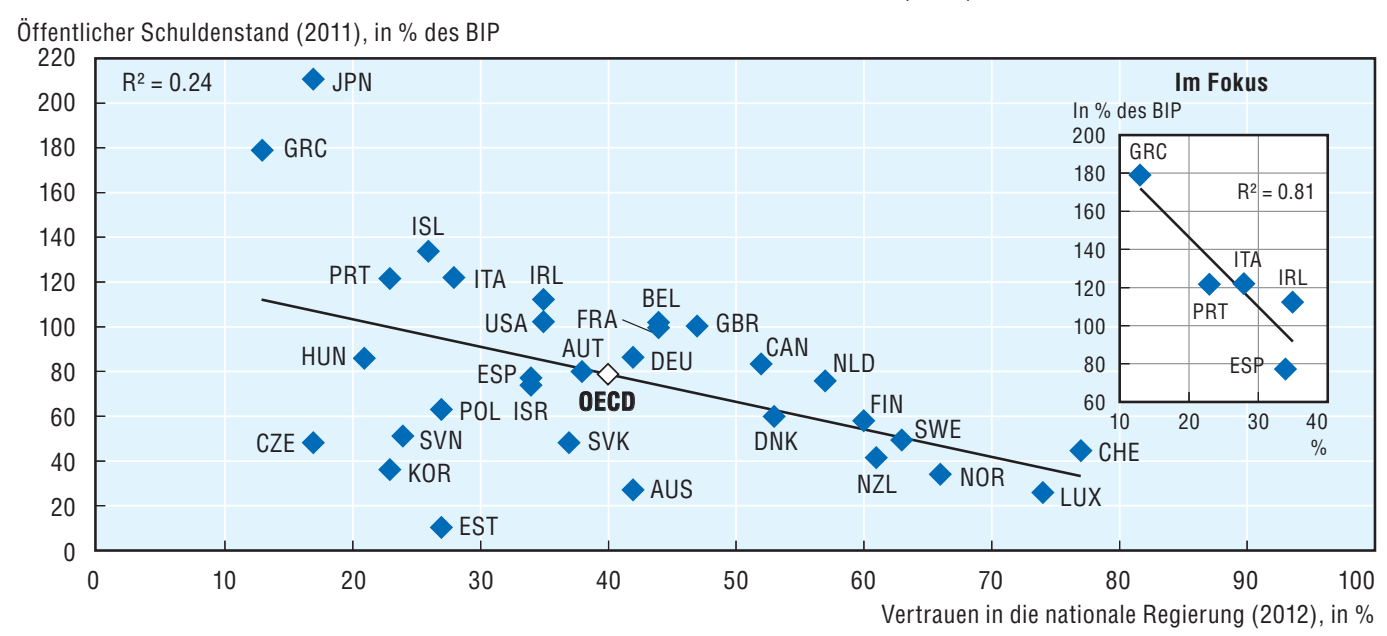

Anmerkung: Die Daten zum Vertrauen in die nationale Regierung beziehen sich auf den Prozentsatz der Befragten, die mit „Ja“ auf die folgende Frage antworteten: „Haben Sie in diesem Land Vertrauen in die folgenden Einrichtungen/ Institutionen oder nicht? Wie sieht es aus mit der nationalen Regierung?" Der öffentliche Schuldenstand bezieht sich auf den Brutto-Gesamtschuldenstand des Staatssektors. Die Daten zum Vertrauen in die nationale Regierung für Chile, Deutschland und das Vereinigte Königreich beziehen sich auf 2011 anstelle von 2012.

Quelle: Gallup World Poll und OECD (2013), „OECD Wirtschaftsausblick 93“, OECD Economic Outlook: Statistics and Projections (Datenbank), Mai 2013, http://dx.doi.org/10.1787/data-00655-en.

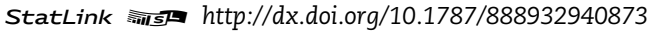

Justizsystem (51\%); am geringsten war das Vertrauen in die nationale Regierung (40\%). Dies unterstreicht, wie wichtig es ist, zu verstehen, was mit „Staat“ bzw. „Regierung“ gemeint ist: Auf welche Elemente des breiten Spektrums an Akteuren, Institutionen und Rechtsvorschriften, die den Staat ausmachen, sowie der Infrastrukturen, die der Erbringung öffentlicher Dienstleistungen dienen, beziehen sich die Bürger, wenn sie Angaben zum Grad ihres Vertrauens in den Staat bzw. die Regierung machen?

Jenseits des allgemeinen Bilds bestehen in Bezug auf den Zusammenhang zwischen dem Vertrauen in die nationale Regierung und der konkreten Zufriedenheit mit den öffentlichen Dienstleistungen erhebliche Unterschiede zwischen den einzelnen Ländern. Der Abstand zwischen den beiden Messgrößen ist in Island, Japan, Slowenien und der Tschechischen Republik, wo die Zufriedenheit mit den öffentlichen Dienstleistungen hoch ist, besonders groß, wohingegen das Vertrauen in die nationale Regierung und die Zufriedenheit mit den öffentlichen Dienstleistungen in der Schweiz, Luxemburg und der Türkei sehr nahe beieinander liegen. Dies bekräftigt die Auffassung, dass die aktuellen Antworten auf Fragen nach dem Vertrauen in die nationale Regierung, wie sie sich in den verfügbaren Daten niederschlagen, in manchen Ländern u.U. eher kurzfristige Wahrnehmungen des politischen Systems als der Regierung und der öffentlichen Verwaltung als Institutionen erfassen.

Das Vertrauen ist auf lokaler Ebene gewöhnlich am größten, wo die Dienstleistungen erbracht werden und der Zusammenhang mit den Ergebnissen des Staats am deutlichsten sichtbar wird. Das Vertrauen ist in der Regel darüber hinaus bei tatsächlichen Nutzern öffentlicher Dienstleistungen größer als bei Personen, die solche Dienstleistungen nicht in Anspruch nehmen. Eine Untersuchung der Unterschiede beim Vertrauen auf den verschiedenen staatlichen Ebenen und in die verschiedenen Arten öffentlicher Einrichtungen würde einige Hinweise auf die Faktoren liefern, die die öffentliche Wahrnehmung des Staats prägen, sowie auf die verschiedenen politischen Hebel, mit denen sich die Wahrnehmung der betreffenden Bereiche der öffentlichen Verwaltung verbessern lässt. 


\section{Abbildung 1.9. Die Zufriedenheit mit den öffentlichen Dienstleistungen ist größer als das Vertrauen in die Regierung (2012)}

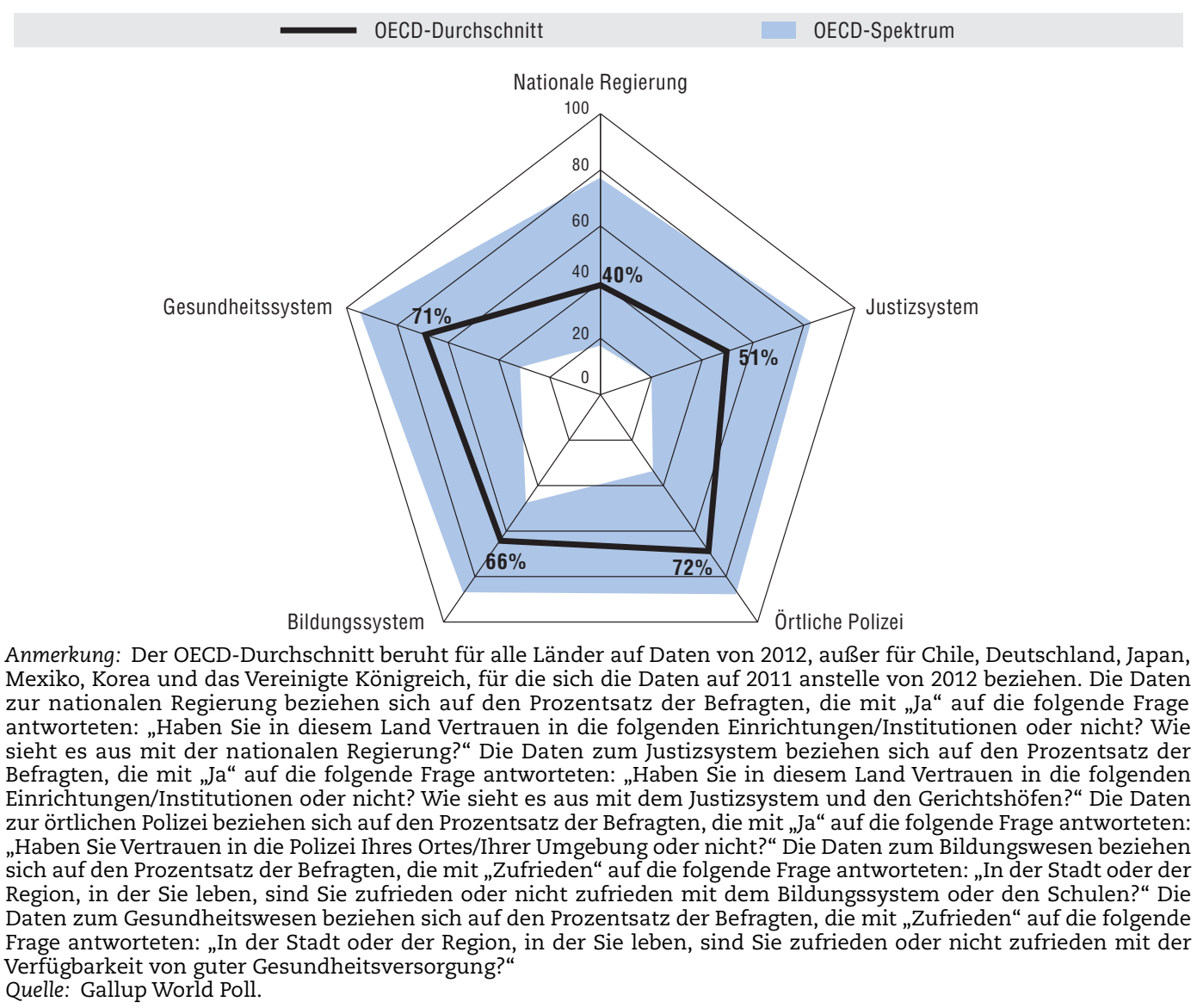

StatLink :

Kapitel 9 zur Qualität der öffentlichen Dienstleistungen beruht auf einem allgemeinen Rahmen für die Dienstleistungsqualität (Tabelle 1.2). Das Kapitel baut auf den bestehenden Indikatoren für die Dienstleistungsqualität auf, die entsprechend den wichtigsten Aspekten der Qualität dargestellt sind: Zugang, Zeitgerechtigkeit, Verlässlichkeit und Zufriedenheit mit den Dienstleistungen. Darüber hinaus werden auch Daten zur Inanspruchnahme internetgestützter staatlicher Dienstleistungen dargestellt, da Regierung und Verwaltung, Unternehmen sowie Bürger solche zunehmend nutzen. Diese Aspekte der Dienstleistungsqualität überschneiden sich mit einigen wichtigen Aspekten der Staats- und Verwaltungsführung, die für Bürger wichtig sind, z.B. Inklusivität (Zugang), Bürgerorientierung und Verlässlichkeit. Da es viele Facetten dieser wichtigen Aspekte der Qualität gibt, wird in einem ersten Versuch für jeden der vier Politikbereiche eine Facette dargestellt, sofern Daten vorliegen: Erschwinglichkeit, Zeitgerechtigkeit, Angemessenheit und geäußerte Zufriedenheit mit den Dienstleistungen.

\section{Integrität: ein Querschnittsthema}

Integrität scheint für das Vertrauen in den Staat von grundlegender Bedeutung zu sein, da die Korrelation zwischen der Wahrnehmung von Korruption und dem Vertrauen in den Staat hoch ist (Abbildung 1.10). Werkzeuge und Mechanismen, die der Schaffung von Integrität dienen und grundlegende Prozesse der Staats- und Verwaltungsführung sind, zielen auf die Vermeidung von Korruption als Ergebnis und auf die Förderung hoher Verhaltensstandards ab, was dazu beiträgt, die Glaubwürdigkeit und Legitimität der an der politischen Entscheidungs- 
Tabelle 1.2. Rahmen für die Dienstleistungsqualität

\begin{tabular}{|c|c|c|c|}
\hline Zugang & Bürgerorientierung & Verlässlichkeit & Zufriedenheit \\
\hline Erschwinglichkeit & Zeitgerechtigkeit & $\begin{array}{l}\text { Angemessenheit/Kompetenz/Kundenrechte } \\
\text { (Möglichkeiten, Beschwerden vorzubringen, } \\
\text { Vorschläge einzureichen, Unterstützung und/ } \\
\text { oder Entschädigung zu erhalten) }\end{array}$ & $\begin{array}{l}\text { Geäußerte Zufriedenheit } \\
\text { (Wahrnehmung) }\end{array}$ \\
\hline Geografische Nähe & $\begin{array}{l}\text { „Anpassung“ der Dienstleistungen } \\
\text { an den Bedarf }\end{array}$ & $\begin{array}{l}\text { Greifbarkeit } \\
\text { (Anlagen, Maschinen usw.) }\end{array}$ & $\begin{array}{l}\text { Geäußertes Vertrauen } \\
\text { (Wahrnehmung) }\end{array}$ \\
\hline $\begin{array}{l}\text { Anpassungsmaßnahmen } \\
\text { für Personen mit Behinderungen }\end{array}$ & $\begin{array}{l}\text { Kundendienst } \\
\text { (Freundlichkeit und Behandlung) }\end{array}$ & Einheitlichkeit/Gerechtigkeit & \\
\hline $\begin{array}{l}\text { Anpassung an andere Kulturen } \\
\text { (z.B. Sprachen usw.) }\end{array}$ & $\begin{array}{l}\text { Integrierte Dienstleistungen } \\
\text { (über die verschiedenen } \\
\text { Erbringungskanäle hinweg) }\end{array}$ & $\begin{array}{l}\text { Sicherheitsmaßnahmen } \\
\text { (Vertraulichkeit, Sicherheit) }\end{array}$ & \\
\hline $\begin{array}{l}\text { Zugang zu elektronischen } \\
\text { Dienstleistungen (digitale Kluft) }\end{array}$ & & & \\
\hline
\end{tabular}

Abbildung 1.10. Augen auf bei Korruption!

Korrelation zwischen dem Vertrauen in die nationale Regierung und der Wahrnehmung staatlicher Korruption (2012)

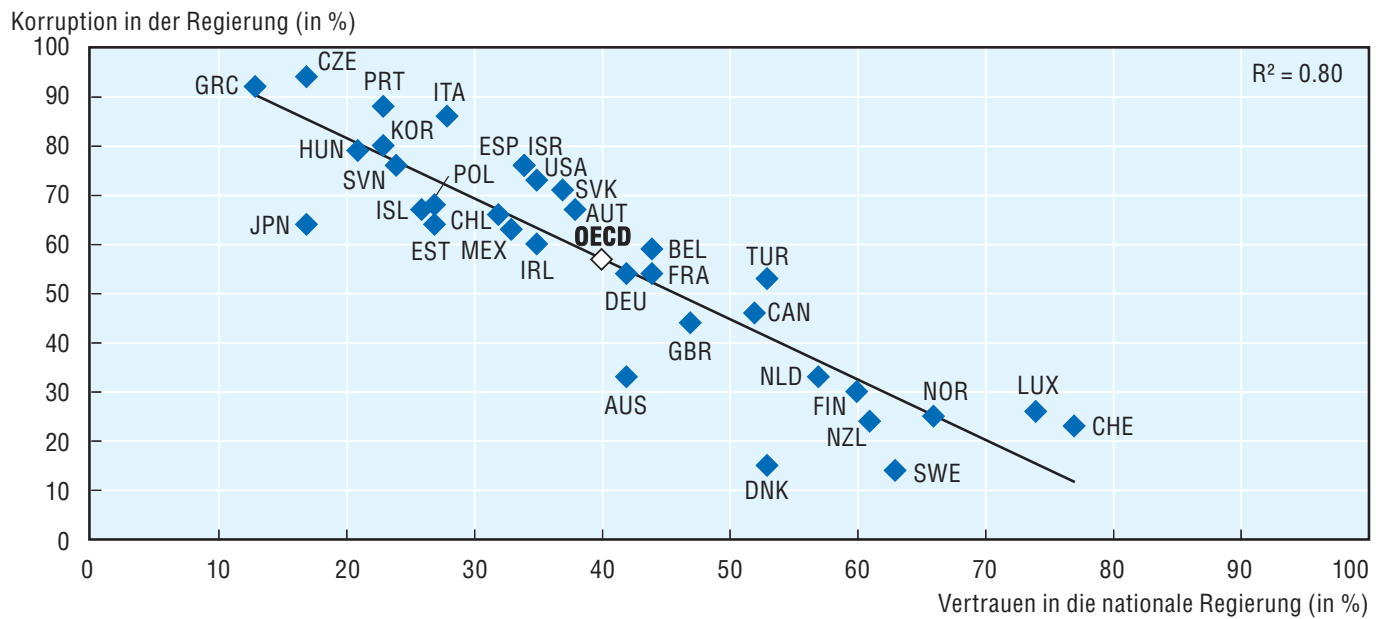

Anmerkung: Die Daten zum Vertrauen in die nationale Regierung beziehen sich auf den Prozentsatz der Befragten, die mit „Ja“ auf die folgende Frage antworteten: „Haben Sie in diesem Land Vertrauen in die folgenden Einrichtungen/ Institutionen oder nicht? Wie sieht es aus mit der nationalen Regierung?" Die Daten zur Wahrnehmung der Korruption in der Regierung beziehen sich auf den Prozentsatz der Befragten, die mit "Ja“ auf die folgende Frage antworteten: „Ist Korruption in der Regierung weit verbreitet oder nicht?“ Die Daten für Chile, Deutschland und das Vereinigte Königreich beziehen sich auf 2011 anstelle von 2012.

Quelle: Gallup World Poll.

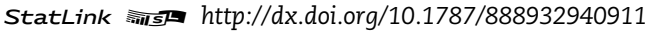

findung beteiligten Akteure zu stärken, die öffentlichen Interessen zu wahren und ein neues Gerechtigkeitsgefühl im Hinblick auf Politikentscheidungen zu bewirken. Politikinstrumente an der Schnittstelle zwischen dem öffentlichen und dem privaten Sektor, wo das Risiko hoch ist - einschließlich eines effektiven Managements von Interessenkonflikten, hoher Verhaltensstandards im öffentlichen Sektor und angemessener Vorschriften für die Lobbyarbeit und die Politikfinanzierung -, können so eingesetzt werden, dass unzulässige Einflussnahme begrenzt wird und Sicherheitsmechanismen eingerichtet werden, um das öffentliche Interesse zu schützen.

Diese Publikation enthält Indikatoren für Praktiken der Staatsführung und -verwaltung, die auf die Verbesserung der Integrität der Regierung und Verwaltung abzielen: Kapitel 8, „Interessenkonflikte und Offenlegung von Vermögen“, Kapitel 8, „Haushaltstransparenz“, und Kapitel 7, „Fairer Wettbewerb im öffentlichen Beschaffungswesen und unter KMU“. 


\section{Schlussbetrachtungen}

Die Erfahrung der institutionellen Herausforderungen der Finanz-, Wirtschafts- und Sozialkrise der jüngsten Vergangenheit hat zur Entstehung einer Vielzahl von Forschungsarbeiten über die Rolle der Staaten in modernen Volkswirtschaften und Gesellschaften beigetragen. Die Rolle, die dem Vertrauen in diesem Zusammenhang zukommt, wird von der politischen Führung wie auch von Wissenschaftlern zunehmend als das potenziell fehlende Element für ein besseres Krisenmanagement und bessere Ergebnisse identifiziert.

Das Vertrauen in den Staat zu verstehen und zu verbessern, scheint eine umfassende, sektorübergreifende Agenda zu erfordern, die mittelfristig ausgerichtet ist und in die zahlreiche Akteure einbezogen werden. Erstens ist es notwendig, eine umfassendere Messgröße für das Vertrauen in den Staat zu entwickeln sowie die Bestimmungsfaktoren für das Vertrauen besser zu identifizieren. Hierfür muss sich unser Verständnis und unsere Kenntnis des Konzepts des Vertrauens und insbesondere des Vertrauens in den Staat verbessern. Darüber hinaus wäre eine regelmäßige international vergleichbare Messung des Vertrauens der Bürger und Unternehmen in den Staat notwendig. Dies ließe sich durch eine (oder mehrere) neue Erhebung(en) erzielen, bei der Elemente bestehender Erhebungen miteinander kombiniert werden, oder durch die Verbesserung bestehender Erhebungen (im Hinblick auf ihre Repräsentativität, das Erhebungsdesign sowie durch Berücksichtigung der im Anhang aufgeführten Fragen und Antwortskalen). Gegenwärtig ist kein nationales Statistikamt mit der Messung des Vertrauens in den Staat befasst.

Zweitens sind weitere Arbeiten an einem analytischen Rahmen erforderlich, gefolgt von weiterentwickelten ökonometrischen Methoden, damit die Zusammenhänge zwischen dem Vertrauen in den Staat und den verschiedenen staatlichen Institutionen sowie Aspekten staatlichen Handelns eingehender untersucht werden können, um Schlüsse zu ziehen, die auf Bereiche hindeuten könnten, in denen staatliches Handeln sinnvoll sein kann. Besonders wichtig ist es, dass wir verstehen, auf welche Art und Weise sich die verschiedenen staatlichen Ebenen und Institutionen auf das Vertrauen in den Staat auswirken, wobei zunächst zu untersuchen wäre, welche Rolle die politische Führung der einzelnen Länder, die verschiedenen Politiksektoren und die für die Dienstleistungserbringung zuständigen Stellen auf Ebene der nachgeordneten Gebietskörperschaften spielen, wenn Entscheidungen getroffen, übermittelt und umgesetzt werden, und welche Verantwortung sie für diese Entscheidungen jeweils tragen. Die OECD könnte bei der Entwicklung internationaler Vergleiche behilflich sein und die Länder dabei unterstützen, sich in Bezug auf Strategien und Maßnahmen gegenseitig auszutauschen, mit denen das Vertrauen in den Staat erfolgreich zurückgewonnen wurde. Ein Vorläufer für diese Arbeiten könnten länderspezifische Fallstudien sein, die u.a. im Rahmen der Public Governance Reviews der OECD durchgeführt werden.

Am wichtigsten ist jedoch, dass ein neuerliches Augenmerk auf Vertrauen in den Staat eine neue Perspektive in die öffentliche Governance einbringen kann, wodurch die Rolle der Bürger gestärkt wird. Auf institutioneller Ebene dürfte dies den Gedanken eines Gesellschaftsuertrags zwischen den Bürgern und dem Staat verstärken, wobei erstere ihren Beitrag nicht nur durch die Entrichtung von Steuern und die Einhaltung der Gesetze leisten, sondern auch durch die Annahme staatlicher Politikmaßnahmen sowie durch Mitwirkung bei ihrer Gestaltung und Umsetzung. Um solche Unterstützung von den Bürgern zu erlangen, müssen Regierung und Verwaltung indessen inklusiver, transparenter, aufnahmefähiger und effizienter werden. Die entscheidende Rolle, die 
Vertrauen bei effektiven staatlichen Maßnahmen zukommt, zu erkennen und besser zu verstehen, dürfte den Regierungen dabei helfen, ihre Politik- und Reformagenden besser zu gestalten, wodurch für alle bessere Ergebnisse erzielt würden.

\section{Anmerkungen}

1. Vgl. GOV/PGC(2013)1 (www2.oecd.org/oecdinfo/info.aspx?app=OLIScoteEN\&Ref=GOV/PGC(2013)1).

2. Die vorliegende Publikation enthält - im online verfügbaren Anhang „Country Contextual Factors Annex“ - grundlegende Informationen über die politischen Institutionen sowie die Staatsstruktur jedes Mitgliedslandes. Wie diese Institutionen funktionieren, ist in den Indikatorenkapiteln dieser Publikation dargestellt.

\section{Literaturverzeichnis}

Aghion, P., Y. Algan, P. Cahuc und A. Shleifer (2010), „Regulation and distrust“, The Quarterly Journal of Economics, Vol. 125(3), Oxford, S. 1015-1049.

Algan, Y. und P. Cahuc (2010), Handbook of Economic Growth, Vol. 1, North-Holland Publishing, Amsterdam.

Blind, P.K. (2007), „Building trust in government in the twenty-first century: Review of literature and emerging issues", in 7th Global Forum on Reinventing Government Building Trust in Government, S. 26-29.

Bouckaert, G. (2012), „Trust and Public Administration“, Administration, Vol. 60, No. 1, S. 91-115.

Bouckaert, G. und S. van de Walle (2003), „Comparing measures of citizen trust and user satisfaction as indicators of 'good governance': Difficulties in linking trust and satisfaction indicators", International Review of Administrative Sciences, Vol. 69(3), S. 329-344.

Dalton, R.J. (2005), „The social transformation of Trust in Government“, International Review of Sociology, Vol. 15, No. 1, März, S. 133-154.

Dasgupta, P. (2009), „A Matter of Trust: Social Capital and Economic Development“, Präsentation für die Annual Bank Conference on Development Economics (ABCDE), Seoul, Juni.

Easton, D. (1965), A Systems Analysis of Political Life, John Wiley, New York.

Fukuyama, F. (1995), Trust: the Social Virtues and the Creation of Prosperity, Free Press, New York.

Gyorffy, D. (2013), Institutional Trust and Economic policy, Central European University Press, Budapest.

Johnston, W., H. Krahn und T. Harrison (2006), „Democracy, Political Institutions, and Trust: The Limits of Current Electoral Reform Proposals“, The Canadian Journal of Sociology, Vol. 31.2, S. 165-182.

Kampen, J.K., S.V. De Walle und G. Bouckaert (2006), „Assessing the relation between satisfaction with public service delivery and trust in government: The impact of the predisposition of citizens toward government on evaluations of its performance“, Public Performance and Management Review, Vol. 29(4), S. 387-404.

Knack, S. und P.J. Zak (2003), „Building trust: Public policy, interpersonal trust, and economic development“, Supreme Court Economic Review, No. 10, S. 91-107.

Knack, S. und P. Keefer (1997), „Does social capital have an economic payoff? A cross country investigation“, The Quarterly Journal of Economics, Vol. 112(4), Oxford, S. 1251-1288.

Murphy, K. (2004), „The role of trust in nurturing compliance: A study of accused tax avoiders“, Law and Human Behavior, Vol. 28(2), S. 187-209.

Nooteboom, B. (2006), Social Capital, Institutions and Trust, Tilburg University.

Putnam, R. (2000), Bowling Alone: The Collapse and Revival of American Community, Simon and Schuster, New York.

Internationale Erhebungen, in denen das Vertrauen in den Staat gemessen wird: Weblinks

Gallup World Poll: www.gallup.com/strategicconsulting/en-us/worldpoll.aspx.

World Values Survey: www.worldvaluessurvey.org.

Eurobarometer: www.ec.europa.eu/public_opinion/index_en.htm.

Edelman-Trust-Barometer: www.edelman.com/insights/intellectual-property/trust-2013.

Latinobarómetro: www.latinobarometro.org. 


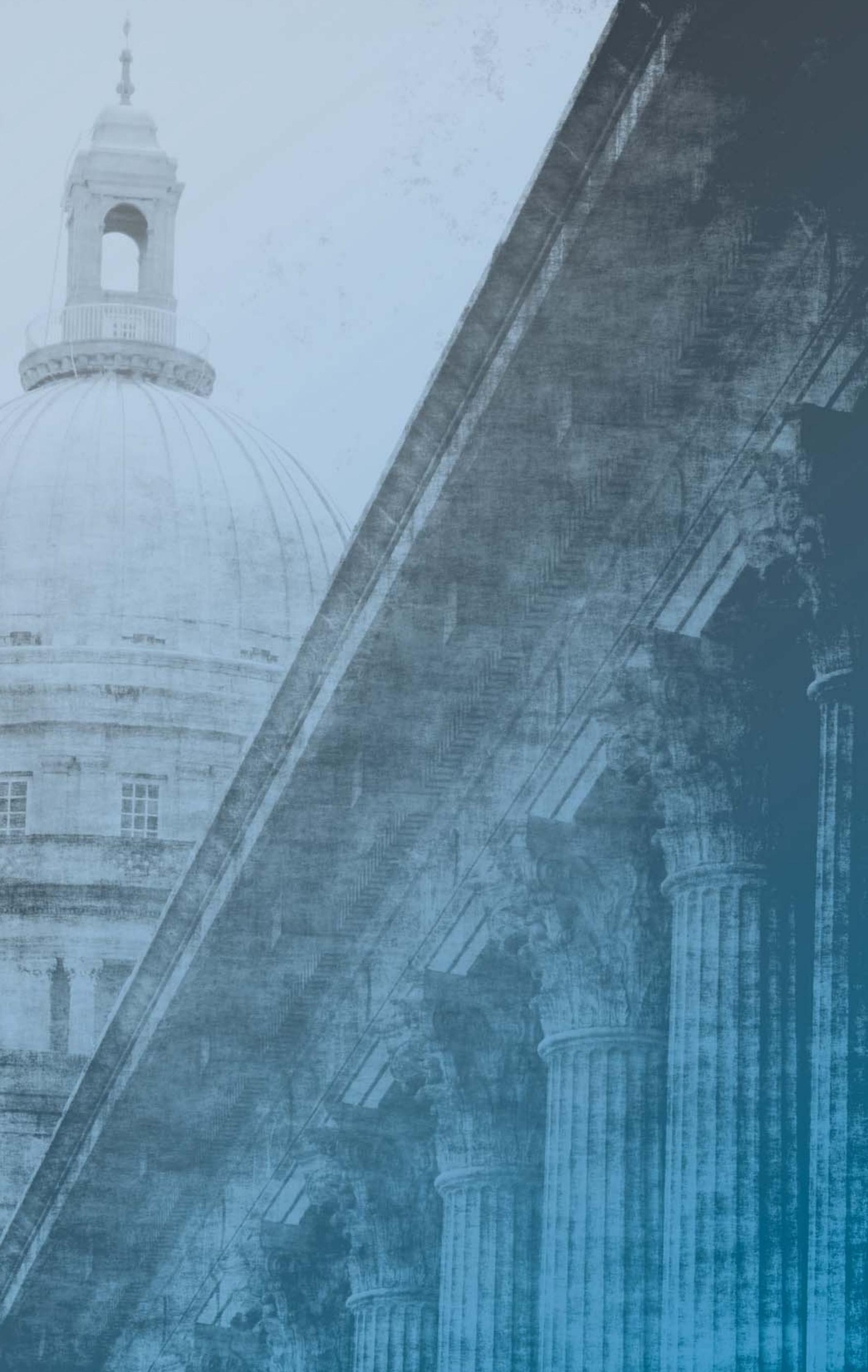


Vertrauen in den Staat bezeichnet die Überzeugung der Bürger und Unternehmen, dass der Staat das tut, was richtig ist und als gerecht empfunden wird. Dies ist eine der entscheidenden Grundlagen, auf denen die Legitimität und Tragfähigkeit politischer Systeme aufbauen. Vertrauen in den Staat ist eine Grundvoraussetzung für den sozialen Zusammenhalt und das gesellschaftliche Wohlergehen, da es die Regierungsfähigkeit der Regierungen beeinflusst und es diesen ermöglicht, ohne Zwangsausübung zu agieren. Es ist daher unabdingbar für das gerechte und effektive Funktionieren staatlicher Institutionen.

Vertrauen in den Staat und seine Institutionen hängt auch von der Kongruenz zwischen den Präferenzen der Bürger und Unternehmen, ihrer Interpretation dessen, was richtig und gerecht und was ungerecht ist, sowie der Wahrnehmung der Leistung des Staates ab. Vertrauen in den Staat ist somit in hohem Maße kulturell geprägt und kontextabhängig. Es gibt Länder mit hohem Vertrauensgrad (High-Trust-Länder), wie z.B. die Schweiz, Luxemburg, Norwegen und Schweden, und Länder mit niedrigem Vertrauensgrad (Low-Trust-Länder), wie z.B. die osteuropäischen Staaten (Abbildung 2.1), und der Grad des Vertrauens in den Staat kann von vielen Kontextfaktoren, wie z.B. der Wirtschaftslage, Naturkatastrophen oder der Verbreitung von Korruption, beeinflusst werden.

Das Vertrauen in den Staat wird vorwiegend durch Meinungsumfragen ermittelt. Auf Grund des Einflusses kultureller und anderer kontextbezogener Faktoren ist bei der Interpretation von Ländervergleichen große Vorsicht geboten. Wertvolle Erkenntnisse lassen sich eher aus der Veränderung des Vertrauensgrads im Zeitverlauf gewinnen als aus der Konzentration auf absolute Vertrauensniveaus. Von 2007 bis 2012 ging das Vertrauen in die nationalen Regierungen im OECDRaum im Durchschnitt um 5 Prozentpunkte von 45\% auf $40 \%$ zurück. Der stärkste Rückgang wurde in Slowenien, Portugal, Griechenland und Irland - Ländern, die sehr stark von der Finanz-, Wirtschafts- und Haushaltskrise betroffen waren verzeichnet. Gleichzeitig stieg in diesem Zeitraum das Vertrauen in die Regierung in der Slowakischen Republik, der Schweiz, Israel, dem Vereinigten Königreich und Frankreich. Weitere Analysen deuten darauf hin, dass die Bürgerinnen und Bürger bei der Frage nach ihrem Vertrauen in die Regierung oder den Staat die politische Führung bewerten.

Der Staat erbringt auch wichtige öffentliche Dienstleistungen in Bereichen wie dem Bildungs- und Gesundheitswesen, der öffentlichen Sicherheit und der Justiz. Die Erbringung dieser Leistungen ist in den meisten OECD-Mitgliedsländern hauptsächlich Verantwortung lokaler Gebietskörperschaften - mit Ausnahme der Justiz, die unabhängig ist. Die Bürgerinnen und Bürger äußern größeres Vertrauen in bzw. höhere Zufriedenheit mit diesen öffentlichen Dienstleistungen als mit dem abstrakteren Konzept der Regierung oder des Staats (Abbildung 2.2). Im OECD-Durchschnitt waren 2012 die Vertrauens-/Zufriedenheitswerte der Polizei am höchsten (72\% der Befragten äußerten Vertrauen in die Polizei), dicht gefolgt vom Gesundheitswesen (71\%). Als nächstes folgte das Bildungswesen (66\%) und schließlich das Justizsystem (50\%).

\section{Methodik und Definitionen}

Die Daten stammen aus dem Gallup World Poll. Der World Poll verwendet proportional geschichtete Zufallsstichproben mit einem Stichprobenumfang von 1000 Einzelpersonen in jedem Land. Weitere Informationen unter: www.gallup.com/strategicconsulting/en-us/worldpoll.aspx.

\section{Weitere Informationen}

OECD (erscheint demnächst), Trust in government, assessing the evidence, understanding the policies, OECD Publishing, Paris.

\section{Anmerkungen zu den Abbildungen}

Die Daten für Chile, Deutschland und das Vereinigte Königreich beziehen sich auf 2011 anstelle von 2012. Die Daten für Island und Luxemburg beziehen sich auf 2008 anstelle von 2007. Die Daten für Finnland, Irland, Norwegen, Österreich, Portugal, die Schweiz, die Slowakische Republik und Slowenien beziehen sich auf 2006 anstelle von 2007.

2.1: Die Daten beziehen sich auf den Prozentsatz der Befragten, die mit "Ja“ auf die folgende Frage antworteten: „Haben Sie Vertrauen in die Regierung?"

2.2: Die Daten für Japan, Korea und Mexiko beziehen sich auf 2011 anstelle von 2012. Die Daten zum Justizsystem beziehen sich auf den Prozentsatz der Befragten, die mit „Ja“ auf die folgende Frage antworteten: „Haben Sie in diesem Land Vertrauen in die folgenden Einrichtungen/Institutionen oder nicht? Wie sieht es aus mit dem Justizsystem und den Gerichtshöfen?" Die Daten zur örtlichen Polizei beziehen sich auf den Prozentsatz der Befragten, die mit „Ja“ auf die folgende Frage antworteten: „Haben Sie Vertrauen in die Polizei Ihres Ortes/Ihrer Umgebung oder nicht?" Die Daten zum Bildungswesen beziehen sich auf den Prozentsatz der Befragten, die mit „Zufrieden“ auf die folgende Frage antworteten: „In der Stadt oder der Region, in der Sie leben, sind Sie zufrieden oder nicht zufrieden mit dem Bildungssystem oder den Schulen?" Die Daten zum Gesundheitswesen beziehen sich auf den Prozentsatz der Befragten, die mit „Zufrieden“ auf die folgende Frage antworteten: „In der Stadt oder der Region, in der Sie leben, sind Sie zufrieden oder nicht zufrieden mit der Verfügbarkeit von guter Gesundheitsversorgung?"

Hinweis zu den Daten für Israel: http://dx.doi.org/10.1787/888932315602. 


\subsection{Vertrauen in die Regierung im Jahr 2012 und Veränderung dieses Werts seit 2007}

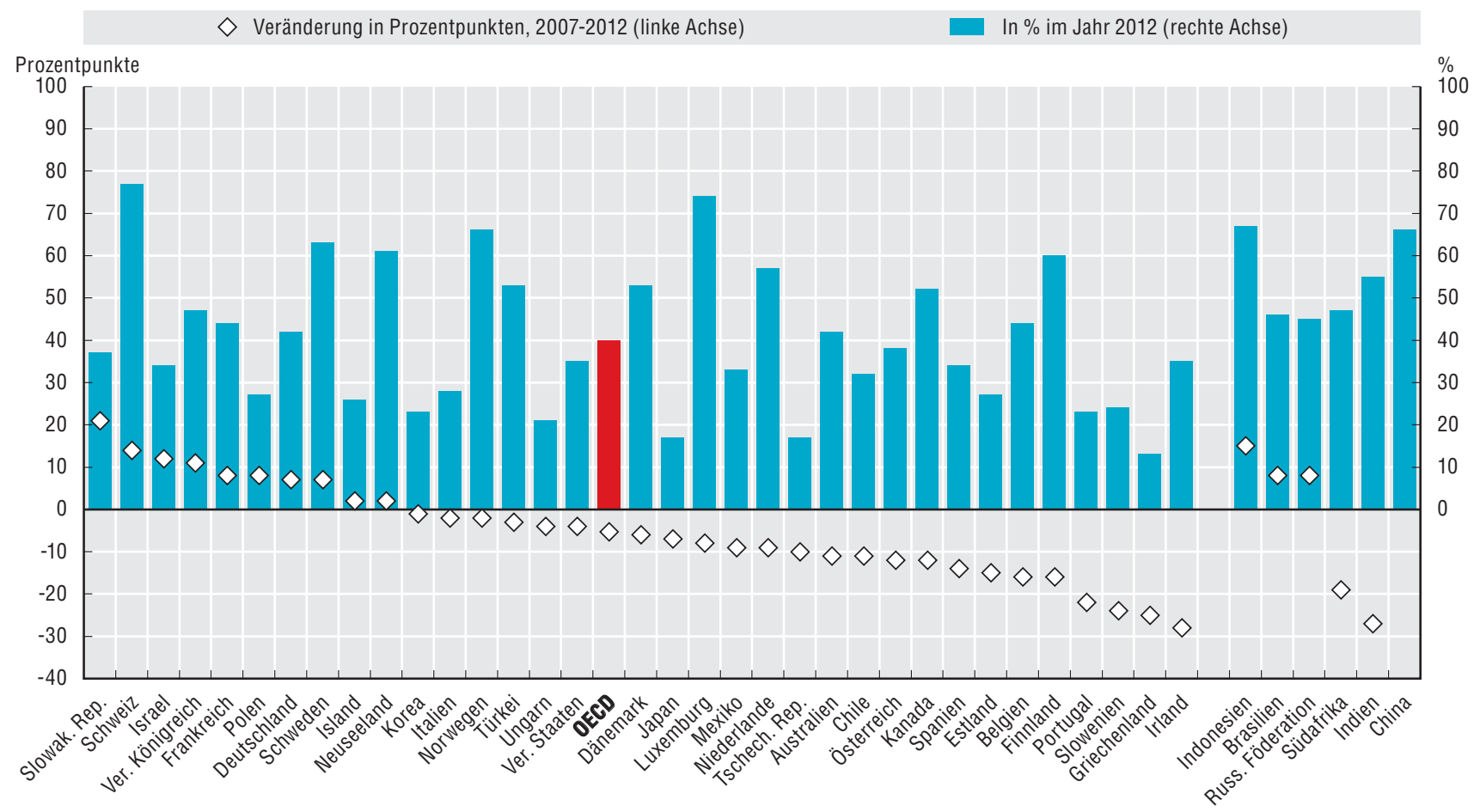

Quelle: Gallup World Poll.

StatLink नinsts http://dx.doi.org/10.1787/888932940740

2.2. Vertrauen in bzw. Zufriedenheit mit staatlichen Institutionen (2012)

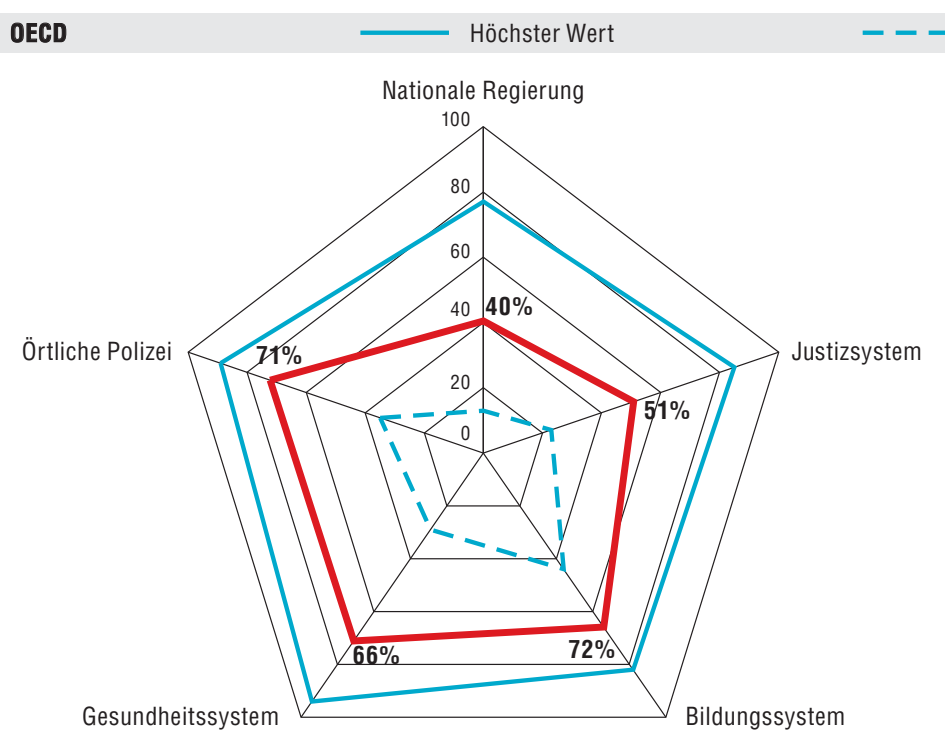

Quelle: Gallup World Poll.

StatLink नillst http://dx.doi.org/10.1787/888932940930 
Eine der zentralen Aufgaben des Staates ist es, gleiche Rahmenbedingungen für seine Bürgerinnen und Bürger sowohl bei den vom Staat befolgten Prozessen - Verfahrensgerechtigkeit als auch bei den erzielten Ergebnissen - Verteilungsgerechtigkeit - zu schaffen. Ein wichtiges Element der Verteilungsgerechtigkeit ist es, Einkommensunterschiede insoweit zuzulassen, als sie Leistung anerkennen und belohnen, und $\mathrm{zu}$ akzeptieren, dass die einzelnen Bürgerinnen und Bürger in unterschiedlichem Maße zum wirtschaftlichen und gesellschaftlichen Wohlstand beitragen. Gleichzeitig sollten die Regierungen eine Minimierung des wirtschaftlichen und gesellschaftlichen Schadens anstreben, der durch Ungleichheit entstehen kann, und den gesellschaftlichen Konsens berücksichtigen. Diese doppelte Zielsetzung hat die Verringerung von Einkommensungleichheiten durch progressive Besteuerung und Einsatz monetärer Transferleistungen (z.B. Renten, Arbeitslosenversicherung) zur Folge.

Seit den 1980er Jahren hat sich das Einkommensgefälle zwischen Armen und Reichen in der Mehrzahl der OECDMitgliedsländer ausgeweitet (OECD, 2011, Divided We Stand: Why Inequality Keeps Rising). Dies zeigt, dass die positiven Effekte des Wirtschaftswachstums nicht allen Bürgerinnen und Bürgern gleichermaßen zugute kommen. Globalisierung, technologischer Fortschritt sowie regulatorische und institutionelle Reformen gelten als Haupttriebfedern zunehmender Einkommensungleichheiten. Die globale Wirtschaftskrise verstärkte diese Entwicklungen weiter, da sie sich unterschiedlich stark auf verschiedene Bevölkerungsgruppen auswirkte; die Hauptlast der Krise wurde von Arbeitslosen und Unterbeschäftigten getragen.

Die meisten OECD-Länder haben eine Reihe von Politikmaßnahmen verabschiedet, um die Einkommensungleichheit in der Gesellschaft und deren langfristige Kosten für die Wirtschaftsentwicklung zu reduzieren. Die sozialen Sicherungssysteme haben dank einer Kombination monetärer Transferleistungen und progressiver Einkommensbesteuerung Wirkung gezeigt. Zusätzlich wurden spezielle Konjunkturpakete zur Ankurbelung der Nachfrage und zur Unterstützung ärmerer Haushalte geschnürt, um so die Auswirkungen der Krise abzufedern. Diese Maßnahmen waren darauf ausgerichtet, der Einkommensungleichheit durch eine Umverteilung von Einkommen zwischen Reichen und Armen zu begegnen, kombiniert mit einer Umverteilung zwischen den Generationen zur Unterstützung bedürftigerer Altersgruppen.

Der Gini-Koeffizient gilt als wichtigster Indikator für den Grad der Einkommensungleichheit eines Landes. Der Einfluss des sozialen Sicherungssystems, das von den Zentralregierungen durch Transferleistungen und Steuern umgesetzt wird, kann durch einen Vergleich der Koeffizienten vor und nach Steuern und Transferzahlungen gemessen werden. Bei Betrachtung einer Mehrheit der OECD-Länder zeigt sich, dass sich im Durchschnitt die Größenordnung der Einkommensungleichheit vor Steuern zwischen 2005 und 2010 nicht verändert hat $(0,47)$. Allerdings war in einigen Ländern, wie z.B. Irland, in den letzten fünf Jahren ein kontinuierlicher Anstieg der Ungleichheit vor Steuern und Transferzahlungen zu beobachten. In diesen Fällen erwiesen sich die staatlichen Umverteilungsmaßnahmen als besonders wichtig - sie bewirkten eine Verringerung des GiniKoeffizienten um rd. 0,26 (verglichen mit einer durchschnittlichen Verringerung von 0,16$)$. Chile dagegen verzeichnete in beiden Jahren die geringste Umverteilung; der Effekt der Umverteilung auf den Gini-Index betrug lediglich 0,02. Im Übrigen scheinen alle Länder an einem progressiven Einkommensteuersystem festzuhalten, wobei Polen und Chile weniger unterschiedliche Steuersätze haben als Irland.

\section{Methodik und Definitionen}

Der Wert des Gini-Koeffizienten liegt zwischen 0 - bei vollkommener Gleichverteilung der Einkommen - und 1 in diesem Fall hätte die reichste Person das gesamte Einkommen. Die Unterschiede zwischen den Ärmsten und den Reichsten werden als das Verhältnis des durchschnittlichen Einkommens der untersten 10\% zum durchschnittlichen Einkommen der obersten 10\% errechnet. Die Umverteilung wird gemessen, indem man die GiniKoeffizienten für das Markteinkommen (d.h. das Bruttoeinkommen vor öffentlichen Transferzahlungen und Steuern) und für das verfügbare Einkommen (d.h. nach Transferzahlungen und Steuern) vergleicht. Die Definition des verfügbaren Haushaltseinkommens berücksichtigt keine nichtmonetären Transferleistungen (Sachleistungen). Die Daten stammen aus der OECD Income Distribution Database. Die in dieser Datenbank erfassten Informationen werden durch ein Netz nationaler Datenlieferanten erhoben, um die Entwicklung der einzelnen Länder im Hinblick auf die Einkommensungleichheit vergleichen zu können.

Bei den Steuerdaten, die aus dem OECD-Bericht Taxing Wages stammen, wurden die für das jeweilige Steuerjahr geltenden Steuersätze angewandt. In Australien, Neuseeland und dem Vereinigten Königreich entspricht das Steuerjahr nicht dem Kalenderjahr. Die Daten zeigen den Unterschied zwischen den Steuersätzen für einen Alleinstehenden ohne wirtschaftlich abhängige Familienangehörige, der $67 \%$ des Durchschnittsverdiensts verdient, und einen Alleinstehenden ohne wirtschaftlich abhängige Familienangehörige, der 167\% des Durchschnittsverdiensts bezieht. Die durchschnittlichen Steuersätze werden als Prozentsatz des Bruttoerwerbseinkommens ausgedrückt. Der Durchschnittsverdienst misst den durchschnittlichen Jahresbruttoverdienst erwachsener vollzeitbeschäftigter Arbeiter und Angestellter in der Industrie.

\section{Weitere Informationen}

OECD (2013), Taxing Wages 2013, OECD Publishing, Paris, http://dx.doi.org/10.1787/tax_wages-2013-en.

OECD (2011), Divided We Stand: Why Inequality Keeps Rising, OECD Publishing, Paris, http://dx.doi.org/10.1787/9789264119536-en.

\section{Anmerkungen zu den Abbildungen}

2.3: Für Mexiko, die Türkei und Ungarn stehen keine Daten zur Verfügung. Für die Schweiz stehen keine Daten für das Jahr 2005 zur Verfügung. 2005: Die Daten für Australien, Deutschland, Finnland, Italien, Norwegen und Schweden beziehen sich auf 2004. Die Daten für Chile, Japan und Korea beziehen sich auf 2006. Die Daten für Neuseeland beziehen sich auf 2003. 2010: Die Daten für Chile, Irland, Japan, Neuseeland und die Schweiz beziehen sich auf 2009.

2.4: Die Lohndaten für die Türkei beruhen auf der alten Definition des Durchschnittsarbeiters (ISIC D, Rev. 3). Die Daten beziehen sich auf die Einkommensteuern zuzüglich Arbeitnehmerbeiträgen zur Sozialversicherung (in \% des Bruttoerwerbseinkommens). In Chile ist der Durchschnittsverdienst von der Einkommensteuer befreit, so dass die Einkommensteuer nur geringen Einfluss auf das Gesamtsteueraufkommen hat.

Hinweis zu den Daten für Israel: http://dx.doi.org/10.1787/888932315602. 
2.3. Differenz zwischen der Einkommensungleichheit vor und nach Steuern und staatlichen Transferzahlungen (2005 und 2010)

\author{
Nach Steuern und Transferzahlungen \\ Vor Steuern und Transferzahlungen
}
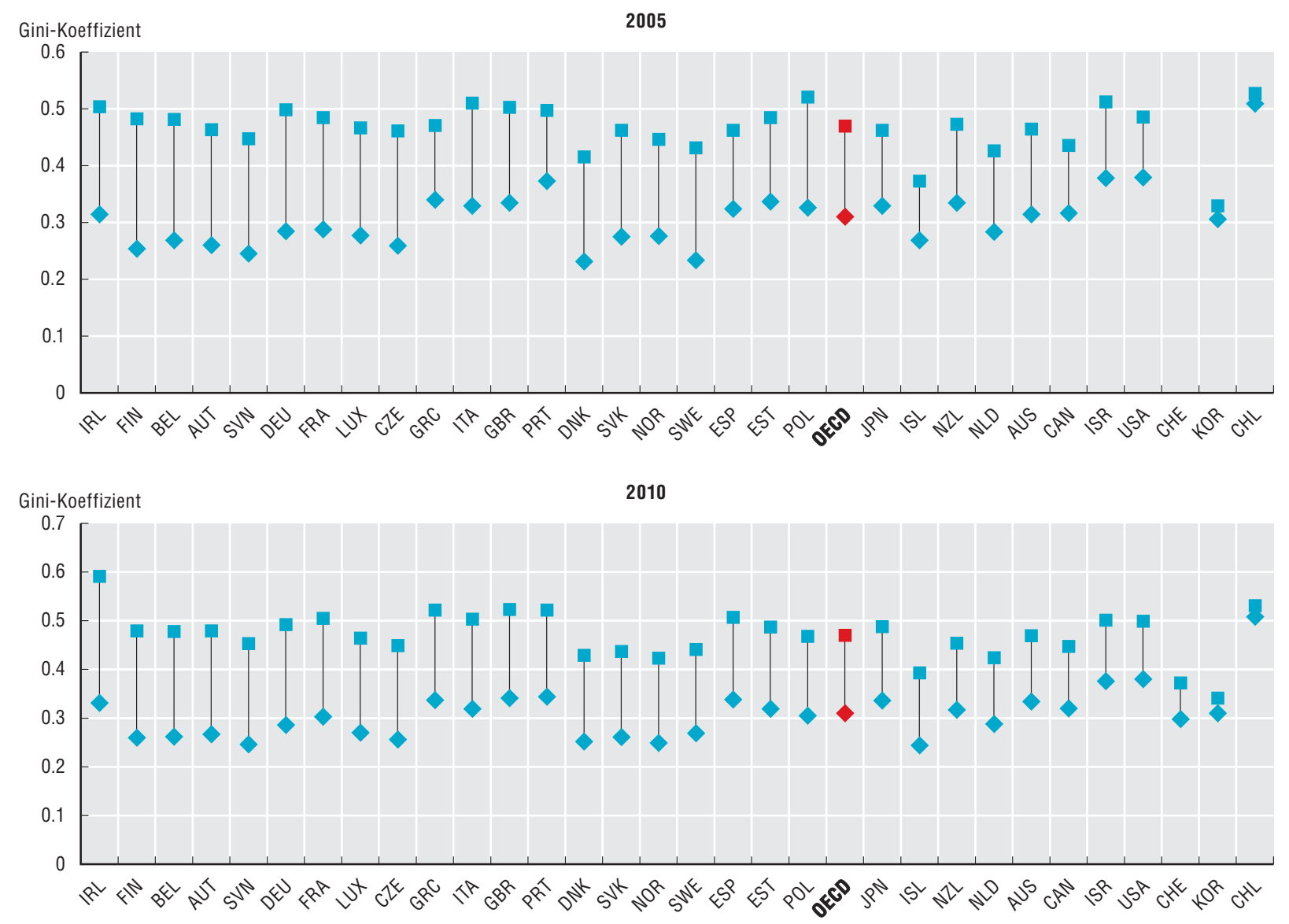

Quelle: OECD Income Distribution Database, www.oecd.org/social/income-distribution-database.htm.

StatLink तiाst http://dx.doi.org/10.1787/888932940949

2.4. Differenz zwischen den durchschnittlichen Einkommensteuersätzen für Alleinstehende (ohne wirtschaftlich abhängige Angehörige) mit einem Verdienst von 167\% und 67\% des Durchschnittsverdiensts (2012)

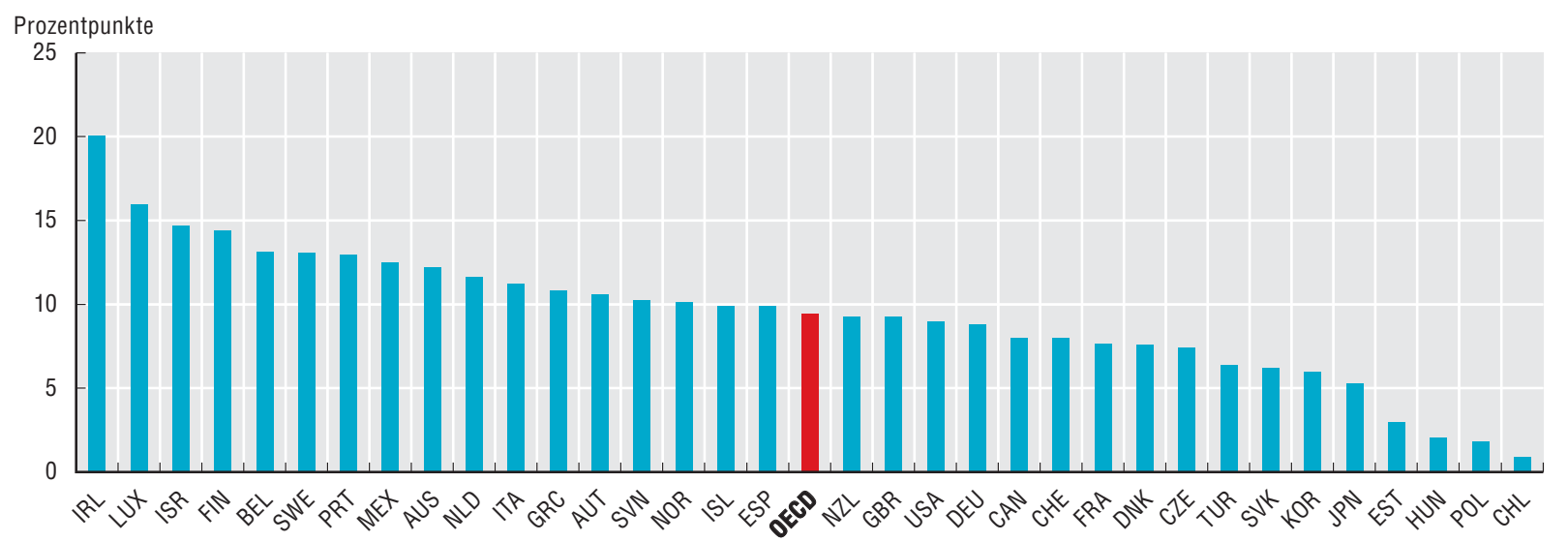

Quelle: OECD (2013), Taxing Wages 2013, OECD Publishing, Paris, http://dx.doi.org/10.1787/tax_wages-2013-en. 
Rechtsstaatlichkeit bedeutet, dass niemand - auch nicht der Staat - über dem Gesetz steht, die Gesetze die Grundrechte schützen und die Gerichtsbarkeit für alle zugänglich ist. Sie unterstellt gemeinsame Handlungsnormen, die durch Gesetze definiert und in der Praxis durch Verfahren und Rechenschaftslegungsmechanismen durchgesetzt werden, wobei Verlässlichkeit, Vorhersehbarkeit und eine „Verwaltung durch das Gesetz“ angestrebt werden. Die Rechtsstaatlichkeit gilt als eine der entscheidenden Dimensionen der Qualität und der verantwortungsvollen Staats- und Regierungsführung eines Landes.

Es gibt verschiedene Interpretationen von Rechtsstaatlichkeit. Wir verwenden die für den Rule of Law Index des World Justice Project (WJP) entwickelte, da es sich hierbei um einen der umfassendsten und systematischsten Ansätze handelt. Demnach umfasst Rechtsstaatlichkeit die folgenden vier universellen Grundsätze: „die Regierung und deren Vertreter sind nach dem Gesetz rechenschaftspflichtig; die Gesetze sind transparent, öffentlich, beständig, gerecht und schützen die Grundrechte, einschließlich der Sicherheit von Personen und Eigentum; der Prozess, mit dem Gesetze erlassen, angewandt und vollzogen werden, ist zugänglich, effizient und gerecht; die Gerichtsbarkeit liegt in Händen kompetenter, ethisch untadeliger und unabhängiger Justizvertreter in hinreichender Zahl, die mit hinreichenden Ressourcen ausgestattet und repräsentativ für die Zusammensetzung der von ihnen vertretenen Bevölkerung sind."

Auf Basis dieser vier Grundsätze wurden im Rahmen des WJP neun Schlüsselfaktoren entwickelt, die als Grundlage für den Rule of Law Index dienen. Vier dieser Faktoren, die für eine verantwortungsvolle Staats- und Regierungsführung besonders wichtig sind, werden im Folgenden näher erläutert. Hierbei handelt es sich um folgende Kategorien: Eingeschränkte Machtbefugnisse der Regierung, Grundrechte, Vollzug der Rechtsvorschriften und Zivilgerichtsbarkeit. Das Thema „Open Government Data“ wird separat behandelt. Die in diesen Faktoren zusammengefassten Informationen stellen die Meinung von Experten und Bürgern dar.

\section{Eingeschränkte Machtbefugnisse der Regierung}

Der Faktor „Eingeschränkte Machtbefugnisse der Regierung“ setzt sich aus sieben maßgeblichen Elementen (Teilfaktoren) zusammen: Die Machtbefugnisse der Regierung sind in den Grundgesetzen definiert; sie werden durch die Legislative wirksam begrenzt; sie werden durch die Judikative wirksam begrenzt; sie werden durch unabhängige Prüf- und Kontrollorgane wirksam begrenzt; Fehlverhalten von Staatsbediensteten wird sanktioniert; die Machtbefugnisse der Regierung unterliegen einer nichtstaatlichen Kontrolle; die Übertragung politischer Machtbefugnisse erfolgt in Einklang mit dem Gesetz. Dieser zusammengesetzte Indikator gibt Aufschluss darüber, ob Machtbefugnisse - sei es durch offizielle Regeln oder auf Grund von Konventionen - so verteilt sind, dass kein einzelnes staatliches Organ in der Lage ist, unkontrollierte Macht auszuüben.

Selbst innerhalb des OECD-Raums bestehen erhebliche Unterschiede zwischen den einzelnen Ländern im Hinblick darauf, wie sehr die Machtbefugnisse der Regierung eingeschränkt sind. In den nordischen Ländern unterliegen die Machtbefugnisse der Regierung den größten Beschränkungen, gefolgt von Australien und Neuseeland, während die Befugnisse der Regierung in der Türkei, Mexiko und Griechenland am wenigsten eingeschränkt sind. Wie erwartet, erreichen die OECD-Länder bei diesem Indikator einen hohen Durch- schnittswert, der besagt, dass die Machtbefugnisse der Regierung einer erheblichen Kontrolle unterliegen. In Partner-, Teilnehmer- und Beitrittsländern, einschließlich der Russischen Föderation, der Ukraine und Chinas, sind die Machtbefugnisse der Regierung weniger stark eingeschränkt.

Der OECD-Durchschnitt für die in diesem zusammengesetzten Indikator aggregierten Teilfaktoren zeigt, dass der am besten entwickelte Teilfaktor mit dem höchsten Wert (wobei ein Wert von 1 für die strikteste Einhaltung der Rechtsstaatlichkeitsprinzipien steht) die Übertragung politischer Machtbefugnisse ist $(0,87)$, während die Sanktionierung des Fehlverhaltens von Staatsbediensteten $(0,67)$ am wenigsten entwickelt ist und die Rolle unabhängiger Prüf- und Kontrollorgane ebenfalls verstärkt werden sollte $(0,73)$.

\section{Grundrechte}

Dieser zusammengesetzte Indikator erfasst den Schutz grundlegender Menschenrechte und ist somit eine normative Größe. Er umfasst die Bewertung acht maßgeblicher Elemente: Gleichbehandlung und Diskriminierungsfreiheit; wirksame Garantien für das Recht auf Leben und Sicherheit der Person; ordnungsgemäße Verfahren und Rechte des Angeklagten; wirksame Garantien für das Recht auf freie Meinungsäußerung; wirksame Garantien für die Glaubens- und Religionsfreiheit; Schutz vor willkürlichen Eingriffen in das Privatleben; wirksame Garantien für die Versammlungs- und Vereinigungsfreiheit und grundlegende Arbeitsrechte. Der Indikator deckt ein relativ eingeschränktes Spektrum von Rechten ab, die fest im internationalen Recht verankert sind und in besonders engem Zusammenhang mit der Rechtsstaatlichkeit und der verantwortungsvollen Staats- und Regierungsführung stehen.

Der durchschnittliche Wert für die OECD-Länder ist mit fast 0,8 hoch, was bedeutet, dass in den meisten Ländern eine starke Garantie für die Grundrechte besteht. Ähnlich wie die Einschränkung der Machtbefugnisse der Regierung werden die Grundrechte von den nordischen Ländern (Schweden, Dänemark, Norwegen und Finnland) am besten garantiert, gefolgt von Neuseeland und Spanien, während die Gewährleistung der Grundrechte erneut in der Türkei, in Mexiko und in Griechenland am geringsten ausgeprägt ist. Dies deutet darauf hin, dass ein starker Zusammenhang zwischen der Beschränkung der Machtfülle der Regierung und der Sicherung von Grundrechten besteht $\left(R^{2}=0,81\right)$. Allerdings ist der Vollzug der Rechtsvorschriften im Vergleich zur Einhaltung der Grundrechte in den OECD-Ländern im Schnitt schwächer entwickelt. Weniger einheitlich stellt sich die Situation in den Partner- und Teilnehmerländern dar, wo in Brasilien und Südafrika eine gute Gewährleistung der Grundrechte - wenn auch unter dem OECD-Durchschnitt - festzustellen ist, während in den übrigen Ländern, insbesondere in China und Ägypten, potenziell Verbesserungsbedarf besteht.

Im Durchschnitt sind in den OECD-Ländern im Bereich der Gleichbehandlung und Diskriminierungsfreiheit weitere Fortschritte erforderlich $(0,7)$, während der Schutz des Rechts auf Leben und die Sicherheit der Person am besten entwickelt ist $(0,86)$.

\section{Vollzug der Rechtsvorschriften}

Der zusammengesetzte Indikator für den Vollzug der Rechtsvorschriften misst, inwiefern Rechtsvorschriften gerecht und wirksam vollstreckt werden. Er bewertet nicht, was und wie der Staat reguliert, sondern lediglich, wie diese Rechtsvorschriften umgesetzt und vollzogen werden. Er untersucht 
Regulierungsbereiche, die von allen Ländern bis zu einem gewissen Grad reguliert werden, wie etwa das öffentliche Gesundheitswesen, den Arbeitsschutz, den Umweltschutz und gewerbliche Aktivitäten. Die Teilfaktoren messen, ob die staatlichen Rechtsvorschriften wirksam vollzogen werden, ob die staatlichen Rechtsvorschriften ohne ungebührliche Einflussnahme angewandt und vollzogen werden; ob Verwaltungsvorgänge ohne unangemessene Verzögerungen durchgeführt werden; ob bei diesen Vorgängen ordnungsgemäße Verfahren eingehalten werden und ob der Staat keine Enteignungen ohne angemessene Entschädigung vornimmt. Dieser Indikator unterscheidet sich somit von den Indikatoren zum Regulierungsmanagement in Regierung und Verwaltung auf einen Blick 2009, die sich auf Konsultationen, Gesetzesfolgenabschätzung und Vereinfachungsstrategien konzentrierten.

Der Vollzug der Rechtsvorschriften ist in Schweden, Japan, Dänemark und Österreich am besten entwickelt, dicht gefolgt von Australien, Norwegen, den Niederlanden, Finnland und Neuseeland. Mexiko, Griechenland, die Türkei und Italien weisen dagegen Verbesserungsbedarf auf. Insgesamt besteht in Anbetracht eines OECD-Durchschnittswerts von 0,71 in vielen OECD-Ländern erheblicher Raum für Verbesserungen. Die Werte der Partner- und Teilnehmerländer liegen alle unter dem OECD-Durchschnitt. Am besten schnitten Brasilien und Südafrika ab, am schlechtesten die Ukraine.

Bei Betrachtung der einzelnen Elemente des Vollzugs der Rechtsvorschriften zeigt sich, dass eine ungebührliche Einflussnahme auf die Anwendung und den Vollzug staatlicher Rechtsvorschriften selten ist, da hierfür der höchste Wert $(0,77)$ verzeichnet wurde, während bei der Effektivität des Vollzugs von Rechtsvorschriften der größte Verbesserungsbedarf besteht $(0,67)$.

\section{Zivilgerichtsbarkeit}

Der Indikator für die Zivilgerichtsbarkeit misst, ob die Bürgerinnen und Bürger Rechtsstreitigkeiten wirksam durch das System der Zivilgerichtsbarkeit klären können. Dies erfordert ein zugängliches, bezahlbares, effektives und unparteiisches System mit interkultureller Kompetenz. Die einzelnen Komponenten messen, ob die Zivilgerichtsbarkeit für die Bevölkerung zugänglich und bezahlbar ist; ob die Zivilgerichtsbarkeit frei von Diskriminierung ist; ob die Zivilgerichtsbarkeit frei von Korruption ist; ob die Zivilgerichtsbarkeit frei von ungebührlicher Einflussnahme der Regierung ist; ob die Zivilgerichtsbarkeit keinen unangemessenen Verzögerungen unterliegt; ob die Zivilgerichtsbarkeit effektiv vollstreckt wird und ob alternative Streitbeilegungsverfahren zugänglich, unparteiisch und effektiv sind.

Den besten Zugang zur Zivilgerichtsbarkeit bieten die nordischen Länder sowie die Niederlande und Deutschland. Italien, Mexiko und die Türkei sind die OECD-Länder mit den niedrigsten Werten für die Zivilgerichtsbarkeit. Von den vier hier untersuchten zentralen Faktoren der Rechtsstaatlichkeit eingeschränkte Machtbefugnisse der Regierung, Grundrechte,
Vollzug der Rechtsvorschriften und Zugang zur Zivilgerichtsbarkeit - ist das durchschnittliche Abschneiden der OECDMitgliedsländer im Fall der Zivilgerichtsbarkeit am niedrigsten $(0,69)$, knapp unter dem Wert für den Vollzug der Rechtsvorschriften $(0,71)$, während Brasilien und Südafrika unter den Partnerländern am besten abschneiden.

Das größte Problem im Zusammenhang mit dem Zugang zur Zivilgerichtsbarkeit ist die Geschwindigkeit der Rechtsprechung $(0,47)$, während bei der Frage, ob die Zivilgerichtsbarkeit frei von Korruption ist, der höchste Wert $(0,8)$ erzielt wurde.

\section{Methodik und Definitionen}

Die Daten werden vom World Justice Project durch eine Reihe von fünf Fragebogen erhoben, die auf dem konzeptuellen Rahmen für den Rule of Law Index beruhen. Die Umfragen werden sowohl in der breiten Bevölkerung als auch unter Experten durchgeführt. Im Schnitt sind mehr als 300 potenzielle lokale Experten pro Land qualifiziert, an der Befragung teilzunehmen. Mit der Bevölkerungsbefragung werden lokal tätige Meinungsforschungsinstitute beauftragt. Es liegen Daten für 28 OECD-Länder sowie 8 Partner- und Teilnehmerländer vor. Alle Variablen für jeden der zusammengesetzten Indikatoren werden kodiert und normalisiert, so dass die Werte in einer Spanne zwischen 0 und 1 liegen, wobei 1 den höchsten und 0 den niedrigsten Wert darstellt. Nähere Informationen zu den ausgewählten Faktoren - Eingeschränkte Machtbefugnisse der Regierung (2.10), Grundrechte (2.11), Vollzug der Rechtsvorschriften (2.12) und Zivilgerichtsbarkeit (2.13) - sind online verfügbar unter: http:// dx.doi.org/10.1787/888932943172, http://dx.doi.org/10.1787/ 888932943191, http://dx.doi.org/10.1787/888932943210, http://dx.doi.org/10.1787/888932943229.

\section{Weitere Informationen}

Aghast, M. et al. (2013), WJP Rule of Law Index 2012-2013, The World Justice Project, Washington.

\section{Anmerkungen zu den Abbildungen}

Für Irland, Island, Israel, Luxemburg, die Schweiz und die Slowakische Republik stehen keine Daten zur Verfügung. Die Daten für Ungarn sind nicht abgebildet.

In den Daten für Italien sind Gesetzesänderungen aus dem Jahr 2013 nicht berücksichtigt.

Hinweis zu den Daten für Israel: http://dx.doi.org/10.1787/888932315602. 


\section{STRATEGISCHE GOVERNANCE}

\section{Rechtsstaatlichkeit}

\subsection{Eingeschränkte Machtbefugnisse der Regierung (2012-13)}

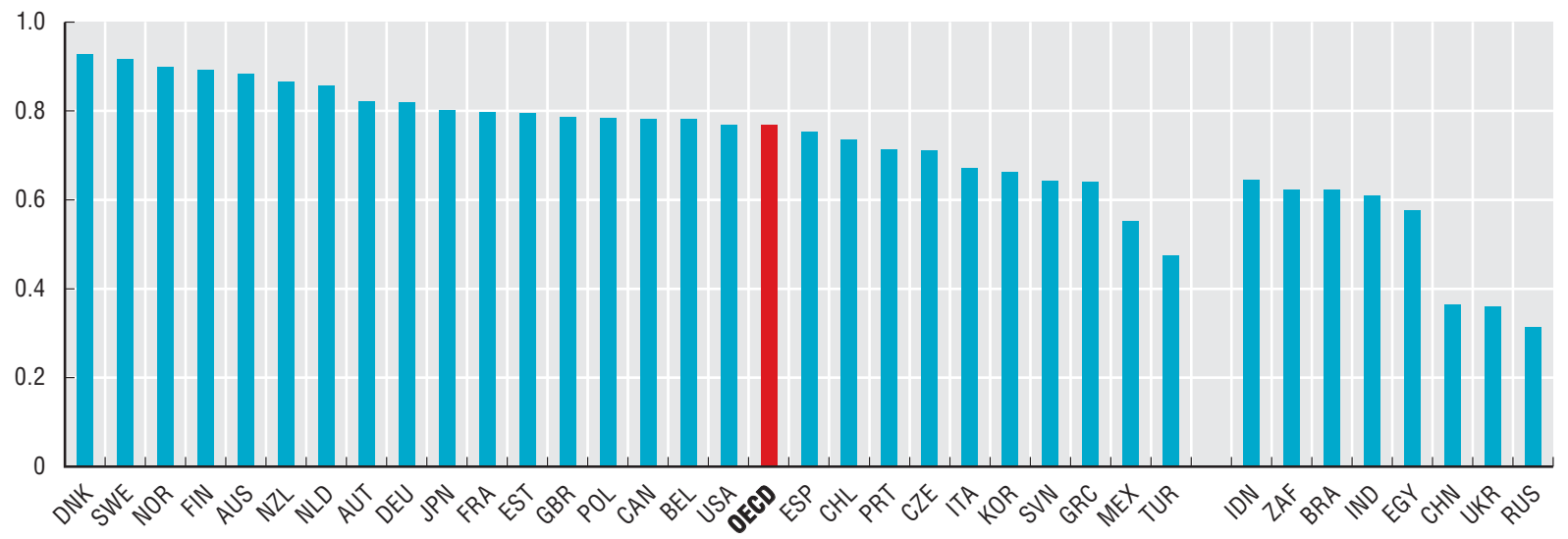

Quelle: The World Justice Project.

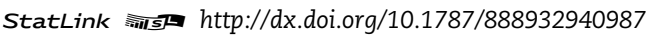

\subsection{Grundrechte (2012-13)}

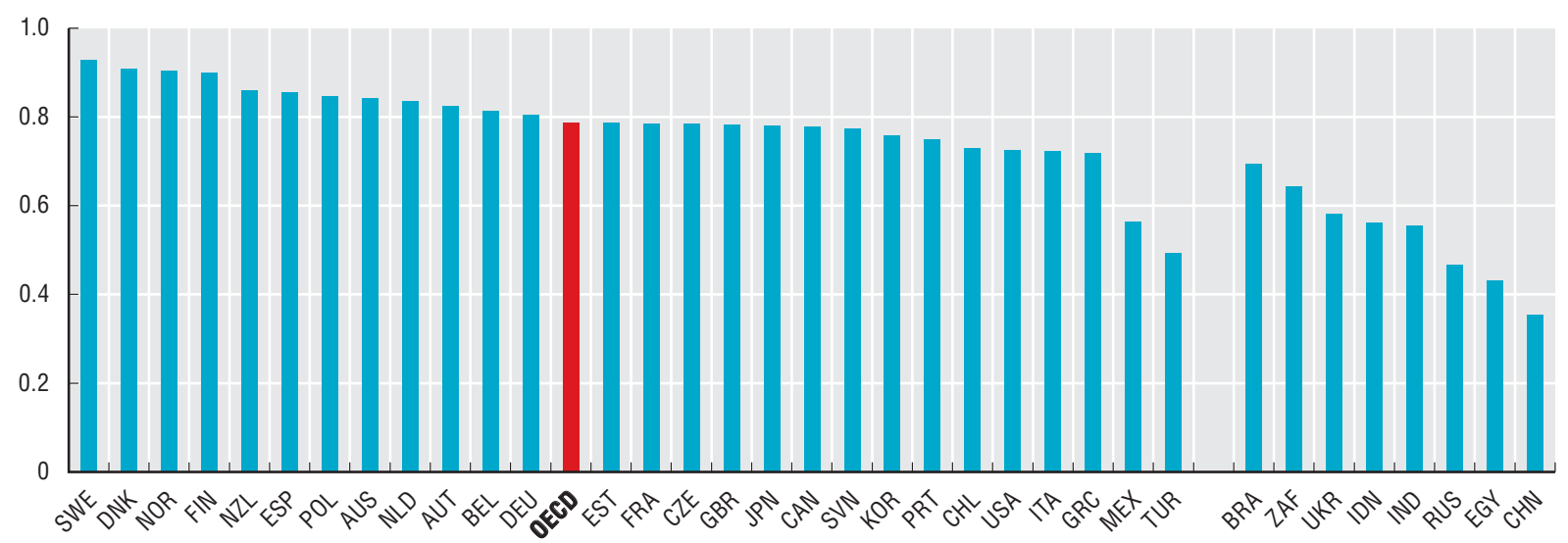

Quelle: The World Justice Project.

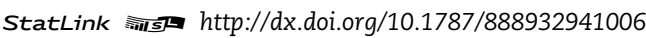

\subsection{Eingeschränkte Machtbefugnisse der Regierung und Grundrechte (2012-13)}

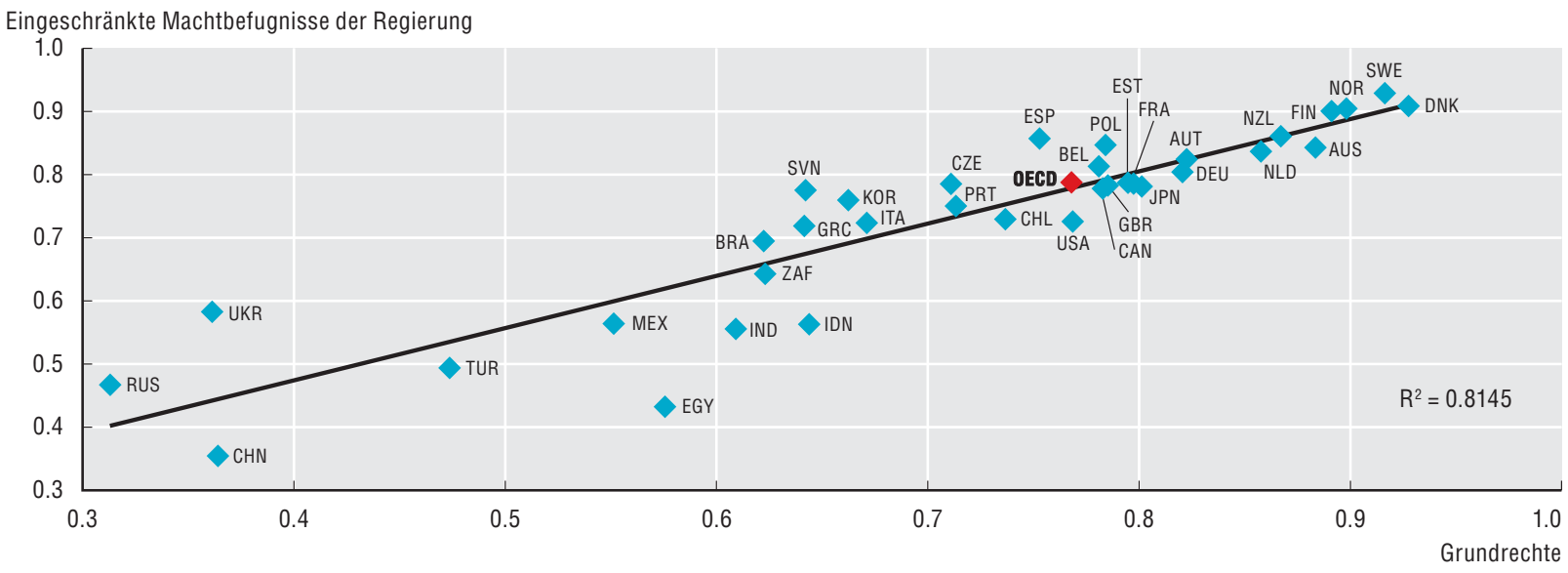

Quelle: The World Justice Project. 
2.8. Vollzug der Rechtsvorschriften (2012-13)

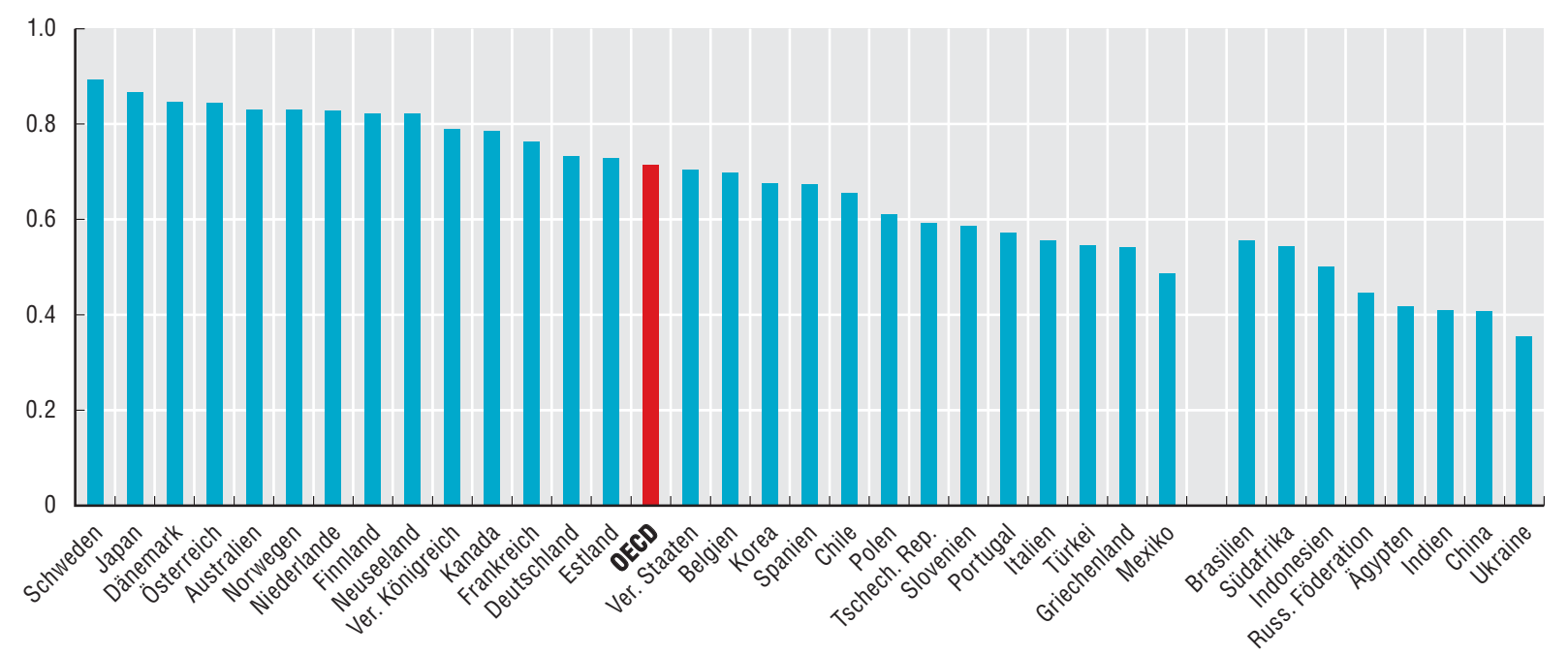

Quelle: The World Justice Project.

StatLink Ailsta $h t t p: / / d x . d o i . o r g / 10.1787 / 888932941044$

\subsection{Zivilgerichtsbarkeit (2012-13)}

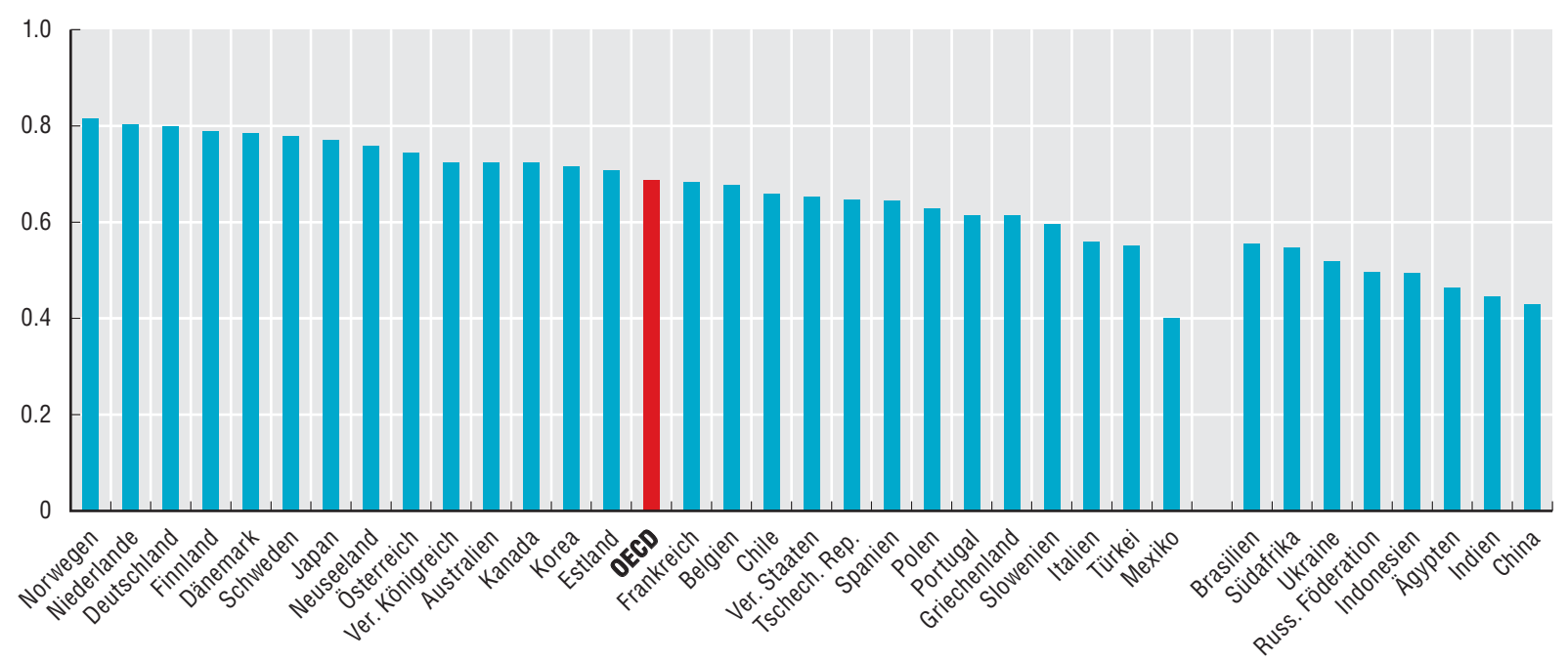

Quelle: The World Justice Project. 
Die Mitgliedsländer der OECD waren in den letzten Jahrzehnten in erheblichem Maße von Katastrophen mit zunehmenden ökonomischen Auswirkungen betroffen.

In den letzten 30 Jahren ist die Zahl der Katastrophen in den OECD-Ländern von ungefähr 100 auf mitunter mehr als 300 pro Jahr gestiegen, mit Schäden in Höhe von Hunderten Milliarden pro Jahr. Derartige Katastrophen stellen die Regierungen vor zahlreiche Herausforderungen, gefährden das Leben vieler Bürgerinnen und Bürger und können sowohl kleine und mittlere als auch international operierende Unternehmen in ihrer Geschäftstätigkeit beeinträchtigen. Auch große kritische Infrastrukturen können bedroht sein - mit teils verheerenden Folgen, wie das schwere Erdbeben in Japan im Jahr 2011 gezeigt hat. Solche Großkatastrophen haben die Länder veranlasst, ihre Risikomanagementstrategien, die u.a. die Risikoidentifizierung und -analyse sowie die Umsetzung von Maßnahmen zur Erhöhung der Resilienz umfassen, zu verstärken.

Für die Bewältigung kritischer Risiken im OECD-Raum ist einerseits Unterstützung auf höchster politischer Ebene erforderlich, aber auch ein Engagement für die Risikovorsorge in allen Sektoren und auf allen territorialen Ebenen des Staates, einschließlich der Kommunalebene. Dies erfordert einen strategischen Rahmen, in dem Strategie, Kapazitäten und Governance zusammengeführt und koordiniert werden, um eine risikoinformierte Politikgestaltung zu ermöglichen. In vier OECD-Ländern liegt die Zuständigkeit für die Risikominderung bei der Regierungszentrale (in den meisten Fällen das Büro des Premierministers). In den meisten anderen OECD-Ländern ist eine zentrale Koordinierung sichergestellt, die oft bei den nationalen Zivilschutzbehörden angesiedelt ist.

Zudem ist die Risikomanagementpolitik durch verschiedene Strategien, Pläne und Instrumente in den einzelnen Politiksektoren integriert worden. Fast alle OECD-Länder, die interdisziplinäre Prüfungen der Fortschritte bei der Integration des Risikomanagements in der staatlichen Politik und Investitionstätigkeit eingeleitet haben, berücksichtigen bei sektoralen öffentlichen Investitionsstrategien und Planungsvorgängen systematisch die Katastrophenvorsorge. Nur zwei Drittel verwenden jedoch Kosten-Nutzen-Analysen des Risikomanagements bei der Konzeption und dem Betrieb großer öffentlicher Investitionsprojekte. Die Bedeutung, die der Lokalebene zugemessen wird, kommt in der Tatsache zum Ausdruck, dass 86\% der OECD-Mitgliedsländer einen rechtlichen Rahmen für lokale Zuständigkeiten geschaffen haben und fast zwei Drittel eine risikosensible Regulierung für die Flächennutzungsplanung und die private Immobilienentwicklung erarbeitet haben. Allerdings ist der Anteil der lokalen Gebietskörperschaften, die regelmäßig Mittel für die Katastrophenvorsorge erhalten, mit nur $62 \%$ deutlich niedriger. Die Stärkung der rechtlichen Rahmenbedingungen wird keine Wirkung zeigen, wenn die lokalen Gebietskörperschaften nicht mit den notwendigen Mitteln für Vorsorgemaßnahmen ausgestattet werden.

Die Staaten stehen vor der anspruchsvollen Aufgabe, integrierte politische Maßnahmen zur Bewältigung multidisziplinärer Herausforderungen zu entwickeln. In diesem Zusammenhang stellen die nationalen Risikoanalysen ein wichtiges Instrument dar, das dazu beitragen kann, eine integrierte Allgefahren-Risikomanagementstrategie zu entwickeln. Über die Hälfte der OECDMitgliedsländer führt die Risikoanalyse in integrierter Form durch, auf Basis eines Allgefahren-Ansatzes und unter Einbeziehung zukünftiger wahrscheinlicher Risiken.

Im Vergleich zu den Herausforderungen, die in Ländern mit niedrigerem Einkommensniveau bestehen, werden im OECDRaum hohe Risikomanagementstandards erreicht. Dennoch nehmen angesichts der wachsenden potenziellen Risiken und der sich verändernden Gefahrenprofile die Verluste an Sachvermögen kontinuierlich zu, obgleich bei der Zahl der Todesopfer ein Abwärtstrend zu beobachten ist. Frühwarnsysteme ermöglichen eine effektive Warnung der betroffenen Bevölkerung, die in der Regel weiß, wie auf diese Warnungen zu reagieren ist. Das Risikobewusstsein ist in vielen Ländern ebenfalls gestiegen, was u.a. auf wirkungsvolle öffentliche Informationskampagnen und die Integration der Risikomanagementthematik in die Lehrpläne von Bildungseinrichtungen des Primar-, Sekundar- und Tertiärbereichs zurückzuführen ist.

\section{Methodik und Definitionen}

Die Daten zu Katastrophen beruhen auf EM-DAT, der OFDA/CRED International Disaster Database (www.emdat.be), die von der Université Catholique de Louvain in Belgien entwickelt wurde. Die Verlustdaten basieren auf Schätzungen von SwissRe. Die von der Internationalen Strategie zur Katastrophenvorsorge (International Strategy for Disaster Reduction - ISDR) der Vereinten Nationen unterhaltene Online-Plattform bietet Zugang zu Länderberichten über die Fortschritte bei der Umsetzung der im Hyogo-Rahmenaktionsplan (Hyogo Framework for Action - HFA) definierten Ziele (www.preventionweb.net/english/ hyogo/). Die hier wiedergegebenen Daten beziehen sich auf den jüngsten Berichtszeitraum (2011-13). Die Fortschrittsberichte basieren auf einer Selbstevaluierung durch MultiStakeholder-Prozesse. Die Informationserhebung erfolgte darüber hinaus durch OECD-Fragebogen, gefolgt von Telefoninterviews im Jahr 2012. Hierbei wurde mit öffentlichen Amtsträgern und anderen Risikoexperten des Forums Großrisiken der OECD zusammengearbeitet.

Abbildung 2.17, Zahl der Katastrophen pro Jahr, 1980-2010, ist online verfügbar unter http://dx.doi.org/10.1787/ 888932941101.

\section{Weitere Informationen}

OECD (2012), Disaster Risk Assessment and Risk Financing: A G20/ OECD Methodological Framework, OECD, Paris, www.oecd.org/ gov/risk/G20disasterriskmanagement.pdf.

SwissRe (2011), „Closing the financial gap: New partnerships between the public and private sectors to finance disaster risks", SwissRe Economic Research and Consulting, Zürich, http://media.swissre.com/documents/pub-closing-thefinancialgap_w1.pdf.

UNISDR (2013), Global Assessment Report on Disaster Risk Reduction - From Shared Risk to Shared Value: The Business Case for Disaster Risk Reduction, Sekretariat der Vereinten Nationen für die Internationale Strategie zur Katastrophenvorsorge, Genf, www.preventionweb.net/english/hyogo/gar/2013/en/home/ index.html.

\section{Anmerkungen zu den Tabellen}

Für Belgien, Dänemark, Estland, Island, Irland, Israel, Luxemburg, Österreich und die Slowakische Republik stehen keine Daten zur Verfügung.

2.16: Die Informationen zu Kanada beruhen auf HFA-Daten für den Zeitraum 2009-11. 
2.14. Gemeinden von $O E C D-M i t g l i e d s l a ̈ n d e r n$ mit Mandat und Budget für die Katastrophenvorsorge

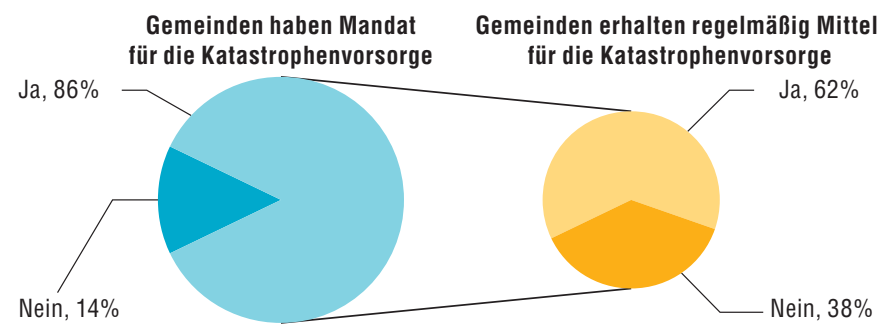

Quelle: Daten aus den HFA-Fortschrittsberichten, veröffentlicht auf: www.preventionweb.net/english/hyogo/progress/?pid:3\&pil:1. StatLink त्नारा http://dx.doi.org/10.1787/888932941082
2.15. Zuständigkeit für die Koordination der Katastrophenvorsorge in OECD-Ländern

\begin{tabular}{ll}
\hline & Zuständigkeit für Koordination der Katastrophenvorsorge: \\
\hline Büro des Premierministers & Australien, Frankreich, Neuseeland, Türkei \\
Zentrale Planungs- und/ & Ägypten, Chile, Griechenland, Japan, Korea, Norwegen, \\
oder Koordinierungsstelle & $\begin{array}{l}\text { Polen, Schweiz, Vereinigtes Königreich } \\
\text { Zivilschutzbehörde }\end{array}$ \\
$\begin{array}{l}\text { Australien, Finnland, Frankreich, Italien, Norwegen, } \\
\text { Portugal, Schweden, Slowenien }\end{array}$ \\
Umweltbehörde & Frankreich, Schweiz \\
Finanzministerium & Frankreich
\end{tabular}

Quelle: Daten aus den HFA-Fortschrittsberichten, veröffentlicht auf: www.preventionweb.net/english/hyogo/progress/?pid:3\&pil:1.

StatLink intste http://dx.doi.org/10.1787/888932943248

\subsection{Nationale Risikomanagementpolitiken im OECD-Raum}

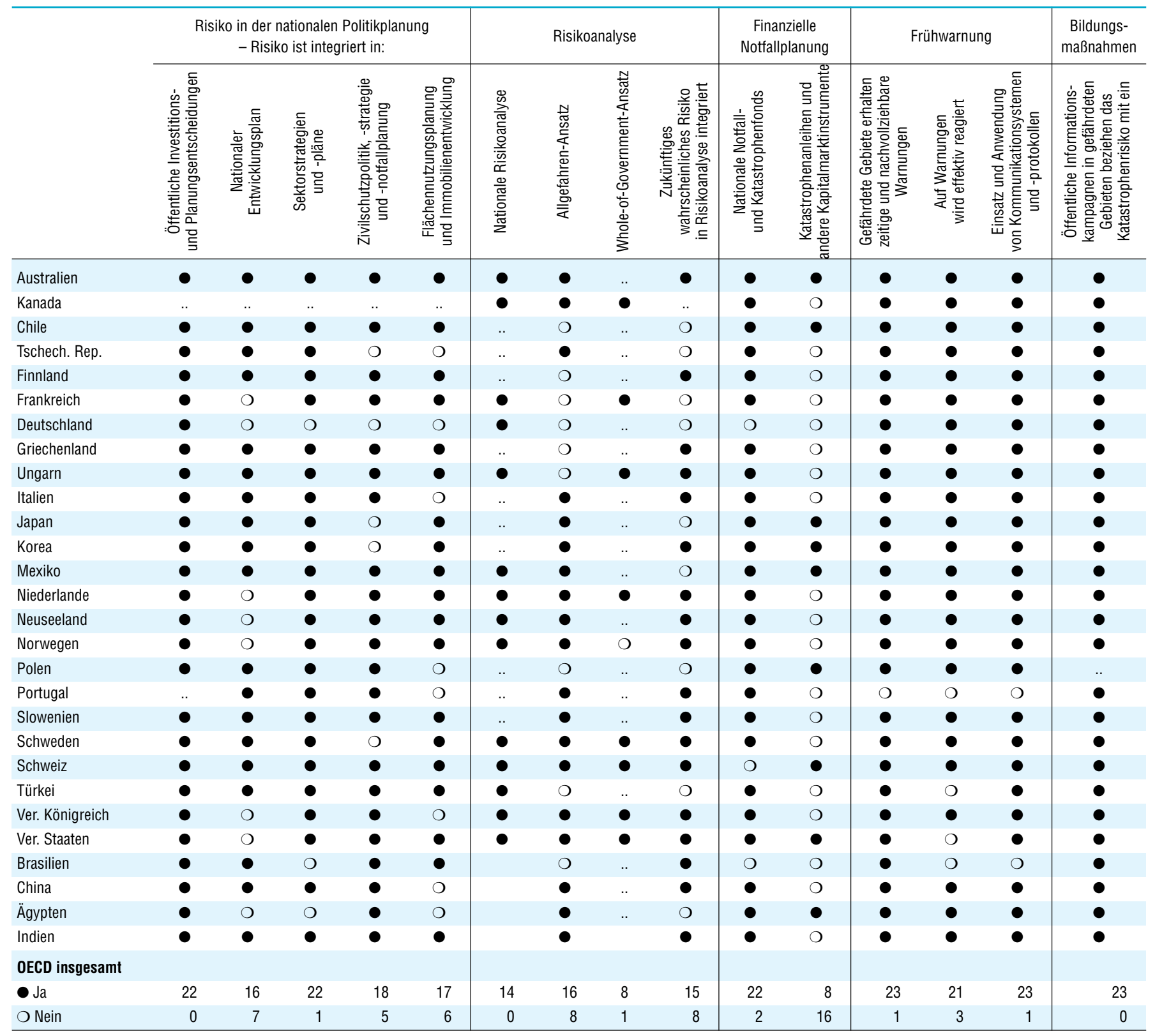

Quelle: Daten aus den HFA-Fortschrittsberichten, veröffentlicht auf: www.preventionweb.net/english/hyogo/progress/?pid:3\&pil:1. 
Unter der Tragfähigkeit der öffentlichen Finanzen wird die Fähigkeit des Staates verstanden, die Staatsfinanzen langfristig auf einem Niveau zu halten, das als solide erachtet wird und es dem Staat ermöglicht, seinen finanziellen Verpflichtungen nachzukommen. Um die langfristige Tragfähigkeit der Staatsfinanzen sicherzustellen, müssen die Regierungen kontinuierliche strategische Prognosen zu den zukünftigen Einnahmen und Verbindlichkeiten des Staates, Umweltfaktoren und sozioökonomischen Trends erstellen, um die Finanzplanung entsprechend anzupassen. Ein hoher und steigender Schuldenstand beeinträchtigt die Finanzlage der öffentlichen Haushalte und kann eine Schuldenspirale auslösen, in der das wirtschaftliche Wachstumspotenzial abnimmt, weil weniger Mittel für produktive Investitionen zur Verfügung stehen. Viele OECD-Länder weisen infolge der Finanz- und Wirtschaftskrise nach wie vor eine steigende Staatsschuldenquote auf. Die durch die aktuelle Konjunkturschwäche verursachten Kosten in Verbindung mit dem erwarteten Anstieg der alterungsbedingten Ausgaben stellen gravierende Herausforderungen für die Tragfähigkeit der öffentlichen Finanzen dar.

Die OECD hat Schätzungen aufgestellt, um wie viel der strukturelle Primärsaldo in den einzelnen Ländern verbessert werden müsste, um die Bruttostaatsverschuldung bis 2030 auf $60 \%$ des BIP zu senken. Diesem Modell zufolge wäre in Griechenland, dem Vereinigten Königreich und den Vereinigten Staaten insgesamt eine durchschnittliche Verbesserung des jeweiligen strukturellen Primärsaldos des Jahres 2012 um mehr als 6\% des Potenzial-BIP (Vollauslastung der Volkswirtschaft) erforderlich, um die Staatsverschuldung innerhalb dieses Zeitraums auf $60 \%$ des BIP zu verringern. In Japan wäre eine Steigerung um $13 \%$ des Potenzial-BIP notwendig, um die Staatsschuldenquote auf $60 \%$ zu reduzieren. Die dafür erforderlichen Konsolidierungsmaßnahmen sind jedoch so umfangreich, dass in diesem Szenario nicht davon ausgegangen wird, dass Japan dieses Ziel bis 2030 erreicht. Dagegen wird angesichts der gegenwärtigen Verfassung der Staatsfinanzen (z.B. hinsichtlich Finanzierungssaldo und Schuldenstand) in Dänemark, Deutschland, Estland, Korea und der Schweiz nicht damit gerechnet, dass diese Länder Überschüsse benötigen, um die Verschuldung auf den Zielwert von 60\% des BIP zu reduzieren.

Im Schnitt entspricht die von den OECD-Mitgliedsländern umgesetzte bzw. angekündigte Haushaltskonsolidierung für den Zeitraum 2009-15 einem Umfang von mehr als 5,5\% des BIP. Zwei Drittel der Konsolidierung werden durch Maßnahmen auf der Ausgabenseite, das verbleibende Drittel durch einnahmeseitige Maßnahmen erzielt. Der Umfang und die Zusammensetzung der Konsolidierungspakete sind jedoch in den einzelnen OECD-Ländern sehr unterschiedlich. In den Ländern mit den größten wirtschaftlichen Ungleichgewichten und der raschesten Verschlechterung der öffentlichen Finanzlage ist eine umfangreichere Konsolidierung erforderlich. Dementsprechend haben Griechenland, Irland und Portugal Konsolidierungspakete im Umfang von mehr als $12 \%$ des BIP angekündigt. Dagegen machen die von Kanada, Schweden und der Schweiz umgesetzten oder angekündigten Konsolidierungsmaßnahmen weniger als $1,6 \%$ des BIP aus. Ausgabenseitige Maßnahmen bilden in den meisten Ländern den größten Anteil an den Konsolidierungspaketen. Einnahmeseitige Maßnahmen stellen lediglich in sieben Ländern - Belgien, Dänemark, Estland, Italien, Polen, der Tschechischen Republik und der Türkei - den höchsten Anteil.

Für fundierte strategische Prognosen sollten die Kosten des demografischen Wandels berücksichtigt werden, insbesondere da sich die meisten OECD-Mitgliedsländer angesichts des erwarteten Anstiegs der durch die Bevölkerungsalterung bedingten Ausgaben und des technischen Fortschritts zunehmenden Budgetengpässen im Gesundheitswesen, in der Langzeitpflege und bei den Renten ausgesetzt sehen. Im Schnitt wird damit gerechnet, dass bei einer unveränderten Fortsetzung der gegenwärtigen Politik die alterungsbedingten Staats- ausgaben in den OECD-Mitgliedsländern zwischen 2014 und 2030 um fast 3 Prozentpunkte des BIP steigen werden.

\section{Methodik und Definitionen}

Die Daten für Abbildungen 2.18 und 2.20 stammen aus dem OECD-Wirtschaftsausblick 93. Der insgesamt erforderliche Konsolidierungsumfang für eine Rückführung der Bruttostaatsschuldenquote auf $60 \%$ des BIP wird in zwei Zeitspannen gemessen: zwischen 2012 und 2014 als die Veränderung des strukturellen Primärsaldos und von 2014 bis 2030 als die Differenz zwischen dem 2014 erreichten Niveau des strukturellen Primärsaldos und seinem Durchschnittswert für den Zeitraum 2014-2030. Die Annahmen, die bei der Ermittlung des zur Verringerung der Schuldenquote auf $60 \%$ erforderlichen Primärsaldos zu Grunde gelegt wurden, sind Kasten 4.5 und Tabelle 4.2 im OECDWirtschaftsausblick 93 zu entnehmen.

Die Daten für Abbildung 2.19 stammen aus dem OECD Fiscal Consolidation Survey 2012.

Bei den meisten Ländern beziehen sich die für die Zwecke dieser Berechnungen verwendeten Daten zur Bruttoverschuldung auf die gesamtstaatlichen (kurz- und langfristigen) Verbindlichkeiten gemäß der Definition im System der Volkswirtschaftlichen Gesamtrechnungen. Diese Definition der Verschuldung weicht von der Definition des Maastricht-Vertrags ab, die zur Bewertung der Lage der öffentlichen Finanzen der EU-Länder verwendet wird.

\section{Weitere Informationen}

OECD (2013), „OECD-Wirtschaftsausblick 93“, OECD Economic Outlook: Statistics and Projections (Datenbank), http://dx.doi.org/ 10.1787/data-00655-en.

OECD (2012), Restoring Public Finances, 2012 Update, OECD Publishing, Paris, http://dx.doi.org/10.1787/9789264179455-en.

\section{Anmerkungen zu den Abbildungen}

2.18 und 2.20: Für Chile, Mexiko und die Türkei stehen keine Daten zur Verfügung. In Dänemark, Deutschland, Estland, Korea, Norwegen und der Schweiz ist keine Konsolidierung erforderlich, um bis 2030 eine Bruttostaatsschuldenquote von $60 \%$ des BIP zu erreichen. Der OECD-Durchschnitt ist ungewichtet. Haushaltsprojektionen resultieren aus der Anwendung eines stilisierten Haushaltskonsolidierungspfads und sollten nicht als Prognose interpretiert werden.

2.19: Die Daten sind die Summe des jährlichen inkrementellen Konsolidierungsumfangs von 2009/2010 bis 2015 gemäß den Angaben der zuständigen nationalen Stellen. Nur die folgenden Länder meldeten eine Konsolidierung im Jahr 2009: Estland, Irland, Polen, Slowenien und Ungarn. Die Haushaltskonsolidierung in Ungarn im Zeitraum 2007-08 wurde nicht berücksichtigt. In Österreich reicht der Konsolidierungszeitraum bis 2016. Die folgenden Länder haben keine Angaben zu einem angekündigten konkreten Konsolidierungsplan gemacht und wurden nicht in die Abbildung aufgenommen: Japan, Korea und die Vereinigten Staaten. Australien vermeldet Konsolidierungsmaßnahmen (insbesondere im Jahr 2013), verwendet jedoch eine breitere Definition des Begriffs „Konsolidierung“ als in dieser Erhebung. Neuseeland und Slowenien haben einige Maßnahmen zur Steigerung der Einnahmen gemeldet, die jedoch nicht in vollem Umfang quantifiziert wurden.

2.20: In Fällen, in denen für die alterungsbedingten Ausgaben keine Projektionen für den Zeitraum 2014-2030 zur Verfügung standen, wurde lineare Interpolation angewendet.

Hinweis zu den Daten für Israel: http://dx.doi.org/10.1787/888932315602. 


\subsection{Zur Verringerung der Bruttostaatsschuldenquote auf $60 \%$ des BIP erforderlicher Gesamtkonsolidierungsbedarf} zwischen 2012 und 2030

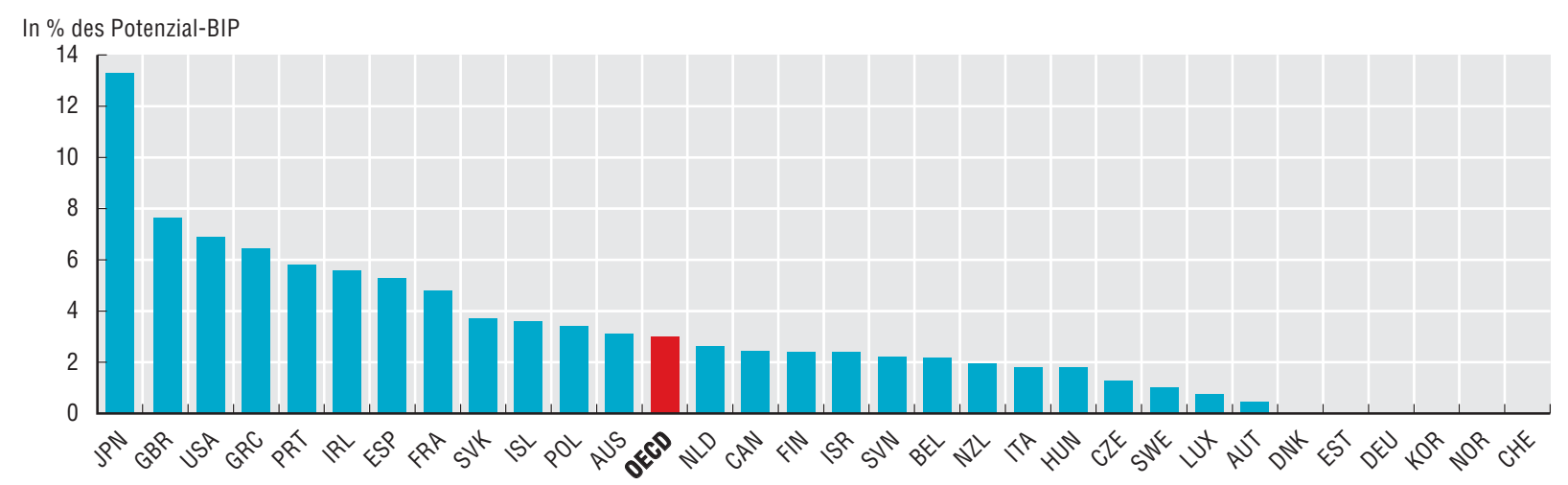

Quelle: OECD-Berechnungen; OECD (2013), „OECD-Wirtschaftsausblick 93“, OECD Economic Outlook: Statistics and Projections (Datenbank), Mai 2013, http://dx.doi.org/10.1787/data-00655-en.

StatLink त्राड़ http://dx.doi.org/10.1787/888932941120

\subsection{Ausgabenseitige und einnahmeseitige Haushaltskonsolidierung in Prozent des BIP (2009-2015)}

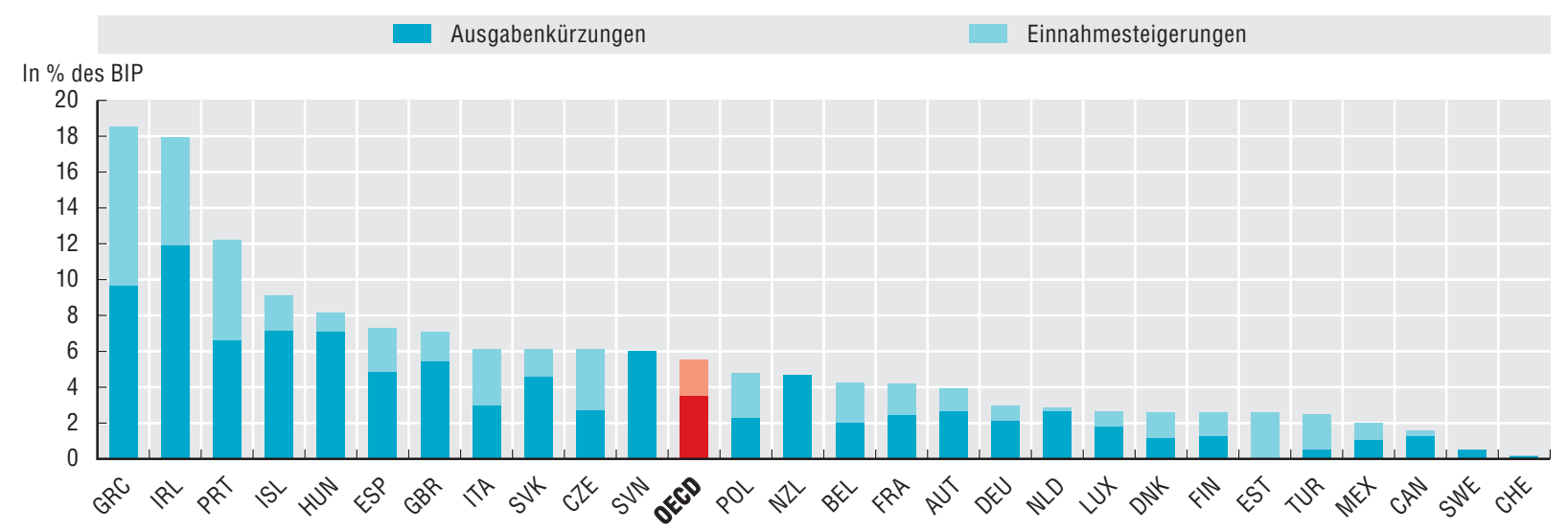

Quelle: OECD Fiscal Consolidation Survey 2012.

\subsection{Haushaltskonsolidierungsbedarf und projektionsgemäßer Anstieg der alterungsbedingten Ausgaben (2014-2030)}

Zur Verringerung der Bruttostaatsschuldenquote auf $60 \%$ des BIP erforderliche Veränderung des strukturellen Primärsaldos (in \% des Potenzial-BIP)

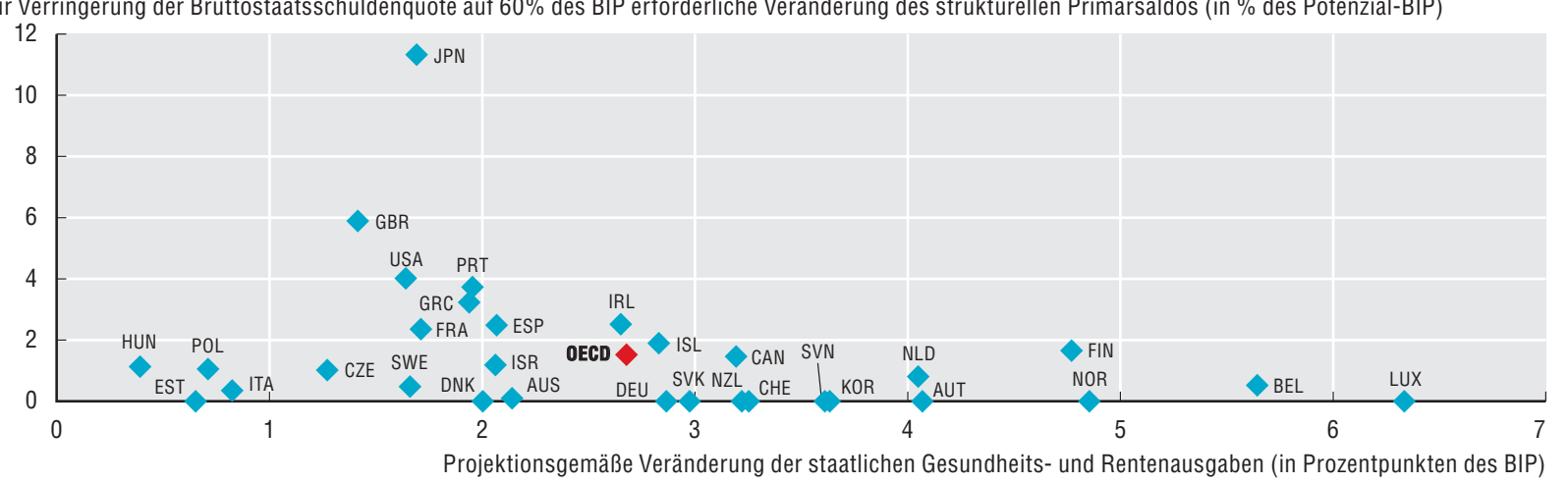

Quelle: OECD-Berechnungen; OECD (2013), „OECD-Wirtschaftsausblick 93“, OECD Economic Outlook: Statistics and Projections (Datenbank), Mai 2013, http://dx.doi.org/10.1787/data-00655-en. 
Bei der Schätzung von Effizienz wird das Verhältnis zwischen dem investierten Input und dem mit diesen Ressourcen erzielten Output analysiert. Die Verbesserung dieser Messgröße als Mittel zur Ausgabenkontrolle ist eine wesentliche Zielsetzung der Regierungen in den OECD-Ländern. Die Haushaltskrisen, denen sich viele Länder sowohl vor als auch nach der großen Wirtschafts- und Finanzkrise ausgesetzt sahen, rückten die Leistungsfähigkeit des öffentlichen Sektors in den Vordergrund.

Effizienzindikatoren vergleichen Outputgrößen mit Inputgrößen. Zusammengenommen können sie Effizienz in ihren beiden Dimensionen abbilden, d.h. technische (oder operationale) und allokative Effizienz. Die Leistungsmessung und -bewertung sollte auf der ökonomischen Effizienz (oder Kosteneffizienz), d.h. dem Produkt aus operationaler und allokativer Effizienz, beruhen.

Im Folgenden werden Effizienzindikatoren für die Bereiche Gesundheit, Bildung, Justiz und Steuerverwaltung präsentiert, wo sowohl Input- als auch Output-Daten zur Verfügung stehen und sich zunehmend ein länderübergreifender Konsens darüber herausbildet, wie Effizienz auf international vergleich bare Weise zu messen ist.

\section{Gesundheitswesen}

Es gibt verschiedene Messgrößen für die Effizienz im Gesundheitswesen. Eine der maßgeblichen Größen ist die durchschnittliche Verweildauer im Krankenhaus. Unter ansonsten gleichen Bedingungen ist davon auszugehen, dass sich bei einer kürzeren Verweildauer die Kosten je Krankenhausfall verringern und eine Verlagerung von der stationären auf weniger kostspielige Anschlussbehandlungen stattfindet. Ein kürzerer Aufenthalt ist in der Tendenz jedoch leistungsintensiver, so dass die Kosten je Krankenhaustag höher sind. Außerdem kann eine zu geringe Verweildauer auch negative Auswirkungen auf den Gesundheitszustand haben oder das Wohlbefinden und die Genesung des Patienten beeinträchtigen. 2011 lag die durchschnittliche Krankenhausverweildauer für alle Behandlungsanlässe im OECD-Durchschnitt bei acht Tagen. In Mexiko und der Türkei war die Verweildauer mit weniger als der Hälfte des OECDDurchschnitts am kürzesten. Am längsten war die Verweildauer in Japan mit fast 18 Tagen, mehr als doppelt so lang wie im OECD-Durchschnitt. In den meisten Ländern ist die durchschnittliche Verweildauer im Verlauf des vergangenen Jahrzehnts zurückgegangen - von durchschnittlich 9,2 Tagen im Jahr 2000 auf 8,0 Tage im Jahr 2011. Auf der Ebene des Gesundheitssystems wird die durchschnittliche Krankenhausverweildauer durch Faktoren wie Praxisrichtlinien oder Zahlungssysteme beeinflusst. In Japan beispielsweise schaffen die hohe Bettenzahl und die Vergütungsstruktur für Krankenhäuser Anreize, Patienten länger zu halten.

\section{Justizsystem}

Die Staaten stehen unter erheblichem Druck, ein effizientes und reaktionsfähiges Justizsystem zu gewährleisten, um Bürgerinnen und Bürgern bei der Durchsetzung ihrer Rechte keinen zusätzlichen zeitlichen und finanziellen Aufwand, u.a. durch die Ausgaben für einen Rechtsbeistand, zu verursachen. Ein maßgeblicher Indikator für die Effizienz des Zivilgerichtswesens setzt die Kosten des Verfahrens in Prozent des Streitwerts (d.h. den Input) ins Verhältnis zur durchschnittlichen erstinstanzlichen Verfahrenslänge in dem jeweiligen Land (d.h. dem Output). Langsamere Gerichte schmälern das Vertrauen in die Justiz; zudem können sie auf lange Sicht die Kosten für Unternehmen in die Höhe treiben und Privatinvestoren abschrecken. Außerdem bedeuten längere Verfahren eine größere wirtschaftliche Belastung sowohl für die Bürger als auch für den Staat. Die Kosten eines Verfahrens belaufen sich in den OECD-Mitgliedsländern im Durchschnitt auf 19\% des Streitwerts, die durchschnittliche Dauer erstinstanzlicher Verfahren liegt bei acht Monaten. Korea, Neuseeland und Norwegen schneiden hierbei am besten ab, während die Verfahren in der Slowakischen Republik länger und teurer sind. In Italien scheinen institutionelle Differenzen und die ungleichmäßige geografische Verteilung der Ressourcen im Justizwesen die Hauptgründe für die frappierend lange Dauer erstinstanzlicher Verfahren zu sein. Allerdings sollten Effizienzvergleiche in diesem Sektor nicht als Maßstab für die Leistungsqualität und die Ordnungsmäßigkeit von Verfahren oder für die Qualität der Gerichtsurteile betrachtet werden.

\section{Bildungswesen}

Die Entwicklung und Akkumulation von Humankapital ist unerlässlich für die Schaffung eines Potenzials an hochqualifizierten Arbeitskräften, die gute Voraussetzungen mitbringen, um auf dem internationalen Arbeitsmarkt zu bestehen und aktive Bürger responsiver Demokratien zu werden. Der Bildungsstand wird als geeignete Output-Messgröße für die Humankapitalproduktion betrachtet. Vergleicht man diesen mit den gesamten Bildungsausgaben pro Schüler (d.h. dem Bildungsinput) in den jeweiligen Ländern, lassen sich Erkenntnisse darüber gewinnen, welche Systeme in der Lage sind, effizientere Leistungen zu erbringen. Die in der internationalen Schulleistungsstudie PISA gemessenen Schülerleistungen in Lesekompetenz und Mathematik weisen eine positive Korrelation mit den Ausgaben sowohl für die Primar- als auch für die Sekundarschulbildung auf, wenngleich dieser Zusammenhang insbesondere bei geringeren Gesamtbildungsausgaben pro Schüler zuzutreffen scheint (OECD PISA im Fokus 13). Neben den Bildungsausgaben hängen die Schülerleistungen auch von der Qualität der Lehrkräfte, dem sozioökonomischen Hintergrund der Schülerinnen und Schüler, der Schulverwaltungspraxis und anderen Faktoren ab. In Ländern wie Finnland, Korea und Neuseeland liegen die Bildungsausgaben pro Schüler unter dem OECD-Durchschnitt, ihre Leistungen sind aber besser. In Luxemburg und Österreich dagegen sind die Ausgaben pro Schüler höher, obwohl die Schülerleistungen unter dem Durchschnitt liegen.

\section{Steuerverwaltung}

Die Steuererhebung von Bürgern und Unternehmen ist für die Staaten die Haupteinnahmequelle, um die Erbringung öffentlicher Dienstleistungen zu finanzieren. Die „Interventionsquote" ist eine häufig von Finanzbehörden verwendete Effizienzmessgröße, die die jährlichen Kosten der Steuerverwaltung dem Gesamtsteueraufkommen des entsprechenden Steuerjahres gegenüberstellt. Ein Abwärtstrend bei der Interventionsquote kann unter ansonsten gleichen Bedingungen Zeichen einer Verringerung der relativen Kosten (verbesserte Effizienz) oder einer besseren Steuerdisziplin (verbesserte Effektivität) sein. In den meisten Ländern war zwischen 2005 und 2008 eine rückläufige oder stabile Entwicklung festzustellen, die aller Wahrscheinlichkeit nach auf verringerte Kosten zurückzuführen war. Andererseits stellten einige Finanzbehörden im Zeitraum 2008-2011 eine Umkehr dieses Trends fest, wobei der Anstieg der Quoten aller Wahrscheinlichkeit nach auf den Rückgang der Steuereinnahmen infolge der Wirtschaftskrise zurückzuführen war.

Beim internationalen Vergleich der Effizienz von Steuerverwaltungen ist jedoch Vorsicht geboten. Die hier dargestellten Effi- 
zienzkennziffern werden durch zahlreiche Faktoren, wie z.B. Unterschiede bei den Steuersätzen und der gesetzlich vorgesehenen Steuerlast insgesamt, Unterschiede in der Bandbreite und der Art der erhobenen Steuern, die makroökonomischen Bedingungen, die sich auf die Steuereinnahmen auswirken, sowie Unterschiede bei den Kostenstrukturen, die aus den institutionellen Gegebenheiten (z.B. Mitwirkung mehrerer Stellen an der Steuerverwaltung, wie in Italien) resultieren, und/oder die Verwaltung nichtsteuerlicher Angelegenheiten (z.B. Zollverwaltung) beeinflusst.

\section{Methodik und Definitionen}

Die durchschnittliche Verweildauer bezieht sich auf die durchschnittliche Zahl der Tage, die Patienten im Krankenhaus verbringen. Sie wird im Allgemeinen durch Division der jährlichen Gesamtzahl der Krankenhaustage aller stationär behandelten Patienten durch die Zahl der Krankenhausfälle ermittelt. Ambulante Patienten werden nicht miteingerechnet. Die Daten umfassen alle stationären Patienten (nicht nur Fälle der kurativen oder Akutbehandlung).

Die Daten zur Leistungsfähigkeit der Justiz bei Zivilverfahren stammen aus OECD, „Judicial Performance and its Determinants: A Cross-Country Perspective“. Die Verfahrenslänge ist geschätzt (weitere Informationen auf Seite 167). Die privat zu tragenden Gesamtkosten eines Verfahrens (als prozentualer Anteil des Streitwerts) nach Berücksichtigung der Wahrscheinlichkeit des Erhalts von Prozesskostenhilfe beziehen sich auf die Kosten für die volle Länge eines spezifischen Zivilrechtsverfahrens. Sie stammen aus der Weltbank-Datenbank Doing Business und umfassen drei verschiedene Arten von Kosten, die für die Regelung eines geschäftlichen Rechtsstreits anfallen: Gerichtskosten, Vollstreckungskosten und durchschnittliche Anwaltshonorare.

Die Daten zu den Bildungsausgaben pro Schüler beziehen sich auf das Finanzjahr 2009. Die Ausgaben pro Schüler entsprechen den Gesamtausgaben der Bildungseinrichtungen (sowohl in staatlicher als auch in privater Trägerschaft, sofern nicht anders angegeben) dividiert durch die Schülerzahl (Vollzeitäquivalent) und umfassen sowohl eigentliche Bildungsdienstleistungen als auch zusätzliche Dienstleistungen. Angesichts von Land zu Land unterschiedlich langer Bildungsgänge entsprechen die jährlichen Ausgaben pro Schüler u.U. nicht genau den insgesamt für einen Schüler angefallenen Ausgaben. Die Punktzahlen für die Lese- und Mathematikleistungen basieren auf der Leistungsmessung bei 15-Jährigen im Rahmen der PISA-Schulleistungsstudie.

Die Daten zur Steuerverwaltung wurden von befragten Finanzbehörden zur Verfügung gestellt oder offiziellen Länderberichten entnommen. Die Kosten der Steuerverwaltung fallen in drei Kategorien: Verwaltungskosten, Lohnkosten und IT-Kosten. Die IT-Ausgaben sind definiert als die Gesamtkosten für die Erbringung von ITDienstleistungen für alle - sowohl steuerbezogene als auch nicht steuerbezogene - Verwaltungsprozesse. Für Vergleichszwecke wurde versucht, die verwendeten Ressourcen und die Kosten steuerbezogener und nichtsteuerbezogener Aufgaben getrennt zu erfassen.

\section{Weitere Informationen}

OECD (2013a), Tax Administration 2013: Comparative Information on OECD and other Advanced and Emerging Economies, OECD Publishing, Paris, http://dx.doi.org/10.1787/9789264200814-en.

OECD (2013b, erscheint demnächst), Health at a Glance 2013: OECD Indicators, OECD Publishing, Paris.

OECD (2012), Bildung auf einen Blick 2012: OECD-Indikatoren, W. Bertelsmann Verlag, Bielefeld, http://dx.doi.org/10.1787/eag2012-de.

Palumbo, G. et al. (2013), „Judicial Performance and its Determinants: A Cross-Country Perspective“, OECD Economic Policy Papers, No. 5, OECD Publishing, Paris, http://dx.doi.org/10.1787/ 5k44x00md5g8-en.

\section{Anmerkungen zu den Abbildungen}

2.21: Die Daten für Japan, Kanada und die Niederlande beziehen sich auf die durchschnittliche Verweildauer für kurative (Akut-) Behandlungen (was in einer Unterschätzung resultiert). Daten für das Jahr 2011: Die Daten für Australien, Belgien, Chile, China, Frankreich, Kanada, Norwegen und die Russische Föderation beziehen sich auf 2010, die Daten für Island auf 2009, die Daten für Griechenland und Indonesien auf 2008. Daten für das Jahr 2000: Die Daten für China und Korea beziehen sich auf 1999, die Daten für Chile und Österreich auf 2001, die Daten für Luxemburg auf 2002.

2.22: Die Daten für das Vereinigte Königreich umfassen nur England und Wales. Weitere Informationen zu den Daten sind der Datenbank Doing Business zu entnehmen.

2.23: Die Daten zu den Ausgaben in Kanada beziehen sich auf 2008. Die Daten zu den Ausgaben in Chile beziehen sich auf 2010. Die Daten zu den Ausgaben in Irland, Italien, Polen, Portugal, der Schweiz, Slowenien, Ungarn, Brasilien und der Russischen Föderation beziehen sich nur auf öffentliche Bildungseinrichtungen.

2.24: Die Daten für Deutschland, Frankreich, Polen, Portugal, die Slowakische Republik und die Tschechische Republik umfassen keine Sozialversicherungsbeiträge und Zölle. Die Daten für Belgien, Japan, Korea, Luxemburg, Mexiko, Österreich und Spanien umfassen keine Sozialversicherungsbeiträge. Die Daten für Finnland, Indonesien, Neuseeland und Slowenien umfassen keine Zölle. Bei den Daten für Brasilien, Irland und Südafrika ist die Zollverwaltung in den Kosten enthalten. Bei den Daten für Estland ist für das Jahr 2005 die Zollverwaltung in den Kosten enthalten. Bei den Daten für Spanien ist für die Jahre 2008 und 2011 die Zollverwaltung in den Kosten enthalten. Bei den Daten für Chile und Schweden sind die Inkassokosten nicht in den Kosten enthalten. Bei den Daten für die Schweiz ist nur die Umsatzsteuerverwaltung berücksichtigt. Die für Island für diese Jahre errechneten Quoten sind unterzeichnet, da anscheinend nicht alle Kosten für die Zwecke der Erhebung quantifiziert wurden. Die für Italien für diese Jahre errechneten Quoten sind stark unterzeichnet, da sie die Ausgaben für steuerbezogene Tätigkeiten anderer Behörden, die nicht quantifiziert wurden, nicht berücksichtigen. Die für die Vereinigten Staaten angegebenen Quoten unterscheiden sich von den von der US-Steuerbehörde IRS veröffentlichten Quoten auf Grund der Verwendung der „Netto-“ anstelle der „Brutto-“ Steuereinnahmen im Nenner. Bei den Daten für Italien ist die laufende Steuerreform zur Rationalisierung des Steuervollzugs nicht berücksichtigt.

Hinweis zu den Daten für Israel: http://dx.doi.org/10.1787/888932315602. 


\section{STRATEGISCHE GOVERNANCE}

\section{Effizienz des öffentlichen Sektors}

2.21. Durchschnittliche Verweildauer für alle Behandlungsanlässe (2000 und 2011)

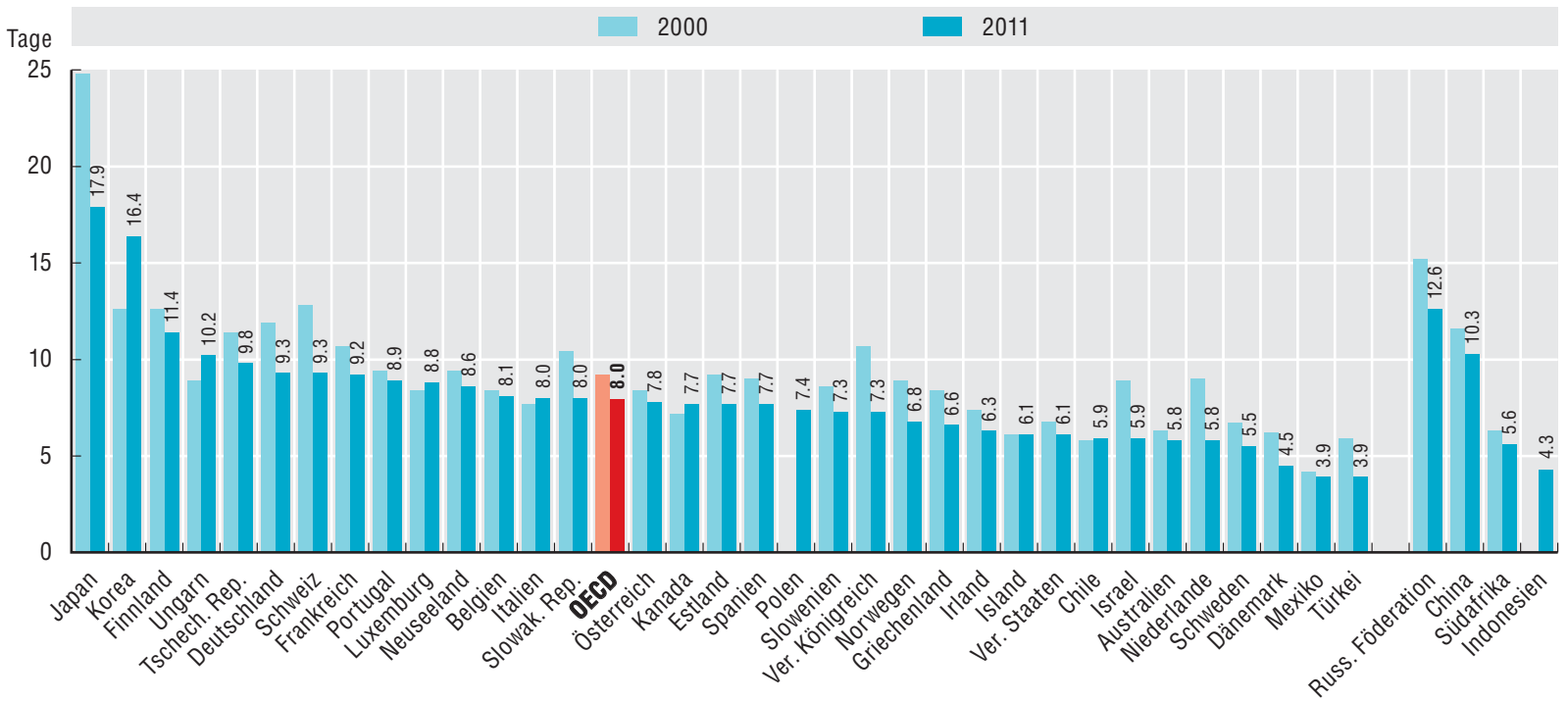

Quelle: OECD Health Statistics 2013.

StatLink त्नाI http://dx.doi.org/10.1787/888932941177

\subsection{Verfahrensdauer in erster Instanz in Tagen sowie Verfahrenskosten (in Prozent des Streitwerts, 2012)}

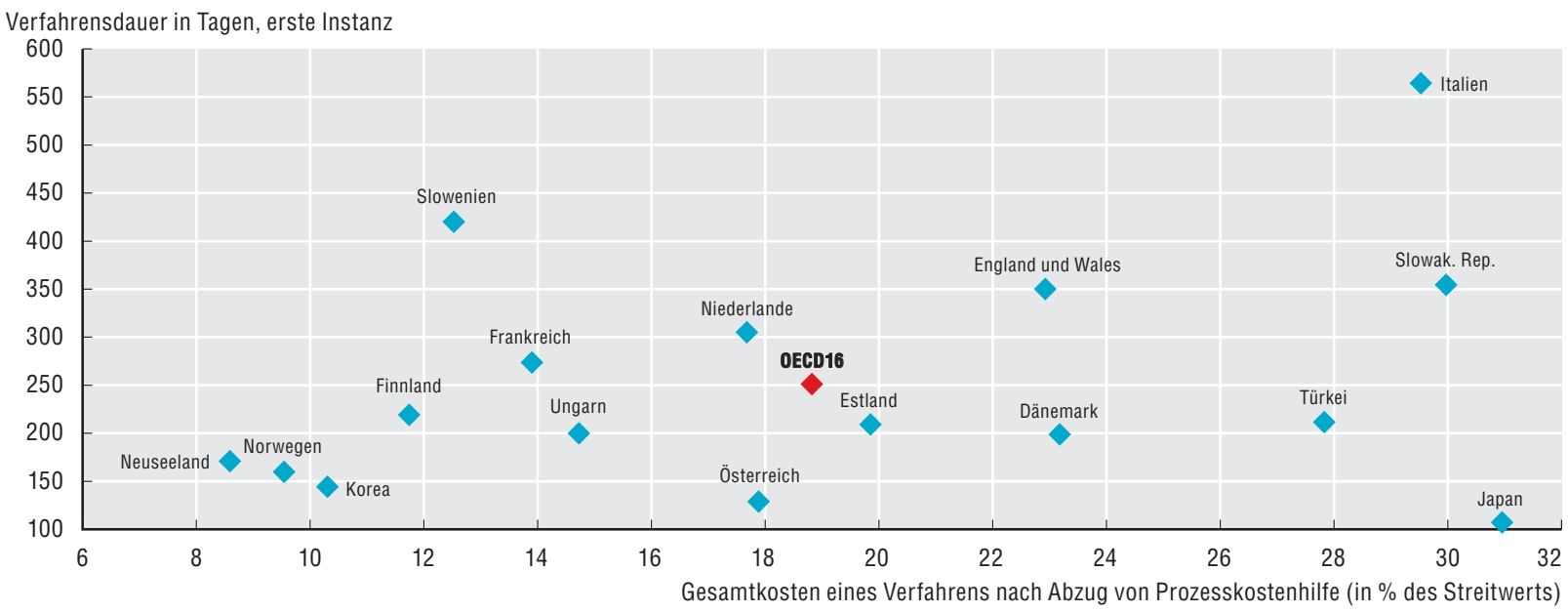

Quelle: Weltbank, Doing Business (Datenbank) sowie G. Palumbo et al. (2013), „Judicial Performance and its Determinants: A Cross-Country Perspective“, OECD Economic Policy Papers, No. 5, OECD Publishing, Paris, http://dx.doi.org/10.1787/5k44x00md5g8-en. 


\section{STRATEGISCHE GOVERNANCE}

Bffizienz des öffentlichen Sektors

\subsection{PISA-Ergebnisse und Gesamt-Bildungsausgaben pro Schüler der Altersstufe 6-15 Jahre in US-\$ KKP (2009)}

Ergebnisse im Bereich Lesekompetenz und Ausgaben pro Schüler
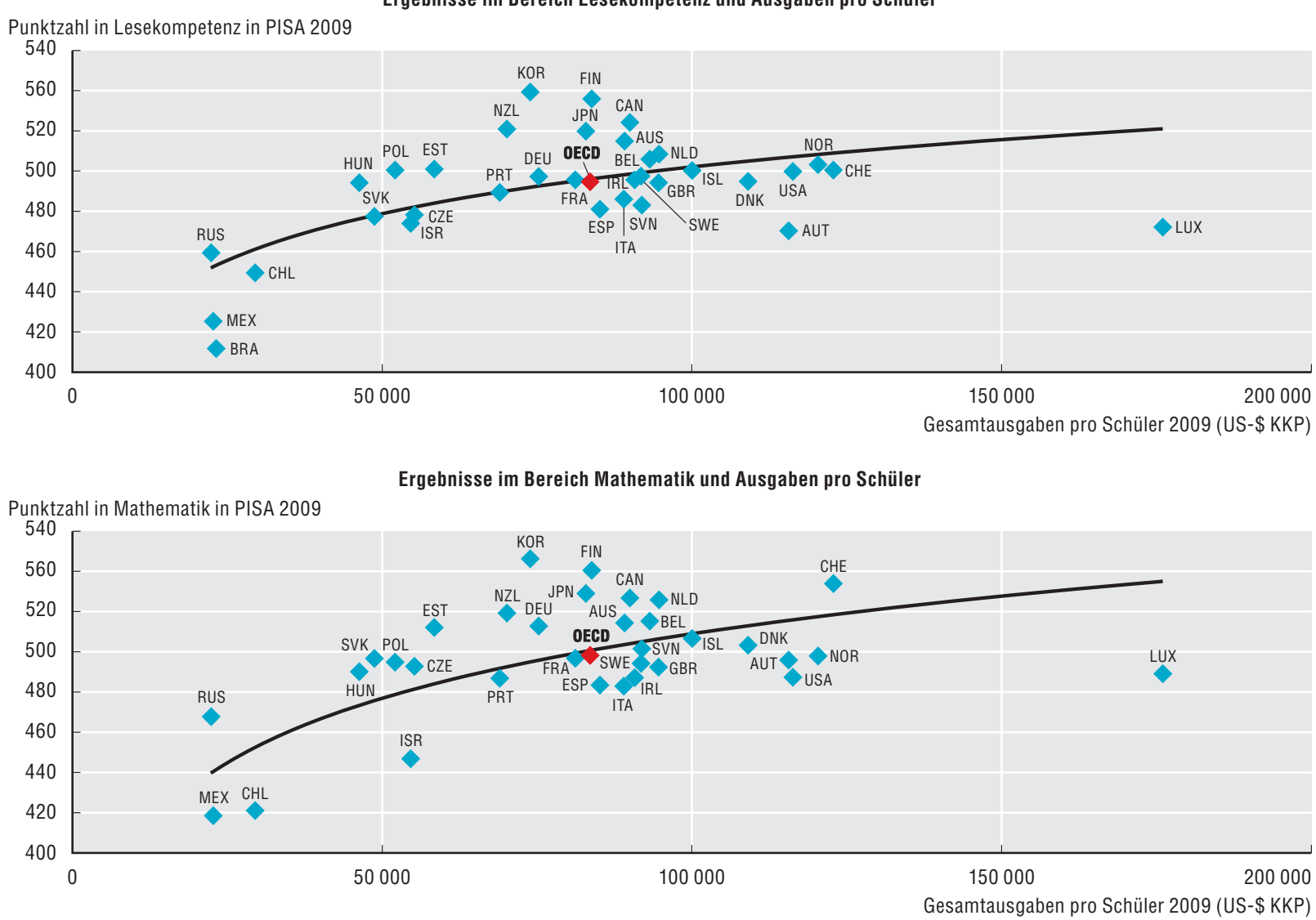

Quelle: OECD (2012), Bildung auf einen Blick 2012: OECD-Indikatoren, W. Bertelsmann Verlag, Bielefeld, http://dx.doi.org/10.1787/eag-2012-de, Tabelle B1.3b; und OECD (2010), PISA 2009 Ergebnisse: Was Schülerinnen und Schüler wissen und können: Schülerleistungen in Lesekompetenz, Mathematik und Naturwissenschaften (Band I), W. Bertelsmann Verlag, Bielefeld, Tabelle I.2.3, http://dx.doi.org/10.1787/9789264095335-de.

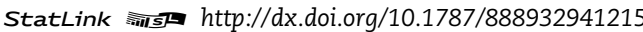

\subsection{Interventionsquoten (Verwaltungskosten/Nettosteuereinnahmen) (2005, 2008 und 2011)}

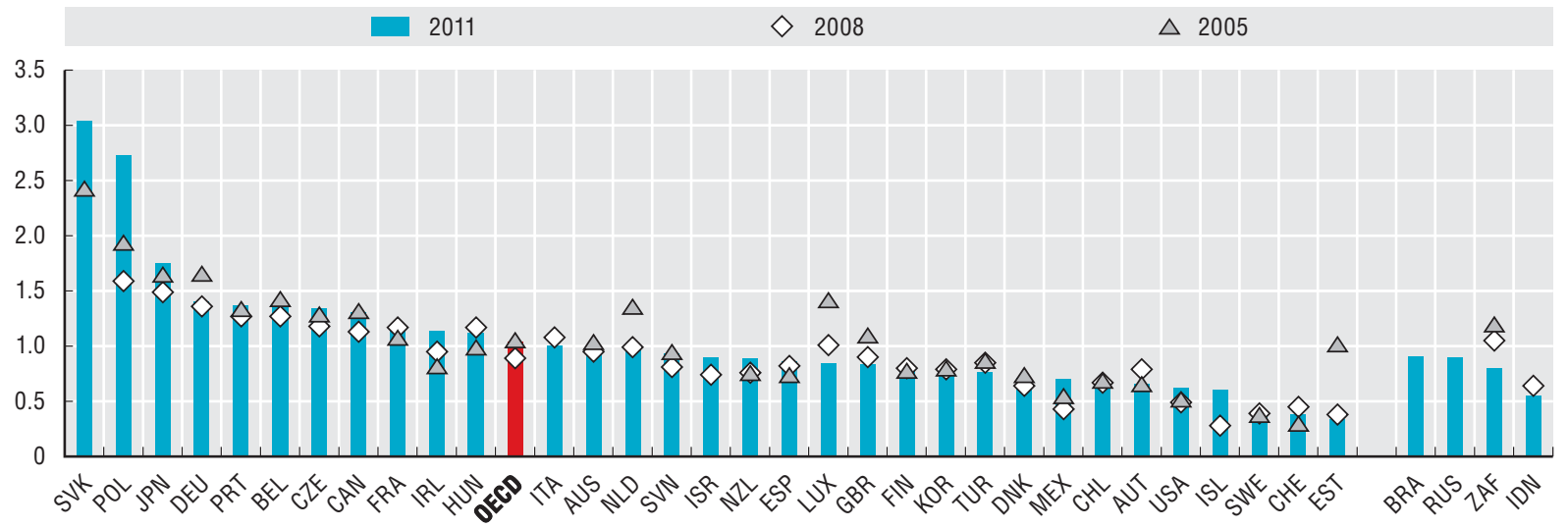

Quelle: OECD (2013), Tax Administration 2013: Comparative Information on OECD and other Advanced and Emerging Economies, OECD Publishing, Paris, http://dx.doi.org/10.1787/9789264200814-en. 
Während das Konzept der Effizienz die Leistung im Hinblick darauf misst, ob die eingesetzten Ressourcen produktiv in den gewünschten Output verwandelt werden, misst die Effektivität, inwiefern mit einer Aktivität die gesetzten Ziele erreicht werden. Die Kosteneffektivität, d.h. das Verhältnis eines Inputs zu einem Zwischen- oder Endergebnis, stellt das Verhältnis zwischen den eingesetzten Ressourcen und den erzielten Ergebnissen dar und ist entscheidend für die Bewertung des Erfolgs staatlicher Politik.

Besonders wichtig ist die Bewertung der staatlichen Leistungen in Sektoren wie dem Bildungs- und Gesundheitswesen, die von zentraler Bedeutung für das Wohlergehen der Bevölkerung und die wirtschaftliche und soziale Entwicklung von Ländern sind. Zudem ist in diesen beiden Sektoren die Messung von Inputs und Outcomes hinreichend entwickelt und international standardisiert, so dass aussagekräftige Effektivitätsvergleiche möglich sind.

\section{Bildungswesen}

Im Bildungswesen sind die Schaffung von Humankapital und die Kompetenzentwicklung zwei zentrale Zielsetzungen für den öffentlichen Sektor, da sie sich positiv auf die Beschäftigungsaussichten und das Lebenseinkommen auswirken. Darüber hinaus trägt eine besser ausgebildete Erwerbsbevölkerung dazu bei, das Wirtschaftswachstum und somit das gesellschaftliche Wohlergehen zu steigern. Dies wirkt sich auch auf die Höhe der Mittel aus, die dem Staat zur Verfügung stehen: Wenn die Zahl der beschäftigungsfähigen und besser qualifizierten Arbeitskräfte zunimmt, können sich dadurch die durch Steuern und Abgaben erzielten Staatseinnahmen erhöhen, während zugleich der Bedarf an staatlichen Sozialausgaben abnimmt.

Eine der wichtigsten Ergebnismessgrößen für den Bildungssektor ist der öffentliche Kapitalwert der Bildung. Der Kapitalwert misst den ökonomischen Ertrag öffentlicher Investitionen in den Bildungssektor nach Berücksichtigung der Investitionskosten. Zusätzliche Bildungsteilnahme schafft einen wirtschaftlichen Nutzen für Staaten, da sie durch höhere Einkommen und neue Arbeitsmarktteilnehmer für zusätzliche Staatseinnahmen sorgt. Im Schnitt beläuft sich der Kapitalwert der Tertiärbildung in den OECD-Mitgliedsländern auf mehr als 100000 US-\$. Vergleicht man dies mit dem Umfang der öffentlichen Investitionen, der im Durchschnitt ein Drittel des Kapitalwerts beträgt, werden die Anreize für Staaten, in Tertiärbildung zu investieren, deutlich. Unter den OECD-Mitglieds- ländern weisen Irland, Ungarn und die Vereinigten Staaten den größten öffentlichen Kapitalwert aus der Tertiärbildung aus. Die Investitionen in Sekundarbereich-II-Bildung erzielen im Schnitt niedrigere Erträge; am ungünstigsten stellt sich das Verhältnis in Estland dar, wo die Kosten höher ausfallen als der Nutzen.

\section{Gesundheitswesen}

Die Regierungen haben außerdem ein starkes Interesse daran, die Kosteneffektivität ihrer Gesundheitssysteme zu evaluieren - nicht nur wegen der hohen Relevanz der im Gesundheitswesen erzielten Ergebnisse, sondern auch weil die Ausgaben für diesen Sektor einen der größten Posten der Staatsausgaben ausmachen, der zudem in der jüngeren Vergangenheit auf Grund des technologischen Fortschritts und der Bevölkerungsalterung immer weiter gestiegen ist.

Zur Evaluierung der Kosteneffektivität wird die Verbesserung der Lebenserwartung (das am häufigsten verwendete und am besten vergleichbare Ergebnis) im Vergleich zu den Pro-KopfGesamtgesundheitsausgaben dieser Länder betrachtet. Bei der Interpretation dieser Daten ist jedoch Vorsicht geboten, da neben den Gesamtgesundheitsausgaben noch viele weitere Faktoren entscheidenden Einfluss auf die Lebenserwartung haben und die Gesamtgesundheitsausgaben sowohl staatliche als auch private Ausgaben umfassen (wobei der private Anteil an den Gesundheitsausgaben in Ländern wie den Vereinigten Staaten und Mexiko besonders hoch ist). Die Resultate zeigen, dass ein positiver Zusammenhang zwischen den Gesamtgesundheitsausgaben pro Kopf und der Lebenserwartung besteht, was darauf hindeutet, dass höhere Gesundheitsausgaben mit besseren Gesundheitsergebnissen der Bevölkerung einhergehen. In Italien, Japan und Spanien ist die Lebenserwartung in Relation zu den Gesundheitsausgaben relativ hoch. In Mexiko, Ungarn und den Vereinigten Staaten dagegen ist die Lebenserwartung in Anbetracht der Gesamtgesundheitsausgaben dieser Länder relativ niedrig. Ähnliche Ergebnisse (vgl. Abbildung online) haben gezeigt, dass der allgemein positive Zusammenhang mit der Lebenserwartung auch dann bestehen bleibt, wenn nur staatliche Gesundheitsausgaben berücksichtigt werden. Das Ausmaß, in dem die relativ niedrige Lebenserwartung im Verhältnis zu den Gesundheitsausgaben in Mexiko und den Vereinigten Staaten vom OECD-Durchschnitt abweicht, verringert sich jedoch geringfügig, wenn nur staatliche Gesundheitsausgaben berücksichtigt werden. 


\section{Methodik und Definitionen}

Die staatlichen ökonomischen Erträge aus Bildung werden anhand des Kapitalwerts gemessen. Die staatlichen Kosten enthalten die entgangenen Einkommensteuern während der Dauer der Bildungsteilnahme. Die staatlichen Ausgaben für Bildung hängen mit dem jeweiligen Bildungsabschluss und der Ausbildungsdauer zusammen. Sie umfassen direkte Bildungsausgaben und öffentlich-private Transferleistungen. Auf der Nutzenseite stehen für den öffentlichen Sektor die Mehreinnahmen von Steuern und Sozialabgaben aus höheren Einkommen sowie Einsparungen bei den Transferleistungen, die der Staat oberhalb eines bestimmten Einkommensniveaus nicht mehr aufbringen muss. Die Datenwerte zur Berechnung des Kapitalwerts einer Sekundarbereich-II-Bildung beruhen auf der Differenz zwischen Personen mit einem Bildungsabschluss des Sekundarbereichs II oder des postsekundären nichttertiären Bereichs und Personen ohne einen derartigen Abschluss. Die Datenwerte zur Berechnung des Kapitalwerts einer Tertiärbildung beruhen auf der Differenz zwischen Personen mit einem Bildungsabschluss des Tertiärbereichs und Personen mit Sekundarbereich-II-Abschluss.

Die Lebenserwartung misst, wie viele Jahre die Menschen basierend auf einer gegebenen Reihe von altersspezifischen Mortalitätsraten im Durchschnitt leben. Die Lebenserwartung bei Geburt für die Gesamtbevölkerung wird als der ungewichtete Durchschnitt der Lebenserwartung von Männern und Frauen berechnet. Die Gesamtausgaben für das Gesundheitswesen messen den Endverbrauch an Waren und Dienstleistungen der Gesundheitsversorgung (d.h. die laufenden Ausgaben) sowie die Investitionsausgaben für die Gesundheitsinfrastruktur. Hierzu zählen sowohl staatliche als auch private Ausgaben für medizinische Leistungen und Waren, öffentliche Gesundheits- und Vorsorgeprogramme sowie Verwaltungsleistungen.

Abbildung 2.27, Lebenserwartung bei Geburt und staatliche Gesundheitsausgaben pro Kopf (2011), ist online verfügbar unter http://dx.doi.org/10.1787/888932941291.

\section{Weitere Informationen}

OECD (2013a), Bildung auf einen Blick 2013: OECD-Indikatoren, W. Bertelsmann Verlag, Bielefeld, http://dx.doi.org/10.1787/eag2013-de.

OECD (2013b, erscheint demnächst), Health at a Glance 2013: OECD Indicators, OECD Publishing, Paris.

\section{Anmerkungen zu den Abbildungen}

2.25: Für Belgien und die Niederlande wurden keine Daten zum Sekundarbereich II berücksichtigt, da dieser Bildungsbereich Teil der Pflichtschulzeit ist. Für Japan wurden mangels Aufschlüsselung in Sekundarbereich I und II keine Daten zum Sekundarbereich II berücksichtigt. Die Daten für Italien, die Niederlande und Polen beziehen sich auf 2008. Die Daten für Japan beziehen sich auf 2007. Die Daten für die Türkei beziehen sich auf 2005. Vgl. Anmerkungen in Anhang 3 (www.oecd.org/edu/eag.htm).

2.26: Die Daten zu den Ausgaben in Belgien und Neuseeland enthalten keine Investitionen. Die Daten zu den Ausgaben in den Niederlanden beziehen sich auf die laufenden Ausgaben. Die Daten zu den Ausgaben in Belgien, Mexiko und Neuseeland beruhen auf einer anderen Methodik. Die Daten zu den Ausgaben in Chile, Israel und Mexiko sind Schätzungen. Die Daten zur Lebenserwartung in Australien, Belgien, Chile, Frankreich, Italien und den Vereinigten Staaten sind Schätzungen. Die Daten zu den Ausgaben in Australien, Israel, Japan und Luxemburg beziehen sich auf 2009. Die Daten zu den Ausgaben in der Türkei beziehen sich auf 2008. Die Daten zur Lebenserwartung in Kanada beziehen sich auf 2009.

Hinweis zu den Daten für Israel: http://dx.doi.org/10.1787/888932315602. 


\section{STRATEGISCHE GOVERNANCE}

\section{Kosteneffektivität des öffentlichen Sektors}

2.25. Staatlicher Kapitalwert bei einem Mann, der im Rahmen der Erstausbildung einen Abschluss im Sekundarbereich II bzw. im postsekundären nichttertiären Bereich (linke Seite) oder im Tertiärbereich (rechte Seite) erwirbt (2009 oder letztes verfügbares Jahr)

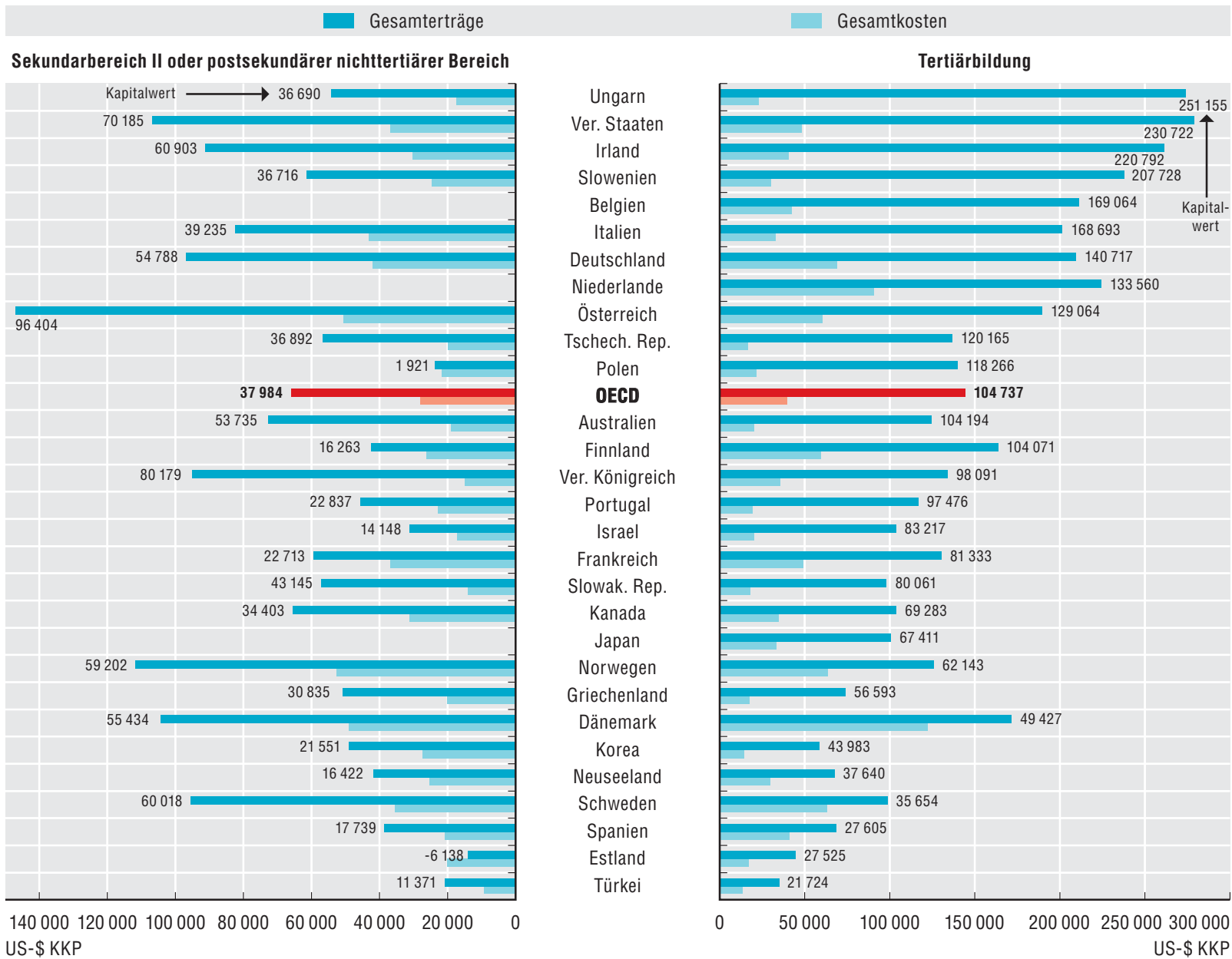

Quelle: OECD (2013), Bildung auf einen Blick 2013: OECD-Indikatoren, W. Bertelsmann Verlag, Bielefeld, Tabelle A7.2a und Tabelle A7.4a, http://dx.doi.org/ 10.1787/eag-2013-de. 
2.26. Lebenserwartung bei Geburt und Gesamtgesundheitsausgaben pro Kopf (2011)

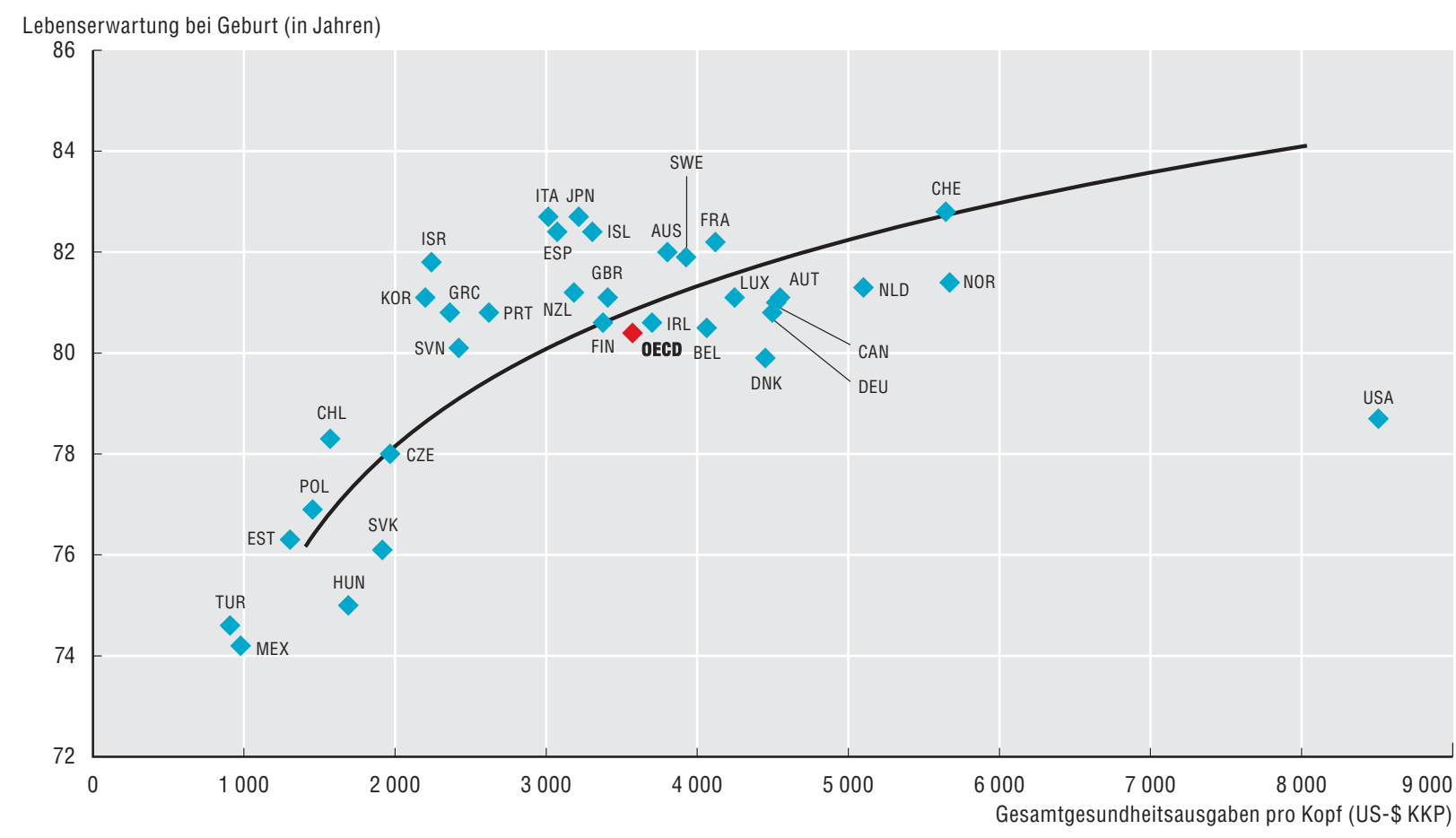

Quelle: OECD Health Statistics 2013.

StatLink न्ता st http://dx.doi.org/10.1787/888932941272 


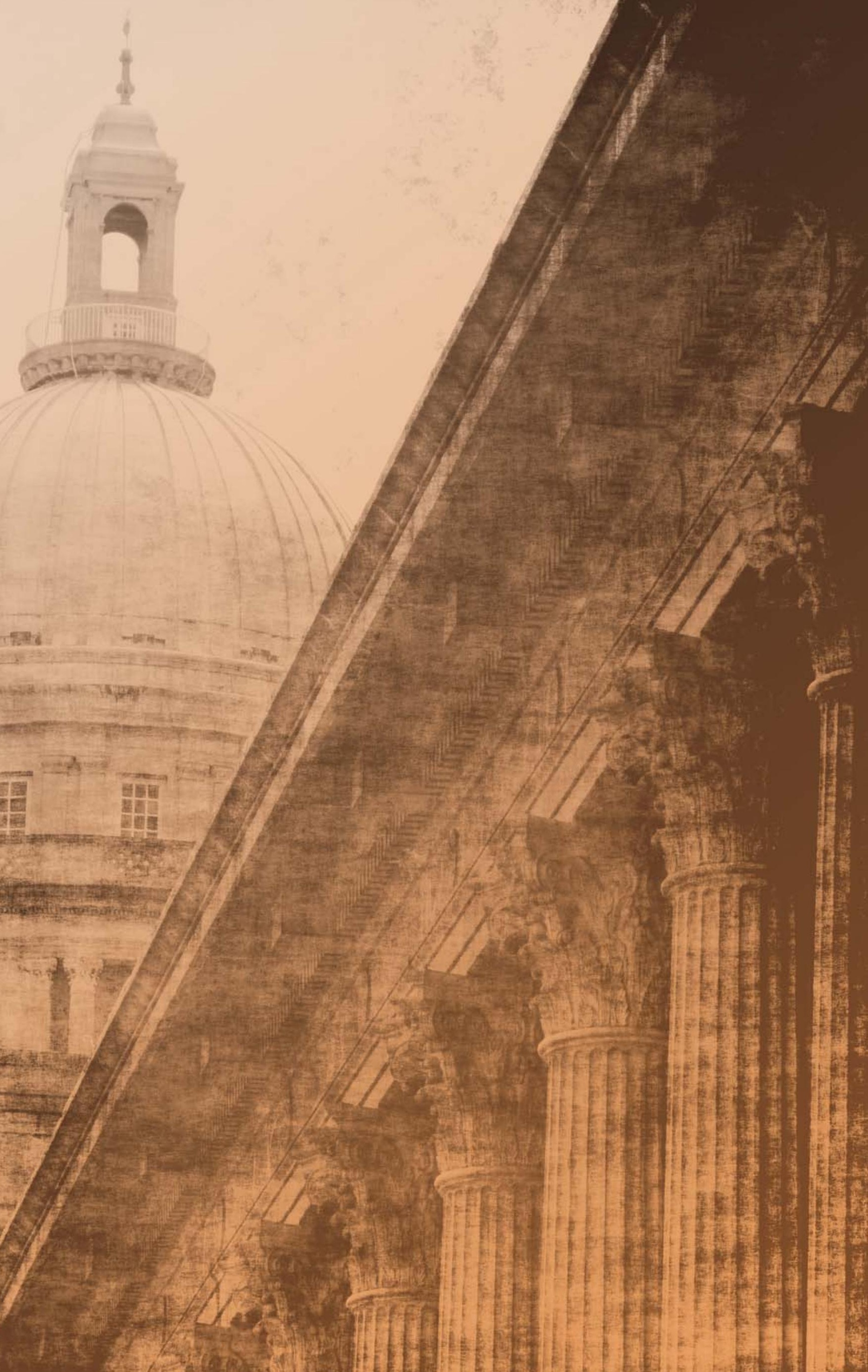


Der Finanzierungssaldo des Staats ist die Differenz zwischen seinen Einnahmen und Ausgaben. Ein Defizit entsteht, wenn der Staat in einem gegebenen Jahr mehr ausgibt, als er einnimmt. Ein Überschuss bildet sich, wenn die staatlichen Einnahmen höher sind als die staatlichen Ausgaben. Wenn über mehrere Jahre hinweg hohe Defizite ausgewiesen werden, wirkt sich dies sehr nachteilig auf die Tragfähigkeit der öffentlichen Finanzen aus, da diese Defizite durch Neuverschuldung finanziert werden müssen. Eine hohe Verschuldung treibt den Staat über steigende Schuldendienstkosten (in Form absoluter Zinszahlungen und höherer Zinssätze) weiter ins Defizit, wodurch die Tragfähigkeit der öffentlichen Finanzen zusätzlich beeinträchtigt wird. Die Regierungen können die künftigen Schuldendienstkosten senken, indem sie den Primärsaldo verbessern, d.h. den Finanzierungssaldo abzüglich der Zinszahlungen.

2011 wiesen die OECD-Länder ein durchschnittliches Finanzierungsdefizit in Höhe von 3,5\% ihres BIP aus. Am höchsten war das Defizit in Irland (13,3\%), den Vereinigten Staaten (10,1\%), Griechenland (9,6\%), Spanien $(9,4 \%)$ und Japan (8,9\%). Nur sechs OECD-Länder konnten einen Finanzierungsüberschuss vorweisen: Norwegen $(13,4 \%)$, Ungarn $(4,2 \%)$, Korea $(2,0 \%)$, Estland (1,2\%), die Schweiz (0,5\%) und Schweden (0,03\%).

Zwischen 2001 und 2009 haben sich die Defizite in allen OECDLändern außer der Schweiz und Deutschland ausgeweitet (von durchschnittlich 0,7\% auf 5,5\% des BIP). Der Großteil dieser Ausweitung entfiel allerdings auf den Zeitraum 2008-09 und war auf die weltweite Finanz- und Wirtschaftskrise zurückzuführen, während der die Staatsausgaben stärker stiegen als die Staatseinnahmen und das BIP (nominal, in allen Ländern mit Ausnahme Israels und Ungarns). Nach 2009 kam es zu einer Wende in diesem Trend, hauptsächlich auf Grund der Haushaltsregeln, neuen Haushaltsverfahren und Pläne zur Konsolidierung der öffentlichen Haushalte, die als Reaktion auf die Krise umgesetzt wurden. Zwischen 2009 und 2011 war die nominale Wachstumsrate der Ausgaben niedriger als die der Einnahmen und des BIP (in allen Ländern außer Japan, Neuseeland, Slowenien und der Schweiz). Dies hatte zur Folge, dass sich die staatlichen Finanzierungssalden im Verhältnis zum BIP um durchschnittlich 2 Prozentpunkte verbesserten. Die stärkste Verbesserung wurde in Ungarn verbucht (wo das Defizit um 8,7 Prozentpunkte abnahm und sich in einen Überschuss von $4,2 \%$ verwandelte), gefolgt von Griechenland (6 Prozentpunkte) und Portugal (5,8 Prozentpunkte). Die erhebliche Verbesserung des ungarischen Haushaltssaldos war auf Vermögenstransfers von den privaten an die öffentlichen Haushalte (in Höhe von 9,7\% des BIP) zurückzuführen, zu denen es im Zuge der Reform des Rentensystems im Jahr 2011 kam. Nur in drei Ländern hat sich der Finanzierungssaldo des Staats zwischen 2009 und 2011 nicht verbessert, in Japan, Slowenien und der Schweiz.

2011 waren die Primärhaushalte der OECD-Mitgliedsländer im Durchschnitt defizitär (mit einem negativen Saldo von 0,8\% des BIP). Der verbleibende Teil der Finanzierungssalden entsprach den Schuldzinszahlungen. Der Saldo der Primärhaushalte fiel in den einzelnen OECD-Ländern sehr unterschiedlich aus: 19 Länder wiesen im Primarhaushalt ein Defizit aus, das von $10 \%$ des BIP in Irland bis zu 0,1\% des BIP in Dänemark reichte, während 14 andere Länder einen Überschuss - im Umfang von $0,2 \%$ des BIP in Österreich bis zu 14,5\% des BIP in Norwegen erwirtschafteten.

Die Bedeutung der Zinszahlungen innerhalb der gesamtstaatlichen Finanzierungssalden unterschied sich ebenfalls deutlich im Ländervergleich. In den 19 Ländern, die ein Defizit im Primärhaushalt auswiesen, machten die Zinszahlungen im Durchschnitt 2,9\% des BIP aus. Am geringsten war dieser Anteil in der Tschechischen Republik mit 1,4\%, am höchsten in Griechenland mit 7,2\%. Griechenlands Primärdefizit war mit 2,4\% des BIP zwar nicht das höchste im OECD-Raum, kein anderes OECD-Land musste jedoch so hohe Zinszahlungen leisten.

\section{Methodik und Definitionen}

Die Daten zu den gesamtstaatlichen Finanzierungssalden stammen aus der OECD-Datenbank National Accounts Statistics, die auf dem System der Volkswirtschaftlichen Gesamtrechnungen (SNA) basiert. Dabei handelt es sich um einen international vereinbarten Katalog von Konzepten, Definitionen, Klassifizierungen und Regeln für Volkswirtschaftliche Gesamtrechnungen. Nach der SNATerminologie setzt sich der Sektor Staat zusammen aus der Zentralregierung, den Gliedstaaten, den Gemeinden und der Sozialversicherung. Der gesamtstaatliche Finanzierungssaldo, auch als Finanzierungsüberschuss (+) bzw. Finanzierungsdefizit (-) der öffentlichen Haushalte bezeichnet, ergibt sich aus den gesamtstaatlichen Einnahmen abzüglich der gesamtstaatlichen Ausgaben. Die Einnahmen umfassen Sozialversicherungsbeiträge, Steuern sowie Zuwendungen und sonstige Einkünfte. Bei den Ausgaben handelt es sich um Vorleistungen, Arbeitnehmerentgelte, Subventionen, Sozialleistungen und sonstige laufende Ausgaben (einschließlich Zinsaufwendungen), Vermögenstransfers und sonstige investive Ausgaben. Der Primärsaldo entspricht dem Finanzierungssaldo des Staats abzüglich der auf die Verschuldung der öffentlichen Haushalte zu zahlenden Zinsen.

Das Bruttoinlandsprodukt (BIP) ist die Standardmessgröße des Werts der von einer Volkswirtschaft in einem bestimmten Zeitraum produzierten Waren und Dienstleistungen.

\section{Literaturhinweise}

OECD (2013a), National Accounts at a Glance 2013, OECD Publishing, Paris, http://dx.doi.org/10.1787/na_glance-2013-en.

OECD (2013b), OECD-Wirtschaftsausblick Vol. 2013/1, OECD Publishing, Paris, http://dx.doi.org/10.1787/eco_outlook-v2013-1-de.

\section{Anmerkungen zu den Abbildungen}

Die Daten für Kanada, Neuseeland und die Russische Föderation beziehen sich auf 2010 anstelle von 2011.

3.1: Für Chile und die Türkei liegen keine Daten für 2001 vor, diese Länder sind im OECD-Durchschnitt daher nicht berücksichtigt. Die Daten für Chile beziehen sich auf 2010 anstelle von 2011. Bei den Daten für Japan und Mexiko für 2001 handelt es sich um Schätzungen. Die Daten für die Russische Föderation beziehen sich auf 2002 anstelle von 2001.

3.2: Für Chile stehen keine Daten zur Verfügung.

Hinweis zu den Daten für Israel: http://dx.doi.org/10.1787/888932315602. 


\section{3. ÖFFENTLICHE FINANZEN UND WIRTSCHAFTLICHES HANDELN DES STAATES}

3.1. Gesamtstaatlicher Finanzierungssaldo in Prozent des BIP (2001, 2009 und 2011)

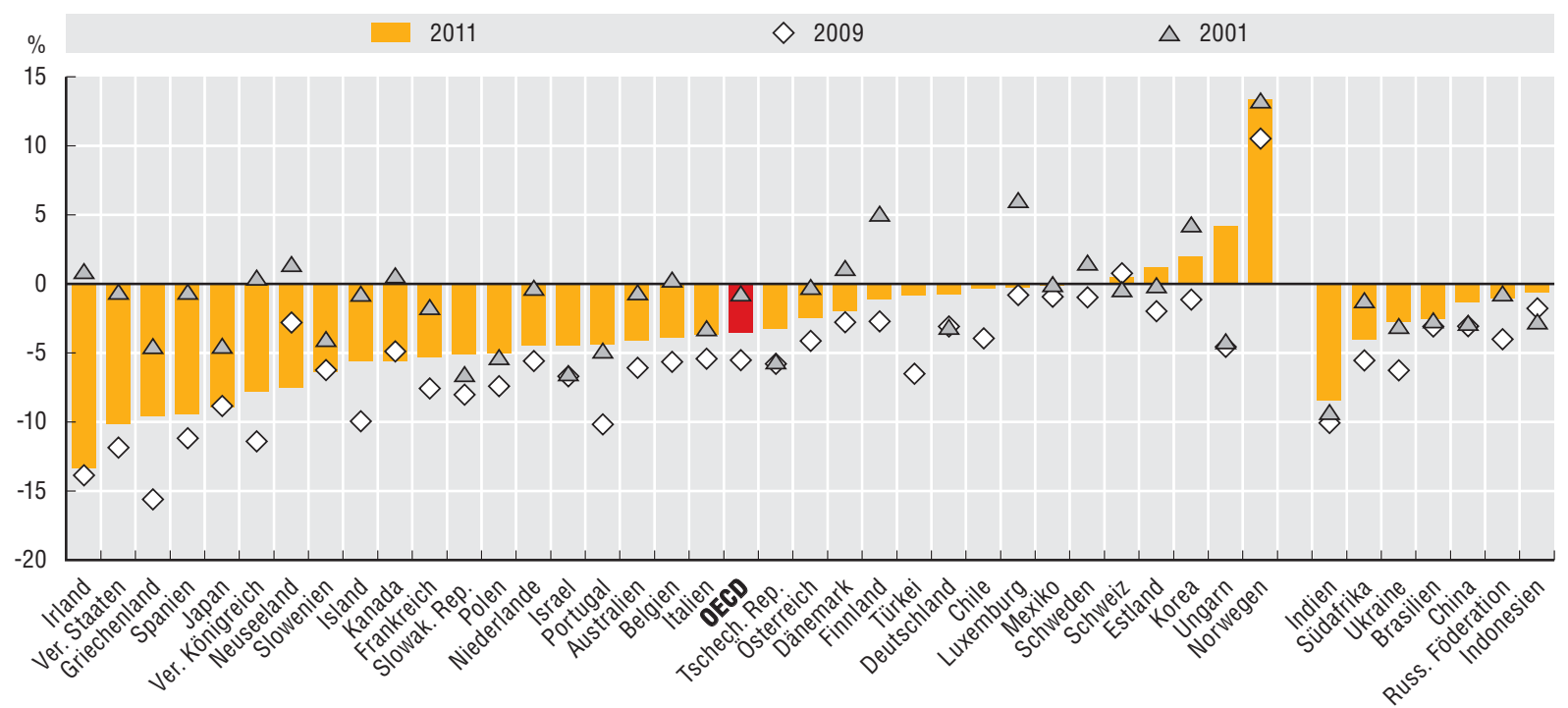

Quelle: Daten zu den OECD-Mitgliedsländern: OECD National Accounts Statistics (Datenbank). Daten zu den anderen großen Volkswirtschaften (ohne Russische Föderation): Internationaler Währungsfonds (2013), Economic Outlook, April 2013, IWF, Washington, DC.

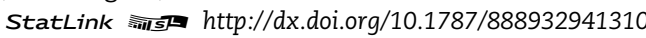

3.2. Primärsaldo und Zinsaufwendungen des Staats in Prozent des BIP (2011)

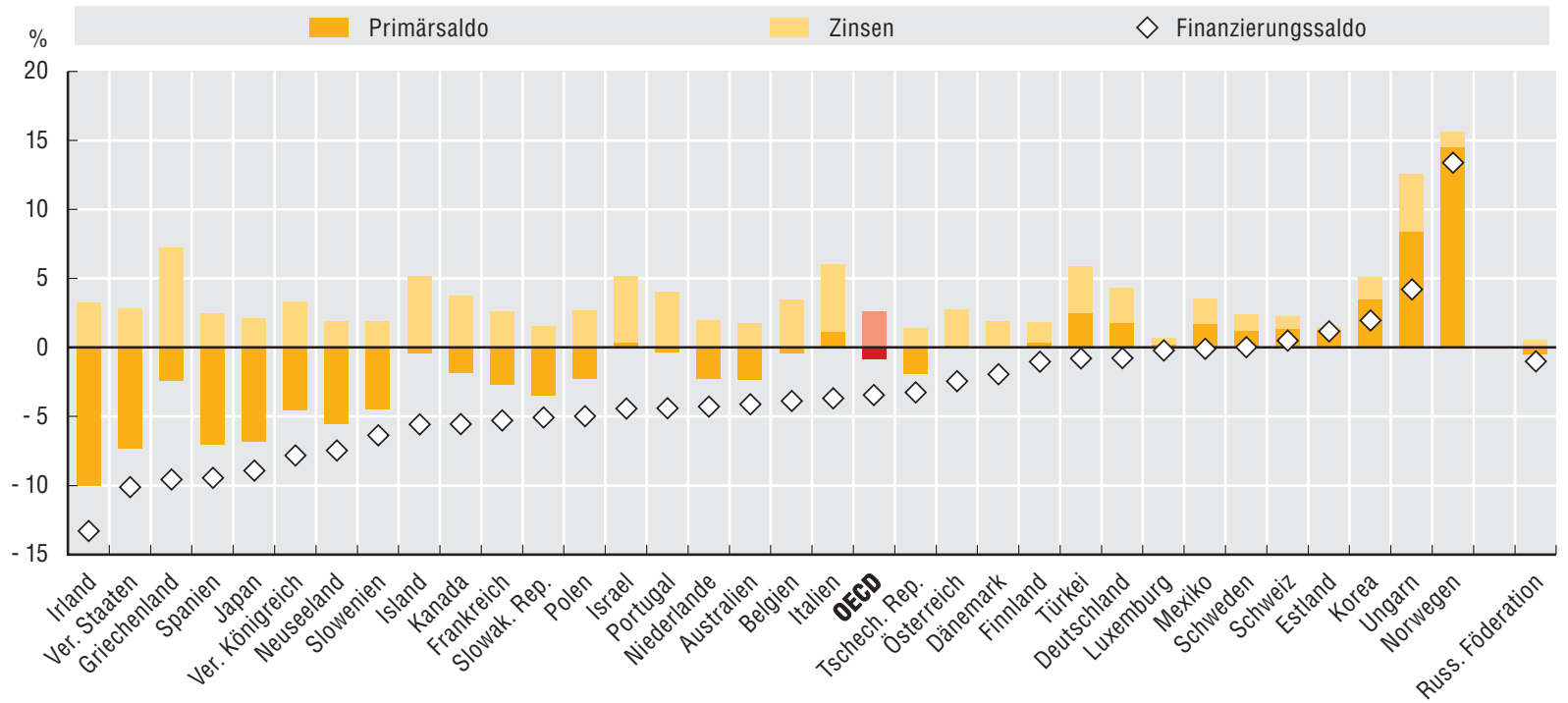

Quelle: OECD National Accounts Statistics (Datenbank). 
Wenn die Ausgaben die Einnahmen übersteigen, muss der Staat zur Finanzierung seines Defizits zusätzliche Mittel in Form von Krediten aufnehmen, womit sich die öffentliche Verschuldung erhöht. Veränderungen des Schuldenstands im zeitlichen Verlauf ergeben sich aus der vergangenen Entwicklung der staatlichen Finanzierungssalden. Werden wiederholt hohe Defizite ausgewiesen, steigt der Schuldenstand. Werden mehrere Jahre in Folge Haushaltsüberschüsse erwirtschaftet, sinkt er hingegen. Je höher die Verschuldung eines Staates, umso höher stufen die Märkte im Allgemeinen das Kreditausfallrisiko dieses Staates ein und umso höher ist folglich der Risikoaufschlag, den die Märkte diesem Staat berechnen, so dass sich dessen Schuldendienstkosten erhöhen.

2011 belief sich die gesamtstaatliche Bruttoverschuldung der OECD-Mitgliedsländer im Durchschnitt auf 78,8\% ihres BIP; am niedrigsten war sie mit 10\% des BIP in Estland, am höchsten mit $228 \%$ des BIP in Japan. In der Mehrzahl der OECD-Länder war die Verschuldung 2011 höher als 2001. Dies war allerdings das Resultat des Zusammenwirkens verschiedener Entwicklungen, da die Schuldenquote bis 2007 rückläufig war, hauptsächlich auf Grund des Wirtschaftswachstums. Seitdem ist die Verschuldung kontinuierlich gestiegen, vor allem wegen der weltweiten Finanzkrise, d.h. konkret wegen geringerer Staatseinnahmen, einer schrumpfenden Wirtschaftstätigkeit und/ oder zusätzlicher Ausgaben für Konjunkturpakete sowie Maßnahmen zur Stützung von Finanzinstituten. Der größte Anstieg wurde in diesem Zeitraum in Japan verzeichnet (76,6 Prozentpunkte), gefolgt von Irland (67 Prozentpunkte), dem Vereinigten Königreich (55,1 Prozentpunkte) und den Vereinigten Staaten (48,1 Prozentpunkte).

Die Pro-Kopf-Staatsverschuldung variiert erheblich, am niedrigsten ist sie mit 2207 US-\$ in Estland, am höchsten mit 77134 US-\$ in Japan. Im OECD-Durchschnitt beläuft sie sich auf 26774 US-\$. In Japan befindet sich der Großteil der Staatsanleihen im Besitz japanischer Staatsbürger; deshalb wird das Kreditausfallrisiko - und damit auch die erforderliche Risikoprämie trotz des hohen Schuldenstands als geringer betrachtet.

Außer in Australien und Estland sind Wertpapiere ohne Anteilsrechte das bevorzugte Finanzierungsinstrument der OECD-Länder. Eine Schuldenstruktur, in der Wertpapiere ohne Anteilsrechte großes Gewicht haben, ist Marktschwankungen unterworfen, die Auswirkungen auf die Schuldendienstkosten haben.

\section{Methodik und Definitionen}

Die Daten stammen aus der OECD-Datenbank OECD National Accounts Statistics, die auf dem System der Volkswirtschaftlichen Gesamtrechnungen (SNA) basiert. Dabei handelt es sich um einen international vereinbarten Katalog von Konzepten, Definitionen, Klassifizierungen und Regeln für Volkswirtschaftliche Gesamtrechnungen. Die Verschuldung ist ein häufig verwendetes Konzept, definiert als eine spezifische, nach den berücksichtigten oder nicht berücksichtigten Arten von Finanzierungsinstrumenten identifizierte Untergruppe von Verbindlichkeiten. Als Verschuldung gelten in der Regel sämtliche Verbindlichkeiten, die es erforderlich machen, dass der Schuldner zu einem Zeitpunkt bzw. mehreren Zeitpunkten in der Zukunft an den Gläubiger Zins- oder Tilgungszahlungen leistet. Alle Schuldtitel sind folglich Verbindlichkeiten; einige Verbindlichkeiten, z.B. Beteiligungen, Aktien und Finanzderivate, gelten jedoch nicht als Schuldtitel.
Die Verschuldung ergibt sich somit aus der Summe der folgenden Kategorien von Verbindlichkeiten (nach dem System der Volkswirtschaftlichen Gesamtrechnungen von 1993), soweit diese im Rahmen der Vermögensbilanz des jeweiligen institutionellen Sektors verfügbar/anwendbar sind: Bargeldbestände und Einlagen, Wertpapiere ohne Anteilsrechte (außer Finanzderivate), Kredite, versicherungstechnische Rückstellungen und sonstige Verbindlichkeiten. Gemäß dem System der volkswirtschaftlichen Gesamtrechnungen werden die meisten Schuldtitel zu Marktpreisen bilanziert (einige Länder weichen jedoch u.U. von dieser Regel ab, insbesondere was Wertpapiere ohne Anteilsrechte außer Finanzderivate betrifft).

Die entsprechenden Daten sind für die einzelnen Länder nicht immer vergleichbar, da die Bestandteile der Verschuldung dort u.U. unterschiedlich definiert oder behandelt werden. Insbesondere enthalten sie in einigen OECD-Ländern (z.B. Australien und Kanada) sowie in den Ländern, deren Daten dem Wirtschaftsausblick des IWF entnommen sind, nicht kapitalgedeckte staatlich geförderte Altersvorsorgepläne. Daher ist der Schuldenstand in diesen Ländern überzeichnet im Vergleich zu Ländern mit großen nicht kapitalgedeckten Rentenverbindlichkeiten, die nicht unter den Hauptposten des Systems der Volkswirtschaftlichen Gesamtrechnungen von 1993 erfasst sind.

Die SNA-Definition der Verschuldung weicht von der Definition des Maastricht-Vertrags ab, die zur Bewertung der Lage der öffentlichen Finanzen der EU-Länder verwendet wird. Abbildung 3.7 zur gesamtstaatlichen Verschuldung in der Maastricht-Abgrenzung, nach Gläubigern (2011) kann online eingesehen werden unter: http://dx.doi.org/ 10.1787/888932941424.

Die Pro-Kopf-Staatsverschuldung wurde ermittelt, indem die Staatsverschuldung unter Verwendung der OECD/ Eurostat-Kaufkraftparitäten (KKP) für das BIP in US-Dollar von 2011 konvertiert und durch die Einwohnerzahl dividiert wurde. Für die Länder, bei denen der Wirtschaftsausblick des IWF als Datenquelle dient, wurde ein implizierter KKP-Umrechnungsfaktor verwendet. Die KKP entsprechen der Zahl der Währungseinheiten von Land B, die erforderlich sind, um eine gleiche Menge an Waren und Dienstleistungen in Land A zu kaufen. Abbildung $3.6 \mathrm{zu}$ den Jahreswachstumsraten der realen Pro-Kopf-Staatsverschuldung (2001-11) kann online eingesehen werden unter: $h t t p: / / d x . d o i . o r g / 10.1787 / 888932941405$.

\section{Literaturhinweise}

OECD (2013), Die OECD in Zahlen und Fakten 2013, OECD Publishing, Paris, http://dx.doi.org/10.1787/factbook-2013-de.

\section{Anmerkungen zu den Abbildungen}

Für Island, Neuseeland und die Türkei stehen keine Daten zur Verfügung. Die Daten für Chile, Japan, Korea und das Vereinigte Königreich sind auf nicht konsolidierter Basis angegeben. Die Daten für die Schweiz beziehen sich auf 2010 anstelle von 2011.

3.3: Für Chile und Luxemburg liegen keine Daten für 2001 und für Mexiko keine Daten für 2011 vor, diese Länder sind im OECD-Durchschnitt daher nicht berücksichtigt. Die Daten für Korea beziehen sich auf 2002 anstelle von 2001. Die Daten für Dänemark beziehen sich auf 2003 anstelle von 2001.

3.4 und 3.5: Für Mexiko stehen keine Daten zur Verfügung.

Hinweis zu den Daten für Israel: http://dx.doi.org/10.1787/888932315602. 


\section{3. ÖFFENTLICHE FINANZEN UND WIRTSCHAFTLICHES HANDELN DES STAATES}

\subsection{Gesamtstaatliche Verschuldung in Prozent des BIP (2001, 2009 und 2011)}

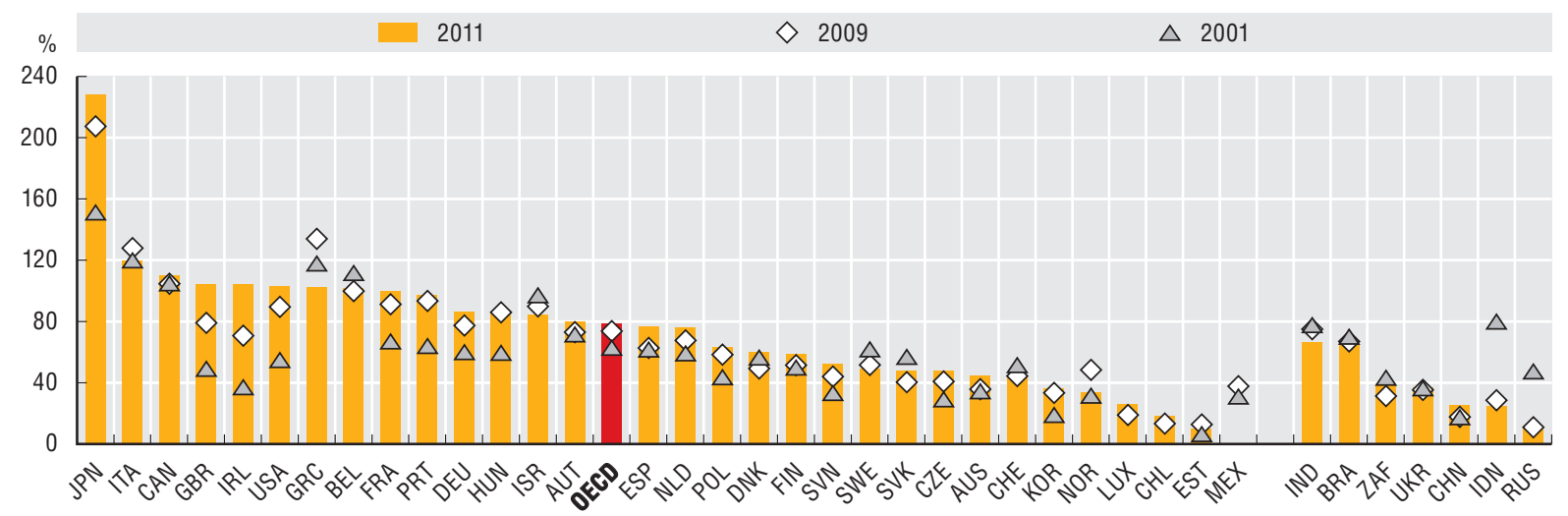

Quelle: Daten zu den OECD-Mitgliedsländern: OECD National Accounts Statistics (Datenbank). Daten zu den anderen großen Volkswirtschaften (ohne Russische Föderation): Internationaler Währungsfonds (2013), Economic Outlook, April 2013, IWF, Washington, DC.

\subsection{Pro-Kopf-Staatsverschuldung (2011)}

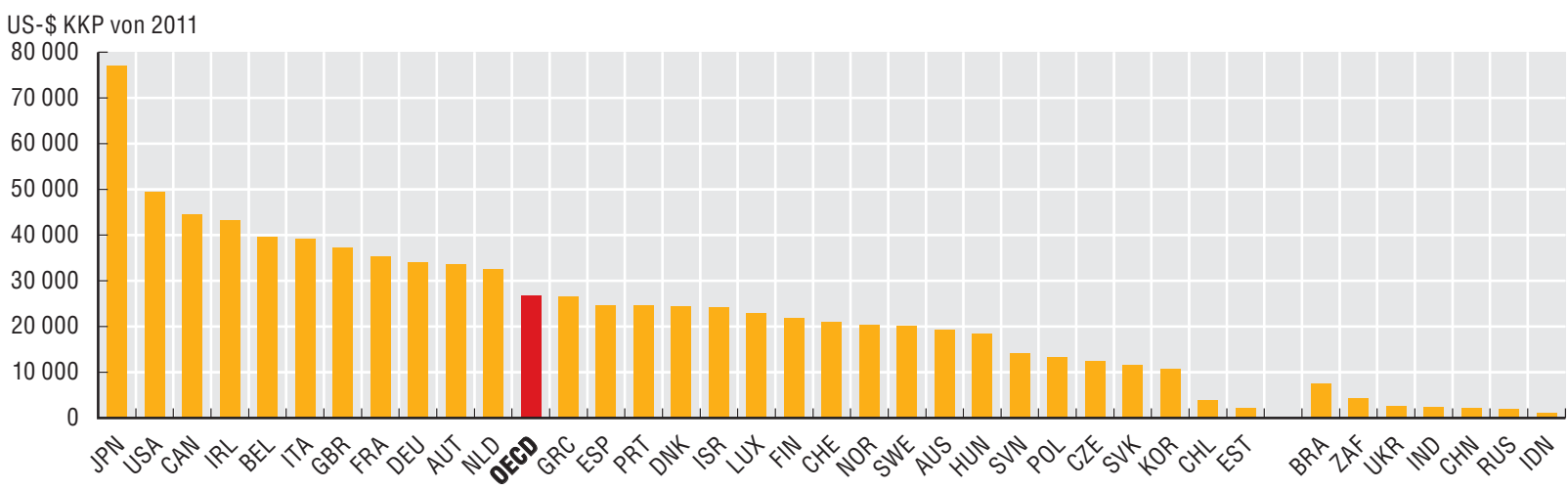

Quelle: Daten zu den OECD-Mitgliedsländern: OECD National Accounts Statistics (Datenbank). Daten zu den anderen großen Volkswirtschaften (ohne Russische Föderation): Internationaler Währungsfonds (2013), Economic Outlook, April 2013, IWF, Washington, DC.

StatLink त्तारs http://dx.doi.org/10.1787/888932941367

\subsection{Zusammensetzung der Staatsverschuldung nach Finanzierungsinstrumenten (2011)}

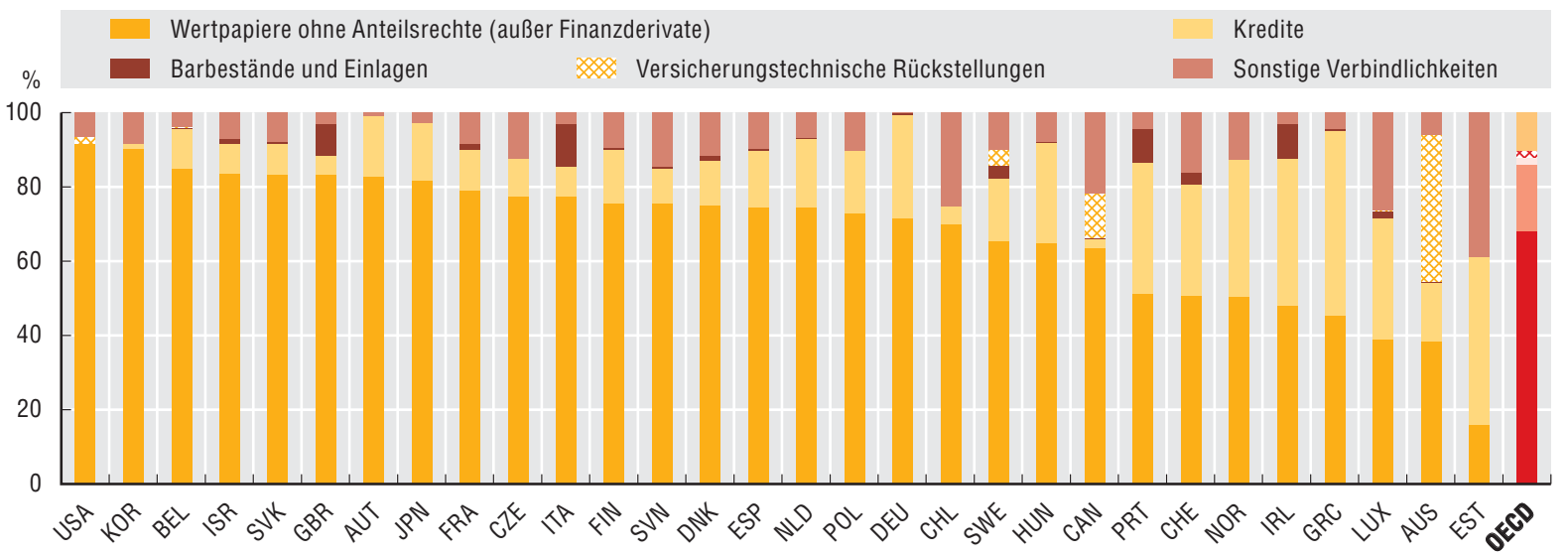

Quelle: OECD National Accounts Statistics (Datenbank). 
Die Zentralregierungen teilen ihre Hoheitsrechte in unterschiedlichem Umfang mit den nachgeordneten Gebietskörperschaften. Daher kann sich die fiskalische Situation der nachgeordneten Gebietskörperschaften recht unterschiedlich darstellen. In verschiedenen politischen Systemen verfügen die zentrale und die nachgeordneten Ebenen jeweils über einen unterschiedlichen Grad an Autonomie in Bezug auf die Aufnahme von Krediten; nachgeordnete Gebietskörperschaften unterliegen im Allgemeinen strengen Haushaltsregeln, insbesondere ist ihre Kapazität zur Aufnahme von Krediten oft begrenzt. Verbindlichkeiten nachgeordneter Gebietskörperschaften, die sich aus der Notwendigkeit der Finanzierung von Defiziten durch Kreditaufnahme ergeben, gelten als Verschuldung der nachgeordneten Gebietskörperschaften. Wenn die Verschuldung einer großen Zahl von Verwaltungseinheiten (z.B. Gliedstaaten oder Kommunen) steigt - und sei es nur um einen geringeren Betrag -, kann sich die gesamtstaatliche Verschuldung (alle staatlichen Ebenen kombiniert) erhöhen, was Auswirkungen auf den Finanzierungssaldo des Staats und potenziell auch auf die Zinssätze hat, die auf die öffentliche Verschuldung gezahlt werden müssen.

Im Vergleich zu den Zentralregierungen verfügen die nachgeordneten Gebietskörperschaften meistens nur über eine geringe Einnahmebasis. Deshalb sind die meisten nachgeordneten Gebietskörperschaften von Transferzahlungen der zentralen Ebene abhängig. 2011 belief sich das Finanzierungsdefizit auf Ebene der Zentralregierungen im OECD-Durchschnitt auf 3,2\% des BIP und war damit nur 0,2 Prozentpunkte geringer als auf gesamtstaatlicher Ebene. In Australien, Kanada, Deutschland und Spanien ergibt sich mehr als ein Drittel des gesamtstaatlichen Finanzierungssaldos aus den Finanzierungssalden der Gliedstaaten.

Die Verschuldung der nachgeordneten Gebietskörperschaften ist im Vergleich zur gesamtstaatlichen Verschuldung im OECDRaum nicht besonders hoch, außer in einigen - mehrheitlich föderal bzw. quasiföderal organisierten - Ländern. 2011 belief sich die Verschuldung der nachgeordneten Ebenen im Durchschnitt auf $11,8 \%$ des BIP, wobei die Verschuldung der Gemeinden in Griechenland mit 1,3\% des BIP am niedrigsten und in Japan mit $38 \%$ des BIP am höchsten war. In Kanada, Deutschland, den Vereinigten Staaten (Bundesstaaten und Gemeinden) und Spanien betrug die Verschuldung der Gliedstaaten $53,3 \%, 26,0 \%, 24,7 \%$ bzw. $18,7 \%$ des BIP.

Bei der Aufschlüsselung der Verschuldung nach staatlichen Ebenen waren in den OECD-Ländern zwischen 2001 und 2011 keine größeren Veränderungen festzustellen. Im Vereinigten Königreich verringerte sich die Verschuldung der nachgeordneten Gebietskörperschaften um 7,1 Prozentpunkte; dieser Trend dürfte sich infolge der Reform der Finanzierung des sozialen Wohnungsbaus, die zu einer erhöhten Kreditaufnahme der Gemeinden führen könnte, in den kommenden Jahren jedoch umkehren. Auch in Japan, den Niederlanden und den Vereinigten Staaten wurde ein erheblicher Rückgang des Anteils der gesamtstaatlichen Verschuldung verzeichnet, der auf die nachgeordneten Gebietskörperschaften entfällt. Da der Schuldenstand in diesen Ländern insgesamt weiter gestiegen ist, kann dieser Rückgang allerdings darauf zurückgeführt werden, dass die Verschuldung auf den nachgeordneten Ebenen langsamer expandiert hat als auf der zentralen Ebene. Außerdem unterliegen die nachgeordneten Gebietskörperschaften häufig strengen Haushaltsregeln und werden von den Zentralregierungen verpflichtet, sich an nationalen Haushaltskonsolidierungsanstrengungen zu beteiligen.

\section{Methodik und Definitionen}

Die Daten stammen aus der OECD-Datenbank National Accounts Statistics, die auf dem System der Volkswirtschaftlichen Gesamtrechnungen (SNA) basiert. Dabei handelt es sich um einen international vereinbarten Katalog von Konzepten, Definitionen, Klassifizierungen und Regeln für Volkswirtschaftliche Gesamtrechnungen. Nach der SNA-Terminologie setzt sich der Sektor Staat zusammen aus der Zentralregierung, den Gliedstaaten, den Gemeinden und der Sozialversicherung. Die Kategorie der Gliedstaaten findet nur für die neun OECD-Länder Anwendung, die föderal organisiert sind: Australien, Österreich, Belgien, Kanada, Deutschland, Mexiko, Spanien (wird als Quasi-Föderalstaat betrachtet), Schweiz und Vereinigte Staaten.

Der gesamtstaatliche Finanzierungssaldo, auch als Finanzierungsüberschuss (+) bzw. Finanzierungsdefizit (-) der öffentlichen Haushalte bezeichnet, ergibt sich aus den gesamtstaatlichen Einnahmen abzüglich der gesamtstaatlichen Ausgaben.

Wegen weiterer Informationen zur Verschuldung vgl. "Methodik und Definitionen“ im Abschnitt zum Indikator "Gesamtstaatliche Verschuldung" auf S. 72.

\section{Literaturhinweise}

Teresa Ter-Minassian (2007), „Fiscal Rules for Subnational Governments: Can They Promote Fiscal Discipline?", OECD Journal on Budgeting, Vol. 6/3, OECD Publishing, Paris, http:// dx.doi.org/10.1787/budget-v6-art17-en.

Vammalle, C. und C. Hulbert (2013), „Sub-National Finances and Fiscal Consolidation: Walking on Thin Ice", OECD Regional Development Working Papers, No. 2013/02, OECD Publishing, Paris, http://dx.doi.org/10.1787/5k49m8cqkcf3-en.

\section{Anmerkungen zu den Abbildungen}

Für Chile stehen keine Daten zur Verfügung. In Australien und den Vereinigten Staaten werden die Gemeinden unter den Gliedstaaten erfasst. In Australien gibt es keine öffentlichen Sozialversicherungssysteme. In Norwegen, dem Vereinigten Königreich und den Vereinigten Staaten wird die Sozialversicherung auf Ebene der Zentralregierung erfasst.

3.8: Die Daten für Kanada und Neuseeland beziehen sich auf 2010 anstelle von 2011.

3.9: Für Mexiko, Neuseeland, die Schweiz und die Türkei stehen keine Daten zur Verfügung. Für Island und Irland liegen keine Daten zur zentralen Ebene vor, diese Länder sind im OECD-Durchschnitt daher nicht berücksichtigt. Die Daten für Japan, Korea, das Vereinigte Königreich und die Vereinigten Staaten sind auf nicht konsolidierter Basis angegeben. Die Daten für die Schweiz beziehen sich auf 2010 anstelle von 2011.

3.10: Für Island, Mexiko, Neuseeland und die Türkei stehen keine Daten zur Verfügung. Für Israel und Luxemburg liegen keine Daten für 2001 vor, diese Länder sind im OECD-Durchschnitt daher nicht berücksichtigt. Die Daten sind auf nicht konsolidierter Basis angegeben (außer für Australien). Die Daten für die Schweiz beziehen sich auf 2010 anstelle von 2011. Die Daten für Korea beziehen sich auf 2002 anstelle von 2001. Die Daten für Dänemark beziehen sich auf 2003 anstelle von 2001.

Hinweis zu den Daten für Israel: http://dx.doi.org/10.1787/888932315602. 


\section{3. ÖFFENTLICHE FINANZEN UND WIRTSCHAFTLICHES HANDELN DES STAATES}

Finanrierungssaldo und Verschuldung nach staatlichen Fibenen

\subsection{Finanzierungssaldo nach staatlichen Ebenen in Prozent des BIP (2011)}

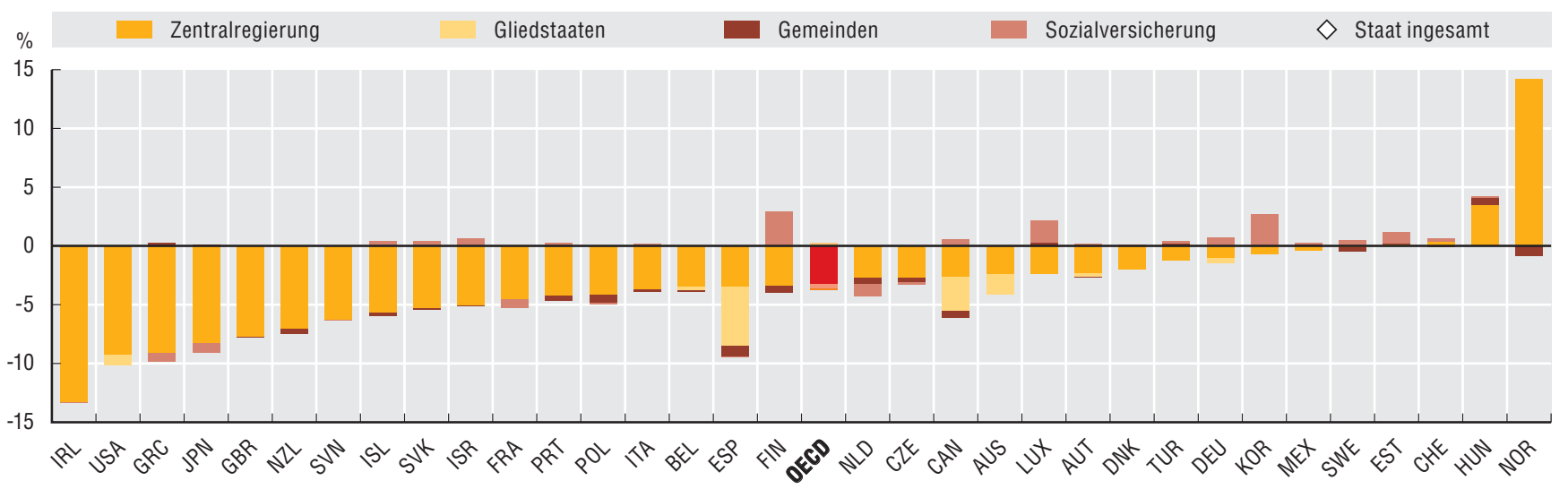

Quelle: OECD National Accounts Statistics (Datenbank).

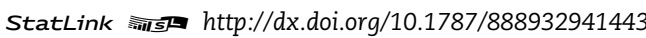

\subsection{Verschuldung nach staatlichen Ebenen in Prozent des BIP (2011)}

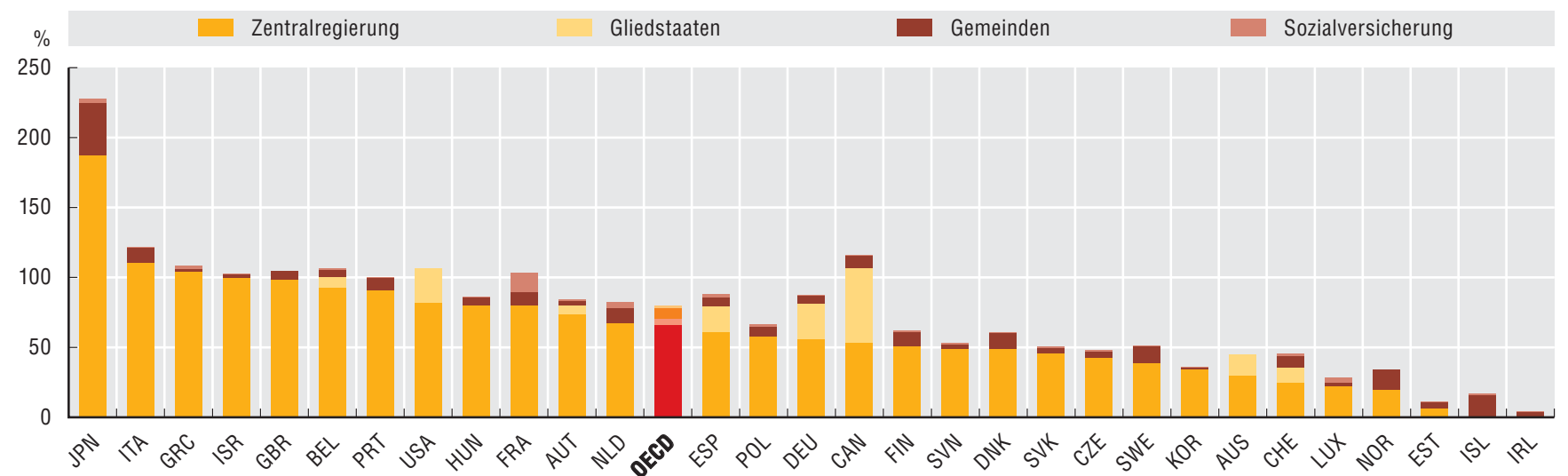

Quelle: OECD National Accounts Statistics (Datenbank).

3.10. Aufschlüsselung der Verschuldung nach staatlichen Ebenen (2001 und 2011)

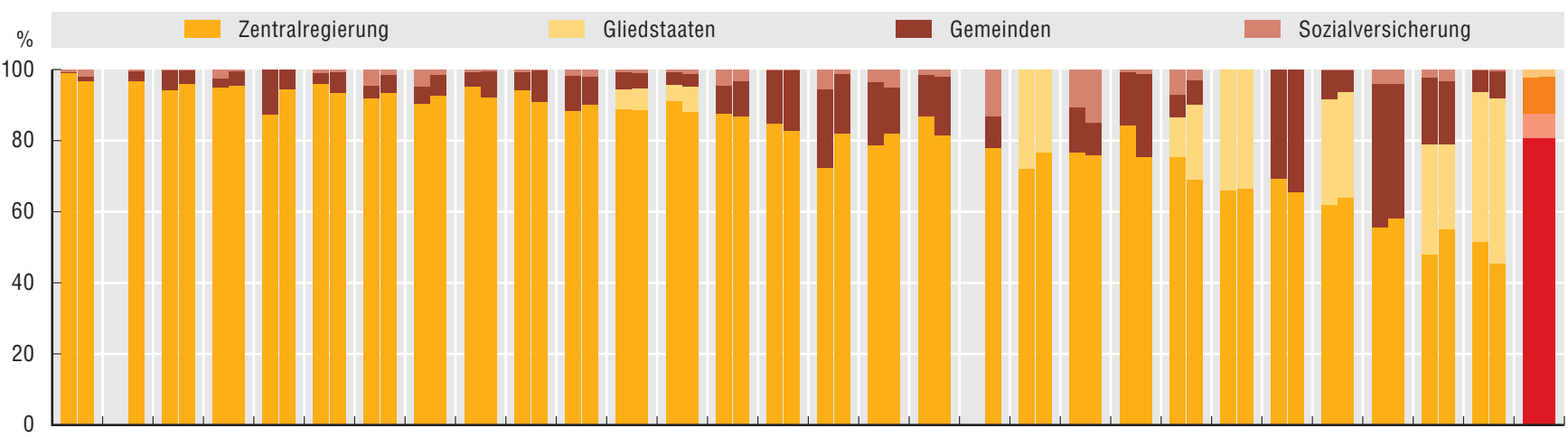

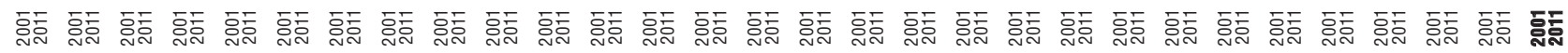
GRC ISR IRL KOR GBR HUN SVN SVK PRT ITA CZE BEL AUT POL DNK JPN NLD FIN LUX USA FRA SWE ESP AUS NOR DEU EST CHE CANOECD Quelle: OECD National Accounts Statistics (Datenbank). 
Staatseinnahmen dienen vor allem zwei Zwecken, zur Finanzierung der Güter und Dienstleistungen, die der Staat für Bürger und Unternehmen bereitstellt, und zur Umverteilung. Die Haupteinnahmequellen des Staats sind Steuern, die bei privaten Haushalten und Unternehmen erhoben werden, und Sozialversicherungsbeiträge. Der Vergleich der Staatseinnahmen der einzelnen OECD-Länder im Verhältnis zu ihrem BIP oder ihrer Einwohnerzahl liefert einen Anhaltspunkt für die Bedeutung, die dem öffentlichen Sektor in der Wirtschaft im Hinblick auf die verfügbaren finanziellen Mittel zukommt. Der Gesamtumfang der Staatseinnahmen wird durch aktuelle und vergangene politische Entscheidungen bestimmt, die ihrerseits durch kulturell geprägte Erwartungen in Bezug auf die soziale Umverteilung, durch Haushaltszwänge, konjunkturelle Schwankungen sowie die Wirtschaftsleistung beeinflusst werden. Daher sind bei der Höhe der Staatseinnahmen erhebliche Unterschiede zwischen den einzelnen OECD-Ländern festzustellen.

2011 beliefen sich die gesamtstaatlichen Einnahmen im OECDDurchschnitt auf 41,9\% des BIP und waren damit nur 0,2 Prozentpunkte höher als zehn Jahre zuvor (im Jahr 2001 machten sie $41,7 \%$ aus). Am höchsten waren sie in Norwegen mit 57,3\%, am niedrigsten in Mexiko mit 22,7\% des BIP. In den nordischen Ländern sind die Staatseinnahmen in der Regel höher als in anderen Ländergruppen, da der Großteil der von den privaten Haushalten bezogenen Sozialleistungen steuerpflichtig ist. Auch wenn die Staatseinnahmen im Verhältnis zum BIP im OECD-Durchschnitt im Zeitraum 2001-11 weitgehend unverändert geblieben sind, waren im Ländervergleich doch deutliche Unterschiede zu verzeichnen. Am stärksten gestiegen sind die Staatseinnahmen in Ungarn (um 10,1 Prozentpunkte) sowie in Portugal (um 6,6 Prozentpunkte), wobei sich dieser Anstieg in beiden Ländern auf den Zeitraum ab 2009 konzentrierte und auf die Finanzkrise zurückzuführen war. Am stärksten zurückgegangen sind die Staatseinnahmen im Verhältnis zum BIP während des Betrachtungszeitraums in Israel (um 7,3 Prozentpunkte) und in Schweden (um 4,9 Prozentpunkte). In Israel haben sie zwischen 2009 und 2011 allerdings zugenommen. Im Zeitraum zwischen 2009 und 2011 sind die Staatseinnahmen in insgesamt zwei Dritteln der OECD-Mitgliedsländer gestiegen.

Pro Kopf beliefen sich die Staatseinnahmen 2011 im OECDDurchschnitt auf 15141 US-\$ KKP. Die Staatseinnahmen des einnahmestärksten OECD-Lands sind bezogen auf die Einwohnerzahl mehr als neunmal so hoch wie die des einnahmeschwächsten (36 800 US-\$ KKP pro Kopf in Luxemburg gegenüber knapp 4000 US-\$ KKP in Mexiko). Bezogen auf das BIP beträgt die Differenz nur ein 2,5-Faches.

Die Pro-Kopf-Staatseinnahmen sind im Durchschnitt der OECD-Länder zwischen 2001 und 2011 jährlich um 1,5\% gestiegen. Die höchsten jahresdurchschnittlichen Zuwachsraten wurden in Estland (5,3\%) und Korea (5,0\%) verzeichnet. In lediglich vier OECD-Ländern sind die Staatseinnahmen im fraglichen Zeitraum gesunken, allerdings nur sehr geringfügig - um jahresdurchschnittlich 0,1-0,3\% -, in Italien, den Vereinigten Staaten, Spanien und Kanada.

\section{Methodik und Definitionen}

Die Daten zu den Staatseinnahmen stammen aus der OECD-Datenbank National Accounts Statistics, die auf dem System der Volkswirtschaftlichen Gesamtrechnungen (SNA) basiert. Dabei handelt es sich um einen international vereinbarten Katalog von Konzepten, Definitionen, Klassifizierungen und Regeln für Volkswirtschaftliche Gesamtrechnungen. Nach der SNA-Terminologie setzt sich der Sektor Staat zusammen aus der Zentralregierung, den Gliedstaaten, den Gemeinden und der Sozialversicherung. Die Einnahmen umfassen Sozialversicherungsbeiträge, Steuern sowie Zuwendungen und sonstige Einkünfte. Das Bruttoinlandsprodukt (BIP) ist die Standardmessgröße des Werts der von einer Volkswirtschaft in einem bestimmten Zeitraum produzierten Waren und Dienstleistungen.

Die Staatseinnahmen pro Kopf wurden ermittelt, indem die Gesamteinnahmen unter Verwendung der OECD/ Eurostat-Kaufkraftparitäten (KKP) für das BIP in US-Dollar von 2011 konvertiert und durch die Einwohnerzahl dividiert wurden. Bei den Ländern, für die der Wirtschaftsausblick des IWF (IMF Economic Outlook) als Datenquelle diente, wurde ein implizierter KKP-Umrechnungsfaktor verwendet. Die KKP entsprechen der Zahl der Währungseinheiten von Land B, die erforderlich sind, um eine gleiche Menge an Waren und Dienstleistungen in Land A zu kaufen.

\section{Literaturhinweise}

OECD (2013), National Accounts at a Glance 2013, OECD Publishing, Paris, http://dx.doi.org/10.1787/na_glance-2013-en.

\section{Anmerkungen zu den Abbildungen}

Für Chile stehen keine Daten zur Verfügung. Die Daten für Kanada, Neuseeland und die Russische Föderation beziehen sich auf 2010 anstelle von 2011. Bei den Daten für Japan und Mexiko für 2001 handelt es sich um Schätzungen. Die Daten für die Russische Föderation beziehen sich auf 2002 anstelle von 2001.

3.11: Für die Türkei liegen keine Daten für 2001 vor, daher ist sie im OECD-Durchschnitt nicht berücksichtigt.

3.12: Für die Türkei stehen keine Daten zur Verfügung.

Hinweis zu den Daten für Israel: http://dx.doi.org/10.1787/888932315602. 


\section{3. ÖFFENTLICHE FINANZEN UND WIRTSCHAFTLICHES HANDELN DES STAATES}

\subsection{Gesamtstaatliche Einnahmen in Prozent des BIP (2001, 2009 und 2011)}

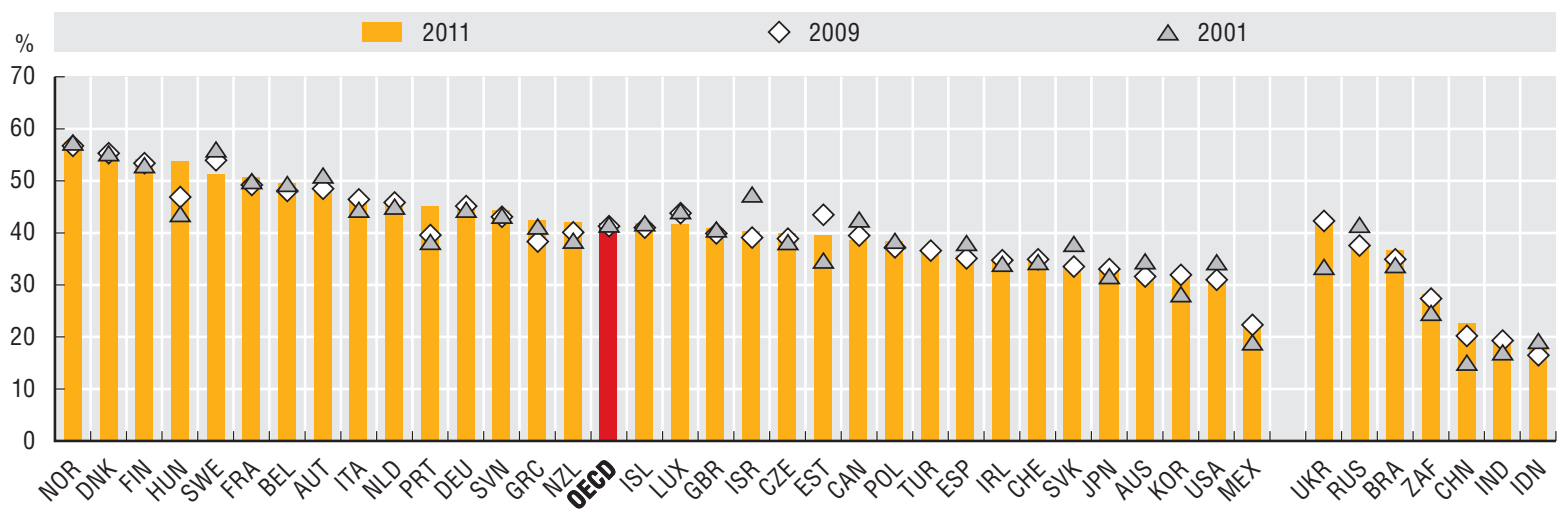

Quelle: Daten zu den OECD-Mitgliedsländern: OECD National Accounts Statistics (Datenbank). Daten zu den anderen großen Volkswirtschaften (ohne Russische Föderation): Internationaler Währungsfonds (2013), Economic Outlook, April 2013, IWF, Washington, DC.

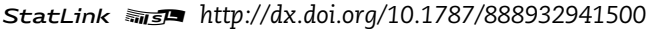

\subsection{Pro-Kopf-Staatseinnahmen (2011)}

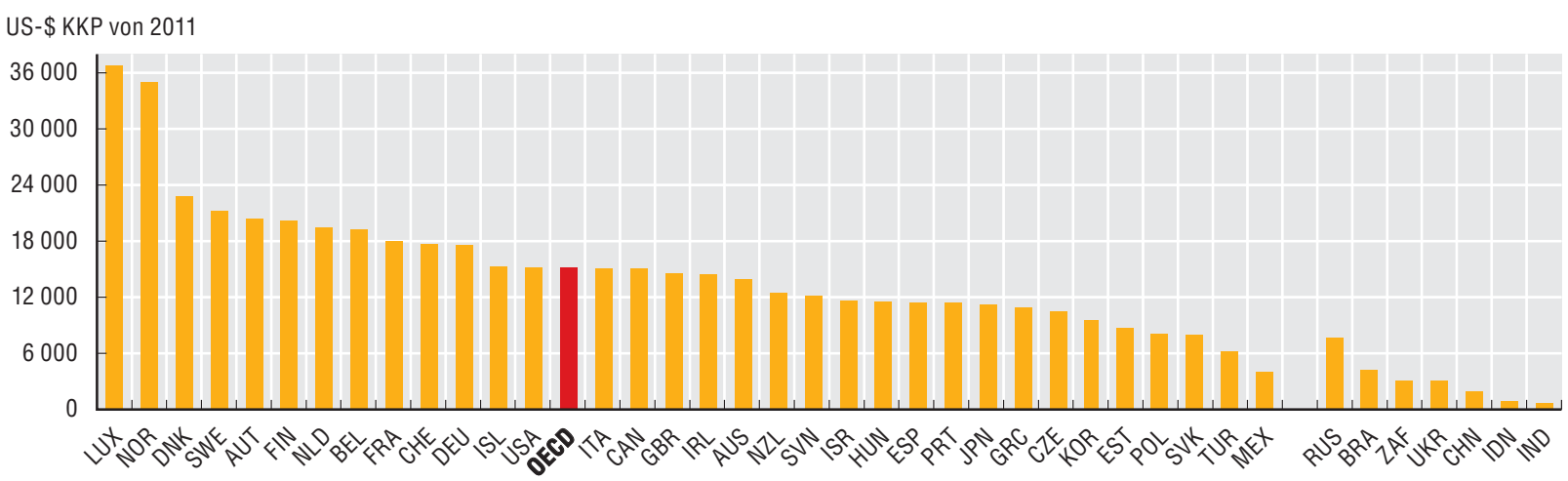

Quelle: Daten zu den OECD-Mitgliedsländern: OECD National Accounts Statistics (Datenbank). Daten zu den anderen großen Volkswirtschaften (ohne Russische Föderation): Internationaler Währungsfonds (2013), Economic Outlook, April 2013, IWF, Washington, DC.

StatLink त्ता $h$ http://dx.doi.org/10.1787/888932941519

\subsection{Jahresdurchschnittliche Wachstumsrate der realen Pro-Kopf-Staatseinnahmen (2001-11)}

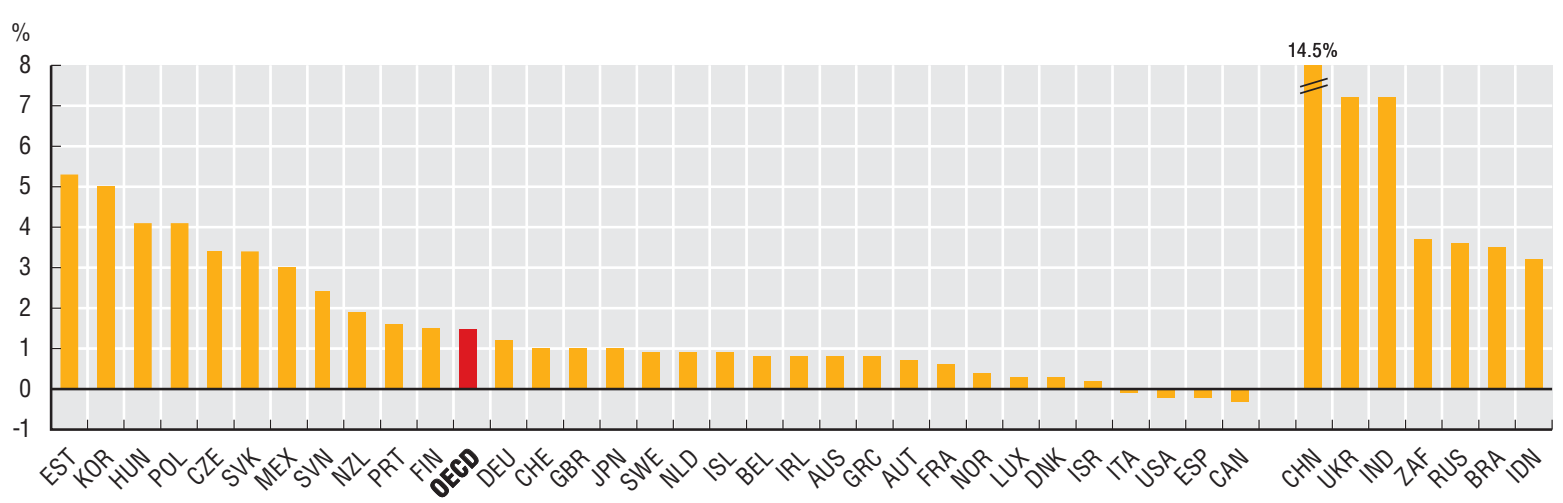

Quelle: Daten zu den OECD-Mitgliedsländern: OECD National Accounts Statistics (Datenbank). Daten zu den anderen großen Volkswirtschaften (ohne Russische Föderation): Internationaler Währungsfonds (2013), Economic Outlook, April 2013, IWF, Washington, DC.

StatLink त्नाज् http://dx.doi.org/10.1787/888932941538 
Eine Analyse der Struktur der gesamtstaatlichen Einnahmen gibt einen Anhaltspunkt für den relativen Umfang des Beitrags, den die Bürger und/oder verschiedene Wirtschaftssektoren zur Finanzierung der Staatsausgaben leisten.

2011 bestanden die gesamtstaatlichen Einnahmen im OECDDurchschnitt zu über 60\% aus Steuern und zu fast $25 \%$ aus Sozialversicherungsbeiträgen; beim Rest handelte es sich um Zuwendungen und sonstige Einkünfte. Die Staatsausgaben werden in den verschiedenen OECD-Ländern jeweils unterschiedlich finanziert. In Dänemark und Australien z.B. spielen Steuern eine größere Rolle (sie machen über $80 \%$ der Gesamteinnahmen aus), so dass die Sozialausgaben dort hauptsächlich aus dem allgemeinen Steueraufkommen finanziert werden. In der Tschechischen Republik, Frankreich, Deutschland, Japan, der Slowakischen Republik und Spanien kommt Sozialversicherungsbeiträgen hingegen vergleichsweise größeres Gewicht zu (sie machen fast 40\% der Gesamteinnahmen aus). Norwegen ist das einzige Land, in dem der Anteil der Zuwendungen und sonstigen Einkünfte 25\% übersteigt (was sich hauptsächlich aus den Dividenden- und Zinseinkünften des norwegischen Staatsfonds erklärt, der sich aus den Nettoeinnahmen der Ölwirtschaft speist).

Zwischen 2009 und 2011 blieb die Zusammensetzung der Staatseinnahmen im OECD-Durchschnitt weitgehend unverändert. Der Anteil der Steuern erhöhte sich um 0,4 Prozentpunkte, der der Sozialversicherungsbeiträge sank um 0,6 Prozentpunkte - weil wegen der Wirtschaftskrise die Beschäftigung schrumpfte und somit auch das Aufkommen an Sozialversicherungsbeiträgen abnahm -, und der Anteil der Zuwendungen und sonstigen Einkünfte stieg um 0,2 Prozentpunkte. Zu den deutlichsten Veränderungen der staatlichen Einnahmestruktur kam es in Ungarn (der Anteil der Zuwendungen und sonstigen Einkünfte stieg um 16,7 Prozentpunkte), in Mexiko (der Steueranteil erhöhte sich um 9,3 Prozentpunkte) und in Portugal (der Anteil der Zuwendungen und sonstigen Einkünfte wuchs um 6,8 Prozentpunkte).

Im Durchschnitt der OECD-Länder entfiel ein Drittel der Einnahmen aus Steuern und Abgaben (einschließlich Sozialversicherungsbeiträge) 2010 auf Einkommen- und Gewinnsteuern, ein weiteres Drittel auf Steuern auf Waren und Dienstleistungen (darunter ein erheblicher Teil Umsatz- und Mehrwertsteuern), über ein Viertel auf Sozialversicherungsbeiträge und der Rest auf Grundsteuern (5,4\%), beschäftigungsbezogene Abgaben (1,0\%) und sonstige Steuern und Abgaben (0,6\%). 2001 war die Einnahmestruktur ziemlich ähnlich. Die einzelnen OECD-Länder messen den verschiedenen Steuerarten allerdings unterschiedliches Gewicht bei. In Dänemark, Australien und Neuseeland handelt es sich beim Großteil der Steuer- und Abgabeneinnahmen um Einnahmen aus Einkommen- und Gewinnsteuern.

\section{Methodik und Definitionen}

Die Einnahmedaten stammen aus der OECD-Datenbank National Accounts Statistics, die auf dem System der Volkswirtschaftlichen Gesamtrechnungen (SNA) basiert. Dabei handelt es sich um einen international vereinbarten Katalog von Konzepten, Definitionen, Klassifizierungen und Regeln für Volkswirtschaftliche Gesamtrechnungen. Nach der SNA-Terminologie setzt sich der Sektor Staat zusammen aus der Zentralregierung, den Gliedstaaten, den Gemeinden und der Sozialversicherung. Bei den Ein nahmearten handelt es sich um Steuern (z.B. Verbrauchsteuern, Einkommensteuern, Vermögensteuern, Grundsteuern und Kapitalsteuern), Sozialversicherungsbeiträge (z.B. zur Rentenversicherung, Krankenversicherung und Arbeitslosenversicherung) sowie $\mathrm{Zu}$ wendungen (von anderen Staaten oder internationalen Organisationen) und sonstige Einkünfte (z.B. Veräußerungsgewinne, Gebühren, Vermögenseinkommen und Subventionen). Da diese Aggregate den OECD National Accounts Statistics nicht direkt entnommen werden können, wurden sie unter Verwendung von Einzelposten der Unterkonten konstruiert (vgl. Anhang A). Die in Abbildung 3.16 wiedergegebenen Daten stammen aus den OECD Revenue Statistics.

In den OECD Revenue Statistics und im System der Volkswirtschaftlichen Gesamtrechnungen (SNA) sind die Steuereinnahmen jeweils anders definiert. Im SNA sind Steuern Geld- oder Sachleistungen, die institutionelle Einheiten ohne Anspruch auf individuelle Gegenleistungen an die öffentlichen Haushalte entrichten müssen. Sozialversicherungsbeiträge sind tatsächliche oder kalkulatorische Zahlungen an Sozialversicherungssysteme, mit denen Sozialversicherungsansprüche erworben werden. Es kann sich dabei um Pflichtbeiträge oder freiwillige Beiträge handeln, und die entsprechenden Systeme können kapitalgedeckt oder nicht kapitalgedeckt sein. In den OECD Revenue Statistics werden Pflichtbeiträge zu Sozialversicherungssystemen als Steuern behandelt, was im SNA nicht der Fall ist, weil der Bezug von Sozialversicherungsleistungen in den meisten Ländern voraussetzt, dass zuvor Beiträge in die entsprechenden Systeme eingezahlt wurden, auch wenn die Höhe der Leistungen nicht zwangsläufig von der Höhe der Beiträge abhängt.

\section{Literaturhinweise}

OECD (2012), Revenue Statistics 2012, OECD Publishing, Paris, http:// dx.doi.org/10.1787/rev_stats-2012-en-fr.

OECD (2010), Tax Policy Reform and Economic Growth, OECD Tax Policy Studies, No. 20, OECD Publishing, Paris, http://dx.doi.org/ 10.1787/9789264091085-en.

\section{Anmerkungen zu den Abbildungen}

3.14 und 3.15: Für Chile stehen keine Daten zur Verfügung. Für Japan und die Türkei liegen keine Daten für 2001 vor, diese Länder sind im OECD-Durchschnitt daher nicht berücksichtigt. Australien bezieht keine Einnahmen in Form von Sozialversicherungsbeiträgen, da es dort keine staatlichen Sozialversicherungssysteme gibt. Für die Russische Föderation liegen keine Angaben zu Kapitalsteuern vor. Die Daten für Kanada, Neuseeland und die Russische Föderation beziehen sich auf 2010 anstelle von 2011. Die Daten für Mexiko beziehen sich auf 2003 anstelle von 2001. Die Daten für die Russische Föderation beziehen sich auf 2002 anstelle von 2001.

3.16: Für die OECD-Mitgliedsländer, die der Europäischen Union angehören, beinhaltet das Gesamtsteueraufkommen auch die im Namen der Europäischen Union erhobenen Zölle.

Hinweis zu den Daten für Israel: http://dx.doi.org/10.1787/888932315602. 


\section{3. ÖFFENTLICHE FINANZEN UND WIRTSCHAFTLICHES HANDELN DES STAATES}

3.14. Struktur der gesamtstaatlichen Einnahmen (2001 und 2011)

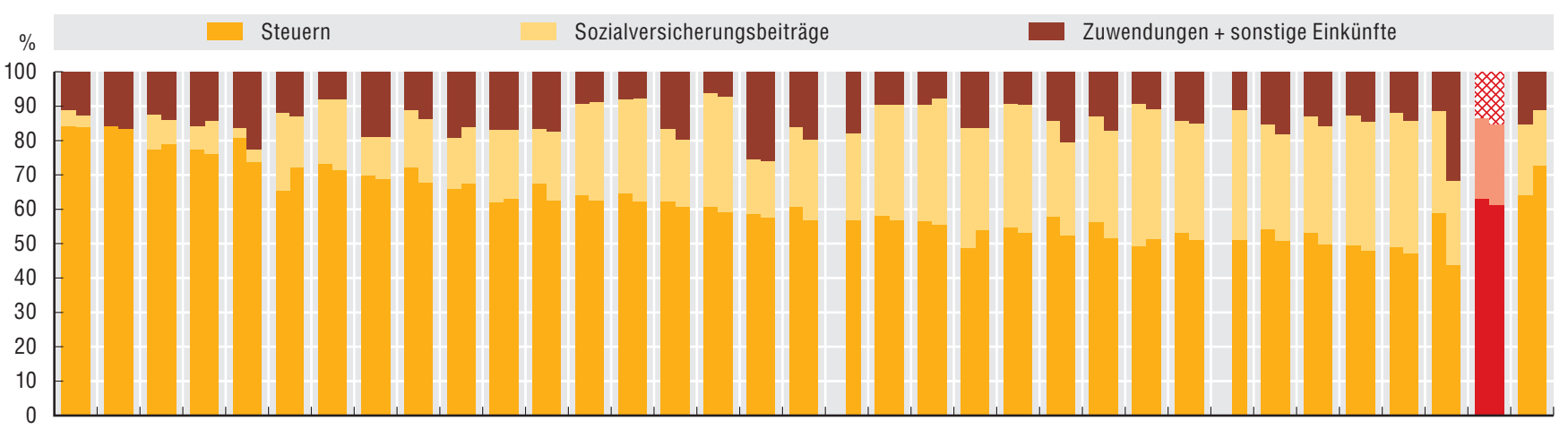

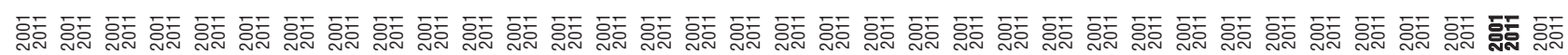
DNK AUS MEX ISL NZL SWE GBR CAN IRL ISR CHE KOR LUX ITA USA BEL NOR FIN TUR AUT ESP POL FRA PRT EST DEU NLD JPN GRC SVN SVK CZE HUN OECD RUS Quelle: OECD National Accounts Statistics (Datenbank).

StatLink तilst http://dx.doi.org/10.1787/888932941557

\subsection{Veränderung der Struktur der gesamtstaatlichen Einnahmen (2009-2011)}

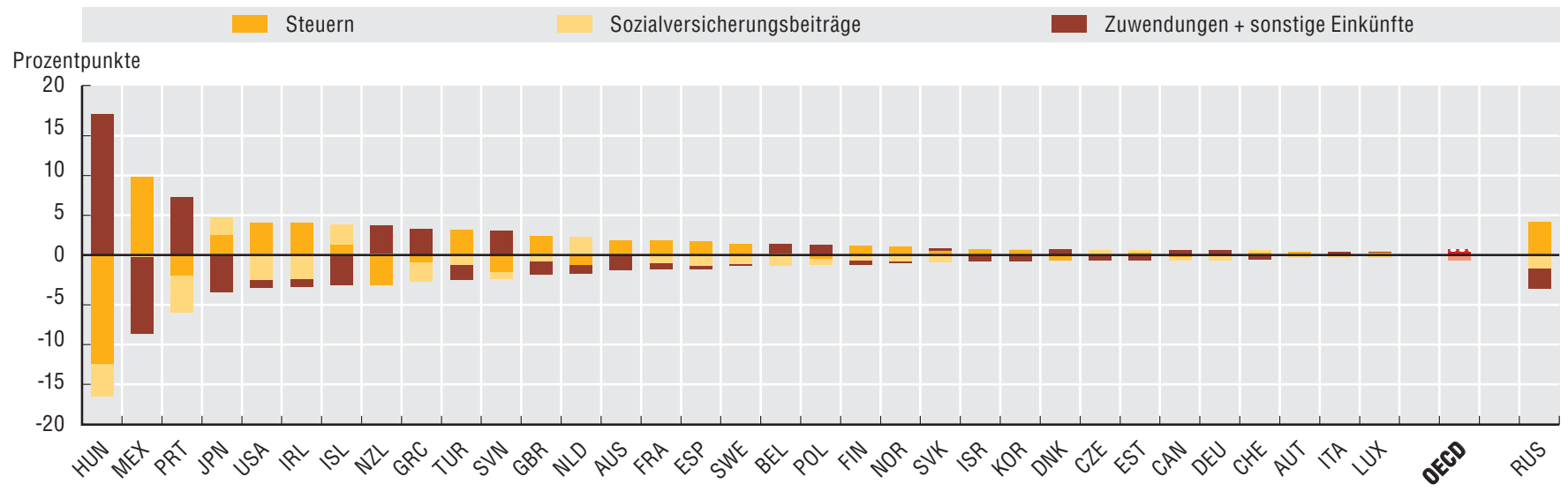

Quelle: OECD National Accounts Statistics (Datenbank).

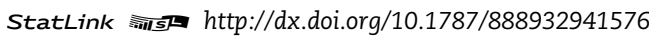

3.16. Aufschlüsselung der Einnahmen aus Steuern und Abgaben in Prozent des Gesamtbetrags (2001 und 2010$)$

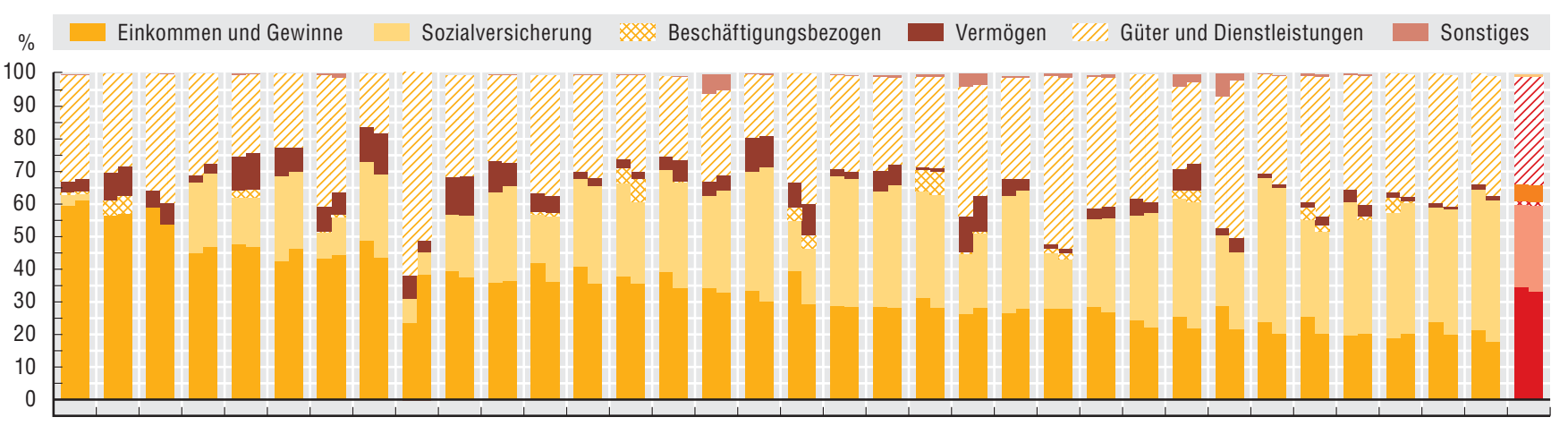

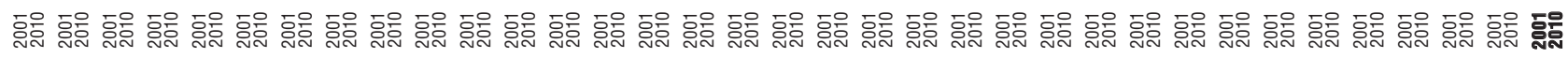
DNK AUS NZL NOR CAN CHE ISL USA CHL GBR LUX IRL FIN SWE BEL ITA JPN ISR DEU ESP AUT KOR NLD MEX PRT GRC FRA TUR CZE HUN POL SVN EST SVKOECD Quelle: OECD (2012), Revenue Statistics 2012, OECD Publishing, Paris, http://dx.doi.org/10.1787/rev_stats-2012-en-fr. 
Die staatliche Einnahmeerhebung erfolgt auf den verschiedenen staatlichen Ebenen unterschiedlich, da Zentralregierung, Gliedstaaten und Gemeinden unterschiedliche Befugnisse zur Erhebung von Steuern und Einziehung von Sozialversicherungsbeiträgen haben. Der Umfang der Einnahmetransfers zwischen den verschiedenen staatlichen Ebenen gibt einen Anhaltspunkt für deren gegenseitige finanzielle Abhängigkeit. Die Höhe der von den nachgeordneten Gebietskörperschaften erhobenen Steuern und Abgaben kann als Ersatzvariable für ihre fiskalische Autonomie betrachtet werden.

Der Großteil der gesamtstaatlichen Einnahmen entfiel $2011 \mathrm{im}$ OECD-Durchschnitt auf die zentralen Ebenen (60,3\%). Auf die nachgeordneten Gebietskörperschaften (Gliedstaaten und Gemeinden) entfielen durchschnittlich 21\% der Gesamteinnahmen, die verbleibenden $18,7 \%$ gingen an Sozialversicherungsträger. Die Aufschlüsselung der Einnahmen nach staatlichen Ebenen unterscheidet sich erheblich zwischen den verschiedenen OECD-Ländern. In drei Ländern betrug der auf die zentrale Ebene entfallende Anteil an den gesamtstaatlichen Einnahmen mehr als 85\%, im Vereinigten Königreich $(90,6 \%)$, in Neuseeland $(89,6 \%)$ und in Norwegen $(86,2 \%)$. Weniger als die Hälfte machte er demgegenüber in acht OECDLändern aus, drei davon keine Föderalstaaten: Finnland $(42,9 \%)$, Frankreich $(34,1 \%)$ und Japan $(24,1 \%)$. In Japan und den nordischen Ländern mit Ausnahme von Norwegen entfällt ein vergleichsweise größerer Anteil an den Gesamteinnahmen auf die Gemeinden: durchschnittlich 31,2\% im Vergleich zu 13,3\% im OECD-Durchschnitt. Unter den neun föderal organisierten OECD-Ländern ist der auf die Gliedstaaten entfallende Einnahmeanteil in den Vereinigten Staaten $(46 \%)$, in Kanada $(43,2 \%)$ und in Australien (38,8\%) am höchsten. In Frankreich entfällt fast die Hälfte der gesamtstaatlichen Einnahmen auf Sozialversicherungsträger.

Zwischen 2001 und 2011 ist der Anteil der Einnahmen, der an die zentralen Ebenen geht, im OECD-Durchschnitt um 1,7 Prozentpunkte zurückgegangen. Der auf die nachgeordneten Gebietskörperschaften entfallende Anteil hat demgegenüber um 1,3 Prozentpunkte zugenommen. Nur in sieben Ländern ist der Einnahmeanteil der zentralen Ebenen gestiegen: in Dänemark (+9,4 Prozentpunkte), Ungarn (+8,2 Prozentpunkte), Norwegen (+4,0 Prozentpunkte), Griechenland (+3,3 Prozentpunkte), Deutschland (+2,4 Prozentpunkte), der Schweiz (+1,6 Prozentpunkte) und Portugal (+0,1 Prozentpunkte).

Die zentralen Ebenen finanzieren sich hauptsächlich über Steuern, die 2011 durchschnittlich 77\% der Einnahmen ausmachten. Im Gegensatz zu der relativ großen Homogenität der Einnahmequellen der Zentralregierungen sind auf den nachgeordneten Ebenen erhebliche Unterschiede festzustellen. Der Großteil der Einnahmen der Gemeinden besteht aus Finanztransfers zwischen verschiedenen Gebietskörperschaften sowie sonstigen Einkünften. Diese beiden Posten machten 2011 im Schnitt mehr als $61 \%$ der kommunalen Einnahmen aus. Von den übrigen 39\% der kommunalen Einnahmen stammt der größte Teil aus Grundsteuern. Die Beschränkungen, denen die Gemeinden bei der Festlegung von Steuerbemessungsgrundlagen, Steuersätzen und Steuererleichterungen unterliegen, können ihre Möglichkeiten zur Erschließung eigener Einnahmequellen und somit u.U. auch ihre Fähigkeit zur Bereitstellung eines bedarfsgerechteren öffentlichen Dienstleistungsangebots verringern.

\section{Methodik und Definitionen}

Die Einnahmedaten stammen aus der OECD-Datenbank National Accounts Statistics, die auf dem System der Volkswirtschaftlichen Gesamtrechnungen (SNA) basiert. Dabei handelt es sich um einen international vereinbarten Katalog von Konzepten, Definitionen, Klassifizierungen und Regeln für Volkswirtschaftliche Gesamtrechnungen. Nach der SNA-Terminologie setzt sich der Sektor Staat zusammen aus der Zentralregierung, den Gliedstaaten, den Gemeinden und der Sozialversicherung. Die Kategorie der Gliedstaaten findet nur für die neun OECD-Länder Anwendung, die föderal organisiert sind: Australien, Österreich, Belgien, Kanada, Deutschland, Mexiko, Spanien (wird als Quasi-Föderalstaat betrachtet), Schweiz und Vereinigte Staaten. In den Daten in Abbildung 3.17 und 3.18 (online verfügbar) sind Finanztransfers zwischen verschiedenen staatlichen Ebenen nicht berücksichtigt, außer für Australien und Japan. Abbildung 3.18 zur Veränderung der Einnahmeverteilung nach staatlichen Ebenen (2009-11) sowie Abbildung 3.19, 3.20 und 3.21 (Struktur der Einnahmen der Zentralregierungen, der Gliedstaaten und der Gemeinden) sind online verfügbar unter: http://dx.doi.org/10.1787/ 888932941633, http://dx.doi.org/10.1787/888932941652, http://dx.doi.org/10.1787/888932941671 bzw. http:// dx.doi.org/10.1787/888932941690 .

Bei den Einnahmearten handelt es sich um Steuern (z.B. Verbrauchsteuern, Einkommensteuern, Vermögensteuern, Grundsteuern und Kapitalsteuern), Sozialversicherungsbeiträge (z.B. zur Renten-, Kranken- und Arbeitslosenversicherung) sowie Zuwendungen und sonstige Einkünfte. Die Zuwendungen können von anderen Staaten, internationalen Organisationen oder anderen innerstaatlichen Verwaltungseinheiten kommen. Sonstige Einkünfte umfassen Veräußerungserlöse, Gebühren, Vermögenseinkommen und Subventionen. Da diese Aggregate den OECD National Accounts Statistics nicht direkt entnommen werden können, wurden sie unter Verwendung von Einzelposten der Unterkonten konstruiert (vgl. Anhang A).

\section{Literaturhinweise}

Blöchliger, H. et al. (2010), „Fiscal Policy Across Levels of Government in Times of Crisis“, OECD Working Papers on Fiscal Federalism, No. 12, OECD Publishing, Paris, http://dx.doi.org/10.1787/ 5k97b10wqn46-en.

\section{Anmerkungen zu den Abbildungen}

Für Chile stehen keine Daten zur Verfügung. Für Japan, Polen und die Türkei liegen keine Daten für 2001 vor, diese Länder sind im OECDDurchschnitt daher nicht berücksichtigt. Finanztransfers zwischen verschiedenen staatlichen Ebenen sind nicht berücksichtigt (außer für Australien, Japan und die Türkei). Die Daten für Kanada und Neuseeland beziehen sich auf 2010 anstelle von 2011. Die Daten für Mexiko beziehen sich auf 2003 anstelle von 2001. In Australien und den Vereinigten Staaten werden die Gemeinden unter den Gliedstaaten erfasst. In Australien gibt es keine staatlichen Sozialversicherungssysteme. In Neuseeland, Norwegen, dem Vereinigten Königreich und den Vereinigten Staaten wird die Sozialversicherung auf Ebene der Zentralregierung erfasst.

Hinweis zu den Daten für Israel: http://dx.doi.org/10.1787/888932315602. 


\section{3. ÖFFENTLICHE FINANZEN UND WIRTSCHAFTLICHES HANDELN DES STAATES}

3.17. Einnahmeverteilung nach staatlichen Ebenen (2001 und 2011)

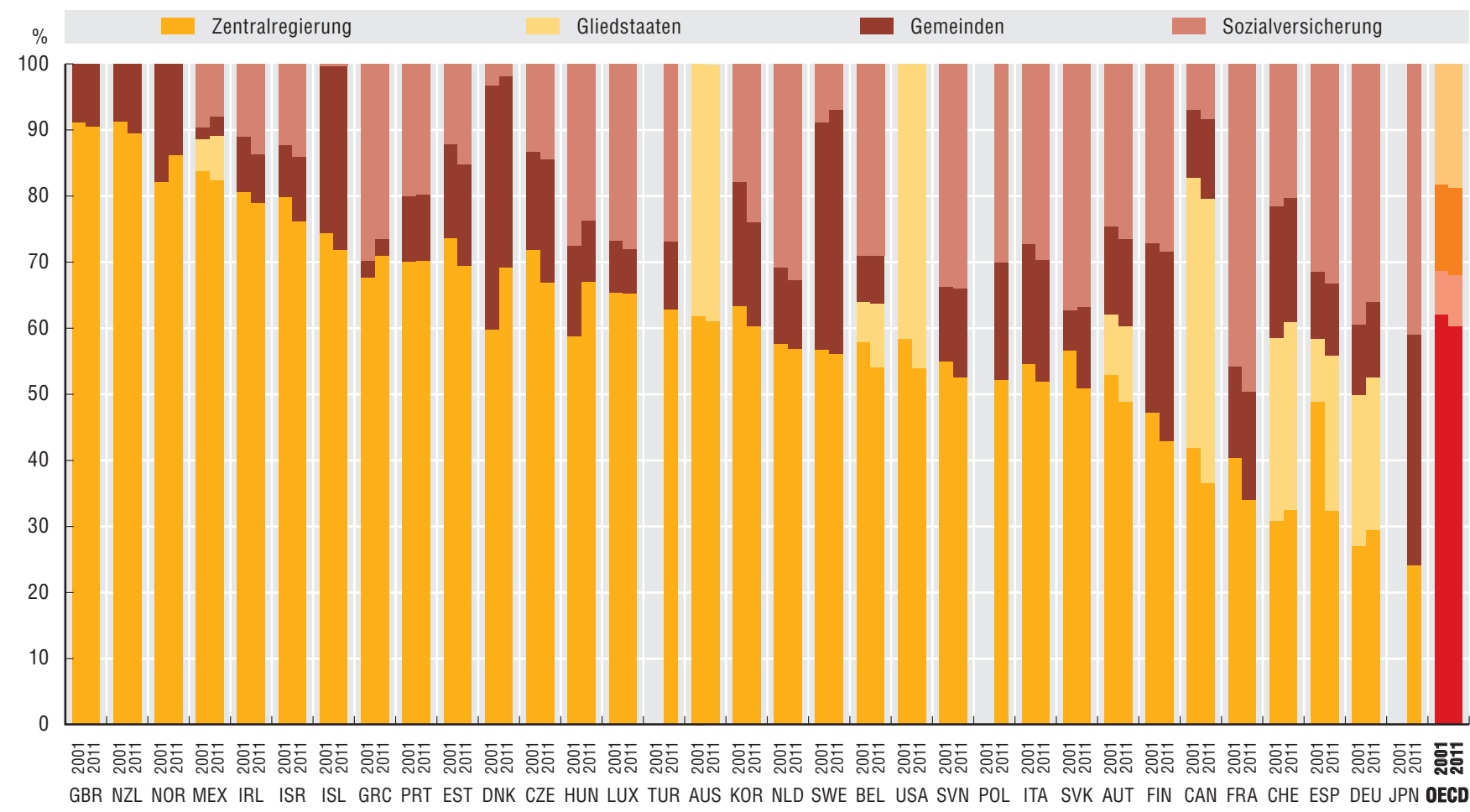

Quelle: OECD National Accounts Statistics (Datenbank). 
Staatsausgaben dienen vor allem zwei Zwecken, zur Herstellung und/oder Bezahlung der Güter und Dienstleistungen, die der Staat für Bürger und Unternehmen bereitstellt, und zur Einkommensumverteilung. Der Vergleich der Staatsausgaben der einzelnen OECD-Länder im Verhältnis zu ihrem BIP oder ihrer Einwohnerzahl liefert einen Anhaltspunkt für den Umfang des staatlichen Sektors innerhalb der Wirtschaft im Hinblick auf die aufgewendeten finanziellen Mittel. Wie die Staatseinnahmen werden auch die Staatsausgaben durch aktuelle und vergangene Entscheidungen bestimmt, die ihrerseits durch kulturell geprägte Erwartungen in Bezug auf die soziale Umverteilung und den Umfang der bereitzustellenden Güter und Dienstleistungen, durch Haushaltszwänge, konjunkturelle Schwankungen sowie die Wirtschaftsleistung beeinflusst werden. Daher sind bei der Höhe der Staatsausgaben starke Unterschiede zwischen den einzelnen OECDLändern festzustellen. Das Ausgabenvolumen gestattet allerdings keine Rückschlüsse auf die Effizienz oder Produktivität des Staates.

2011 beliefen sich die gesamtstaatlichen Ausgaben im OECDDurchschnitt auf $45,4 \%$ des BIP. Am höchsten waren sie mit $57,6 \%$ des BIP in Dänemark, am niedrigsten mit 22,8\% des BIP in Mexiko. In den OECD-Ländern, die der EU angehören, ist der Anteil der Staatsausgaben am BIP in der Regel höher. Am größten ist der staatliche Sektor in Dänemark, Frankreich und Finnland, wo sich die Staatsausgaben auf mindestens 55\% des BIP belaufen, am kleinsten in Korea und Mexiko, wo sie $30 \%$ bzw. 23\% des BIP ausmachen.

Im Zeitraum 2001-11 waren im OECD-Raum starke Veränderungen der Staatsausgaben im Verhältnis zum BIP festzustellen. Zwischen 2001 und 2009 stiegen die Staatsausgaben deutlich, um durchschnittlich 4,5 Prozentpunkte, vor allem in Irland (+15,4 Prozentpunkte), im Vereinigten Königreich (+11,1 Prozentpunkte) und in Estland (+10,7 Prozentpunkte). Der stärkste Anstieg war allerdings nach 2007 zu beobachten: Wegen der schrumpfenden Wirtschaftstätigkeit begannen die automatischen Stabilisatoren zu wirken, während zugleich besondere Ausgabenprogramme eingeführt wurden. Nur drei Länder verringerten im Zeitraum 2001-09 die Staatsausgaben im Verhältnis zum BIP: Israel (-8,1 Prozentpunkte), die Slowakische Republik (-2,9 Prozentpunkte) und die Schweiz (-0,7 Prozentpunkte). Zwischen 2009 und 2011 kam es insgesamt zu einer Trendwende, und die Staatsausgaben sanken im Verhältnis zum BIP um durchschnittlich 1,4 Prozentpunkte, hauptsächlich auf Grund der Verlangsamung des Wachstums der Ausgaben (teilweise sogar ihrer Verringerung) im Vergleich zum BIP-Wachstum. Am stärksten war der Rückgang in Estland (7,2 Prozentpunkte), Schweden, Island und der Slowakischen Republik (jeweils über 3 Prozentpunkte). Ein Ausgabenanstieg im Verhältnis zum BIP wurde während dieses Zeitraums nur in drei Ländern verzeichnet, in Neuseeland (6,6 Prozentpunkte zwischen 2009 und 2010 infolge von Konjunkturprogrammen), Slowenien (1,4 Prozentpunkte) und Japan (0,1 Prozentpunkte).

Pro Kopf beliefen sich die Staatsausgaben 2011 im OECDDurchschnitt auf 16240 US-\$ KKP. Bezogen auf die Einwohnerzahl sind die Staatsausgaben im OECD-Land mit dem höchsten Ausgabenniveau (Luxemburg mit 37000 US-\$ KKP pro Kopf) neunmal höher als in dem mit dem niedrigsten Ausgabenniveau (Mexiko mit 4000 US-\$ KKP pro Kopf). Im Verhältnis zum BIP beläuft sich diese Differenz nur auf ein 2,5-Faches.
Zwischen 2001 und 2011 sind die Pro-Kopf-Staatsausgaben im OECD-Durchschnitt um 2,2\% jährlich gestiegen. Am stärksten war der jahresdurchschnittliche Anstieg in Korea $(6,1 \%)$ und Estland (5\%). Gesunken sind die Pro-Kopf-Ausgaben nur in Israel und auch dort nur geringfügig (um 0,1\% pro Jahr).

\section{Methodik und Definitionen}

Die Daten über die Staatsausgaben stammen aus der OECD-Datenbank Annual National Accounts, die auf dem System der Volkswirtschaftlichen Gesamtrechnungen (SNA) basiert. Dabei handelt es sich um einen international vereinbarten Katalog von Konzepten, Definitionen, Klassifizierungen und Regeln für Volkswirtschaftliche Gesamtrechnungen. Nach der SNA-Terminologie setzt sich der Sektor Staat zusammen aus der Zentralregierung, den Gliedstaaten, den Gemeinden und der Sozialversicherung. Bei den Ausgaben handelt es sich um Vorleistungen, Arbeitnehmerentgelte, Subventionen, Sozialleistungen und sonstige laufende Ausgaben (einschließlich Zinsaufwendungen), Vermögenstransfers und sonstige investive Ausgaben.

Das Bruttoinlandsprodukt (BIP) ist die Standardmessgröße des Werts der von einer Volkswirtschaft in einem bestimmten Zeitraum produzierten Waren und Dienstleistungen. Die Staatsausgaben pro Kopf wurden ermittelt, indem die Gesamtausgaben unter Verwendung der OECD/ Eurostat-Kaufkraftparitäten (KKP) für das BIP in US-Dollar von 2011 konvertiert und durch die Einwohnerzahl dividiert wurden (bei den Ländern, für die der Wirtschaftsausblick des IWF als Datenquelle diente, wurde ein implizierter KKP-Umrechnungsfaktor verwendet). Die KKP entsprechen der Zahl der Währungseinheiten von Land B, die erforderlich sind, um eine gleiche Menge an Waren und Dienstleistungen in Land A zu kaufen.

\section{Literaturhinweise}

OECD (2013), National Accounts at a Glance 2013, OECD Publishing, Paris, http://dx.doi.org/10.1787/na_glance-2013-en.

\section{Anmerkungen zu den Abbildungen}

Für Chile stehen keine Daten zur Verfügung. Die Daten für Kanada, Neuseeland und die Russische Föderation beziehen sich auf 2010 anstelle von 2011. Bei den Daten für Japan und Mexiko für 2001 handelt es sich um Schätzungen. Die Daten für die Russische Föderation beziehen sich auf 2002 anstelle von 2001.

3.22: Für die Türkei liegen keine Daten für 2001 vor, daher ist sie im OECD-Durchschnitt nicht berücksichtigt.

3.24: Für die Türkei stehen keine Daten zur Verfügung.

Hinweis zu den Daten für Israel: http://dx.doi.org/10.1787/888932315602. 


\section{3. ÖFFENTLICHE FINANZEN UND WIRTSCHAFTLICHES HANDELN DES STAATES}

\subsection{Gesamtstaatliche Ausgaben in Prozent des BIP (2001, 2009 und 2011)}

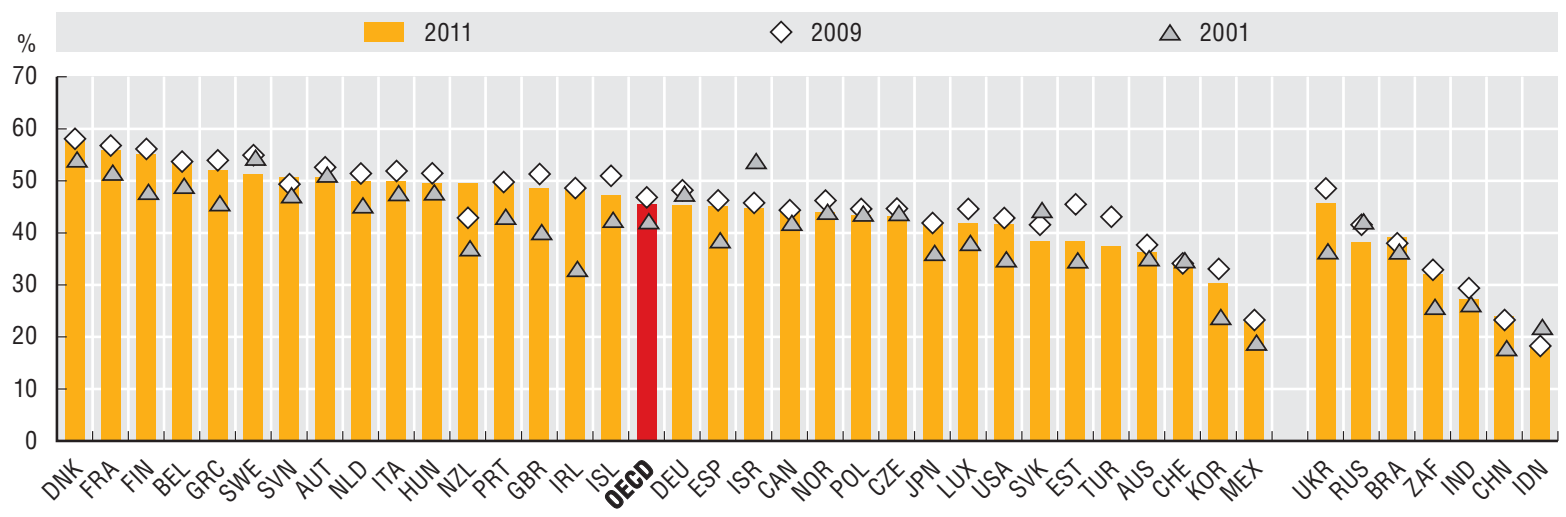

Quelle: Daten zu den OECD-Mitgliedsländern: OECD National Accounts Statistics (Datenbank). Daten zu den anderen großen Volkswirtschaften (ohne Russische Föderation): Internationaler Währungsfonds (2013), Economic Outlook, April 2013, IWF, Washington, DC.

StatLink ants http://dx.doi.org/10.1787/888932941709

\subsection{Pro-Kopf-Staatsausgaben (2011)}

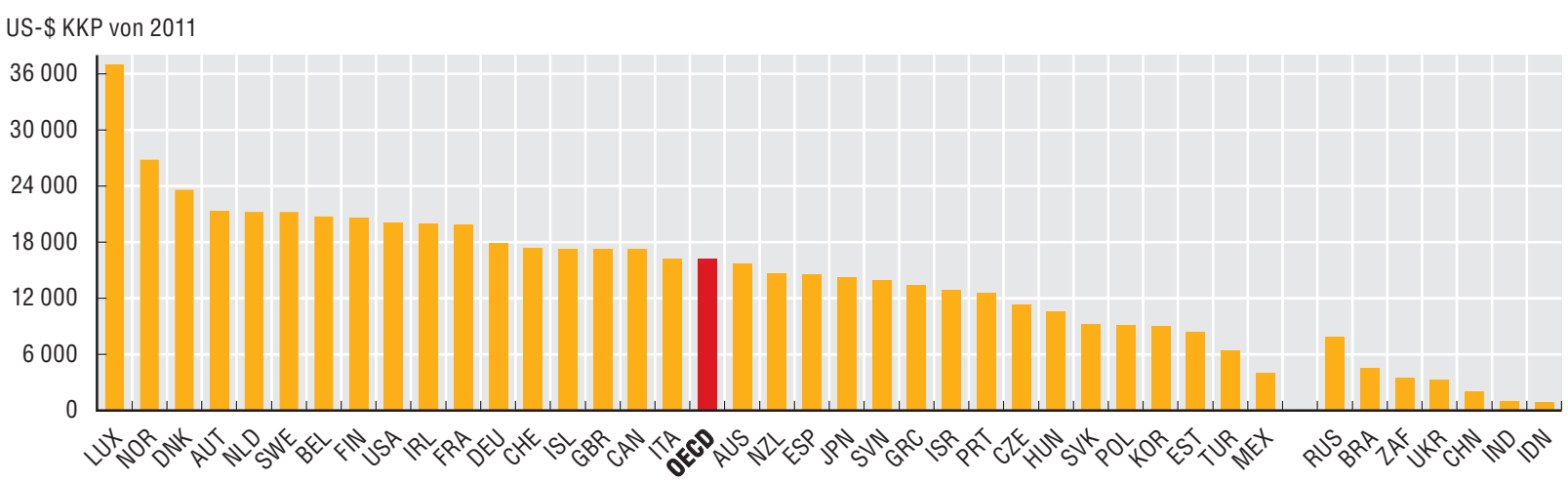

Quelle: Daten zu den OECD-Mitgliedsländern: OECD National Accounts Statistics (Datenbank). Daten zu den anderen großen Volkswirtschaften (ohne Russische Föderation): Internationaler Währungsfonds (2013), Economic Outlook, April 2013, IWF, Washington, DC.

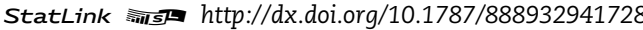

\subsection{Jahresdurchschnittliche Wachstumsrate der realen Pro-Kopf-Staatsausgaben (2001-11)}

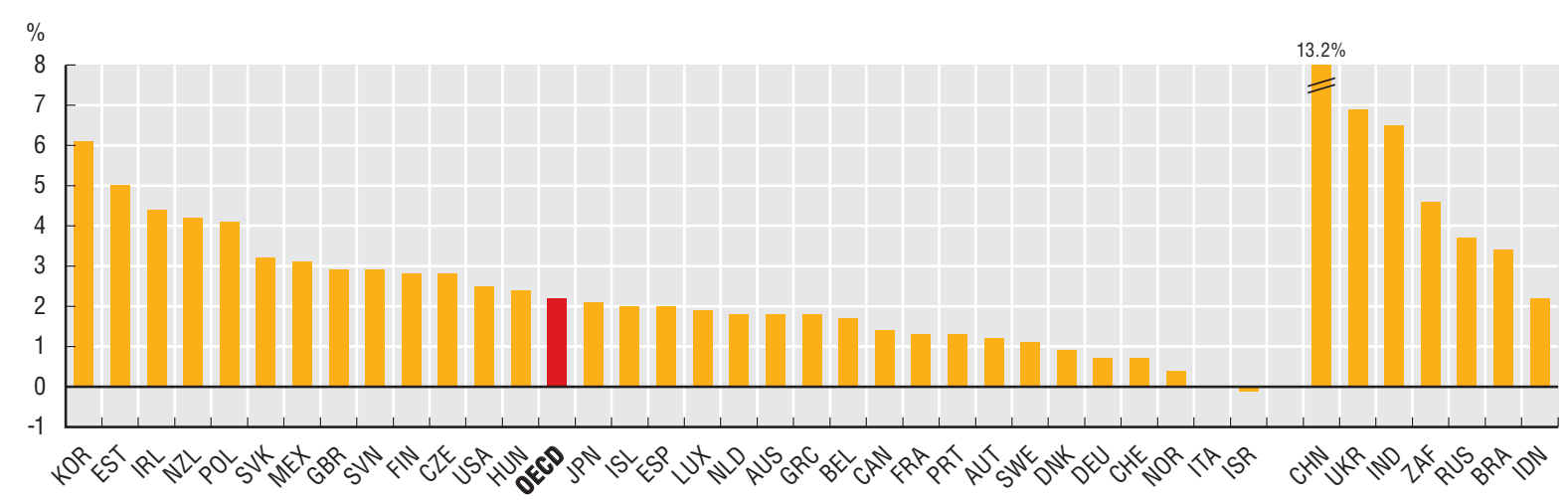

Quelle: Daten zu den OECD-Mitgliedsländern: OECD National Accounts Statistics (Datenbank). Daten zu den anderen großen Volkswirtschaften (ohne Russische Föderation): Internationaler Währungsfonds (2013), Economic Outlook, April 2013, IWF, Washington, DC.

StatLink त्नाज्ञ http://dx.doi.org/10.1787/888932941747 
Staatliche Stellen geben für eine Vielzahl verschiedener Güter und Dienstleistungen Geld aus, von der Kinderbetreuung über den Brückenbau bis zur Subventionierung alternativer Energiequellen. Internationale Verpflichtungen auf gemeinsame Politikziele können ebenfalls Einfluss auf die Ausgabenstruktur haben, so z.B. im Fall der in der EU vertretenen OECD-Länder, die u.a. gemeinsame Ziele im Hinblick auf Wirtschaftswachstum, Landwirtschaft, Energie, Infrastruktur sowie Forschung und Entwicklung verfolgen.

Außer in Korea und den Vereinigten Staaten ist die Finanzierung der sozialen Sicherung - d.h. vor allem der Alterssicherung, aber auch der Arbeitslosenunterstützung, der Erwerbsminderungsrenten usw. - im gesamten OECD-Raum die wichtigste Funktion der öffentlichen Ausgaben. Auf diesen Posten entfielen 2011 durchschnittlich 35,6\% der Gesamtausgaben. In Korea ist der größte Teil der öffentlichen Ausgaben für wirtschaftliche Angelegenheiten bestimmt (was die Förderung einzelner Wirtschaftszweige beinhaltet); in den Vereinigten Staaten ist das Gesundheitswesen der größte Ausgabenposten, auf ihn entfielen 2011 21,4\% der Gesamtausgaben.

In der Struktur der Staatsausgaben drücken sich soziale und politische Präferenzen aus, sie wird aber auch durch Faktoren wie die Bevölkerungsalterung oder eine hohe Verschuldung, die zu hohen Zinszahlungen führt, beeinflusst. Zwischen 2001 und 2011 veränderte sich die Verteilung der Ausgaben auf die verschiedenen Politikbereiche. Der Anteil der Ausgaben für die soziale Sicherung und für das Gesundheitswesen stieg im OECD-Durchschnitt um 2 bzw. 1,2 Prozentpunkte. Auch der Anteil der Ausgaben für Freizeitgestaltung, Sport, Kultur und Religion nahm leicht zu (um 0,05 Prozentpunkte). Die Ausgabenverschiebung zu Gunsten der sozialen Sicherung erklärt sich hauptsächlich aus den Auswirkungen der Finanz- und Wirtschaftskrise (Anstieg der Arbeitslosenversicherungs- und sonstigen Sozialleistungen), während das gestiegene Gewicht der Gesundheitsausgaben wohl neuen medizinischen Techniken, Innovationen und einer alternden Bevölkerung zuzuschreiben ist. Der Anteil der anderen Politikbereiche war rückläufig: allgemeine öffentliche Verwaltung (-1,1 Prozentpunkte), wirtschaftliche Angelegenheiten (-0,6 Prozentpunkte), Verteidigung sowie Wohnungswesen und kommunale Einrichtungen (jeweils -0,5 Prozentpunkte), Bildung (-0,3 Prozentpunkte), öffentliche Ordnung und Sicherheit sowie Umweltschutz (jeweils -0,1 Prozentpunkte).

Das Gesundheitswesen ist ein gutes Beispiel für Veränderungen des Ausgabenniveaus innerhalb eines Politikbereichs. Im Zeitraum 2001-11 wurde in allen OECD-Ländern, außer in Island, Irland, Luxemburg, Portugal und Slowenien, ein Anstieg des Anteils der Gesundheitsausgaben verzeichnet. In den letzten Jahren hat sich dieser Trend infolge des Konjunkturabschwungs sowie aktiver Maßnahmen zur Eindämmung des Wachstums der Gesundheitsausgaben jedoch verlangsamt oder umgekehrt. In Irland z.B. wurde der Großteil des Rückgangs durch Lohnkürzungen, Personalabbau im Gesundheitssektor sowie geringere Ärztehonorare und Arzneimitteltarife erzielt. In Island wurden Investitionen in die Gesundheitsinfrastruktur auf Eis gelegt und Effizienzsteigerungen durch die Zusammenlegung von Krankenhäusern erzielt.

\section{Methodik und Definitionen}

Die Ausgabendaten stammen aus der OECD-Datenbank National Accounts Statistics, die auf dem System der Volkswirtschaftlichen Gesamtrechnungen (SNA) basiert. Dabei handelt es sich um einen international vereinbarten Katalog von Konzepten, Definitionen, Klassifizierungen und Regeln für Volkswirtschaftliche Gesamtrechnungen. Die Ausgabendaten werden gemäß der Klassifikation der Ausgaben des Staats nach Aufgabenbereichen (Classification of the Functions of Government - COFOG) aufgeschlüsselt, nach der die Staatsausgaben in zehn Funktionen aufgeteilt werden: allgemeine öffentliche Verwaltung; Verteidigung; öffentliche Ordnung und Sicherheit; wirtschaftliche Angelegenheiten; Umweltschutz; Wohnungswesen und kommunale Einrichtungen; Gesundheitswesen; Freizeitgestaltung, Sport, Kultur und Religion; Bildungswesen; soziale Sicherung. Weitere Informationen zu den Ausgabenarten, die unter den verschiedenen Kategorien erfasst sind, können Anhang B entnommen werden. Der Sektor Staat setzt sich zusammen aus der Zentralregierung, den Gliedstaaten, den Gemeinden und der Sozialversicherung. Aus Tabelle 3.27 (online verfügbar unter http://dx.doi.org/10.1787/ 888932943324 ) und Tabelle 3.28 (online verfügbar unter http://dx.doi.org/10.1787/888932943343) ist die Aufteilung der gesamtstaatlichen Ausgaben nach Verwendungszweck im Verhältnis zum BIP im Jahr 2011 sowie ihre Entwicklung im Zeitraum 2001-11 ersichtlich.

Über die Aufschlüsselung der Staatsausgaben nach ausgewählten Aufgabenbereichen auf COFOG-II-Ebene informieren Abbildung 3.29 (allgemeine öffentliche Verwaltung), Abbildung 3.30 (öffentliche Ordnung und Sicherheit), Abbildung 3.31 (wirtschaftliche Angelegenheiten), Abbildung 3.32 (Gesundheitswesen), Abbildung 3.33 (Bildungswesen) und Abbildung 3.34 (soziale Sicherung). Diese Abbildungen sind online verfügbar unter: http://dx.doi.org/10.1787/888932941766, http://dx.doi.org/ 10.1787/888932941785, http://dx.doi.org/10.1787/ 888932941804, http://dx.doi.org/10.1787/888932941823, http://dx.doi.org/10.1787/888932941842bzw. http:// dx.doi.org/10.1787/888932941861.

\section{Literaturhinweise}

OECD (2012), Health at a Glance: Europe 2012, OECD Publishing, http://dx.doi.org/10.1787/9789264183896-en.

\section{Anmerkungen zu den Tabellen}

Für Kanada, Chile, Mexiko und Neuseeland sind keine Daten verfügbar.

3.26: Für Japan, die Schweiz und die Türkei stehen keine Zeitreihen zur Verfügung. Die Daten für Polen beziehen sich auf 2002 anstelle von 2001.

Hinweis zu den Daten für Israel: http://dx.doi.org/10.1787/888932315602. 


\section{3. ÖFFENTLICHE FINANZEN UND WIRTSCHAFTLICHES HANDELN DES STAATES}

Struktur der gesamtstaatlichen Ausgaben nach Verwendungszweck (COFOG)

3.25. Struktur der gesamtstaatlichen Ausgaben nach Verwendungszweck (2011)
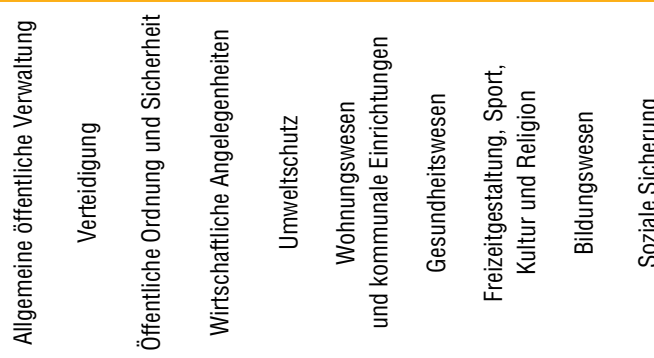

\begin{tabular}{|c|c|c|c|c|c|c|c|c|c|c|}
\hline Australien & 12.5 & 4.1 & 4.8 & 11.4 & 2.6 & 1.8 & 19.2 & 2.1 & 14.5 & 27.1 \\
\hline Österreich & 13.1 & 1.4 & 2.9 & 10.5 & 1.0 & 1.2 & 15.3 & 2.0 & 11.0 & 41.6 \\
\hline Belgien & 15.0 & 1.8 & 3.4 & 12.3 & 1.4 & 0.7 & 14.8 & 2.4 & 11.6 & 36.6 \\
\hline Tschech. Rep. & 10.7 & 2.1 & 4.3 & 13.9 & 3.1 & 1.9 & 18.1 & 2.9 & 11.4 & 31.7 \\
\hline Dänemark & 13.7 & 2.4 & 2.0 & 6.1 & 0.7 & 0.6 & 14.5 & 2.8 & 13.5 & 43.8 \\
\hline Estland & 8.4 & 4.1 & 5.6 & 12.0 & -0.9 & 1.6 & 13.3 & 5.0 & 16.9 & 34.2 \\
\hline Finnland & 13.3 & 2.6 & 2.7 & 8.8 & 0.5 & 1.0 & 14.2 & 2.2 & 11.6 & 43.1 \\
\hline Frankreich & 11.5 & 3.2 & 3.1 & 6.3 & 1.9 & 3.4 & 14.7 & 2.5 & 10.8 & 42.6 \\
\hline Deutschland & 13.6 & 2.4 & 3.5 & 7.8 & 1.5 & 1.2 & 15.5 & 1.8 & 9.4 & 43.3 \\
\hline Griechenland & 24.6 & 4.6 & 3.3 & 6.2 & 1.0 & 0.4 & 11.6 & 1.2 & 7.9 & 39.3 \\
\hline Ungarn & 17.5 & 2.3 & 3.9 & 14.4 & 1.5 & 1.6 & 10.4 & 3.5 & 10.5 & 34.5 \\
\hline Island & 17.8 & 0.1 & 3.1 & 12.4 & 1.3 & 0.7 & 16.1 & 7.0 & 17.1 & 24.6 \\
\hline Irland & 11.4 & 0.9 & 3.7 & 16.4 & 2.1 & 1.3 & 15.6 & 1.8 & 10.9 & 35.9 \\
\hline Israel & 14.7 & 14.7 & 3.8 & 5.8 & 1.5 & 1.0 & 12.3 & 3.9 & 16.5 & 25.9 \\
\hline Italien & 17.3 & 3.0 & 4.0 & 7.1 & 1.8 & 1.4 & 14.7 & 1.1 & 8.5 & 41.0 \\
\hline Japan & 11.0 & 2.2 & 3.1 & 9.8 & 2.9 & 1.8 & 17.3 & 0.8 & 8.4 & 42.7 \\
\hline Korea & 15.2 & 8.6 & 4.2 & 20.1 & 2.4 & 3.3 & 15.2 & 2.2 & 15.8 & 13.1 \\
\hline Luxemburg & 11.4 & 1.0 & 2.5 & 9.9 & 2.8 & 1.8 & 11.4 & 4.0 & 12.1 & 43.2 \\
\hline Niederlande & 11.2 & 2.7 & 4.2 & 10.9 & 3.3 & 1.2 & 17.0 & 3.5 & 11.6 & 34.5 \\
\hline Norwegen & 9.7 & 3.6 & 2.2 & 9.6 & 1.5 & 1.6 & 16.5 & 2.9 & 12.6 & 39.8 \\
\hline Polen & 13.4 & 2.7 & 4.2 & 13.0 & 1.6 & 2.0 & 10.9 & 3.0 & 12.8 & 36.6 \\
\hline Portugal & 17.1 & 2.7 & 4.0 & 8.2 & 1.1 & 1.3 & 13.8 & 2.2 & 12.9 & 36.7 \\
\hline Slowak. Rep. & 15.4 & 2.7 & 6.4 & 9.8 & 2.7 & 2.6 & 15.5 & 3.0 & 10.6 & 31.3 \\
\hline Slowenien & 12.4 & 2.3 & 3.3 & 11.4 & 1.6 & 1.3 & 13.5 & 3.7 & 13.2 & 37.3 \\
\hline Spanien & 12.5 & 2.3 & 4.8 & 11.6 & 2.1 & 1.3 & 14.1 & 3.3 & 10.5 & 37.4 \\
\hline Schweden & 14.4 & 2.9 & 2.7 & 8.2 & 0.7 & 1.5 & 13.7 & 2.2 & 13.3 & 40.5 \\
\hline Schweiz & 9.9 & 2.9 & 5.0 & 13.7 & 2.3 & 0.6 & 6.1 & 2.6 & 17.9 & 39.0 \\
\hline Türkei & 16.4 & 4.1 & 5.2 & 11.9 & 1.1 & 3.5 & 12.1 & 2.3 & 11.4 & 31.9 \\
\hline Ver. Königreich & 11.6 & 5.1 & 5.3 & 5.3 & 2.0 & 1.8 & 16.5 & 2.1 & 13.4 & 36.8 \\
\hline Ver. Staaten & 12.4 & 11.7 & 5.5 & 9.4 & 0.0 & 2.1 & 21.4 & 0.7 & 15.5 & 21.3 \\
\hline OECD & 13.6 & 3.6 & 3.9 & 10.5 & 1.6 & 1.6 & 14.5 & 2.7 & 12.5 & 35.6 \\
\hline
\end{tabular}

Quelle: OECD National Accounts Statistics (Datenbank). Die Daten für Australien gründen sich auf die Government Finance Statistics des Australian Bureau of Statistics.

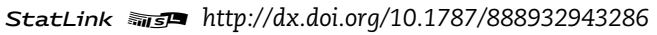

3.26. Veränderung der Struktur der gesamtstaatlichen Ausgaben nach Verwendungszweck (2001 und 2011)

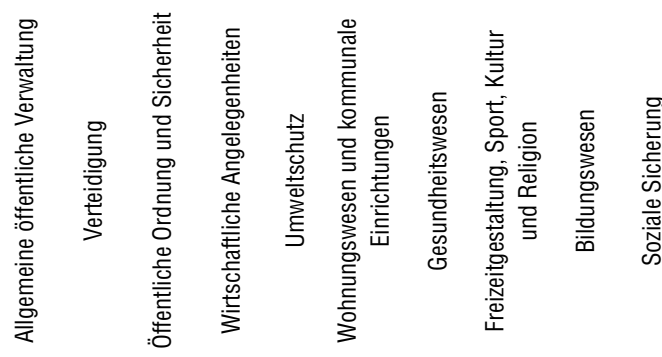

\begin{tabular}{|c|c|c|c|c|c|c|c|c|c|c|}
\hline Australien & 0.4 & -0.4 & 0.3 & -1.4 & 1.0 & -0.7 & 2.4 & -0.1 & 0.0 & -1.5 \\
\hline Österreich & -2.5 & -0.3 & 0.0 & 0.3 & 0.0 & -0.3 & 1.7 & 0.1 & 0.2 & 0.8 \\
\hline Belgien & -6.3 & -0.7 & 0.2 & 3.4 & -0.1 & 0.1 & 1.5 & 0.5 & -0.2 & 1.6 \\
\hline Tschech. Rep. & 1.6 & -1.4 & -0.6 & -6.4 & 1.0 & -0.7 & 2.2 & 0.5 & 1.5 & 2.3 \\
\hline Dänemark & -1.6 & -0.6 & 0.2 & 0.1 & -0.4 & -0.7 & 1.9 & -0.3 & -0.1 & 1.5 \\
\hline Estland & -0.9 & 0.2 & -1.4 & 1.4 & -3.0 & -0.2 & 1.6 & -0.7 & -2.1 & 5.0 \\
\hline Finnland & -1.1 & -0.1 & -0.1 & -0.8 & -0.2 & 0.1 & 2.0 & -0.1 & -0.9 & 1.2 \\
\hline Frankreich & -2.7 & -0.7 & 0.2 & -0.5 & 0.3 & -0.2 & 1.0 & 0.4 & -0.6 & 2.9 \\
\hline Deutschland & 1.0 & 0.0 & 0.1 & -1.4 & 0.0 & -0.9 & 1.4 & 0.0 & 0.7 & -0.8 \\
\hline Griechenland & 1.1 & -2.8 & 0.7 & -3.8 & -0.2 & -0.5 & 0.3 & 0.4 & 1.8 & 2.8 \\
\hline Ungarn & -4.6 & -0.3 & -0.5 & 1.9 & -0.1 & -0.2 & 0.1 & 0.2 & -0.6 & 4.0 \\
\hline Island & 2.5 & 0.0 & -0.4 & -4.0 & -0.4 & -0.2 & -2.5 & -0.1 & -1.1 & 6.1 \\
\hline Irland & 0.4 & -1.0 & -1.1 & 3.1 & -0.7 & -4.3 & -3.1 & -0.2 & -2.6 & 9.5 \\
\hline Israel & -4.3 & -1.2 & 0.7 & 0.3 & 0.3 & -0.7 & 1.2 & 0.5 & 1.8 & 1.6 \\
\hline Italien & -2.9 & 0.6 & 0.1 & -1.9 & 0.0 & -0.3 & 1.6 & -0.7 & -1.3 & 4.8 \\
\hline Korea & 1.8 & -1.9 & -0.9 & -3.2 & -0.3 & -0.6 & 4.2 & -0.1 & -2.2 & 3.2 \\
\hline Luxemburg & -0.6 & 0.2 & 0.1 & 2.7 & -0.5 & -0.3 & -1.3 & -0.4 & 0.1 & 0.0 \\
\hline Niederlande & -3.6 & -0.8 & 0.4 & -1.4 & 0.1 & -0.5 & 5.8 & -0.2 & 0.2 & 0.0 \\
\hline Norwegen & -1.8 & -0.7 & -0.1 & -1.0 & 0.3 & 0.8 & 0.3 & 0.5 & -0.7 & 2.4 \\
\hline Polen & -0.1 & 0.0 & 0.8 & 5.0 & 0.2 & -1.7 & 1.0 & 0.6 & -1.0 & -4.9 \\
\hline Portugal & 3.0 & -0.5 & 0.0 & -4.8 & -0.5 & -1.0 & -1.1 & -0.8 & -2.2 & 7.8 \\
\hline Slowak. Rep. & -1.7 & -2.3 & 0.2 & -5.2 & 1.0 & 0.8 & 4.5 & 0.9 & 3.3 & -1.5 \\
\hline Slowenien & -2.0 & -0.4 & -0.7 & 2.0 & 0.0 & -0.1 & -0.4 & 1.1 & -0.6 & 1.1 \\
\hline Spanien & -2.1 & -0.5 & 0.0 & -0.2 & -0.2 & -1.3 & 0.8 & -0.1 & -0.6 & 4.2 \\
\hline Schweden & -0.5 & -1.0 & 0.2 & 1.0 & 0.1 & -0.3 & 1.8 & 0.2 & 0.1 & -1.6 \\
\hline Ver. Königreich & 1.0 & -0.6 & -0.4 & -1.0 & 0.3 & 0.0 & 1.8 & -0.4 & 0.3 & -1.0 \\
\hline Ver. Staaten & -2.5 & 2.3 & -0.4 & -1.7 & 0.0 & 0.5 & 2.2 & -0.2 & -2.0 & 1.7 \\
\hline OECD & -1.1 & -0.5 & -0.1 & -0.6 & -0.1 & -0.5 & 1.2 & 0.0 & -0.3 & 2.0 \\
\hline
\end{tabular}

Quelle: OECD National Accounts Statistics (Datenbank). Die Daten für Australien gründen sich auf die Government Finance Statistics des Australian Bureau of Statistics.

StatLink तiाs http://dx.doi.org/10.1787/888932943305 
Die Zuständigkeiten für die Finanzierung öffentlicher Güter und Dienstleistungen und die Einkommensumverteilung sind auf mehrere staatliche Ebenen verteilt. Inwieweit welche Ebenen für welche Ausgabenbereiche zuständig sind, hängt von der institutionellen Struktur der einzelnen Länder und der Verteilung der Ausgabenbefugnisse zwischen den verschiedenen staatlichen Ebenen ab. Verfügen die nachgeordneten Gebietskörperschaften über mehr Autonomie, sind sie u.U. besser imstande, Maßnahmen und Programme zu gestalten.

2011 wurden im OECD-Durchschnitt 46\% der gesamtstaatlichen Ausgaben auf Ebene der Zentralregierung getätigt. Die nachgeordneten Gebietskörperschaften (Gliedstaaten und Gemeinden) kamen für 32\% auf, der Rest entfiel auf Sozialversicherungsträger. Der Grad der fiskalischen Dezentralisierung unterscheidet sich allerdings erheblich im Ländervergleich. In Irland wurden z.B. 76,4\% der Gesamtausgaben von der zentralen Ebene getätigt, was einem Anstieg gegenüber 2001 um 27,1 Prozentpunkte entsprach. In Deutschland und der Schweiz, beides Föderalstaaten, entfielen hingegen weniger als 20\% der Gesamtausgaben auf die zentrale Ebene.

Die Zentralregierungen wenden im Allgemeinen einen vergleichsweise großen Teil ihrer Mittel für die Bereiche soziale Sicherung (z.B. Altersrenten und Arbeitslosenunterstützung), allgemeine öffentliche Verwaltung (z.B. Organe der Exekutive und Legislative, Staatsschuldentransaktionen) und Verteidigung auf. In über der Hälfte der OECD-Länder ist die soziale Sicherung der größte Posten im Budget der Zentralregierungen. In Belgien und Spanien werden mehr als $60 \%$ der auf zentraler Ebene zur Verfügung stehenden Mittel für die allgemeine öffentliche Verwaltung aufgewandt.

Außer in Österreich ist der größte Ausgabenposten auf Ebene der Gliedstaaten ebenso wie der Gemeinden die Bildung. Auch Umweltschutz, Wohnungswesen und kommunale Einrichtungen sowie Freizeitgestaltung, Sport, Kultur und Religion werden hauptsächlich von den nachgeordneten Gebietskörperschaften finanziert, worin zum Ausdruck kommt, dass diese Ebenen unmittelbarer und auf greifbarere Weise mit den Bürgern in Kontakt stehen.

Im OECD-Vergleich ist ein positiver Zusammenhang zwischen den Ausgaben und Einnahmen der nachgeordneten Gebietskörperschaften festzustellen. Der Grad der Steuerautonomie der nachgeordneten Verwaltungsebenen variiert jedoch erheblich. Beschränkungen der Kapazität der nachgeordneten Gebietskörperschaften zur Festlegung eigener steuerlicher Bemessungsgrundlagen, Steuersätze und Steuererleichterungen verringern deren Fähigkeit, eigene Einnahmequellen zu erschließen und auf wirtschaftliche Schocks durch Steuerbzw. Abgabenerhöhungen zu reagieren, und könnten auch ihre Möglichkeiten zur Bereitstellung eines bedarfsgerechteren öffentlichen Dienstleistungsangebots begrenzen. Folglich sind die Gemeinden in der Regel stark von Finanztransfers der Zentralregierung abhängig. Ein klarer Trend zur fiskalischen Dezentralisierung zeichnet sich im OECD-Raum nicht ab.

\section{Methodik und Definitionen}

Die Ausgabendaten stammen aus der OECD-Datenbank National Accounts Statistics, die auf dem System der Volkswirtschaftlichen Gesamtrechnungen (SNA) basiert. Dabei handelt es sich um einen international vereinbarten Katalog von Konzepten, Definitionen, Klassifizierungen und Regeln für Volkswirtschaftliche Gesamtrechnungen. Die Ausgabendaten werden gemäß der Klassifikation der Ausgaben des Staats nach Aufgabenbereichen (Classification of the Functions of Government - COFOG) aufgeschlüsselt, nach der die Staatsausgaben in zehn Funktionen aufgeteilt werden: allgemeine öffentliche Verwaltung; Verteidigung; öffentliche Ordnung und Sicherheit; wirtschaftliche Angelegenheiten; Umweltschutz; Wohnungswesen und kommunale Einrichtungen; Gesundheitswesen; Freizeitgestaltung, Sport, Kultur und Religion; Bildungswesen; soziale Sicherung. Weitere Informationen zu den Ausgabenarten, die unter den verschiedenen Kategorien erfasst sind, können Anhang B entnommen werden. Der Sektor Staat setzt sich zusammen aus der Zentralregierung, den Gliedstaaten, den Gemeinden und der Sozialversicherung. Die Kategorie der Gliedstaaten findet nur für die neun OECD-Länder Anwendung, die föderal organisiert sind: Australien, Österreich, Belgien, Kanada, Deutschland, Mexiko, Spanien (wird als Quasi-Föderalstaat betrachtet), Schweiz und Vereinigte Staaten.

In den Daten von Abbildung 3.35 und 3.37 (Entwicklung der Ausgabenverteilung auf die einzelnen staatlichen Ebenen, 2009-11) sind Transferzahlungen zwischen verschiedenen staatlichen Ebenen ausgeklammert. Sie geben daher Aufschluss darüber, wie die Zuständigkeiten für die Bereitstellung von Gütern und Dienstleistungen in etwa zwischen den einzelnen staatlichen Ebenen verteilt sind. Die Daten zu den Ausgaben auf Ebene der Zentralregierung, der Gliedstaaten und der Gemeinden (Tabelle 3.38, 3.39 und 3.40) beinhalten demgegenüber Finanztransfers zwischen den verschiedenen staatlichen Ebenen. Sie verdeutlichen somit, wie viel auf den jeweiligen Ebenen für die verschiedenen Aufgabenbereiche aufgewendet wird. Abbildung 3.37 (http:/ /dx.doi.org/10.1787/888932941918) sowie Tabelle 3.38 (http:// dx.doi.org/10.1787/888932941937), 3.39 (http://dx.doi.org/ 10.1787/888932941956) und 3.40 (http://dx.doi.org/10.1787/ $888932941975)$ sind online verfügbar.

\section{Literaturhinweise}

Blöchliger, H. und C. Vammalle (2012), Reforming Fiscal Federalism and Local Government: Beyond the Zero-Sum Game, OECD Fiscal Federalism Studies, OECD Publishing, Paris, http:// dx.doi.org/10.1787/9789264119970-en.

OECD (2013), National Accounts at a Glance 2013, OECD Publishing, Paris, http://dx.doi.org/10.1787/na_glance-2013-en.

\section{Anmerkungen zu den Abbildungen}

Für Chile stehen keine Daten zur Verfügung. Finanztransfers zwischen verschiedenen staatlichen Ebenen sind nicht berücksichtigt (außer für Australien, Japan und die Türkei). Die Daten für Kanada und Neuseeland beziehen sich auf 2010 anstelle von 2011.

3.35: Für Japan, Polen und die Türkei liegen keine Daten für 2001 vor, diese Länder sind im OECD-Durchschnitt daher nicht berücksichtigt. Die Daten für Mexiko beziehen sich auf 2003 anstelle von 2001. In Australien und den Vereinigten Staaten werden die Gemeinden unter den Gliedstaaten erfasst. In Australien gibt es keine staatlichen Sozialversicherungssysteme. In Neuseeland, Norwegen, dem Vereinigten Königreich und den Vereinigten Staaten wird die Sozialversicherung auf Ebene der Zentralregierung erfasst.

Hinweis zu den Daten für Israel: http://dx.doi.org/10.1787/888932315602. 


\section{3. ÖFFENTLICHE FINANZEN UND WIRTSCHAFTLICHES HANDELN DES STAATES}

3.35. Verteilung der Ausgaben auf die verschiedenen staatlichen Ebenen (2001 und 2011)

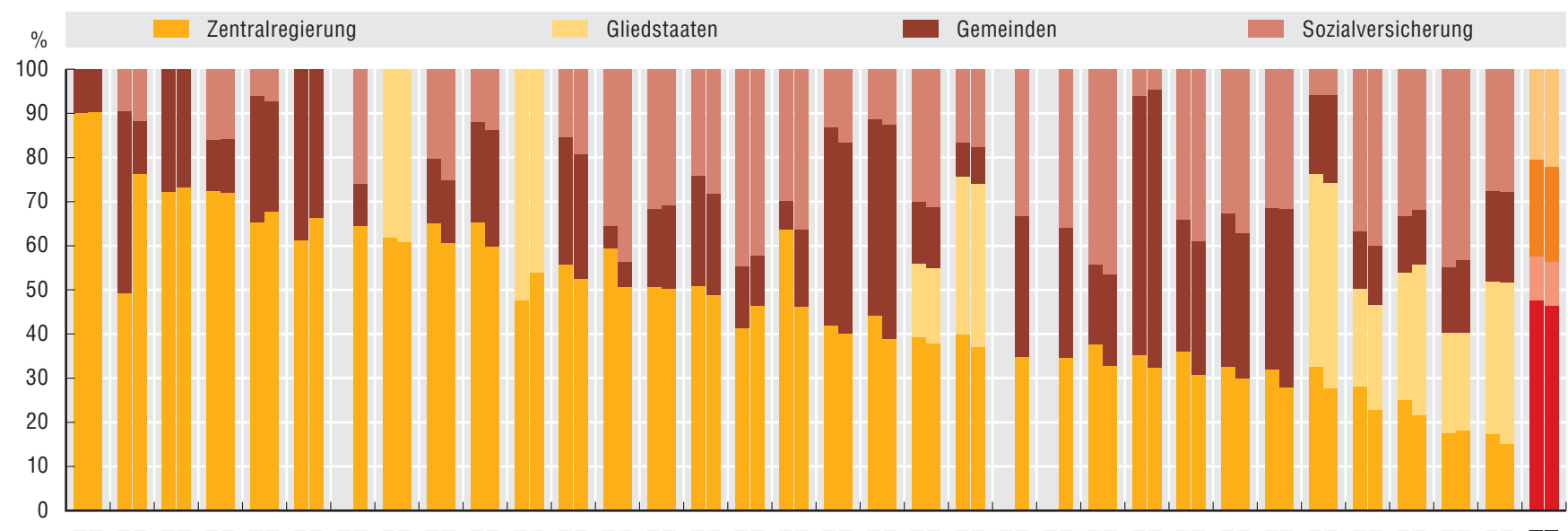

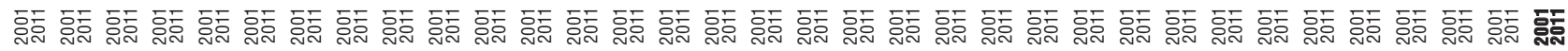
NZL IRL GBR ISR EST NOR TUR AUS PRT CZE USA ISL GRC SVN HUN LUX SVK KOR SWE AUT MEX POL JPN FRA DNK ITA NLD FIN CAN BEL ESP DEU CHE OECD

Quelle: OECD National Accounts Statistics (Datenbank).

StatLink तilst http://dx.doi.org/10.1787/888932941880

\subsection{Fiskalische Dezentralisierung: Auf die nachgeordneten Gebietskörperschaften entfallender Anteil an den gesamtstaatlichen Ausgaben und Einnahmen (2011)}

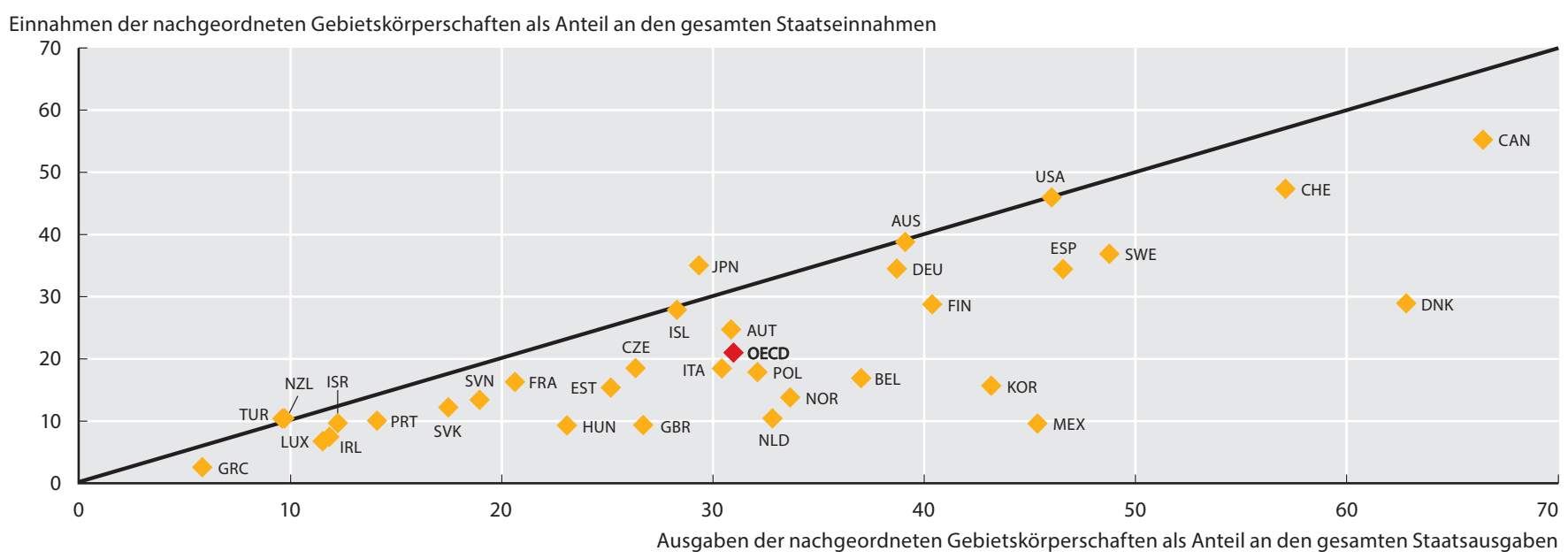

Quelle: OECD National Accounts Statistics (Datenbank). 
Einer der Hauptgründe, warum der Staat Investitionen tätigt, ist die Förderung des Wirtschaftswachstums. Diesem Zweck dient z.B. die Finanzierung öffentlicher Infrastrukturvorhaben (Bau von Straßen, Wohnungen, Schulen, Krankenhäusern und Kommunikationsnetzen). Außerdem sind einige Vorhaben, die auf soziale Ziele ausgerichtet sind (Investitionen in öffentliche Güter), für den privaten Sektor nicht rentabel, weshalb sie vom öffentlichen Sektor durchgeführt werden müssen. Direkte öffentliche Investitionen können vom Staat eingesetzt werden, um antizyklische Effekte zu erzielen. Der jüngste Konjunkturabschwung veranlasste viele Regierungen im OECD-Raum z.B. dazu, im Rahmen von Konjunkturprogrammen die Investitionen in weiche und harte Infrastrukturen zu erhöhen.

2011 machten die staatlichen Ausgaben für direkte Investitionen im OECD-Durchschnitt 15,5\% der Gesamtinvestitionen aus. In 23 OECD-Ländern war dieser Wert niedriger als 2009, was auf die Umsetzung von Spar- und Sanierungsprogrammen zurückzuführen war. Weiter gestiegen ist der Anteil der direkten staatlichen Investitionen an den Gesamtinvestitionen im Zeitraum 2009-11 in Polen (+3,6 Prozentpunkte), Irland (+1,9 Prozentpunkte), Dänemark (+1,8 Prozentpunkte), Ungarn (+1,8 Prozentpunkte), Kanada (+1,3 Prozentpunkte), Australien (+0,4 Prozentpunkte), Belgien und der Schweiz (jeweils +0,1 Prozentpunkte).

Der Anteil der direkten Investitionen an den gesamtstaatlichen Ausgaben variiert stark im Ländervergleich. Diese Unterschiede hängen mit dem existierenden Infrastrukturbestand zusammen. 2011 entfielen im OECD-Durchschnitt 6,7\% der gesamtstaatlichen Ausgaben auf direkte Investitionen. In vier OECD-Ländern war sowohl im Zeitraum 2001-09 als auch 2009-11 ein kontinuierlicher Anstieg des Anteils der direkten Investitionen an den gesamtstaatlichen Ausgaben zu beobachten, und zwar in Polen (+5,4 Prozentpunkte), Kanada (+3,5 Prozentpunkte), Schweden (+1,3 Prozentpunkte) und Dänemark (+0,3 Prozentpunkte). In 11 OECD-Ländern ist der Anteil der direkten Investitionen hingegen in beiden Zeiträumen kontinuierlich gesunken. Am stärksten war der Rückgang in Irland (-7,5 Prozentpunkte), Korea (-7,2 Prozentpunkte) und in Island (-6,6 Prozentpunkte). In den Ländern, in denen die Krise besonders heftig war, fiel der proportionale Rückgang der direkten staatlichen Investitionen während der anschließenden Konsolidierungsphase am stärksten aus. Dies erklärte sich aus dem kombinierten Effekt der höheren Ausgaben für andere Posten (Arbeitslosenversicherung und andere automatisch gezahlte Sozialleistungen) sowie der Konsolidierungsmaßnahmen, die die Investitionstätigkeit beeinträchtigten.

Die Verteilung der direkten Investitionsausgaben auf die verschiedenen staatlichen Ebenen ist stark an die politische Struktur der Länder geknüpft. Investitionen auf Ebene der Gliedstaaten spielen nur in föderal organisierten Ländern eine Rolle. Sie machen dort im Schnitt über ein Viertel der direkten öffentlichen Investitionen aus; in den Vereinigten Staaten erreicht ihr Anteil allerdings 84\% und in Australien 66\%. Im OECD-Durchschnitt werden $\mathrm{rd}$. $62 \%$ der direkten staatlichen Investitionen von nachgeordneten Gebietskörperschaften vorgenommen; 37,3\% werden auf Ebene der Zentralregierungen getätigt. Im Zeitraum 2001-11 war kein einheitlicher Trend hin $z u$ einer Dezentralisierung der Investitionstätigkeit zu erkennen. Während es in einigen Ländern, wie der Slowakischen Republik und Ungarn, zu einer deutlichen Umverteilung von der zentralen Ebene hin zu den lokalen Gebietskörperschaften kam, war in anderen Ländern, Irland und Polen z.B., eine umgekehrte Entwicklung zu beobachten.

\section{Methodik und Definitionen}

Die Daten stammen aus der OECD-Datenbank OECD National Accounts Statistics, die auf dem System der Volkswirtschaftlichen Gesamtrechnungen (SNA) basiert. Dabei handelt es sich um einen international vereinbarten Katalog von Konzepten, Definitionen, Klassifizierungen und Regeln für Volkswirtschaftliche Gesamtrechnungen. Die gesamtstaatlichen Investitionen unterteilen sich in direkte Investitionen (die anhand der Bruttoanlageinvestitionen gemessen werden) und indirekte Investitionen (die anhand der Vermögenstransfers gemessen werden). In dieser Analyse wurden nur die direkten Investitionen berücksichtigt. Bei den Bruttoanlageinvestitionen handelt es sich vor allem um Straßeninfrastrukturen, sie umfassen aber auch Infrastrukturen wie Büro- und Wohngebäude, Schulen und Krankenhäuser.

Die Gesamtinvestitionen beziehen sich auf die Investitionsausgaben der gesamten Volkswirtschaft, einschließlich Staatssektor, Nichtfinanzunternehmen, Finanzunternehmen, private Haushalte und Organisationen ohne Erwerbszweck.

Der Sektor Staat setzt sich zusammen aus der Zentralregierung, den Gliedstaaten, den Gemeinden und der Sozialversicherung. Die Kategorie der Gliedstaaten findet nur für die neun OECD-Länder Anwendung, die föderal organisiert sind: Australien, Österreich, Belgien, Kanada, Deutschland, Mexiko, Spanien (wird als Quasi-Föderalstaat betrachtet), Schweiz und Vereinigte Staaten. Abbildung 3.44, Veränderung der Verteilung der Investitionsausgaben auf die verschiedenen staatlichen Ebenen (2001-11), ist online verfügbar unter: $h t t p: / / d x$.doi.org/10.1787/888932942051.

\section{Literaturhinweise}

OECD (2013, erscheint demnächst), Investing Together: Working Effectively across Levels of Government, OECD Publishing, Paris, http://dx.doi.org/10.1787/9789264197022-en.

OECD (2011), Making the Most of Public Investment in a Tight Fiscal Environment: Multi-level Governance Lessons from the Crisis, OECD Publishing, Paris, http://dx.doi.org/10.1787/9789264114470-en.

OECD/Korea Institute of Public Finance (2012), Institutional and Financial Relations across Levels of Government, OECD Fiscal Federalism Studies, OECD Publishing, Paris, http:// dx.doi.org/10.1787/9789264167001-en.

\section{Anmerkungen zu den Abbildungen}

Die Daten für Kanada und die Russische Föderation beziehen sich auf 2010 anstelle von 2011. Die Daten für Mexiko beziehen sich auf 2003 anstelle von 2001. Die Daten für die Russische Föderation beziehen sich auf 2002 anstelle von 2001. Unterschiede hinsichtlich der vorhandenen Daten zwischen Abbildungen 3.41 und 3.42 erklären sich aus der Verwendung unterschiedlicher Datentabellen in der OECDDatenbank National Accounts Statistics.

3.41: Für Island sind keine Daten verfügbar. Folgende Länder sind auf Grund fehlender Zeitreihen im OECD-Durchschnitt nicht berücksichtigt: Chile (2001), Griechenland (2001), Türkei (2001), Israel (2009-11) und Neuseeland (2009-11). Die Daten für Australien und Chile beziehen sich auf 2010 anstelle von 2011. Die Daten für Irland beziehen sich auf 2002 anstelle von 2001.

3.42: Für Chile stehen keine Daten zur Verfügung. Für Japan und die Türkei liegen keine Daten für 2001 vor, diese Länder sind im OECDDurchschnitt daher nicht berücksichtigt. Die Daten für Neuseeland beziehen sich auf 2010 anstelle von 2011.

3.43: Für Chile stehen keine Daten zur Verfügung. Die Daten für Neuseeland beziehen sich auf 2010 anstelle von 2011. In Australien und den Vereinigten Staaten werden die Gemeinden unter den Gliedstaaten erfasst. In Australien gibt es keine staatlichen Sozialversicherungssysteme. In Neuseeland, Norwegen, dem Vereinigten Königreich und den Vereinigten Staaten wird die Sozialversicherung auf Ebene der Zentralregierung erfasst.

Hinweis zu den Daten für Israel: http://dx.doi.org/10.1787/888932315602. 


\section{3. ÖFFENTLICHE FINANZEN UND WIRTSCHAFTLICHES HANDELN DES STAATES}

3.41. Staatliche Investitionen in Prozent der Gesamtinvestitionen (2001, 2009 und 2011)

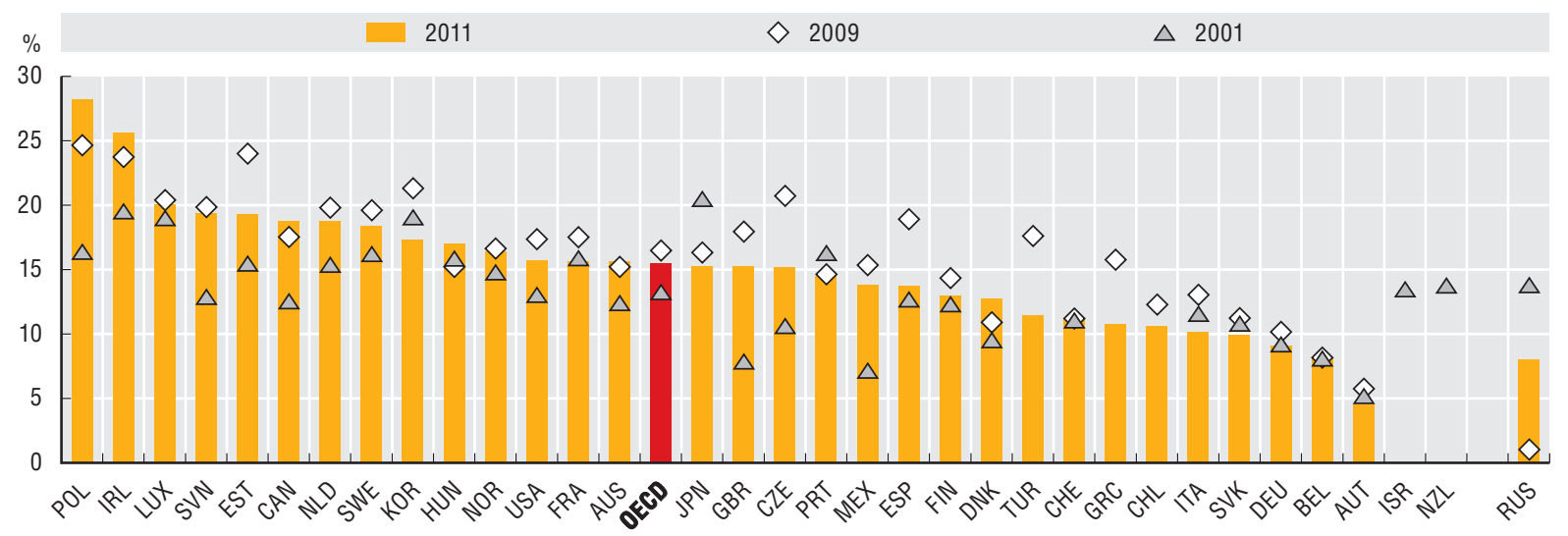

Quelle: OECD National Accounts Statistics (Datenbank).

StatLink त्राड़ http://dx.doi.org/10.1787/888932941994

3.42. Staatliche Investitionen in Prozent der gesamtstaatlichen Ausgaben (2001, 2009 und 2011)

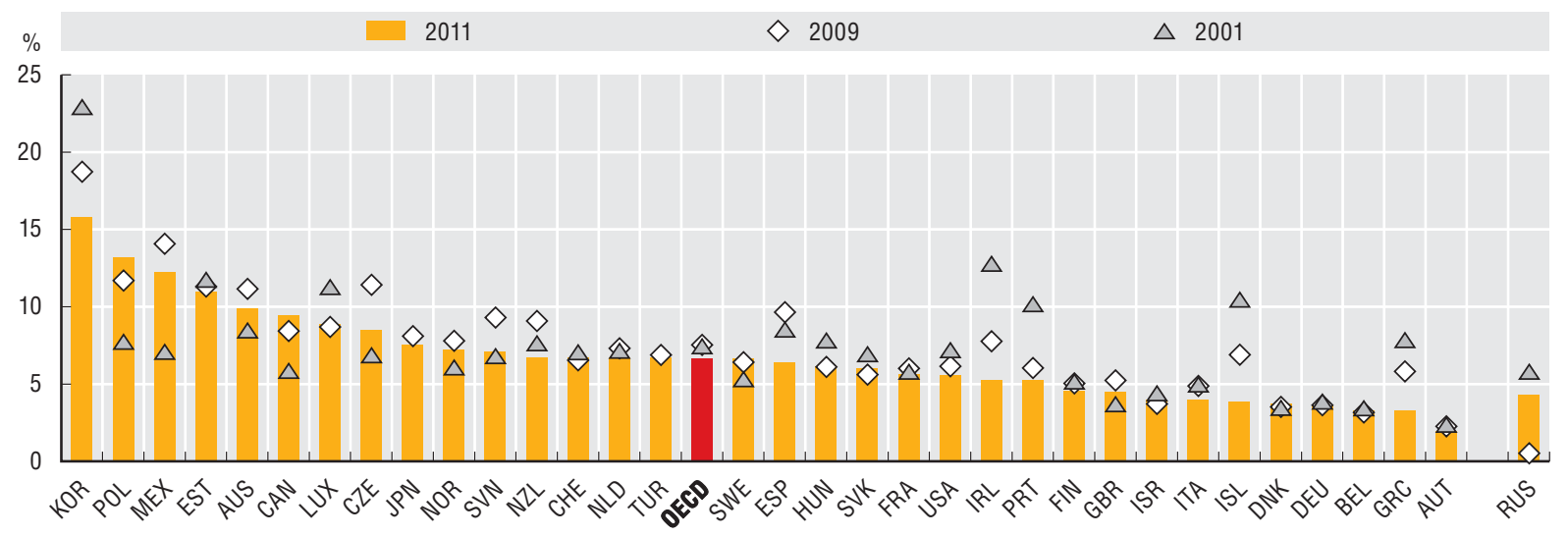

Quelle: OECD National Accounts Statistics (Datenbank).

StatLink त्राड़ http://dx.doi.org/10.1787/888932942013

3.43. Verteilung der Investitionsausgaben auf die verschiedenen staatlichen Ebenen (2011)

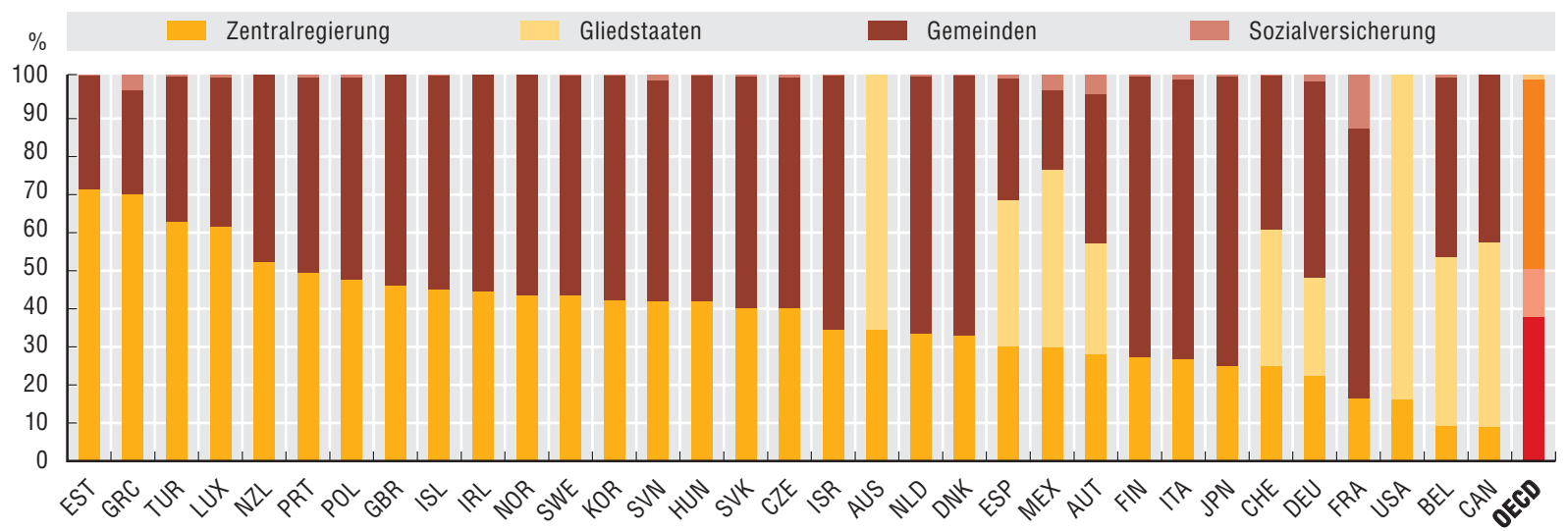

Quelle: OECD National Accounts Statistics (Datenbank). 
Bei den Produktionskosten handelt es sich um den Anteil der staatlichen Ausgaben, der für die Produktion von Gütern und Dienstleistungen bestimmt ist. Einige staatliche Stellen produzieren die meisten ihrer Güter und Dienstleistungen selbst, andere hingegen übertragen einen großen Teil ihrer Güter- und Dienstleistungsproduktion an Organisationen ohne Erwerbszweck oder Privatunternehmen. Dieses Outsourcing staatlicher Aufgaben kann auf zweierlei Weise erfolgen. Die staatlichen Stellen können entweder Güter und Dienstleistungen zur eigenen Weiterverwendung einkaufen (vom Staat genutzte Güter und Dienstleistungen, d.h. Vorleistungen) oder sie können Organisationen ohne Erwerbszweck oder Privatunternehmen mit der direkten Bereitstellung von Gütern und Dienstleistungen für die Endverbraucher betrauen (vom Staat finanzierte Güter und Dienstleistungen, d.h. über Marktproduzenten bereitgestellte soziale Sachtransfers).

Staatliche Entscheidungen über Umfang und Art der zu produzierenden Güter und Dienstleistungen sowie darüber, wie diese am besten produziert werden können, haben Einfluss darauf, wie sie den Bürgern angeboten werden. Das Outsourcing staatlicher Aufgaben ist eine Methode, um externes Fachwissen zu gewinnen und Güter und Dienstleistungen kostengünstiger bereitzustellen (wie vorteilhaft diese Methode ist, unterscheidet sich allerdings von Fall zu Fall). Zudem kann der Staat durch dieses Outsourcing einen stärkeren Einfluss auf die Nachfrage und die Beschäftigung im nichtstaatlichen Sektor ausüben. Der Umfang des Outsourcing kann an der Höhe der Ausgaben der Zentralregierung, der Gliedstaaten und der Gemeinden für den Kauf von Waren und Dienstleistungen gemessen werden.

2011 machten die Kosten der Produktion staatlicher Güter und Dienstleistungen fast ein Viertel des BIP aus; am höchsten war dieser Anteil mit 32\% in Dänemark und den Niederlanden, am niedrigsten mit $12 \%$ in Mexiko. Im Zeitraum 2001-11 ist der Anteil der staatlichen Produktionskosten am BIP im OECDDurchschnitt um 1,6 Prozentpunkte gestiegen. Nach 2009 kam es allerdings zu einer Wende in diesem Trend, und der Anteil schrumpfte um 1 Prozentpunkt. Etwa 56\% der Korrektur erfolgten über eine Abnahme des Anteils der Personalvergütungen.

Was die Zusammensetzung der staatlichen Produktionskosten betrifft, so entfiel fast die Hälfte (47\%) auf Personalvergütungen; der Anteil des Outsourcing (vom Staat genutzte oder finanzierte Güter und Dienstleistungen) war mit $44 \%$ geringer. Die übrigen 9\% entsprachen der Abschreibung von Anlagevermögen.

2011 machte das Outsourcing staatlicher Aufgaben im OECDDurchschnitt $10 \%$ des BIP aus. Dieser Anteil variierte jedoch erheblich: Vergleichsweise gering war er in Mexiko und der Schweiz mit 2,8\% bzw. 5,4\%, vergleichsweise hoch mit 14,2\% bzw. 19\% in Finnland und den Niederlanden. In Belgien, Japan und Deutschland entfielen weniger als $40 \%$ der OutsourcingAusgaben auf Vorleistungen, woraus sich schließen lässt, dass die Mittel dort großenteils zur Bezahlung Dritter verwendet werden, die vom Staat übertragene Aufgaben erledigen. In der Schweiz, Dänemark, Finnland und Estland entfielen demgegenüber mehr als $80 \%$ der Outsourcing-Ausgaben auf Vorleistungen, was bedeutet, dass sich der Staat dort weiter direkt um die Bereitstellung von Gütern und Dienstleistungen kümmert.

\section{Methodik und Definitionen}

Konzept und Methodik der Produktionskosten stützen sich auf die bestehende Klassifizierung der Staatsausgaben im System der Volkswirtschaftlichen Gesamtrechnungen (SNA). Nach der SNA-Terminologie setzt sich der Sektor Staat zusammen aus der Zentralregierung, den Gliedstaaten, den Gemeinden und der Sozialversicherung. Bei den Produktionskosten des Staats handelt es sich im Einzelnen um:

- Personalvergütungen, darunter Geld- und Sachleistungen sowie alle obligatorischen (und kalkulatorischen) Arbeitgeberbeiträge zur Sozialversicherung sowie freiwilligen Beiträge, die für Staatsbedienstete entrichtet werden.

- Kosten von Gütern und Dienstleistungen, die vom Staat genutzt werden, was die erste Komponente des staatlichen Outsourcing ist. Nach der SNA-Terminologie umfasst dies Vorleistungen, die zur Produktion staatlicher Güter und Dienstleistungen benötigt werden, z.B. Buchhaltungs- und IT-Dienstleistungen.

- Kosten von Gütern und Dienstleistungen, die vom Staat finanziert werden, was die zweite Komponente des staatlichen Outsourcing ist. Nach der SNA-Terminologie beinhaltet dies vom Staat finanzierte, über Marktproduzenten bereitgestellte soziale Sachtransfers (auch solche, die zunächst von den Bürgern bezahlt, deren Kosten aber dann vom Staat zurückerstattet werden, z.B. von der öffentlichen Krankenkasse erstattete ärztliche Behandlungen).

- Abschreibungen von Anlagevermögen (nutzungsbedingte Wertminderungen).

Die Daten umfassen die Beschäftigung im Staatssektor und die Vorleistungen für Produkte, die vom Staat zur eigenen Verwendung produziert wurden, z.B. für Straßen oder sonstige Anlagen, die von Staatsbediensteten gebaut werden. Die hier wiedergegebenen Produktionskosten entsprechen nicht dem Produktionswert in den Volkswirtschaftlichen Gesamtrechnungen. Tabelle 3.48, Entwicklung der Produktionskosten in Prozent des BIP (2009-11), ist online verfügbar unter: http://dx.doi.org/10.1787/ 888932943362. Abbildung 3.49, Struktur der gesamtstaatlichen Outsourcing-Ausgaben (2011), ist online verfügbar unter: http://dx.doi.org/10.1787/888932942127.

\section{Literaturhinweise}

OECD (2013), National Accounts at a Glance 2013, OECD Publishing, Paris, http://dx.doi.org/10.1787/na_glance-2013-en.

\section{Anmerkungen zu den Abbildungen}

Die Daten für Kanada, Neuseeland und die Russische Föderation beziehen sich auf 2010 anstelle von 2011. Die Daten für Mexiko beziehen sich auf 2003 anstelle von 2001. Die Daten für die Russische Föderation beziehen sich auf 2002 anstelle von 2001. Kanada, Island, Israel, Mexiko, das Vereinigte Königreich und die Vereinigten Staaten weisen vom Staat finanzierte Güter und Dienstleistungen in ihren Volkswirtschaftlichen Gesamtrechnungen nicht gesondert aus.

3.45: Für Japan und die Türkei liegen keine Daten für 2001 vor, diese Länder sind im OECD-Durchschnitt daher nicht berücksichtigt. Für Chile liegen Daten für 2010 anstatt für 2011 vor, und sie beziehen sich nur auf die Personalvergütungen (sie sind im OECD-Durchschnitt nicht berücksichtigt).

3.46 und 3.47: Für Chile stehen keine Daten zur Verfügung.

Hinweis zu den Daten für Israel: $h t t p: / / d x . d o i . o r g / 10.1787 / 888932315602$. 


\section{3. ÖFFENTLICHE FINANZEN UND WIRTSCHAFTLICHES HANDELN DES STAATES}

Produktionskosten des Staates und Outsourcing staatlicher Aufgaben

\subsection{Produktionskosten des Staats in Prozent des BIP (2001 und 2011)}

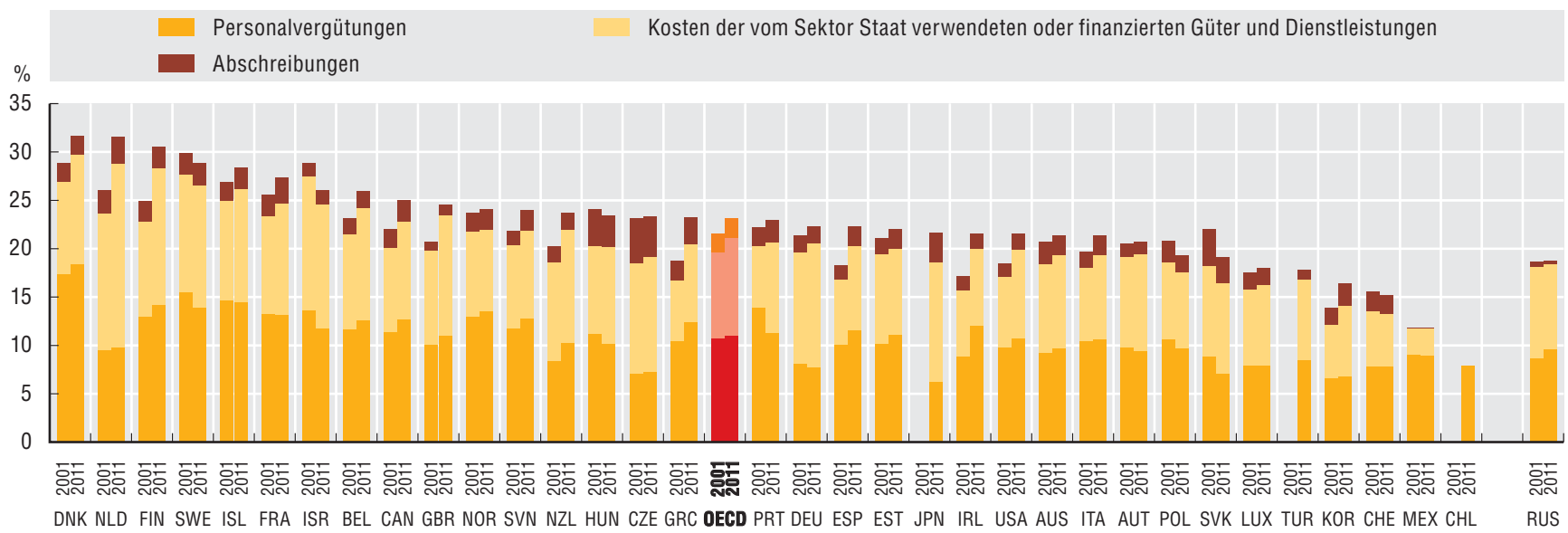

Quelle: OECD National Accounts Statistics (Datenbank). Die Daten für Australien gründen sich auf die Government Finance Statistics sowie vom Australian Bureau of Statistics zur Verfügung gestellte Daten aus der australischen Volkswirtschaftlichen Gesamtrechnung.

StatLink त्ताsम $h t t p: / / d x . d o i . o r g / 10.1787 / 888932942070$

\subsection{Struktur der Produktionskosten (2011)}

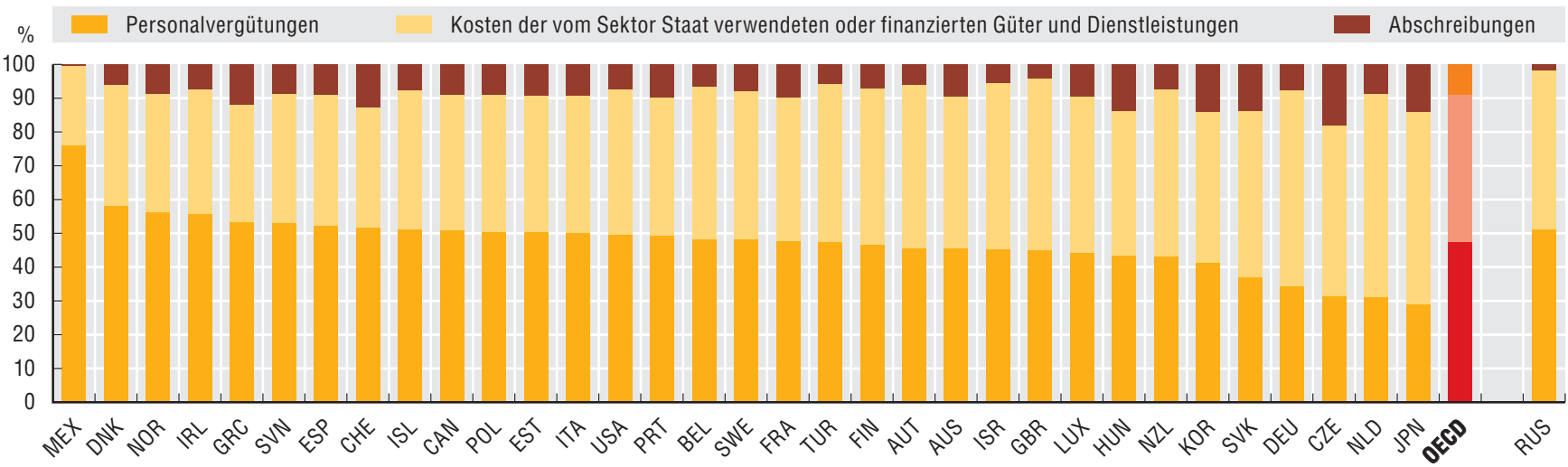

Quelle: OECD National Accounts Statistics (Datenbank). Die Daten für Australien gründen sich auf die Government Finance Statistics sowie vom Australian Bureau of Statistics zur Verfügung gestellte Daten aus der australischen Volkswirtschaftlichen Gesamtrechnung.

StatLink त्ताज़ http://dx.doi.org/10.1787/888932942089

\subsection{Ausgaben für das Outsourcing staatlicher Aufgaben in Prozent des BIP (2011)}

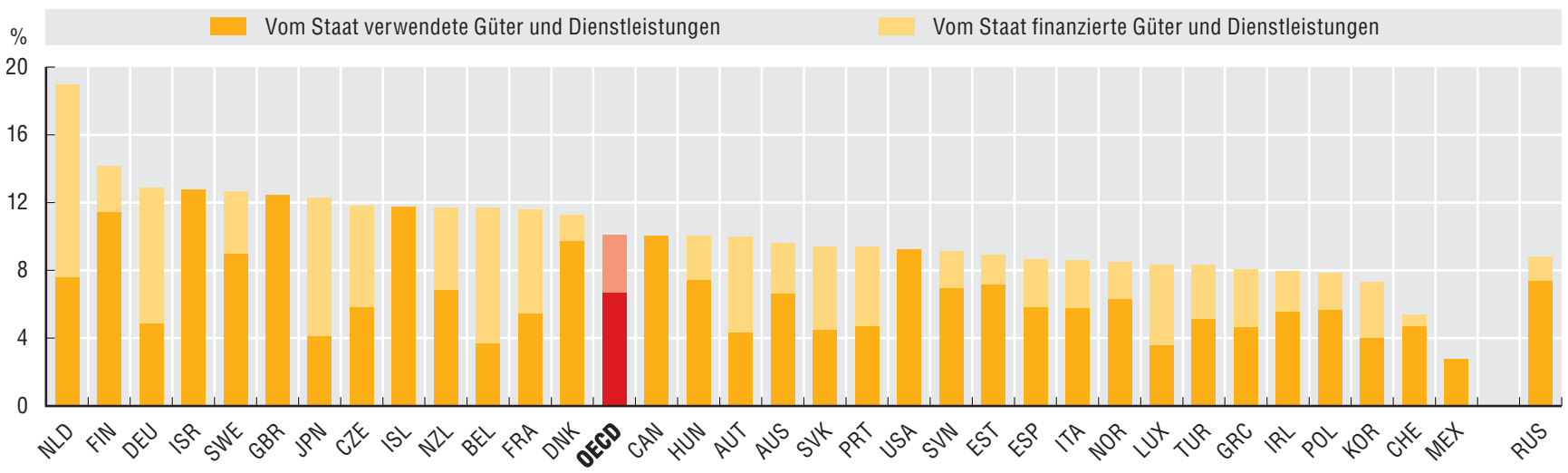

Quelle: OECD National Accounts Statistics (Datenbank). Die Daten für Australien gründen sich auf die Government Finance Statistics sowie vom Australian Bureau of Statistics zur Verfügung gestellte Daten aus der australischen Volkswirtschaftlichen Gesamtrechnung.

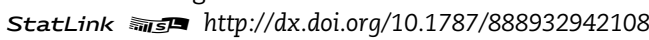


Bei den gesamtstaatlichen IKT-Ausgaben handelt es sich um den Anteil der gesamtstaatlichen Mittel, der für Informationsund Kommunikationstechnologien verwendet wird (z.B. Hardware- und Softwareinvestitionen, Betriebskosten von IKTInfrastrukturen, Personalkosten von IKT-Spezialisten und Schulungen). Für staatliche Stellen ist die Nutzung moderner Technologien und insbesondere des Internets ein Instrument, um die internen Abläufe effizienter zu gestalten, die Qualität des öffentlichen Dienstleistungsangebots zu erhöhen und den Prozess der politischen Entscheidungsfindung zu verbessern und stärker zu öffnen.

Weil für IKT beträchtliche Summen aufgewendet werden, sind auch die Erwartungen hoch, dass damit politikrelevante Ergebnisse erzielt werden. Beispiele des absoluten Umfangs dieser Ausgaben machen deutlich, warum es wichtig ist, die $z u$ Grunde liegenden Muster zu verstehen und den IKT-Einsatz zu optimieren: 75 Mrd. US-\$ auf Ebene der Zentralregierung in den Vereinigten Staaten, 10 Mrd. US-\$ im Vereinigten Königreich, 4-5 Mrd. US-\$ in Kanada, Frankreich und Australien. In manchen Fällen können die IKT-Ausgaben mehr als 2\% des Etats der Zentralregierung ausmachen. Dennoch wäre es nicht richtig, einen hohen bzw. niedrigen Anteil an IKT-Ausgaben als einen Anhaltspunkt für Prioritätensetzung, Leistung oder Effizienz zu betrachten. Wichtige Kontextfaktoren müssen ebenfalls berücksichtigt werden, auch wenn deren Rolle noch nicht ganz klar ist. Bei den Ländern, in denen mehr als 1,5\% des Etats der Zentralregierung für IKT aufgewendet werden, handelt es sich ebenso um stark zentralisierte Länder (Neuseeland, Frankreich) wie um Föderalstaaten (Vereinigte Staaten, Kanada, Schweiz) und ebenso um Länder mit geringer wie mit hoher Einwohnerzahl.

Auch der absolute Umfang der öffentlichen Verwaltung kann Einfluss auf die Ausgabenmuster haben. In Slowenien und Estland ist die Zahl der Beschäftigten des Staatssektors in etwa gleich hoch, und die Kosten für IKT-Kapital und -Betrieb je Beschäftigten sind ebenfalls vergleichbar; in Italien, Spanien und Deutschland ist die öffentliche Verwaltung größer, und die IKT-Ausgaben je Beschäftigten sind ebenfalls höher (rd. 3 0004000 US-\$ KKP). Am höchsten sind die durchschnittlichen IKTAusgaben je Beschäftigten in den Vereinigten Staaten (mehr als 26000 US-\$ KKP), gefolgt von der Schweiz, dem Vereinigten Königreich, Korea und Australien.

Allerdings erklärt der Umfang der öffentlichen Verwaltung nicht alles. Trotz einer ähnlich umfangreichen Verwaltung auf Ebene der Zentralregierung sind die IKT-Ausgaben je Beschäftigten auf dieser Ebene in Australien und Korea ungefähr dreimal so hoch wie in Chile oder Finnland. Frankreich und die Vereinigten Staaten fallen innerhalb der Länderstichprobe beide durch ein vergleichsweise hohes Beschäftigungsvolumen auf Ebene der Zentralregierung auf, in den Vereinigten Staaten sind die IKT-Ausgaben je Beschäftigten jedoch deutlich höher. Es wäre zu erwarten, dass die durchschnittlichen Ausgaben je Beschäftigten in größeren Verwaltungen auf Grund von Skaleneffekten niedriger sind; allerdings kann die Politikumsetzung in größeren Verwaltungen komplexer sein, wodurch sich die Skalenvorteile wieder verringern.

In einigen Ländern wurden spezielle IKT-Ausgabenberichte erstellt, um die Ausgabenmuster besser zu verstehen, inländische Kontextfaktoren zu untersuchen und die Erträge aus der staatlichen IKT-Nutzung zu steigern.

Genauere Informationen über die IKT-Ausgaben, z.B. Vergleiche aufgeschlüsselter Daten, könnten die Entscheidungsfindung in Bezug auf die IKT-Ausgaben verbessern. Analysen solcher Daten könnten zeigen, wie sich einzelne IKT-Ausga- benposten im Zeitverlauf entwickeln - z.B. die Personalkosten im Staatssektor im Vergleich zu den Outsourcingkosten -, welche Wechselbeziehungen zwischen den IKT-Ausgaben der Zentralregierung und denen der lokalen Gebietskörperschaften, auf deren Ebene der Großteil der öffentlichen Dienstleistungen erbracht wird, bestehen und wie der Technologieeinsatz die Verwirklichung von Politikzielen in Bereichen wie Gesundheitsversorgung, Bildung oder Justiz unterstützt.

\section{Methodik und Definitionen}

IKT-Ausgabendaten (Kapital-, Betriebs- und Personalaufwendungen) liegen für 21 Länder vor. Die Daten stammen aus einer Umfrage zu den staatlichen IKT-Ausgaben, die die OECD 2010 und 2011 bei auf Ebene der Zentralregierungen tätigen Staatsbediensteten durchgeführt hat, die am OECD-Netzwerk E-Government teilnehmen. Weitere Daten wurden allgemein zugänglichen amtlichen Datenquellen entnommen. Dies ist das erste Mal, dass eine Datenerhebung und -harmonisierung für eine derart große Zahl von Ländern vorgenommen wurde. Die hier präsentierten Daten haben daher vorläufigen Charakter und müssen weiter harmonisiert werden.

\section{Literaturhinweise}

OECD (2010), OECD E-government Studies: Indicators Project, verfügbar unter: www.oecd.org/governance/public-innovation.

\section{Anmerkungen zu den Abbildungen}

Die Daten für Australien, Deutschland, Italien, Korea, Spanien und die Schweiz beziehen sich auf 2010 anstelle von 2011. Die Daten für Belgien und Slowenien beziehen sich auf 2009 anstelle von 2011. Die Daten für Frankreich, Neuseeland und das Vereinigte Königreich beziehen sich auf 2008 anstelle von 2011.

3.50: Die IKT-Gesamtausgaben umfassen im Allgemeinen Kapital-, Betriebs- und Personalaufwendungen. In folgenden Ländern sind jedoch keine Daten zu den Personalaufwendungen verfügbar: Österreich, Belgien, Estland, Deutschland, Island, Slowenien, Schweiz und Vereinigtes Königreich. Die Daten für Österreich und Portugal beziehen sich auf 2010 anstelle von 2011. Die Daten für Island beziehen sich auf 2008 anstelle von 2011. Chile legte detaillierte Daten zu den IKT-Ausgaben vor, konnte aber nicht in die Abbildung aufgenommen werden, weil Daten zu den Ausgaben auf Ebene der Zentralregierung fehlen.

3.51: Die Ausgabendaten in dieser Abbildung beziehen sich nur auf Aufwendungen für IKT-Kapital und -Betrieb, weil dies die Bereiche sind, in denen am ehesten Skaleneffekte zu erwarten sind (die Daten für die Niederlande, Neuseeland und die Vereinigten Staaten umfassen Personalaufwendungen). Für Österreich, Island und Portugal stehen keine Daten zur Verfügung. Die Daten zur Beschäftigung auf Ebene der Zentralregierungen beziehen sich im Allgemeinen auf die Zahl der Beschäftigten. Für Frankreich, Korea, Neuseeland, die Schweiz und das Vereinigte Königreich ist die Beschäftigung allerdings in Vollzeitäquivalenten ausgedrückt. Für diese fünf Länder unterzeichnet der Vergleich daher die Beschäftigtenzahlen, während die Gesamtausgaben für IKT-Kapital und -Betrieb je Beschäftigten überzeichnet sind. 


\section{3. ÖFFENTLICHE FINANZEN UND WIRTSCHAFTLICHES HANDELN DES STAATES}

3.50. IKT-Ausgaben insgesamt in Prozent der Ausgaben der Zentralregierungen (2011 oder letztes verfügbares Jahr)

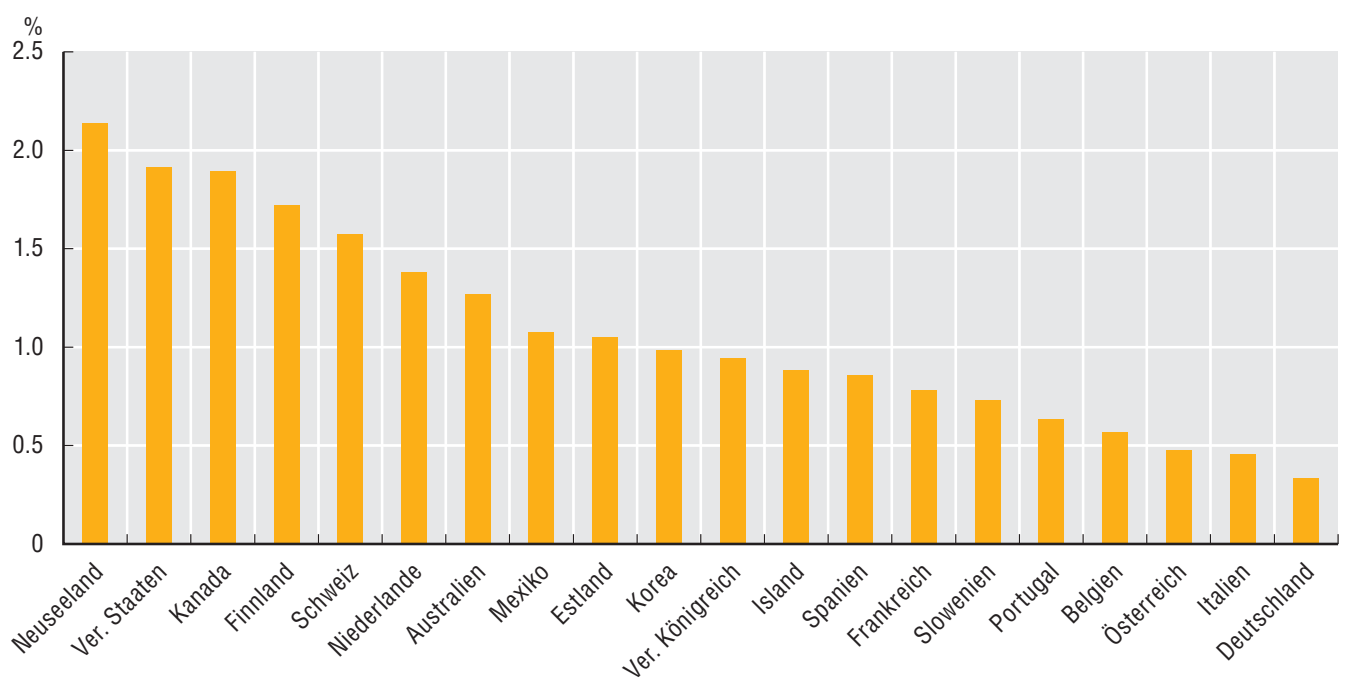

Quelle: OECD Survey of ICT Expenditures, 2010-11; OECD National Accounts Statistics (Datenbank).

StatLink त्ञाजम http://dx.doi.org/10.1787/888932942146

\subsection{Ausgaben für IKT-Kapital und -Betrieb je Beschäftigten im Vergleich zur Gesamtbeschäftigung auf Ebene der Zentralregierungen (2011 oder letztes verfügbares Jahr)}

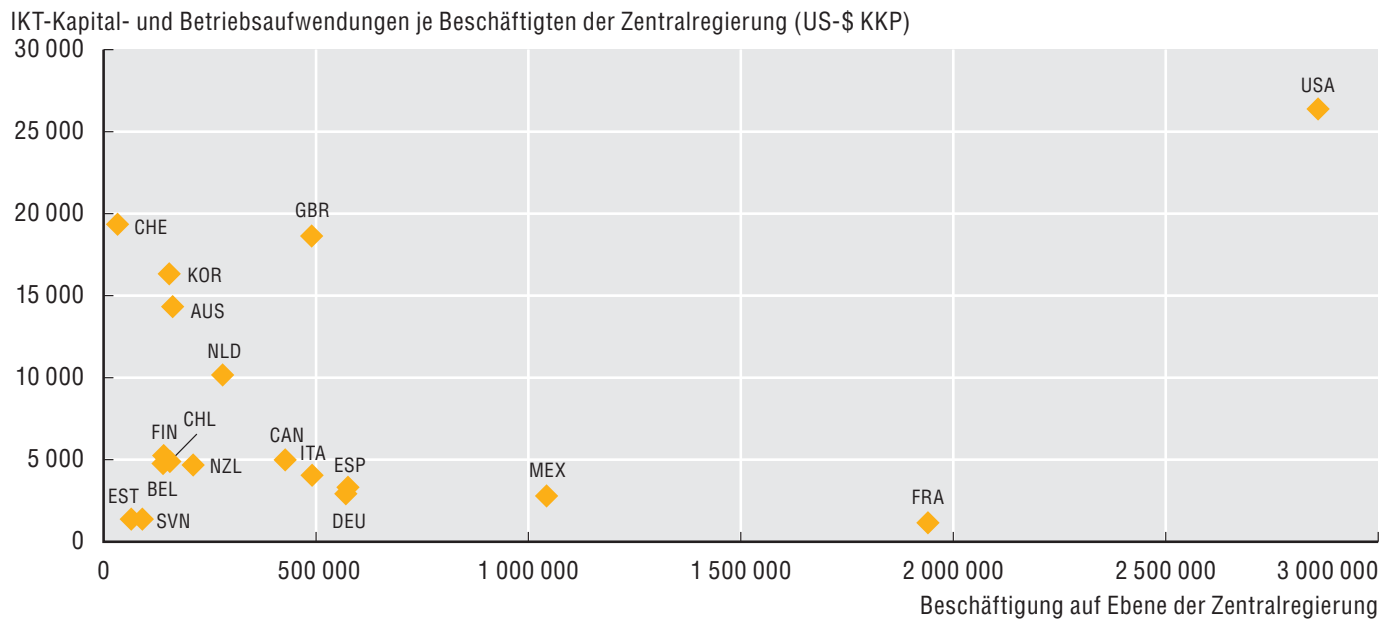

Quelle: OECD Survey of ICT Expenditures, 2010-11; Internationale Arbeitsorganisation (ILO), LABORSTA (Datenbank). Die Beschäftigungsdaten für Chile, Frankreich, Korea, die Schweiz und das Vereinigte Königreich stammen aus dem OECD Public Employment Survey 2010.

StatLink त्ताइ http://dx.doi.org/10.1787/888932942165 


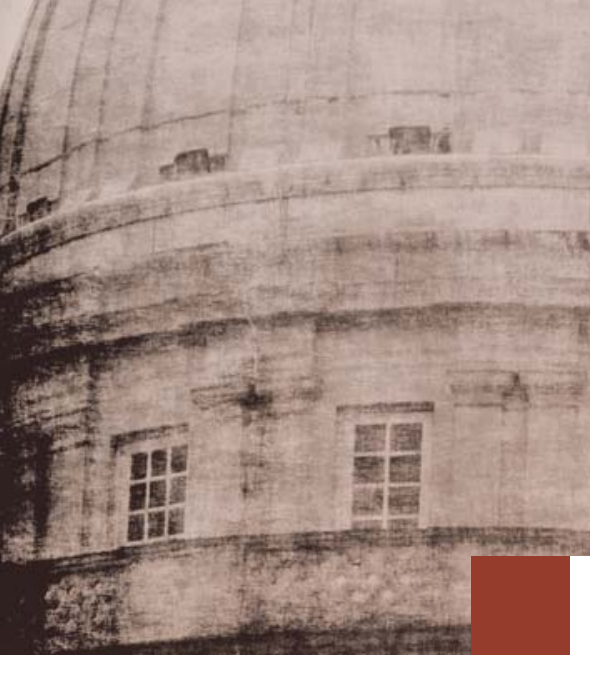

4. HAUSHALTSPRAXIS UND -VERFAHREN

Positive Haushaltsergebnisse ergeben sich vor allem aus dem Zusammenspiel von drei Faktoren: der allgemeinen Wirtschaftsleistung, dem politischen Engagement zur Haushaltsdisziplin und den institutionellen Vorkehrungen für den Haushaltsplanungsprozess. In diesem Kapitel liegt der Schwerpunkt auf dem letzten Merkmal. Gut funktionierende Haushaltsinstitutionen sind eine unabdingbare Voraussetzung für die Schaffung einer solideren Haushaltsposition, Erreichung eines stabilen Steueraufkommens sowie Wahrung der Generationengerechtigkeit. Außerdem muss das Staatsausgabensystem eines Landes die Fiskaldisziplin, die bestmögliche Ressourcenallokation und das effiziente Funktionieren des Staats fördern.

Die in diesem Kapitel aufgeführten Indikatoren befassen sich mit den wesentlichen institutionellen Haushaltsmerkmalen. Hierzu zählen die Existenz von Fiskalregeln als Instrument zur Erreichung tragfähiger öffentlicher Finanzen durch die Einführung von Angabenbeschränkungen, das Ausmaß, in dem in den Haushalten eine mittelfristige Perspektive einbezogen ist, die sicherstellt, dass die Konsequenzen von ausgabenpolitischen Maßnahmen über mehrere Jahre berücksichtigt werden, die Autonomie, die den staatlichen Stellen bei Budgetentscheidungen eingeräumt wird, die Frage, ob bei der Aufstellung des Haushalts leistungs- und ergebnisbezogene Informationen berücksichtigt werden, die Beurteilung öffentlich-privater Partnerschaften (im Vergleich zu herkömmlichen Infrastrukturprojekten) und schließlich die Frage, ob Länder eine unabhängige fiskalische Institution als Unterstützungsmechanismus eingerichtet haben, um einen umsichtigen Umgang mit den Staatsfinanzen sicherzustellen. Wenngleich alle diese Elemente als gesonderte Merkmale einer soliden Haushaltsgestaltung präsentiert werden, bauen sie dennoch aufeinander auf und sollten daher als Paket verstanden werden.

Die in diesem Kapitel vorgestellten Ergebnisse entstammen den Antworten der Länder auf OECD-Erhebungen und stellen deren eigene Einschätzung dar. Die Mischindizes basieren auf Berechnungen von OECD-Mitarbeitern und dienen der Zusammenfassung diskreter, qualitativer Informationen zur Haushaltspraxis in aggregierten Indikatoren, die einfacher zu interpretieren sind als mehrere getrennte Variablen; Mischindizes werden als Diskussionsbeitrag berechnet und können sich dementsprechend im Zeitverlauf verändern. 
Fiskalregeln schränken die finanzpolitische Handlungsfähigkeit durch quantitative Begrenzungen der Haushaltsaggregate langfristig ein. Ohne eine globale Budgetdeckelung kann eine schrittweise Haushaltsplanung zu einem nach oben nicht begrenzten Verfahren werden, in dessen Rahmen die Regierungen den Forderungen gerecht werden, indem sie mehr ausgeben, als ihnen zur Verfügung steht. Fiskalregeln zeichnen sich durch zwei grundlegende Merkmale aus. Erstens stellen sie eine Einschränkung dar, der die politischen Entscheidungen der Legislative und Exekutive unterliegen. Zweitens dienen sie als konkreter Indikator für die Finanzverwaltung der Exekutive. Auch wenn Haushaltsgrundsätze den Regierungen helfen können, fiskalische Ziele zu erreichen und Haushaltsdisziplin herzustellen, gibt es keinen einheitlichen Grundsatz, der für alle Länder passt.

Fiskalregeln können bei unterschiedlichen Aspekten der staatlichen Haushaltsergebnisse ansetzen: Einnahmen, Ausgaben, Haushaltssaldo und Staatsschulden. Bezogen auf die OECDMitgliedsländer sind die gebräuchlichsten Formen von Fiskalregeln die Regeln für einen ausgeglichenen Haushalt (28 Mitgliedsländer) und die Verschuldung (23 Mitgliedsländer), die für die Länder der Europäischen Union gelten. Einnahmeregeln sind die seltensten, und nur in fünf OECD-Mitgliedsländern in Kraft: Australien, Frankreich, Griechenland, die Niederlande und die Slowakische Republik.

Fiskalregeln können in den einzelnen Ländern unterschiedliche gesetzliche Grundlagen haben und in der Verfassung oder in der Primär- oder Sekundärgesetzgebung verankert sein. Es gibt auch Länder, die ihre Haushaltsregeln in öffentlichen Politikzusagen oder vom Finanzministerium aufgestellten internen Regeln festschreiben. Australien ist insofern ein interessantes Beispiel, als in diesem Land alle vier Formen von Haushaltsregeln in Kraft sind. Die Rechtsgrundlage für drei dieser Regeln ist der Budget Honesty Act, der ein starkes politisches Engagement zum Ausdruck bringt. Was die Schuldenregel anbelangt, so beruht diese auf einem Gesetz. In Japan und Korea existieren nur Ausgabenregeln, in beiden Fällen als behördeninterne Vorschriften und Politikmaßnahmen.

Schließlich müssen sich einige Länder auch an Haushaltsregeln halten, die im internationalen Recht verankert sind. Für Länder der Europäischen Union beispielsweise stellt der MaastrichtVertrag eine Schuldenregel und zwei Regeln zur Erreichung eines ausgeglichenen Haushalts auf. Infolge des neuen Fiskalpakts und der sog. „Six-Pack“-Maßnahmen zur Haushaltskonsolidierung haben die EU-Behörden gefordert, als Mittel zur Erhöhung der Politikkosten der Verfehlung der Haushaltsregeln diesen Verfassungsrang zu verleihen. Zehn Länder (Österreich, Belgien, die Tschechische Republik, Deutschland, Ungarn, Italien, Polen, die Slowakische Republik, Spanien und die Schweiz) haben Haushaltsregeln in ihren Verfassungen verankert.

Eine der wichtigsten Erkenntnisse, die sich aus Erfahrungen der Vergangenheit ziehen lassen, besteht darin, dass übermäßig strenge Regeln in der Praxis im Allgemeinen nicht funktionieren und sich u.U. nicht eignen, um angemessen auf Veränderungen der wirtschaftlichen und politischen Rahmenbedingungen reagieren zu können. Demgegenüber dürften sich Systeme mit strengen Haushaltsregeln eher auf die Stärke politischer Verpflichtungen, das Monitoring durch unabhängige fiskalische Institutionen und andere Akteure sowie klare und effiziente Durchsetzungsverfahren bei Nichteinhaltung der Vorschriften stützen. Im Hinblick auf die Durchsetzungsverfahren lassen sich verschiedene Arten von Maßnahmen umsetzen, von der Notwendigkeit, dem Gesetzgeber Korrekturvorschläge zu unterbreiten bis hin zum Einsatz automatischer Korrekturmechanismen und Sanktionen. EU-Länder unterliegen dem Verfahren bei einem übermäßigen Defizit (EDP), einem aus mehreren Schritten bestehenden Prozess zur Prüfung der Haushaltslage eines Landes, der Sanktionen nach sich ziehen kann. Mit den jüngsten Veränderungen in der wirtschaftspolitischen Steuerung wurden die Voraussetzungen für die Einleitung eines Verfahrens bei einem übermäßigen Defizit gelockert. Abgesehen von den Verfahren bei einem übermäßigen Defizit sind automatische Korrekturmechanismen das in den OECD-Mitgliedsländern am häufigsten eingesetzte Durchsetzungsinstrument für die Regeln, die den Haushaltssaldo betreffen, während bei den Verschuldungsregeln der geläufigste Korrekturmechanismus darin besteht, die verschuldete Einrichtung zur Ergreifung von Abhilfemaßnahmen zu verpflichten. Auf automatische Sanktionen bei einem Verstoß gegen Haushaltsregeln wird nur in fünf OECD-Ländern zurückgegriffen (Niederlande, Vereinigte Staaten, Schweiz, Polen und die Slowakische Republik).

\section{Methodik und Definitionen}

Die Daten beziehen sich auf das Jahr 2012 und entstammen den Antworten der Länder auf den OECD Survey of Budgeting Practices and Procedures von 2012. Die Befragten waren vornehmlich hochrangige Haushaltsverantwortliche in den OECD-Ländern. Die Antworten stellen die Selbsteinschätzung der Länder zu den derzeitigen Praktiken und Verfahren dar. Die Daten beziehen sich nur auf die Zentral-/Bundesregierungen, die Haushaltspraxis auf der Ebene der Gliedstaaten/Kommunen ist ausgeklammert.

Bei der Primärgesetzgebung (die auch als grundlegende Rechtsakte oder Primärrecht bezeichnet wird) handelt es sich um Rechtsvorschriften, die von der Legislative genehmigt werden müssen, und bei der Sekundärgesetzgebung um Rechtsvorschriften, die vom Regierungschef, einem einzelnen Minister oder dem Kabinett genehmigt werden können - d.h. einer anderen Kontrollinstanz als der Legislative. Sekundäre Rechtsvorschriften können von der Legislative abgelehnt werden.

\section{Literaturhinweise}

Anderson, B. und J. Sheppard (2010), „Fiscal futures, institutional budget reforms, and their effects: What can be learned?" OECD Journal on Budgeting, Vol. 9/3. http://dx.doi.org/10.1787/ budget-9-5kmh6dnl056g.

Schick, A. (2010), „Post-Crisis Fiscal Rules: Stabilising Public Finance while Responding to Economic Aftershocks" OECD Journal on Budgeting, Vol. 10/2. http://dx.doi.org/10.1787/budget-10-5km7rqpkqts1.

\section{Anmerkungen zu den Tabellen}

4.1 und 4.2: Für Island stehen keine Daten zur Verfügung. Die Daten geben Auskunft über die Vielfalt der Haushaltsregeln. In Neuseeland und der Türkei sind keine Fiskalregeln in Kraft, und entsprechend enthält die Tabelle für diese beiden Länder auch keine Einträge.

4.1: In Italien wurden mit dem Ausführungsgesetz Nr. 243/2012 die Budgetregel bezüglich des strukturellen Defizits sowie die Ausgabenund Schuldenregel gemäß den EU-Vorgaben umgesetzt. Die beiden ersten Regeln treten 2014 in Kraft, die Schuldenregel 2015.

Hinweis zu den Daten für Israel: $h t t p: / / d x . d o i . o r g / 10.1787 / 888932315602$. 


\subsection{Arten und Rechtsgrundlage für Fiskalregeln (2012)}

\begin{tabular}{|c|c|c|c|c|}
\hline & Ausgabenregel(n) & $\begin{array}{c}\text { Regeln bezüglich des Haushaltssaldos } \\
\text { (Defizit/Überschuss) }\end{array}$ & Schuldenregel(n) & Einnahmeregel(n) \\
\hline Australien & O & O & $\square$ & O \\
\hline Österreich & $\square$ & $\square$ & $\mathbf{\square}$ & \\
\hline Belgien & & $\bullet \diamond \square$ & $\mathbf{\square}$ & \\
\hline Kanada & & $\diamond$ & & \\
\hline Chile & $\square$ & $\square$ & & \\
\hline Tschech. Rep. & $\square$ & $\bullet$ & $\mathbf{\square}$ & \\
\hline Dänemark & $\square$ & $\square \mathbf{a}$ & $\mathbf{\square}$ & \\
\hline Estland & $\diamond$ & $\mathbf{\square}$ & $\square$ & \\
\hline Finnland & $\diamond$ & $\square$ & $\mathbf{\square}$ & \\
\hline Frankreich & $\square$ & $\mathbf{\square}$ & $\mathbf{\square}$ & $\square$ \\
\hline Deutschland & & 미 & $\boldsymbol{\square}$ & \\
\hline Griechenland & $\mathbf{\square}$ & $\neg \boldsymbol{\square}$ & $\mathbf{\square}$ & $\mathbf{\square}$ \\
\hline Ungarn & & $\square \square$ & $\mathbf{\square}$ & \\
\hline Irland & $\diamond$ & $\square \mathbf{\square}$ & $\square \mathbf{\square}$ & \\
\hline Israel & $\square$ & $\square$ & & \\
\hline Italien & & 口 & $\mathbf{\square}$ & \\
\hline Japan & $\diamond$ & & & \\
\hline Korea & $\diamond$ & & & \\
\hline Luxemburg & $\diamond$ & 口 & $\square$ & \\
\hline Mexiko & & $\square$ & & \\
\hline Niederlande & $\diamond$ & $\square \mathbf{a}$ & $\neg \mathbf{\square}$ & $\diamond$ \\
\hline Norwegen & & $\diamond$ & & \\
\hline Polen & $\square$ & $\mathbf{\square}$ & ロ๑ロ & \\
\hline Portugal & $\square$ & $\mathbf{\square}$ & $\mathbf{\square}$ & \\
\hline Slowak. Rep. & & $\mathbf{\square}$ & $\bullet$ & $\square$ \\
\hline Slowenien & $\square$ & $\mathbf{\square}$ & $\boldsymbol{\square}$ & \\
\hline Spanien & $\square$ & $\bullet$ & $\bullet$ & \\
\hline Schweden & $\square$ & $\square \square$ & $\boldsymbol{\square}$ & \\
\hline Schweiz & & $\bullet$ & & \\
\hline Ver. Königreich & & $\square \mathbf{a}$ & $\square \mathbf{\square}$ & \\
\hline Ver. Staaten & $\square$ & & $\square$ & \\
\hline Russ. Föderation & $\square$ & & & $\square$ \\
\hline OECD insgesamt & 21 & 28 & 23 & 5 \\
\hline
\end{tabular}

- Verfassung

Internationaler Vertrag

$\square$ Primär- und/oder Sekundärgesetzgebung

↔ Interne Regeln oder Maßnahmen

P Politische Verpflichtung

Quelle: 2012 OECD Survey on Budgeting Practices and Procedures.

StatLink :

\subsection{Durchsetzungsmechanismen für Fiskalregeln (2012)}

\begin{tabular}{|c|c|c|c|c|c|c|}
\hline $\begin{array}{l}\text { Art der Regel/ } \\
\text { Korrekturmechanismen }\end{array}$ & $\begin{array}{l}\text { Automatische } \\
\text { Korrekturmechanismen }\end{array}$ & $\begin{array}{l}\text { Dem Gesetzgeber } \\
\text { vorgelegter Vorschlag mit } \\
\text { Korrekturmaßnahmen }\end{array}$ & $\begin{array}{l}\text { Einrichtung muss } \\
\text { Maßnahmen } \\
\text { umsetzen }\end{array}$ & $\begin{array}{l}\text { Automatische } \\
\text { Sanktionen }\end{array}$ & $\begin{array}{c}\text { Verfahren bei einem } \\
\text { übermäßigen Defizit des } \\
\text { Stabilitäts- und Wachstumspakts }\end{array}$ & Keine \\
\hline Ausgaben & DNK, GRC, USA, ESP & $\begin{array}{l}\text { EST, FRA, ISR, } \\
\text { NLD, SWE, ESP }\end{array}$ & $\begin{array}{l}\text { AUT, GRC, NLD, } \\
\text { SWE, CHL, ESP }\end{array}$ & USA & & $\begin{array}{l}\text { AUS, CZE, FIN, } \\
\text { FRA, IRL, JPN, } \\
\text { KOR, LUX, POL, } \\
\text { PRT, RUS, SVN }\end{array}$ \\
\hline Haushaltssaldo & $\begin{array}{l}\text { AUT, BEL, DNK, FIN, FRA, } \\
\text { DEU, GRC, IRL, ITA, LUX, } \\
\text { PRT, SVK, SVN, ESP, CHE }\end{array}$ & ISR, ITA, MEX, ESP & GRC, ESP, CHL & NLD, CHE & $\begin{array}{c}\text { AUT, BEL, CZE, DNK, EST, FIN, } \\
\text { FRA, DEU, GRC, HUN, IRL, ITA, } \\
\text { LUX, NLD, POL, PRT, SVK, SVN, } \\
\text { ESP, SWE, GBR }\end{array}$ & AUS, CAN, NOR \\
\hline Schulden & POL, SVK, ESP & POL, SVK, ESP & $\begin{array}{l}\text { GRC, HUN, POL, } \\
\text { SVK, ESP }\end{array}$ & NLD, POL, SVK & $\begin{array}{c}\text { AUT, BEL, CZE, DNK, EST, FIN, } \\
\text { FRA, DEU, GRC, HUN, IRL, ITA, } \\
\text { LUX, NLD, POL, PRT, SVK, SVN, } \\
\text { ESP, SWE, GBR }\end{array}$ & AUS, USA \\
\hline Einnahmen & GRC & NLD & & & & AUS, FRA, RUS, SVK \\
\hline
\end{tabular}

Quelle: 2012 OECD Survey on Budgeting Practices and Procedures. 
Mittelfristige Ausgabenrahmen (MTEF) ermöglichen es den staatlichen Stellen auf der Ebene der Zentral-/Bundesregierung, statt lediglich einer Jahresvorausschau für den Haushalt auch eine mittelfristige Haushaltsvorausschau einzuführen. Mittelfristige Ausgabenrahmen erstrecken sich in der Regel auf einen Drei- bis Fünfjahreszeitraum, einschließlich des bereits veranschlagten Finanzjahres, und bestehen aus einer Kombination aus verbindlichen jährlichen Ausgabenobergrenzen und deskriptiven Vorausschätzungen.

Auf internationaler Ebene herrscht Einigkeit über die Bedeutung einer mittelfristigen Vorausschau im Haushaltsprozess. Zunächst einmal sind mittelfristige Ausgabenrahmen für die zentralen Haushaltsbehörden ein wichtiges Instrument, da sie ihnen die Ausgabensteuerung auf der Ebene der Zentralregierung und die Gewährleistung der Haushaltsdisziplin erleichtern: Ausgabenschätzungen enthalten Informationen über die Kosten bestehender Maßnahmen und Programme und bilden die Grundlagen der Haushalte für die folgenden Jahre, während Ausgabenobergrenzen eine Top-down-Beschränkung der Ausgaben in den kommenden Jahren bedeuten. Mittelfristige Ausgabenrahmen tragen ferner zur Ausgabenkontrolle bei, indem sie die Einbeziehung von Maßnahmen ermöglichen, die sich über mehrere Jahre erstrecken und deren Umsetzung u.U. einen längeren Zeithorizont in Anspruch nimmt, wie dies beispielsweise bei großen Investitionsvorhaben, neuen Programmen oder organisatorischen Umstrukturierungen der Fall ist. Aus der Sicht der Leitung von Fachministerien und Behörden zeigt die mittelfristige Haushaltsvorausschau die Politikrichtung und Finanzierungsveränderungen an und gibt ihnen dadurch Zeit, sich anzupassen und ihre Prozesse besser zu planen.

Die Auswirkungen einer mittelfristigen Haushaltsvorausschau hängen indessen letztlich von der Glaubwürdigkeit der Ausgabenschätzungen und -obergrenzen sowie davon ab, wie diese Informationen von den Entscheidungsträgern und Teilen aus der Zivilgesellschaft verwendet werden. Wenn mittelfristige Haushaltsziele nicht erreicht werden, liegt dies oft an schwachen Regelungen hinsichtlich der Vorbereitung, der gesetzlichen Verankerung und der Umsetzung der Haushaltsziele.

Mittelfristige Ausgabenobergrenzen sind in allen außer vier OECD-Ländern (Belgien, Israel, Ungarn und Luxemburg) festgelegt worden und umspannen in den meisten Fällen einen Zeitraum von vier Jahren. Die Durchschlagskraft dieser Rahmenkonzepte weicht in den OECD-Mitgliedsländern sehr stark ab, je nachdem inwieweit sie in der Gesetzgebung verankert sind, von der Exekutive oder Legislative beschlossen werden und ihre Einhaltung in der Folgezeit von Gesetzgebungsorganen oder unabhängigen Einrichtungen überwacht wird. Meistens beziehen sich Ausgabenobergrenzen auf die Gesamtausgaben. In einigen Ländern (Österreich, Deutschland, Italien, Korea, die Niederlande und Neuseeland) existieren je Programm, Sektor und/oder Organisation zusätzliche Obergrenzen. Damit mittelfristige Ausgabenprogramme wirkungsvoll sind, sollten Beobachtungs- und Durchsetzungsmechanismen in Kraft sein, über die die Exekutive der Legislative oder einer unabhängigen fiskalischen Institution Bericht über die Befolgung erstattet.

\section{Methodik und Definitionen}

Die Daten beziehen sich auf das Jahr 2012 und entstammen den Antworten der Länder auf den OECD Survey of Budgeting Practices and Procedures von 2012. Die Befragten waren vornehmlich hochrangige Haushaltsverantwortliche in den OECD-Ländern. Die Antworten stellen die Selbsteinschätzung der Länder zu den derzeitigen Praktiken und Verfahren dar. Die Daten beziehen sich nur auf die Zentral-/Bundesregierungen, die Haushaltspraxis auf der Ebene der Gliedstaaten/Kommunen ist ausgeklammert.

Als Rahmen für die Integration von Fiskalpolitik und Haushaltsplanung auf mittlere Sicht wurde ein mittelfristiges Ausgabenprogramm definiert, indem gesamtwirtschaftliche fiskalische Prognosen mit einem disziplinierten Verfahren der Aufrechterhaltung detaillierter mittelfristiger Budgetschätzungen auf der Ebene der Ministerien verknüpft wurden, die die bestehenden Politikmaßnahmen widerspiegeln. Vorausschätzungen der Ausgaben werden zur Grundlage der Budgetverhandlungen in den Jahren nach der Budgetaufstellung, und die Prognosen werden mit den endgültigen Ergebnissen in den Berichten über die Haushaltsergebnisse in Einklang gebracht.

Der Mischindex in Abbildung 4.4 enthält 10 Variablen, die Informationen zur Existenz einer mittelfristigen Haushaltsvorausschau im Haushaltsprozess, zur Anzahl der in der Schätzung berücksichtigten Jahre, zur Möglichkeit der Übertragung ungenutzter Mittel von einem in das nächste Jahr sowie zu den Monitoring-Modalitäten liefern. $\mathrm{Zu}$ beachten ist, dass der Index nicht die Gesamtqualität des mittelfristigen Ausgabenrahmens messen, sondern vielmehr seinen deskriptiven Charakter beurteilen soll. Anhang C enthält eine Beschreibung der Methodik, die zur Erstellung dieses Indikators verwendet wurde, einschließlich der spezifischen Gewichtungen der einzelnen Variablen.

\section{Literaturhinweise}

OECD (2013, erscheint demnächst), Budgeting Practices and Procedures in OECD Countries, OECD Publishing, Paris.

OECD (2002) „OECD Best Practices for Budget Transparency“, OECD Journal on Budgeting, Vol. 1/3, S. 7-14, OECD Publishing, Paris, http://dx.doi.org/10.1787/budget-v1-art14-en.

Weltbank (2013), Beyond the Annual Budget - Global Experience with Medium-Term Expenditure Frameworks, Internationale Bank für Wiederaufbau und Entwicklung, Weltbank, Washington, DC, http://dx.doi.org/10.1596/978-0-8213-9625-4.

\section{Anmerkungen zu den Abbildungen und Tabellen}

Für Island stehen keine Daten zur Verfügung.

4.3: Im Falle Deutschlands ist der Finanzplan die Rechtsgrundlage des mittelfristigen Ausgabenplans, der parallel zum Beschluss des Haushalts im Bundestag diskutiert wird und die Budgetobergrenzen enthält. Im Falle der Niederlande sind die Verwaltungsausgaben in den Gesamtund sektorspezifischen Ausgaben für die Niederlande inbegriffen.

4.4: Die Indexwerte für Belgien, Ungarn, Israel und Luxemburg sind gleich null, da diese Länder angegeben haben, keinen mittelfristigen Ausgabenplan erstellt zu haben.

Hinweis zu den Daten für Israel: http://dx.doi.org/10.1787/888932315602. 
4.3. Mittelfristige Vorausschau im Haushaltsprozess auf Ebene der Zentralregierung (2012)

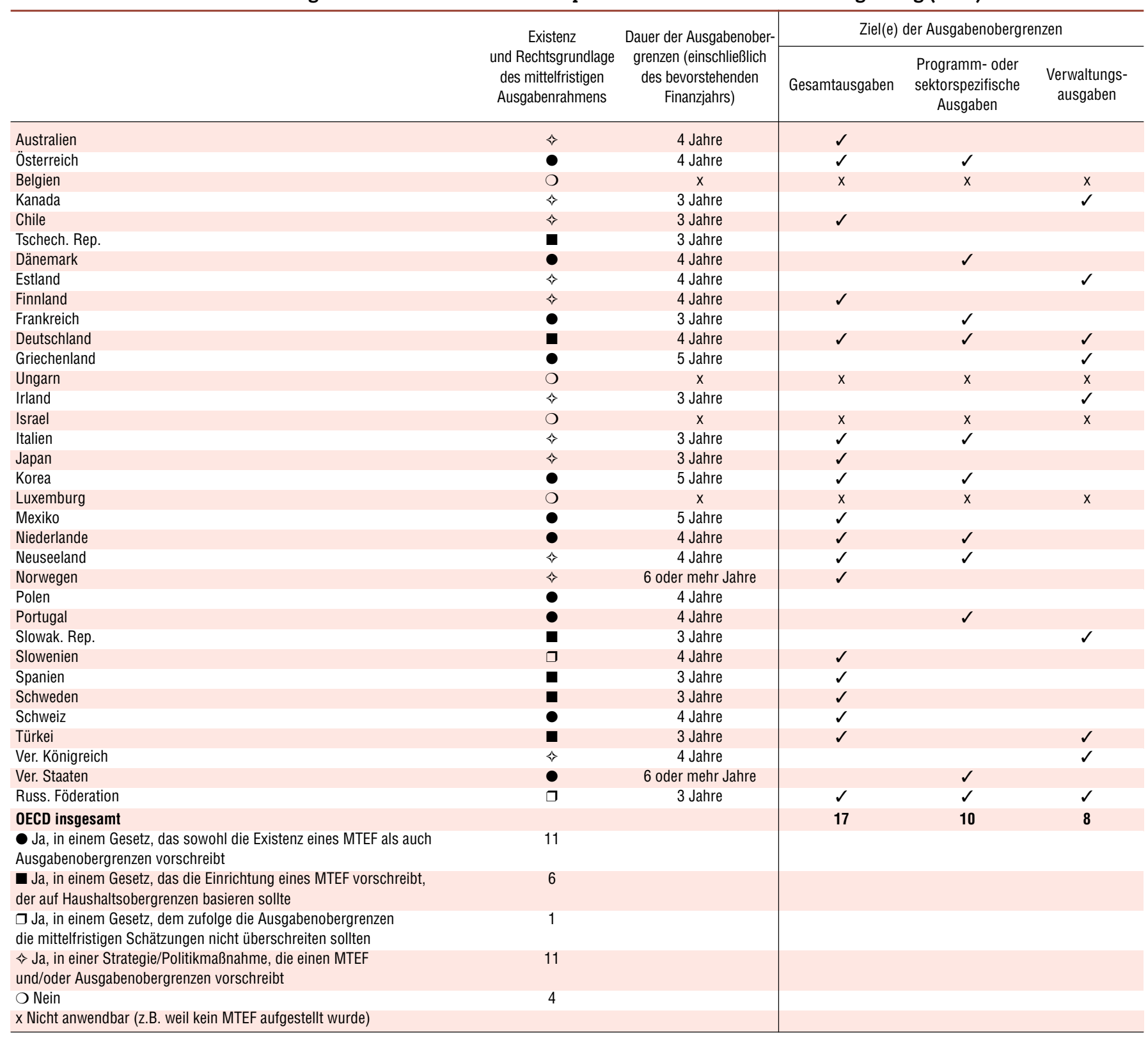

Quelle: 2012 OECD Survey on Budgeting Practices and Procedures.

\subsection{Verwendung einer mittelfristigen Vorausschau im Haushaltsprozess (2012)}

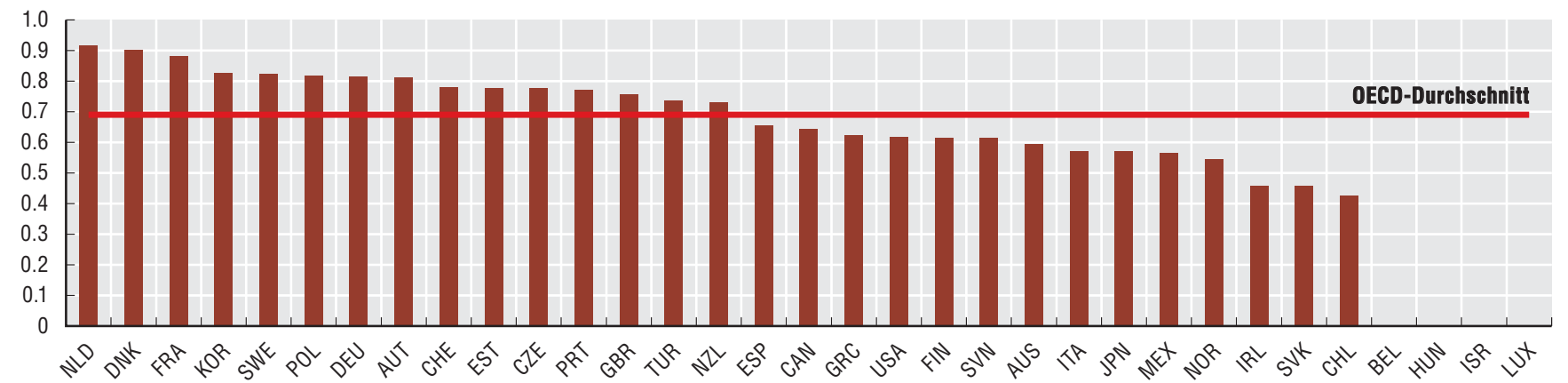

Quelle: 2012 OECD Survey on Budgeting Practices and Procedures. 
Ein Merkmal der Haushaltsreformen in vielen OECD-Ländern ist die Lockerung der Input-Kontrollen auf Seiten der zentralen Haushaltsbehörde, um den staatlichen Stellen für die effiziente und effektive Erreichung ihrer Ziele mehr Flexibilität und Autonomie einzuräumen. Bei dieser Maßnahme wird davon ausgegangen, dass die Leitungen der einzelnen staatlichen Stellen am besten platziert sind, um ihre Politik- und Programmziele durchzusetzen (let managers manage - dezentrale Ressourcenverantwortung). Und auch bei einem soliden Prozess der Haushaltsaufstellung können sich nachträglich die ökonomischen Annahmen verändern, die Vorleistungspreise schwanken und veränderte politische Prioritäten eine Reallokation zugewiesener Ressourcen erfordern. Mehr Flexibilität für nachgeordnete Führungsebenen ermöglicht es diesen, die Ausgaben den sich verändernden Rahmenbedingungen anzupassen.

Wenn diese Verfügungsgewalt jedoch uneingeschränkt ist und keiner Kontrolle unterliegt, kann sie die Tragfähigkeit der öffentlichen Finanzen gefährden. Zu den potenziellen Risiken gehören Möglichkeiten des Machtmissbrauchs durch leitende Staatsbedienstete, erhöhte staatliche Defizite und geringere Effizienz. Zu viel Flexibilität kann auch der Absicht der gesetzgebenden Organe und den Zielen der Mittelbewilligungen zuwiderlaufen, da Ressourcen für die von diesen gesetzten Prioritäten für andere Zwecke abgezweigt werden könnten. Daher schaffen die meisten OECD-Mitgliedsländer ein Gleichgewicht zwischen Top-down-Direktiven und Ergebniskontrolle, mit einem unterschiedlichen Grad an Flexibilität für die Ministerien, wobei allerdings verschiedene Modelle angewendet werden. Auf der Grundlage der Politikprioritäten und mittelfristigen Ausgabenerwägungen setzt die zentrale Haushaltsbehörde die Budgetbeschränkungen für die nachgeordneten Ebenen fest, während den Fachministerien, Behörden und sonstigen öffentlichen Einrichtungen die Verantwortung für die Allokation und Verwendung dieser Mittel zur Erreichung vorab vereinbarter Programmziele übertragen wird. Mit einer derartigen Freiheit wächst zugleich aber auch der Bedarf an Rechenschaftspflicht und ergebnisorientiertem Management (vgl. den Indikator zur ergebnisorientierten Budgetierung). Hierdurch lassen sich Effizienz und Effektivität im Hinblick auf neue Prioritäten, Rahmenbedingungen und Erkenntnisse steigern. Zugleich erhöht diese Freiheit die Anreize für Politiker, sich stärker auf die Ergebnisse und Wirkungen als allein auf die Inputs zu konzentrieren.

Ein wichtiger Aspekt der Haushaltsflexibilität der Exekutive ist die Verwendung von Pauschalzuweisungen, die der jeweiligen Leitung mehr Flexibilität einräumt, die Mittel zwischen und innerhalb von Programmen so zuzuordnen, wie sie es für richtig hält. Die Mehrzahl der Mitgliedsländer legt im Rahmen dieser Pauschalzuweisungen Obergrenzen für einzelne Bereiche fest, am häufigsten bei den Lohnaufwendungen und Arbeitnehmerentgelten. Desgleichen verleiht eine begrenzte Zahl von Zuweisungen für bestimmte Einzelposten im genehmigten Haushalt der Exekutive mehr Flexibilität. Nur ein Viertel der OECD-Mitgliedsländer weist weniger als 300 Einzelposten auf. Die Mehrzahl der zentralen Haushaltsbehörden in den Mitgliedsländern gestattet den Fachministerien und nachgeordneten Behörden außerdem, nicht genutzte Mittel von einem auf das nächste Jahr zu übertragen, wenn auch mit gewissen Auflagen, wie die Einhaltung von Schwellenwerten, die Beantragung der erforderlichen Zustimmung oder beides. In der Regel ist die Flexibilität bei Investitionsausgaben größer als bei
Sachausgaben, da sich kapitalintensive Projekte häufig über mehrere Jahre erstrecken. Außerdem gestatten es einige Länder der Exekutive, Mittel bereits im Vorgriff auf künftige Bewilligungen auszugeben. Von den sieben Ländern, die von dieser Praxis Gebrauch machen, haben indessen alle für die Haushaltsüberschreitungen im laufenden Finanzjahr einen Schwellenwert festgesetzt. In manchen Fällen hat die Exekutive ferner das Recht, die Ausgaben während des Finanzjahrs ohne Vorabgenehmigung der Legislative zu erhöhen oder zu senken. Diese zusätzliche Flexibilität wird oft in der Erwartung gewährt, dass sie die optimale Verwendung öffentlicher Ressourcen erleichtern und Anreize zur Effizienzsteigerung der öffentlichen Ausgaben schaffen kann.

\section{Methodik und Definitionen}

Die Daten beziehen sich auf das Jahr 2012 und entstammen den Antworten der Länder auf den OECD Survey of Budgeting Practices and Procedures von 2012. Die Befragten waren vornehmlich hochrangige Haushaltsverantwortliche in den OECD-Ländern. Die Antworten stellen die Selbsteinschätzung der Länder zu den derzeitigen Praktiken und Verfahren dar. Die Daten beziehen sich nur auf die Zentral-/Bundesregierungen, die Haushaltspraxis auf der Ebene der Gliedstaaten/Kommunen ist ausgeklammert.

\section{Literaturhinweise}

OECD (2013, erscheint demnächst), Budgeting Practices and Procedures in OECD Countries, OECD Publishing, Paris.

\section{Anmerkungen zu den Tabellen}

4.5: Für Island sind keine Daten verfügbar. In Deutschland können die Fachministerien auf künftige Mittel vorgreifen, sofern der Haushalt des nachfolgenden Finanzjahrs einen Ausgabenposten enthält, der demselben Zweck dient, und die Absicht besteht, die Kassenbestände im laufenden Finanzjahr auszugleichen. Wenn zusätzliche Ausgaben nicht als vorgezogene Ausgaben verbucht werden können, müssen sie wie außerplanmäßige Ausgaben behandelt werden. In Spanien erhalten die Fachministerien keine Pauschalzuweisungen, können aber dennoch innerhalb bestimmter Grenzen Mittel umverteilen. In Finnland, Korea und Slowenien können nur bestimmte Arten von Ausgaben in das folgende Finanzjahr übertragen werden. In Australien erlöschen die jährlichen Zuweisungen am Ende des Finanzjahres nicht und können im Rahmen der zur Verfügung gestellten Mittel bezogen werden, d.h. sie sind generell verfügbar, bis sie vollständig ausgegeben sind, im Einklang mit den Reduzierungsbestimmungen in den jährlichen Bewilligungsgesetzen gesenkt werden oder das gültige Bewilligungsgesetz durch ein anderes Gesetz aufgehoben wird. In den Vereinigten Staaten beziehen sich die Daten nur auf große Ministerien/Fachministerien. In der Türkei können einige Zuweisungen ausnahmsweise in das nächste Jahr übertragen werden, die Höhe wird aber alljährlich im Haushaltsgesetz für den Haushalt der Zentralregierung festgelegt, und die Entscheidungsbefugnis wird dem Finanzministerium übertragen.

Hinweis zu den Daten für Israel: http://dx.doi.org/10.1787/888932315602. 
4.5. Recht der Fachministerien, ungenutzte Mittel zu übertragen und auf künftige Mittel vorzugreifen (2012)

\begin{tabular}{|c|c|c|c|c|c|}
\hline & \multirow{2}{*}{$\begin{array}{l}\text { Zahl der Einzelgrenzen bei } \\
\text { Pauschalzuweisungen } \\
\text { an Fachministerien }\end{array}$} & \multicolumn{2}{|c|}{$\begin{array}{l}\text { Fähigkeit der Fachministerien, } \\
\text { auf künftige Mittel vorzugreifen }\end{array}$} & \multicolumn{2}{|c|}{$\begin{array}{l}\text { Fähigkeit der Fachministerien, ungenutzte Mittel oder } \\
\text { Zuweisungen von einem in das nächste Jahr zu übertragen }\end{array}$} \\
\hline & & Sachausgaben & Investitionsausgaben & Sachausgaben & Investitionsausgaben \\
\hline Australien & 0 & $\mathrm{O}$ & $\mathrm{O}$ & $x$ & $x$ \\
\hline Österreich & x (keine Pauschalbeträge) & O & O & ○ & ○ \\
\hline Belgien & 2 & O & O & O & O \\
\hline Kanada & 2 & ○ & $\bigcirc$ & $\mathbf{\square}$ & $\mathbf{\square}$ \\
\hline Chile & 3 oder mehr & O & O & O & O \\
\hline Tschech. Rep. & 3 oder mehr & O & O & ○ & - \\
\hline Dänemark & 1 & $\mathbf{\square}$ & $O$ & $\bullet$ & $\bullet$ \\
\hline Estland & 1 & O & O & $\mathbf{\square}$ & $\bullet$ \\
\hline Finnland & 0 & O & O & 0 & 0 \\
\hline Frankreich & 0 & O & O & $\mathbf{\square}$ & $\mathbf{\square}$ \\
\hline Deutschland & x (keine Pauschalbeträge) & $\mathbf{\square}$ & $\mathbf{\square}$ & $\bigcirc$ & 0 \\
\hline Griechenland & 3 oder mehr & $O$ & $O$ & $O$ & O \\
\hline Ungarn & 1 & O & $\mathbf{\square}$ & $\bullet$ & $\bullet$ \\
\hline Irland & 0 & ○ & $\bigcirc$ & O & $\mathbf{\square}$ \\
\hline Israel & 3 oder mehr & O & O & $\bullet$ & $\bullet$ \\
\hline Italien & 1 & O & $\mathbf{a}$ & O & $\mathbf{\square}$ \\
\hline Japan & 0 & O & O & $\bullet$ & $\bullet$ \\
\hline Korea & 3 oder mehr & O & O & 0 & - \\
\hline Luxemburg & 1 & O & $\mathbf{\square}$ & 0 & 0 \\
\hline Mexiko & 3 oder mehr & O & O & O & O \\
\hline Niederlande & 0 & O & O & $\mathbf{\square}$ & $\mathbf{\square}$ \\
\hline Neuseeland & 0 & $\mathbf{\square}$ & $\mathbf{\square}$ & $\mathbf{\square}$ & $\mathbf{\square}$ \\
\hline Norwegen & 0 & ○ & $O$ & $\mathbf{\square}$ & $\mathbf{\square}$ \\
\hline Polen & 0 & O & O & O & O \\
\hline Portugal & 1 & O & $O$ & ○ & $\bullet$ \\
\hline Slowak. Rep. & 2 & O & $O$ & O & 0 \\
\hline Slowenien & 1 & O & O & 0 & $\bullet$ \\
\hline Spanien & x (keine Pauschalbeträge) & O & O & $\mathbf{\square}$ & $\boldsymbol{\square}$ \\
\hline Schweden & 0 & $\mathbf{\square}$ & $\mathbf{\square}$ & $\mathbf{\square}$ & $\mathbf{\square}$ \\
\hline Schweiz & 0 & ○ & $O$ & $\bullet$ & $\bullet$ \\
\hline Türkei & x (keine Pauschalbeträge) & O & O & O & O \\
\hline Ver. Königreich & x (keine Pauschalbeträge) & O & O & $\mathbf{\square}$ & $\boldsymbol{\square}$ \\
\hline Ver. Staaten & 3 oder mehr & O & $O$ & $\mathbf{\square}$ & $\mathbf{\square}$ \\
\hline Russ. Föderation & 3 oder mehr & $\mathbf{\square}$ & $\mathbf{\square}$ & $\mathbf{\square}$ & - \\
\hline \multicolumn{6}{|l|}{ OECD insgesamt } \\
\hline Ja, ohne Schwellenwert & & 0 & 0 & 11 & 14 \\
\hline - Ja, bis zu einem gewissen Schwellenwert & & 4 & 6 & 10 & 11 \\
\hline Nein, nicht zulässig & & 29 & 27 & 11 & 7 \\
\hline
\end{tabular}

Quelle: 2012 OECD Survey on Budgeting Practices and Procedures. 
Die Regierungen beziehen leistungs- und ergebnisbezogene Informationen zunehmend in die Prozesse der Haushaltsaufstellung sowie der Staats- und Verwaltungsführung ein, um bessere Ergebnisse zu erzielen, die Kosteneffizienz zu steigern und die Transparenz der Ausgabenentscheidungen zu erhöhen. Dank guter ergebnisbezogener Informationen lassen sich sowohl im Hinblick auf den Ressourceneinsatz als auch die Frage, wie bestimmte Programme am besten umzusetzen sind, fundiertere Entscheidungen treffen. Mehr Transparenz hinsichtlich der Ergebnisse und der Ressourcenallokation erhöht ferner die Rechenschaftspflicht der Behörden in Bezug auf ihre Ausgaben.

Wenngleich die Praxis der ergebnisorientierten Budgetierung in den OECD-Mitgliedsländern weit verbreitet ist, gibt es große Unterschiede zwischen den gewählten Ansätzen, und es herrscht kein Konsens hinsichtlich der optimalen Methode, die angewendet werden soll. Gleichwohl hat die OECD drei große Kategorien ergebnisorientierter Haushaltsplanungssysteme identifiziert: a) Darstellung von Ergebnisinformationen im Budget (presentational performance budgeting), bei der ergebnisbezogene Daten erstellt und zusammen mit Finanzzuweisungen aufgeführt, aber nicht unbedingt für Ausgabenentscheidungen herangezogen werden; b) Budgetierung unter Berücksichtigung von Ergebnisinformationen (performance-informed budgeting), bei der diese Informationen die Ressourcenallokation ausdrücklich beeinflussen sowie c) direkte ergebnisorientierte Budgetierung (direct performance budgeting), bei der die Mittelbereitstellung streng an die Ergebnisse und Wirkungen (Outputs und Outcomes) gekoppelt ist. Die meisten OECD-Mitgliedsländer fallen mit ihrem Ansatz in die erste oder zweite Kategorie, wobei einige wenige die letztgenannte Methode für ausgewählte Ausgabenarten anwenden (z.B. Finanzierung von Hochschulbildung oder Krankenhäusern).

Die Ergebnisse des OECD Survey on Performance Budgeting von 2011 deuten darauf hin, dass die Praxis innerhalb der Zentral-/Bundesregierungen im Allgemeinen dezentralisiert ist, was bedeutet, dass Fachministerien bei der Zuteilung ihrer eigenen Haushaltsmittel an die Behörden/Ressorts häufig die Praxis der ergebnisorientierten Budgetierung anwenden. Ausnahmen sind Ausgabenprüfungen, bei denen die zentralen Haushaltsbehörden und Geschäftsführer eine zentralere Rolle spielen. Einige Länder wählen indessen einen stärker zentralisierten Ansatz und haben einen umfassenden, den gesamten Staat berücksichtigenden Rahmen eingerichtet (Evaluierungen und Leistungsmessgrößen), indem sie leistungs- und ergebnisorientierte Informationen in den Haushalts- und Rechenschaftsprozess integrieren, sie bei der Entscheidungsfindung berücksichtigen und die Ergebnisse überwachen und über sie Bericht erstatten. Länder wie Korea, Mexiko und Kanada beispielsweise verfügen für die Fachministerien und Behörden über einen standardisierten und umfassenden Rahmen, der auch Elemente wie Richtlinien, Vorlagen für die Berichterstattung und Systeme der Ergebnisbeurteilung einbezieht. In der Mehrzahl der Länder hat das Verfehlen von Leistungszielen meistens jedoch keine finanziellen Folgen, sondern zieht vielmehr die Veröffentlichung der schlechten Ergebnisse und ein intensiveres Monitoring in der Zukunft nach sich.

Leistungs- und ergebnisbezogene Informationen können auf vielerlei Weise dargestellt werden, insbesondere in Form von Finanz- und operationellen Daten, Evaluierungen und sogar unabhängigen Statistiken und Berichten von Stellen außerhalb des Staatssektors. Die in den Budgetverhandlungen mit Abstand am häufigsten verwendeten Informationen sind Input-Messgrößen, wie Finanz- und operationelle Daten (Tabelle 4.6).

\section{Methodik und Definitionen}

Die Daten beziehen sich auf das Jahr 2011 und entstammen den Antworten der Länder auf den OECD Survey of Budgeting Practices and Procedures von 2011. Die Befragten waren vornehmlich hochrangige Haushaltsverantwortliche in den OECD-Ländern. Die Antworten stellen die Selbsteinschätzung der Länder zu den derzeitigen Praktiken und Verfahren dar. Die Daten beziehen sich nur auf die Zentral-/Bundesregierungen, die ergebnisorientierte Haushaltspraxis auf der Ebene der Gliedstaaten/ Kommunen ist ausgeklammert. Für die EU-Mitgliedsländer sind alle EU-Zuweisungen ausgenommen.

Ausgabenprüfungen stellen eine spezifische Form der Evaluierung der Regierungsarbeit dar und werden mit dem ausdrücklichen Ziel in Auftrag gegeben, auf den verschiedenen Verwaltungsebenen Haushaltseinsparungen $\mathrm{zu}$ identifizieren. Im OECD-Projekt Value for Money (Kosteneffizienz) werden Ausgabenprüfungen anhand von drei Hauptmerkmalen von anderen Evaluierungsformen unterschieden:

1. Ausgabenprüfungen untersuchen nicht nur die Wirksamkeit und Effizienz der Programme mit dem aktuellen Finanzierungsniveau, sondern auch die Folgen einer alternativen Finanzausstattung für Ergebnisse und Wirkungen. Sie können funktionalen Charakter haben (d.h. sich auf die operationelle Effizienz konzentrieren) und/oder strategischer Natur sein (d.h. schwerpunktmäßig auf die Frage der Effizienz und Übereinstimmung der Initiativen mit den hochrangigen Politikprioritäten ausgerichtet sein).

2. Die Verantwortung für das Verfahren der Ausgabenprüfung liegt entweder beim Finanzministerium oder beim Büro des Regierungschefs.

3. Die Fortsetzung der Ausgabenprüfungen wird im Haushaltsprozess beschlossen.

Dieser Mischindex in Abbildung 4.7 enthält 11 Variablen, die Angaben zur Verfügbarkeit und Art der erstellten leistungs- und ergebnisorientierten Informationen, den Prozessen zur Überwachung und Berichterstattung wie auch dazu enthalten, ob (und wie) die Leistungsinformationen in Haushaltsverhandlungen und bei der Entscheidungsfindung durch die zentralen Haushaltsbehörden, Fachministerien und Politiker genutzt werden. Zu beachten ist, dass der Index nicht die Gesamtqualität des Verfahrens der ergebnisorientierten Budgetierung messen soll, sondern eher deskriptiven Charakter hat. Anhang C enthält eine Beschreibung der Methodik, die zur Erstellung dieses Indikators verwendet wurde, einschließlich der spezifischen Gewichtungen der einzelnen Variablen.

\section{Literaturhinweise}

OECD (2008), „Ergebnisorientierte Budgetierung: Leitfaden für die Praxis", OECD Policy Brief, OECD Publishing, Paris, www.oecd.org/governance/budgeting/40357919.pdf.

OECD (2007), Performance Budgeting in OECD Countries, OECD Publishing, Paris, http://dx.doi.org/10.1787/9789264034051-en.

\section{Anmerkungen zu den Abbildungen und Tabellen}

Für Island und Israel stehen keine Daten zur Verfügung. In Österreich kamen leistungs- und ergebnisorientierte Informationen in den Budgetverhandlungen bisher nicht zum Einsatz, jedoch beim Haushaltsvollzug und zur Steigerung der Effizienz. 
4.6. Ergebnisorientiertes Haushaltsplanungssystem auf Ebene der Zentralregierung (2011)

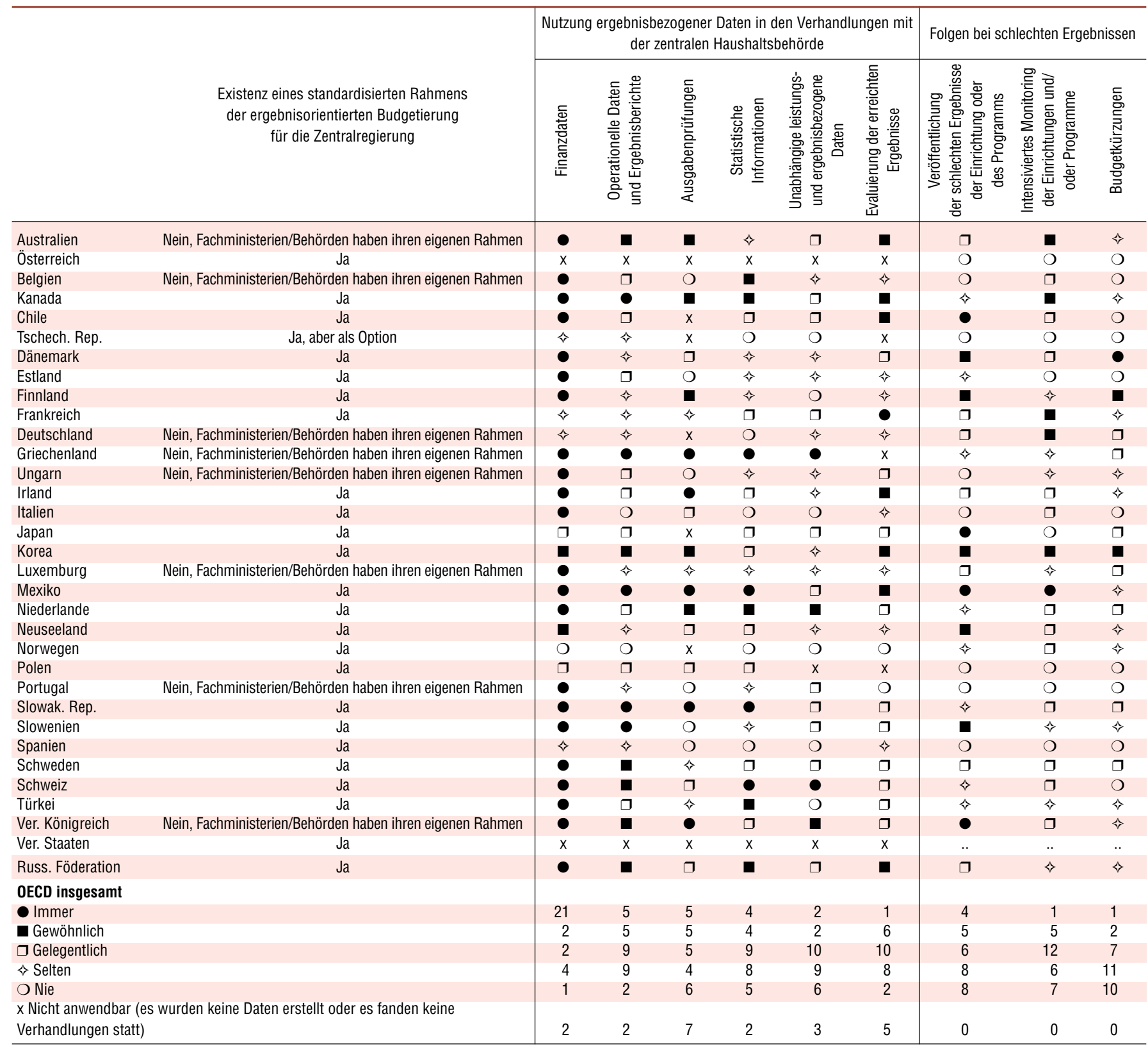

Quelle: 2011 OECD Survey on Performance Budgeting.

4.7. Anwendung eines ergebnisorientierten Haushaltsplanungssystems auf Ebene der Zentralregierung (2011)

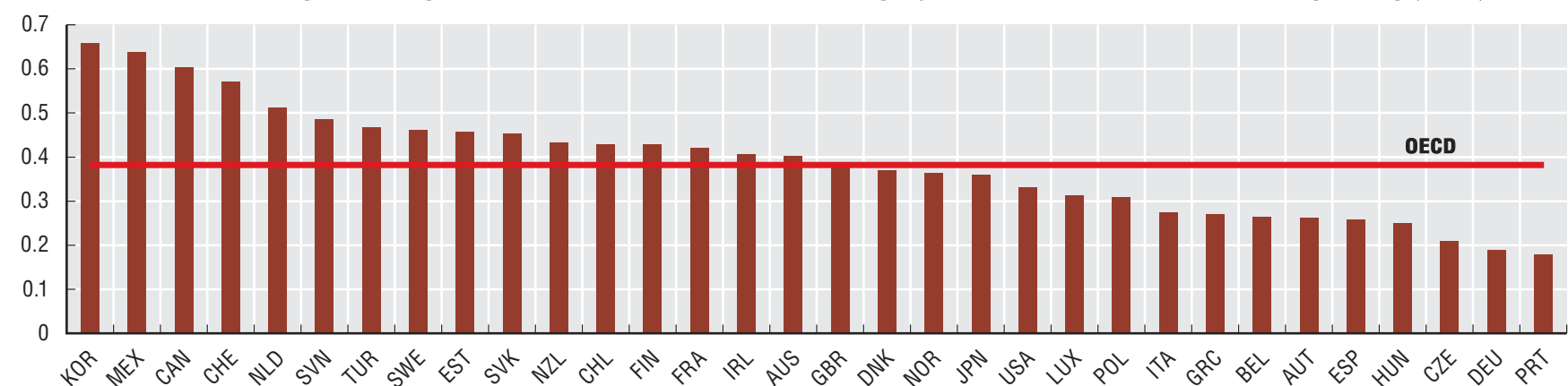

Quelle: 2011 OECD Survey on Performance Budgeting. 
Bei den öffentlich-privaten Partnerschaften (ÖPP) handelt es sich um langfristige vertragliche Vereinbarungen zwischen dem Staat und einem privaten Partner, in deren Rahmen der private Partner in der Regel durch die Nutzung von Anlagegütern (z.B. Verkehrs- oder Energieinfrastruktur, Krankenhäuser oder Schulgebäude) öffentliche Dienstleistungen finanziert und erbringt. Die private Partei kann mit der Konzipierung, dem Bau, der Finanzierung, dem Betrieb, der Verwaltung und der Bereitstellung der Dienstleistung für einen vorab definierten Zeitraum beauftragt werden, das Entgelt besteht in einer regelmäßigen einheitlichen Zahlung an den Auftragnehmer oder den Nutzern in Rechnung gestellten Gebühren. Auf öffentlich-private Partnerschaften (ÖPP) entfallen weniger als $15 \%$ der jährlichen Investitionsausgaben der Zentralregierung, und das Ausmaß, in dem Länder auf diese Partnerschaften zurückgreifen, ist sehr unterschiedlich: Das Vereinigte Königreich weist die meisten dieser Projekte auf (648), gefolgt von Korea (567) und Australien (127).

Staatliche Stellen entscheiden sich möglicherweise für den Abschluss von ÖPP, da diese Partnerschaften es ihnen im Vergleich zu konventionelleren Formen der Auftragsvergabe für die Bereitstellung von Infrastruktur ermöglichen, das Fachwissen des privaten Sektors besser auszuschöpfen, indem Konzeption und Betrieb von Vorhaben kombiniert werden, so dass Dienstleistungen auf effizientere Weise erbracht werden können. Staatliche Behörden mit hinreichend Erfahrung und ausreichend Datenmaterial, um sich ein Urteil über ÖPP zu bilden, geben an, dass diese die traditionellen Infrastrukturprojekte in Bezug auf Fristeinhaltung, Baukosten und Qualität überbieten (Abbildung 4.8). Allerdings legen die Erfahrungen einiger OECD-Mitgliedsländer den Schluss nahe, dass nicht alle ÖPP gut verwaltet sind und daher nicht den erwarteten Nutzen bringen dürften. Langzeitverträge für bestimmte Dienstleistungen können sich angesichts der sich wandelnden Bedürfnisse des öffentlichen Sektors und technologischer Veränderungen als zu unflexibel erweisen, und das ÖPP-Vergabeverfahren war häufig für den öffentlichen wie auch privaten Sektor langwierig, komplex und kostspielig. Die Länder geben ferner an, dass die Transaktionskosten für die öffentlichen und privaten Teilnehmer an ÖPP höher sind als bei den konventionellen Formen der Auftragsvergabe von Infrastrukturprojekten. Schließlich wurden in einigen Ländern Anreize zur Nutzung öffentlich-privater Partnerschaften geschaffen, um außeretatmäßige Vermögenswerte $z u$ finanzieren. Mit einer derartigen Absicht konzipierte ÖPP-Projekte können aus der Nettoverschuldung des öffentlichen Sektors ausgeklammert werden, was wiederum einen Mangel an Transparenz bei den künftigen Verbindlichkeiten und Finanzrisiken nach sich zieht.

Als Reaktion auf diese Herausforderungen hat die OECD die Grundsätze für die öffentliche Governance von öffentlich-privaten Partnerschaften formuliert. Die Grundsätze bieten genaue Orientierungshilfen in drei breit gefassten Themenbereichen: a) Schaffung eines klaren, vorhersehbaren und legitimierten institutionellen Rahmens auf der Basis kompetenter und gut ausgestatteter Behörden; b) Orientierung der Auswahl von öffentlich-privaten Partnerschaften am Kosten-Nutzen-Verhältnis und c) transparente Nutzung des Haushaltsprozesses, um fiskalische Risiken zu minimieren und die Integrität des Beschaffungsprozesses zu sichern.

Es gibt keine klare Antwort auf die Frage, ob eine der Methoden der öffentlichen Auftragsvergabe die anderen in jeder Hinsicht übertrifft, wenn in den Berechnungen die gesamte Lebensdauer des Projekts zu Grunde gelegt wird. Ein stärkerer Einsatz von Beurteilungen der Kosteneffizienz wird empfohlen, um ex ante beurteilen zu können, ob sich ein bestimmtes Projekt gut für den
Abschluss einer ÖPP-Vereinbarung eignet. Die Kosteneffizienz eines Projekts sollte in allen Projektphasen evaluiert werden, mit einer Fokussierung auf die Kosten über den gesamten Projektlebenszyklus und die potenziellen Risiken, die das Projekt für den öffentlichen Sektor darstellt. Auch wenn die Mehrzahl der Mitgliedsländer (21) bei öffentlich-privaten Partnerschaften anders als bei konventionellen Infrastrukturprojekten Beurteilungen der relativen Kosteneffizienz vornimmt, gilt dies in den meisten Ländern nur für gewisse Projekte (Tabelle 4.9). Beurteilungen der absoluten Kosteneffizienz sind geläufiger. Einige Länder haben spezielle ÖPP-Einheiten eingerichtet, um solide Evaluierungen der Kosteneffizienz von ÖPP sicherzustellen, die aufgestellten Ziele mit den Gewinnzielen des privaten Sektors in Einklang zu bringen und zu gewährleisten, dass sie auf transparente Art und Weise verwaltet werden. 2010 hatten 17 OECDLänder derartige Einheiten eingerichtet, wobei derzeit immer mehr Länder diesem Trend folgen.

\section{Methodik und Definitionen}

Die Daten stammen aus dem OECD Survey on Budgeting Practices and Procedures von 2012. Die Befragten waren vornehmlich hochrangige Haushaltsverantwortliche. Die Antworten stellen die Selbsteinschätzung der Länder zu den derzeitigen Praktiken und Verfahren dar. Die Daten beziehen sich nur auf die Zentral-/Bundesregierungen, die Haushaltspraxis auf der Ebene der Gliedstaaten/Kommunen ist ausgeklammert.

Das ÖPP-Konzept umfasst sowohl reine ÖPP, d.h. Vorhaben, in denen die Zahlungen des Staats die Haupteinnahmequelle der privaten Partner darstellen (in Form von regelmäßigen Zahlungen oder einer einheitlichen Gebühr) als auch Konzessionen (wo die Haupteinnahmequelle aus den Benutzungsentgelten besteht, die von den privaten Partnern bei den Nutznießern der Leistungen erhoben werden).

In den Tests der relativen Kosteneffizienz werden mehrere Formen der Auftragsvergabe verglichen, um herauszufinden, welche am kosteneffizientesten ist. In den Tests der absoluten Kosteneffizienz wird ermittelt, ob ein Vorhaben (z.B. ein Damm, ein Flughafen, eine Autobahn) global betrachtet für die Gesellschaft kosteneffizient ist. Die Umsetzungsmethoden für beide Formen der Evaluierung variieren in den einzelnen Ländern.

\section{Literaturhinweise}

Burger, P. und I. Hawkesworth (2013), „Capital budgeting and procurement practices - towards an integrated approach?“, vorgestellt auf dem OECD Annual Network Meeting of Senior Public-Private Partnership Officials, 15.-16. April 2013.

OECD (2012), Grundsätze für die öffentliche Governance von öffentlich-privaten Partnerschaften, Mai 2012, OECD, Paris, www.oecd.org/gou/budgeting/PPPnoSG.pdf.

OECD (2010), Dedicated Public-Private Partnership Units: A Survey of Institutional and Governance Structures, OECD Publishing, Paris, http://dx.doi.org/10.1787/9789264064843-en.

\section{Anmerkungen zu den Abbildungen und Tabellen}

4.8 und 4.9: Für Island sind keine Daten verfügbar.

Hinweis zu den Daten für Israel: http://dx.doi.org/10.1787/888932315602. 
4.8. Länderbeurteilungen der ÖPP im Vergleich zu konventionellen Infrastrukturprojekten unter verschiedenen Gesichtspunkten

$\begin{array}{lll}\text { Kann sich auf Grund von mangelndem } & \text { Besser als bei konventionellen } & \text { Wie bei konventionellen } \\ \text { Datenmaterial oder fehlender } & \text { Infrastrukturprojekten } & \text { Infrastrukturprojekten } \\ \text { Erfahrung mit ÖPP kein Urteil bilden } & & \text { Schlechter als bei } \\ \text { Infrastrukturprojekten }\end{array}$

Anteil der Länder, die an der Erhebung teilnahmen (in \%)

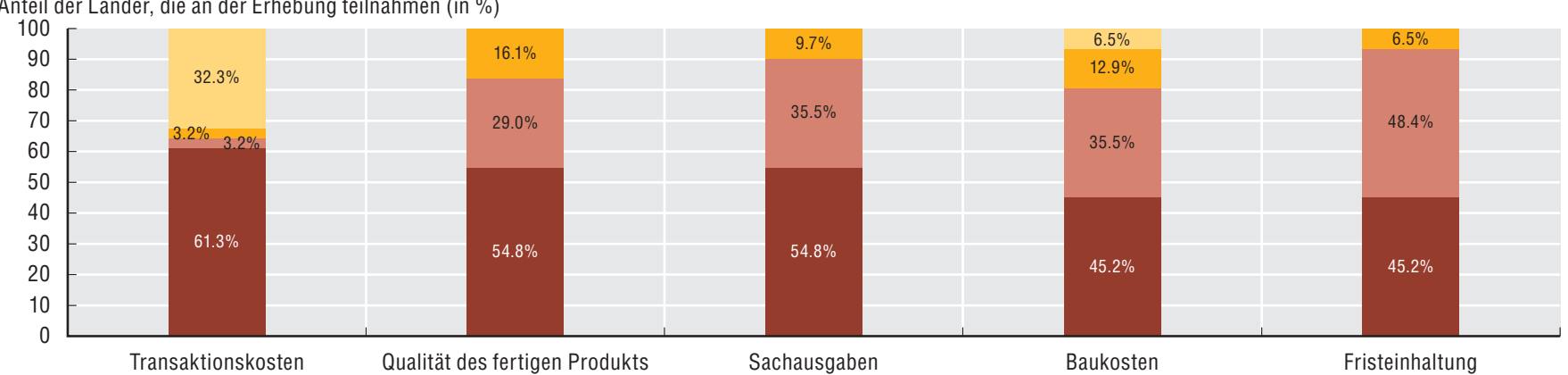

Quelle: 2012 OECD Survey on Budgeting Practices and Procedures.

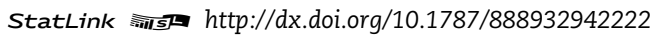

4.9. Evaluierungen der Kosteneffizienz von ÖPP und konventionellen Infrastrukturprojekten sowie spezifischen ÖPP-Einheiten

\begin{tabular}{|c|c|c|c|c|c|c|}
\hline & \multirow{2}{*}{$\begin{array}{c}\begin{array}{c}\text { Einsatz von Methoden zur } \\
\text { Beurteilung der relativen } \\
\text { Kosteneffizienz }\end{array} \\
\text { für ÖPP }\end{array}$} & \multicolumn{2}{|c|}{$\begin{array}{c}\text { Einsatz von Methoden } \\
\text { zur Beurteilung der absoluten } \\
\text { Kosteneffizienz }\end{array}$} & \multirow{2}{*}{\multicolumn{2}{|c|}{$\begin{array}{l}\text { Berichterstattung } \\
\text { spezifischer ÖPP- Öpezifische } \\
\text { Einheiten an das OPP-Einheiten } \\
\text { Finanzministerium in Fachministerien }\end{array}$}} & \multirow{2}{*}{$\begin{array}{l}\text { Auf der Ebene der } \\
\text { Zentral-/Bundesre- } \\
\text { gierung existieren } \\
\text { keine spezifischen } \\
\text { ÖPP-Einheiten }\end{array}$} \\
\hline & & \multicolumn{2}{|c|}{ für ÖPP $\begin{array}{c}\text { für konventionelle } \\
\text { Infrastrukturprojekte }\end{array}$} & & & \\
\hline Australien & 0 & 0 & $\mathbf{\square}$ & & & $\checkmark$ \\
\hline Österreich & $x$ & $x$ & O & & & $\checkmark$ \\
\hline Belgien & $x$ & $x$ & $x$ & & & $\checkmark$ \\
\hline Kanada & $\bullet$ & 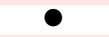 & $\square$ & $\checkmark$ & $\checkmark$ & \\
\hline Chile & $\bullet$ & $\bullet$ & $\mathbf{\square}$ & $\checkmark$ & $\checkmark$ & \\
\hline Tschech. Rep. & .. &.. & $\square$ & $\checkmark$ & & \\
\hline Dänemark & 0 & $\mathbf{\square}$ & $\mathbf{\square}$ & & $\checkmark$ & \\
\hline Estland & $x$ & $x$ & $\square$ & & & $\checkmark$ \\
\hline Finnland & $\square$ & $\mathbf{\square}$ & 口 & & & $\checkmark$ \\
\hline Frankreich & $\square$ & - & $\square$ & $\checkmark$ & & \\
\hline Deutschland & 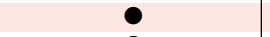 & - & - & $\checkmark$ & $\checkmark$ & \\
\hline Griechenland & 0 & 0 & $\square$ & .. & .. & .. \\
\hline Ungarn & $\square$ & $\square$ & $x$ & & $\checkmark$ & \\
\hline Island &.. & .. & .. & .. & .. & .. \\
\hline Irland & 0 & $\bullet$ & - & $\checkmark$ & & \\
\hline Israel & $\mathbf{\square}$ & $\boldsymbol{\square}$ & $\boldsymbol{\square}$ & $\checkmark$ & & \\
\hline Italien & O & $\square$ & $\square$ & & & $\checkmark$ \\
\hline Japan & O & 0 & $\mathbf{\square}$ & & $\checkmark$ & \\
\hline Korea & $\mathbf{\square}$ & $\bullet$ & $\mathbf{\square}$ & $\checkmark$ & $\checkmark$ & \\
\hline Luxemburg & $\square$ & $\square$ & $\square$ & & & $\checkmark$ \\
\hline Mexiko & $\bullet$ & $\bullet$ & $\bullet$ & & & $\checkmark$ \\
\hline Niederlande & $\mathbf{\square}$ & 0 & $\mathbf{\square}$ & $\checkmark$ & $\checkmark$ & \\
\hline Neuseeland & $\bullet$ & $\bullet$ & $\mathbf{\square}$ & $\checkmark$ & & \\
\hline Norwegen & $x$ & $x$ & $\mathbf{\square}$ & & & $\checkmark$ \\
\hline Polen & $x$ & $\bullet$ & $\bullet$ & $\checkmark$ & $\checkmark$ & \\
\hline Portugal & ○ & $\bullet$ & ○ & $\checkmark$ & & \\
\hline Slowak. Rep. & $x$ & $x$ & 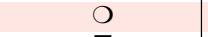 & & & $\checkmark$ \\
\hline Slowenien & $\mathbf{\square}$ & $\bullet$ & $\mathbf{\square}$ & & & $\checkmark$ \\
\hline Spanien & $\square$ & $\square$ & $\square$ & & & $\checkmark$ \\
\hline Schweden & $\square$ & $\mathbf{\square}$ & $\mathbf{\square}$ & & & $\checkmark$ \\
\hline Schweiz & $\square$ & O & $O$ & & & $\checkmark$ \\
\hline Türkei & $O$ & - & - & $\checkmark$ & & \\
\hline Ver. Königreich & $\bullet$ & $\bullet$ & - & $\checkmark$ & & \\
\hline Ver. Staaten & $\square$ & $\square$ & $\mathbf{\square}$ & & & $\checkmark$ \\
\hline Russ. Föderation & $O$ & - & ○ & $\checkmark$ & $\checkmark$ & \\
\hline \multicolumn{7}{|l|}{ OECD insgesamt } \\
\hline Ja, bei allen Projekten & 10 & 17 & 7 & 14 & 9 & 15 \\
\hline - Ja, bei Projekten über einem gewissen monetären Schwellenwert & 4 & 4 & 13 & & & \\
\hline$\square \mathrm{Ja}$, auf Ad-hoc-Basis & 8 & 5 & 8 & & & \\
\hline Nein & 4 & 1 & 3 & & & \\
\hline x Nicht anwendbar & 6 & 5 & 2 & & & \\
\hline
\end{tabular}

Quelle: 2012 OECD Survey on Budgeting Practices and Procedures. 
Mitte der 1990er Jahre verbreiteten Wirtschaftswissenschaftler die Idee, dass die Länder einige der guten Erfahrungen mit unabhängigen Zentralbanken in den fiskalischen Bereich übertragen könnten. Den Beschluss zur Schaffung unabhängiger Fiskalinstitutionen (UFI, häufig als Finanzrat oder parlamentarische Haushaltsbehörde bezeichnet) fasste eine wachsende Zahl von OECD-Mitgliedsländern aber erst nach dem sprunghaften Anstieg der Haushaltsdefizite und der Staatsverschuldung im Anschluss an die jüngste Krise.

Mit der Gründung dieser Gremien wollen die Regierungen die Haushaltsregeln stärken, die sich isoliert betrachtet als unzureichend herausgestellt hatten, um ein umsichtiges Management der öffentlichen Finanzen zu gewährleisten sowie ihre Entschlossenheit zu signalisieren, nach der Krise verantwortungsbewusst zu handeln. Dies trifft insbesondere auf die Europäische Union zu, wo neue Rechtsvorschriften die Mitgliedsländer dazu verpflichten, über unabhängige Organe zu verfügen, die die Einhaltung der Haushaltsregeln überprüfen und makroökonomische Prognosen erstellen oder diese validieren. Weitere neue Beispiele dieser Art finden sich in Kanada und Australien, wo auf diese Weise die Haushaltstransparenz erhöht und die Rolle des Gesetzgebers im Haushaltsprozess gestärkt werden soll. Zusätzlich zu dieser neuen Generation unabhängiger Fiskalinstitutionen existiert eine Handvoll sehr viel älterer Einrichtungen, wie das US-Congressional Budget Office (CBO) in den Vereinigten Staaten und das Niederländische Planungsamt (CPB).

Die Landschaft der unabhängigen Fiskalinstitutionen von heute ist äußerst vielfältig: Ihre Rollen, Ressourcen und Strukturen sind je nach Land sehr unterschiedlich. Zusätzlich zu einer Analyse der Fiskalpolitik und der Haushaltsentwürfe zählen zu den gemeinsamen Funktionen die Mitwirkung bei der Erstellung von Prognosen, die Kontrolle der Einhaltung der Haushaltsregeln, die Analyse der langfristigen finanziellen Tragfähigkeit, die Berechnung der Kosten vorgeschlagener Maßnahmen sowie analytische Studien zu ausgewählten Themen.

Die in den neuen EU-Verordnungen enthaltenen Auflagen deuten auf ein dominierendes Modell in der Europäischen Union hin, wenngleich die Bedürfnisse einzelner Länder und das jeweilige institutionelle Umfeld vor Ort viele der gewählten Optionen auch weiterhin bestimmen. Mit Ausnahme des Niederländischen Planungsamts (CPB) und (bis vor kurzem des dänischen Wirtschaftsrats) sind alle derartigen Finanzräte in den OECD-Mitgliedsländern, die der EU angehören, mit der Kontrolle der Einhaltung der Haushaltsregeln beauftragt. Keine der Einrichtungen in OECD-Mitgliedsländern außerhalb der EU erfüllt diese Aufgabe.

Die Institutionen spielen bei der Erstellung von Prognosen unterschiedliche Rollen. Für das Niederländische Planungsamt (CPB) und das Office for Budget Responsibility (OBR) im Vereinigten Königreich ist die Erstellung amtlicher Prognosen Teil ihres Auftrags. In anderen Ländern (z.B. die Vereinigten Staaten und Kanada) liefern die Einrichtungen alternative Prognosen, und in wiederum anderen geben sie Stellungnahmen zu den Prognosen der Regierung ab (z.B. Frankreich, Irland und Schweden). Die überwiegende Mehrzahl der Institutionen erstellt auch Analysen der langfristigen fiskalischen Tragfähigkeit. Knapp die Hälfte der Institutionen in OECD-Mitgliedsländern spielt bei der Kostenberechnung von Politikvorschlägen eine Rolle, wobei diese Funktion in der Regel eher von parlamentarischen Haushaltsbehörden ausgeübt wird. Sowohl das Niederländische Planungsamt (CPB) als auch der Australische Parliamentary Budget Officer (PBO) sind mit der Kostenberechnung von Wahlprogrammen beauftragt.
Bei den Strukturen und Ressourcen der unabhängigen Fiskalinstitutionen findet sich eine ähnliche Vielfalt. Einige stehen kraft Gesetz unter der Kontrolle der Exekutive, und eine geringere Zahl untersteht der Legislative (Australien, Kanada, Italien [laut einem im Dezember 2012 verabschiedeten Gesetz, das 2014 in Kraft treten soll] und die Vereinigten Staaten). Zwei sind als autonome Einheiten innerhalb der jeweiligen nationalen Kontrollbehörde eingerichtet worden (Finnland und Frankreich). Ihre Führungsstruktur ist individuell oder kollegial (Rat). Das Congressional Budget Office (CBO) der Vereinigten Staaten verfügt mit 45 Mio. US-\$ über das größte Budget und hat etwa 250 Mitarbeiter, während der irische Finanzbeirat mit weniger als 1 Mio. Euro auskommt und in seinem Sekretariat weniger als fünf Mitarbeiter hat. Institutionen, die bei der Kostenberechnung von Politikmaßnahmen eine Rolle spielen eine der ressourcenintensivsten Aufgaben, die Mitarbeiter mit spezifischen Programmkenntnissen voraussetzt -, haben in der Regel einen größeren Mitarbeiterstab. Drei Institutionen verfügen über Haushalte mit mehrjährigen Finanzierungszusagen, eine Praxis, die die Unabhängigkeit verstärken und dabei helfen kann, die Einrichtung vor politischem Druck abzuschirmen (Australien, die Niederlande und das Vereinigte Königreich).

\section{Methodik und Definitionen}

Die Daten, die anhand eines Fragenkatalogs für detaillierte Länderstudien über unabhängige Fiskalinstitutionen im Zeitraum 2012-13 erhoben wurden, konzentrieren sich auf Institutionen in 17 OECD-Mitgliedsländern. Bei der Ausarbeitung der Länderbeiträge beriet sich das OECD-Sekretariat ausgiebig mit Vertretern der ausgewählten unabhängigen Fiskalinstitutionen wie auch mit Abgeordneten, Staatsbediensteten, Akademikern und im Einzelfall auch mit anderen Akteuren. Weitere Daten zu den neuen Institutionen werden derzeit zusammengetragen.

Chile richtete Anfang 2013 einen Finanzbeirat ein. Spanien hat Gesetzesvorschläge zur Einrichtung einer unabhängigen Finanzaufsichtsbehörde eingebracht, die Ende 2013 verabschiedet werden sollten.

\section{Literaturhinweise}

Calmfors, L. und S. Wren-Lewis (2011), „What Should Fiscal Councils Do?", Centre for Economic Studies and IFO Institute for Economic Research (CESifo), Vol. 26, No. 68, S. 649-695, London.

Hagemann, R. (2011), „How Can Fiscal Councils Strengthen Fiscal Performance?“, OECD Journal: Economic Studies, Vol. 2011/1, http://dx.doi.org/10.1787/eco_studies-2011-5kg2d3gx4d5c.

Kopits, G. (2011), „Independent Fiscal Institutions: Developing Good Practices", OECD Journal on Budgeting, Vol. 11/3, S. 35-14, OECD Publishing, Paris, http://dx.doi.org/10.1787/budget-11$5 k g 3 p d g c p n 42$.

OECD (2013, erscheint demnächst), Principles for Independent Fiscal Institutions and Country Notes, OECD Publishing, Paris.

\section{Anmerkungen zu den Tabelle}

Vgl. StatLink wegen länderspezifischer Anmerkungen. 
4.10. Rolle und Ressourcen unabhängiger fiskalischer Institutionen in OECD-Ländern (2013)

\begin{tabular}{|c|c|c|c|c|c|c|c|c|c|}
\hline & $\begin{array}{c}\text { Name } \\
\text { der Institution }\end{array}$ & Gegründet & Budget & Mitarbeiter & $\begin{array}{c}\text { Rolle bei der } \\
\text { Aufstellung } \\
\text { makroökonomi- } \\
\text { scher Prognosen }\end{array}$ & $\begin{array}{l}\text { Analyse der langfri- } \\
\text { stigen Tragfähigkeit } \\
\text { der öffentlichen } \\
\text { Finanzen }\end{array}$ & $\begin{array}{l}\text { Rolle bei der } \\
\text { Kontrolle der } \\
\text { Einhaltung der } \\
\text { Haushaltsregeln }\end{array}$ & $\begin{array}{c}\text { Rolle bei der } \\
\text { Kostenberech- } \\
\text { nung von Politik- } \\
\text { maßnahmen }\end{array}$ & $\begin{array}{c}\text { Rolle bei der } \\
\text { Kostenberech- } \\
\text { nung von } \\
\text { Wahlprogrammen }\end{array}$ \\
\hline Österreich & $\begin{array}{l}\text { Staatsschuldenaus- } \\
\text { schuss (GDC) }\end{array}$ & 1970 & $\begin{array}{l}\text { Keine Daten } \\
\text { verfügbar }\end{array}$ & $\begin{array}{c}15 \text { Ausschussmitglieder, } \\
3 \text { Mitarbeiter }\end{array}$ & च & - & - & O & O \\
\hline Belgien & $\begin{array}{l}\text { Hoher Finanzrat } \\
\text { (HCF) }\end{array}$ & 1936 & $\begin{array}{l}\text { Keine Daten } \\
\text { verfügbar }\end{array}$ & $\begin{array}{l}27 \text { Ratsmitglieder, } \\
14 \text { Mitarbeiter }\end{array}$ & - & $\bullet$ & $\bullet$ & O & O \\
\hline Kanada & $\begin{array}{l}\text { Parliamentary Bud- } \\
\text { get Officer (PBO) }\end{array}$ & 2008 & 2,8 Mio. kan\$ & 15 & $\square$ & $\bullet$ & O & $\bullet$ & O \\
\hline Frankreich & $\begin{array}{c}\text { Hoher Rat } \\
\text { für öffentliche } \\
\text { Finanzen (HCFP) }\end{array}$ & 2013 & $\begin{array}{c}782000 \text { Euro (Finanz- } \\
\text { jahr 2013) }\end{array}$ & $\begin{array}{l}10 \text { Ratsmitglieder, } \\
\text { Einstellung von } \\
5 \text { Mitarbeitern im Gange }\end{array}$ & घ & O & - & 0 & O \\
\hline Irland & Fiskalischer Beirat & 2011 & $\begin{array}{c}800000 \text { Euro (Finanz- } \\
\text { jahr 2013) }\end{array}$ & $\begin{array}{c}5 \text { Ratsmitglieder, } \\
\text { 3 Sekretariatsmitarbeiter }\end{array}$ & घ & O & $\bullet$ & O & O \\
\hline Korea & $\begin{array}{l}\text { National Assembly } \\
\text { Budget Office } \\
\text { (NABO) }\end{array}$ & 2003 & 12,7 Mio. US-\$ & 125 & $\square$ & $\bullet$ & O & $\bullet$ & 0 \\
\hline Mexiko & $\begin{array}{l}\text { Centro de Estudios } \\
\text { de las Finanzas } \\
\text { Públicas (CEFP) }\end{array}$ & 1998 & $\begin{array}{l}\text { 50,9 Mio. Peso } \\
\text { (Finanzjahr 2009) }\end{array}$ & 59 & $\square$ & $\bullet$ & O & $\bullet$ & O \\
\hline Niederlande & Planungsamt & 1945 & 13,5 Mio. Euro & 117 & 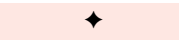 & $\bullet$ & O & $\bullet$ & $\bullet$ \\
\hline Schweden & $\begin{array}{l}\text { Fiscal Policy Council } \\
\text { (FPC) }\end{array}$ & 2007 & 7,55 Mio. SEK & $\begin{array}{c}6 \text { Ratsmitglieder, } \\
5 \text { Sekretariatsmitarbeiter }\end{array}$ & घ & $\bullet$ & $\bullet$ & O & O \\
\hline $\begin{array}{l}\text { Ver. } \\
\text { Königreich }\end{array}$ & $\begin{array}{l}\text { Office for Budget } \\
\text { Responsibility } \\
\text { (OBR) }\end{array}$ & 2010 & 1,75 Mio. £ & $\begin{array}{l}3 \text { köpfiger Ausschuss für } \\
\text { verantwortungsvolle } \\
\text { Haushaltsführung, } \\
2 \text { Nicht-Exekutiv- } \\
\text { Mitglieder, } \\
17 \text { Sekretariatsmitarbeiter }\end{array}$ & r & $\bullet$ & $\bullet$ & $\bullet$ & O \\
\hline Ver. Staaten & $\begin{array}{l}\text { Congressional Bud- } \\
\text { get Office } \\
\text { (CBO) }\end{array}$ & 1974 & 45,2 Mio. US-\$ & 250 & $\square$ & $\bullet$ & 0 & $\bullet$ & 0 \\
\hline
\end{tabular}

Ja.

Nein.

$\diamond$ Keine Bedeutung.

$\downarrow$ Erstellung amtlicher Prognosen.

nur Prognosebeurteilung.

$\square$ Erstellung alternativer Prognosen.

Quelle: OECD (2013, erscheint demnächst), Principles for Independent Fiscal Institutions and Country Notes, OECD Publishing, Paris. 


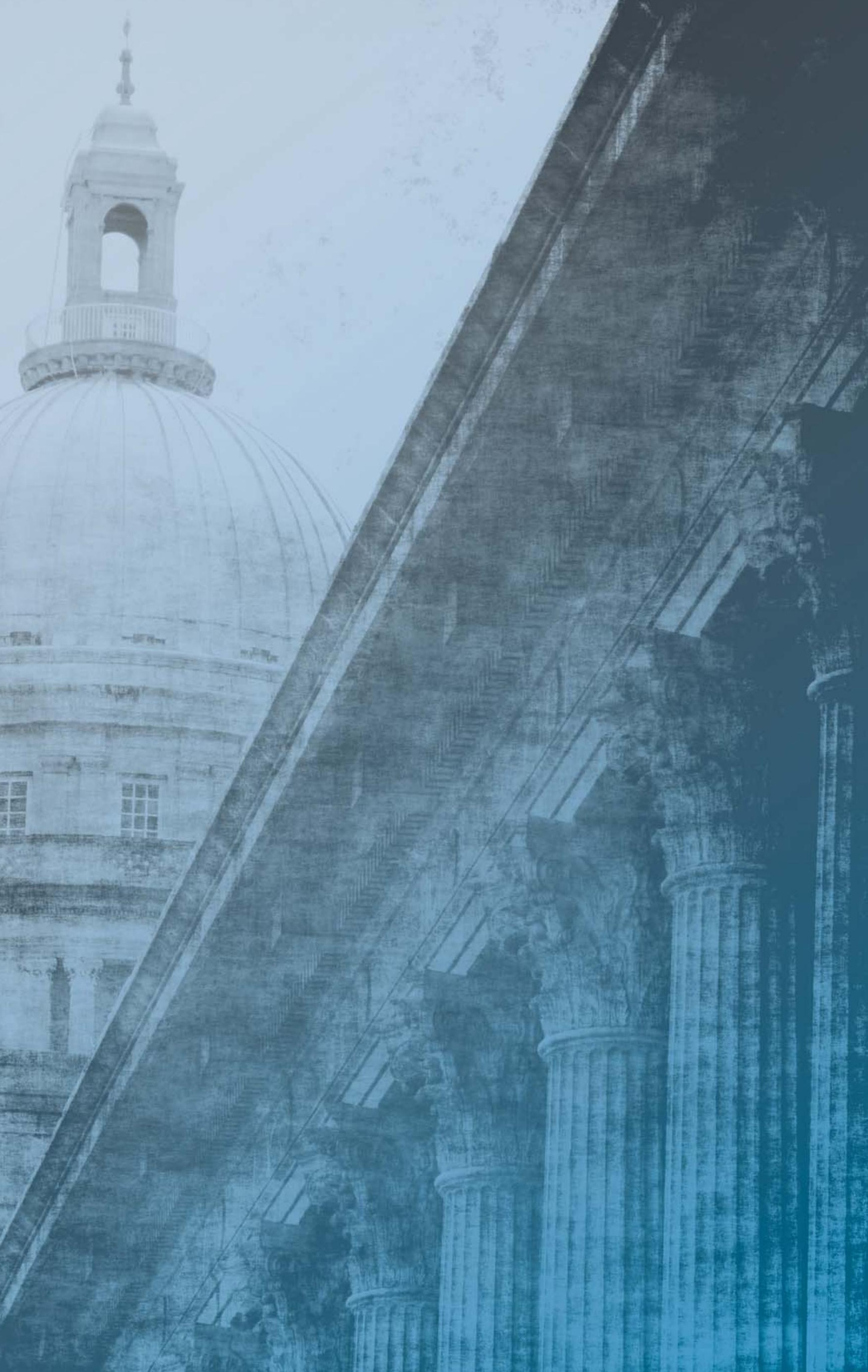



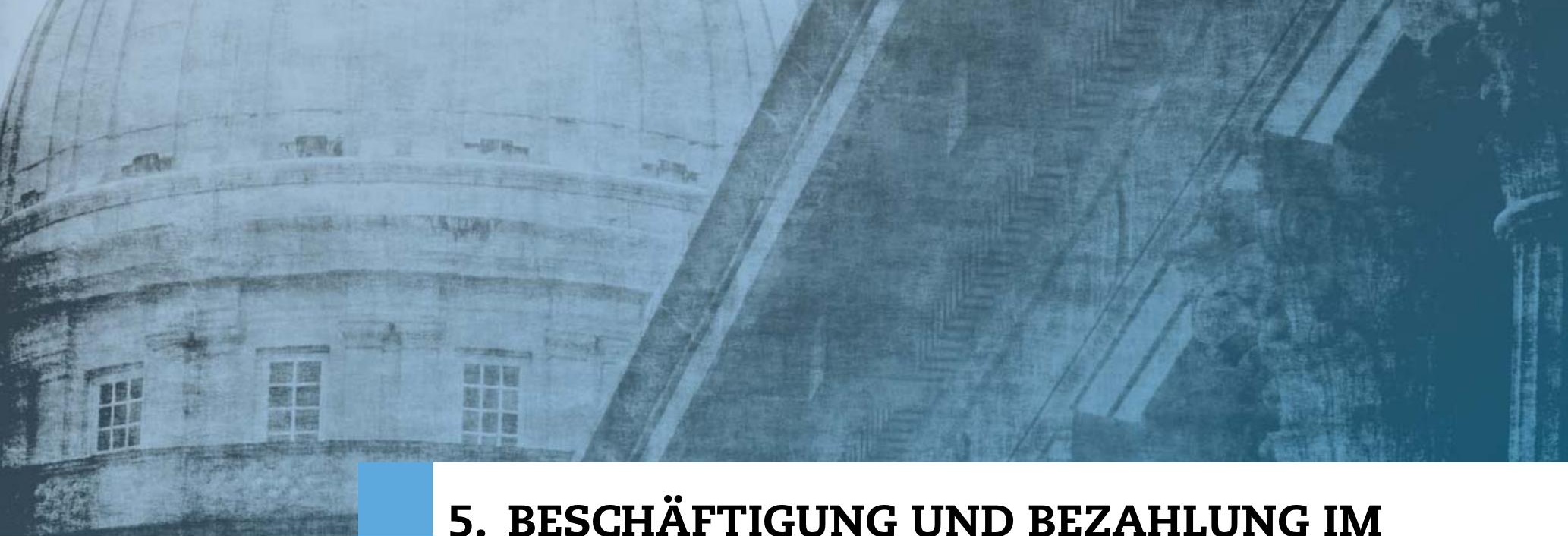

\section{BESCHÄFTIGUNG UND BEZAHLUNG IM ÖFFENTLICHEN SEKTOR}

Die Größe des Staatssektors ist häufig mit der Beschäftigtenzahl verbunden, obgleich die öffentliche Beschäftigung infolge des zunehmenden Outsourcing staatlicher Aufgaben immer weniger eine präzise Messgröße für die Reichweite der Staatstätigkeit ist. In bestimmten Schlüsselberufen - wie Lehrer/in, Krankenpfleger/in, Arzt/Ärztin und Polizeibeamter/ -beamtin - ist der Staat jedoch nach wie vor der größte Arbeitgeber.

In diesem Kapitel werden die Beschäftigungstrends im öffentlichen Sektor in den Jahren 2001 und 2011 untersucht. Die Daten zeigen erneut, dass die öffentliche Beschäftigung „unbeweglich“ ist. Zwar haben viele OECD-Mitgliedsländer im Rahmen ihrer Haushaltskonsolidierungspläne Einstellungsstopps bzw. Stellenkürzungen angekündigt, einen umfangreichen Beschäftigungsabbau im öffentlichen Sektor konnten jedoch nur wenige Länder aufrechterhalten. Da die Nachfrage nach öffentlichen Dienstleistungen nicht sinkt, gibt es zwei Möglichkeiten, die Beschäftigung auf lange Sicht zu reduzieren: Produktivitätssteigerungen, die selten quantifizierbar sind, von denen aber angenommen wird, dass sie eher gering sind, und Outsourcing, bei dem der Staat weiterhin für die Dienstleistungen bezahlt, diese jedoch vom privaten Sektor erbracht werden.

Neben den Daten zur öffentlichen Beschäftigung wecken wenig andere Themen mehr Interesse als die Frage, wie viel Beschäftigte im öffentlichen Dienst verdienen. Gegenüber der Ausgabe 2011 werden hier die bislang umfassendsten, international vergleichbaren Daten zur Vergütung aufgeführt. Die Daten wurden anhand eines verbesserten Erhebungsinstruments für eine erweiterte Gruppe von Schlüsselberufen auf der Ebene der Zentralregierung gesammelt. Außer der Vergütung von leitenden und mittleren Führungskräften, akademischen Fachkräften und Sekretariatskräften wird auch die Vergütung für wichtige Dienstleistungsberufe wie Lehrer/in, Beamter/Beamtin der Grenzpolizei, Steuerbeamter/-beamtin, Zollbeamter/-beamtin und Polizeibeamter/-beamtin gezeigt. Die Zahl der Länder, die an der Datenerhebung teilgenommen haben, ist zwischen 2011 und 2013 von 20 auf 26 Länder gestiegen. Allerdings konnten nicht alle teilnehmenden Länder Daten für alle Berufe vorlegen. 
Jeder Staat entscheidet darüber, welche öffentlichen Dienstleistungen er anbietet und wie sie zu erbringen sind. Einige Länder setzen zur Erbringung von Dienstleistungen mehr öffentlich Bedienstete ein als andere Länder: Lehrkräfte, Erbringer von Gesundheitsleistungen, Arbeitnehmer im Sicherheitsbereich und beim Katastrophenschutz sowie Verwaltungsangestellte gelten z.B. in vielen Mitgliedsländern als öffentlich Bedienstete und erbringen wichtige Dienstleistungen. Andere Länder hingegen nutzen verstärkt den privaten und den gemeinnützigen Sektor. Der Anteil der Beschäftigten im öffentlichen Sektor spiegelt diese Entscheidungen wider und ist ein Faktor zur Bestimmung der tatsächlichen Kosten der Dienstleistungserbringung für die Steuerzahler. Der relative Umfang der öffentlichen Beschäftigung kann auch einen Effekt auf den Arbeitsmarkt haben, da er sich auf die Produktivität der Wirtschaft auswirken kann.

Der Umfang der öffentlichen Beschäftigung unterscheidet sich zwischen den einzelnen OECD-Mitgliedsländern erheblich, wobei der Staat in den nordischen Ländern einen höheren Anteil der Erwerbsbevölkerung beschäftigt als es in anderen Ländern der Fall ist. So beschäftigte beispielsweise der Staat in Norwegen und Dänemark 2011 rd. 30\% der Erwerbsbevölkerung, im Vergleich zu höchstens $9 \%$ in Korea, Japan, Griechenland und Mexiko.

In den einzelnen OECD-Mitgliedsländern ist die Beschäftigung im Sektor Staat als Anteil an der Erwerbsbevölkerung zwischen 2001 und 2011 mit durchschnittlich knapp unter 16\% relativ stabil geblieben. Insgesamt ist der Anteil in diesem Zeitraum nur in nominaler Rechnung gesunken, wobei die stärksten Rückgänge von knapp über 2 Prozentpunkten in der Slowakischen Republik, Schweden, Mexiko und Portugal verzeichnet wurden. Die Beschäftigtenzahl in öffentlichen Unternehmen - wie bei Post und Bahn in manchen OECD-Ländern - stellt nur einen kleinen Anteil der Erwerbsbevölkerung dar und ist in der Regel geringer als im Sektor Staat. Im Allgemeinen beschäftigen öffentliche Unternehmen in mittel- und osteuropäischen Ländern trotz der in diesen Ländern Anfang der 1990er Jahre erfolgten starken Privatisierungswelle einen verhältnismäßig größeren Anteil der Erwerbsbevölkerung als dies in anderen OECD-Mitgliedsländern der Fall ist. Insgesamt ist die Beschäftigung in öffentlichen Unternehmen in Prozent der Erwerbsbevölkerung in der Mehrheit der OECD-Mitgliedsländer zwischen 2001 und 2011 von durchschnittlich 5,7\% auf 4,7\% zurückgegangen.

\section{Methodik und Definitionen}

Die Daten beziehen sich auf 2001 und 2011 und wurden von der Internationalen Arbeitsorganisation (ILO) und der OECD erhoben. Die Daten basieren auf den Definitionen des Systems der Volkswirtschaftlichen Gesamtrechnungen (SNA) und beziehen sich auf die Beschäftigung im Sektor Staat und in den öffentlichen Unternehmen. Der Sektor Staat umfasst alle staatlichen Ebenen (Zentralregierung, Gliedstaaten, Gemeinden und Sozialversicherungsträger). und erstreckt sich auf die wichtigsten Ministerien, Ämter und Behörden sowie gemeinnützige Einrichtungen, die staatlicher Kontrolle unterliegen und zum Großteil von der öffentlichen Hand finanziert werden. Öffentliche Unternehmen sind Rechtspersonen, deren Eigentümer und Kontrollorgan in erster Linie der Staat ist und die Waren und Dienstleistungen für den Absatz auf dem Markt produzieren. Zu den öffentlichen Unternehmen gehören auch Quasi-Unternehmen.
Die Daten beziehen sich auf die Zahl der Beschäftigten, mit Ausnahme Österreichs, der Tschechischen Republik, Italiens, der Niederlande und Neuseelands, wo es sich um Vollzeitäquivalente (VZÄ) handelt. Infolgedessen sind die Beschäftigtenzahlen für diese fünf Länder im Vergleich zu niedrig angegeben. Die Erwerbsbevölkerung bzw. die ökonomisch aktive Bevölkerung umfasst alle Personen, die die Voraussetzungen für die Berücksichtigung als Beschäftigte oder Arbeitslose erfüllen. Für Zwecke der internationalen Vergleichbarkeit wird die Bevölkerung im erwerbsfähigen Alter gemeinhin definiert als Personen ab 15 Jahre, obgleich dies in manchen Ländern variieren kann. Erwerbsbevölkerung bezieht sich auf alle Personen im Erwerbsalter, die in einem bestimmten Referenzzeitraum das Arbeitsangebot für die Produktion von Waren und Dienstleistungen stellen.

\section{Weitere Informationen}

OECD (2011), Public Servants as Partners for Growth: Toward a Stronger, Leaner and More Equitable Workforce, OECD Publishing, Paris, http://dx.doi.org/10.1787/9789264166707-en.

Pilichowski, E. und E. Turkisch (2008), „Employment in Government in the Perspective of the Production Costs of Goods and Services in the Public Domain“, OECD Working Papers on Public Governance, Nr. 8, OECD Publishing, Paris, http://dx.doi.org/ $10.1787 / 245160338300$.

\section{Anmerkungen zu den Abbildungen}

Für Island sind keine Daten verfügbar. Die Daten für Australien und Chile beziehen sich auf den öffentlichen Sektor (Sektor Staat und öffentliche Unternehmen). Die Daten für Deutschland, Irland, Schweden, das Vereinigte Königreich und die Ukraine beziehen sich auf 2010 anstelle von 2011. Die Daten für Ungarn, Japan, Mexiko, Brasilien und die Russische Föderation beziehen sich auf 2009 anstelle von 2011. Die Daten für Chile, Griechenland, Portugal und die Schweiz beziehen sich auf 2008 anstelle von 2011. Die Daten für Frankreich beziehen sich auf 2006 anstelle von 2011. Die Daten für die Ukraine beziehen sich auf 2002 anstelle von 2001.

5.1: Daten für 2001 sind für Korea und die Türkei nicht verfügbar, diese Länder sind daher nicht im OECD-Durchschnitt berücksichtigt. Die Daten für Norwegen beziehen sich auf 2010 anstelle von 2011. Die Daten für Südafrika beziehen sich auf 2006 anstelle von 2011.

5.2: Für Österreich, Belgien, Ungarn, Israel, Italien, Japan, Korea, Portugal und die Vereinigten Staaten sind keine Daten über öffentliche Unternehmen vorhanden, und daher sind diese Länder nicht aufgeführt. Die Daten für die Tschechische Republik beziehen sich auf 2010 anstelle von 2011. Die Daten für Finnland beziehen sich auf 2008 anstelle von 2011. Die Daten für Norwegen beziehen sich auf 2007 anstelle von 2011. Die Daten für die Niederlande beziehen sich auf 2005 anstelle von 2011.

Hinweis zu den Daten für Israel: http://dx.doi.org/10.1787/888932315602. 


\section{BESCHÄFTIGUNG UND BEZAHLUNG IM ÖFFENTLICHEN SEKTOR}

\subsection{Beschäftigung im Sektor Staat in Prozent der Erwerbsbevölkerung (2001 und 2011)}

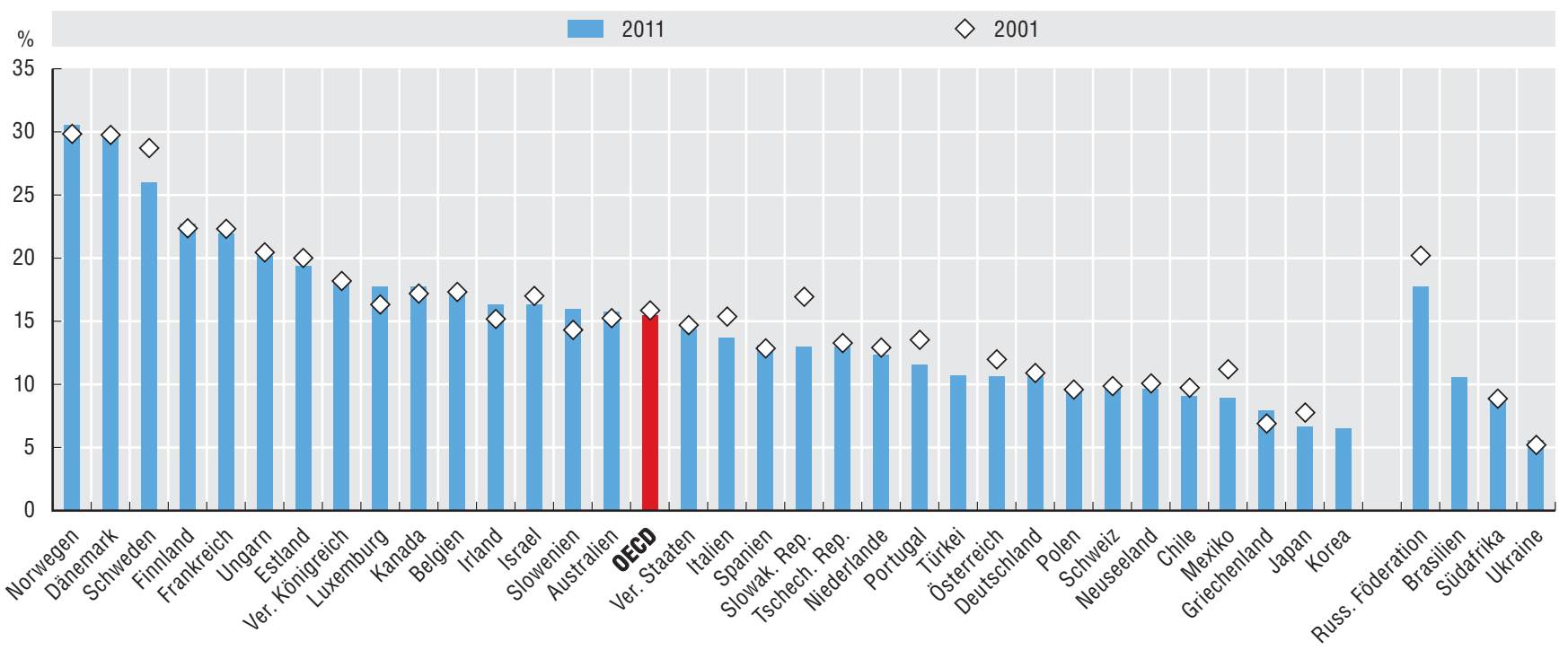

Quelle: Internationale Arbeitsorganisation (ILO), LABORSTA (Datenbank); OECD Labour Force Statistics (Datenbank). Die Daten für Korea wurden von Regierungsvertretern zur Verfügung gestellt.

StatLink त्राड़ http://dx.doi.org/10.1787/888932942241

5.2. Beschäftigung im Sektor Staat und in öffentlichen Unternehmen in Prozent der Erwerbsbevölkerung (2001 und 2011)

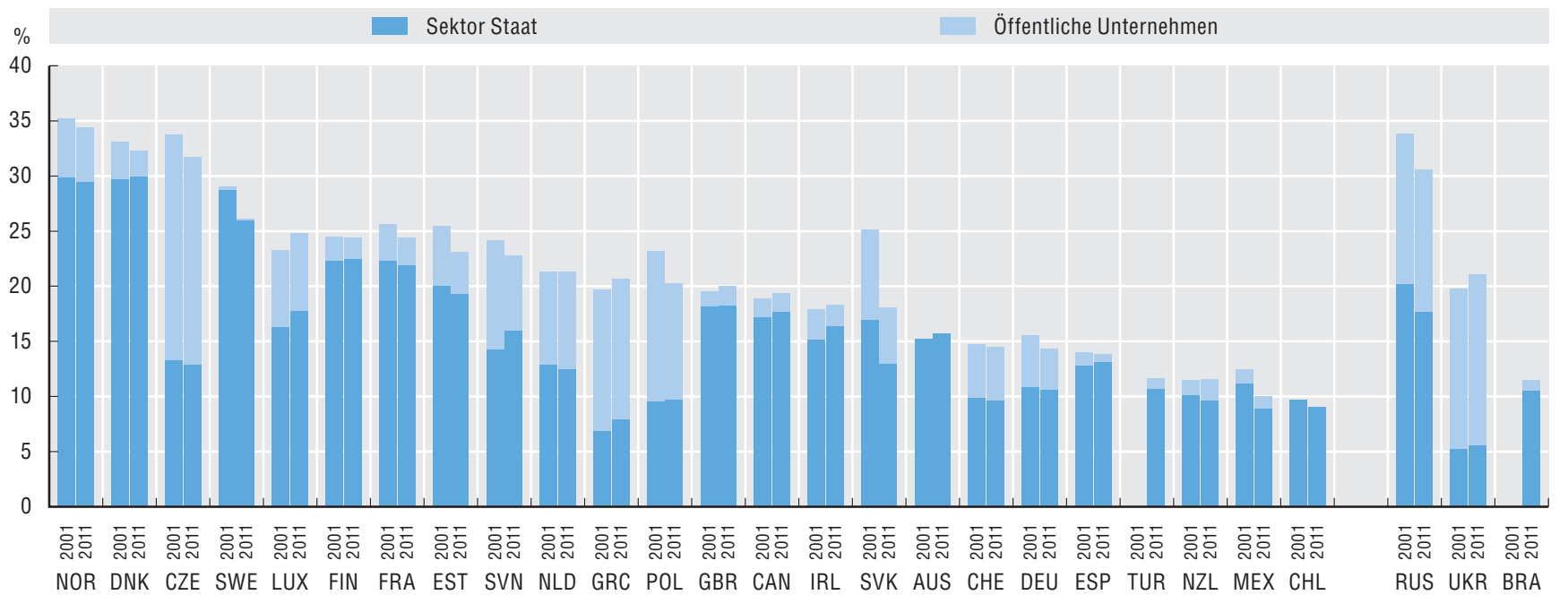

Quelle: Internationale Arbeitsorganisation (ILO), LABORSTA (Datenbank); OECD Labour Force Statistics (Datenbank).

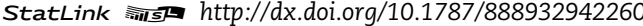


Der Anteil der auf den nachgeordneten Verwaltungsebenen tätigen öffentlich Bediensteten ist ein Indikator für den Grad der Dezentralisierung der öffentlichen Verwaltung. In der Regel deutet ein größerer Anteil des Personalbestands auf nachgeordneter staatlicher Ebene darauf hin, dass mehr Zuständigkeiten bei der Erbringung öffentlicher Dienstleistungen bei den Regionen und Gemeinden liegen. Die Dezentralisierung kann zwar die Art und Weise verbessern, wie der Staat auf die lokalen Bedürfnisse und Prioritäten eingeht, doch kann sie innerhalb der einzelnen Länder auch zu Unterschieden bei der Erbringung von Dienstleistungen führen.

2011 hatten die meisten Länder mehr Beschäftigte auf nachgeordneter als auf zentraler Ebene. In Föderal- bzw. Bundesstaaten ist weniger als ein Drittel aller öffentlich Bediensteten auf Ebene der Zentralregierung beschäftigt, ein Hinweis darauf, dass der Dezentralisierungsgrad dort höher ist. Bei Einheitsstaaten variieren die Prozentsätze öffentlich Bediensteter in der Zentralverwaltung wesentlich stärker, sie reichen von weniger als $20 \%$ in Japan und Schweden bis rd. $90 \%$ in Irland, der Türkei und Neuseeland.

Der Anteil der öffentlich Bediensteten ist zwischen 2001 und 2011 auf Ebene der Zentralregierung relativ stabil geblieben, wobei in der Mehrheit der OECD-Mitgliedsländer ein leichter Trend hin zu einer stärkeren Dezentralisierung zu erkennen ist. In ein paar Ländern hat sich in diesem Zeitraum eine starke Dezentralisierung vollzogen, so in Spanien, der Tschechischen Republik und in Japan, wo der Anteil der auf nachgeordneter staatlicher Ebene tätigen öffentlich Bediensteten um mindestens 10 Prozentpunkte gestiegen ist. Im Fall Spaniens war dieser Anstieg durch die Übertragung von Zuständigkeiten an die nachgeordneten Gebietskörperschaften sowie den seit 2010 umgesetzten Personalabbau bedingt. Nur in einem Land Norwegen - war in diesem Zeitraum eine bemerkenswerte Zentralisierung des Personalbestands im öffentlichen Dienst zu beobachten und dort hat sich der Anteil des Personalbestands auf Ebene der Zentralregierung um 13 Prozentpunkte erhöht, obgleich nahezu zwei Drittel der öffentlich Bediensteten nach wie vor auf Ebene der nachgeordneten Gebietskörperschaften beschäftigt sind.

\section{Methodik und Definitionen}

Die Daten wurden von der Internationalen Arbeitsorganisation (ILO) und der OECD erhoben und beziehen sich auf 2001 und 2011, sofern nicht anders angegeben. Die Daten basieren auf den Definitionen des Systems der Volkswirtschaftlichen Gesamtrechnungen (SNA) und beziehen sich auf die Beschäftigung auf der Ebene der Zentralregierung und der nachgeordneten Gebietskörperschaften. Bei den nachgeordneten Gebietskörperschaften handelt es sich um Gliedstaaten und lokale Gebietskörperschaften, einschließlich Regionen, Provinzen und Kommunen. Zusammen bilden die Zentralregierung und die nachgeordneten Gebietskörperschaften den Sektor Staat. Zusätzlich übermittelten die Länder Beschäftigungsdaten zu den ebenfalls zum Sektor Staat zählenden Sozialversicherungsträgern, zu denen alle institutionellen Einheiten auf zentraler, gliedstaatlicher und lokaler Ebene gehören, deren Haupttätigkeit die Bereitstellung von Sozialleistungen ist. Da Sozialversicherungsträger auf verschiedenen staatlichen Ebenen tätig sind, wurde die Beschäftigung in dieser Kategorie separat erfasst, sofern nicht anders angegeben. In den meisten Ländern, außer in Frankreich, Mexiko und Deutschland, ist die Beschäftigtenzahl der Sozialversicherungsträger gering und stellt nur einen kleinen Prozentsatz des gesamten Personalbestands dar. In den Datensätzen handelt es sich bei den folgenden Ländern um Föderal- bzw. Bundesstaaten: Belgien, Brasilien, Kanada, Deutschland, Mexiko, Russische Föderation, Spanien (wird als Quasi-Föderalstaat betrachtet), Schweiz und Vereinigte Staaten.

Die Daten beziehen sich auf die Zahl der Beschäftigten, mit Ausnahme Italiens, der Niederlande und Neuseelands, wo es sich um Vollzeitäquivalente (VZÄ) handelt. Infolgedessen sind die Beschäftigtenzahlen für diese drei Länder im Vergleich zu niedrig angegeben.

\section{Weitere Informationen}

Charbit, C. und M. Michalun (2009), „Mind the Gaps: Managing Mutual Dependence in Relations among Levels of Government", OECD Working Papers on Public Governance, Nr. 14, OECD Publishing, Paris, http://dx.doi.org/10.1787/221253707200.

OECD (2011), Public Servants as Partners for Growth: Toward a Stronger, Leaner and More Equitable Workforce, OECD Publishing, Paris, http://dx.doi.org/10.1787/9789264166707-en.

\section{Anmerkungen zu den Abbildungen}

Für Australien, Österreich, Chile, Island, Korea, Polen, die Slowakische Republik und das Vereinigte Königreich stehen keine Daten zur Verfügung. Die Beschäftigtenzahl der Sozialversicherungsträger wurde für Kanada, Estland, Irland, Japan, Neuseeland, Norwegen, Spanien, die Schweiz und die Vereinigten Staaten nicht separat erfasst (d.h. sie wurde in die Beschäftigtenzahl der Zentralregierung und/ oder der nachgeordneten Gebietskörperschaften eingerechnet). Die Daten für Deutschland, Irland, Norwegen und Schweden beziehen sich auf 2010 anstelle von 2011. Die Daten für Japan, Mexiko und die Russische Föderation beziehen sich auf 2009 anstelle von 2011. Die Daten für Griechenland, Ungarn und die Schweiz beziehen sich auf 2008 anstelle von 2011. Die Daten für die Tschechische Republik beziehen sich auf 2007 anstelle von 2011. Die Daten für Frankreich und Südafrika beziehen sich auf 2006 anstelle von 2011.

5.3: Die Daten für Brasilien beziehen sich auf 2009. Die Daten für Portugal beziehen sich auf 2008.

5.4: Für Portugal stehen keine Daten zur Verfügung.

Hinweis zu den Daten für Israel: http://dx.doi.org/10.1787/888932315602. 


\section{BESCHÄFTIGUNG UND BEZAHLUNG IM ÖFFENTLICHEN SEKTOR}

Beschäftigung nach staatlichen Fbenen

\subsection{Verteilung der Beschäftigung auf die verschiedenen staatlichen Ebenen (2011)}

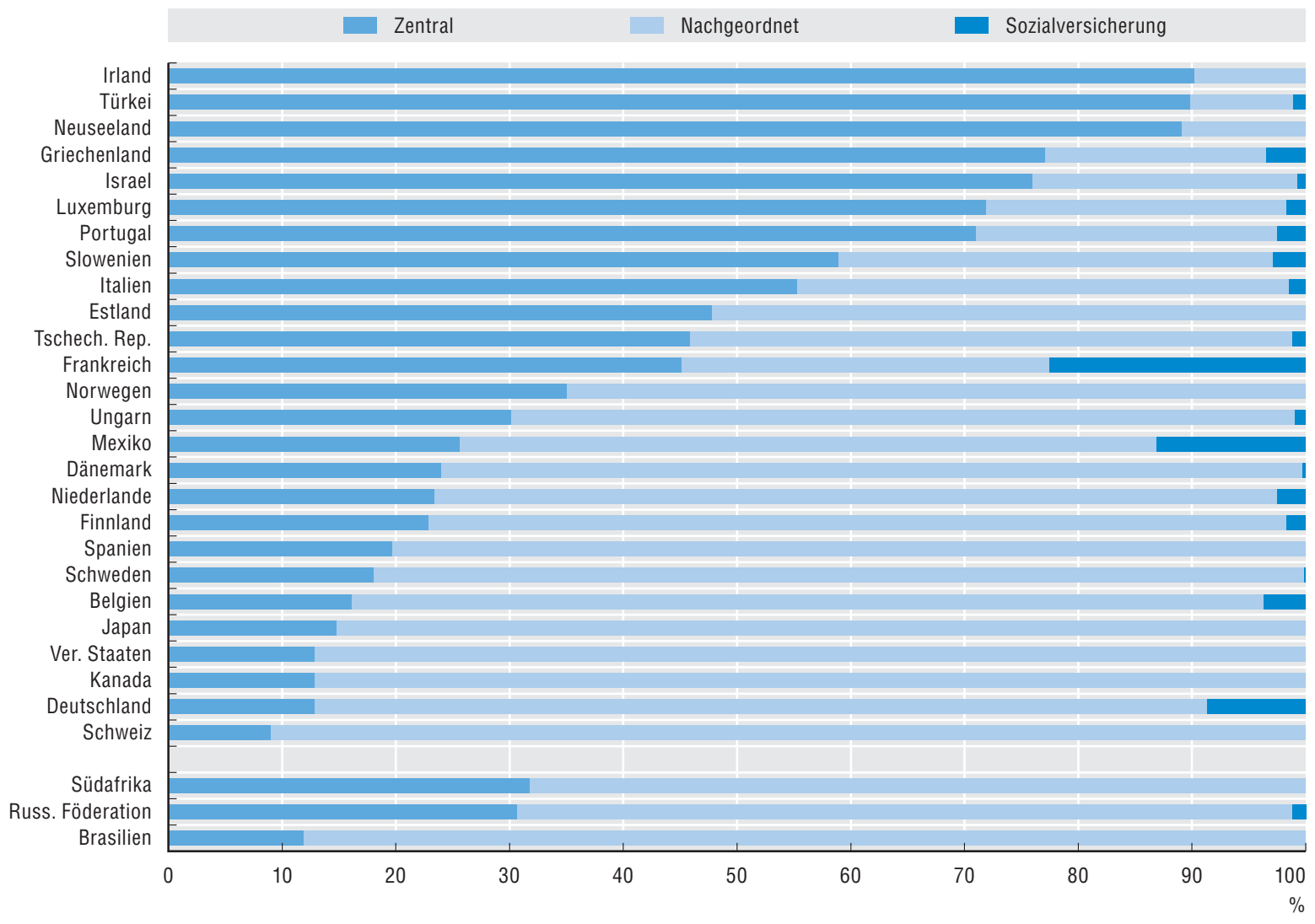

Quelle: Internationale Arbeitsorganisation (ILO), LABORSTA (Datenbank).

\subsection{Veränderung des Prozentsatzes der öffentlich Bediensteten auf Ebene der Zentralregierung (2001 und 2011$)$}

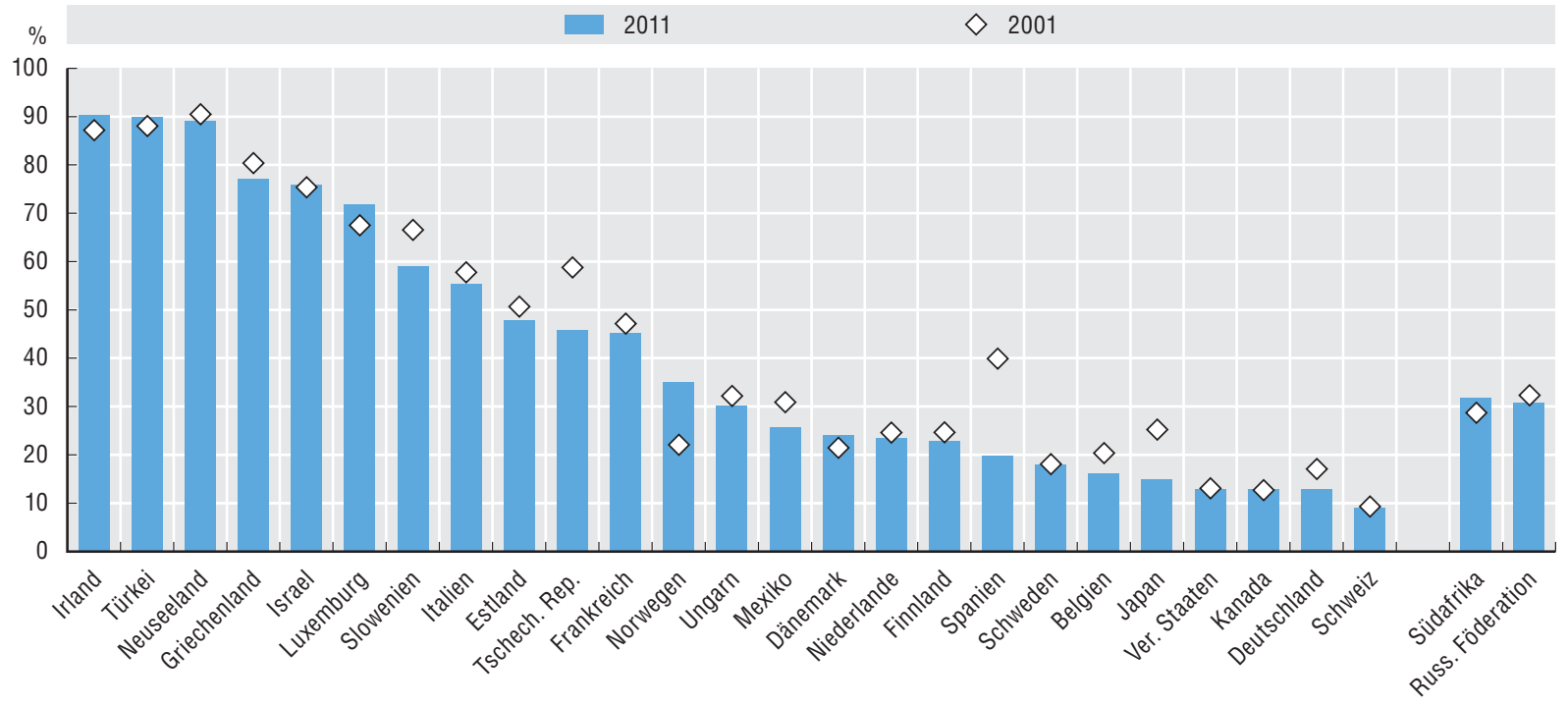

Quelle: Internationale Arbeitsorganisation (ILO), LABORSTA (Datenbank). 
Das Niveau der Gesamtvergütung leitender Führungskräfte im öffentlichen Sektor ist ein Indikator für die Attraktivität des Staatsdienstes und seine Fähigkeit, hochqualifizierte Kräfte in Positionen mit viel Verantwortung in Regierung und Verwaltung zu binden. Die Vergütung in diesen Positionen entspricht zwar einem minimalen Anteil der öffentlichen Ausgaben, sie hat aber einen symbolischen Wert, da sie Staatsbedienstete betrifft, die bei der Politikgestaltung und -umsetzung eine führende Rolle spielen und deren Ernennung häufig auf Ermessensbasis erfolgt.

Führungskräfte der Stufe D1 sind öffentliche Amtsträger in Spitzenpositionen unterhalb des Ministers oder des Staatssekretärs, und Führungskräfte der Stufe D2 sind direkt unter Stufe D1 angesiedelt (vgl. Anhang D wegen Einzelheiten). Führungskräfte der Stufe D1 verdienen im Durchschnitt 32\% mehr als Führungskräfte der Stufe D2, in manchen Fällen kann es aber auch sein, dass sie weniger Gehalt beziehen als Führungskräfte der Stufe D2, weil sie weniger Dienstjahre vorweisen können, z.B. wenn sie ihre Stelle seit einem kürzeren Zeitraum innehaben.

Im Durchschnitt beläuft sich die Vergütung von leitenden Führungskräften der Stufe D1 auf rd. 230000 US-\$ KKP, in der rd. 36000 US-\$ KKP für Arbeitgeber-Sozialversicherungsbeiträge und 31000 US-\$ KKP für Arbeitszeitanpassungen inbegriffen sind. Die Gesamtvergütung auf Stufe D2 liegt bei rd. 175000 US-\$ KKP (einschließlich der Arbeitgeber-Sozialversicherungsbeiträge und bezahlter Urlaubstage). Differenzen bei den Vergütungsniveaus zwischen den einzelnen Ländern resultieren aus Unterschieden beim Anteil der hochqualifizierten Bediensteten, der Dienstaltersstufe und dem Anteil an Frauen in leitenden Positionen. Differenzen können auch auf unterschiedliche Organisationsstrukturen innerhalb der Länder zurückzuführen sein. Im Verhältnis zum Pro-Kopf-BIP (nach Berücksichtigung der Unterschiede bei der wirtschaftlichen Entwicklung der einzelnen Länder) erhielten leitende Führungskräfte der Stufe D1 in Italien, Neuseeland und Chile die höchsten Vergütungen, während D1-Führungskräfte in Island, der Slowakischen Republik und Norwegen die niedrigsten Vergütungsniveaus aufwiesen.

Differenzen beim Vergütungsniveau können auch durch Unterschiede auf den nationalen Arbeitsmärkten bedingt sein, insbesondere die Entlohnung im privaten Sektor für vergleichbare Kompetenzen. Dieser Effekt wird teilweise erfasst, indem die durchschnittliche Vergütung leitender Führungskräfte mit dem durchschnittlichen Arbeitsentgelt von Beschäftigten mit Tertiärabschluss verglichen wird. Im Durchschnitt ist das Gehalt einer Führungskraft der Stufe D1 3,4-mal höher als das durchschnittliche Arbeitsentgelt eines Beschäftigten mit Tertiärabschluss.

\section{Methodik und Definitionen}

Die Daten beziehen sich auf 2011 und wurden 2012 durch den von der OECD durchgeführten Survey on the Compensation of Employees in Central/Federal Governments erhoben. Bedienstete von Ministerien und Behörden auf Ebene der Zentralregierung nahmen durch das OECD Network on Public Employment and Management an der Erhebung teil.
Die Daten beziehen sich auf sechs Ministerien der Zentralregierung (Inneres, Finanzen, Justiz, Bildung, Gesundheit und Umwelt bzw. auf das jeweils entsprechende Ressort). Die Klassifizierung und die Definition der Berufe wurde anhand der Internationalen Standardklassifikation der Berufe (ISCO) der Internationalen Arbeitsorganisation (ILO) adaptiert. Die Vergütungsniveaus wurden errechnet, indem die durchschnittliche Vergütung des beschäftigten Personals ermittelt wurde.

Die Gesamtvergütung umfasst Löhne und Gehälter sowie die Arbeitgeber-Sozialversicherungsbeiträge, wobei die entsprechenden Systeme sowohl kapitalgedeckt als auch nicht kapitalgedeckt sein können. Die Sozialversicherungsbeiträge beschränken sich auf das Gesundheitsund das Rentensystem, damit für die einzelnen Länder konsistente Daten vorliegen.

Die Vergütung wurde anhand des BIP auf KKP-Basis mit Hilfe von Daten aus der OECD-Datenbank OECD National Accounts Statistics in US-Dollar umgerechnet. Die Daten wurden nicht um die Wochenarbeitsstunden bereinigt, da von Führungskräften offiziell oder inoffiziell erwartet wird, dass sie mehr Stunden arbeiten, sie wurden aber um die durchschnittliche Zahl an bezahlten Urlaubstagen bereinigt.

Vgl. Anhang D wegen der vollständigen Methodik.

\section{Weitere Informationen}

OECD (2012), Public Sector Compensation in Times of Austerity, OECD Publishing, Paris, http://dx.doi.org/10.1787/9789264177758-en.

\section{Anmerkungen zu den Abbildungen}

Für Kanada, die Tschechische Republik, Ungarn, Irland, Luxemburg, Mexiko, die Schweiz und die Türkei sind keine Daten verfügbar.

In Finnland und Slowenien sind die Daten zur Vergütung für D2-Positionen mit denen für D1-Positionen vermischt. Belgien: Das Bildungsund das Umweltministerium wurden nicht einbezogen, da sie nicht auf föderaler Ebene agieren. Dänemark: Das Bildungsministerium wurde nicht berücksichtigt, weil viele seiner Zuständigkeiten anderen Ministerien übertragen wurden. Estland: Für das Bildungs- und das Umweltministerium sind keine Daten verfügbar. Griechenland, Neuseeland: Es liegen lediglich Daten zum Anfangs- und zum Höchstgehalt vor, der Durchschnitt entspricht daher nicht dem tatsächlichen Durchschnitt, sondern dem Mittelwert zwischen Anfangs- und Höchstgehalt. Island: Das Justizministerium ist Teil des Innenministeriums. Italien: Ein Gesetz von 2011 begrenzt das Vergütungsniveau für leitende Führungskräfte seit 2012 auf maximal 370000 US-\$ KKP. Japan: Daten wurden in Bezug auf die unterste und die höchste Stufe der Gesamtvergütung zur Verfügung gestellt, daher wurde im OECD-Durchschnitt das arithmetische Mittel berücksichtigt. Slowakische Republik: Aus statistischen Gründen wurde nur die Hälfte der im Justizministerium Beschäftigten berücksichtigt (Konsistenz der ISCO-Codes). Zur Deckung von in der Behörde anfallenden unvermeidbaren Kosten und sonstigen persönlichen Aufwendungen hat der Leiter der Verwaltungsbehörde (ein Bediensteter je Ministerium, D1) Anspruch auf eine steuerfreie monatliche Pauschalvergütung in Höhe von 121\% des höchsten Gehaltstarifs (der höchste Gehaltstarif beträgt 935,50 Euro). Dieser Betrag wurde nicht in die Daten einbezogen. Spanien: Die Daten für D1-Positionen umfassen keine Anreizzahlungen. Schweden: Das Innenministerium ist Teil des Amts des Ministerpräsidenten und wurde nicht in den Daten berücksichtigt. Vereinigtes Königreich: Die Daten beziehen sich auf 2012 (unter Verwendung von KKP aus dem Jahr 2012), und der Durchschnitt entspricht eher dem Medianwert als dem arithmetischen Mittel.

Vgl. auch Anhang D wegen weiterer Anmerkungen.

Hinweis zu den Daten für Israel: http://dx.doi.org/10.1787/888932315602. 
5.5. Durchschnittliche jährliche Vergütung von leitenden Führungskräften auf Ebene der Zentralregierung (2011) Bereinigt um Unterschiede bei den bezahlten Urlaubstagen

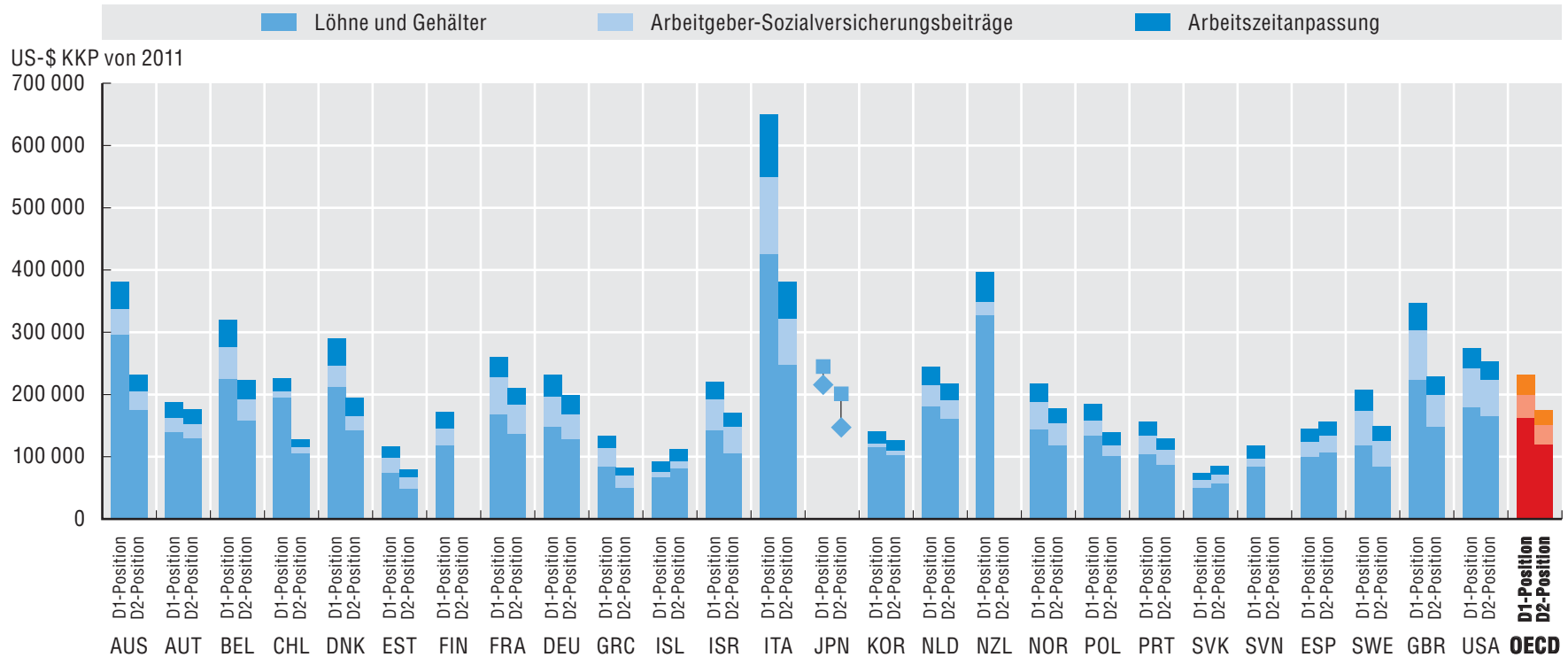

Quelle: 2012 OECD Survey on Compensation of Employees in Central/Federal Governments; OECD STAN/National Accounts Statistics (Datenbank).

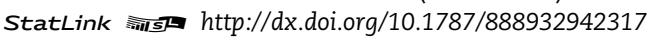

\subsection{Durchschnittliche jährliche Vergütung von leitenden Führungskräften auf Ebene der Zentralregierung im Verhältnis} zum Pro-Kopf-BIP und Arbeitsentgelt von Beschäftigten mit Tertiärabschluss

Verhältnis 2011

$$
\text { D1-Position }
$$

\section{D1/D2-Position gegenüber Arbeitsentgelt Beschäftigter mit Tertiärabschluss}

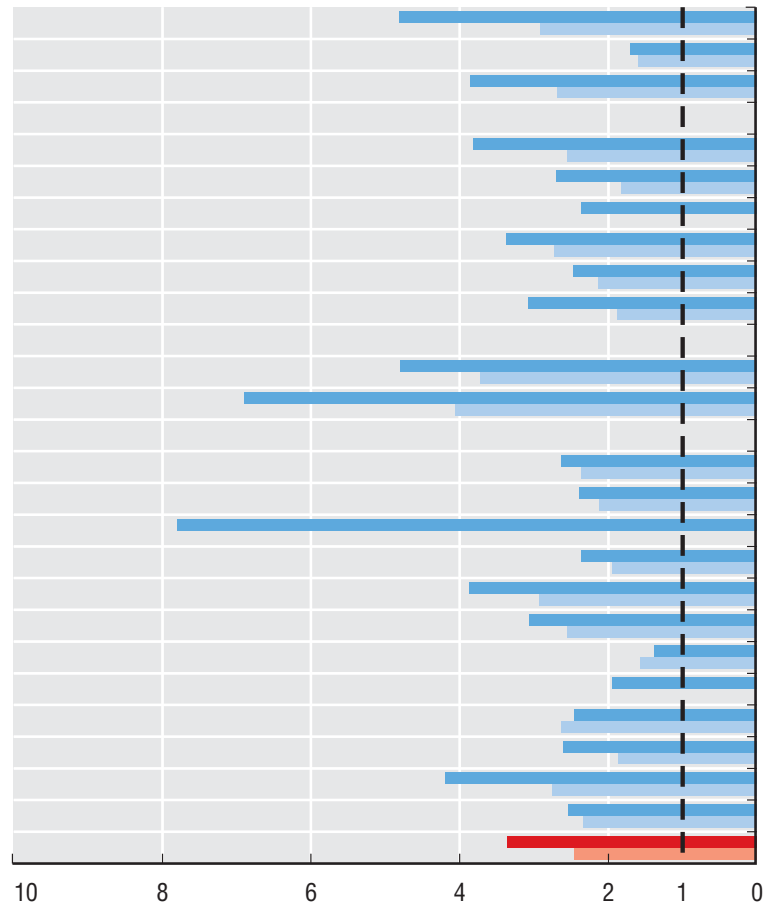

\section{D2-Position}

Australien
Österreich
Belgien
Chile
Dänemark
Estland
Finnland
Frankreich
Deutschland
Griechenland
Island
Israel
Italien
Japan
Korea
Niederlande
Neuseeland
Norwegen
Polen
Portugal
Slowak. Rep.
Slowenien
Spanien
Schweden
Ver. Königreich
Ver. Staaten
OECD

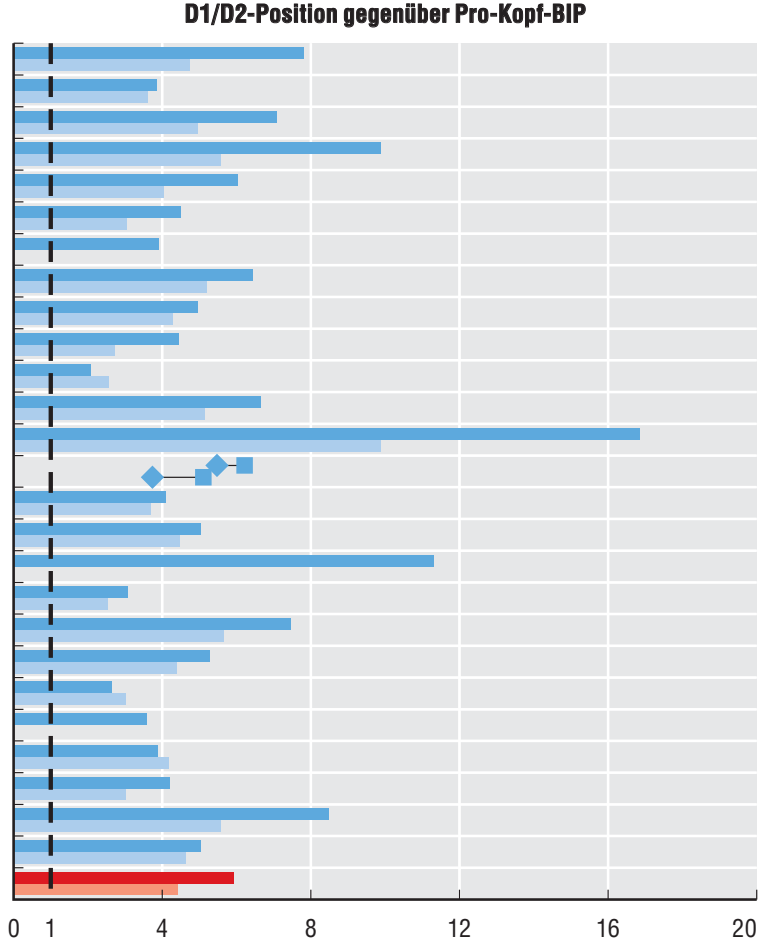

Quelle: 2012 OECD Survey on Compensation of Employees in Central/Federal Governments; OECD STAN/National Accounts Statistics (Datenbank); OECD (2013), Bildung auf einen Blick 2013: OECD-Indikatoren, W. Bertelsmann Verlag, Bielefeld, http://dx.doi.org/10.1787/eag-2013-de.

StatLink त्ताs http://dx.doi.org/10.1787/888932942336 
Mittlere Führungskräfte sind in der Hierarchie der öffentlich Bediensteten auf der Ebene der Zentralregierung zwischen den leitenden Führungskräften und den akademischen Fachkräften angesiedelt. Sie nehmen folglich eine wichtige Mittelstellung ein, um Personalmanagementreformen umzusetzen, mit allen Staatsbediensteten zu interagieren und dabei Vertrauen sowie einen sozialen Dialog aufzubauen.

Im Durchschnitt beläuft sich die Vergütung von mittleren Führungskräften der Stufe D3 auf rd. 126000 US-\$ KKP, wovon 17\% auf die Arbeitgeber-Sozialversicherungsbeiträge und 14\% auf Arbeitszeitanpassungen entfallen. Die Gesamtvergütung auf Stufe D4 liegt bei rd. 113000 US-\$ KKP (einschließlich der Arbeitgeber-Sozialversicherungsbeiträge und bezahlter Urlaubstage).

Führungskräfte der Stufe D3 planen, bestimmen und koordinieren die Arbeitsweise einer Abteilung/Verwaltungseinheit innerhalb eines Ministeriums, und Führungskräfte der Stufe D4 sind direkt unter Stufe D3 angesiedelt (vgl. Anhang D wegen Einzelheiten). Die Differenz bei der Vergütung zwischen D3und D4-Positionen ist kleiner als die Differenz zwischen den beiden leitenden Positionen. Führungskräfte der Stufe D3 verdienen im Durchschnitt 10\% mehr als Führungskräfte der Stufe D4. Außerdem ist die Vergütung der mittleren Führungskräfte deutlich niedriger als die der leitenden Führungskräfte - leitende Führungskräfte der Stufe D1 verdienen $84 \%$ mehr als mittlere Führungskräfte der Stufe D3 und fast doppelt so viel wie Führungskräfte der Stufe D4. Diese zwischen D1- und D4Positionen bestehende Differenz ist in Italien, Australien, dem Vereinigten Königreich und Israel am höchsten und in den Vereinigten Staaten und Korea am niedrigsten.

Im Verhältnis zum Pro-Kopf-BIP erreichten D3-Führungskräfte in Polen und Chile die höchsten Vergütungsniveaus, während sie in Norwegen und Estland in dieser Position am wenigsten verdienten. Desgleichen wurden die höchsten Vergütungsquoten für mittlere Führungskräfte der Stufe D4 in den Vereinigten Staaten verzeichnet, wohingegen die niedrigsten Quoten in Norwegen, Israel und Griechenland anzutreffen waren.

Differenzen beim Vergütungsniveau können auch durch Unterschiede auf den nationalen Arbeitsmärkten bedingt sein. Im Durchschnitt ist das Gehalt einer Führungskraft der Stufe D3 1,8-mal höher als die Vergütung eines Beschäftigten mit Tertiärabschluss. D3-Positionen scheinen in Polen vergleichsweise besser vergütet zu werden, dagegen sind sie in Österreich, Estland und der Slowakischen Republik offenbar weniger attraktiv. Im Vergleich zur Vergütung von Beschäftigten mit Tertiärabschluss waren D4-Positionen in den Vereinigten Staaten und Belgien im öffentlichen Sektor wettbewerbsfähiger, im Gegensatz dazu war dies in Österreich, Israel und Norwegen nicht der Fall.

\section{Methodik und Definitionen}

Die Daten beziehen sich auf 2011 und wurden 2012 durch den von der OECD durchgeführten Survey on the Compensation of Employees in Central/Federal Governments erhoben. Bedienstete von Ministerien und Behörden auf Ebene der Zentralregierung nahmen durch das OECD Network on Public Employment and Management an der Erhebung teil.
Die Daten beziehen sich auf sechs Ministerien der Zentralregierung (Inneres, Finanzen, Justiz, Bildung, Gesundheit und Umwelt bzw. auf das jeweils entsprechende Ressort). Die Klassifizierung und die Definition der Berufe wurde anhand der Internationalen Standardklassifikation der Berufe (ISCO) der Internationalen Arbeitsorganisation (ILO) adaptiert. Die Vergütungsniveaus wurden errechnet, indem die durchschnittliche Vergütung des beschäftigten Personals ermittelt wurde.

Die Gesamtvergütung umfasst die Bruttolöhne und -gehälter sowie die Arbeitgeber-Sozialversicherungsbeiträge, wobei die entsprechenden Systeme sowohl kapitalgedeckt als auch nicht kapitalgedeckt sein können. Die Sozialversicherungsbeiträge beschränken sich auf das Gesundheits- und das Rentensystem, damit für die einzelnen Länder konsistente Daten vorliegen.

Die Vergütung wurde anhand des BIP auf KKP-Basis mit Hilfe von Daten aus der OECD-Datenbank OECD National Accounts Statistics in US-Dollar umgerechnet. Die Daten wurden nicht um die Wochenarbeitsstunden bereinigt, da von Führungskräften offiziell oder inoffiziell erwartet wird, dass sie mehr Stunden arbeiten, sie wurden aber um die durchschnittliche Zahl an bezahlten Urlaubstagen bereinigt.

Vgl. Anhang D wegen der vollständigen Methodik.

\section{Weitere Informationen}

OECD (2012), Public Sector Compensation in Times of Austerity, OECD Publishing, Paris, http://dx.doi.org/10.1787/9789264177758-en.

\section{Anmerkungen zu den Abbildungen}

Für Kanada, die Tschechische Republik, Ungarn, Irland, Luxemburg, Mexiko, Neuseeland, die Schweiz und die Türkei sind keine Daten verfügbar.

In Estland, Finnland, Japan und Slowenien sind die Daten zur Vergütung für D4-Positionen mit denen für D3-Positionen vermischt. Belgien: Das Bildungs- und das Umweltministerium wurden nicht einbezogen, da sie nicht auf föderaler Ebene agieren. Dänemark: Das Bildungsministerium wurde nicht berücksichtigt, weil viele seiner Zuständigkeiten anderen Ministerien übertragen wurden. Estland: Für das Bildungs- und das Umweltministerium sind keine Daten verfügbar. Griechenland: Es liegen lediglich Daten zum Anfangs- und zum Höchstgehalt vor, der Durchschnitt entspricht daher nicht dem tatsächlichen Durchschnitt, sondern dem Mittelwert zwischen Anfangs- und Höchstgehalt. Island: Das Justizministerium ist Teil des Innenministeriums. Japan: Daten zu D3-Positionen wurden in Bezug auf die unterste und die höchste Stufe der Gesamtvergütung zur Verfügung gestellt, daher wurde im OECD-Durchschnitt das arithmetische Mittel berücksichtigt. Slowakische Republik: Aus statistischen Gründen wurde nur die Hälfte der im Justizministerium Beschäftigten berücksichtigt (Konsistenz der ISCO-Codes). Schweden: Das Innenministerium ist Teil des Amts des Ministerpräsidenten und wurde nicht in den Daten berücksichtigt. Vereinigtes Königreich: Die Daten beziehen sich auf 2012 (unter Verwendung von KKP aus dem Jahr 2012), und der Durchschnitt entspricht eher dem Medianwert als dem arithmetischen Mittel.

Vgl. auch Anhang D wegen weiterer Anmerkungen.

Hinweis zu den Daten für Israel: http://dx.doi.org/10.1787/888932315602. 
5.7. Durchschnittliche jährliche Vergütung von mittleren Führungskräften auf Ebene der Zentralregierung (2011) Bereinigt um Unterschiede bei den bezahlten Urlaubstagen

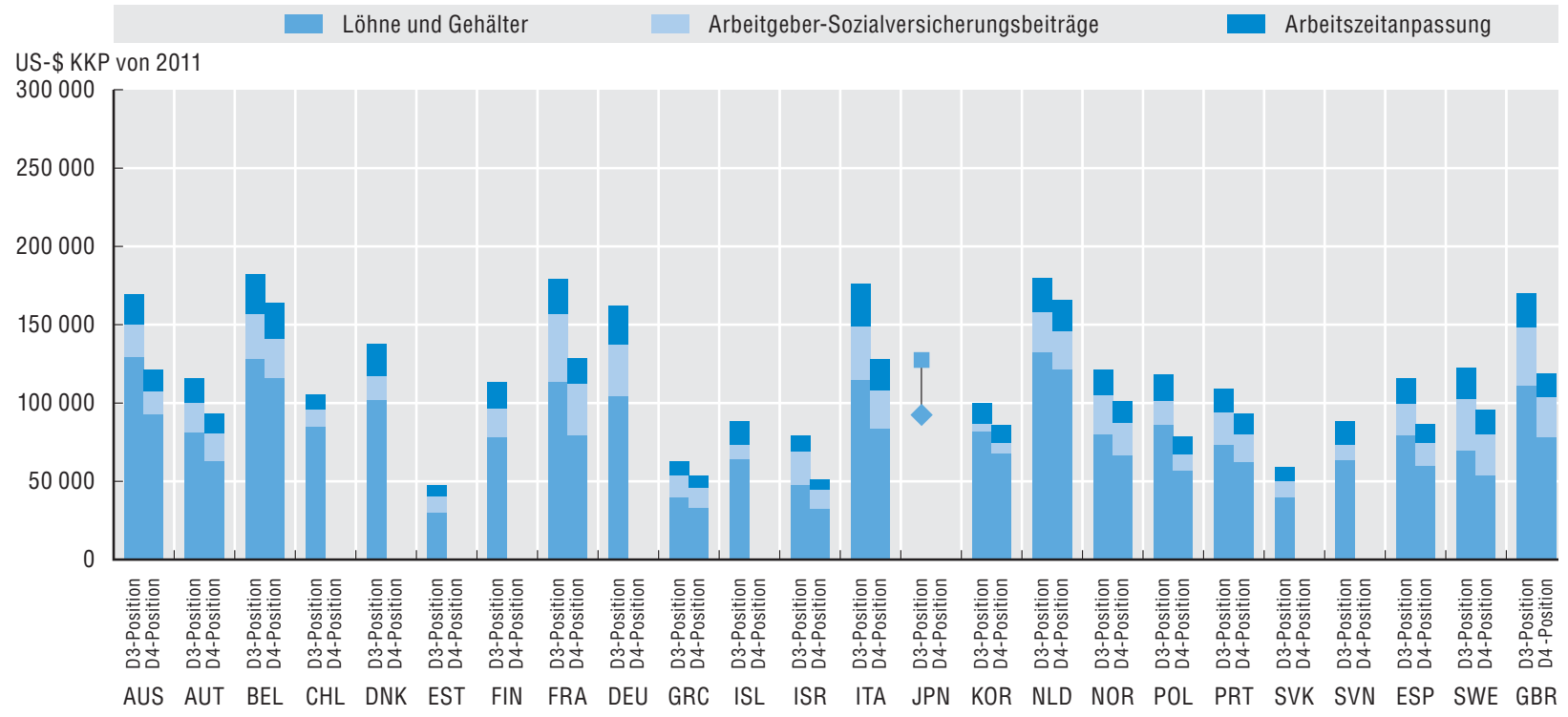

Quelle: 2012 OECD Survey on Compensation of Employees in Central/Federal Governments; OECD STAN/National Accounts Statistics (Datenbank).

StatLink ints http://dx.doi.org/10.1787/888932942355

\subsection{Durchschnittliche jährliche Vergütung von mittleren Führungskräften auf Ebene der Zentralregierung im Verhältnis} zum Pro-Kopf-BIP und Arbeitsentgelt von Beschäftigten mit Tertiärabschluss

Verhältnis 2011

D3-Position

D3/D4-Position gegenüber Arbeitsentgelt Beschäftigter mit Tertiärabschluss

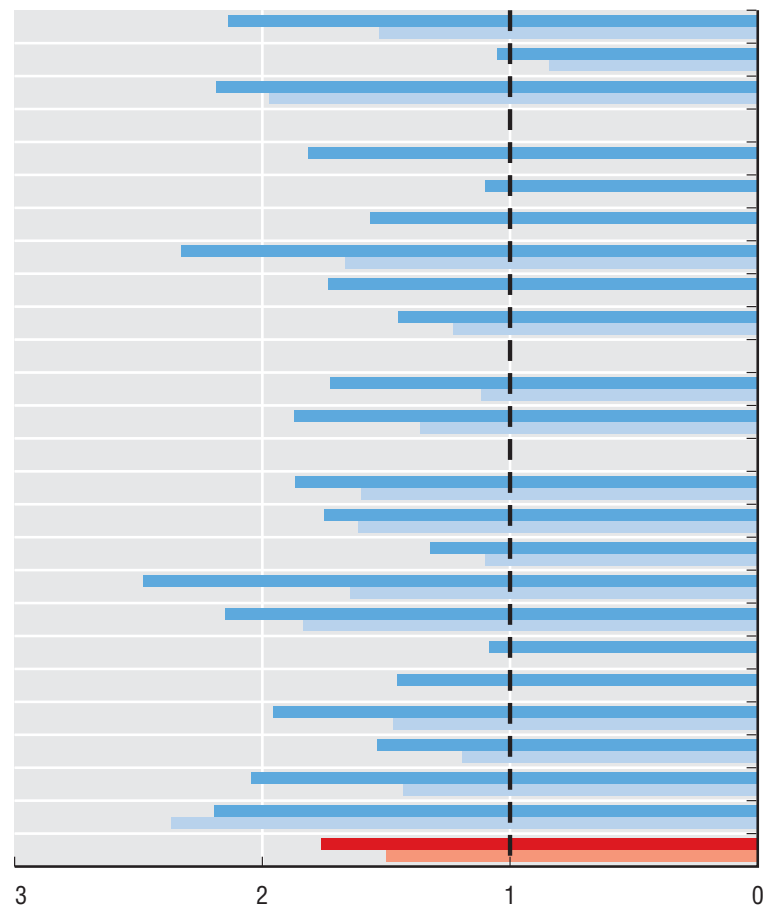

D4-Position

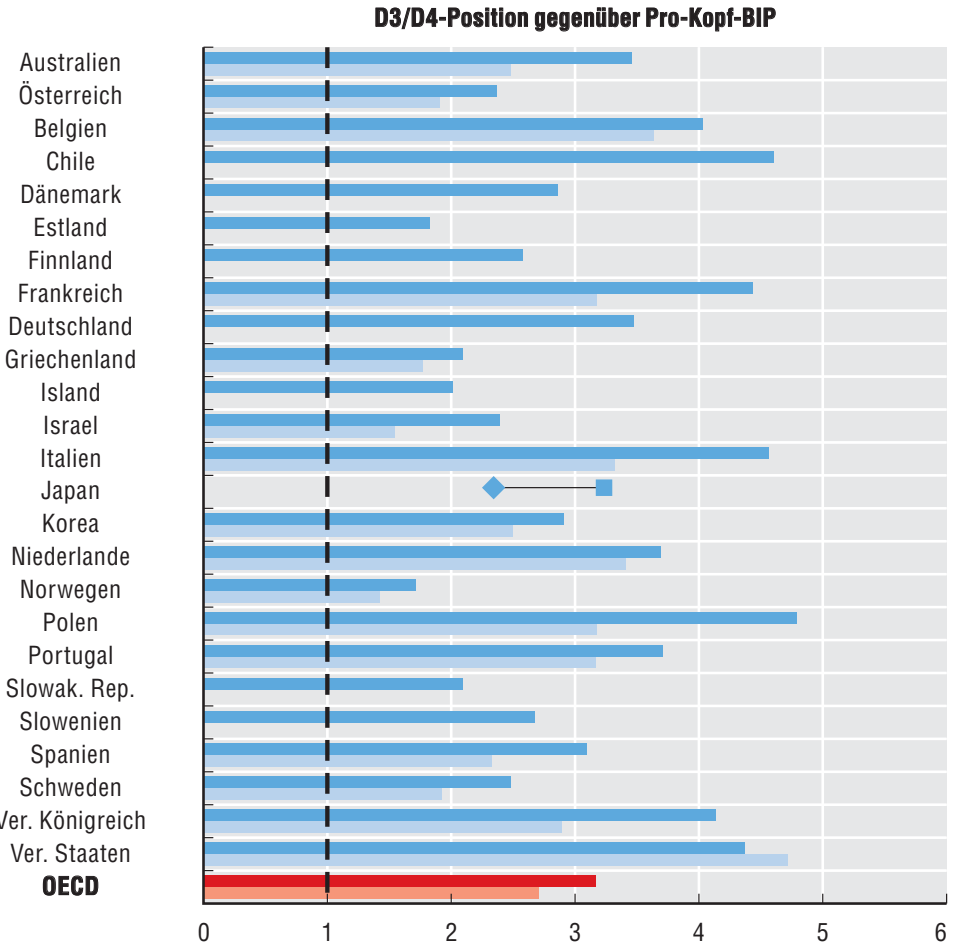

Quelle: 2012 OECD Survey on Compensation of Employees in Central/Federal Governments; OECD STAN/National Accounts Statistics (Datenbank); OECD (2013), Bildung auf einen Blick 2013: OECD-Indikatoren, W. Bertelsmann Verlag, Bielefeld, $h$ ttp://dx.doi.org/10.1787/eag-2013-de.

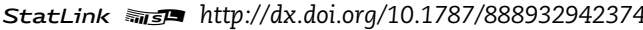


Die Analysekompetenz von Volkswirten und Politikspezialisten ist von entscheidender Bedeutung, um die Fähigkeit der Regierung zu verbessern, evidenzbasierte Entscheidungen zu treffen. Akademische Fachkräfte nehmen keine Führungsaufgaben wahr (sie haben höchstens drei Mitarbeiter unter sich) und sind rangmäßig über den Verwaltungs-/Sekretariatskräften angesiedelt (vgl. Anhang D wegen Einzelheiten). Daten werden für erfahrene und junge akademische Fachkräfte präsentiert.

Im Durchschnitt beläuft sich die Vergütung von erfahrenen akademischen Fachkräften auf rd. 89000 US-\$ KKP, wovon jeweils $17 \%$ auf die Arbeitgeber-Sozialversicherungsbeiträge und Arbeitszeitanpassungen entfallen. Die Vergütung von Nachwuchskräften liegt bei nahezu 72000 US-\$ KKP (einschließlich der Arbeitgeber-Sozialversicherungsbeiträge sowie der geleisteten Arbeitsstunden und bezahlter Urlaubstage).

Erfahrene akademische Fachkräfte verdienen im Durchschnitt 24\% mehr als Nachwuchskräfte. Die Differenz zwischen diesen beiden Stufen ist in Belgien, Chile und Dänemark am höchsten und in Korea am niedrigsten. Leitende Führungskräfte der Stufe D1 verdienen im Durchschnitt 2,6-mal mehr als erfahrene akademische Fachkräfte. Die Differenz zwischen leitenden Führungskräften der Stufe D1 und erfahrenen akademischen Fachkräften ist in Italien und im Vereinigten Königreich am höchsten und in der Slowakischen Republik, Spanien und Island am niedrigsten. Die Erhebung legt den Schluss nahe, dass die Differenzen beim Vergütungsniveau im Ländervergleich zwischen diesen beiden Berufsgruppen in den einzelnen Ländern weniger stark ausgeprägt sind als zwischen den leitenden und den mittleren Führungskräften.

Differenzen bei den Vergütungsniveaus können auf verschiedene Faktoren zurückzuführen sein, die nicht berücksichtigt wurden. Im Verhältnis zum Pro-Kopf-BIP erreichten akademische Fachkräfte in Chile die höchsten Vergütungsniveaus, während in Griechenland, Estland und Island in dieser Berufsgruppe das niedrigste relative Erwerbseinkommen verzeichnet wurde. Im Vergleich zum Arbeitsentgelt von Beschäftigten mit Tertiärabschluss weist die Kategorie der akademischen Nachwuchsfachkräfte im Durchschnitt nahezu dasselbe Vergütungsniveau auf.

\section{Methodik und Definitionen}

Die Daten beziehen sich auf 2011 und wurden 2012 durch den von der OECD durchgeführten Survey on the Compensation of Employees in Central/Federal Governments erhoben. Bedienstete von Ministerien und Behörden auf Ebene der Zentralregierung nahmen durch das OECD Network on Public Employment and Management an der Erhebung teil.

Die Daten beziehen sich auf sechs Ministerien der Zentralregierung (Inneres, Finanzen, Justiz, Bildung, Gesundheit und Umwelt bzw. auf das jeweils entsprechende Ressort). Die Klassifizierung und die Definition der Berufe wurde anhand der Internationalen Standardklassifikation der Berufe (ISCO) der Internationalen Arbeitsorganisation (ILO) adaptiert. Die Vergütungsniveaus wurden errechnet, indem die durchschnittliche Vergütung des beschäftigten Personals ermittelt wurde.
Die Gesamtvergütung umfasst die Bruttolöhne und -gehälter sowie die Arbeitgeber-Sozialversicherungsbeiträge, wobei die entsprechenden Systeme sowohl kapitalgedeckt als auch nicht kapitalgedeckt sein können. Die Sozialversicherungsbeiträge beschränken sich auf das Gesundheits- und das Rentensystem, damit für die einzelnen Länder konsistente Daten vorliegen.

Die Vergütung wurde anhand des BIP auf KKP-Basis mit Hilfe von Daten aus der OECD-Datenbank OECD National Accounts Statistics in US-Dollar umgerechnet. Arbeitszeitanpassungen gleichen Unterschiede bei der Arbeitszeit aus, dabei wird sowohl die durchschnittliche Zahl der Arbeitstage/geleisteten Arbeitsstunden als auch die durchschnittliche Zahl der bezahlten Urlaubstage berücksichtigt.

Vgl. Anhang D wegen der vollständigen Methodik.

\section{Weitere Informationen}

OECD (2012), Public Sector Compensation in Times of Austerity, OECD Publishing, Paris, http://dx.doi.org/10.1787/9789264177758-en.

\section{Anmerkungen zu den Abbildungen}

Für Australien, Kanada, die Tschechische Republik, Ungarn, Irland, Japan, Luxemburg, Mexiko, Neuseeland, Norwegen, die Schweiz und die Türkei sind keine Daten verfügbar.

In Österreich, Estland, Griechenland, Island, Israel, Italien, Portugal, der Slowakischen Republik, Spanien und den Vereinigten Staaten sind die Daten zur Vergütung von erfahrenen akademischen Fachkräften mit denen von Nachwuchsfachkräften vermischt. Belgien: Das Bildungs- und das Umweltministerium wurden nicht einbezogen, da sie nicht auf föderaler Ebene agieren. Dänemark: Das Bildungsministerium wurde nicht berücksichtigt, weil viele seiner Zuständigkeiten anderen Ministerien übertragen wurden. Estland: Für das Bildungs- und das Umweltministerium sind keine Daten verfügbar. Deutschland: Die Unterscheidung zwischen den beiden Kategorien beruht auf dem unterschiedlichen Bildungsniveau und nicht auf der Länge der Berufserfahrung. Griechenland: Es liegen lediglich Daten zum Anfangs- und zum Höchstgehalt vor, der Durchschnitt entspricht daher nicht dem tatsächlichen Durchschnitt, sondern dem Mittelwert zwischen Anfangs- und Höchstgehalt. Italien: Die Zahl der Beschäftigten umfasst Vollzeit- und Teilzeitbeschäftigte. Island: Das Justizministerium ist Teil des Innenministeriums. Slowakische Republik: Aus statistischen Gründen wurde nur die Hälfte der im Justizministerium Beschäftigten berücksichtigt (Konsistenz der ISCO-Codes). Schweden: Das Innenministerium ist Teil des Amts des Ministerpräsidenten und wurde nicht in den Daten berücksichtigt. Vereinigtes Königreich: Die Daten beziehen sich auf 2012 (unter Verwendung von KKP aus dem Jahr 2012), und der Durchschnitt entspricht eher dem Medianwert als dem arithmetischen Mittel.

Vgl. Anhang D wegen weiterer Anmerkungen.

Hinweis zu den Daten für Israel: http://dx.doi.org/10.1787/888932315602. 


\section{BESCHÄFTIGUNG UND BEZAHLUNG IM ÖFFENTLICHEN SEKTOR}

Vergütung von akademischen Fachkräften auf Fbene der Zentralregierung

5.9. Durchschnittliche jährliche Vergütung von erfahrenen akademischen Fachkräften und Nachwuchsfachkräften auf Ebene der Zentralregierung (2011)

Bereinigt um Unterschiede bei den geleisteten Arbeitsstunden und den bezahlten Urlaubstagen

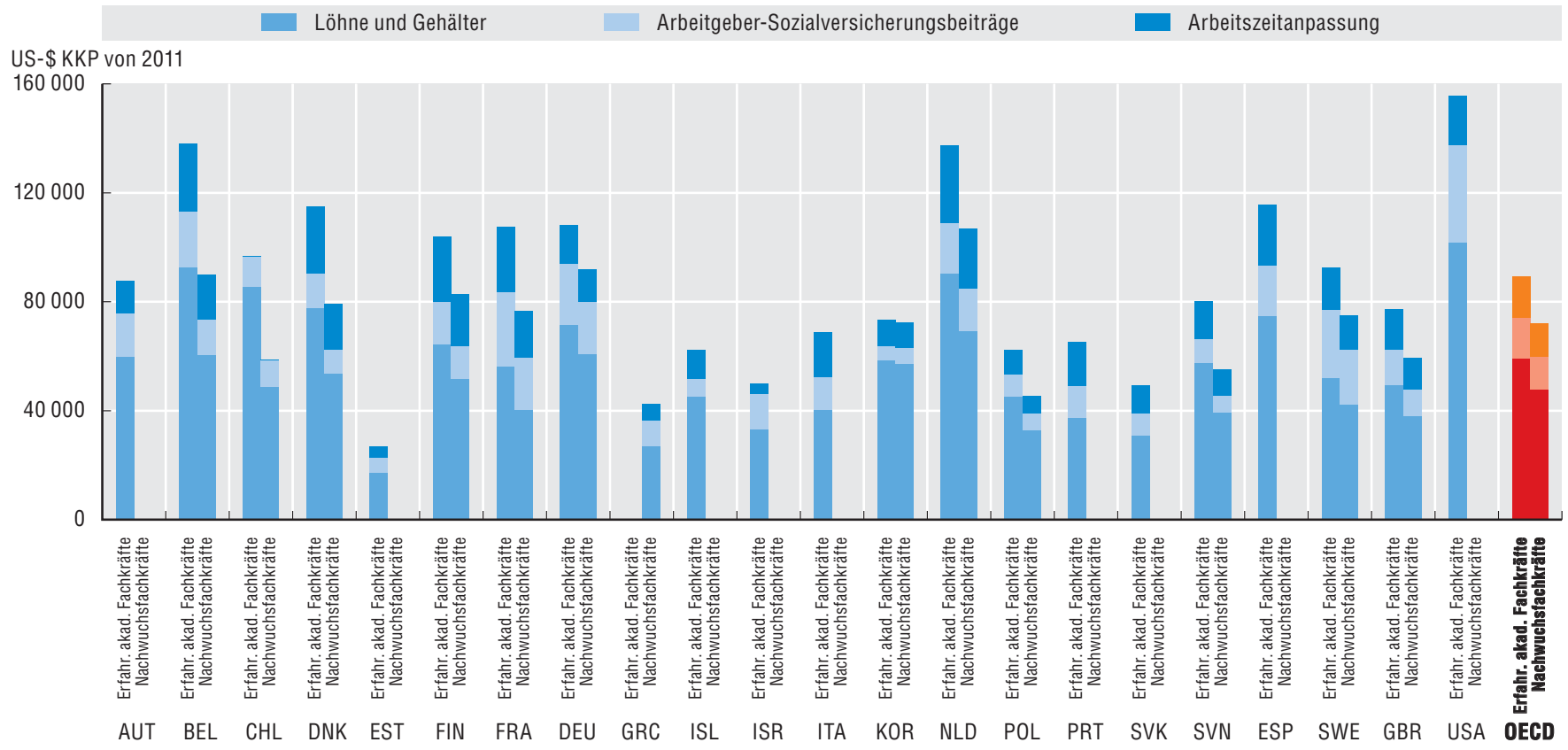

Quelle: 2012 OECD Survey on Compensation of Employees in Central/Federal Governments; OECD STAN/National Accounts Statistics (Datenbank).

StatLink iनाIsta http://dx.doi.org/10.1787/888932942393

5.10. Durchschnittliche jährliche Vergütung von erfahrenen akademischen Fachkräften und Nachwuchsfachkräften auf Ebene der Zentralregierung im Verhältnis zum Pro-Kopf-BIP und Arbeitsentgelt von Beschäftigten mit Tertiärabschluss

Verhältnis 2011

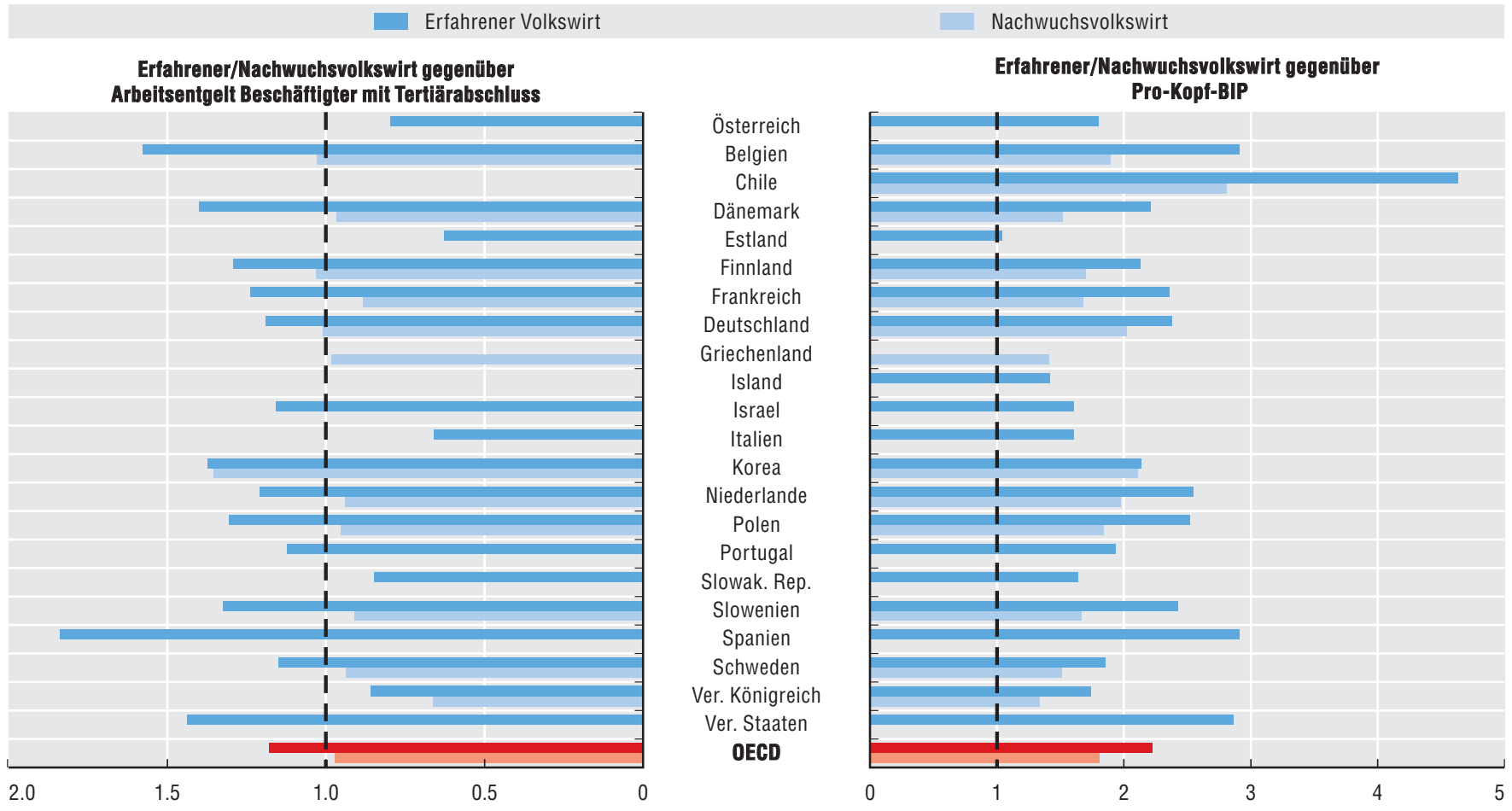

Quelle: 2012 OECD Survey on Compensation of Employees in Central/Federal Governments; OECD STAN/National Accounts Statistics (Datenbank); OECD (2013), Bildung auf einen Blick 2013: OECD-Indikatoren, W. Bertelsmann Verlag, Bielefeld, http://dx.doi.org/10.1787/eag-2013-de.

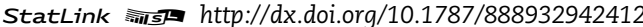


Unter den verschiedenen Berufen auf Ebene der Zentralregierung scheint die Vergütung von Sekretariatskräften zwischen den einzelnen OECD-Mitgliedsländern am wenigsten zu variieren. Im Durchschnitt beläuft sich die Vergütung von Sekretariatskräften auf rd. 50000 US-\$ KKP, worin nahezu 8600 US-\$ KKP für Arbeitgeber-Sozialversicherungsbeiträge und 8500 US\$ KKP für Arbeitszeitanpassungen enthalten sind. Damit liegen die Bruttogehälter bei $66 \%$ der Gesamtvergütung. Um dem Gesamtbetrag der Beiträge zu den Sozialversicherungssystemen Rechnung zu tragen, müssen die Arbeitnehmer-Sozialversicherungsbeiträge hinzugerechnet werden, die im Bruttogehalt enthalten sind. Wie bei den anderen Berufen gestaltet sich die Struktur der Gesamtvergütung in Bezug auf Gehälter und Arbeitgeber-Sozialversicherungsbeiträge von Land zu Land unterschiedlich. Diese Unterschiede sind auf einen historischen, kulturellen und politischen Konsens bei der Finanzierung der Sozialversicherungssysteme zurückzuführen. Leitende Führungskräfte der Stufe D1 verdienen im Durchschnitt 4,6-mal mehr als Sekretariatskräfte. Diese Differenz ist in Italien, Australien und dem Vereinigen Königreich am größten und in Island, der Slowakischen Republik und Spanien am geringsten.

Nach Berücksichtigung des Pro-Kopf-BIP ist die Vergütung von Sekretariatskräften in Polen und den Niederlanden am höchsten, wohingegen die verhältnismäßig niedrigsten Vergütungen in der Slowakischen Republik und Estland anzutreffen sind.

Differenzen beim Vergütungsniveau können auch durch Unterschiede auf den nationalen Arbeitsmärkten bedingt sein, insbesondere bei der Entlohnung im privaten Sektor für vergleichbare Kompetenzen. In diesem Vergleich scheinen Sekretariatskräfte in Korea und Spanien im Vergleich zu Beschäftigten mit Tertiärabschluss verhältnismäßig besser entlohnt zu werden. Im Gegensatz dazu sind diese Positionen in der Slowakischen Republik offenbar weniger attraktiv. Es ist zu beachten, dass das Verhältnis zwischen der Vergütung von Sekretariatskräften und dem durchschnittlichen Arbeitsentgelt von Beschäftigten mit Tertiärabschluss in allen Ländern unter 1 liegt und im Durchschnitt 0,7 entspricht. Das erklärt sich möglicherweise daraus, dass für Sekretariatstätigkeiten nicht zwangsläufig ein Tertiärabschluss erforderlich ist, es kann jedoch auch darauf hindeuten, dass dies aus den Unterschieden auf den nationalen Arbeitsmärkten resultiert, insbesondere der Entlohnung im privaten Sektor für ähnliche Stellen.

\section{Methodik und Definitionen}

Die Daten beziehen sich auf 2011 und wurden 2012 durch den von der OECD durchgeführten Survey on the Compensation of Employees in Central/Federal Governments erhoben. Bedienstete von Ministerien und Behörden auf Ebene der Zentralregierung nahmen durch das OECD Network on Public Employment and Management an der Erhebung teil.

Die Daten beziehen sich auf sechs Ministerien der Zentralregierung (Inneres, Finanzen, Justiz, Bildung, Gesundheit und Umwelt bzw. auf das jeweils entsprechende Ressort). Die Klassifizierung und die Definition der Berufe wurde anhand der Internationalen Standardklassifikation der Berufe (ISCO) der Internationalen Arbeitsorganisation (ILO) adaptiert. Die Vergütungsniveaus wurden errechnet, indem die durchschnittliche Vergütung des beschäftigten Personals ermittelt wurde.

Die Gesamtvergütung umfasst die Bruttolöhne und -gehälter sowie die Arbeitgeber-Sozialversicherungsbeiträge, wobei die entsprechenden Systeme sowohl kapitalgedeckt als auch nicht kapitalgedeckt sein können. Die Sozialversicherungsbeiträge beschränken sich auf das Gesundheits- und das Rentensystem, damit für die einzelnen Länder konsistente Daten vorliegen.

Die Vergütung wurde anhand des BIP auf KKP-Basis mit Hilfe von Daten aus der OECD-Datenbank OECD National Accounts Statistics in US-Dollar umgerechnet. Arbeitszeitanpassungen gleichen Unterschiede bei der Arbeitszeit aus, dabei wird sowohl die durchschnittliche Zahl der Arbeitstage/geleisteten Arbeitsstunden als auch die durchschnittliche Zahl der bezahlten Urlaubstage berücksichtigt.

Vgl. Anhang D wegen der vollständigen Methodik.

\section{Weitere Informationen}

OECD (2012), Public Sector Compensation in Times of Austerity, OECD Publishing, Paris, http://dx.doi.org/10.1787/9789264177758-en.

\section{Anmerkungen zu den Abbildungen}

Für Kanada, die Tschechische Republik, Griechenland, Ungarn, Irland, Japan, Luxemburg, Mexiko, Neuseeland, die Schweiz und die Türkei sind keine Daten verfügbar.

Belgien: Das Bildungs- und das Umweltministerium wurden nicht einbezogen, da sie nicht auf föderaler Ebene agieren. Dänemark: Das Bildungsministerium wurde nicht berücksichtigt, weil viele seiner Zuständigkeiten anderen Ministerien übertragen wurden. Estland: Für das Bildungs- und das Umweltministerium sind keine Daten verfügbar. Island: Das Justizministerium ist Teil des Innenministeriums. Italien: Die Zahl der Beschäftigten umfasst Vollzeit- und Teilzeitbeschäftigte. Slowakische Republik: Aus statistischen Gründen wurde nur die Hälfte der im Justizministerium Beschäftigten berücksichtigt (Konsistenz der ISCO-Codes). Schweden: Das Innenministerium ist Teil des Amts des Ministerpräsidenten und wurde nicht in den Daten berücksichtigt. Vereinigtes Königreich: Die Daten beziehen sich auf 2012 (unter Verwendung von KKP aus dem Jahr 2012), und der Durchschnitt entspricht eher dem Medianwert als dem arithmetischen Mittel.

Vgl. Anhang D wegen weiterer Anmerkungen.

Hinweis zu den Daten für Israel: http://dx.doi.org/10.1787/888932315602. 


\subsection{Durchschnittliche jährliche Vergütung von Sekretariatskräften (2011)}

Bereinigt um Unterschiede bei den geleisteten Arbeitsstunden und den bezahlten Urlaubstagen

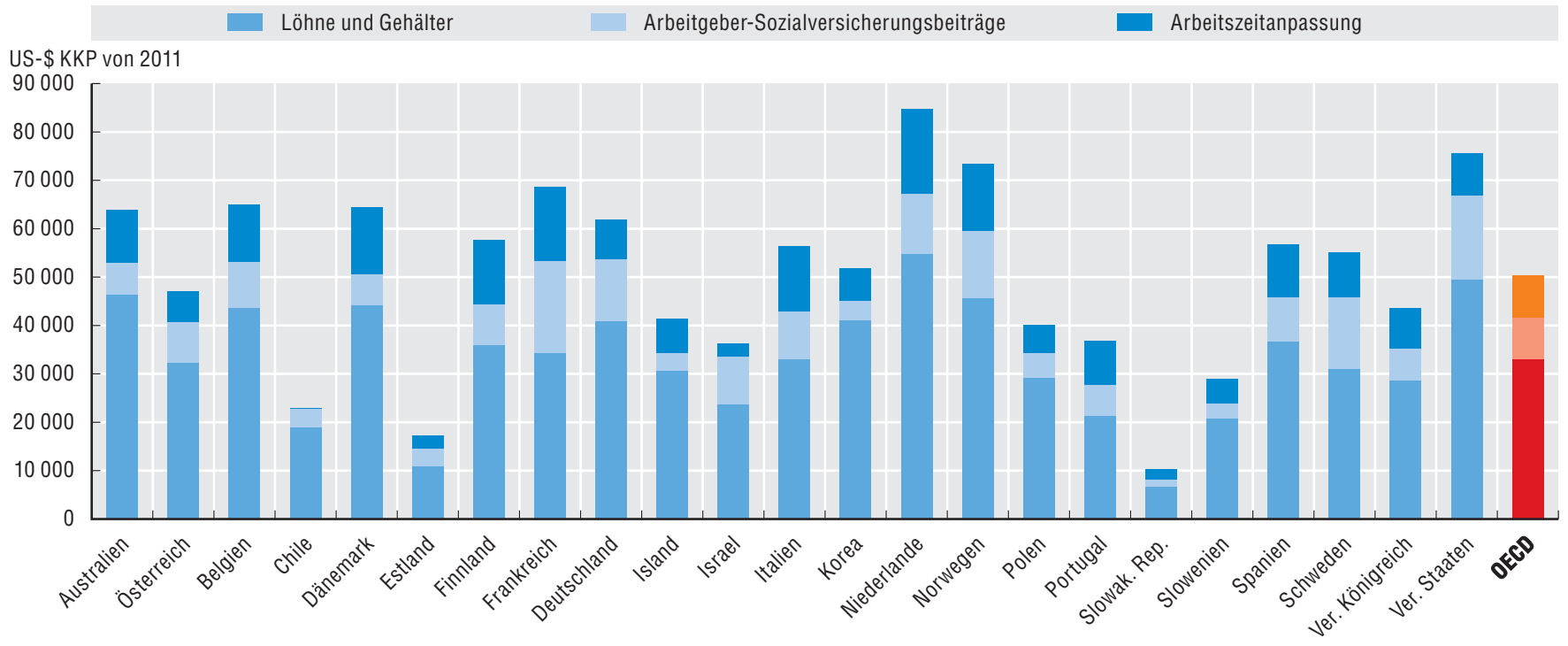

Quelle: 2012 OECD Survey on Compensation of Employees in Central/Federal Governments; OECD STAN/National Accounts Statistics (Datenbank).

StatLink 角sla $h$ ttp://dx.doi.org/10.1787/888932942431

\subsection{Durchschnittliche jährliche Vergütung von Sekretariatskräften im Verhältnis zum Pro-Kopf-BIP und Arbeitsentgelt von Beschäftigten mit Tertiärabschluss}

Verhältnis 2011

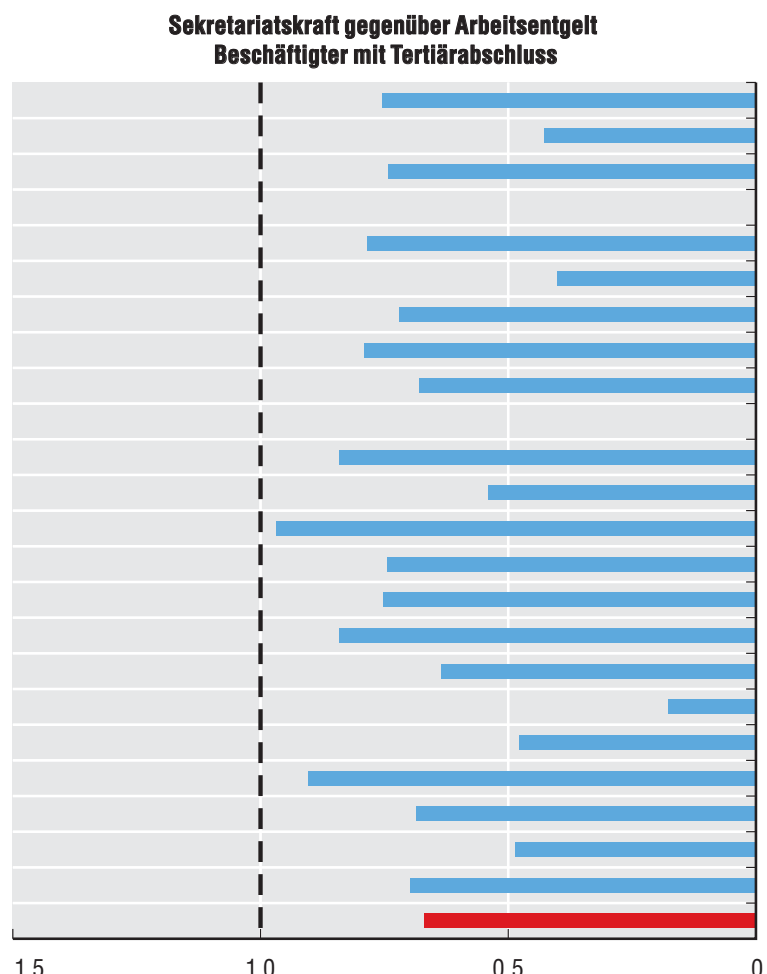

Australien
Österreich
Belgien
Chile
Dänemark
Estland
Finnland
Frankreich
Deutschland
Island
Israel
Italien
Korea
Niederlande
Norwegen
Polen
Portugal
Slowak. Rep.
Slowenien
Spanien
Schweden
Ver. Königreich
Ver. Staaten
OECD

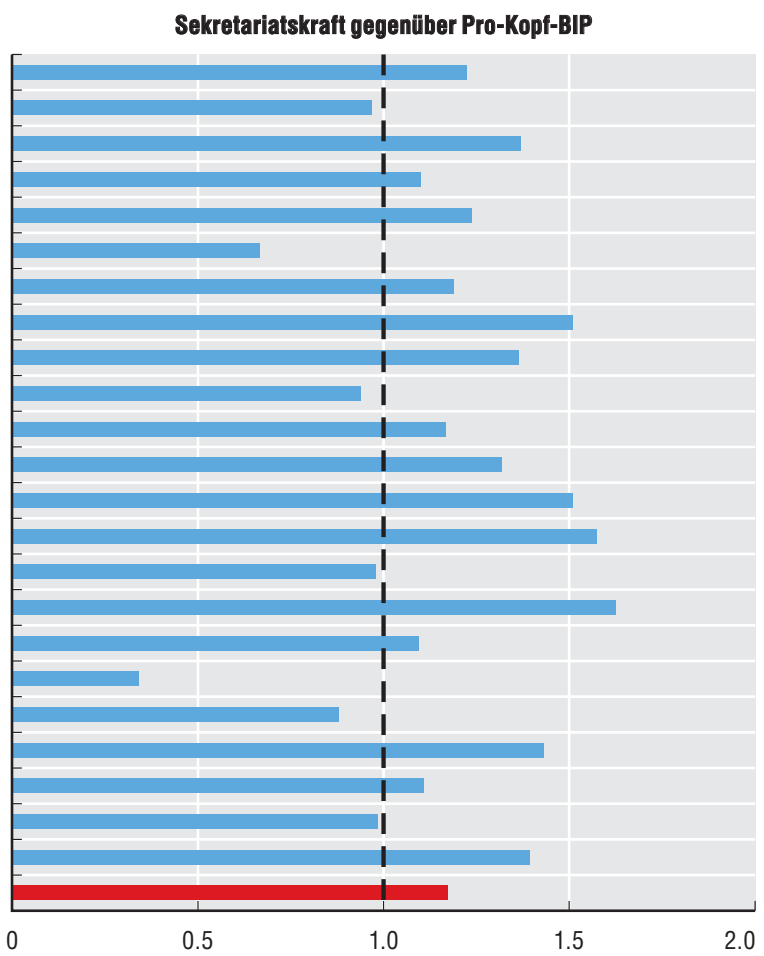

Quelle: 2012 OECD Survey on Compensation of Employees in Central/Federal Governments; OECD STAN/National Accounts Statistics (Datenbank); OECD (2013), Bildung auf einen Blick 2013: OECD-Indikatoren, W. Bertelsmann Verlag, Bielefeld, http://dx.doi.org/10.1787/eag-2013-de. 
Die auf Ebene der Zentralregierung in Dienstleistungsberufen tätigen öffentlich Bediensteten arbeiten an vorderster Front, da sie mit Nutzern von öffentlichen Dienstleistungen interagieren. Die aufgeführten Daten beziehen sich auf Berufe im Bereich Recht und Ordnung und in der Steuerverwaltung: Polizeikommissare und Kriminalbeamte (hier als Kriminalbeamte bezeichnet), Polizisten, Beamte der Grenzpolizei, Zollbeamte und Steuerprüfer. Obgleich alle Länder Beschäftigte haben, die für diese Aufgaben zuständig sind, werden einige dieser Funktionen auch von nachgeordneten Gebietskörperschaften ausgeführt. In einigen Ländern wird keine Unterscheidung nach spezifischen Funktionen (Grenzkontrolle oder Steuern) vorgenommen.

Im Durchschnitt beläuft sich die Vergütung von Kriminalbeamten auf rd. 83000 US-\$ KKP, wovon 18\% auf Arbeitgeber-Sozialversicherungsbeiträge und $19 \%$ auf Arbeitszeitanpassungen entfallen. Die Vergütung von Polizisten liegt bei rd. 63000 US-\$ KKP (einschließlich der Arbeitgeber-Sozialversicherungsbeiträge sowie der geleisteten Arbeitsstunden und bezahlter Urlaubstage).

Die Differenzen bei den Vergütungsniveaus sind bei diesen fünf Berufsgruppen ziemlich gering. Ein Kriminalbeamter verdient im Durchschnitt 33\% mehr als ein gewöhnlicher Polizist. Die Vereinigten Staaten und das Vereinigte Königreich investieren im Verhältnis mehr als andere Länder in ihre Kriminalbeamten als in ihre Polizisten. Im Gegensatz dazu verdienen Polizisten und Kriminalbeamte in Island, den Niederlanden und Norwegen fast gleich viel.

Vor allem in den Niederlanden, Slowenien, Portugal und Spanien scheint die Zentralregierung Steuerprüfer besser zu entlohnen als Polizisten. Darüber hinaus wenden Portugal, das Vereinigte Königreich und die Vereinigten Staaten offenbar weitaus mehr Mittel für Zollbeamte als für Polizisten auf. Die gleiche Gruppe von Ländern und die Niederlande bezahlen Beamte der Grenzpolizei anscheinend besser als Polizisten.

Wie im Fall der anderen Berufe gestaltet sich die Struktur der Gesamtvergütung in Bezug auf Löhne und Gehälter sowie Arbeitgeber-Sozialversicherungsbeiträge von Land zu Land unterschiedlich. Diese Unterschiede sind auf einen historischen, kulturellen und politischen Konsens bei der Finanzierung der Sozialversicherungssysteme zurückzuführen.

Im Verhältnis zum Pro-Kopf-BIP und im Vergleich zum durchschnittlichen Gehalt von Beschäftigten mit Tertiärabschluss wurden zwei Berufssparten analysiert: Kriminalbeamte und Polizisten. Im Durchschnitt entspricht die Vergütung von Kriminalbeamten nahezu dem durchschnittlichen Arbeitsentgelt von Beschäftigten mit Tertiärabschluss und für Polizisten ist das Verhältnis geringfügig niedriger, was bedeutet, dass das Gehalt für diese Positionen in gleichem Maße attraktiv ist bzw. unter dem in der Gesamtwirtschaft üblichen durchschnittlichen Arbeitsentgelt liegt.

\section{Methodik und Definitionen}

Die Daten beziehen sich auf 2011 und wurden 2012 durch den von der OECD durchgeführten Survey on the Compensation of Employees in Central/Federal Governments erhoben. Bedienstete von Ministerien und Behörden auf Ebene der Zentralregierung nahmen durch das OECD Network on Public Employment and Management an der Erhebung teil.

Die Daten beziehen sich auf Beamte, die Dienstleistungen an vorderster Front erbringen (Polizeikommissare/ Kriminalbeamte, Polizisten, Beamte der Grenzpolizei, Zollbeamte und Steuerprüfer). Die Klassifizierung und die Definition der Berufe wurde anhand der Internationalen Standardklassifikation der Berufe (ISCO) der Internationalen Arbeitsorganisation (ILO) adaptiert.

Die Gesamtvergütung umfasst die Bruttolöhne und -gehälter sowie die Arbeitgeber-Sozialversicherungsbeiträge, wobei die entsprechenden Systeme sowohl kapitalgedeckt als auch nicht kapitalgedeckt sein können, einschließlich Rentenzahlungen, die aus dem Staatshaushalt und nicht durch die Arbeitgeber-Sozialversicherungsbeiträge bezahlt werden (was vor allem bei einigen umlagefinanzierten Systemen der Fall ist). Die Sozialversicherungsbeiträge beschränken sich auf das Gesundheits- und das Rentensystem, damit für die einzelnen Länder konsistente Daten vorliegen.

Die Vergütung wurde anhand des BIP auf KKP-Basis mit Hilfe von Daten aus der OECD-Datenbank OECD National Accounts Statistics in US-Dollar umgerechnet. Arbeitszeitanpassungen gleichen Unterschiede bei der Arbeitszeit aus, dabei wird sowohl die durchschnittliche Zahl der Arbeitstage/geleisteten Arbeitsstunden als auch die durchschnittliche Zahl der bezahlten Urlaubstage berücksichtigt.

Vgl. Anhang D wegen der vollständigen Methodik.

\section{Weitere Informationen}

OECD (2012), Public Sector Compensation in Times of Austerity, OECD Publishing, Paris, http://dx.doi.org/10.1787/9789264177758-en.

\section{Anmerkungen zu den Abbildungen}

Für Kanada, Chile, die Tschechische Republik, Deutschland, Griechenland, Ungarn, Irland, Japan, Luxemburg, Mexiko, Neuseeland, die Schweiz und die Türkei sind keine Daten verfügbar.

In Österreich, Dänemark, Island, Italien, Norwegen, der Slowakischen Republik und Spanien sind die Angaben zu den Beamten der Grenzpolizei in den Daten zu den Polizisten enthalten. In Israel, Korea und Schweden sind die Daten zu Kriminalbeamte mit denen zu Polizisten vermischt. In der Slowakischen Republik zählen Zollbeamte zu den Polizisten. In Island fallen Steuerprüfer und Beamte der Grenzpolizei in die Kategorie der Polizisten. Australien: Kommunale und örtliche Polizeibeamte und -inspektoren stellen den größten Anteil an Polizeikräften und wurden nicht berücksichtigt. Österreich: Es wurden lediglich Vertragsbedienstete berücksichtigt, da sie die Mehrheit der Dienstleistungen erbringenden Arbeitskräfte ausmachen. Polen: Dienstleistungsberufe sind nicht Teil des öffentlichen Diensts, mit Ausnahme der Steuerprüfer. Vereinigtes Königreich: Die Daten beziehen sich auf 2012 (unter Verwendung von KKP aus dem Jahr 2012). Es liegen lediglich Daten zum Anfangs- und zum Höchstgehalt vor, der Durchschnitt entspricht daher nicht dem tatsächlichen Durchschnitt, sondern dem Mittelwert zwischen Anfangs- und Höchstgehalt.

Vgl. Anhang D wegen weiterer Anmerkungen.

Hinweis zu den Daten für Israel: http://dx.doi.org/10.1787/888932315602. 


\section{BESCHÄFTIGUNG UND BEZAHLUNG IM ÖFFENTLICHEN SEKTOR}

Vergütung in ausgewählten Dienstleistungsberufen

5.13. Durchschnittliche jährliche Vergütung von Beschäftigten in Dienstleistungsberufen (2011)

Bereinigt um Unterschiede bei den geleisteten Arbeitsstunden und den bezahlten Urlaubstagen

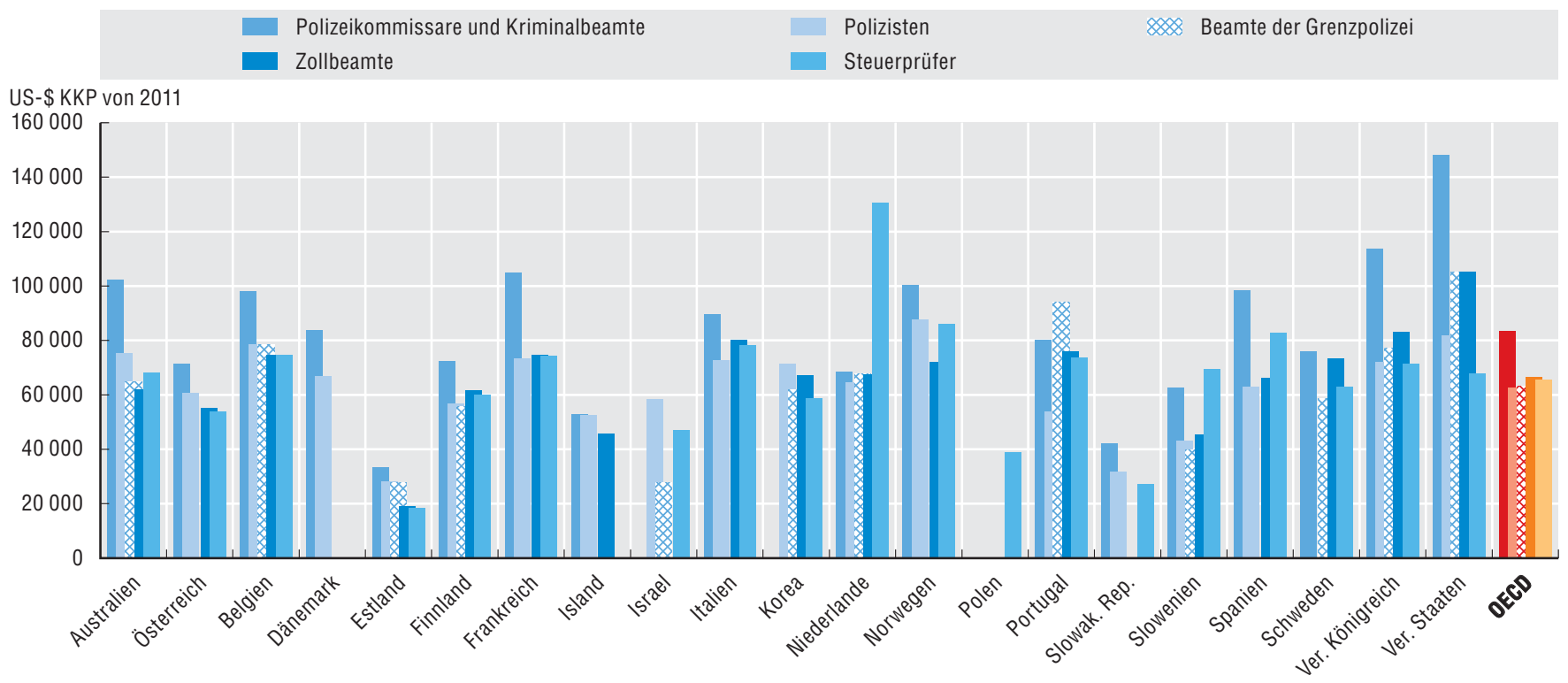

Quelle: 2012 OECD Survey on Compensation of Employees in Central/Federal Governments; OECD STAN/National Accounts Statistics (Datenbank).

StatLink ints $h t t p: / / d x . d o i . o r g / 10.1787 / 888932942469$

5.14. Durchschnittliche jährliche Vergütung von Kriminalbeamten und Polizisten auf Ebene der Zentralregierung im Verhältnis zum Pro-Kopf-BIP und Arbeitsentgelt von Beschäftigten mit Tertiärabschluss

Verhältnis 2011

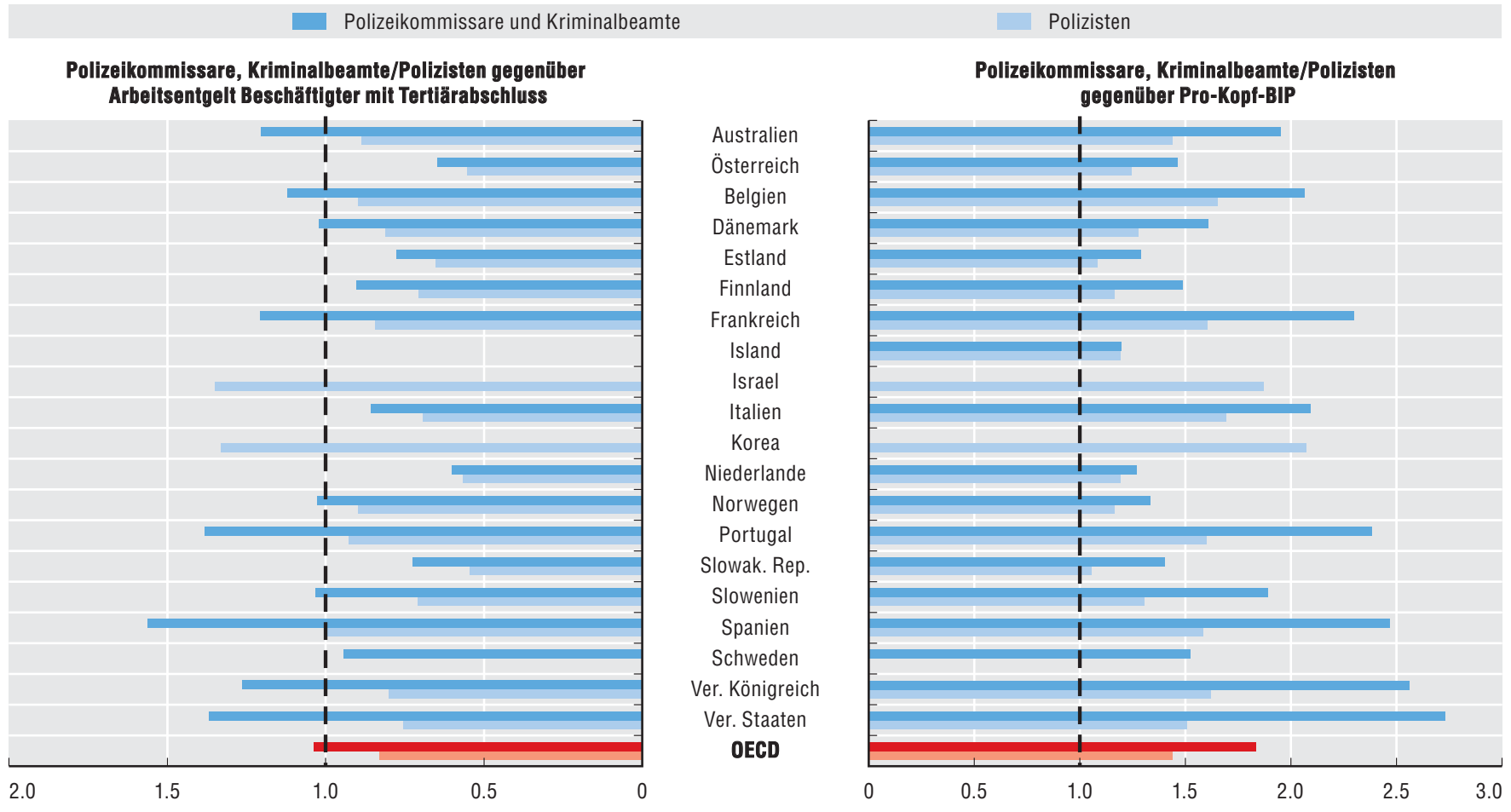

Quelle: 2012 OECD Survey on Compensation of Employees in Central/Federal Governments; OECD STAN/National Accounts Statistics (Datenbank); OECD (2013), Bildung auf einen Blick 2013: OECD-Indikatoren, W. Bertelsmann Verlag, Bielefeld, http://dx.doi.org/10.1787/eag-2013-de.

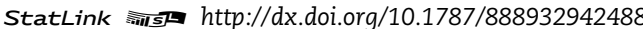


Lehrkräfte sind das Rückgrat des Bildungssektors, der ein entscheidender Bestimmungsfaktor für die Produktivität und das Wachstum ist. Die Gehälter der Lehrkräfte sind der größte Einzelposten bei den Kosten des arbeitsintensiven Bildungssystems. Gehalt und Arbeitsbedingungen spielen eine wichtige Rolle, um qualifizierte Lehrkräfte anzuwerben, zu motivieren und zu binden.

In den meisten Ländern gibt es drei Kategorien von Lehrkräften: Lehrkräfte im Primarbereich, im Sekundarbereich I und im Sekundarbereich II. Die Gehälter steigen mit den Qualifikationen, der Erfahrung und den Arbeitsplatzanforderungen der Bildungsstufe, auf der sie unterrichten. Anhand der hier aufgeführten Daten werden die gesetzlich bzw. vertraglich vereinbarten Bruttogehälter von Lehrkräften im Sekundarbereich I mit der für öffentliche Einrichtungen erforderlichen Mindestausbildung am Anfang und in der Mitte ihrer beruflichen Laufbahn sowie auf der höchsten Stufe der Gehaltsskala miteinander verglichen. Bei internationalen Vergleichen sollte jedoch berücksichtigt werden, dass gesetzlich bzw. vertraglich vereinbarte Gehälter nur eine, wenn auch eine wichtige, Komponente der Gesamtvergütung von Lehrkräften sind. Unterschiede zwischen den Ländern bei den Sozialleistungen, bei den Arbeitgeber- wie auch den Arbeitnehmer-Sozialversicherungsbeiträgen, ebenso wie bei den Bonuszahlungen und den Zulagen können zu Differenzen in der Gesamtvergütung führen. Darüber hinaus wurden die Lehrergehälter nicht um die Unterschiede bei der vertraglich vereinbarten Arbeitszeit und den bezahlten Urlaubstagen bereinigt, die für nationale und internationale Vergleiche von Bedeutung sein können. Diese Daten können allerdings als Anhaltspunkt für die Differenzen bei den Erträgen aus der Lehrerfahrung in den einzelnen OECD-Mitgliedsländern dienen.

Die gesetzlichen bzw. vertraglich vereinbarten Bruttojahresgehälter von Lehrkräften im Sekundarbereich I mit 15 Jahren Berufserfahrung reichten 2011 von weniger als 15000 US-\$ KKP in Estland, der Slowakischen Republik und Ungarn bis zu über 60000 US-\$ KKP in Luxemburg, Deutschland und den Niederlanden. Der OECD-Durchschnitt liegt nahezu bei 40000 US-\$ KKP. In Korea, Japan und Mexiko sind die Gehälter auf der obersten Gehaltsstufe mehr als doppelt so hoch wie die Anfangsgehälter. Die Gehälter auf der obersten Gehaltsstufe sind im Durchschnitt rd. 60\% höher als die Anfangsgehälter.

Im Allgemeinen sind die Bruttogehälter von Lehrkräften niedriger als die durchschnittlichen Bruttojahresgehälter von Beschäftigten mit ähnlichem Bildungsniveau (ganzjährig Vollzeitbeschäftigte mit Tertiärabschluss im Alter von 25-64 Jahren). In Spanien, Korea, Luxemburg und Portugal verdienen die Lehrkräfte mehr. In Neuseeland, Kanada, Deutschland und Finnland entsprechen die gesetzlich bzw. vertraglich vereinbarten Gehälter der Lehrkräfte fast dem durchschnittlichen Arbeitsentgelt von Beschäftigten mit Tertiärabschluss. In der Slowakischen Republik, Island und der Tschechischen Republik, Ungarn, Italien und Österreich liegen die Lehrergehälter jedoch deutlich unter dem durchschnittlichen Arbeitsentgelt von Beschäftigten mit Tertiärabschluss.

\section{Methodik und Definitionen}

Gesetzliche bzw. vertraglich vereinbarte Gehälter sind die regulären Gehälter gemäß amtlichen Gehaltsskalen. Die Gehälter sind auf Bruttobasis angegeben (die vom Arbeitgeber vor Steuern gezahlte Gesamtsumme) abzüglich der Arbeitgeberbeiträge zur Sozial- und Rentenversicherung (entsprechend den gültigen Gehaltsskalen). Die Gehälter beziehen sich auf Vollzeitlehrkräfte mit der für eine Lehrerqualifikation erforderlichen Mindestausbildung am Anfang der Lehrerlaufbahn, nach 15 Jahren Berufserfahrung und auf der höchsten Stufe der Gehaltsskala (maximales Jahresgehalt).

Die in diesem Indikator angegebenen gesetzlichen bzw. vertraglich vereinbarten Gehälter sind zu unterscheiden sowohl von den tatsächlichen Ausgaben für Gehälter, die von staatlicher Seite zu leisten sind, als auch von den Durchschnittsgehältern der Lehrkräfte.

Die Bruttolehrergehälter wurden auf KKP-Basis mit Hilfe von Daten aus der OECD-Datenbank OECD National Accounts Statistics in US-Dollar umgerechnet.

Der Indikator zu den relativen Gehältern ist für das letzte Jahr berechnet, für das Daten zu den Gehältern vorliegen. In diesem Fall entsprechen die Lehrergehälter den Gehältern, die tatsächlich nach 15 Jahren Berufserfahrung bezahlt werden. Die Gehälter von Beschäftigten mit einem Abschluss im Tertiärbereich sind die durchschnittlichen Gehälter von 25- bis 64-jährigen ganzjährig Vollzeitbeschäftigten mit einem Abschluss der ISCEDStufen $5 \mathrm{~A} / 5 \mathrm{~B} / 6$.

\section{Weitere Informationen}

OECD (2013), Bildung auf einen Blick 2013: OECD-Indikatoren, W. Bertelsmann Verlag, Bielefeld, http://dx.doi.org/10.1787/eag2013-de.

\section{Anmerkungen zu den Abbildungen}

Belgien ist als Belgien (frz.) und Belgien (fläm.) abgebildet. Das Vereinigte Königreich wird als England und Schottland aufgeführt.

5.15: Für die Schweiz und die Türkei sind keine Daten verfügbar.

5.16: Für Griechenland, Japan, Mexiko, die Schweiz und die Türkei sind keine Daten verfügbar. Die Daten für Australien, Kanada, Finnland, Irland, Italien, Luxemburg, die Niederlande, Norwegen, Polen, Portugal und Spanien beziehen sich auf 2010. Die Daten für Frankreich beziehen sich auf 2009. Die Daten für Island beziehen sich auf 2006.

Hinweis zu den Daten für Israel: http://dx.doi.org/10.1787/888932315602. 


\section{BESCHÄFTIGUNG UND BEZAHLUNG IM ÖFFENTLICHEN SEKTOR}

\subsection{Gehälter von Lehrkräften im Sekundarbereich I in öffentlichen Einrichtungen (2011)}

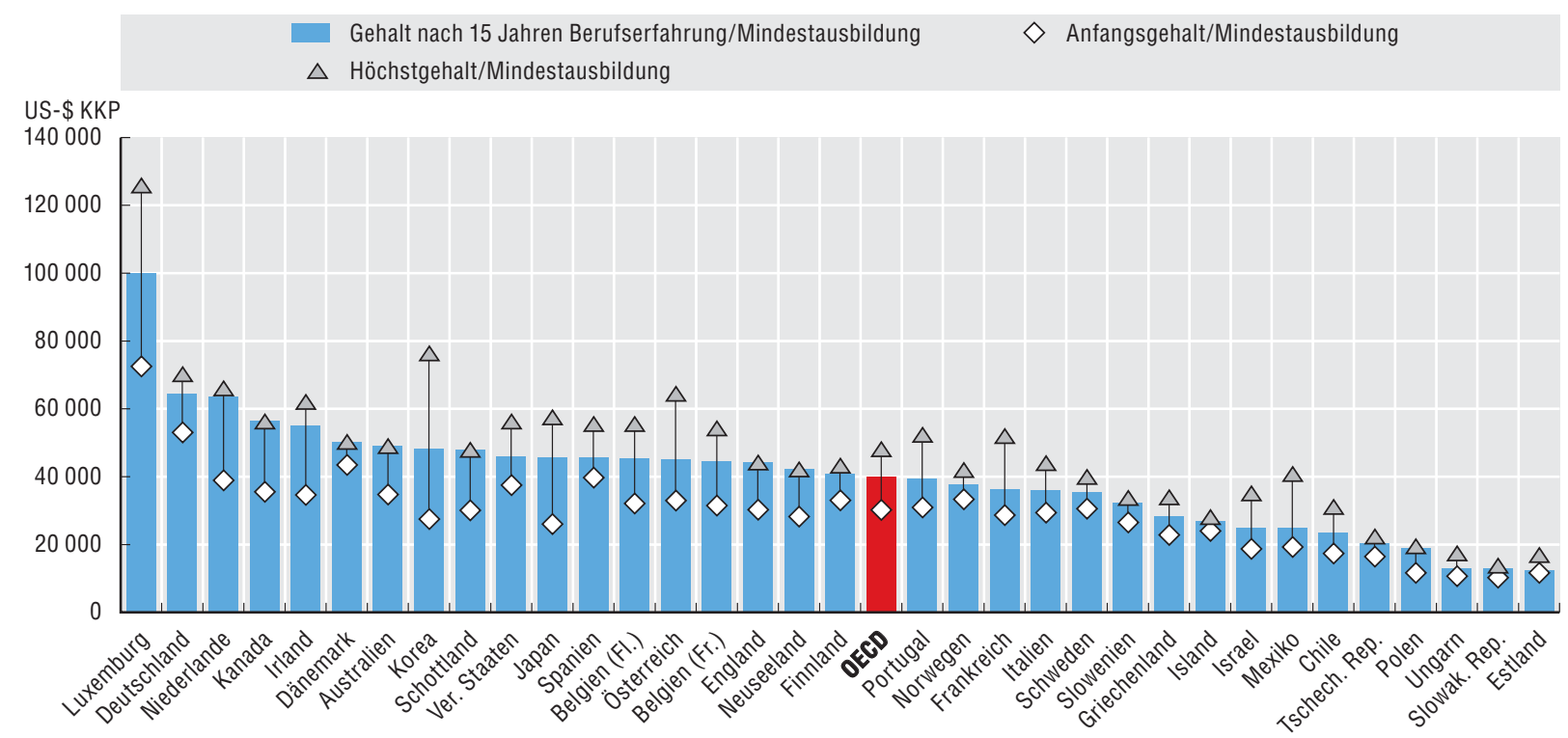

Quelle: OECD (2013), Bildung auf einen Blick 2013: OECD-Indikatoren, W. Bertelsmann Verlag, Bielefeld, http://dx.doi.org/10.1787/eag-2013-de.

StatLink नillst http://dx.doi.org/10.1787/888932942507

\subsection{Verhältnis der Lehrergehälter zum Arbeitsentgelt von Beschäftigten mit Tertiärabschluss (2011)}

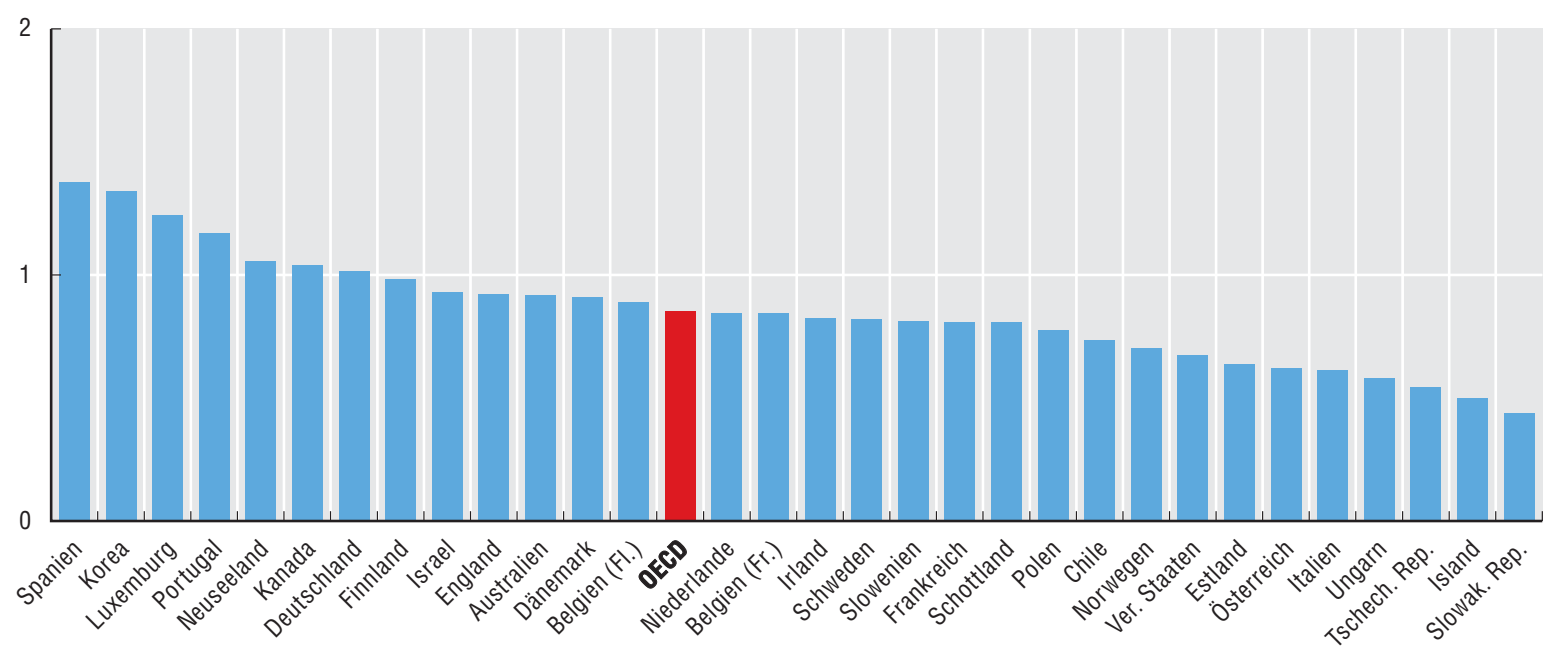

Quelle: OECD (2013), Bildung auf einen Blick 2013: OECD-Indikatoren, W. Bertelsmann Verlag, Bielefeld, http://dx.doi.org/10.1787/eag-2013-de.

StatLink त्राडा http://dx.doi.org/10.1787/888932942526 


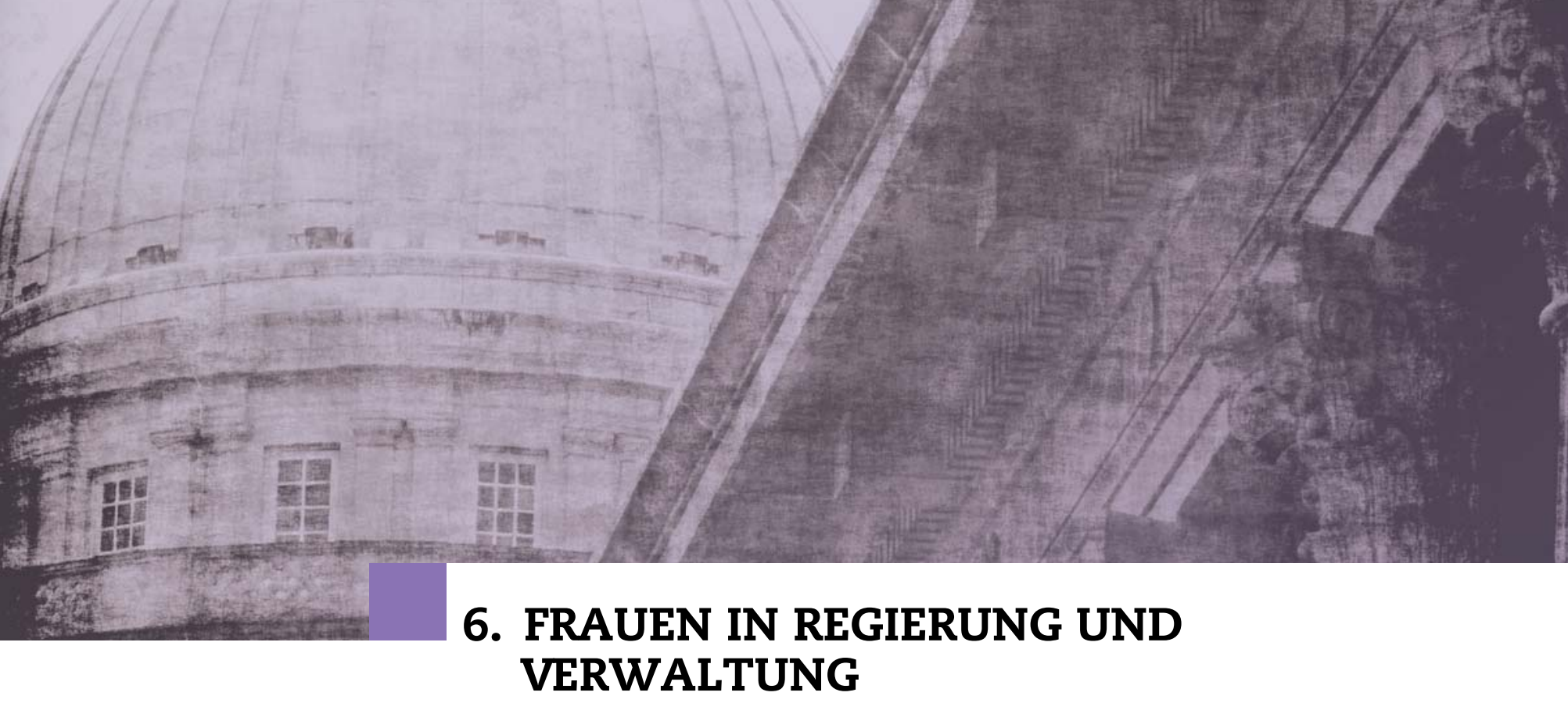




\section{FRAUEN IN REGIERUNG UND VERWALTUNG}

\section{Die Beschäftigung von Frauen im Staatssektor}

Der Anteil der Frauen unter den Beschäftigten des Staatssektors ist ein wichtiger Indikator für die Offenheit und Gerechtigkeit öffentlicher Institutionen. Da die Regierungen grundlegenden Prinzipien des öffentlichen Dienstes, wie Leistung und Transparenz, immer mehr Gewicht beimessen, erkennen sie zunehmend die Bedeutung von Maßnahmen zur Förderung der Diversität und damit auch zur Stärkung der Rolle der Frauen. Durch die Schaffung eines öffentlichen Sektors, der repräsentativ für die Bevölkerung ist, in deren Dienst er steht, kann zudem die Qualität der staatlichen Politik und die Bürgernähe der Dienstleistungen gesteigert werden, da sie ein besseres Verständnis der Bedürfnisse der Bürgerinnen und Bürger ermöglicht. Außerdem kann so die Produktivität des öffentlichen Sektors erhöht werden, indem sichergestellt wird, dass der Staat die in der Bevölkerung vorhandenen Kompetenzen und Talente optimal nutzt. Darüber hinaus kann die Erhöhung des Frauenanteils unter den öffentlich Bediensteten auch ein Mittel sein, die soziale Mobilität zu verbessern.

Der Frauenanteil ist im OECD-Durchschnitt unter den Beschäftigten des Staatssektors (56\%) höher als in der Gesamterwerbsbevölkerung, wo er rd. $40-50 \%$ beträgt. In einigen Ländern wird im Staatssektor eine Beschäftigungspolitik verfolgt, die diesen Sektor für Frauen besonders attraktiv macht, z.B. weil im Vergleich zum Privatsektor flexiblere Arbeitsbedingungen, zusätzlicher bezahlter Erziehungsurlaub und/oder stärker subventionierte Kinderbetreuung oder sonstige Leistungen angeboten werden. Die Situation stellt sich jedoch von Land zu Land anders dar: Am größten ist die Differenz in den nordischen Ländern, Estland und Slowenien, wo der Frauenanteil im Staatssektor 20 Prozentpunkte höher ist als in der Gesamtbeschäftigung. Im Gegensatz dazu sind Frauen in Griechenland, Japan, den Niederlanden und der Türkei in der Erwerbsbevölkerung insgesamt etwas stärker repräsentiert als im Staatssektor.

Der Frauenanteil unter den Beschäftigten des Staatssektors ist zwischen 2001 und 2010 in allen 21 OECD-Mitgliedsländern, für die Daten vorliegen, gestiegen. Estland verzeichnete in diesem Zeitraum den größten Anstieg der Frauenbeschäftigung; 2010 waren drei Viertel der Beschäftigten des Staatssektors Frauen, mehr als in jedem anderen OECD-Mitgliedsland. Im Gegensatz dazu betrug der Frauenanteil unter den Beschäftigten des Staatssektors in der Türkei und in Griechenland neuesten Zahlen zufolge nur $24 \%$ bzw. $36 \%$.

\section{Methodik und Definitionen}

Die Daten stammen von der Internationalen Arbeitsorganisation (ILO) und beziehen sich auf 2001 und 2010. Der Sektor Staat umfasst alle staatlichen Ebenen, darunter: a) alle Organe der zentralen, gliedstaatlichen und lokalen Ebene, b) alle Sozialversicherungsträger der verschiedenen staatlichen Ebenen und c) alle nicht marktorientierten Einrichtungen ohne Erwerbszweck, die staatlicher Kontrolle unterliegen und größtenteils von staatlichen Stellen finanziert werden. Die Erwerbsbevölkerung umfasst alle Personen, die die Voraussetzungen für die Berücksichtigung als Beschäftigte oder Arbeitslose erfüllen.

Die Daten beziehen sich auf die Zahl der Beschäftigten, außer für die Niederlande und Neuseeland, wo es sich um Vollzeitäquivalente (VZÄ) handelt. Infolgedessen sind die Beschäftigtenzahlen für diese beiden Länder im Vergleich unterzeichnet. Die Daten für Kanada, Chile, Finnland, Polen und die Türkei beziehen sich auf den öffentlichen Sektor (Sektor Staat und öffentliche Unternehmen) und nicht nur auf den Sektor Staat.

\section{Literaturhinweise}

OECD (2013), Gleichstellung der Geschlechter - Zeit zu handeln, OECD Publishing, Paris, http://dx.doi.org/10.1787/9789264190344-de.

OECD (2011), Public Servants as Partners for Growth: Toward a Stronger, Leaner and More Equitable Workforce, OECD Publishing, Paris, http://dx.doi.org/10.1787/9789264166707-en.

\section{Anmerkungen zu den Abbildungen}

Die Daten für Japan beziehen sich auf 2009 anstelle von 2010. Die Daten für Kanada, Chile, Finnland, Griechenland, Polen und die Schweiz beziehen sich auf 2008 anstelle von 2010. Die Daten für Norwegen und Schweden beziehen sich auf 2007 anstelle von 2010. Die Daten für die Türkei beziehen sich auf 2006 anstelle von 2010. Die Daten für die Niederlande beziehen sich auf 2005 anstelle von 2010.

6.1: Die Daten über den Frauenanteil im Staatssektor stammen aus der ILO-Datenbank LABORSTA. Die Daten über den Frauenanteil in der Erwerbsbevölkerung stammen aus der OECD-Datenbank Labour Force Statistics. 


\subsection{Frauenanteil im Staatssektor im Vergleich zum Frauenanteil in der Erwerbsbevölkerung (2010)}

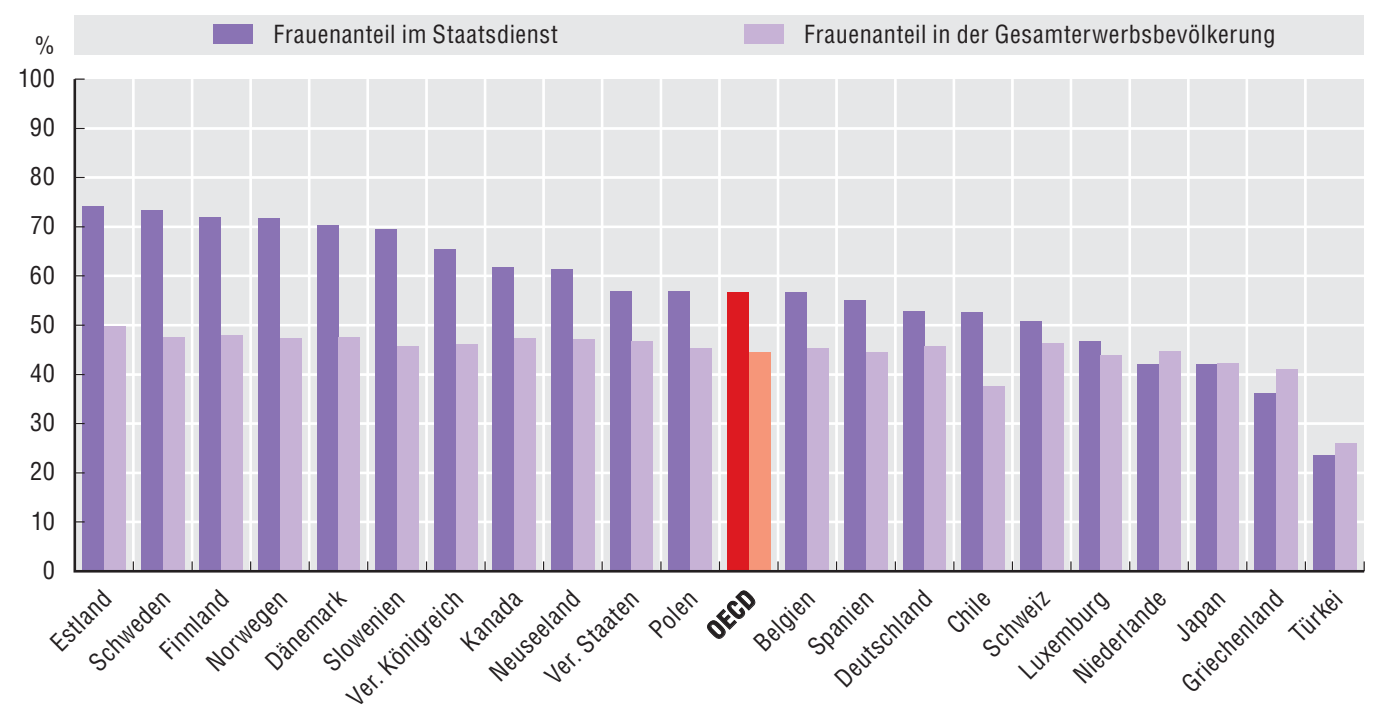

Quelle: Internationale Arbeitsorganisation (ILO), LABORSTA (Datenbank) und OECD Labour Force Statistics (Datenbank).

StatLink नinsts http://dx.doi.org/10.1787/888932942545

\subsection{Frauenanteil im Staatssektor (2001 und 2010)}

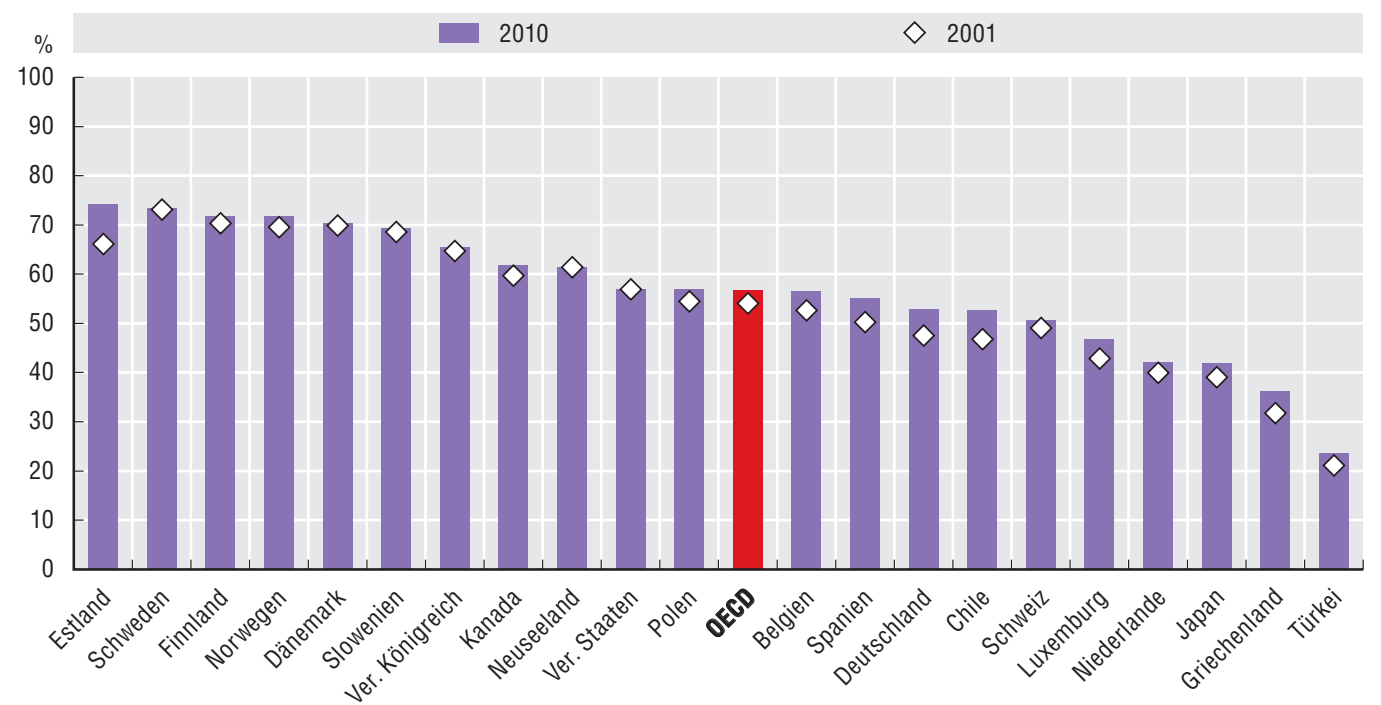

Quelle: Internationale Arbeitsorganisation (ILO), LABORSTA, (Datenbank). 
Die Beschäftigung auf der Ebene der Zentralregierung ist eine Unterkategorie der Beschäftigung im Staatssektor und umfasst eine Reihe von akademischen Berufen, Führungspositionen und Sekretariatstätigkeiten in Ministerien und sonstigen Organen der zentralen Verwaltungsebenen. Der Frauenanteil auf der Ebene der Zentralregierung, einschließlich höherer Verwaltungspositionen, ist deshalb ein wichtiger Indikator für die Rolle, die Frauen in den OECD-Mitgliedsländern in Politikgestaltung und -umsetzung spielen.

2010 belief sich der Frauenanteil unter den öffentlich Bediensteten auf Ebene der Zentralregierung in den 22 Ländern, für die Daten zur Verfügung stehen, auf durchschnittlich knapp über $50 \%$. Er war damit etwas niedriger als der Anteil der Frauen unter den Beschäftigten des Staatssektors insgesamt (57\%, Abbildung 6.2), was darauf zurückzuführen ist, dass typische Frauenberufe wie Lehrerin oder Krankenschwester häufiger auf Ebene der Gliedstaaten oder der lokalen Gebietskörperschaften angesiedelt sind. Der Frauenanteil auf Ebene der Zentralregierung ist mit rd. 70\% in Chile, Italien und Polen nach wie vor am höchsten, während er in Japan (16\%), gefolgt von Deutschland (39\%), am niedrigsten ist. Der Frauenanteil ist unter den Beschäftigten auf Ebene der Zentralregierung weiterhin etwas höher als in der Erwerbsbevölkerung insgesamt, da im öffentlichen Dienst in vielen Ländern flexiblere Arbeitszeiten und mehr bezahlter Elternurlaub angeboten werden.

Der Frauenanteil unter den Beschäftigten auf Ebene der Zentralregierung ist zwischen 2000 und 2010 in fast allen 15 OECDMitgliedsländern, für die Daten zur Verfügung stehen, gestiegen (in zwei Ländern - Estland und Japan - ist der Frauenanteil geringfügig um weniger als 1 Prozentpunkt gesunken). Trotz des in den letzten zehn Jahren auf Ebene der Zentralregierung zu beobachtenden Anstiegs des Frauenanteils sind Frauen auf den unteren Ebenen nach wie vor überrepräsentiert. In 15 von 19 Ländern werden über $50 \%$ der Sekretariatstätigkeiten von Frauen ausgeübt - in Slowenien und Österreich handelt es sich bei rd. 90\% aller Sekretariatskräfte um Frauen. Auf den höheren Ebenen sind Frauen wesentlich weniger stark vertreten: Auf sie entfallen nur $40 \%$ der Posten der mittleren Führungsebene und nur 29\% der Posten der oberen Führungsebene (Abbildung 6.4).

Darüber hinaus handelt es sich bei einem überproportional hohen Anteil der Teilzeitbeschäftigten auf Ebene der Zentralregierung um Frauen. In allen 16 OECD-Mitgliedsländern, die Daten vorgelegt haben, waren 2010 mindestens zwei Drittel der Teilzeitbeschäftigten Frauen, wobei der Frauenteil in Deutschland, Luxemburg und Frankreich bei über 85\% lag. Teilzeitbeschäftigung kann zwar eine attraktive Option für Beschäftigte sein, die eine größere Flexibilität wünschen, um berufliche und familiäre Pflichten miteinander in Einklang zu bringen, sie wird jedoch generell weniger gut bezahlt und verschlechtert langfristig die beruflichen Aufstiegsmöglichkeiten. Eine Möglichkeit, allen Beschäftigten Flexibilität zu bieten, ohne ihre langfristigen Karriereaussichten zu beeinträchtigen, besteht darin, Übergänge von der Teilzeit- in die Vollzeitbeschäftigung zu schaffen.

\section{Methodik und Definitionen}

Die Daten wurden im Rahmen des 2011 durchgeführten OECD Survey on Gender in Public Employment erhoben. Befragt wurden vornehmlich Führungskräfte aus Personalabteilungen von Behörden auf Ebene der Zentralregierung.

Der Begriff Zentralregierung (bzw. nationale Regierung oder Bundesregierung) bezieht sich auf die Ministerien und Behörden, die von der zentralen staatlichen Ebene kontrolliert und finanziert werden. Die Beschäftigungsdaten beziehen sich auf die Staatsbediensteten, für die die allgemeinen Beschäftigungsbedingungen gelten, was für die meisten öffentlich Bediensteten sowie die meisten externen Mitarbeiter, die dem Gesetz nach als Angestellte behandelt werden, der Fall ist. Als Teilzeitbeschäftigungen gelten Stellen mit einer geringeren Wochenarbeitszeit als Vollzeitstellen. Die Definition von Teilzeitbeschäftigung variiert von Land zu Land, beträgt generell aber weniger als 30 oder 35 Wochenstunden.

Die Posten der oberen Führungsebene sind definiert als Positionen unterhalb der Minister oder stellvertretenden Minister. Die mittlere Führungsebene beginnt unmittelbar unter der oberen Führungsebene und umfasst Personen, die Personalverantwortung für mindestens drei Mitarbeiter tragen. Dabei handelt es sich normalerweise um die Abteilungs-, Unterabteilungs- und Referatsleiter in einem Ministerium. Als akademische Berufe werden die Berufsgruppen zwischen den Führungskräften und den Sekretariatskräften behandelt. Sekretariatskräfte sind Mitarbeiter, die für Verwaltungsaufgaben und allgemeine Büroarbeiten zuständig sind.

\section{Literaturhinweise}

OECD (2013), Gleichstellung der Geschlechter - Zeit zu handeln, OECD Publishing, Paris, http://dx.doi.org/10.1787/9789264190344-de.

OECD (2011), Public Servants as Partners for Growth: Toward a Stronger, Leaner and More Equitable Workforce, OECD Publishing, Paris, http://dx.doi.org/10.1787/9789264166707-en.

\section{Anmerkungen zu den Abbildungen}

Die Daten für Luxemburg, Slowenien und Schweden beziehen sich auf 2011 anstelle von 2010. Die Daten für Frankreich beziehen sich auf 2009 anstelle von 2010. Die Daten für die Niederlande entsprechen Vollzeitäquivalenten.

6.3: Die Daten für Estland, Japan und Spanien beziehen sich nur auf Vollzeitbeschäftigte. Für Finnland stehen keine Daten für das Jahr 2010 zur Verfügung.

6.4: Die Daten zu Sekretariatskräften in der Schweiz umfassen auch Stellen im technischen Bereich. 


\subsection{Frauenanteil unter den Beschäftigten auf Ebene der Zentralregierung (2000 und 2010)}

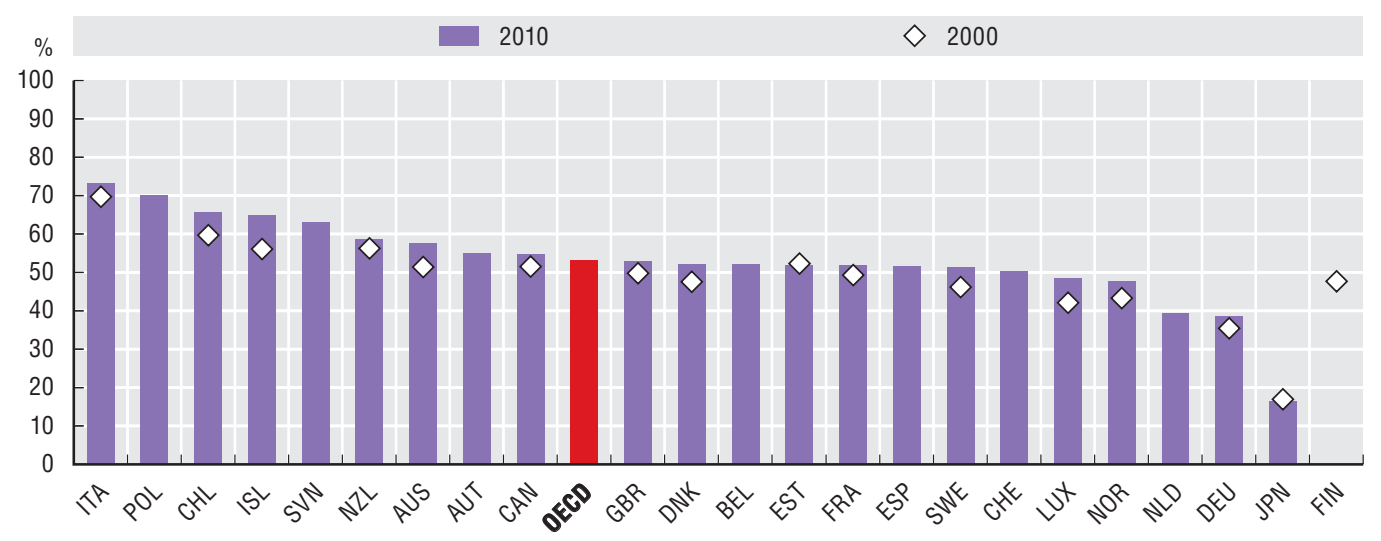

Quelle: 2011 OECD Survey on Gender in Public Employment.

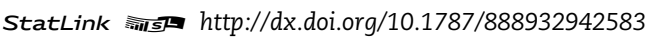

\subsection{Frauenanteil unter den Beschäftigten auf Ebene der Zentralregierung nach Berufsgruppen (2010)}

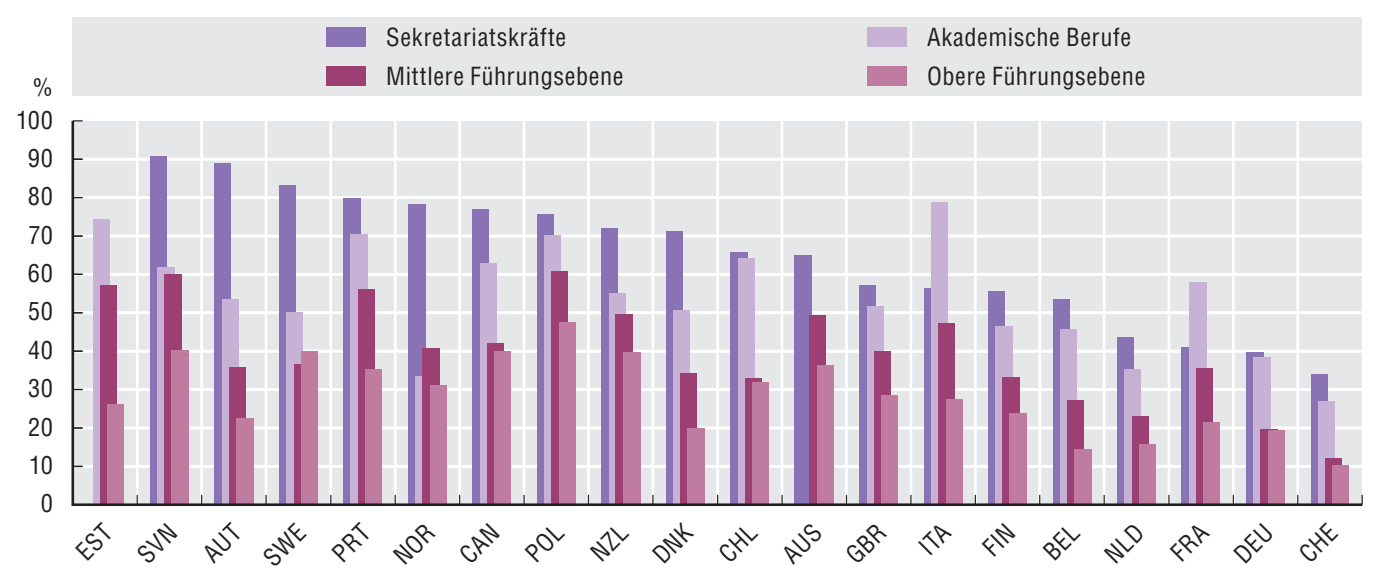

Quelle: 2011 OECD Survey on Gender in Public Employment.

6.5. Frauenanteil in Teilzeitstellen auf Ebene der Zentralregierung (2010)

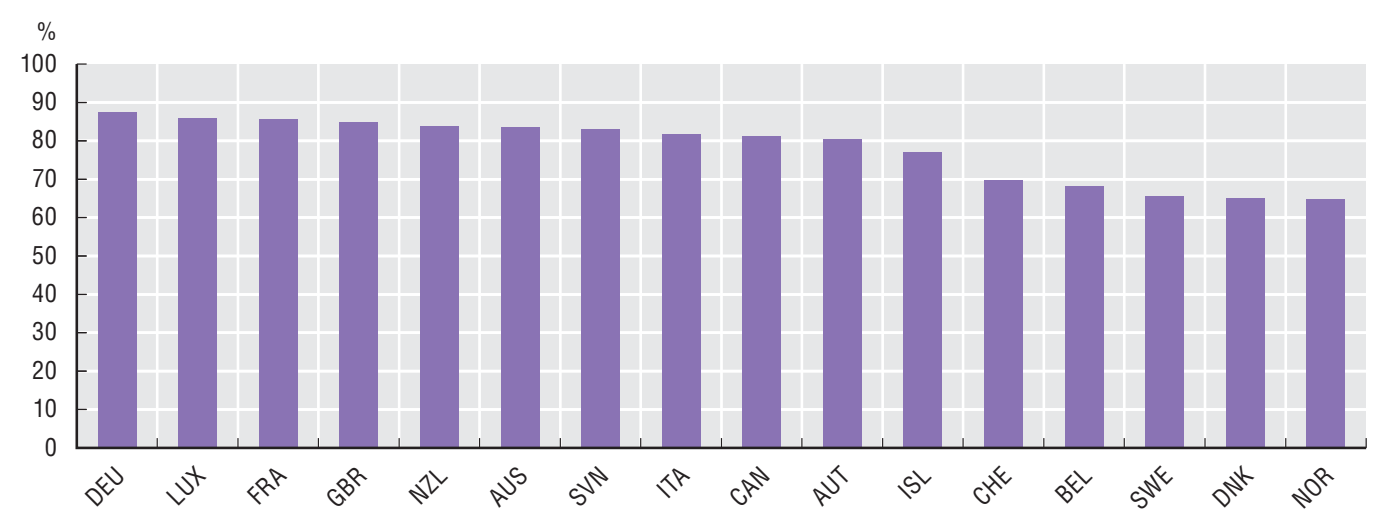

Quelle: 2011 OECD Survey on Gender in Public Employment. 
Trotz des wachsenden Anteils der Frauen unter den Beschäftigten auf Ebene der Zentralregierung (Abbildung 6.3) sind Frauen in leitenden Verwaltungs- oder Führungspositionen nach wie vor unterrepräsentiert. Das auf Ebene der Zentralregierung in den Führungsetagen zu beobachtende Ungleichgewicht zwischen den Geschlechtern begrenzt den Einfluss der Frauen im Entscheidungsprozess, was negative Auswirkungen auf die Fairness staatlicher Maßnahmen und Gesetze haben kann. Ein deutliches Beispiel hierfür ist die Geschlechterverteilung im Justizsystem, die von entscheidender Bedeutung ist, um gleiche Rechte zu sichern und zu verhindern, dass es bei Gerichtsurteilen zu Geschlechtsdiskriminierungen kommt.

Im OECD-Durchschnitt sind über 50\% der Stellen auf Ebene der Zentralregierung mit Frauen besetzt, auf der oberen Führungsebene beträgt dieser Anteil in den 18 Ländern, für die entsprechende Daten vorliegen, aber nur noch $29 \%$. Der Frauenanteil auf der oberen Führungsebene der Behörden der Zentralregierung variiert zwischen den Mitgliedsländern erheblich. In Polen sind fast $50 \%$ der Posten der oberen Führungsebene mit Frauen besetzt, während diese Zahl in Ländern wie Belgien und der Schweiz weniger als halb so hoch ist. Am größten sind die Unterschiede zwischen dem Anteil der Frauen in der Zentralregierung insgesamt und auf der oberen Führungsebene in Österreich, Belgien und Italien.

Ein ähnlicher Trend ist in vielen Justizsystemen im OECDRaum festzustellen. In den Mitgliedsländern, für die Daten vorliegen, waren 2010 etwas weniger als die Hälfte der Berufsrichter Frauen, jedoch waren nur 29\% der Präsidentenposten der Gerichte erster und zweiter Instanz mit Frauen besetzt. In den Obersten Gerichtshöfen waren Frauen ebenfalls vergleichsweise weniger stark repräsentiert (28\% der Richter). In lediglich fünf Mitgliedsländern, für die Daten vorliegen (Tschechische Republik, Irland, Griechenland, Finnland und Schweden), handelte es sich bei den Vorsitzenden der Obersten Gerichtshöfe um Frauen.

Einige Länder ergreifen aktive politische Maßnahmen, um diese Ungleichgewichte abzubauen und gleiche Beschäftigungsmöglichkeiten für Männer und Frauen sicherzustellen. Dazu gehören Diversitäts- und Genderquoten, Vorschriften bzw. Leitlinien zur Sicherung von gleicher Bezahlung für gleiche Arbeit, Schulungen für Führungskräfte und MentoringProgramme sowie Maßnahmen zur besseren Vereinbarkeit von Berufs- und Privatleben, wie z.B. flexible Arbeitszeiten. Einige Länder wie das Vereinigte Königreich und Kanada haben spezielle Rechtskommissionen eingesetzt, um das Gleichgewicht zwischen Männern und Frauen unter den Kandidaten für Richterämter zu erhöhen.

\section{Methodik und Definitionen}

Die Daten über den Frauenanteil auf der oberen Führungsebene und auf Ebene der Zentralregierung insgesamt stammen aus dem 2011 von der OECD durchgeführten Survey on Gender in Public Employment. Befragt wurden Staatsbedienstete, die auf Ebene der Zentral-/Bundesregierungen für Personalmanagement und Beschäftigungspolitik zuständig sind.

Die Daten über die Zahl der Berufsrichterinnen und Gerichtspräsidentinnen stammen von der Europäischen Kommission für die Wirksamkeit der Justiz (CEPEJ) des Europarats. Sofern nicht anders angegeben, beziehen sich die letztgenannten Daten auf Gerichte der ersten und zweiten Instanz. Die Daten über Richterinnen in den Obersten Gerichtshöfen stammen aus der Datenbank der Europäischen Kommission über Frauen in Führungspositionen. Die Europäische Kommission definiert den Obersten Gerichtshof als das höchste Rechtsorgan der einzelnen Länder im Bereich des Zivil- und Strafrechts. In einigen Ländern ist der Oberste Gerichtshof jedoch auch für Verwaltungs- und/oder Verfassungsrecht zuständig. Unter den Richtern sind auch die Präsidenten der Obersten Gerichtshöfe erfasst.

\section{Literaturhinweise}

CEPEJ (2012), Evaluation of European Judicial Systems - 2012 edition (2010 data), CEPEJ Studies No. 18, Council of Europe Publishing, Straßburg.

OECD (2013), Gleichstellung der Geschlechter - Zeit zu handeln, OECD Publishing, Paris, http://dx.doi.org/10.1787/9789264190344-de.

\section{Anmerkungen zu den Abbildungen}

6.6: Für Portugal und Finnland liegen keine Daten über den Anteil der Frauen unter den Beschäftigten auf Ebene der Zentralregierung vor. Die Daten für Luxemburg, Slowenien und Schweden beziehen sich auf 2011 anstelle von 2010. Die Daten für Frankreich beziehen sich auf 2009 anstelle von 2010. Die Daten für Estland, Japan und Spanien beziehen sich nur auf Vollzeitbeschäftigte. Die Daten für die Niederlande entsprechen Vollzeitäquivalenten.

6.7: Die Daten über den Anteil der Gerichtspräsidentinnen in Island, Irland, der Schweiz und der Türkei beziehen sich nur auf Gerichte der ersten Instanz. Die Daten für Spanien beziehen sich nur auf Gerichte der zweiten Instanz.

6.8: Die Daten für Australien, Kanada, Korea, Mexiko, Neuseeland, die Schweiz und die Ukraine beziehen sich auf 2010. Die Daten für Chile, Norwegen und Israel beziehen sich auf 2011. Die Daten für die Vereinigten Staaten und Japan beziehen sich auf 2013.

Hinweis zu den Daten für Israel: http://dx.doi.org/10.1787/888932315602. 


\subsection{Frauenanteil auf der oberen Führungsebene im Vergleich zum Frauenanteil auf Ebene der Zentralregierung insgesamt (2010)}

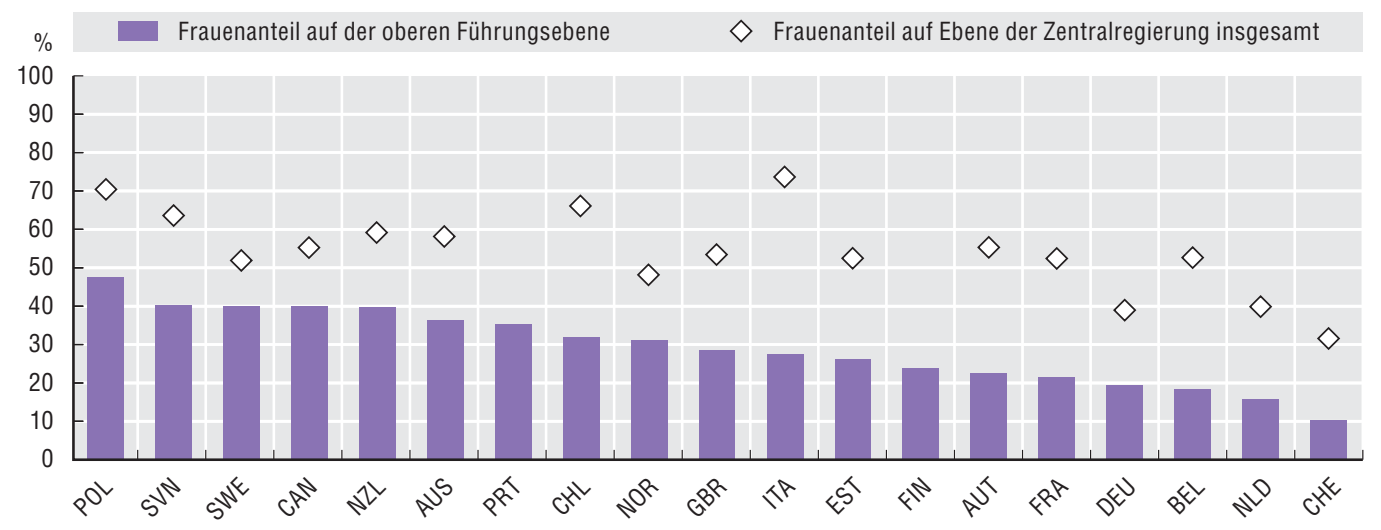

Quelle: 2011 OECD Survey on Gender in Public Employment.

\subsection{Frauenanteil unter den Gerichtspräsidenten im Vergleich zum Frauenanteil unter den Berufsrichtern (2010)}

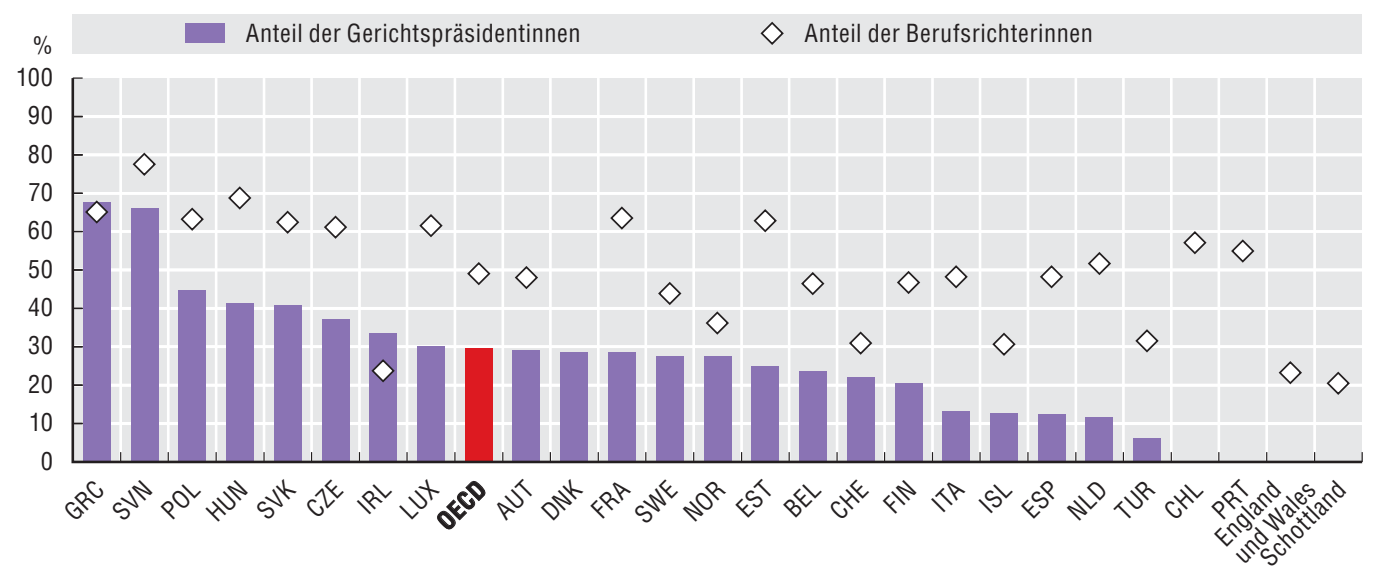

Quelle: CEPEJ (2012), Evaluation of European Judicial Systems - 2012 edition (2010 data), CEPEJ Studies No. 18, Council of Europe Publishing, Straßburg. Die Daten für Chile wurden von nationalen Behörden bereitgestellt.

\subsection{Frauenanteil unter den Richtern der Obersten Gerichtshöfe (2012 oder letztes verfügbares Jahr)}

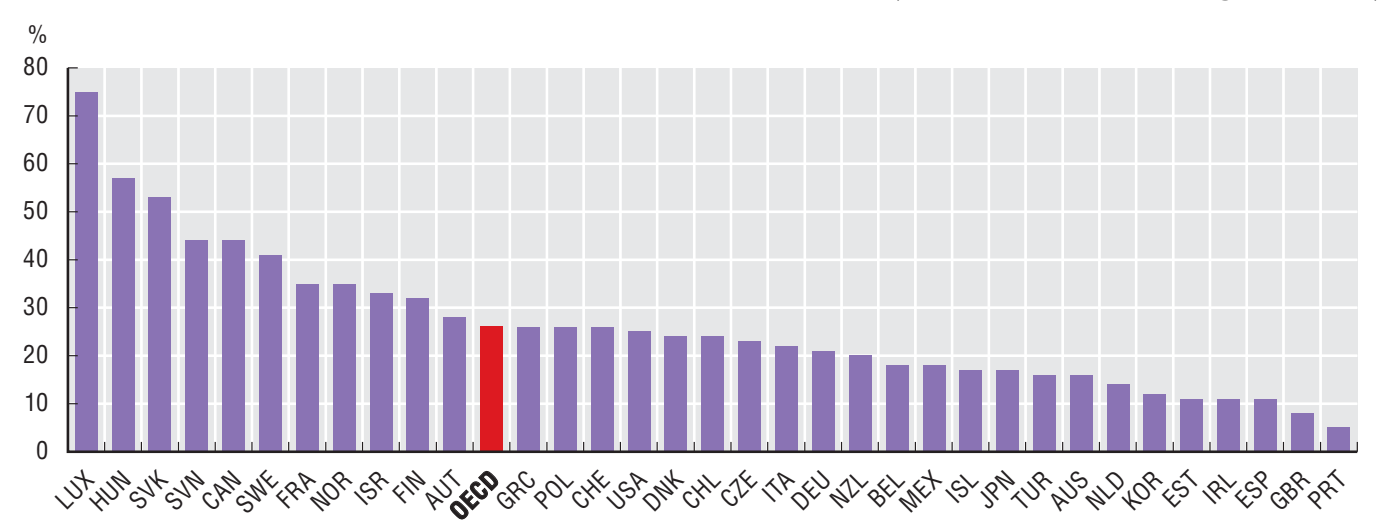

Quelle: EU-Länder: Europäische Kommission, Datenbank über Frauen in Führungspositionen. Nicht-EU-Länder: 2011 OECD Survey on Women in Public Leadership. 
Frauen in der Politik sind nicht nur wichtig, um die Gleichstellung der Geschlechter im politischen Prozess zu gewährleisten, sondern auch um die Aufmerksamkeit auf wichtige sozioökonomische Fragen zu lenken, z.B. menschliche Entwicklung, geschlechtsbasierte Gewalt, familienfreundliche Maßnahmen, Lohngleichheit, Renten, Reform des Wahlrechts sowie Dienstleistungserbringung. Für Frauen gibt es jedoch nach wie vor eine "gläserne Decke“ , die ihre volle Teilnahme am politischen Leben in der Legislative und der Exekutive verhindert, und sie sind in der Politik generell immer noch unterrepräsentiert.

Anfang 2012 entfiel im OECD-Raum knapp über ein Viertel der Unterhaussitze bzw. der Sitze in Einkammerparlamenten auf Frauen, und in lediglich 12 Mitgliedsländern wurde der von den Vereinten Nationen und der Interparlamentarischen Union empfohlene Mindestfrauenanteil von 30\% erreicht oder überschritten. Im Vergleich zu 2002 bedeutet dies einen geringfügigen Anstieg um durchschnittlich 6 Prozentpunkte. Der Frauenanteil in den Parlamenten war im OECD-Raum in den nordischen Ländern generell am höchsten, und in Schweden, Finnland, Island und Norwegen hatten Frauen mindestens 40\% der Sitze inne. In der Türkei sowie in Japan, Chile und Ungarn entfiel weniger als ein Viertel der Sitze auf Frauen.

In Ländern mit Verhältniswahlrecht ist der Frauenanteil in der Legislative tendenziell größer, was möglicherweise darauf zurückzuführen ist, dass bei der Kandidatenauswahl für die Parteilisten stärker auf ein ausgewogenes Geschlechterverhältnis geachtet wird. In neun der zwölf OECD-Länder, die die 30\%Marke für den Frauenanteil erreichen, werden die Parlamentsabgeordneten in einem Verhältniswahlsystem gewählt. Einfluss auf die Präsenz der Frauen in der aktiven Politik hat jedoch nicht nur die Art des Wahlsystems; auch kulturelle und finanzielle Faktoren können ein Hindernis darstellen, ebenso wie Schwierigkeiten bei der Vereinbarung von politischen und familiären Pflichten. Um diesem Defizit entgegenzuwirken, haben neun OECD-Länder Genderquoten zur Förderung der Gleichstellung der Geschlechter in den Parlamenten eingeführt. Bei der Anwendung dieser Quoten bestehen jedoch Unterschiede, so gibt es beispielsweise Quoten für den Nominierungsprozess (die z.B. einen bestimmten Frauenanteil auf den Parteilisten oder einen bestimmten Anteil weiblicher Kandidaten für die Wahlbezirke vorsehen) ebenso wie Ergebnisquoten, mit denen ein bestimmter Anteil der Parlamentssitze für Frauen reserviert wird.

In der politischen Exekutive der OECD-Mitgliedsländer ist der Prozentsatz der Ministerinnen von 21\% im Jahr 2005 auf 25\% im Jahr 2012 gestiegen. Daten von 2012 zeigen, dass diesbezüglich jedoch große Unterschiede zwischen den Ländern bestehen: Während in Norwegen, Schweden, Finnland und Island über $50 \%$ der Ministerposten mit Frauen besetzt sind, beträgt der Anteil der Ministerinnen in Ungarn, Estland, Slowenien, Griechenland und der Türkei weniger als 10\%. Frauen haben oft Ministerposten im Bereich der Sozial- und Kulturpolitik. Der Prozess der Ernennung der Minister unterscheidet sich zwar je nach dem politischen System der einzelnen Länder (Wahl oder Ernennung durch das Parlament vs. Ernennung durch den Staatspräsidenten mit oder ohne Zustimmung des Parlaments), eine gleich starke Repräsentation von Männern und Frauen ist jedoch in keinem System gewährleistet.

\section{Methodik und Definitionen}

Die Daten über Parlamentarierinnen beziehen sich auf Unterhaussitze bzw. Sitze in Einkammerparlamenten und stammen aus der PARLINE-Datenbank der Interparlamentarischen Union. Die Daten beziehen sich auf den Frauenanteil in den Parlamenten zum 31. Oktober 2012 und zum 25. Oktober 2002.

Die Balken in einem helleren Lilaton stellen Länder dar, in denen es mit Stand von Januar 2013 gesetzliche Quoten für den Anteil der Frauen unter den Kandidaten für die Wahl zum Unterhaus oder zum Einkammerparlament gab. Solche Quotenregelungen für die Wahl der Legislative sind im Wahlrecht, im Parteiengesetz oder in anderen vergleichbaren Gesetzen eines Landes festgelegt. Beide Formen basieren per definitionem auf Rechtsvorschriften, die alle an Wahlen teilnehmenden politischen Organisationen verpflichten, die Quotenregelungen gleichermaßen anzuwenden. Politische Organisationen, die die Quotenregelungen nicht einhalten, können mit Strafen belegt werden. Die Daten zu den Frauenquoten stammen aus der Global Database on Quotas for Women des Institute for Democracy and Electoral Assistance (IDEA).

Die Daten zum Anteil der Ministerinnen basieren auf den Übersichtstafeln der Interparlamentarischen Union zum Thema „Frauen in der Politik“ (Women in Politics). Die Daten beziehen sich auf die zum 1. Januar 2012 und 1. Januar 2005 amtierenden Ministerinnen. Sie zeigen den Frauenanteil unter den Ministern insgesamt, einschließlich der stellvertretenden Premierminister und Minister. Premierminister bzw. Regierungschefs wurden ebenfalls berücksichtigt, wenn sie ein Ministeramt ausübten. Vizepräsidenten und Leiter von Regierungsstellen oder Behörden wurden in der Gesamtzahl nicht berücksichtigt.

\section{Literaturhinweise}

International IDEA (2007), Designing for Equality, Stockholm, Schweden.

OECD (2013), Gleichstellung der Geschlechter - Zeit zu handeln, OECD Publishing, Paris, http://dx.doi.org/10.1787/9789264190344-de.

\section{Anmerkungen zu den Abbildungen}

6.9: Die Daten beziehen sich auf den Frauenanteil in den Parlamenten, Stand 31. Oktober 2012 und 25. Oktober 2002. Die Prozentsätze entsprechen der Zahl der Parlamentarierinnen im Verhältnis zur Zahl der insgesamt besetzten Parlamentssitze. Für die Slowakische Republik liegen für 2002 keine Daten vor.

6.10: Die Daten beziehen sich auf die mit Stand vom 1. Januar 2012 und 1. Januar 2005 amtierenden Ministerinnen. In der Gesamtzahl sind stellvertretende Premierminister und Minister berücksichtigt. Premierminister bzw. Regierungschefs wurden ebenfalls berücksichtigt, wenn sie ein Ministeramt ausübten. Vizepräsidenten und Leiter von Regierungsstellen oder Behörden wurden nicht berücksichtigt.

Hinweis zu den Daten für Israel: http://dx.doi.org/10.1787/888932315602. 


\subsection{Frauenanteil in den Parlamenten und gesetzliche Frauenquoten (2012 und 2002)} Unterhaus oder Einkammerparlament

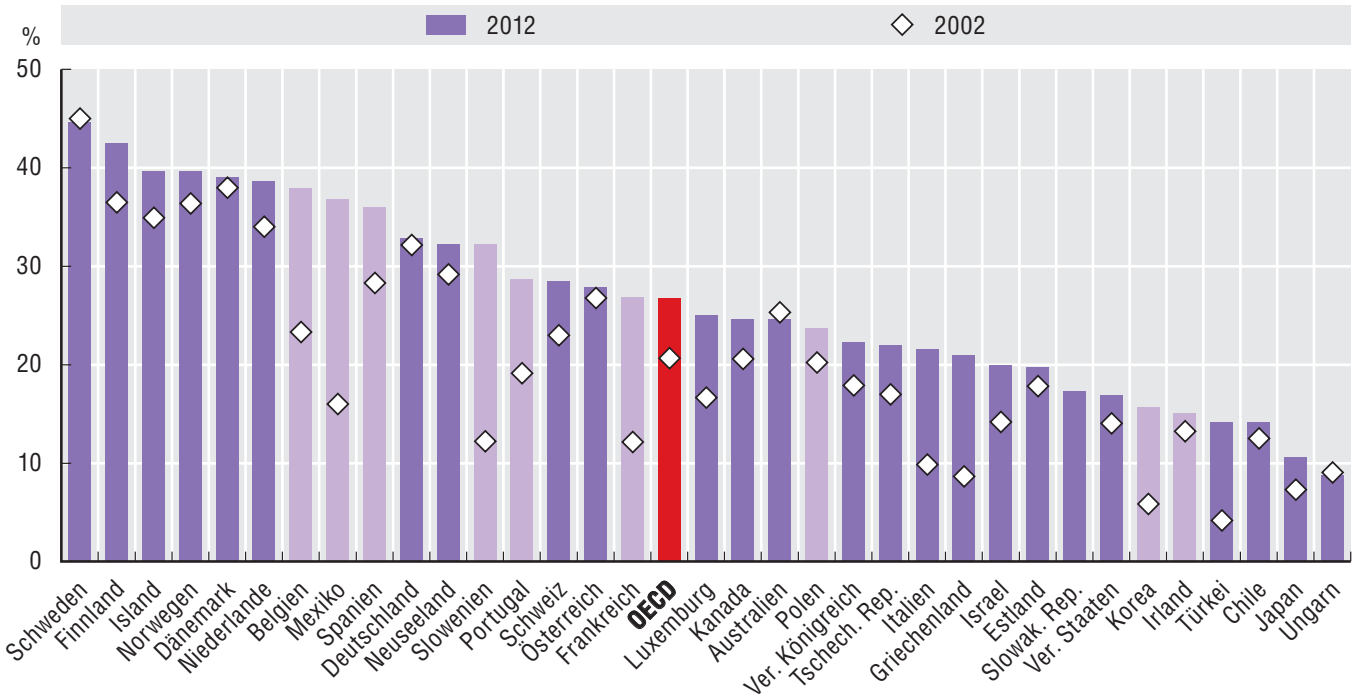

Anmerkung: Die Balken in einem helleren Lilaton stellen die Länder dar, in denen es zum 21. Januar 2013 gesetzliche Quoten für den Anteil der Frauen unter den Kandidaten für die Wahl zum Unterhaus oder zum Einkammerparlament gab.

Quelle: Interparlamentarische Union (IPU), PARLINE (Datenbank), und IDEA, Quota Project (Datenbank).

StatLink तillst http://dx.doi.org/10.1787/888932942697

\subsection{Anteil der Ministerinnen (2005 und 2012)}

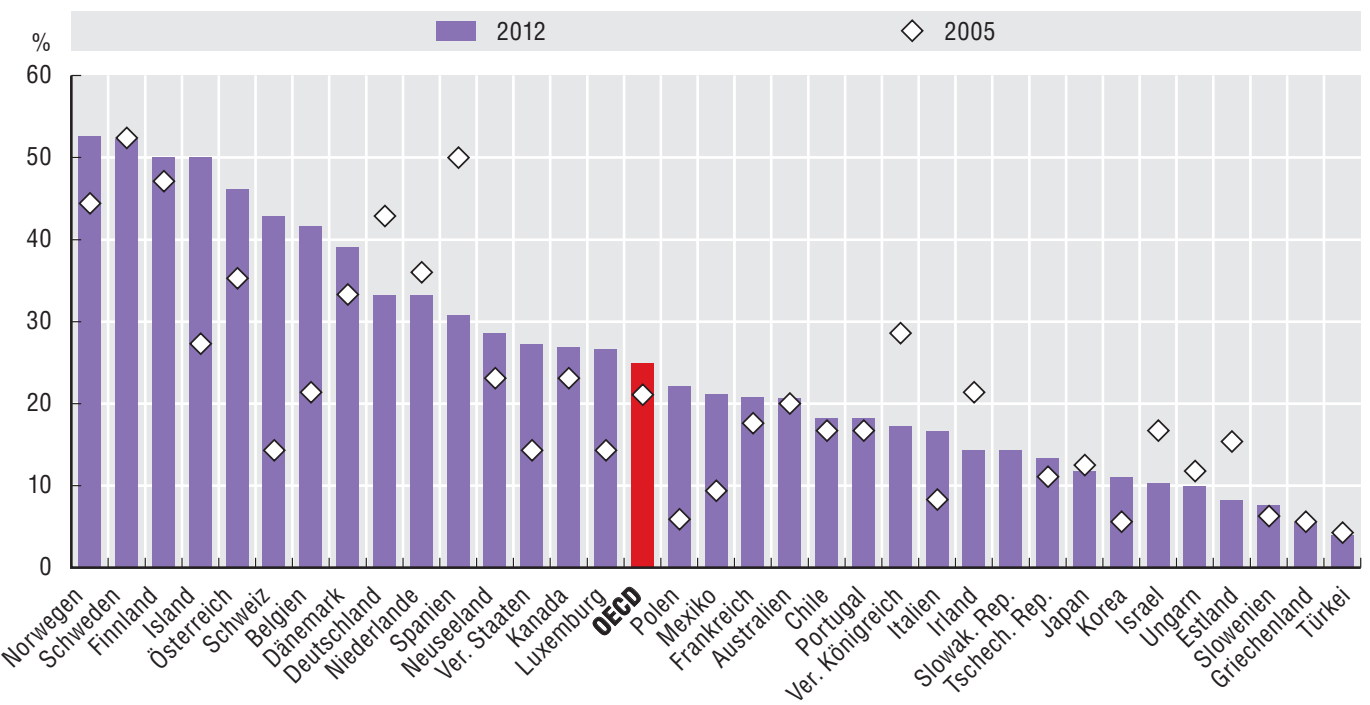

Quelle: Interparlamentarische Union (IPU), „Women in Politics“, 2012 und 2005. 


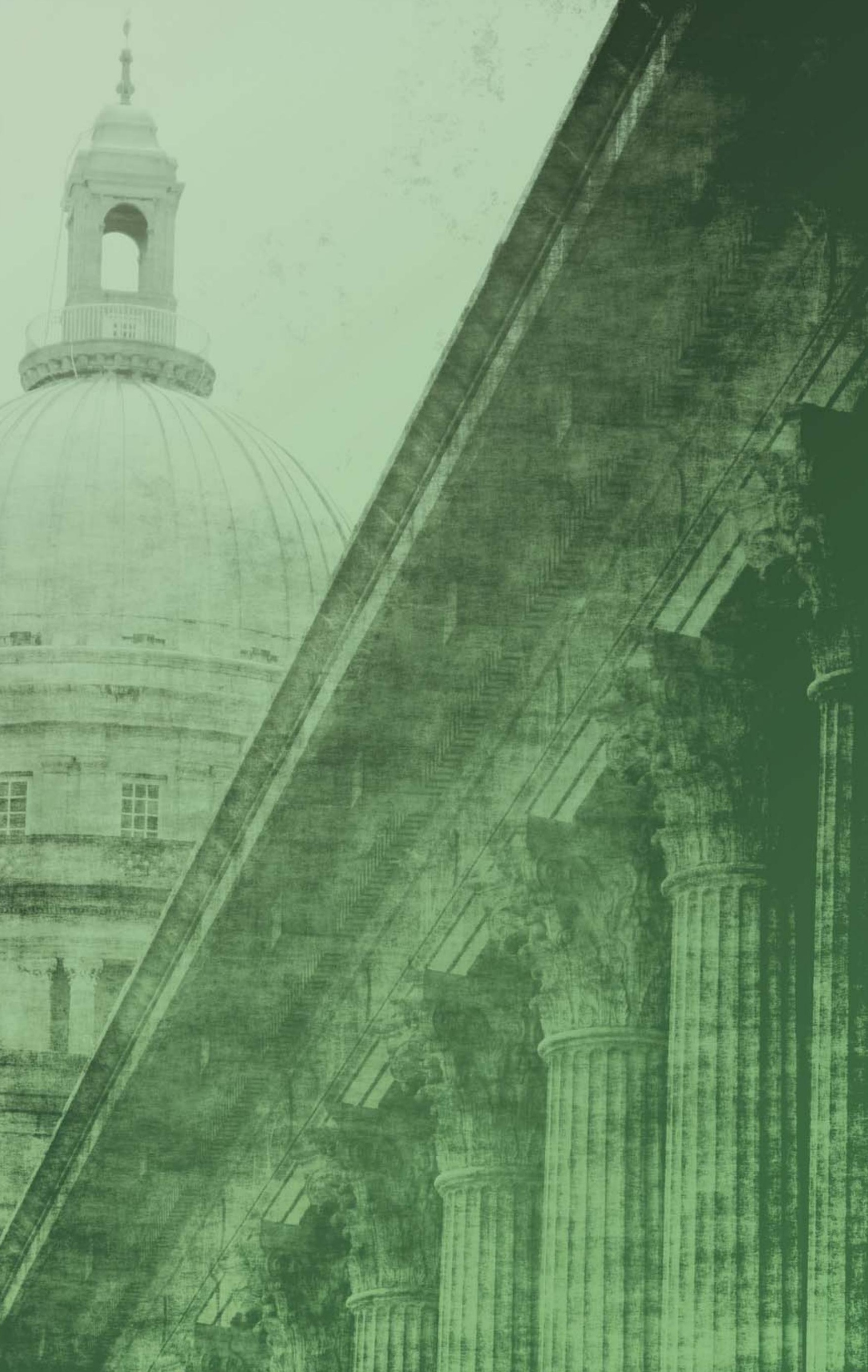




\section{-}

I5

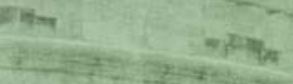

\section{7. ÖFFENTLICHES BESCHAFFUNGSWESEN}

Das öffentliche Beschaffungswesen, auf das im OECD-Durchschnitt 13\% des BIP und $29 \%$ der gesamtstaatlichen Ausgaben entfallen, ist ein sehr wichtiger Bereich staatlichen Handelns. Dieses Kapitel präsentiert eine Schätzung der Kaufkraft des öffentlichen Beschaffungswesens und zeigt die Möglichkeiten, dieses Instrument als politischen Hebel einzusetzen, um einerseits wirtschaftliche, soziale und ökologische Ziele zu verfolgen und andererseits das Preis-Leistungs-Verhältnis und die Ausgabeneffizienz zu verbessern.

Eine der Hauptaufgaben der Regierungen des OECD-Raums besteht heute darin, Einsparungen zu erzielen, um die öffentlichen Finanzen zu konsolidieren und fiskalischen Spielraum für andere Politikmaßnahmen zu schaffen. Viele OECD-Länder greifen deshalb auf innovative Instrumente zurück, um Skalenvorteile zu erzielen, indem sie die Beschaffungsabteilungen neu strukturieren, die Beschaffung konsolidieren und im Beschaffungsprozess von Informationstechnologien (IKT) Gebrauch machen.

Darüber hinaus nutzen zahlreiche OECD-Mitgliedsländer die öffentliche Auftragsvergabe nicht nur, um das Preis-Leistungs-Verhältnis zu verbessern, sondern auch um andere Politikziele zu verfolgen. Bei diesen Politikzielen geht es darum, Anreize für Innovationen zu setzen, nachhaltiges Wachstum zu fördern, die Entwicklung von KMU zu unterstützen und den Zugang zu wirtschaftlichen Chancen durch die Sicherung gleicher Wettbewerbsbedingungen zu verbessern. Neben dieser Thematik werden in diesem Kapitel auch andere strategische Ansätze im Bereich des öffentlichen Beschaffungswesens erfasst.

Die Regierungen werden in den kommenden Jahren zunehmend mit der Herausforderung konfrontiert sein, die verschiedenen durch das Beschaffungswesen verfolgten Ziele miteinander in Einklang zu bringen, die Überwachung und Evaluierung der Beschaffungsausgaben und -ergebnisse zu verstärken und die Professionalisierung im Beschaffungswesen voranzubringen. 
Öffentliche Beschaffung ist der Erwerb von Waren, Dienstleistungen und Bauleistungen durch den Staat und staatseigene Unternehmen. Auf diesen Bereich entfällt ein erheblicher Anteil der gesamtstaatlichen Ausgaben. 2011 betrugen die gesamtstaatlichen Beschaffungsausgaben durchschnittlich $29 \%$ der Gesamtstaatsausgaben (13\% des BIP).

Angesichts der Kaufkraft des öffentlichen Beschaffungswesens können Länder, denen es gelingt, die Effizienz der Beschaffungsausgaben zu verbessern, erhebliche Einsparungen erreichen und folglich den fiskalischen Spielraum für die Wirtschafts- und Sozialpolitik vergrößern. So würde beispielsweise ein durch Effizienzsteigerungen herbeigeführter Rückgang der Beschaffungsausgaben um 10\% (bei unverändertem Waren- und Dienstleistungskorb) im OECD-Durchschnitt zu einer Reduzierung der gesamtstaatlichen Ausgaben um 2,9\% führen, was 2011 1,3\% des BIP entsprach. Die Effizienz kann durch verschiedene Instrumente verbessert werden, u.a. durch den Einsatz von IKT und die Konsolidierung der Beschaffung, um Skalenvorteile zu nutzen.

Die nachgeordneten Gebietskörperschaften sollten ebenfalls Anstrengungen unternehmen, um die Effizienz der Beschaffungsausgaben zu verbessern, da die staatlichen Beschaffungsausgaben auf regionaler und kommunaler Ebene im OECDDurchschnitt $55 \%$ der gesamtstaatlichen Beschaffungsausgaben ausmachen. Dies gilt insbesondere für föderal organisierte Staaten - Österreich, Belgien, Kanada, Deutschland, Mexiko, Spanien, die Schweiz und die Vereinigten Staaten -, da durchschnittlich $76 \%$ der öffentlichen Aufträge auf der Ebene der Gliedstaaten und Kommunen vergeben werden. Einheitsstaaten sollten auf der Ebene der nachgeordneten Gebietskörperschaften, deren Anteil an den Beschaffungsausgaben durchschnittlich $48 \%$ beträgt, aber ebenfalls Maßnahmen ergreifen, insbesondere Italien (80\%), Finnland (72\%), Dänemark (69\%), Japan $(69 \%)$ und Schweden (69\%).

\section{Methodik und Definitionen}

Die für die Schätzung der gesamtstaatlichen Beschaffungsausgaben verwendeten Daten stammen aus der OECD-Datenbank OECD National Accounts Statistics, die auf dem System der Volkswirtschaftlichen Gesamtrechnungen (SNA) basiert. Das öffentliche Beschaffungswesen wird definiert als die Summe aus Vorleistungen (Waren und Dienstleistungen, die vom Staat zur eigenen Verwendung gekauft werden, namentlich Buchführung und ITDienstleistungen), Bruttoanlageinvestitionen (Differenz aus dem Erwerb und der Veräußerung von Anlagegütern, insbesondere der Bau neuer Straßen) und über Marktproduzenten bereitgestellten sozialen Sachtransfers (vom Staat gekaufte Waren und Dienstleistungen, die von Marktproduzenten hergestellt und an Haushalte geliefert werden). Abbildung 7.3, General government procurement as a percentage of GDP (2011), (Öffentliche Auftragsvergabe in Prozent des BIP), ist online verfügbar unter http://dx.doi.org/10.1787/888932942773).

Das öffentliche Beschaffungswesen umfasst die Auftragsvergabe auf der Ebene der Zentralregierung, der Gliedstaaten und der Gemeinden. Der Anteil der nachgeordneten Gebietskörperschaften bezieht sich auf die Gliedstaaten und die Gemeinden. Falls in den Anmerkungen nicht anders vermerkt, wurden die Sozialversicherungsträger bei dieser Analyse ausgeklammert (Abbildung 7.4, Government procurement by levels of government including social security funds - Anteil der staatlichen Ebenen an der öffentlichen Auftragsvergabe unter Einbeziehung der Sozialversicherungsträger - ist online verfügbar unter http://dx.doi.org/10.1787/ 888932942792). Die Kategorie der Gliedstaaten findet nur für die neun OECD-Länder Anwendung, die föderal organisiert sind: Australien, Österreich, Belgien, Kanada, Deutschland, Mexiko, Spanien (wird als Quasi-Föderalstaat betrachtet), Schweiz und Vereinigte Staaten. Öffentliche Unternehmen wurden bei der Schätzung der Beschaffungsausgaben ebenfalls ausgeklammert.

\section{Weitere Informationen}

OECD (2013, erscheint demnächst), Principles for Integrity in Public Procurement: Progress in OECD Countries, OECD Publishing, Paris.

OECD (2011), National Accounts at a Glance 2011, OECD Publishing, Paris, http://dx.doi.org/10.1787/na_glance-2011-en.

\section{Anmerkungen zu den Abbildungen}

Für Chile stehen keine Daten zur Verfügung. Die Daten für Kanada und Neuseeland beziehen sich auf 2010 anstelle von 2011.

7.2: Für Australien stehen keine Daten zur Verfügung. In Neuseeland, Norwegen, dem Vereinigten Königreich und den Vereinigten Staaten umfasst die zentralstaatliche Ebene auch die Sozialversicherungsträger. In Japan beziehen sich die Daten zu den nachgeordneten Gebietskörperschaften auf Finanzjahre.

Hinweis zu den Daten für Israel: http://dx.doi.org/10.1787/888932315602. 


\section{7. ÖFFENTLICHES BESCHAFFUNGSWESEN}

7.1. Öffentliche Auftragsvergabe als Anteil der gesamtstaatlichen Ausgaben (2011)

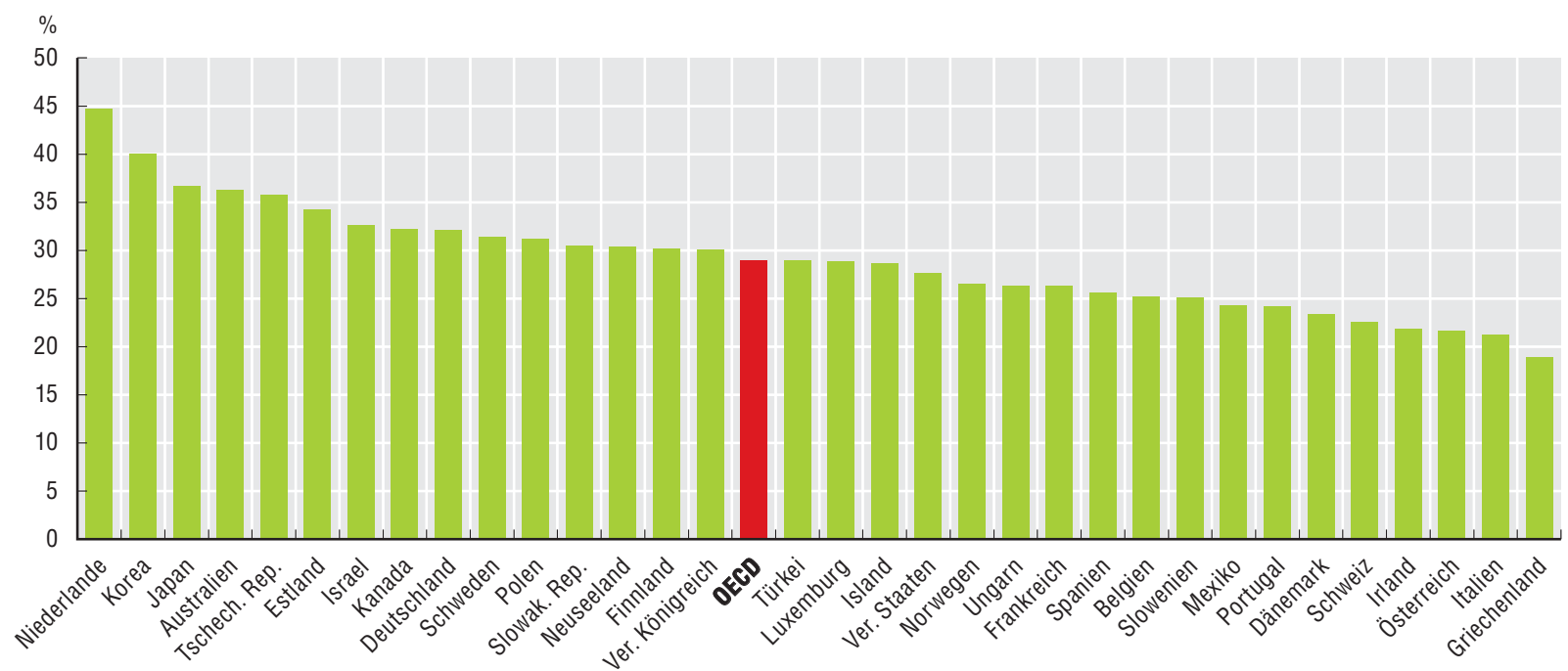

Quelle: OECD National Accounts Statistics (Datenbank). Die Daten für Australien gründen sich auf die Government Finance Statistics sowie vom Australian Bureau of Statistics zur Verfügung gestellte Daten aus der australischen Volkswirtschaftlichen Gesamtrechnung.

StatLink 羿ISL http://dx.doi.org/10.1787/888932942735

7.2. Anteil der staatlichen Ebenen an der öffentlichen Auftragsvergabe bei Ausklammerung der Sozialversicherungsträger (2011)

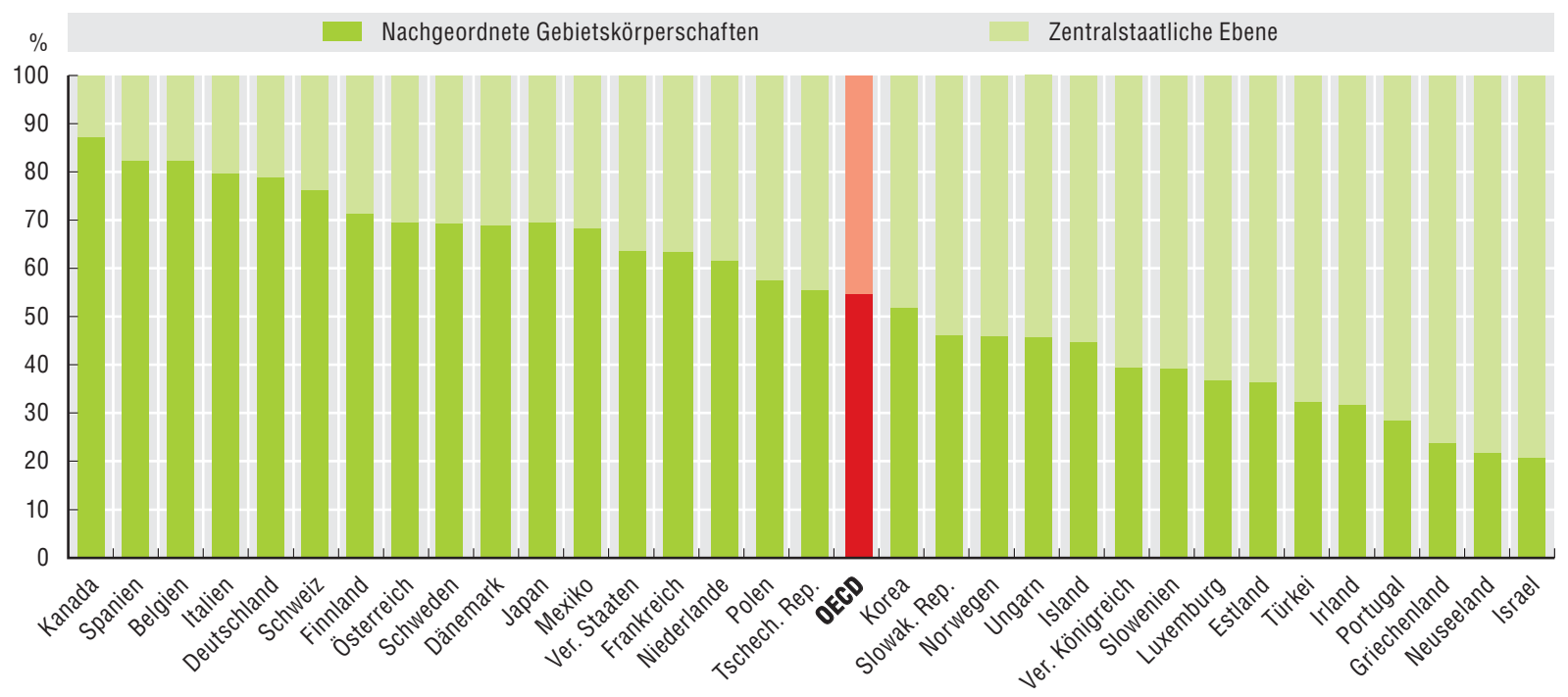

Quelle: OECD National Accounts Statistics (Datenbank). 
Da Produktivitätssteigerungen in Zeiten der Sparpolitik dringend geboten sind, investieren viele OECD-Mitgliedsländer in innovative Instrumente, um die Vergabeverfahren zu straffen und das Preis-Leistungs-Verhältnis der Beschaffungsausgaben zu verbessern. Zu diesen Instrumenten gehört insbesondere der verstärkte Einsatz von elektronischen Vergabeplattformen, Rahmenvereinbarungen, Präqualifikationssystemen, inversen elektronischen Auktionen und Verträgen mit Optionen.

Elektronische Beschaffung - der Einsatz von Informations- und Kommunikationstechnologien im öffentlichen Beschaffungswesen - kann den Zugang zu öffentlichen Ausschreibungen erleichtern und den Wettbewerb erhöhen. Darüber hinaus können E-Procurement-Instrumente dazu beitragen, durch eine Reduzierung des Verwaltungsaufwands, eine Verkürzung der Vergabezyklen und eine Erhöhung des Compliance-Niveaus die Kosten für die öffentliche Hand zu senken. Viele Zentralregierungen haben in elektronische Beschaffungssysteme investiert, die staatlichen Stellen haben die potenziellen Nutzeffekte dieser Instrumente jedoch noch nicht voll ausgeschöpft. In den OECD-Mitgliedsländern werden E-Procurement-Systeme beispielsweise nach wie vor hauptsächlich als Plattform zur Veröffentlichung von Informationen und nicht als Instrument zur wechselseitigen Kommunikation mit den Lieferanten benutzt. Während fast alle OECD-Mitgliedsländer (97\%) die Ausschreibungen in einem nationalen elektronischen Beschaffungssystem ankündigen, bieten nur $48 \%$ den interessierten Lieferanten die Möglichkeit, ihre Angebote elektronisch einzureichen. Die öffentliche Beschaffungsbehörde Koreas stellt eine Ausnahme von diesem Trend dar, da sie 2011 ein neues System eingeführt hat, das es ermöglicht, das Bietungsverfahren über neu entwickelte Sicherheitstoken und Zusatzanwendungen mit Smartphones abzuwickeln.

Darüber hinaus entwickeln die Länder neue Vergabeverfahren, um die Kosten zu senken. Fast alle OECD-Mitgliedsländer (94\%) verwenden Rahmenvereinbarungen, um Skaleneffekte zu erreichen. Aber nur etwa ein Drittel dieser Länder berechnet die Einsparungen, die sich aus dem Einsatz dieser Mechanismen ergeben, um nachzuprüfen, ob Skaleneffekte erreicht wurden. Im Gegensatz dazu nutzt weniger als die Hälfte (42\%) der OECD-Mitgliedsländer, die an der Erhebung teilnahmen, regelmäßig inverse elektronische Auktionen. Beim Einsatz dieses Beschaffungsinstruments müssen sowohl die Erfolgsbedingungen als auch die möglichen negativen Auswirkungen berücksichtigt werden. Durch eine Verschärfung des Wettbewerbs können zwar Einsparungen erzielt werden, damit sind jedoch auch Risiken verbunden, namentlich ein erhöhter Wettbewerbsdruck für kleine und mittlere Unternehmen, die häufig ein niedrigeres Produktionsvolumen und geringere Gewinnmargen haben.

\section{Methodik und Definitionen}

Die Daten stammen aus dem 2011 durchgeführten OECD Survey on Reporting Back on the 2008 Procurement Recommendation (29 OECD-Mitgliedsländer haben daran teilgenommen) und dem 2012 durchgeführten OECD Survey on Public Procurement (33 OECD-Mitgliedsländer haben daran teilgenommen). In beiden Erhebungen wurden Länderdelegierte befragt, die auf der Ebene der Zentralregierung für die Beschaffungspolitik zuständig sind.
Der in Abbildung 7.6 verwendete Begriff elektronische Ausschreibung bezieht sich auf Unternehmen, die das Internet nutzen, um Waren oder Dienstleistungen in den elektronischen Beschaffungssystemen der öffentlichen Behörden ihres Landes anzubieten.

Ein Vertrag mit Optionen ist ein Vertrag, der der Vergabestelle die Option sichert, gemäß vertraglich festgelegten Bedingungen bestimmte zusätzliche Waren und Dienstleistungen zu erhalten oder den Vertrag um einen bestimmten Zeitraum zu verlängern. Eine inverse elektronische Auktion ist ein in Echtzeit im Internet durchgeführtes Beschaffungsverfahren, das die Vergabestelle nutzt, um das erfolgreiche Angebot auszuwählen. Dabei legen Lieferanten oder Auftragnehmer innerhalb eines bestimmten Zeitraums nacheinander Angebote mit rückläufigen Preisen vor, die automatisch geprüft werden.

Die Rahmenvereinbarungen variieren von Land zu Land, es handelt sich jedoch generell um Vereinbarungen zwischen Vergabestellen und Lieferanten, die bestimmte Bedingungen festlegen, was den Zuschlag für künftige Aufträge erleichtern kann. Rahmenvereinbarungen werden in zwei Stufen durchgeführt: In einem ersten Schritt wählt die Vergabestelle einen bzw. mehrere Lieferanten oder einen bzw. mehrere Auftragnehmer aus, mit denen die Rahmenvereinbarung abgeschlossen wird. In einem zweiten Schritt erhält ein an dieser Rahmenvereinbarung beteiligter Lieferant oder Auftragnehmer den Zuschlag für einen Auftrag.

Ein Präqualifikationssystem zielt darauf, die Lieferanten und Auftragnehmer, die für die Durchführung des Auftrags geeignet sind, in einem frühen Stadium zu identifizieren.

\section{Weitere Informationen}

Europäische Kommission (2011), Evaluation Report: Impact and Effectiveness of EU Public Procurement Legislation Part 1, Arbeitspapier der Kommissionsdienststellen, SEC(2011)853 Final, Brüssel. Europäische Kommission (2010), Grünbuch zum Ausbau der eBeschaffung in der EU, KOM(2010)571 Final, Brüssel.

OECD (2013, erscheint demnächst), Principles for Integrity in Public Procurement: Progress in OECD Countries, OECD Publishing, Paris.

\section{Anmerkungen zu den Abbildungen und Tabellen}

7.5: Für Griechenland liegen keine Daten vor. Alle Länder der Europäischen Union sind verpflichtet, auf der Website der Europäischen Union für das öffentliche Auftragswesen (www.ted.europa/eu) Auftragsvergabepläne zu veröffentlichen. Die Angaben für Japan beziehen sich auf die Funktionen eines elektronischen Beschaffungssystems, das 2013 in Betrieb genommen werden sollte.

7.6: Alle Unternehmen beschäftigen mindestens 10 Personen (mit Ausnahme des Finanzsektors). Für Australien, Kanada, Chile, Israel, Japan, Korea, Mexiko, Neuseeland, die Schweiz, die Türkei und die Vereinigten Staaten liegen keine Daten vor. Die Daten für die Türkei beziehen sich auf 2011 anstelle von 2012.

Hinweis zu den Daten für Israel: $h t t p: / / d x . d o i . o r g / 10.1787 / 888932315602$. 


\section{7. ÖFFENTLICHES BESCHAFFUNGSWESEN}

7.5. Einsatz innovativer Vergabeinstrumente auf der Ebene der Zentralregierung (2012)

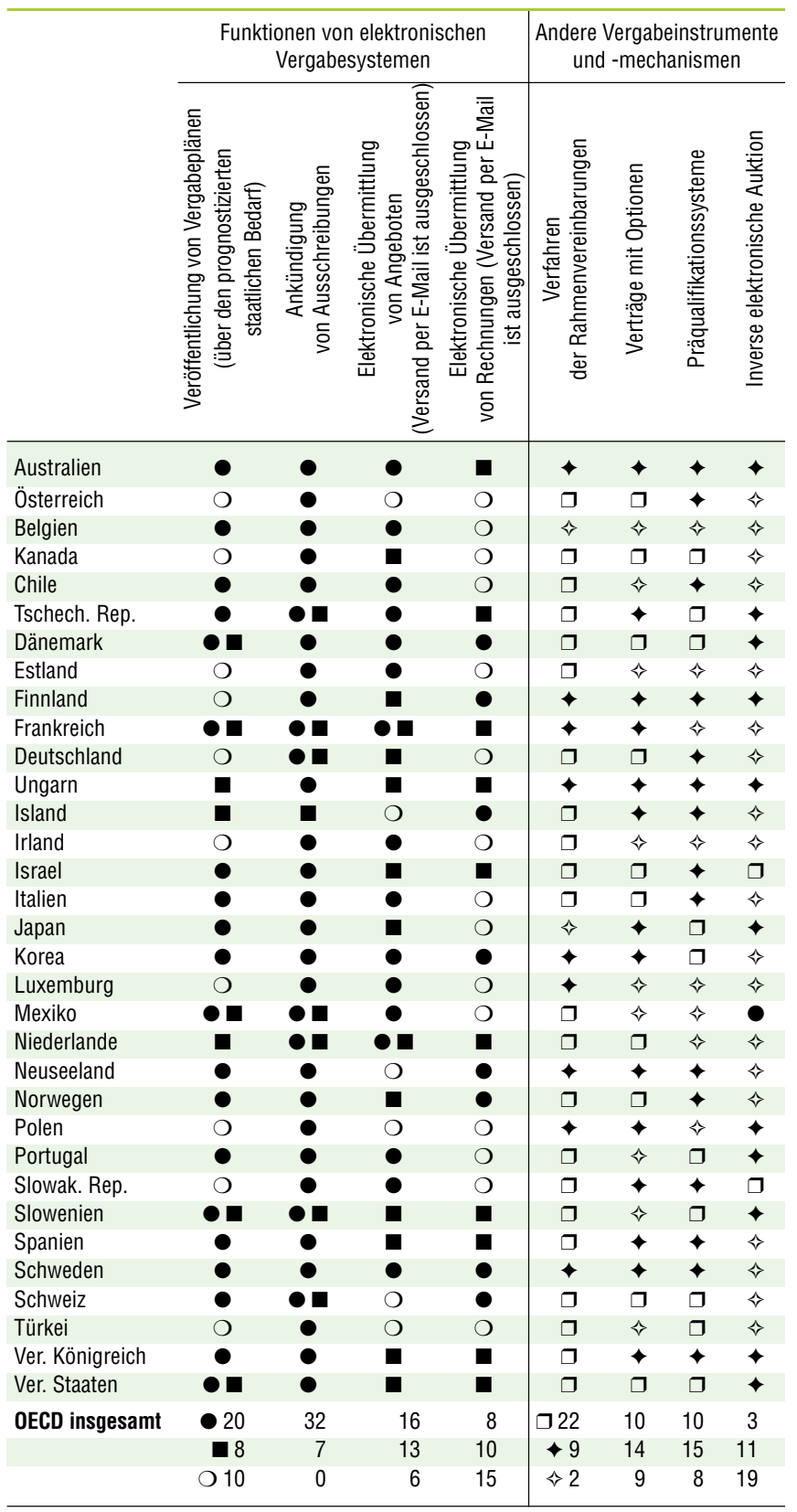

- Ja, in einem nationalen zentralen elektronischen Vergabesystem.

- Ja, in elektronischen Vergabesystemen bestimmter Vergabestellen.

O Nein.

$\square$ Das Instrument wird regelmäßig in allen Vergabestellen eingesetzt.

$\downarrow$ Das Instrument wird regelmäßig in einigen Vergabestellen eingesetzt.

$\diamond$ Das Instrument wird nicht regelmäßig eingesetzt.

Quelle: 2012 OECD Survey on Public Procurement.

StatLink Ailst http://dx.doi.org/10.1787/888932943514

\subsection{Prozentsatz der Unternehmen, die elektronische Vergabesysteme nutzen (2012)}

Zugang zu Ausschreibungsunterlagen und -spezifikationen

$\diamond$ Elektronische Ausschreibungen im Inland

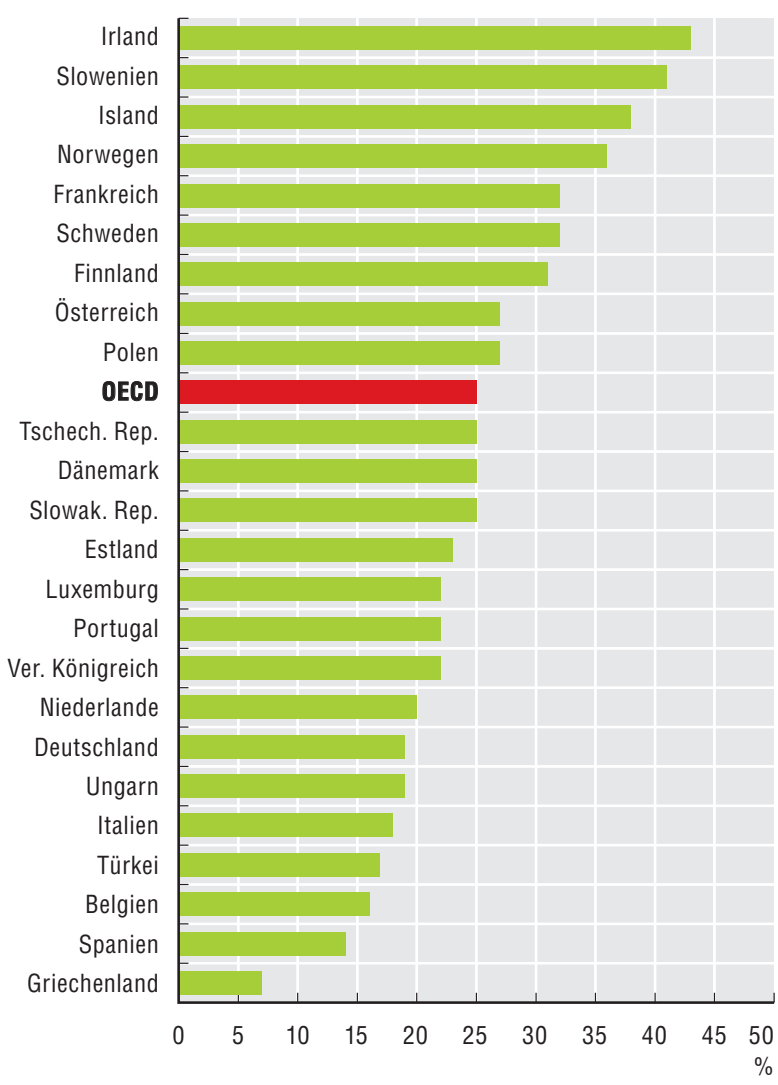

Quelle: Eurostat.

StatLink त्नाIst $h t t p: / / d x . d o i . o r g / 10.1787 / 888932942811$ 
Die OECD-Mitgliedsländer halten ein gutes Preis-LeistungsVerhältnis nicht mehr für das einzige Ziel der öffentlichen Auftragsvergabe. Sie berücksichtigen zunehmend auch stärker strategisch ausgerichtete Ziele wie die Förderung kleiner und mittlerer Unternehmen (KMU), Innovation und Umweltfragen.

Deshalb wird die Priorisierung der Ziele für die staatlichen Stellen immer wichtiger. Fast 70\% der OECD-Mitgliedsländer (23 von 33) haben auf zentralstaatlicher Ebene eine Strategie oder Politik entwickelt, die darauf zielt, die öffentliche Auftragsvergabe verstärkt für die Förderung von KMU einzusetzen. In der Hälfte dieser Länder ist die Förderung von KMU durch das öffentliche Beschaffungswesen gesetzlich geregelt, und in einem Drittel dieser Länder gibt es unverbindliche Regeln mit freiwilligen Zielvorgaben. Darüber hinaus haben 76\% der OECD-Länder auf zentralstaatlicher Ebene eine Strategie oder Politik zur Förderung eines umweltfreundlichen öffentlichen Beschaffungswesens entwickelt.

In den meisten OECD-Mitgliedsländern werden die Opportunitätskosten, die sozioökonomische und ökologische Ziele mit sich bringen, jedoch nicht immer gemessen, was dazu führt, dass den staatlichen Stellen manchmal die geeigneten Instrumente zur Priorisierung widersprüchlicher Ziele fehlen (z.B. ein gutes Preis-Leistungs-Verhältnis im Gegensatz zur Förderung sozioökonomischer und umweltpolitischer Ziele). Die staatlichen Stellen nutzen deshalb die öffentlichen Mittel bei der Auftragsvergabe nicht immer optimal. Obwohl die weitaus meisten OECD-Mitgliedsländer eine KMU-Strategie verfolgen, wird die Zahl oder der Wert der an KMU vergebenen Aufträge in $61 \%$ der OECD-Mitgliedsländer nicht ausgewiesen. Ohne diese Informationen ist die Messung der Effektivität überaus schwierig.

Vor diesem Hintergrund wird von den Fachangestellten in den Beschaffungsämtern erwartet, einerseits immer komplexere Vorschriften einzuhalten und Zielvorgaben in Bezug auf das Preis-Leistungs-Verhältnis zu erfüllen und andererseits strategische Erwägungen zu berücksichtigen. Dennoch geben fast $50 \%$ der OECD-Mitgliedsländer an, dass unzureichende Kapazitäten das größte Problem des Beschaffungswesens darstellen, sowohl in Bezug auf die Personalausstattung, als auch hinsichtlich der mangelhaften Fachkenntnisse über die verfügbaren Technologien, Innovationen und Marktentwicklungen.

Das öffentliche Beschaffungswesen wird in vielen Ländern nach wie vor als Verwaltungsfunktion betrachtet, und mehr als ein Drittel der Länder (39\%) gibt an, dass die Tätigkeit im Beschaffungsamt nicht als eigenständiger Beruf anerkannt wird. Von den 18 OECD-Mitgliedsländern, die die Tätigkeit im Beschaffungsamt als eigenständigen Beruf anerkennen, haben elf Länder eine formelle Stellenbeschreibung für die Fachangestellten im Beschaffungsamt, und in acht Ländern gibt es spezielle Zertifizierungs- oder Lizenzierungsprogramme (Australien, Kanada, Chile, Irland, Neuseeland, Slowakische Republik, Schweiz und Vereinigte Staaten). Allerdings gibt es nur in fünf Ländern spezielle Integritätsleitlinien (z.B. Verhaltenskodizes) für Fachangestellte im Beschaffungswesen.

\section{Methodik und Definitionen}

Die Daten stammen aus dem 2011 durchgeführten OECD Survey on Reporting Back on the 2008 Procurement Recommendation (29 OECD-Mitgliedsländer haben daran teilgenommen) und dem 2012 durchgeführten OECD Survey on Public Procurement (33 OECD-Mitgliedsländer haben daran teilgenommen). In beiden Erhebungen wurden Länderdelegierte befragt, die auf der Ebene der Zentralregierung für die Beschaffungspolitik zuständig sind.

Die Tätigkeit im Beschaffungswesen wird als eigenständiger Beruf anerkannt, wenn dieser Beruf durch ein Zertifizierungs- oder Lizenzierungsprogramm, genau definierte Lehrpläne (z.B. formelle Stellen- bzw. Aufgabenbeschreibung) und/oder Integritätsleitlinien (z.B. spezielle Verhaltenskodizes für Fachangestellte im Beschaffungswesen) anerkannt wurde.

Umweltorientierte Beschaffung wird von der Europäischen Kommission definiert als „ein Prozess, bei dem Behörden Produkte, Dienstleistungen oder Auftragsarbeiten erwerben, die gemessen über ihren gesamten Lebenszyklus geringere Umweltbelastungen verursachen als vergleichbare, aber auf andere Weise beschaffte Produkte, Dienstleistungen und Auftragsarbeiten“.

\section{Weitere Informationen}

OECD (2013, erscheint demnächst), Principles for Integrity in Public Procurement: Progress in OECD Countries, OECD Publishing, Paris.

OECD (2011), Making the Most of Public Investment in a Tight Fiscal Environment: Multi-level Governance Lessons from the Crisis, OECD Publishing, Paris, http://dx.doi.org/10.1787/9789264114470-en.

OECD (2007) „Improving the Environmental Performance of Public Procurement: Report on Implementation of the Council Recommendation“, OECD Papers, Vol. 7/9, http://dx.doi.org/ 10.1787/oecd_papers-u7-art26-en.

\section{Anmerkungen zu den Abbildungen und Tabellen}

7.7: Für Dänemark, Griechenland und Korea liegen keine Daten vor.

7.8: Für Griechenland liegen keine Daten vor. Australien hat eine Vergabepolitik entwickelt, durch die die Gleichstellung der Geschlechter in den Organisationen, die an öffentlichen Ausschreibungen teilnehmen, gefördert wird. In Deutschland beziehen sich die Angaben auf die Bundesebene. Es sollte jedoch darauf hingewiesen werden, dass die Länder eine Vielzahl von Maßnahmen ergriffen haben, um ein umweltorientiertes öffentliches Beschaffungswesen $\mathrm{zu}$ fördern und KMU zu unterstützen. In Dänemark sollte 2013 eine Strategie für intelligente öffentliche Auftragsvergabe eingeführt werden, die strategische Herausforderungen wie Innovation, umweltorientiertes Beschaffungswesen und Förderung von KMU berücksichtigt. Die Daten für die Türkei wurden vom Statistikinstitut der Türkei bereitgestellt.

Hinweis zu den Daten für Israel: http://dx.doi.org/10.1787/888932315602. 


\section{7. ÖFFENTLICHES BESCHAFFUNGSWESEN}

Strategische öffentliche Auftragsvergabe

7.7. Anerkennung der Tätigkeit im Beschaffungswesen als eigenständiger Beruf (2010)

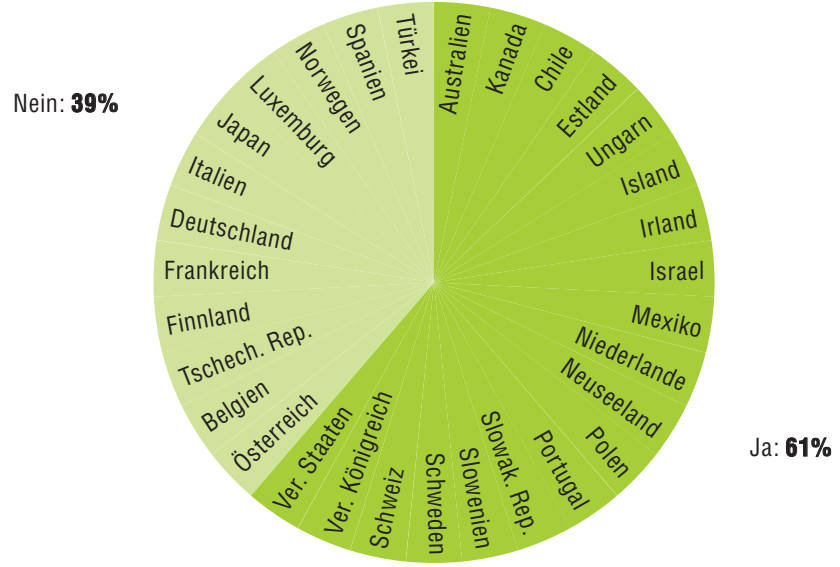

Quelle: 2011 OECD Survey on Reporting Back on the 2008 Procurement Recommendation.

StatLink 게sम http://dx.doi.org/10.1787/888932942830

\subsection{Maßnahmen und Strategien für die Förderung der öffentlichen Auftragsvergabe zu Gunsten sozioökonomischer und/oder ökologischer Ziele (2011)}

\begin{tabular}{|c|c|c|c|c|c|c|}
\hline & \multicolumn{3}{|c|}{$\begin{array}{l}\text { Maßnahmen oder Strategien } \\
\text { zur Förderung der öffentlichen } \\
\text { Auftragsvergabe für sozioökono- } \\
\text { mische und/oder ökologische } \\
\text { Ziele wurden entwickelt }\end{array}$} & \multicolumn{3}{|c|}{$\begin{array}{l}\text { Ergebnisse der Maßnahmen/ } \\
\text { Strategien zur Förderung } \\
\text { der öffentlichen Auftragsvergabe } \\
\text { für sozioökonomische } \\
\text { und/oder ökologische Ziele } \\
\text { werden gemessen }\end{array}$} \\
\hline & 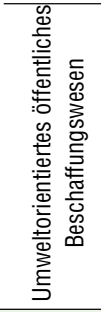 & 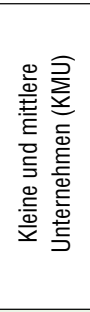 & 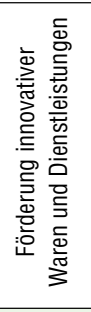 & 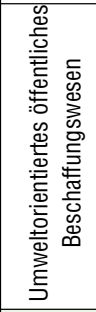 & 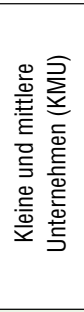 & 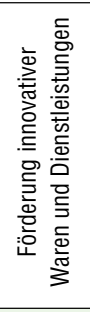 \\
\hline Australien & - & - & - & $\diamond$ & $\diamond$ & $\diamond$ \\
\hline Österreich & - & - & - & $*$ & 口 & $\leftarrow$ \\
\hline Belgien & - & O & O & $*$ & $x$ & $x$ \\
\hline Kanada & - & - & - & च & घ & घ \\
\hline Chile & $\square$ & - & $\square$ & $x$ & घ & $x$ \\
\hline Tschech. Rep. & - & $\square$ & $\square$ & $\downarrow$ & $x$ & $x$ \\
\hline Dänemark & - & - & O & $\diamond$ & $\diamond$ & $x$ \\
\hline Estland & O & O & O & $x$ & $x$ & $x$ \\
\hline Finnland & - & $\square$ & - & च & $x$ & $*$ \\
\hline Frankreich & • & $\bullet$ & - व & घ & $\mathbf{\square}$ & $\diamond$ \\
\hline Deutschland & - & - & - & $\diamond$ & $\diamond$ & $\diamond$ \\
\hline Ungarn & $\square$ & - & O & $x$ & 口 & $x$ \\
\hline Island & - & O & O & $\diamond$ & $x$ & $x$ \\
\hline Irland & - & - & - & 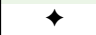 & 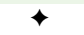 & 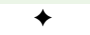 \\
\hline Israel & - & - & O & च & ש & $*$ \\
\hline Italien & - & - & - & च & घ & घ \\
\hline Japan & - & - & O & च & च & $x$ \\
\hline Korea & - & - & - & घ & घ & घ \\
\hline Luxemburg & $\square$ & $\square$ & O & $x$ & $x$ & $x$ \\
\hline Mexiko & - & - & O & $\diamond$ & घ & $x$ \\
\hline Niederlande & - & - & - & a & च & $*$ \\
\hline Neuseeland & O & O & O & $x$ & $x$ & $x$ \\
\hline Norwegen & • 口 & - & - & 口 & $\diamond$ & $\diamond$ \\
\hline Polen & - & - & - & घ & घ & घ \\
\hline Portugal & - & - & $\square$ & घ & $*$ & $x$ \\
\hline Slowak. Rep. & $\square$ & O & O & $x$ & $x$ & $x$ \\
\hline Slowenien & • व & • व & O & घ & घ & $x$ \\
\hline Spanien & - & - & - & $\diamond$ & $\diamond$ & $\diamond$ \\
\hline Schweden & $\square$ & 口 & $\square$ & $x$ & $x$ & $x$ \\
\hline Schweiz & • व & - & - & a & $\downarrow$ & घ \\
\hline Türkei & 0 & O & O & $\mathrm{x}$ & $x$ & $\mathrm{x}$ \\
\hline Ver. Königreich & • 口 & - & - & घ & घ & $x$ \\
\hline Ver. Staaten & $\bullet$ & $\bullet$ & $\bullet$ & $\mathbf{\square}$ & $\mathbf{\square}$ & - \\
\hline \multirow[t]{5}{*}{ OECD insgesamt } & - 25 & 23 & 16 & च 15 & 15 & 6 \\
\hline & $\square 10$ & 7 & 6 & $\bullet 2$ & 1 & 3 \\
\hline & O3 & 6 & 13 & $\uparrow 2$ & 2 & 2 \\
\hline & & & & $\diamond 6$ & 5 & 5 \\
\hline & & & & $\times 8$ & 10 & 17 \\
\hline
\end{tabular}

- Ja, es wurde eine Strategie/Politik auf Ebene der Zentralregierung entwickelt.

$\square$ Ja, einige Vergabestellen haben eine interne Politik entwickelt.

O Nein, es gibt keine derartige Strategie/Politik.

- Ja, regelmäßig.

* Ja, auf Ad-hoc-Basis.

$\uparrow$ Nicht bekannt.

$\diamond$ Nein.

$\mathrm{x}$ Nicht anwendbar.

Quelle: 2012 OECD Survey on Public Procurement.

StatLink Aillsta $h t t p: / / d x . d o i . o r g / 10.1787 / 888932943533$ 
Es ist nach wie vor schwierig, bei öffentlichen Ausschreibungen für alle interessierten Lieferanten gleiche Rahmenbedingungen sicherzustellen, insbesondere auf internationaler Ebene. Die grenzüberschreitende öffentliche Auftragsvergabe in einem integrierten Markt wie der Europäischen Union betrifft weniger als $4 \%$ des Gesamtwerts der vergebenen Aufträge.

Auf nationaler Ebene wird der Wettbewerb durch Ausnahmen vom Grundsatz der wettbewerblichen Ausschreibung eingeschränkt. Infolge des Konjunkturpakets im Anschluss an die Finanzkrise ist die Auftragsvergabe ohne wettbewerbliche Ausschreibung zwischen 2008 und 2011 in 18\% der OECD-Mitgliedsländer gestiegen, was hauptsächlich auf beschleunigte Verfahren zurückzuführen war. Um den Wettbewerb optimal zu nutzen und die Effizienz des Vergabeverfahrens zu sichern, ist es von entscheidender Bedeutung, dass Ausnahmen nur unter bestimmten genau festgelegten Bedingungen gewährt werden. Ausnahmen vom Grundsatz der wettbewerblichen Ausschreibungen können zu Missbrauch führen, wodurch die Verwaltungseffizienz des Beschaffungswesens unterwandert wird.

Auf KMU entfällt zwar ein erheblicher Anteil der Weltwirtschaft und des Arbeitsmarkts, ihr Anteil an den öffentlichen Aufträgen ist jedoch wesentlich niedriger. Um die Harmonisierung der Rahmenbedingungen zu fördern, haben $85 \%$ der OECD-Mitgliedsländer Maßnahmen ergriffen, die direkt auf KMU abzielen, da diese bei der Teilnahme an Ausschreibungen einen komparativen Nachteil haben. Zu den verbreitetsten Maßnahmen gehört die Durchführung von Schulungen und Workshops für KMU (58\% der OECD-Länder) und die Bereitstellung von Unterlagen und Anleitungen für KMU im Internet (51\%). In weniger als einem Drittel der OECD-Mitgliedsländer (30\%) wurden die Verwaltungsverfahren vereinfacht, um die Teilnahme von KMU an Ausschreibungen zu erleichtern.

In einem Drittel der OECD-Mitgliedsländer (33\%) wurden spezifische Rechtsvorschriften oder Politikmaßnahmen eingeführt (z.B. Kontingente), um die Teilnahme von KMU an Ausschreibungen zu fördern. Derartige Präferenzregelungen gibt es z.B. in Australien, Frankreich, Korea und den Vereinigten Staaten. Nur in 6\% der OECD-Mitgliedsländer werden zusätzlich zu Regulierungsmaßnahmen finanzielle Vergünstigungen (z.B. Verzicht auf Gebühren) für KMU gewährt.

\section{Methodik und Definitionen}

Die Daten wurden in zwei Erhebungen über das öffentliche Beschaffungswesen auf der zentralstaatlichen Ebene gesammelt. An dem 2011 von der OECD durchgeführten Survey on Reporting Back on Progress made since the 2008 Procurement Recommendation nahmen 29 OECDMitgliedsländer sowie Brasilien, Ägypten, Marokko und die Russische Föderation teil. Für Dänemark, Griechenland, Korea und Spanien liegen keine Daten vor. An dem 2012 von der OECD durchgeführten Survey on Public Procurement nahmen 33 OECD-Mitgliedsländer sowie Brasilien und Kolumbien teil. Für Griechenland liegen keine Daten vor. In beiden Erhebungen wurden Länderdelegierte befragt, die auf der Ebene der Zentralregierung für die Beschaffungspolitik zuständig sind. Tabelle 7.11, Public procurement in central government by procedure: Availability of data for number and value of contracts (Das öffentliche Beschaffungswesen auf der Ebene der Zentralregierung nach Verfahren: Verfügbarkeit von Daten über die Zahl und den Wert der Aufträge) ist online verfügbar unter $h t t p: / / d x$.doi.org/10.1787/888932943571.

\section{Weitere Informationen}

Europäische Kommission (2010), EU Public Procurement Legislation: Delivering Results, Amt für Gebäude, Anlagen und Logistik, Brüssel. OECD (erscheint demnächst), OECD Review of the United States Federal Public Procurement, OECD Publishing, Paris.

OECD (2013, erscheint demnächst), Principles for Integrity in Public Procurement: Progress in OECD Countries, OECD Publishing, Paris.

OECD (2009), Principles for Integrity in Public Procurement: Progress in OECD Countries, OECD Publishing, Paris. http://dx.doi.org/ 10.1787/9789264056527-en.

OECD (2008), OECD Framework for the Evaluation of SME and Entrepreneurship Policies and Programmes, OECD Publishing, Paris, http://dx.doi.org/10.1787/9789264040090-en.

\section{Anmerkungen zu den Abbildungen und Tabellen}

7.9: In der Tschechischen Republik sind die Auftraggeber verpflichtet, diskriminierungsfreie Ausschreibungsbedingungen festzulegen. In Dänemark hat die Wettbewerbs- und Verbraucherschutzbehörde eine schrittweise Anleitung mit Informationen über Vorschriften, Verfahren und Kernfragen in Bezug auf die Gründung von KMU-Konsortien veröffentlicht. In Estland gibt es keine speziellen Maßnahmen zur Förderung von KMU, weil die meisten estnischen Unternehmen als KMU eingestuft werden. In Finnland plant das zentrale Beschaffungsamt die Ausschreibungen so, dass die Teilnahme von KMU am Ausschreibungsverfahren gefördert wird. In Neuseeland werden die meisten Unternehmen als KMU eingestuft. Es gibt zwar keine spezielle Vorzugsbehandlung für KMU, die Unterstützung erfolgt jedoch, indem die Befolgungskosten der Lieferanten gesenkt werden (z.B. durch die Vereinfachung von Verfahren, die Entwicklung von Online-Anleitungen und Formularen sowie durch Schulungen und Workshops für Lieferanten und Fachkräfte im Beschaffungswesen). In Spanien steht die für die Beurteilung des öffentlichen Beschaffungswesens zuständige zentrale Behörde (Junta Consultativa de Contratación Administrativa) in ständigem Kontakt mit KMU und KMU-Verbänden, um ihre Anliegen in dieser Frage anzuhören. Im Vereinigten Königreich gibt es ein Arbeitsprogramm, das die Ministerien ermutigt, mehr Aufträge an KMU zu vergeben, wenn so Steuergelder kosteneffizienter eingesetzt werden. Anleitungen für KMU bietet beispielsweise der Kurs „Winning the Contract", der auf der Website LearnDirect verfügbar ist. Darüber hinaus wurde das Vergabeverfahren erheblich vereinfacht: So verzichten die Ministerien beispielsweise inzwischen bei den meisten Ausschreibungen unter der EU-Schwelle von rd. 100000 Britischen Pfund (£) auf den Einsatz von Präqualifizierungs-Fragebögen.

7.10: Für Belgien und Griechenland liegen keine Daten vor.

Hinweis zu den Daten für Israel: http://dx.doi.org/10.1787/888932315602. 


\section{7. ÖFFENTLICHES BESCHAFFUNGSWESEN}

Tabelle 7.9. Konzepte zur Förderung eines fairen Zugangs der KMU zu öffentlichen Aufträgen auf der Ebene der Zentralregierung

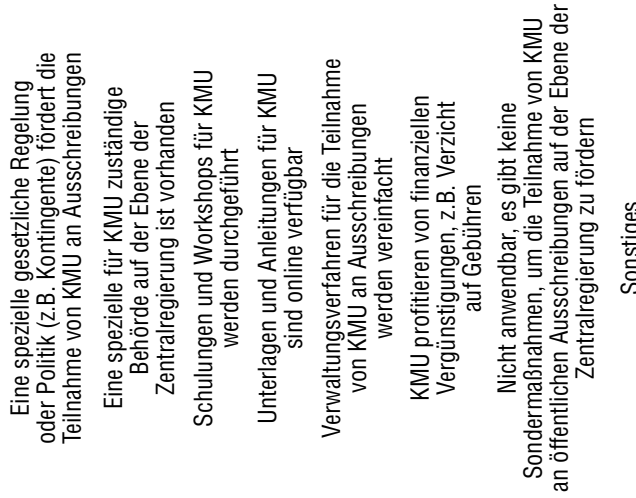

\begin{tabular}{|c|c|c|c|c|c|c|c|c|}
\hline Australien & O & $\bullet$ & O & O & O & O & O & O \\
\hline Österreich & O & $\bullet$ & $\bullet$ & $\bullet$ & $O$ & O & O & O \\
\hline Belgien & O & $\bullet$ & $\bullet$ & O & $\bullet$ & O & O & O \\
\hline Kanada & $O$ & $\bullet$ & $\bullet$ & $\bullet$ & O & O & O & O \\
\hline Chile & O & O & $\bullet$ & - & O & O & O & O \\
\hline Tschech. Rep. & O & O & O & O & $O$ & O & $\bullet$ & O \\
\hline Dänemark & O & $\bullet$ & $\bullet$ & • & O & 0 & O & $\bullet$ \\
\hline Estland & O & O & O & O & O & 0 & O & $\bullet$ \\
\hline Finnland & O & O & O & O & O & O & O & $\bullet$ \\
\hline Frankreich & $\bullet$ & O & $\bullet$ & $\bullet$ & $O$ & O & O & $\bullet$ \\
\hline Deutschland & $\bullet$ & O & O & O & $O$ & O & 0 & O \\
\hline Ungarn & $\bullet$ & O & $\bullet$ & - & $\bullet$ & O & O & O \\
\hline Island & O & O & 0 & O & O & 0 & $\bullet$ & O \\
\hline Irland & $O$ & O & $\bullet$ & $\bullet$ & $\bullet$ & O & O & O \\
\hline Israel & $O$ & $\bullet$ & $O$ & - & $O$ & O & 0 & O \\
\hline Italien & O & O & $\bullet$ & - & O & 0 & O & O \\
\hline Japan & $\bullet$ & $\bullet$ & 0 & $\bullet$ & O & O & O & O \\
\hline Korea & $\bullet$ & $\bullet$ & $\bullet$ & $\bullet$ & O & - & O & O \\
\hline Luxemburg & $O$ & O & O & $O$ & $\bullet$ & O & O & O \\
\hline Mexiko & $\bullet$ & $\bullet$ & - & $\bullet$ & O & - & $O$ & O \\
\hline Niederlande & $\bullet$ & O & $\bullet$ & O & - & O & O & O \\
\hline Neuseeland & O & O & • & O & - & O & O & O \\
\hline Norwegen & O & • & O & O & O & O & O & O \\
\hline Polen & $\bullet$ & $\bullet$ & $\bullet$ & $\bullet$ & O & O & O & O \\
\hline Portugal & $O$ & O & $\bullet$ & $O$ & O & 0 & O & O \\
\hline Slowak. Rep. & O & O & O & O & O & O & $\bullet$ & O \\
\hline Slowenien & $\bullet$ & O & O & O & $\bullet$ & O & O & O \\
\hline Spanien & $\bullet$ & $\bullet$ & O & • & - & O & O & $\bullet$ \\
\hline Schweden & O & O & O & O & O & O & $\bullet$ & O \\
\hline Schweiz & $\bullet$ & O & $\bullet$ & 0 & $\bullet$ & O & O & O \\
\hline Türkei & O & $\bullet$ & $\bullet$ & O & O & O & O & O \\
\hline Ver. Königreich & $O$ & $\bullet$ & $\bullet$ & $\bullet$ & $\bullet$ & O & O & $\bullet$ \\
\hline Ver. Staaten & $\bullet$ & $\bullet$ & $\bullet$ & $\bullet$ & - & 0 & O & O \\
\hline \multicolumn{9}{|c|}{ OECD insgesamt } \\
\hline - Ja & 12 & 15 & 19 & 17 & 11 & 2 & 4 & 6 \\
\hline Nein & 21 & 18 & 14 & 16 & 22 & 31 & 29 & 27 \\
\hline
\end{tabular}

Quelle: 2012 OECD Survey on Public Procurement. StatLink तilst http://dx.doi.org/10.1787/888932943552
7.10. Beurteilungen/Prüfungen sind vorgeschrieben, um ex post die Gewährung von Ausnahmen für die direkte Zuschlagserteilung auf der Ebene der Zentralregierung zu evaluieren

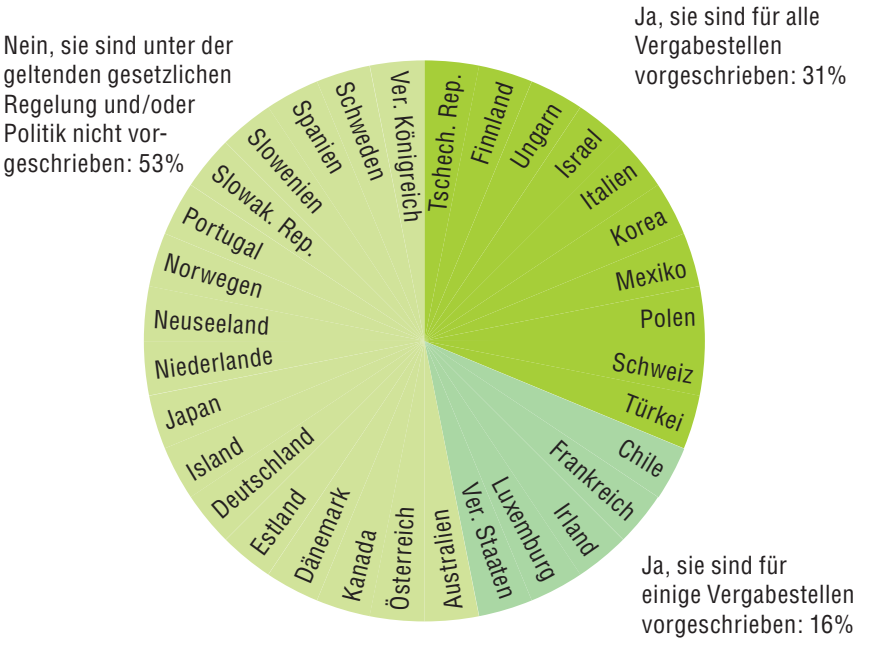

Quelle: 2012 OECD Survey on Public Procurement.

StatLink त्नाIst $h t t p: / / d x . d o i . o r g / 10.1787 / 888932942849$ 
Öffentliche Einrichtungen erstellen und erheben ein breites Spektrum von Daten, um ihre Aufgaben zu erfüllen. Durch die Veröffentlichung dieser Daten erhöht sich die Transparenz und kann die Öffentlichkeit stärker für das staatliche Handeln sensibilisiert werden. Offene Verwaltungsdaten (Open Government Data - OGD) können darüber hinaus dazu beitragen, Erkenntnisse darüber zu gewinnen, wie die Leistungsfähigkeit des Staates verbessert bzw. wie Regierungen zur Rechenschaft gezogen werden können. Außerdem schaffen offene Verwaltungsdaten die Voraussetzungen für eine sinnvolle Beteiligung und Mitwirkung der Öffentlichkeit bei der Einrichtung innovativer, bürgerorientierter Dienstleistungen und Politikmaßnahmen, die einen Mehrwert bringen, und dürften letztlich die Entscheidungsfindung sowohl der staatlichen Stellen als auch der Bürger verbessern. Die Öffentlichkeit sollte die von staatlichen Stellen erhobenen Daten nutzen können, um sachkundigere Entscheidungen zu treffen, die ihre Lebensqualität erhöhen können; die staatlichen Stellen wiederum dürften leichteren Zugang zu einer breiteren Palette von Datensätzen erhalten, was die evidenzbasierte Entscheidungsfindung unterstützen dürfte. Außerdem gelten offene Verwaltungsdaten als potenzielle Quelle von Wirtschaftswachstum sowie als Grundlage für neue Formen der Unternehmertätigkeit und sozialen Innovation.

Allerdings sind offene Verwaltungsdaten immer noch Neuland, und was die Vergleichbarkeit der Beurteilung der staatlichen Ergebnisse in Bezug auf die Bereitstellung und Qualität offener Daten betrifft, müssen noch einige Hindernisse überwunden werden. Erstens werden die Strategien und Politikmaßnahmen bezüglich offener Verwaltungsdaten ständig weiterentwickelt. Zweitens wird die Verwaltung und Erstellung offener Verwaltungsdaten oftmals an die nachgeordneten staatlichen Ebenen delegiert. Drittens gibt es keine gemeinsam vereinbarten internationalen Definitionen, z.B. von Datensätzen.

Die OECD-Mitgliedsländer verfolgen zunehmend einen strategischen Ansatz im Hinblick auf die Offenlegung von Verwaltungsdaten: $56 \%$ der Länder verfügen über eine nationale Strategie, $12 \%$ gaben an, dass für die einzelnen Fachministerien jeweils unterschiedliche Strategien existieren, und in $28 \%$ werden sowohl nationale als auch ressortspezifische Strategien verfolgt. Nur in $4 \%$ der Länder gibt es keine Strategie für die Offenlegung von Verwaltungsdaten.

Die strategischen Hauptziele im Zusammenhang mit der Offenlegung von Verwaltungsdaten variieren zwischen den einzelnen OECD-Ländern. Für die meisten Mitgliedsländer scheinen Transparenz, Offenheit, Steigerungen des Geschäftsvolumens von Unternehmen des privaten Sektors sowie Unternehmensgründungen Priorität zu haben. Das Potenzial offener Verwaltungsdaten für eine bessere Dienstleistungserbringung scheint ebenfalls geschätzt zu werden, wohingegen die Beteiligung der Bürger an öffentlichen Debatten und Entscheidungsprozessen offenbar nicht zu den obersten Prioritäten gehört. Die wichtigsten Ziele zu kennen, ist von grundlegender Bedeutung, um festzulegen, welche Arten von Daten in welchem Format veröffentlicht werden sollten, da die Erreichung unterschiedlicher Ziele die Veröffentlichung unterschiedlicher Datensätze erfordert. Während Rechenschaftslegung und Transparenz durch die Veröffentlichung aggregierter Daten gewährleistet werden können, müssen zur Ankurbelung des Wirtschaftswachstums u.U. konkretere Datensätze, die detailliertere Informationen enthalten, veröffentlicht werden.

Die Verfügbarkeit von Daten, ihre Zugänglichkeit und ihre Relevanz für die Nutzer sind für die Wertschöpfung entscheidend. Was die Verfügbarkeit betrifft, so scheinen Kanada, die Niederlande, Slowenien und die Vereinigten Staaten die größte
Zahl von Datensätzen in ihrem zentralen Portal für offene Verwaltungsdaten anzubieten. Bei länderübergreifenden Vergleichen des Angebots an Datensätzen ist indessen Vorsicht geboten, da den zwischen den Ländern bestehenden Unterschieden bei der Konzipierung der Datensätze sowie beim gewählten Publikationsmodell Rechnung getragen werden muss, so u.a. der Frage, ob es sich um zentrale Portale oder um von den Kommunalbehörden betriebene Portale zur Offenlegung von Verwaltungsdaten handelt.

\section{Methodik und Definitionen}

Die Daten wurden im Rahmen des 2013 von der OECD durchgeführten Survey on Open Government Data gesammelt, in deren Mittelpunkt die Verfügbarkeit von Informationen über den öffentlichen Sektor auf Zentralstaats-/ Bundesebene stand. Dabei wurden in 25 OECD-Ländern sowie Ägypten, der Russischen Föderation und der Ukraine Daten erhoben. An der Erhebung nahmen Länderdelegierte teil, die für E-Government und Informationen öffentlicher Stellen verantwortlich sind.

Die Definition offener Verwaltungsdaten umfasst sowohl alle Daten und Informationen, deren Erhebung von öffentlichen Stellen durchgeführt bzw. in Auftrag gegeben wurde, als auch Daten, die von allen frei verwendet, wiederverwendet und verbreitet werden können, wobei höchstens die Quelle angegeben und auf eine Weitergabe in gleicher Form geachtet werden muss. Ein Datensatz ist eine Datensammlung, die für gewöhnlich in Form einer Tabelle dargestellt ist.

Die Politikziele entsprechen der Politik/Strategie für die Offenlegung von Verwaltungsdaten auf Zentralstaats-/ Bundesebene im jeweiligen Land. Die Länder wurden in der Erhebung gebeten, ihre fünf wichtigsten Politikziele aufzuführen; sie können u.U. weitere nicht genannte Ziele verfolgen. Die Wiederverwendung von Verwaltungsdaten bezieht sich auf die Nutzung veröffentlichter Daten und Informationen in anderen Kontexten als dem, in dem sie ursprünglich verwendet wurden (z.B. zur Softwareentwicklung und für Pressemitteilungen); sie kann durch urheberrechtliche Bestimmungen untersagt oder durch Lizenzvereinbarungen gestattet sein.

Tabelle 8.3 zum Angebot an offenen Daten auf den Websites der Fachministerien (2013) kann online abgerufen werden unter $h t t p: / / d x . d o i . o r g / 10.1787 / 888932943609$.

\section{Weitere Informationen}

Ubaldi, B. (2013), „Open Government Data: Towards Empirical Analysis of Open Government Data Initiatives“, OECD Working Papers on Public Governance, Nr. 22, OECD Publishing, Paris, http://dx.doi.org/10.1787/5k46bj4f03s7-en.

\section{Anmerkungen zu den Abbildungen und Tabellen}

8.1: Daten sind für Länder nicht verfügbar, die kein „zentrales Portal“ für offene Verwaltungsdaten haben: Estland, Finnland, Irland, Japan und die Schweiz. Für Dänemark und Frankreich liegen keine Daten vor. Die Daten für Australien beziehen sich lediglich auf die Dateninitiative der Bundesregierung (http://data.gov.au). Die Daten für Spanien beziehen sich lediglich auf die Dateninitiative der Zentralregierung (http://datos.gob.es).

8.2: Die Schweiz und Ägypten verfolgen derzeit keine Politik/Strategie für die Offenlegung von Verwaltungsdaten auf Zentral-/Bundesebene.

Hinweis zu den Daten für Israel: http://dx.doi.org/10.1787/888932315602. 
8.1. Zahl der Datensätze in zentralen Portalen für offene Verwaltungsdaten (2013)

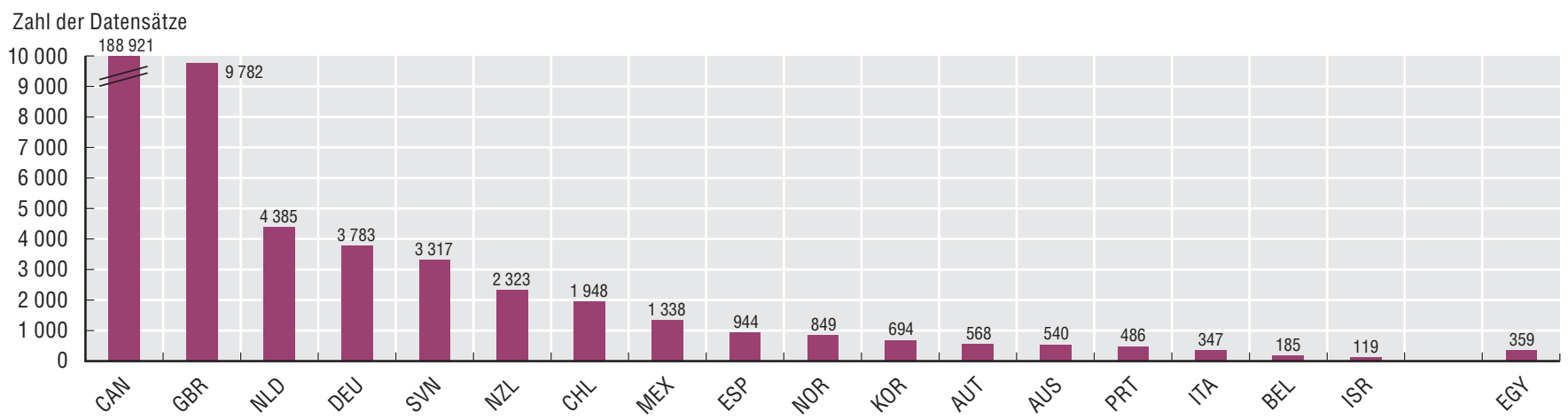

Quelle: OECD Survey on Open Government Data von 2013.

8.2. Die fünf wichtigsten Ziele der Strategien für die Offenlegung von Verwaltungsdaten auf Ebene der Zentral-/Bundesregierungen (2013)

\begin{tabular}{|c|c|c|c|c|c|c|c|c|c|c|}
\hline & $\begin{array}{c}\text { Transparenz } \\
\text { erhöhen }\end{array}$ & $\begin{array}{c}\text { Öffnung } \\
\text { verstärken }\end{array}$ & $\begin{array}{c}\text { Leistung } \\
\text { des öffentlichen } \\
\text { Sektors steigern } \\
\text { durch Stärkung } \\
\text { der Rechenschafts- } \\
\text { pflicht in Bezug auf } \\
\text { die Ergebnisse/ } \\
\text { Wirkungen }\end{array}$ & $\begin{array}{c}\text { Erbringung } \\
\text { öffentlicher } \\
\text { Dienstleistungen } \\
\text { effektiver und } \\
\text { effizienter gestalten } \\
\text { durch Verbesse- } \\
\text { rung der internen } \\
\text { Arbeitsabläufe und } \\
\text { Zusammenarbeit }\end{array}$ & $\begin{array}{l}\text { Erbringung } \\
\text { öffentlicher } \\
\text { Dienstleistungen } \\
\text { effektiver und } \\
\text { effizienter gestalten } \\
\text { durch Ermögli- } \\
\text { chung der } \\
\text { Leistungserbrin- } \\
\text { gung durch den } \\
\text { privaten Sektor } \\
\text { unter Wiederver- } \\
\text { wendung von } \\
\text { Daten }\end{array}$ & $\begin{array}{l}\text { Ökonomischen } \\
\text { Mehrwert } \\
\text { für den } \\
\text { öffentlichen } \\
\text { Sektor } \\
\text { schaffen }\end{array}$ & $\begin{array}{l}\text { Ökonomischen } \\
\text { Mehrwert } \\
\text { für den privaten } \\
\text { Sektor schaffen/ } \\
\text { Geschäftsvolu- } \\
\text { men des privaten } \\
\text { Sektors steigern }\end{array}$ & $\begin{array}{l}\text { Unternehmens- } \\
\text { gründungen } \\
\text { erleichtern }\end{array}$ & $\begin{array}{l}\text { Bürgerbeteili- } \\
\text { gung an } \\
\text { öffentlichen } \\
\text { Debatten } \\
\text { erleichtern }\end{array}$ & $\begin{array}{c}\text { Mitwirkung } \\
\text { der Bürger an } \\
\text { Entscheidungs- } \\
\text { prozessen } \\
\text { ermöglichen }\end{array}$ \\
\hline Australien & 0 & ○ & O & 0 & 0 & O & 0 & O & O & 0 \\
\hline Österreich & - & ○ & $\mathrm{O}$ & O & 0 & O & 0 & $\mathrm{O}$ & 0 & O \\
\hline Belgien & O & $\bullet$ & O & $\bullet$ & $\bullet$ & 0 & 0 & 0 & 0 & 0 \\
\hline Kanada & $O$ & - & - & - & $O$ & $O$ & - & $O$ & - & O \\
\hline Chile & $\bullet$ & O & - & O & 0 & O & $O$ & 0 & $\bullet$ & - \\
\hline Dänemark & O & O & O & $\bullet$ & 0 & O & 0 & $\bullet$ & $O$ & O \\
\hline Estland & $\bullet$ & 0 & 0 & 0 & 0 & 0 & 0 & 0 & 0 & 0 \\
\hline Finnland & $\bullet$ & O & $\bullet$ & O & $\bullet$ & O & $\bullet$ & $\bullet$ & O & O \\
\hline Frankreich & $\bullet$ & O & $\bullet$ & $\bullet$ & 0 & O & $\bullet$ & $\bullet$ & O & 0 \\
\hline Deutschland & 0 & O & O & 0 & $O$ & O & O & $\bullet$ & 0 & - \\
\hline Irland & 0 & $\bullet$ & O & $\bullet$ & 0 & O & O & $\bullet$ & O & O \\
\hline Israel & $\bullet$ & $\bullet$ & O & $\bullet$ & $\bullet$ & O & O & 0 & O & O \\
\hline Italien & 0 & $\bullet$ & $\bullet$ & $\bullet$ & 0 & 0 & 0 & - & 0 & 0 \\
\hline Japan & - & ○ & O & O & ○ & O & $\bullet$ & $\bullet$ & O & O \\
\hline Korea & $\bullet$ & $\bullet$ & O & O & $\bullet$ & O & $\bullet$ & $\bullet$ & O & O \\
\hline Mexiko & ○ & 0 & - & O & $\mathrm{O}$ & O & O & $\mathrm{O}$ & - & O \\
\hline Niederlande & 0 & 0 & $\bullet$ & O & O & O & $\bullet$ & O & $\bullet$ & O \\
\hline Neuseeland & - & O & O & $\bullet$ & O & O & $\bullet$ & $\mathrm{O}$ & $\bullet$ & $\bullet$ \\
\hline Norwegen & 0 & O & O & 0 & $\bullet$ & O & $\bullet$ & $\bullet$ & 0 & O \\
\hline Portugal & O & $\bullet$ & O & $\bullet$ & O & O & O & $\bullet$ & O & $\bullet$ \\
\hline Slowenien & $\bullet$ & $\bullet$ & O & O & $\bullet$ & O & O & $\bullet$ & O & $\bullet$ \\
\hline Spanien & - & - & - & O & O & O & - & - & $O$ & O \\
\hline Schweden & ○ & ○ & ○ & ○ & O & O & O & ○ & O & O \\
\hline Schweiz & $x$ & $x$ & $x$ & $x$ & $x$ & $x$ & $x$ & $x$ & $x$ & $x$ \\
\hline Ver. Königreich & - & O & 0 & 0 & O & O & ○ & O & O & 0 \\
\hline Ägypten & $x$ & $x$ & $x$ & $x$ & $x$ & $x$ & $x$ & $x$ & $x$ & $x$ \\
\hline Russ. Föderation & 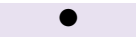 & 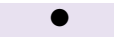 & O & O & 0 & 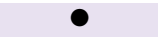 & O & $\bullet$ & $\bullet$ & O \\
\hline Ukraine & $\bullet$ & O & O & $\bullet$ & O & $\bullet$ & $\bullet$ & $\mathrm{O}$ & $\bullet$ & O \\
\hline OECD insgesamt & 17 & 16 & 11 & 14 & 13 & 0 & 16 & 15 & 7 & 7 \\
\hline
\end{tabular}

Ja

Nein

Quelle: OECD Survey on Open Government Data von 2013. 
Die vor allem seit der Finanz- und Wirtschaftskrise zunehmende Erwartungshaltung in Bezug auf eine offene und faire öffentliche Entscheidungsfindung zwingt die Regierungen immer stärker sicherzustellen, dass öffentliche Entscheidungen nicht in unzulässiger Weise durch private Interessen beeinflusst werden. Gleichzeitig wird es für Politikverantwortliche und Führungskräfte des öffentlichen Sektors infolge neuer Formen der Partnerschaft zwischen staatlichen Stellen und dem privaten sowie dem nicht gewinnorientierten Sektor schwieriger, diese Integrität zu gewährleisten. Die Sicherung der Integrität der staatlichen Entscheidungsprozesse ist daher von grundlegender Bedeutung, um das Vertrauen in den Staat wiederherzustellen.

Interessenkonflikte entstehen, wenn die privaten Interessen eines öffentlich Bediensteten seine Amtsausübung beeinträchtigen können. Werden Situationen, in denen Interessenkonflikte bestehen, nicht angemessen identifiziert und geregelt, können sie u.U. zu Korruption führen. Übermäßig strenge Regelungen können jedoch kostspielig und in der Praxis nicht umsetzbar sein; außerdem können sie erfahrene und kompetente potenzielle Kandidaten davon abhalten, ein öffentliches Amt anzunehmen oder in den öffentlichen Dienst einzutreten.

In der Praxis zeigt sich, dass die Offenlegung der Vermögensverhältnisse und Privatinteressen von Entscheidungsträgern ein wichtiges Instrument im Umgang mit Interessenkonflikten bleibt. Abbildung 8.4 bietet einen Überblick über den Grad der Offenlegung sowie der öffentlichen Zugänglichkeit der von hochrangigen Entscheidungsträgern der drei Staatsgewalten (Exekutive, Legislative und Judikative) offengelegten Informationen. Der Offenlegungsgrad bestimmt sich danach, ob hochrangige Entscheidungsträger private Interessen wie Vermögen, Verbindlichkeiten, Einkommensquellen und -höhe, bezahlte und unbezahlte Nebenbeschäftigungen, Geschenke sowie vorherige Beschäftigungsverhältnisse offenlegen müssen.

Die Offenlegung von Vermögen und privaten Interessen durch Entscheidungsträger ist in den OECD-Ländern zwar gängige Praxis, der Offenlegungsgrad fällt für die drei Säulen des Staats jedoch unterschiedlich aus. Die Praxis der Offenlegung ist in der Exekutive und in der Legislative weitaus stärker ausgeprägt als in der Judikative. So sind Richter und Staatsanwälte in der Tschechischen Republik, in Frankreich, Luxemburg und Neuseeland nicht zur Offenlegung verpflichtet. In Luxemburg gelten für keine der drei Staatsgewalten Offenlegungspflichten für Entscheidungsträger. Unter den verschiedenen Formen von privaten Interessen gilt die größte Aufmerksamkeit den bezahlten Nebentätigkeiten sowie der Annahme von Geschenken; sie sind entweder verboten oder müssen offengelegt werden.

Große Unterschiede bestehen in den OECD-Mitgliedsländern darüber hinaus im Hinblick auf die öffentliche Zugänglichkeit von durch die Entscheidungsträger offengelegten Informationen. In den meisten Ländern werden solche Informationen der Öffentlichkeit nur teilweise zugänglich gemacht. In manchen Ländern erklärt sich dies aus der Bedeutung, die dem Schutz der Privatsphäre beigemessen wird. Die überwiegende Mehrheit der Länder macht Informationen über Vermögen und Einkommensquellen öffentlich zugänglich. Vorherige Beschäftigungsverhältnisse und Verbindlichkeiten werden nur in wenigen Ländern veröffentlicht.

Knapp über 80\% der OECD-Länder, in denen Offenlegungspflichten bestehen, prüfen, ob die entsprechenden Offenlegungsformulare tatsächlich eingereicht wurden (Tabelle 8.5). Allerdings werden in weniger als die Hälfte der Länder interne Prüfungen der Richtigkeit der übermittelten Informationen durchgeführt. Irland, Italien, die Schweiz und die Türkei ergreifen nach Einholung der Offenlegungsformulare keinerlei Maßnahmen. In Irland und Italien wird der Großteil der offengelegten Informationen jedoch veröffentlicht, so dass die Bürger die übermittelten Informationen selbst prüfen können.

\section{Methodik und Definitionen}

Die Daten beziehen sich auf 2012 und wurden im Rahmen des OECD Survey on Managing Conflict of Interest erhoben. In der Erhebung wurden Länderdelegierte befragt, die auf Zentralstaats-/Bundesebene für Maßnahmen im Bereich Integrität zuständig sind.

Der Begriff „öffentliche Amtsträger“ bezieht sich auf Personen, die in einem Land ein Amt in den Bereichen Legislative, Exekutive, Verwaltung oder Judikative innehaben, unabhängig davon ob sie ernannt oder gewählt wurden, das Amt dauerhaft oder befristet innehaben, das Amt bezahlt oder unbezahlt ist bzw. wie hochrangig ihre Stellung ist; ferner bezieht sich der Begriff auf alle sonstigen Personen, die eine öffentliche Funktion ausüben, z.B. für eine öffentliche Stelle oder ein öffentliches Unternehmen, oder die gemäß der gesetzlich festgelegten Definition des betreffenden Landes eine öffentliche Dienstleistung erbringen. Im Hinblick auf Entscheidungsträger bezieht sich der Begriff „Exekutive“ auf den Präsidenten, den Premierminister und die Minister bzw. Kabinettsmitglieder. Der Begriff „Legislative“ bezieht sich auf die Mitglieder der gesetzgebenden Körperschaften (beide Kammern). Der Begriff „Judikative“ bezieht sich auf Richter und Staatsanwälte.

Bei der Berechnung eines Aggregats aus den länderspezifischen Daten wurden alle privaten Interessen und alle Positionen als gleichermaßen wichtig betrachtet und deshalb gleich gewichtet. Anhang E enthält detaillierte Daten über die Offenlegung von Interessenkonflikten.

\section{Weitere Informationen}

OECD (2010), Post-Public Employment: Good Practices for Preventing Conflict of Interest, OECD Publishing, Paris, http://dx.doi.org/ 10.1787/9789264056701-en.

OECD (2007), OECD Guidelines for Managing Conflict of Interest in the Public Service: Report on Implementation, OECD Publishing, Paris.

OECD (2003), Recommendation of the Council on Guidelines for Managing Conflict of Interest in the Public Service, OECD Publishing, Paris.

\section{Anmerkungen zu den Abbildungen und Tabellen}

8.4: Die Daten für Brasilien, die Tschechische Republik, Griechenland, Israel und die Russische Föderation beziehen sich auf 2010 anstelle von 2012. Die Daten beziehen sich auf die Praktiken in Mitgliedsländern. Länderspezifische Daten und Anmerkungen finden sich in Anhang E.

8.5: Für die Tschechische Republik, Griechenland und Israel sind keine Daten verfügbar. Für Australien, Österreich, Kanada, Chile, Dänemark, Estland, Deutschland, Ungarn, Italien, Japan, Korea, Luxemburg, Mexiko, Norwegen, die Slowakische Republik, Spanien, die Schweiz, die Türkei und die Vereinigten Staaten beziehen sich die Informationen lediglich auf die Exekutive.

Hinweis zu den Daten für Israel: http://dx.doi.org/10.1787/888932315602. 
8.4. Offenlegung von Vermögenswerten: Grad der Offenlegung privater Interessen und öffentliche Zugänglichkeit der Informationen (2012)

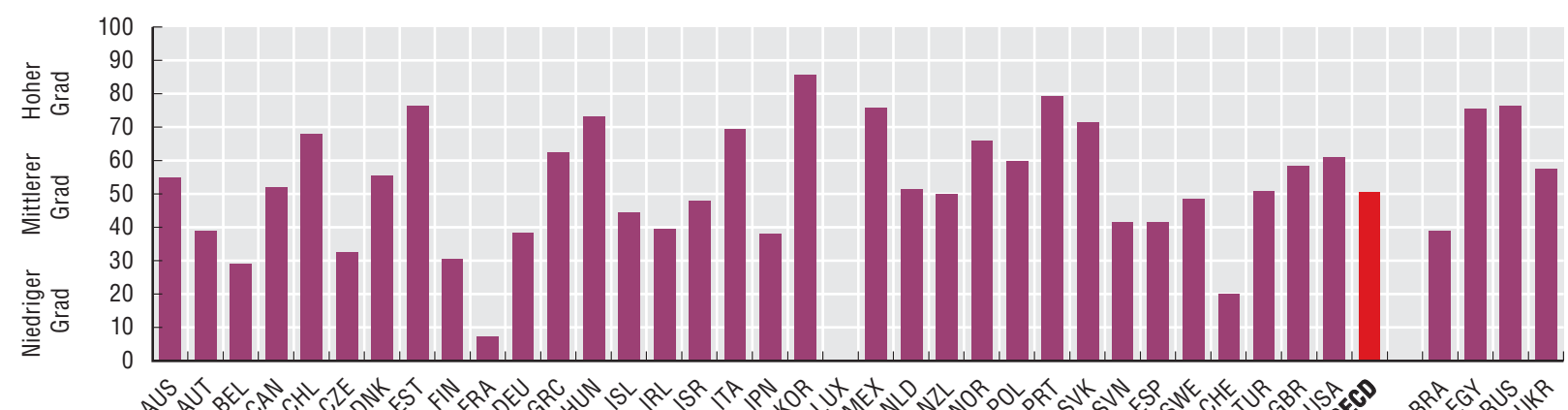

Quelle: OECD Survey on Managing Conflict of Interest von 2012.

StatLink त्तारी http://dx.doi.org/10.1787/888932942887

8.5. Maßnahmen bezüglich der Offenlegung privater Interessen durch öffentliche Amtsträger (2012)

\begin{tabular}{|c|c|c|c|}
\hline & $\begin{array}{l}\text { Prüfung der Übermittlung } \\
\text { des Offenlegungsformulars }\end{array}$ & $\begin{array}{l}\text { Prüfung der Übermittlung } \\
\text { aller vorgeschriebenen } \\
\text { Informationen }\end{array}$ & $\begin{array}{l}\text { Interne Prüfung der } \\
\text { Richtigkeit der übermittelten } \\
\text { Informationen }\end{array}$ \\
\hline Australien & $\bullet$ & ○ & O \\
\hline Österreich & ○ & ○ & 0 \\
\hline Belgien & $\bullet$ & $\bullet$ & 0 \\
\hline Kanada & $\bullet$ & 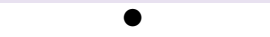 & $O$ \\
\hline Chile & $\bullet$ & $\bullet$ & O \\
\hline Dänemark & ○ & ○ & O \\
\hline Estland & $\bullet$ & $\square$ & $\square$ \\
\hline Finnland & ○ & ○ & O \\
\hline Frankreich & $\bullet$ & $\square$ & $\square$ \\
\hline Deutschland & - & - & $\square$ \\
\hline Ungarn & $\bullet$ & O & O \\
\hline Island & ○ & O & O \\
\hline Irland & O & O & 0 \\
\hline Italien & O & O & O \\
\hline Japan & O & $\bullet$ & $\bullet$ \\
\hline Korea & $\bullet$ & $\bullet$ & $\bullet$ \\
\hline Luxemburg & $x$ & $x$ & $x$ \\
\hline Mexiko & - & ○ & $\square$ \\
\hline Niederlande & $\bullet$ & $\bullet$ & 0 \\
\hline Neuseeland & - & ○ & $\square$ \\
\hline Norwegen & ○ & $\square$ & 0 \\
\hline Polen & $\bullet$ & $\bullet$ & $\square$ \\
\hline Portugal & - & - & - \\
\hline Slowak. Rep. & $\bullet$ & $\bullet$ & O \\
\hline Slowenien & 0 & $\square$ & $\square$ \\
\hline Spanien & $\bullet$ & $\bullet$ & $\bullet$ \\
\hline Schweden & ○ & ○ & $\square$ \\
\hline Schweiz & O & O & O \\
\hline Türkei & O & O & 0 \\
\hline Ver. Königreich & $\bullet$ & $\bullet$ & $\bullet$ \\
\hline Ver. Staaten & ○ & ○ & 0 \\
\hline Ägypten & $\bullet$ & $\bullet$ & 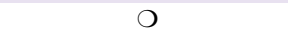 \\
\hline Ukraine & $\square$ & $\square$ & $O$ \\
\hline \multicolumn{4}{|l|}{ OECD insgesamt } \\
\hline $\begin{array}{l}\text { - Verfahren für alle Personen durchgeführt, die ein Offenlegungsformular einreichen } \\
\text { müssen }\end{array}$ & 25 & 19 & 6 \\
\hline $\begin{array}{l}\square \text { Verfahren lediglich für einige Personen durchgeführt, die ein Offenlegungsformular } \\
\text { einreichen müssen }\end{array}$ & 0 & 4 & 8 \\
\hline Verfahren nicht durchgeführt & 5 & 7 & 16 \\
\hline
\end{tabular}

Quelle: OECD Survey on Managing Conflict of Interest von 2012. 
Der nationale Haushalt ist eines der wesentlichen Politikdokumente der Regierung, aus dem ihre Politikziele und Ausgabenprioritäten hervorgehen. Haushaltstransparenz - die Offenlegung und Zugänglichkeit wichtiger fiskalischer und budgetärer Informationen - steht daher im Zentrum einer guten Staats- und Verwaltungsführung. Die Wirtschafts- und Sozialkrise hat deutlich gemacht, dass die Transparenz des Haushalts erhöht werden muss, was sich zu einer Kernkomponente der Open-Government-Strategien der Länder entwickelt hat. In den „OECD Best Practices for Budget Transparency“ wird explizit anerkannt, wie wichtig die zeitnahe und systematische Offenlegung von Haushaltsinformationen der Regierung ist und dass es erforderlich ist, die Qualität, Integrität und vor allem Zugänglichkeit dieser Informationen zu gewährleisten, um die Bürger und den Gesetzgeber zu informieren und ihnen die Möglichkeit zu geben, die Regierung zur Rechenschaft zu ziehen.

Öffentlich zugängliche, umfassende Informationen über den Haushalt können es der Öffentlichkeit erleichtern, die fiskalpolitischen Maßnahmen und die Prioritäten der Regierung zu verstehen. Die Offenlegung der Haushalte kann zu Haushaltsdisziplin, zu einer effektiven Ressourcenallokation und zu operativer Effizienz beitragen. Sie kann zudem gewährleisten, dass die Regierungen für die Erstellung realistischer und tragfähiger Haushalte sowie die sozialen und wirtschaftlichen Auswirkungen geplanter Politikmaßnahmen zur Rechenschaft gezogen werden können. Weil die Zugänglichkeit der in den Haushaltsplänen enthaltenen Informationen nicht zwangsläufig deren Richtigkeit garantiert, ermöglicht ein transparenter Haushalt Bürgern, Mitgliedern der gesetzgebenden Körperschaften und Organisationen der Zivilgesellschaft darüber hinaus, die Haushaltsinformationen zu nutzen, um die Regierung diesbezüglich $\mathrm{zu}$ einer großen Disziplin zu verpflichten. Auf diese Weise trägt die Haushaltstransparenz auch zum Vertrauen in die Regierung bei.

Ein wichtiger Aspekt der Transparenz ist die Frage, inwieweit der von der Exekutive erstellte Haushalt Informationen über den Haushaltsrahmen sowie die Politiken und Prioritäten der Regierung enthält. Umfang und Art der Informationen unterscheiden sich von einem Land zum anderen. Während die haushaltspolitischen Ziele, die Annahmen zur makroökonomischen Entwicklung und eine mittelfristige Haushaltsvorausschau (vgl. den Indikator zu den mittelfristigen Ausgabenrahmen) in den Haushalten aller OECD-Länder enthalten sind, werden Informationen über Steuervergünstigungen, leistungs- und ergebnisbezogene Daten sowie langfristige Haushaltsprojektionen in weniger Ländern geliefert. Kein anderer Einzelfaktor dürfte stärker dazu beitragen, dass Haushaltsziele verfehlt werden und irreführende Defizit- oder Überschussprojektionen erstellt werden, als die Verwendung mangelhafter makroökonomischer Annahmen. Umfrageergebnissen zufolge veröffentlichen rd. 95\% der OECD-Mitgliedsländer die mittelfristigen Ziele der Fiskalpolitik, den Haushaltsentwurf sowie den verabschiedeten Haushalt. Die Transparenz außeretatmäßiger Ausgaben ist ebenfalls wichtig, da Anreize bestehen könnten, um die entsprechenden Mittel nicht in den Bilanzen zu berücksichtigen. Die häufigsten außeretatmäßigen Ausgaben in den Mitgliedsländern sind Sozialversicherungsfonds (13 Länder), öffentliche Gesundheitsfonds (10 Länder) sowie Kreditbürgschaften (9 Länder). In der Mehrzahl dieser Länder sind diese Ausgaben im Haushalt berücksichtigt, auch wenn es hier Ausnahmen gibt (vgl. die online verfügbare Tabelle 8.8).

Die Offenlegung von Haushaltsdaten der Regierungen ist zwar von grundlegender Bedeutung und wird zunehmend ausgeweitet, sie kann jedoch komplex sein und Transparenz sowie Rechnungslegung unbeabsichtigt beeinträchtigen. Tatsächlich können Laien durch Fachbegriffe und die Vielzahl an Haushaltsdaten, die dem Gesetzgeber vorgelegt werden, abgeschreckt werden und Schwierigkeiten dabei haben, Rolle und Umfang der außeretatmäßigen Aktivitäten zu verstehen. 14 OECD-Mitgliedsländer veröffentlichen für die Bürger leicht verständliche Zusammenfassungen der wesentlichen Merkmale des dem Gesetzgeber vorgelegten Jahreshaushalts, die Erläuterungen und Definitionen von Fachbegriffen enthalten.

\section{Methodik und Definitionen}

Die Daten beziehen sich auf das Jahr 2012 und entstammen den Antworten der Länder auf den OECD Survey of Budget Practices and Procedures von 2012. Die Befragten waren vornehmlich hochrangige Haushaltsverantwortliche in den OECD-Ländern. Die Antworten stellen die Selbsteinschätzung der Länder zu den derzeitigen Praktiken und Verfahren dar. Die Daten beziehen sich nur auf die Zentral-/ Bundesregierungen, die Haushaltspraxis auf der Ebene der Gliedstaaten/Kommunen ist ausgeklammert.

Außeretatmäßige Fonds sind Sonderfonds, deren Eigentümer der Staat ist und die nicht Teil des Haushalts sind und mit Einnahmen aus zweckgebundenen Abgaben gespeist werden, zu denen Einnahmen aus anderen Quellen wie Gebühren und Zahlungen aus dem allgemeinen Steueraufkommen hinzukommen können. Zweckgebundene Abgaben unterscheiden sich von Gebühren insofern, als sie nicht den Marktwert der Dienstleistungen widerspiegeln, die mit den entsprechenden Einnahmen finanziert werden. Insbesondere auf Grund sozialer Erwägungen können sie auf einem niedrigen oder höheren Niveau angesetzt sein.

Haushaltsleitfäden für die Bürger sind hier als leicht verständliche Zusammenfassungen der wesentlichen Merkmale des dem Gesetzgeber vorgelegten Jahreshaushalts definiert. Es sollte sich um in sich schlüssige Unterlagen handeln, in denen die Inhalte des Jahreshaushaltsentwurfs sowie die voraussichtlichen Wirkungen erläutert werden. Sie können zwar Links oder Verweise $\mathrm{zu}$ ausführlicheren Unterlagen enthalten, der Leser sollte jedoch nicht gezwungen sein, in diesen Dokumenten nachzuschlagen bzw. mit ihren Inhalten vertraut zu sein, um die Leitfäden zu verstehen.

Tabelle 8.8 zur Aufnahme außeretatmäßiger Ausgaben in die Haushaltsunterlagen kann online abgerufen werden unter http://dx.doi.org/10.1787/888932943666.

\section{Weitere Informationen}

IWF (2001, 2007), Manual on Fiscal Transparency, Internationaler Währungsfonds, Washington, DC.

OECD (2013, erscheint demnächst), Budgeting Practices and Procedures in OECD Countries, OECD Publishing, Paris.

OECD (2002), „OECD Best Practices for Budget Transparency“, OECD Journal on Budgeting, Vol. 1/3, S. 7-14, http://dx.doi.org/ 10.1787/budget-v1-art14-en.

\section{Anmerkungen zu den Abbildungen und Tabellen}

Für Island stehen keine Daten zur Verfügung.

Hinweis zu den Daten für Israel: http://dx.doi.org/10.1787/888932315602. 
8.6. Haushaltsleitfäden für die Bürger in OECD-Ländern (2012)

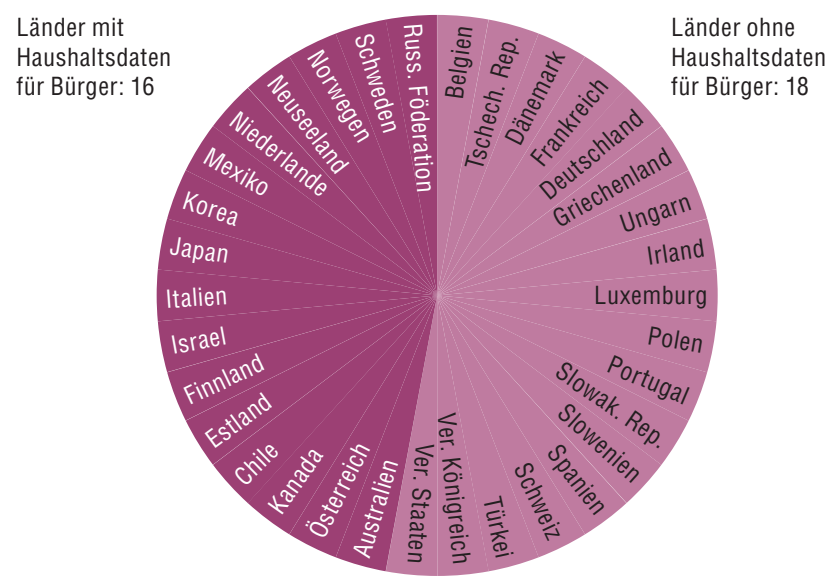

Quelle: OECD Survey on Budgeting Practices and Procedures von 2012.

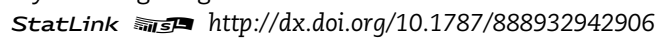

8.7. Veröffentlichte Haushaltsdaten (2012)

\begin{tabular}{|c|c|c|c|c|c|c|c|c|c|}
\hline & 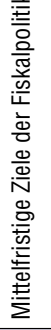 & 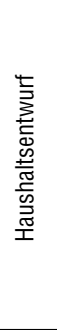 & 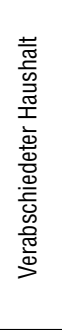 & 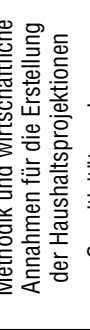 & 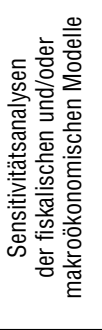 & 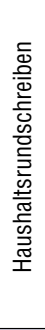 & 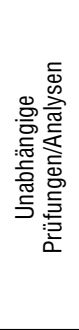 & 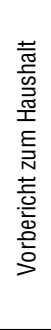 & 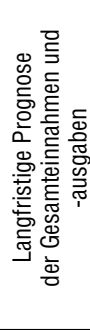 \\
\hline Australien & - & - & - & - & - & O & O & $x$ & - \\
\hline Österreich & $\bullet$ & $\bullet$ & - & - & O & O & 0 & $x$ & - \\
\hline Belgien & $\bullet$ & $\bullet$ & - & - & $x$ & $\bullet$ & - & $x$ & $x$ \\
\hline Kanada & $\bullet$ & $\bullet$ & $\bullet$ & $\bullet$ & $\bullet$ & $x$ & $\bullet$ & $x$ & 0 \\
\hline Chile & $\bullet$ & $\bullet$ & $\bullet$ & $\bullet$ & $O$ & $\bullet$ & $x$ & $\bullet$ & $x$ \\
\hline Tschech. Rep. & $\bullet$ & $\bullet$ & - & $\bullet$ & - & O & - & $x$ & O \\
\hline Dänemark & $\bullet$ & $\bullet$ & - & $\bullet$ & O & O & O & $x$ & $x$ \\
\hline Estland & $\bullet$ & $\bullet$ & - & - & - & O & O & • & $x$ \\
\hline Finnland & $\bullet$ & $\bullet$ & - & - & O & $\bullet$ & $x$ & $x$ & $\bullet$ \\
\hline Frankreich & $\bullet$ & $\bullet$ & - & $\bullet$ & - & $\bullet$ & - & $\bullet$ & O \\
\hline Deutschland & $\bullet$ & $\bullet$ & - & - & 0 & O & - & $x$ & 0 \\
\hline Griechenland & $\bullet$ & $\bullet$ & $x$ & O & $x$ & O & $x$ & $\bullet$ & $x$ \\
\hline Ungarn & $\bullet$ & $\bullet$ & - & $\bullet$ & $O$ & $\bullet$ & O & $O$ & $x$ \\
\hline Irland & $\bullet$ & $\bullet$ & - & $\bullet$ & - & $\bullet$ & - & $\bullet$ & $x$ \\
\hline Israel & $\bullet$ & $\bullet$ & - & - & O & O & O & $\bullet$ & $x$ \\
\hline Italien & $x$ & $\bullet$ & - & $\bullet$ & $\bullet$ & $\bullet$ & - & $x$ & $x$ \\
\hline Japan & $\bullet$ & $\bullet$ & - & - & - & $\bullet$ & $x$ & 0 & 0 \\
\hline Korea & $\bullet$ & $\bullet$ & - & O & O & $\bullet$ & - & $x$ & $x$ \\
\hline Luxemburg & $\bullet$ & $\bullet$ & - & $\bullet$ & - & O & $\bullet$ & $\bullet$ & $\bullet$ \\
\hline Mexiko & $\bullet$ & $\bullet$ & - & - & - & O & $x$ & 0 & $x$ \\
\hline Niederlande & $\bullet$ & $\bullet$ & - & - & - & O & - & $x$ & - \\
\hline Neuseeland & $\bullet$ & - & - & - & - & O & O & $\bullet$ & - \\
\hline Norwegen & $\bullet$ & $\bullet$ & - & O & O & $\bullet$ & O & $x$ & $x$ \\
\hline Polen & $\bullet$ & $\bullet$ & - & $\bullet$ & • & $\bullet$ & O & $x$ & $x$ \\
\hline Portugal & $\bullet$ & $\bullet$ & - & O & 0 & $\bullet$ & - & 0 & $x$ \\
\hline Slowak. Rep. & $\bullet$ & $\bullet$ & $\bullet$ & $O$ & $O$ & $\bullet$ & $\bullet$ & $x$ & $x$ \\
\hline Slowenien & O & $\bullet$ & - & $\bullet$ & $\bullet$ & $\bullet$ & $x$ & O & - \\
\hline Spanien & $\bullet$ & $\bullet$ & $\bullet$ & O & O & $\bullet$ & $\bullet$ & $O$ & $x$ \\
\hline Schweden & $\bullet$ & $\bullet$ & - & $\bullet$ & - & O & - & $\bullet$ & - \\
\hline Schweiz & $\bullet$ & $\bullet$ & - & - & - & O & $x$ & $x$ & - \\
\hline Türkei & $\bullet$ & $\bullet$ & $\bullet$ & • & O & $\bullet$ & O & 0 & O \\
\hline Ver. Königreich & $\bullet$ & O & $\bullet$ & $\bullet$ & $\bullet$ & $x$ & - & $\bullet$ & $\bullet$ \\
\hline Ver. Staaten & $\bullet$ & $\bullet$ & $\bullet$ & $\bullet$ & $\bullet$ & $\bullet$ & $x$ & $x$ & $\bullet$ \\
\hline Russ. Föderation & $\bullet$ & $\bullet$ & $\bullet$ & $\bullet$ & $\bullet$ & $\bullet$ & $\bullet$ & $\bullet$ & $\bullet$ \\
\hline \multicolumn{10}{|l|}{ OECD insgesamt } \\
\hline $\begin{array}{l}\text { - Öffentlich } \\
\text { verfügbar }\end{array}$ & 31 & 32 & 32 & 27 & 20 & 17 & 16 & 14 & 14 \\
\hline $\begin{array}{l}\text { O Nicht öffentlich } \\
\text { verfügbar }\end{array}$ & 1 & 0 & 0 & 6 & 11 & 14 & 9 & 3 & 3 \\
\hline $\begin{array}{l}\text { x Nicht anwend- } \\
\text { bar }\end{array}$ & 1 & 1 & 1 & 0 & 2 & 2 & 8 & 16 & 16 \\
\hline
\end{tabular}

Quelle: OECD Survey on Budgeting Practices and Procedures von 2012. StatLink न्insts http://dx.doi.org/10.1787/888932943647 
Eine offene und inklusive Politikgestaltung ist transparent, evidenzbasiert, zugänglich und geht auf die Bedürfnisse möglichst breiter Bevölkerungsschichten ein. Sie versucht, eine Vielzahl von Akteuren und Ansichten in den Politikgestaltungsprozess einzubeziehen, darunter auch Vertreter kultureller Minderheiten. Um erfolgreich zu sein, muss sie diesen Anforderungen in jeder Phase der Ausgestaltung und Umsetzung öffentlicher Maßnahmen und Dienstleistungen gerecht werden. Eine inklusive Politikgestaltung verbessert nicht nur die Transparenz, die Rechenschaftslegung und die Mitwirkung der Öffentlichkeit und fördert den Aufbau zivilgesellschaftlicher Kapazitäten, sondern eröffnet den staatlichen Stellen auch eine Möglichkeit, durch die Arbeit mit Bürgerinnen und Bürgern, zivilgesellschaftlichen Organisationen (ZGO), Unternehmen und anderen Beteiligten konkrete Verbesserungen ihrer Politikergebnisse und der Qualität der öffentlichen Dienstleistungen zu erzielen und dadurch ihre Politikleistung zu steigern.

Gender Impact Assessments bzw. Gleichstellungsverträglichkeitsprüfungen sind ein Instrument, das die Politikverantwortlichen nutzen können, um nach geschlechterrelevanten Kriterien zu prüfen, welche Auswirkungen neue Gesetze bzw. Politikmaßnahmen auf Frauen haben könnten. In verschiedenen Bereichen ist es von entscheidender Bedeutung für eine inklusive Politikgestaltung, die Politikverantwortlichen für die potenziell unterschiedlichen Effekte politischer Entscheidungen auf Männer und Frauen zu sensibilisieren. Scheinbar geschlechtsneutrale Politikentscheidungen können beabsichtigte oder unbeabsichtigte Auswirkungen auf die Chancen von Frauen haben, gleichberechtigt am Leben der Gesellschaft teilzuhaben. Sie können es für sie u.U. schwieriger machen, Arbeit zu finden, sich zu bilden, ein Unternehmen zu gründen, den Bedarf ihrer Familie zu dekken oder ihre Rechte durchzusetzen. So werden z.B. arbeitsrechtliche Regelungen, die beiden Elternteilen gestatten, Urlaub zu nehmen, um ein krankes Kind zu pflegen, mit größerer Wahrscheinlichkeit von Frauen genutzt werden, da es zumeist sie sind, die sich um die Kinder kümmern. Gender Impact Assessments können ex ante (d.h. vor Verabschiedung bzw. Inkrafttreten des Gesetzes oder der Maßnahme) und ex post (d.h. nach der Umsetzung) erfolgen. Laut dem OECD Survey on Gender Public Policies and Leadership sind Ex-ante-Evaluierungen üblicher. Von den OECD-Ländern, die an dieser Umfrage teilgenommen haben, gaben z.B. 84\% (16 Länder) an, dass bei ihnen Ex-ante-Evaluierungen für gesetzliche Bestimmungen vorgesehen sind, während in 37\% (7 Länder) Ex-post-Evaluierungen vorgeschrieben sind. Im Allgemeinen scheint es jedoch, dass Gender Impact Assessments bei der Politikgestaltung nicht systematisch durchgeführt werden; in der Mehrzahl der Länder, die an der Erhebung teilnahmen, kommen sie nur in der Primärund Sekundärgesetzgebung zur Anwendung, nicht jedoch bei Maßnahmen und Programmen.

Das Gender Responsive Budgeting (GRB), also eine Haushaltsplanung unter geschlechtsspezifischen Gesichtspunkten, ist wahrscheinlich die bekannteste Form des Gender Impact Assessment. Beim Gender Responsive Budgeting wird der Frage der Gleichstellung von Mann und Frau in allen Etappen des Haushaltszyklus Rechnung getragen. Dadurch sollen "geschlechtsblinde" vermieden und die Wirksamkeit staatlicher Programme durch die Identifizierung von in geschlechtsspezifischer Hinsicht unangemessenen Folgen der Mittelverwendung verbessert werden. Etwas über die Hälfte (10) der Länder, die an der Erhebung teilnahmen, gaben an, Vorschriften über Gender Responsive Budgeting zu haben. Belgien, Finnland, Frankreich, Israel, Korea, Mexiko, Norwegen, die Slowakische Republik, Spanien und Schweden gaben an, derartige Evaluierungen bei der Planung ihrer Haushalte auf Zentralstaats-/Bundesebene immer durchzuführen.
Die Konsultation der Bürger ist eine zweite Möglichkeit, um den Politikgestaltungsprozess für die Bürger zu öffnen. Den OECD Guidelines on Open and Inclusive Policy Making zufolge sollten alle Bürgerinnen und Bürger bei Informationszugang, Konsultation und Partizipation gleiche Chancen haben und vielfältige Kanäle nutzen können. Es sollten alle zumutbaren Anstrengungen unternommen werden, um ein möglichst breites Bevölkerungsspektrum einzubeziehen. Deshalb nutzen die Regierungen in den OECD-Ländern die Möglichkeiten der neuen Informations- und Kommunikationstechnologien (IKT), um die Sensibilisierung und Partizipation der Bürger zu erhöhen. Die Nutzung von IKT-Werkzeugen für die Konsultation unterscheidet sich erheblich von einem Land zum anderen, und die Inanspruchnahme seitens der Bürger ist in der Europäischen Union im Durchschnitt nach wie vor gering. Laut der Eurostat-Datenbank Information Society Statistics gaben im Durchschnitt unter $10 \%$ der Bürger an, das Internet zu verwenden, um sich an Online-Konsultationen zu beteiligen oder über den Umgang mit zivilgesellschaftlichen bzw. politischen Problemen abzustimmen (z.B. Abstimmungen über Stadtplanungsfragen, Petitionen). Am stärksten werden OnlineWerkzeuge für Konsultations- oder Abstimmungszwecke in den nordischen Ländern genutzt.

\section{Methodik und Definitionen}

Die Daten beziehen sich auf das Jahr 2012 und entstammen den Antworten der Länder auf den OECD Survey on Gender Public Policies and Leadership von 2011. Die Befragten waren vornehmlich hochrangige Haushaltsverantwortliche der OECD-Länder. Die Antworten stellen die Selbsteinschätzung der Länder zu den derzeitigen Praktiken und Verfahren dar. Die Daten beziehen sich nur auf die Ebene der Zentral-/Bundesregierungen.

Die Indikatoren zu Bürgern und Unternehmen wurden der Eurostat-Datenbank Information Society Statistics entnommen, in der der Anteil der Bürger und Unternehmen evaluiert wird, die das Internet für Online-Konsultationen oder zur Abstimmung über den Umgang mit zivilgesellschaftlichen bzw. politischen Problemen nutzen (z.B. Abstimmungen über Stadtplanungsangelegenheiten, Petitionen). Die Daten werden von den nationalen Statistikämtern auf der Grundlage der jährlichen Gemeinschaftserhebung über IKT-Einsatz und E-Commerce in Unternehmen sowie der jährlichen Gemeinschaftserhebung zur IKT-Nutzung in Privathaushalten und durch Privatpersonen von Eurostat erhoben.

\section{Weitere Informationen}

OECD (2013), Gleichstellung der Geschlechter - Zeit zu handeln, OECD Publishing, Paris. http://dx.doi.org/10.1787/9789264190344-de.

OECD (2009), Focus on Citizens: Public Engagement for Better Policy and Services, OECD Studies on Public Engagement, OECD Publishing, Paris, http://dx.doi.org/10.1787/9789264048874-en.

\section{Anmerkungen zu den Abbildungen und Tabellen}

8.9: Für Österreich, Kanada, Dänemark, Estland, Ungarn, Island, Italien, Japan, die Niederlande, Polen, Portugal, Slowenien, die Türkei, das Vereinigte Königreich und die Vereinigten Staaten sind keine Daten verfügbar.

8.10: Für Australien, Kanada, Chile, Israel, Japan, Korea, Mexiko, Neuseeland, die Schweiz, die Türkei und die Vereinigten Staaten liegen keine Daten vor.

Hinweis zu den Daten für Israel: http://dx.doi.org/10.1787/888932315602. 
8.9. Gender Impact Assessments auf Ebene der Zentral-/Bundesregierungen (2011)

\begin{tabular}{|c|c|c|c|c|c|c|c|}
\hline & \multicolumn{3}{|c|}{$\begin{array}{l}\text { Ministerien/staatliche Stellen müssen ex ante } \\
\text { Gender Impact Assessments durchführen für: }\end{array}$} & \multicolumn{3}{|c|}{$\begin{array}{l}\text { Ministerien/staatliche Stellen müssen ex post } \\
\text { Gender Impact Assessments durchführen für: }\end{array}$} & \multirow{2}{*}{$\begin{array}{l}\text { Auf zentraler } \\
\text { Ebene ist ein Gender } \\
\text { Responsive } \\
\text { Budgeting vorgesehen }\end{array}$} \\
\hline & Primärgesetzgebung & Sekundärgesetzgebung & $\begin{array}{c}\text { staatliche } \\
\text { Programme } \\
\text { und Initiativen }\end{array}$ & Primärgesetzgebung & Sekundärgesetzgebung & $\begin{array}{c}\text { staatliche } \\
\text { Programme } \\
\text { und Initiativen }\end{array}$ & \\
\hline Australien & $\diamond$ & $\diamond$ & $\mathbf{\square}$ & $\diamond$ & $\diamond$ & $\diamond$ & $\Leftrightarrow$ \\
\hline Belgien & O & O & $\mathbf{\square}$ & O & O & O & $\bullet$ \\
\hline Chile & $\mathbf{\square}$ & $\mathbf{\square}$ & $\mathbf{\square}$ & $\diamond$ & $\diamond$ & $\diamond$ & $\mathrm{O}$ \\
\hline Tschech. Rep. & $\mathbf{\square}$ & $\mathbf{\square}$ & $\square$ & $\mathbf{\square}$ & $\mathbf{\square}$ & $\mathbf{\square}$ & $\diamond$ \\
\hline Finnland & 0 & $\mathbf{\square}$ & $\mathbf{\square}$ & $\mathbf{\square}$ & $\mathbf{\square}$ & $\mathbf{\square}$ & 0 \\
\hline Frankreich & $O$ & $O$ & $\bigcirc$ & O & O & O & ○ \\
\hline Deutschland & ○ & - & - & $\diamond$ & $\diamond$ & $\diamond$ & $\diamond$ \\
\hline Griechenland & $\mathbf{\square}$ & $\boldsymbol{\square}$ & $\boldsymbol{\square}$ & $\mathbf{\square}$ & $\mathbf{\square}$ & $\mathbf{\square}$ & $O$ \\
\hline Irland & $\mathbf{\square}$ & $\diamond$ & $\mathbf{\square}$ & $\diamond$ & $\diamond$ & $\diamond$ & $\diamond$ \\
\hline Israel & ○ & - & $\diamond$ & $\mathbf{\square}$ & $\square$ & $\mathbf{\square}$ & - \\
\hline Korea & $\bullet$ & $\bullet$ & $\bullet$ & $\bullet$ & $\bullet$ & $\bullet$ & $\bullet$ \\
\hline Luxemburg & - & - & $\diamond$ & $\diamond$ & $\diamond$ & $\diamond$ & $\diamond$ \\
\hline Mexiko & 口 & $\diamond$ & $\square$ & $\diamond$ & O & $\mathbf{\square}$ & - \\
\hline Neuseeland & $\mathbf{\square}$ & $\square$ & $\square$ & $\diamond$ & $\diamond$ & $\diamond$ & $\diamond$ \\
\hline Norwegen & - & $\diamond$ & $\square$ & $\bullet$ & $\bullet$ & $\mathbf{\square}$ & $\bullet$ \\
\hline Slowak. Rep. & ○ & $\diamond$ & $\diamond$ & $\diamond$ & $\diamond$ & $\diamond$ & $\diamond$ \\
\hline Spanien & ○ & - & $\square$ & $\diamond$ & $\diamond$ & $\diamond$ & ○ \\
\hline Schweden & • & • & $\bullet$ & $\bullet$ & • & • & $\bullet$ \\
\hline Schweiz & $\bullet$ & $\diamond$ & $\diamond$ & O & O & O & $\mathbf{\square}$ \\
\hline \multicolumn{8}{|l|}{ OECD insgesamt } \\
\hline Ja, immer & 10 & 6 & 3 & 3 & 3 & 2 & 9 \\
\hline - Ja, manchmal & 6 & 5 & 11 & 4 & 4 & 6 & 1 \\
\hline Nein, aber geplant & 2 & 2 & 1 & 3 & 4 & 3 & 2 \\
\hline$\diamond$ Nein, auch nicht geplant & 1 & 6 & 4 & 9 & 8 & 8 & 7 \\
\hline
\end{tabular}

Quelle: OECD Survey on National Gender Frameworks, Gender Public Policies and Leadership von 2011.

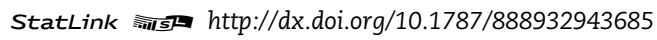

\subsection{Prozentsatz der Bürger, die an einer Online-Konsultation oder -Abstimmung teilgenommen haben}

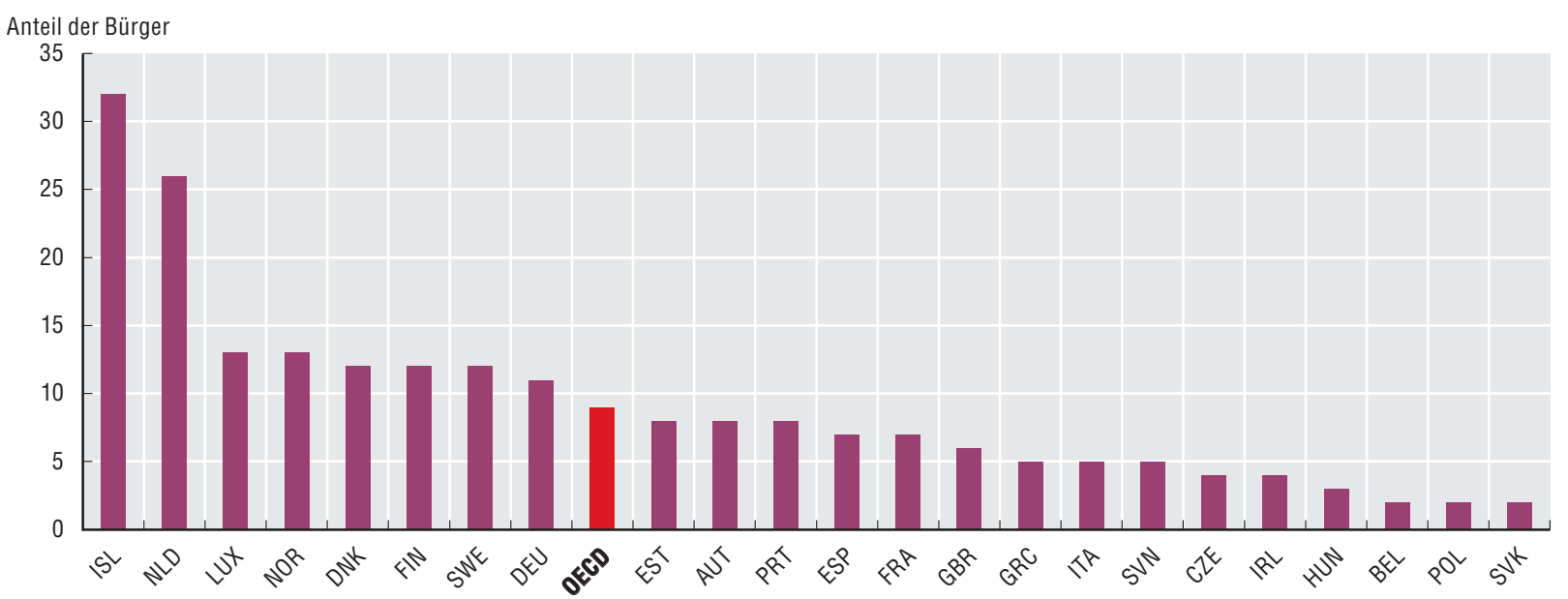

Quelle: Eurostat, Information Society Statistics (Datenbank). 


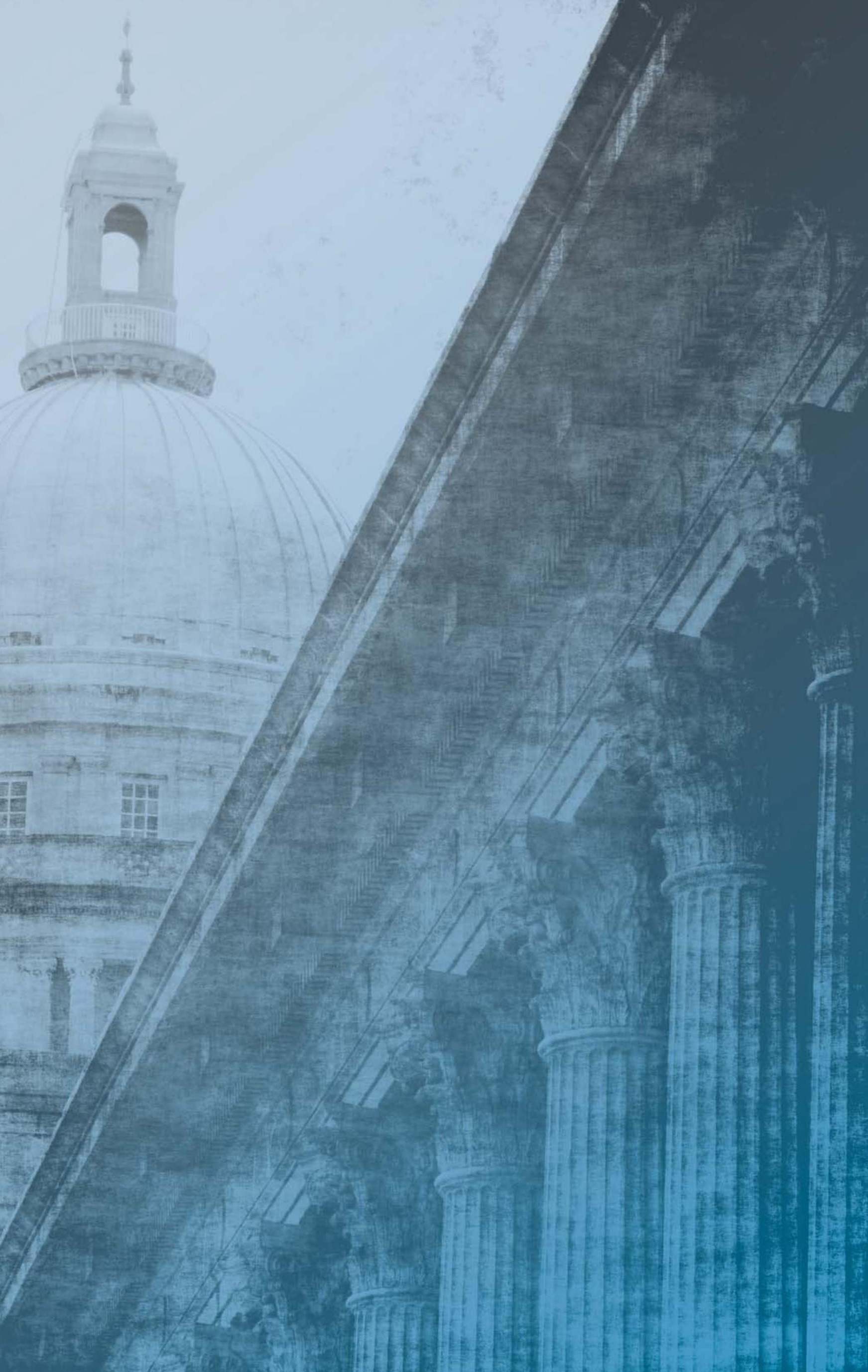




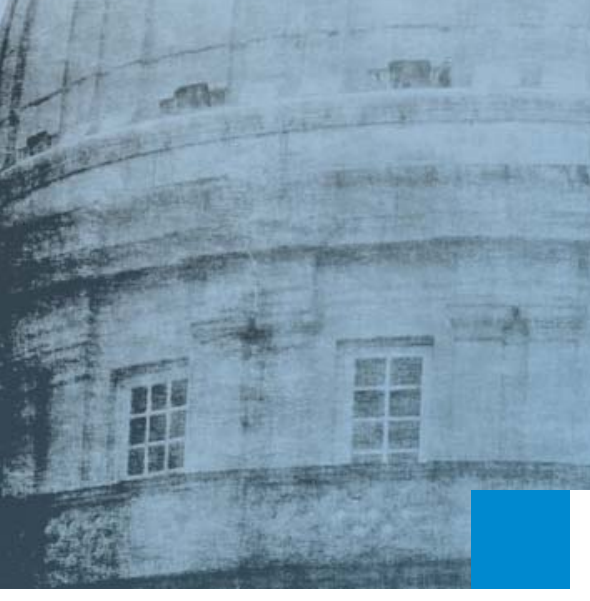

\section{SONDERBEITRAG - IM DIENSTE DER BÜRGER: ZUGÄNGLICHKEIT UND QUALITÄT ÖFFENTLICHER DIENSTLEISTUNGEN}

Die Regierungen der OECD-Mitgliedsländer verfolgen zunehmend das Ziel, möglichst breiten Kreisen der Bevölkerung qualitativ hochwertige öffentliche Güter und Dienstleistungen zugänglich zu machen und zugleich die Anpassungsfähigkeit dieser Güter und Dienstleistungen an verschiedene individuelle Bedürfnisse zu verbessern. In vielen Ländern werden strengere Leistungs- und Erfolgsmaßstäbe eingeführt und Mechanismen eingesetzt, mit denen Feedback von der Bevölkerung eingeholt und in den Prozess der Leistungserbringung integriert werden kann.

Die Ausrichtung auf eine verstärkte Bürgerorientierung beruht auf verschiedenen Motiven. Gebildetere, gut informierte Bürger beurteilen die Regierung nach ihrem Erfolg, für den die Qualität der öffentlichen Dienstleistungen einen wichtigen Teilaspekt ausmacht. Die Erfahrungen der Bürger im Umgang mit den Behörden - und ihre Zufriedenheit mit den erhaltenen Gütern und Dienstleistungen sowie ihrer Behandlung durch die Behörden - können ihre Wahrnehmung der Kompetenz und Gerechtigkeit staatlicher Institutionen und ihre Meinung darüber prägen, ob die Leistungen in einem angemessenen Verhältnis zu den von ihnen entrichteten Steuern und Abgaben stehen. Zudem ist es angesichts der gegenwärtigen Sparpolitik auch deshalb notwendig, die Qualität öffentlicher Dienstleistungen zu messen, um die Auswirkungen von Ausgabenkürzungen auf die Leistungen zu evaluieren und zu ermitteln, ob die staatlichen Behörden und Einrichtungen Effizienzsteigerungen ohne negative Auswirkungen auf diejenigen Elemente erzielen, die den Bürgern am wichtigsten sind.

Dieses Kapitel stellt einen ersten Versuch dar, Dimensionen der Qualität öffentlicher Dienstleistungen nicht nur zwischen verschiedenen Ländern, sondern auch zwischen einzelnen Schlüsselbereichen öffentlicher Dienstleistungen - Bildung, Gesundheitswesen, Justiz und Steuerverwaltung - zu vergleichen. Es behandelt eine Auswahl bürgerorientierter Indikatoren, die verschiedene Dimensionen von Zugänglichkeit und Qualität darstellen: Erschwinglichkeit, Verfügbarkeit von Online-Kanälen, Zeitgerechtigkeit und Verlässlichkeit. Darüber hinaus werden Daten aus Meinungsumfragen zur Zufriedenheit der Bürger mit diesen Dienstleistungen untersucht. Jede dieser Dimensionen hat mehrere Facetten. Beispielsweise kann sich „Zugänglichkeit“ auf verschiedene Faktoren beziehen, die von der räumlichen Nähe zu den Dienstleistern bis zur Anpassung von Einrichtungen oder Kanälen an unterschiedliche Bedürfnisse reichen. Dieses Kapitel enthält jedoch lediglich eine Auswahl von Indikatoren, die auf Basis der Datenverfügbarkeit und der Vergleichbarkeit zwischen verschiedenen Mitgliedsländern getroffen wurde. 
Der Einfluss grundlegender Güter und Dienstleistungen, beispielsweise im Bildungs-, Gesundheits- und Justizbereich, auf das Leben der Bürger hängt in entscheidendem Maße davon ab, in welchem Umfang die vorgesehenen Empfänger Zugang zu ihnen haben und sie in Anspruch nehmen können. Die Zugänglichkeit öffentlicher Dienstleistungen kann als Erfolgskriterium für die staatliche Verwaltung betrachtet werden, das ihre Fähigkeit widerspiegelt, die Vielfalt und das Wesen der unterschiedlichen Bedürfnisse richtig zu erfassen, die Einrichtung und Anpassung von Service- und Kommunikationskanälen darauf abzustimmen und bei der Erbringung und Verteilung öffentlicher Dienstleistungen für Chancengleichheit und Gerechtigkeit zu sorgen.

Es gibt verschiedene Formen von Zugangshindernissen, wie z.B. die räumliche Entfernung, ungeeignete Einrichtungen für Nutzer mit besonderen Bedürfnissen, eine unzureichende Anzahl an Servicekanälen (Internet, Telefon, persönlich usw.), unzureichende Informationen oder die Verwendung einer komplizierten Ausdrucksweise, ein Mangel an Dolmetschern oder Übersetzern für Nichtmuttersprachler, ungünstige Öffnungszeiten oder übermäßiger bürokratischer Aufwand. Derartige Hindernisse können dazu führen, dass potenzielle Leistungsempfänger abgeschreckt werden oder viele von ihnen entweder nicht wissen, dass sie Anspruch auf bestimmte Dienstleistungen hätten oder dass die Leistungen überhaupt existieren. Die Erschwinglichkeit - d.h. die ökonomischen Kosten des Erwerbs eines Gutes oder einer Dienstleistung im Verhältnis zum Einkommen - kann ein bedeutendes Zugangshindernis darstellen. Die finanzielle Zugänglichkeit kann nicht nur ausschlaggebend dafür sein, ob ein Gut oder eine Dienstleistung in Anspruch genommen wird, sondern sie kann sich auch auf das Vertrauen der Bürger in die Gerechtigkeit der staatlichen Institutionen auswirken.

Es liegt zwar im Eigeninteresse der Staaten, die Erschwinglichkeit grundlegender Dienstleistungen für die Bürger - insbesondere Angehörige niedriger Einkommensschichten oder anderer benachteiligter Gruppen - zu gewährleisten, doch neben ihrem Einsatz für Gerechtigkeit und das Wohlergehen ihrer Bürger müssen sie auch Anstrengungen unternehmen, um die Wahlfreiheit der Nutzer zu erhalten und Anreize für Leistungssteigerungen der öffentlichen Dienstleister zu bieten. Subventionierte oder unter Marktpreisen angebotene Leistungen können eine Inanspruchnahme nicht benötigter Güter oder eine Verringerung des Wettbewerbs bewirken, wodurch die Zahl der Anbieter und die Anreize für Qualitätsverbesserungen sinken würden.

\section{Gesundheitswesen}

In den meisten OECD-Mitgliedsländern gibt es zwar einen universellen Krankenversicherungsschutz für einen Grundkatalog an Gesundheitsleistungen, doch die Bürgerinnen und Bürger müssen u.U. dennoch für einige Leistungen oder Arzneimittel selbst aufkommen. Diese Kosten könnten einige Bürger veranlassen, auf Arztbesuche oder Behandlungen zu verzichten oder diese aufzuschieben und somit den Zugang zu Gesundheitsleistungen insgesamt verringern oder zu einem späteren Zeitpunkt einen kostspieligeren Krankenhausaufenthalt erforderlich machen. Zudem müssen Bürger, die einen höheren Bedarf an Gesundheitsleistungen haben, möglicherweise eine höhere Kostenlast tragen, sofern sie nicht von einigen dieser Kosten befreit sind oder eine Ausgabendeckelung besteht. Beim Anteil der Selbstzahlungen am Endverbrauch der privaten Haushalte sind im OECDVergleich deutliche Unterschiede zwischen den einzelnen Ländern festzustellen. In Frankreich, den Niederlanden, der Türkei und dem Vereinigten Königreich beliefen sich die Selbstzahlungen nur auf 1,5\% des Endverbrauchs der privaten Haushalte, während es in Chile, Korea, Mexiko und Portugal mehr als $4 \%$ waren
(Abbildung 9.1). Entscheidender ist jedoch die Verteilung dieser Selbstzahlungen nach Einkommensgruppen. In vielen Ländern gibt es beispielsweise Regelungen, die Niedrigeinkommensbezieher von Selbstzahlungen befreien oder diese Zahlungen deckeln, um den Zugang zu Gesundheitsleistungen für diese Einkommensgruppe zu sichern.

\section{Justizwesen}

Die Klärung von Rechtsstreitigkeiten auf juristischem Weg ist für die Bürgerinnen und Bürger mit zeitlichem und finanziellem Aufwand verbunden. Für Beklagte oder Beschuldigte entstehen zudem Kosten im Zusammenhang mit der rechtlichen Vertretung. Der Zugang zur Justiz kann durch Prozesskostenhilfe erleichtert werden, die dafür sorgt, dass selbst Personen ohne ausreichende finanzielle Mittel ihr Recht auf ein faires Verfahren ausüben können. Prozesskostenhilfe kann gemäß der hier zu Grunde gelegten Definition in Form unentgeltlicher oder bezuschusster rechtlicher Vertretung, Rechtsberatung und Gebührenbefreiung erfolgen. Der Anteil der Rechtssachen, für die Prozesskostenhilfe gewährt wird, kann als Anhaltspunkt dafür dienen, in welchem Umfang öffentliche Finanzhilfen bereitgestellt werden. In vier der elf OECD-Mitgliedsländer, für die Daten zur Verfügung stehen - Finnland, Frankreich, die Niederlande und Norwegen - erhielten die Bürgerinnen und Bürger in mehr als 10\% der erstinstanzlichen Verfahren Prozesskostenhilfe. In den übrigen Ländern erhielten die Bürger in maximal 6\% der erstinstanzlichen Verfahren Prozesskostenhilfe (Abbildung 9.2). In einigen Ländern, wie z.B. Österreich, besteht bei unstreitigen Verfahren u.U. nicht immer Anspruch auf Prozesskostenhilfe, so dass der Anteil der Fälle, in denen eine staatliche Unterstützung gewährt wird, möglicherweise nicht exakt den tatsächlichen Gegebenheiten entspricht.

\section{Bildungswesen}

Der Zugang zu öffentlichen Dienstleistungen kann für die Bürgerinnen und Bürger nicht nur mit direkten, sondern auch mit indirekten Kosten verbunden sein. Beispielsweise müssen bei der Entscheidung für eine Hochschulbildung neben den Studiengebühren auch die Lebenshaltungskosten sowie das potenziell entgangene Einkommen einkalkuliert werden. In fünf Ländern - Chile, Japan, Neuseeland, das Vereinigte Königreich und die Vereinigten Staaten - betrugen die Studiengebühren an öffentlichen Bildungseinrichtungen im Tertiärbereich mindestens $14 \%$ des verfügbaren Einkommens pro Person (Abb. 9.3). In fünf Ländern - Italien, die Niederlande, Österreich, die Schweiz und Spanien - stellten die Studiengebühren mit 3-7\% des verfügbaren Einkommens pro Person eine deutlich geringere Belastung dar. In fünf Ländern - Dänemark, Finnland, Mexiko, Norwegen und Schweden - erhoben die öffentlichen Bildungseinrichtungen des Tertiärbereichs keine Studiengebühren.

In zehn Ländern erhielt mindestens die Hälfte der Studierenden finanzielle Unterstützung. Zu diesen Ländern zählten fünf der sechs Länder mit überdurchschnittlich hohen Studiengebühren (Australien, Chile, Neuseeland, das Vereinigte Königreich und die Vereinigten Staaten) sowie ein Land, in dem die Studiengebühren im Verhältnis zum verfügbaren Einkommen pro Person relativ moderat waren (die Niederlande), und die meisten Länder ohne Studiengebühren (Dänemark, Finnland, Norwegen und Schweden). In diesen Ländern wählten im Schnitt rd. 71\% der Bürgerinnen und Bürger der entsprechenden Alterskohorte einen Bildungsgang auf Universitätsniveau. In Ländern, in denen weniger als die Hälfte der Studierenden finanzielle Unterstützung erhielt, entschieden sich durchschnittlich rd. 45\% der entsprechenden Alterskohorte für einen Bildungsgang auf Universitätsniveau. 


\section{Methodik und Definitionen}

Die Daten zu Selbstzahlungen stammen aus OECD Health Statistics 2013. Selbstzahlungen sind Ausgaben, die von Patienten direkt getragen werden, wenn die Kosten der Gesundheitsprodukte oder -leistungen weder durch öffentliche noch durch private Versicherungen in vollem Umfang gedeckt werden. $\mathrm{Zu}$ den Selbstzahlungen gehören Kostenbeteiligungen und andere Direktzahlungen der privaten Haushalte. Ebenfalls berücksichtigt werden Schätzungen der in einigen Ländern entrichteten informellen Zahlungen an Gesundheitsdienstleister. Die hier dargestellten Daten umfassen nur medizinische Ausgaben (d.h. die laufenden Gesundheitsausgaben abzüglich der Ausgaben für den Gesundheitsversorgungsanteil der Langzeitpflege), da die Möglichkeiten der einzelnen Länder, die privaten Ausgaben für Langzeitpflege zu schätzen, sehr unterschiedlich sind.

Die Daten zur Prozesskostenhilfe und zu erstinstanzlichen Verfahren beruhen auf der Bewertung europäischer Justizsysteme, die von der Europäischen Kommission für die Wirksamkeit der Justiz (European Commission for the Efficiency of Justice - CEPEJ) durchgeführt wird. Prozesskostenhilfe bezieht sich auf Fälle, in denen öffentliche Mittel zur Finanzierung oder Bezuschussung der rechtlichen Vertretung gewährt wurden. Dies kann auch Fälle einschließen, in denen unentgeltliche Rechtsberatung geleistet wurde oder in denen eine Befreiung von bestimmten Gebühren erfolgte. Die Anspruchskriterien für den Erhalt von Prozesskostenhilfe sind von Land zu Land unterschiedlich; beispielsweise kann der Anspruch auf Unterstützung an bestimmte Personenkreise oder an bestimmte Arten von Rechtssachen gebunden sein. Die Daten zu den geschätzten Kosten eines Verfahrens stammen aus der Weltbank-Datenbank Doing Business. Die Kosten werden als prozentualer Anteil des Streitwerts erfasst, für den ein Wert von $200 \%$ des Pro-Kopf-Einkommens angenommen wird. Bestechungsgelder werden nicht berücksichtigt. Es werden drei Arten von Kosten erfasst: Gerichtskosten, Vollstreckungskosten und durchschnittliche Anwaltshonorare.

Die Daten zu Studiengebühren, Finanzhilfen und Studienanfängerquoten für Bildungsgänge auf Universitätsniveau im Tertiärbereich A stammen aus Bildung auf einen Blick 2013: OECD-Indikatoren (OECD, 2013). Die Studiengebühren (in US-\$ auf KKP-Basis) umfassen ausschließlich $\mathrm{zu}$ einem ersten Abschluss führende Studiengänge an öffentlichen Bildungseinrichtungen im Tertiärbereich A für das Studienjahr 2010/2011. Das bereinigte verfügbare Einkommen ist definiert als der um staatliche Transferleistungen bereinigte Höchstbetrag, den eine Einheit für den Konsum von Waren und Dienstleistungen ausgeben kann, ohne ihre finanziellen oder nichtfinanziellen Vermögenswerte reduzieren oder ihre Verschuldung erhöhen zu müssen. Studienanfängerquoten beziehen sich auf den geschätzten prozentualen Anteil einer Alterskohorte, der zum ersten Mal ein Studium im Tertiärbereich A aufnimmt.

\section{Weitere Informationen}

CEPEJ (2012), European Judicial Systems - Edition 2012 - Efficiency and Quality of Justice, Council of Europe Publishing, Straßburg.

OECD (2013a), Bildung auf einen Blick 2013: OECD-Indikatoren, W. Bertelsmann Verlag, Bielefeld, http://dx.doi.org/10.1787/eag2013-de.

OECD (2013b, erscheint demnächst), Health at a Glance 2013: OECD Indicators, OECD Publishing, Paris.

\section{Anmerkungen zu den Abbildungen}

9.1: Die Daten für Australien, Japan, Kanada, Mexiko, Neuseeland, Norwegen und Österreich beziehen sich auf 2010. Die Daten für Israel beziehen sich auf 2009. Die Daten für die Türkei beziehen sich auf 2008.

9.2: Strafrechtliche und nichtstrafrechtliche Rechtssachen für alle Länder außer Frankreich und Österreich, wo sich die Daten lediglich auf nichtstrafrechtliche Rechtssachen beziehen.

9.3: In den Vereinigten Staaten beziehen sich die Zahlen zu den Studienanfängerquoten und zum Prozentsatz der Studierenden, die öffentliche Darlehen/Stipendien erhalten, auf alle Studierenden (inlänische und nicht inländische/ausländische Studierende im Vollzeitstudium), und die Daten zu den Studienanfängerquoten beziehen sich sowohl auf Tertiärbereich A als auch auf Tertiär-bereich B. In Frankreich liegen die durchschnittlichen Studiengebühren für universitäre Studiengänge zwischen 200 US-\$ und 1402 US-\$ (abhängig vom Bildungsministerium). In Japan beziehen sich die Studiengebühren auf öffentliche Bildungseinrichtungen, aber mehr als zwei Drittel der Studierenden sind an privaten Bildungseinrichtungen eingeschrieben. In Chile beläuft sich der Anteil der Studierenden, die öffentliche Studiendarlehen und/oder Stipendien/Zuschüsse erhalten, auf $68 \%$, wenn nur öffentliche Bildungseinrichtungen berücksichtigt werden. Daten zu Darlehen/Stipendien: Die Daten für Australien umfassen keine ausländischen Studierenden. Die Daten für Mexiko und das Vereinigte Königreich beziehen sich auf das Studienjahr 2008/2009. Die Daten für Dänemark, Frankreich, Mexiko, die Niederlande und die Vereinigten Staaten umfassen nur öffentliche Universitäten, einschließlich Tertiärbereich B in Frankreich. Vgl. Anhang 3 von Bildung auf einen Blick 2013: OECD-Indikatoren (OECD, 2013) wegen weiterer Anmerkungen (www.oecd.org/edu/eag.htm).

Hinweis zu den Daten für Israel: http://dx.doi.org/10.1787/888932315602. 


\section{SONDERBEITRAG - IM DIENSTE DER BÜRGER: ZUGÄNGLICHKEIT UND QUALITÄT}

\section{Zugang zu öffentlichen Dienstleistungen: Erschwinglichkeit}

9.1. Anteil der Selbstzahlungen am Endverbrauch der privaten Haushalte (2011 oder letztes verfügbares Jahr)

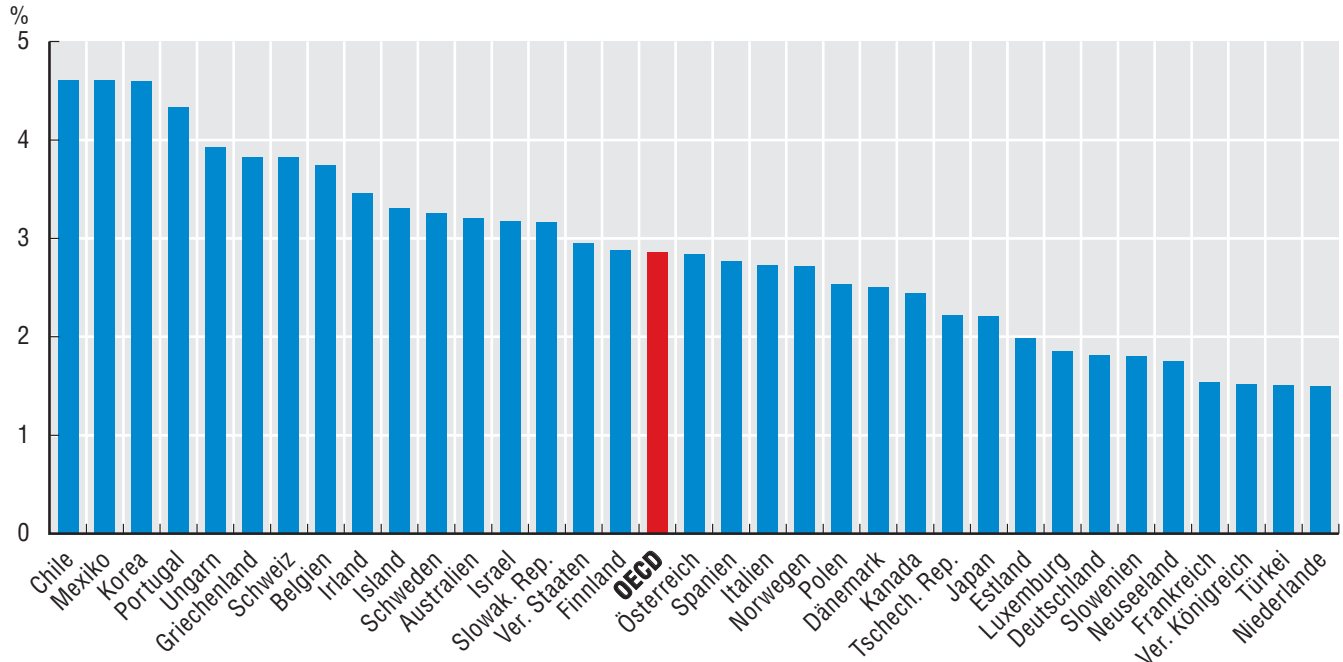

Quelle: OECD Health Statistics 2013.

9.2. Rechtssachen, für die Prozesskostenhilfe gewährt wurde (2010)

In Prozent der erstinstanzlichen Rechtssachen

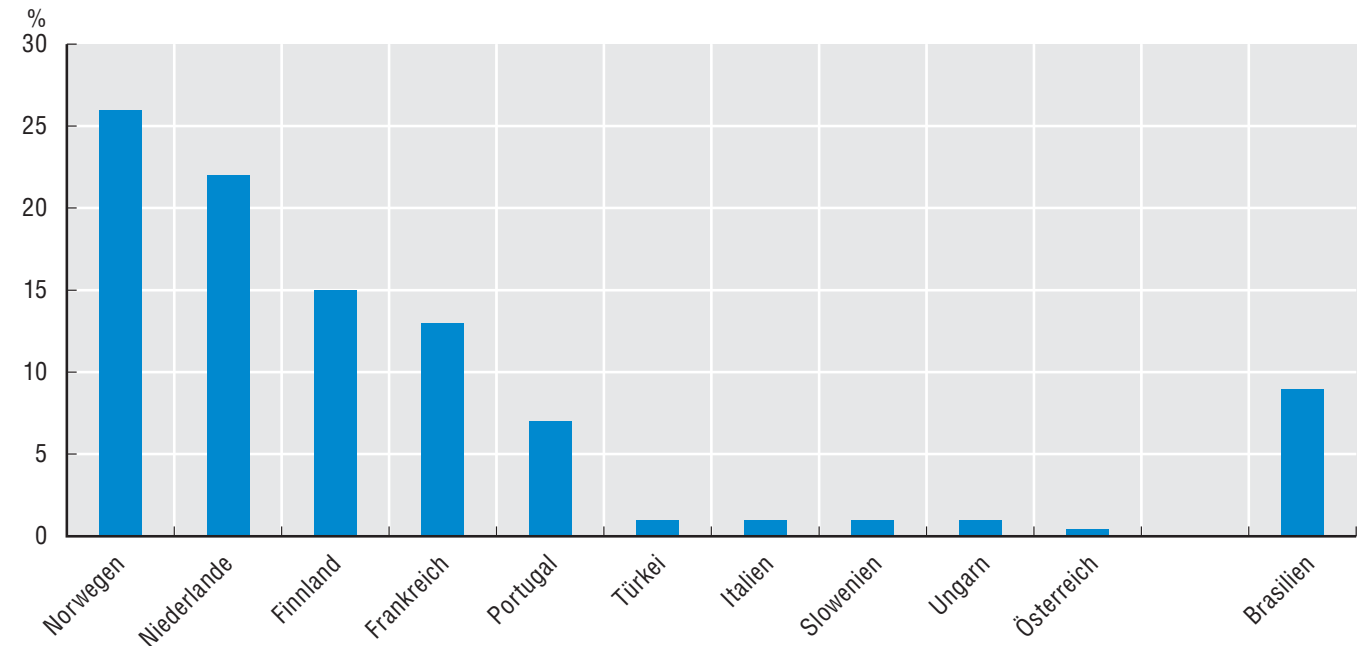

Quelle: CEPEJ (2012), European Judicial Systems (Datenbank). OECD-Berechnungen basierend auf Frage 91, 94, 97, 98, 99 und 100. Die Daten für Brasilien wurden von nationalen Stellen bereitgestellt. 


\subsection{Studiengebühren und Finanzhilfen (2010-11)}

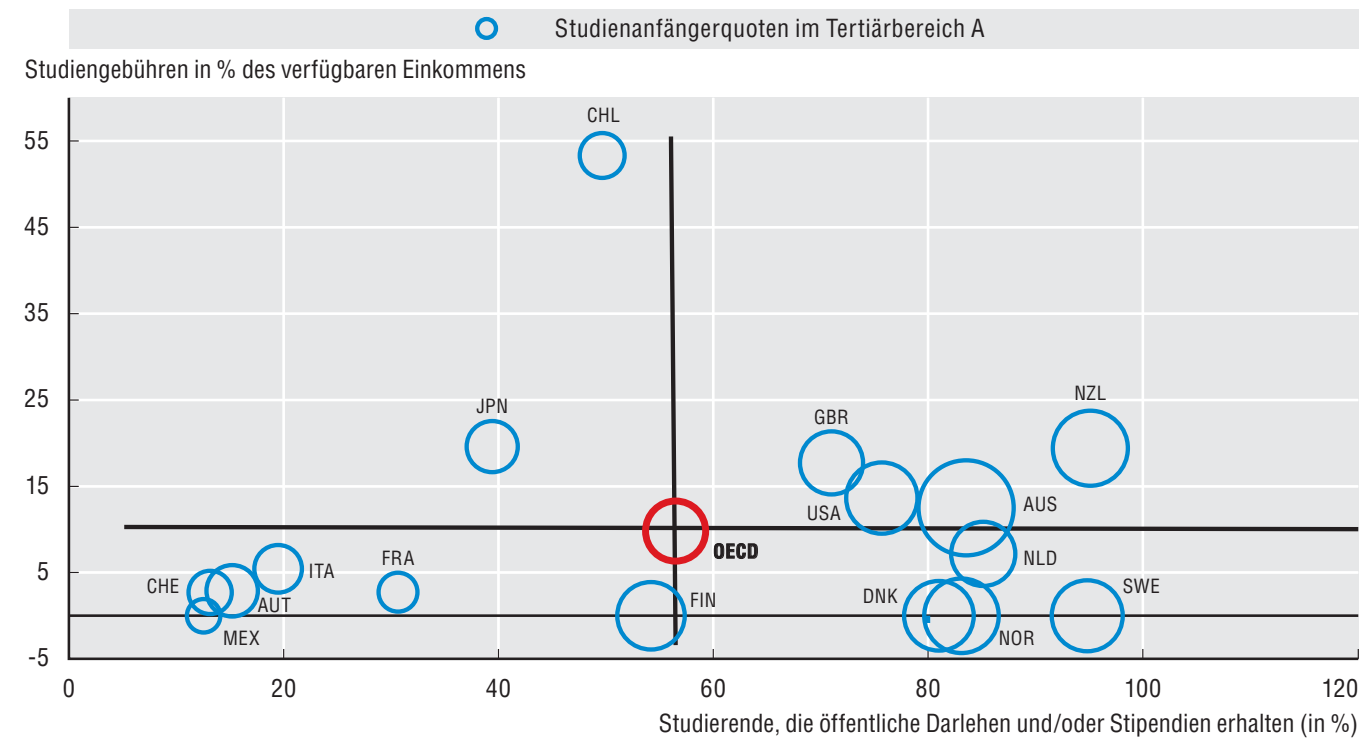

Quelle: OECD (2013), Bildung auf einen Blick 2013: OECD-Indikatoren, W. Bertelsmann Verlag, Bielefeld, http://dx.doi.org/10.1787/eag-2013-de (Tabelle B5.1, B5.2 und C3.1a); sowie OECD (2013), National Accounts at a Glance 2013, OECD Publishing, Paris, http://dx.doi.org/10.1787/na_glance-2013-en.

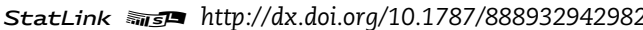


Sowohl die Bürgerinnen und Bürger als auch die Unternehmen bevorzugen und nutzen zunehmend digitale Kanäle für die Interaktion mit Behörden und den Zugang zu öffentlichen Dienstleistungen. Online-Kanäle können einem größeren Nutzerkreis den Zugang zu öffentlichen Dienstleistungen erleichtern und einen nutzerfreundlicheren Service bieten, zugleich aber auch die Kosten für alle Beteiligten, einschließlich der staatlichen Verwaltung, senken. Obwohl die Nutzung von Online-Kanälen generell zunimmt, bestehen weiterhin verschiedenartige Lücken in der Inanspruchnahme von E-Government-Diensten in vielen OECD-Mitgliedsländern, in denen Online-Dienste nach wie vor in geringerem Umfang genutzt werden. Die Staaten müssen weiter an der Verringerung dieser Disparitäten arbeiten, während sie ihr Angebot an OnlineDiensten weiterentwickeln.

\section{Inanspruchnahme öffentlicher Online- Dienstleistungen durch Unternehmen}

Durch die Verfügbarkeit öffentlicher Dienstleistungen über das Internet können Unternehmen Zeit sparen und ihren Verwaltungsaufwand verringern; zugleich erhöht sich dadurch auch die Wahrscheinlichkeit, dass in den Unternehmen Informations- und Kommunikationstechnologien (IKT) zur Produktivitätssteigerung eingesetzt werden. Da den Unternehmen eine höhere E-Government-Bereitschaft unterstellt wird als den Bürgern, ergreifen die Länder zunehmend Maßnahmen, um die Inanspruchnahme der Online-Verwaltungsdienstleistungen weiter zu steigern, indem sie beispielsweise bei bestimmten Transaktionen die Online-Abwicklung verbindlich vorschreiben.

Island, Finnland und die Slowakische Republik gehören zur Spitzengruppe der Länder mit einem besonders hohen Grad an allgemeiner Online-Interaktion zwischen Unternehmen und Behörden. Ein anderes Bild ergibt sich allerdings, wenn man die Nutzung vollständig transaktionaler Dienstleistungen, wie z.B. die elektronische Abwicklung von Verwaltungsvorgängen, untersucht. In diesem Bereich verzeichnen Australien, Frankreich und Slowenien die höchsten Werte unter den Mitgliedsländern. Die Unterschiede zwischen den einzelnen Ländern lassen sich unter anderem auf länderspezifische Gesetzgebung, unterschiedliche Vorschriften für die Nutzung von Online-Dienstleistungen sowie den Einsatz von Intermediären bei der Leistungserbringung zurückführen.

Großunternehmen (ab 250 Beschäftigten) zeichnen sich im Allgemeinen durch eine starke Inanspruchnahme von E-Government-Dienstleistungen aus; sie machen jedoch im Durchschnitt weniger als 1\% der Gesamtzahl der Unternehmen im OECD-Raum aus. In mittleren Unternehmen (50-249 Beschäftigte) liegt die Inanspruchnahme von E-GovernmentServices eher in der Nähe des in Großunternehmen erreichten Niveaus, während zwischen Großunternehmen und kleinen Unternehmen (10-49 Beschäftigte) in den einzelnen Ländern große Lücken klaffen. In Ländern wie Australien, Italien, Korea, Spanien und Ungarn sind die Unterschiede zwischen großen und kleinen Unternehmen bei der Nutzung von E-Government-Diensten besonders stark ausgeprägt.

Eine Steigerung der Inanspruchnahme dieser Dienste durch die Unternehmen bringt zweierlei Vorteile mit sich. Zum einen kann die verstärkte Nutzung von Online-Kanälen bei der Erbringung öffentlicher Dienstleistungen für Unternehmen dazu beitragen, die Staatsausgaben zu senken. Daher setzen die Regierungen zunehmend auf Dienstleistungen, bei denen die digitale Interaktion für Unternehmen verbindlich vorgeschrieben werden kann (z.B. für Umsatzsteuererklärungen, Lizenzen oder Registrierungsvorgänge). Zum anderen ist zwar die Akzeptanz öffentlicher Online-Dienstleistungen in kleineren Unternehmen geringer, doch kleine und mittlere Unternehmen sind nach wie vor wichtige Wachstumsmotoren der OECD-Länder. Somit kann eine besondere Fokussierung auf die Steigerung der Zugangs- und IKT-Kapazitäten das Wirtschaftswachstum insgesamt fördern, nicht nur in internetnahen Bereichen.

\section{Inanspruchnahme öffentlicher Online- Dienstleistungen durch die Bürger}

Im Vergleich zu den Unternehmen nutzen die Bürgerinnen und Bürger seltener Online-Kanäle, um öffentliche Dienstleistungen in Anspruch zu nehmen. Die nordischen Länder sind in dieser Hinsicht führend, sowohl bei der Nutzung des Internets für die allgemeine Interaktion mit staatlichen Stellen als auch für anspruchsvollere Vorgänge wie z.B. das Einschicken ausgefüllter Formulare. Auch in Frankreich, Irland und den Niederlanden nutzen viele Bürger das Internet, um ausgefüllte Formulare bei Behörden einzureichen.

Hinter den durchschnittlichen Nutzungsraten der E-Government-Dienste durch die Bürger können sich erhebliche alters-, bildungs- oder einkommensabhängige Disparitäten verbergen. Beispielsweise verzeichnet die Altersgruppe der 25- bis 34-Jährigen die stärkste Interaktion mit den Behörden. Im Vergleich mit der jüngeren Bevölkerungsgruppe der 16- bis 24-Jährigen ist eine „Jugendlücke“ bei der Inanspruchnahme von E-GovernmentDiensten festzustellen. Hierfür sind mehrere Gründe denkbar: Beispielsweise könnte es sein, dass 16- bis 24-Jährige generell weniger öffentliche Dienstleistungen nutzen, weil sie u.U. immer noch abhängige Haushaltsmitglieder sind. In manchen Ländern sind einige der am weitesten verbreiteten E-Government-Dienste, wie z.B. Steuererklärungen, für die Angehörigen dieser jüngeren Altersgruppe entweder nicht relevant oder werden nicht unbedingt von ihnen selbst erledigt. Andererseits erfordern Angelegenheiten wie z.B. die Eintragung in ein Wählerverzeichnis, die Ausstellung eines Führerscheins, Anträge auf staatliche Sozialleistungen sowie administrative Vorgänge im Zusammenhang mit der Bildung und Arbeitsplatzwahl in vielen Ländern eine Interaktion mit staatlichen Stellen, was auf tatsächlich bestehende Herausforderungen auch für die jüngere Altersgruppe hindeutet.

Bei älteren Bürgern ist die Wahrscheinlichkeit, dass sie das Internet für den Zugang zu öffentlichen Dienstleistungen nutzen, ebenfalls geringer. In einer Reihe von Ländern sind erhebliche Disparitäten bei der Nutzung des Internets für E-Government-Dienstleistungen zwischen den 25- bis 34-Jährigen und den 65- bis 74-Jährigen festzustellen. Hierfür sind erneut mehrere Erklärungen denkbar. Ein entscheidendes Hemmnis für die ältere Altersgruppe ist mangelnde digitale Kompetenz. Dies hat auf kurze bis mittlere Sicht auch negative Auswirkungen für Staaten, die zur Bewältigung des demografischen Wandels vermehrt digitale soziale Dienstleistungen (z.B. E-Health-Dienste wie Telemedizin) einsetzen wollen.

Die Regierungen ergreifen eine Reihe von Maßnahmen, um die Nutzung ihrer Online-Dienste durch die Bürger zu steigern. Beispielsweise werden Dienstleistungen identifiziert, bei denen eine Schließung der physischen Erbringungskanäle aus Nutzersicht vertretbar ist, oder es werden Anreize für die Nutzung der digitalen Kanäle geboten, z.B. durch längere Bearbeitungszeiten bei papiergebundenen Verfahren oder sogar die Erhebung von Bearbeitungsgebühren bei direktem persönlichem Kontakt. Bestimmte Bevölkerungsgruppen, die das Internet intensiv nutzen, wie z.B. Studierende, können eher zur Nutzung elektronischer Kanäle verpflichtet werden, wie 
beispielsweise in Dänemark. In Kanada wird den Bürgerinnen und Bürgern Unterstützung beim Wechsel auf digitale Kanäle angeboten, z.B. durch soziale Medien oder Videoclips, wodurch sich die Inanspruchnahme von Online-Diensten erhöht hat. Einige Länder verfolgen einen „Digital-by-Default“-Ansatz durch verbindliche Festlegung bestimmter Kommunikationskanäle. Andere Länder, wie das Vereinigte Königreich, setzen auf eine „Pull“-Strategie, d.h. sie versuchen, durch qualitativ hochwertige Online-Dienste dafür zu sorgen, dass die Bürger bevorzugt digitale Kanäle nutzen.

Die einseitige Interaktion mit staatlichen Stellen (z.B. zur Informationsbeschaffung) wird sowohl von Bürgern als auch von Unternehmen nach wie vor häufiger genutzt als anspruchsvollere transaktionale Interaktionen (z.B. vollständige elektronische Vorgangsbearbeitung oder Einschicken ausgefüllter Formulare). Um das Effizienzsteigerungspotenzial von Informations- und Kommunikationstechnologien bei der Dienstleistungserbringung in vollem Umfang auszuschöpfen, ist es für die Staaten von zentraler Bedeutung, eine geeignete Infrastruktur zu implementieren und die Nutzer beim Erreichen der letzteren Stufe der Online-Interaktion zu unterstützen.

\section{Methodik und Definitionen}

Die Indikatoren für Bürger und Unternehmen basieren auf der Eurostat-Datenbank Information Society Statistics und der ICT Database der OECD. Die Indikatoren messen den Anteil der Bürger und Unternehmen, die das Internet für die Interaktion mit staatlichen Stellen verwenden. Die beiden Quellen sind aus methodischer Sicht vergleichbar. Die Daten werden von den nationalen Statistikämtern auf der Grundlage der jährlichen Gemeinschaftserhebung über den IKT-Einsatz und E-Commerce in Unternehmen sowie der jährlichen Gemeinschaftserhebung zur IKT-Nutzung in Privathaushalten und durch Privatpersonen von Eurostat erhoben.

Die Daten zeigen die Interaktion mit staatlichen Stellen über das Internet. Staatliche Stellen bezieht sich auf öffentliche Dienstleistungen sowie Verwaltungsangelegenheiten auf allen staatlichen Ebenen. Die Interaktion ist ein abgeleiteter Indikator, der verschiedene Unterkategorien von online erledigten Aktivitäten abdeckt, wie z.B. Informationsbeschaffung, Herunterladen und Versenden von Formularen oder die Abwicklung von Verwaltungsverfahren und Vorgangsbearbeitung. Die Nutzung von Intermediären ist zwar in einer Reihe von Ländern weitverbreitet, dies ist jedoch in den Erhebungsdaten nicht erfasst.

Der Indikator der Inanspruchnahme von E-GovernmentDiensten misst den Anteil der Unternehmen oder Bürger, die in den letzten 12 Monaten vor der Erhebung das Internet zur Interaktion mit staatlichen Stellen genutzt haben. Der 12-Monats-Zeitraum ermöglicht Ländervergleiche trotz unterschiedlicher länderspezifischer Zeitrahmen für Dienstleistungen verschiedener Sektoren. In der Erhebung werden nur Unternehmen außerhalb des Finanzsektors mit mindestens 10 Beschäftigten berücksichtigt. Der erfasste Personenkreis erstreckt sich auf Bürgerinnen und Bürger im Alter von 16-74 Jahren.

\section{Weitere Informationen}

OECD/Internationale Fernmeldeunion (2011), M-Government: Mobile Technologies for Responsive Governments and Connected Societies, OECD Publishing, Paris, http://dx.doi.org/10.1787/ 9789264118706-en.

OECD (2009), Rethinking e-Government Services: User-Centred Approaches, OECD Publishing, Paris, http://dx.doi.org/10.1787/ 9789264059412-en.

\section{Anmerkungen zu den Abbildungen}

9.4: Für Chile, Israel, Japan, Kanada, Mexiko, die Türkei und die Vereinigten Staaten stehen keine Daten zur Verfügung. Für die Schweiz liegen keine Daten zur vollständigen elektronischen Interaktion, für Australien keine Daten zur Informationsbeschaffung vor. Die Daten für Australien, Chile, Korea, Neuseeland und die Schweiz beziehen sich nur auf Informationsbeschaffung, nicht auf allgemeine Interaktion, und beinhalten auch den Finanzsektor. Die Daten für Australien beziehen sich auf 2010, die Daten für Island, das Vereinigte Königreich und Neuseeland auf 2012. Die Daten für Belgien und Finnland zur Online-Interaktion beziehen sich auf 2012; die Daten für Belgien, Finnland, Island und das Vereinigte Königreich zu elektronischen Verwaltungsverfahren beziehen sich auf die vollständige elektronische Vorgangsbearbeitung im Jahr 2010.

9.5: Für Chile, Israel, Japan, Kanada, Mexiko, die Türkei und die Vereinigten Staaten stehen keine Daten zur Verfügung. Die Daten für Australien, Korea, Neuseeland und die Schweiz beziehen sich nur auf Informationsbeschaffung, nicht auf allgemeine Interaktion, und beinhalten auch den Finanzsektor. Die Daten für Korea und die Schweiz beziehen sich auf 2011. Die Daten für Australien beziehen sich auf 2010 und umfassen die Internetnutzung zum Ausfüllen von Online-Formularen.

9.6: Für Korea, Mexiko, die Türkei und die Vereinigten Staaten stehen keine Daten zur Verfügung. Für Australien, Israel und die Schweiz stehen keine Daten zum Versenden ausgefüllter Formulare zur Verfügung; für Japan liegen keine Daten zur Interaktion mit staatlichen Stellen vor. Die Daten für Kanada, die Schweiz und Neuseeland beziehen sich nur auf die Informationsbeschaffung, nicht auf allgemeine Interaktion. Die Daten für Israel beziehen sich auf Bürgerinnen und Bürger ab 20 Jahren und umfassen sowohl die Informationsbeschaffung als auch das Ausfüllen von Online-Formularen. Die Daten für Kanada beziehen sich auf 2009, die Daten für Australien, Israel und die Schweiz auf 2010. Die Daten für Neuseeland beziehen sich auf das Herunterladen von Formularen, nicht auf das Versenden ausgefüllter Formulare.

9.7: Für Japan, Korea, Mexiko, die Türkei und die Vereinigten Staaten stehen keine Daten zur Verfügung. Die Daten für Australien, Kanada, Neuseeland, Israel und die Schweiz beziehen sich nur auf die Informationsbeschaffung, nicht auf allgemeine Interaktion. In diesen Ländern bezieht sich die Altersgruppe der „25- bis 34-Jährigen“ auf 25- bis 44-Jährige. Die Daten für Chile beziehen sich auf die Altersgruppe der 25- bis 64-Jährigen. Die Daten für Israel beziehen sich auf Bürger ab 20 Jahren und umfassen sowohl die Informationsbeschaffung als auch das Ausfüllen von Online-Formularen. Die Daten für Israel, Kanada und die Schweiz beziehen sich auf 2010.

Hinweis zu den Daten für Israel: http://dx.doi.org/10.1787/888932315602. 
9.4. Unternehmen, die das Internet zur Interaktion mit staatlichen Stellen nutzen, nach Art der Aktivität (2011)

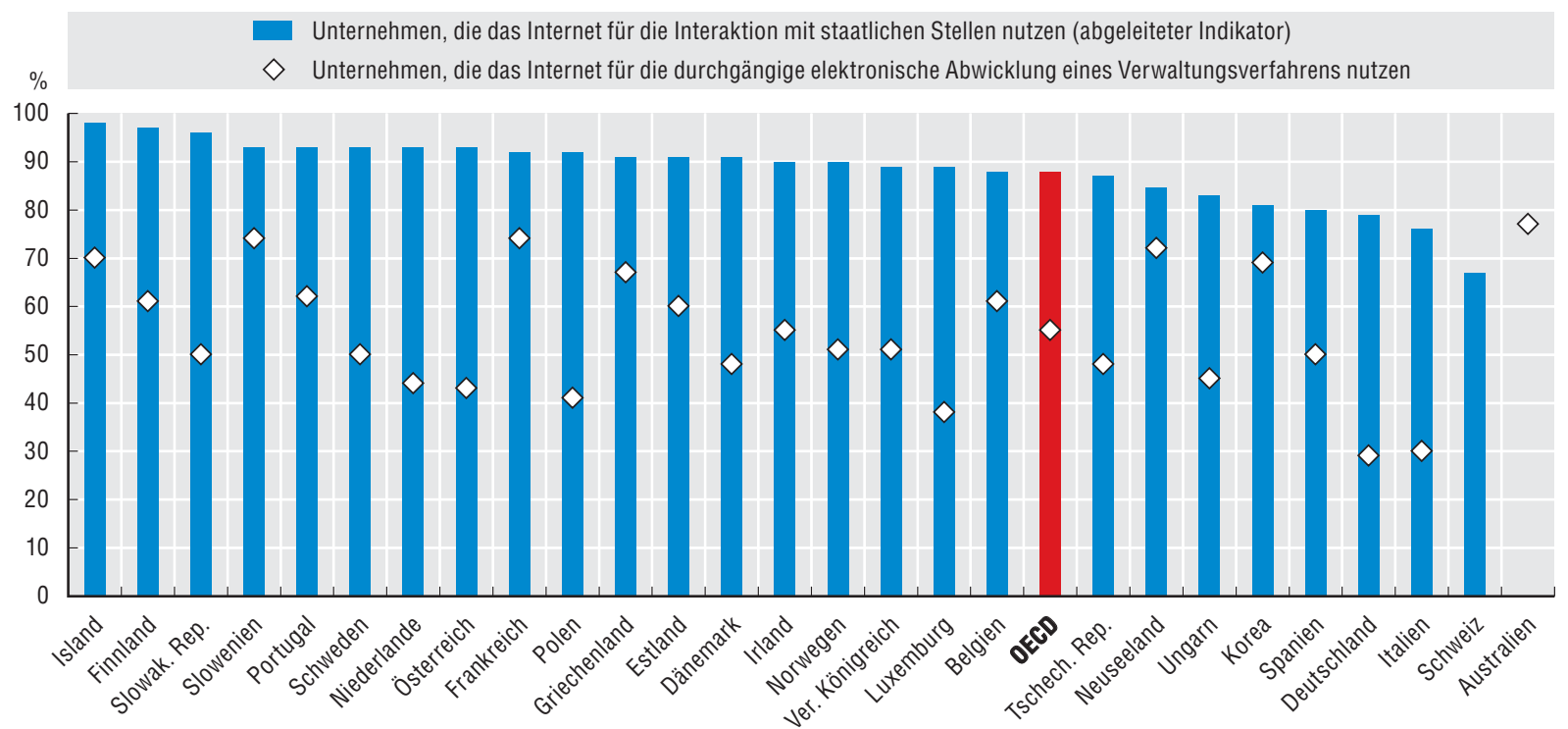

Quelle: OECD, ICT Database; Eurostat, Information Society Statistics (Datenbank).

9.5. Unternehmen, die das Internet zur Interaktion mit staatlichen Stellen nutzen, nach Unternehmensgröße (2012)

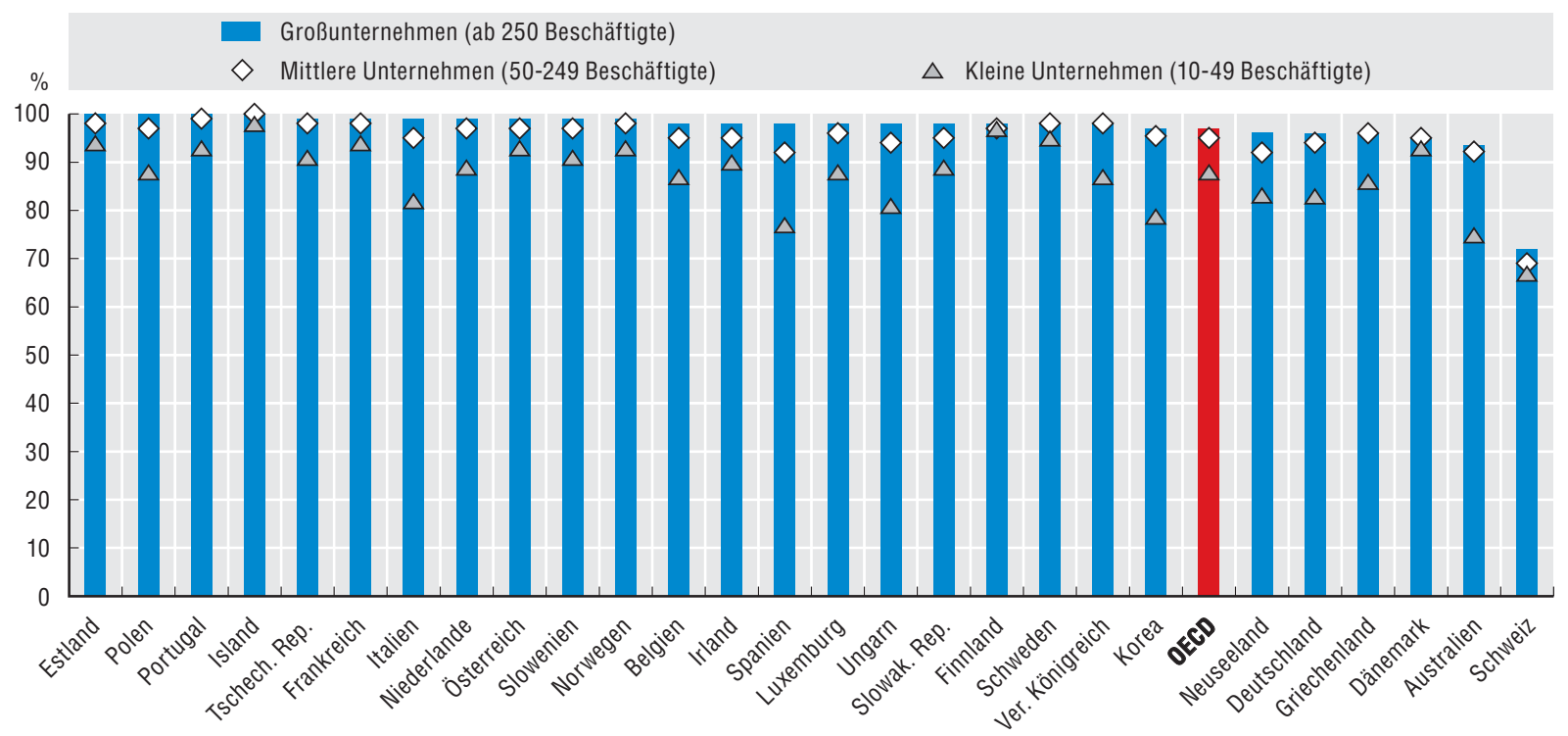

Quelle: OECD, ICT Database; Eurostat, Information Society Statistics (Datenbank). 


\section{SONDERBEITRAG - IM DIENSTE DER BÜRGER: ZUGÄNGLICHKEIT UND QUALITÄT}

9.6. Bürger, die das Internet zur Interaktion mit staatlichen Stellen nutzen, nach Art der Aktivität (2012)

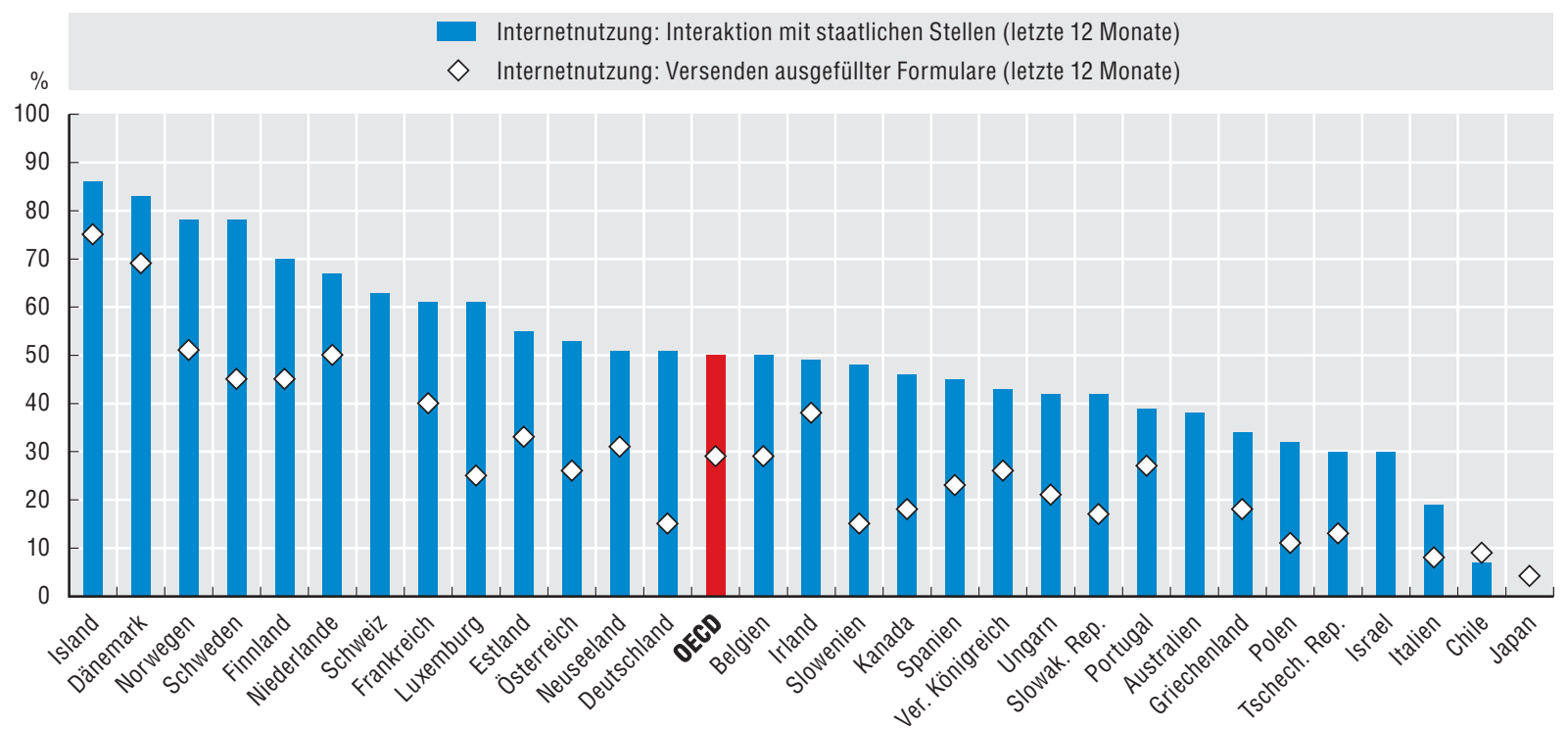

Quelle: OECD, ICT Database; Eurostat, Information Society Statistics (Datenbank).

StatLink न्ताड़ी http://dx.doi.org/10.1787/888932943039

9.7. Bürger, die das Internet zur Interaktion mit staatlichen Stellen nutzen, nach Altersgruppe (2012)

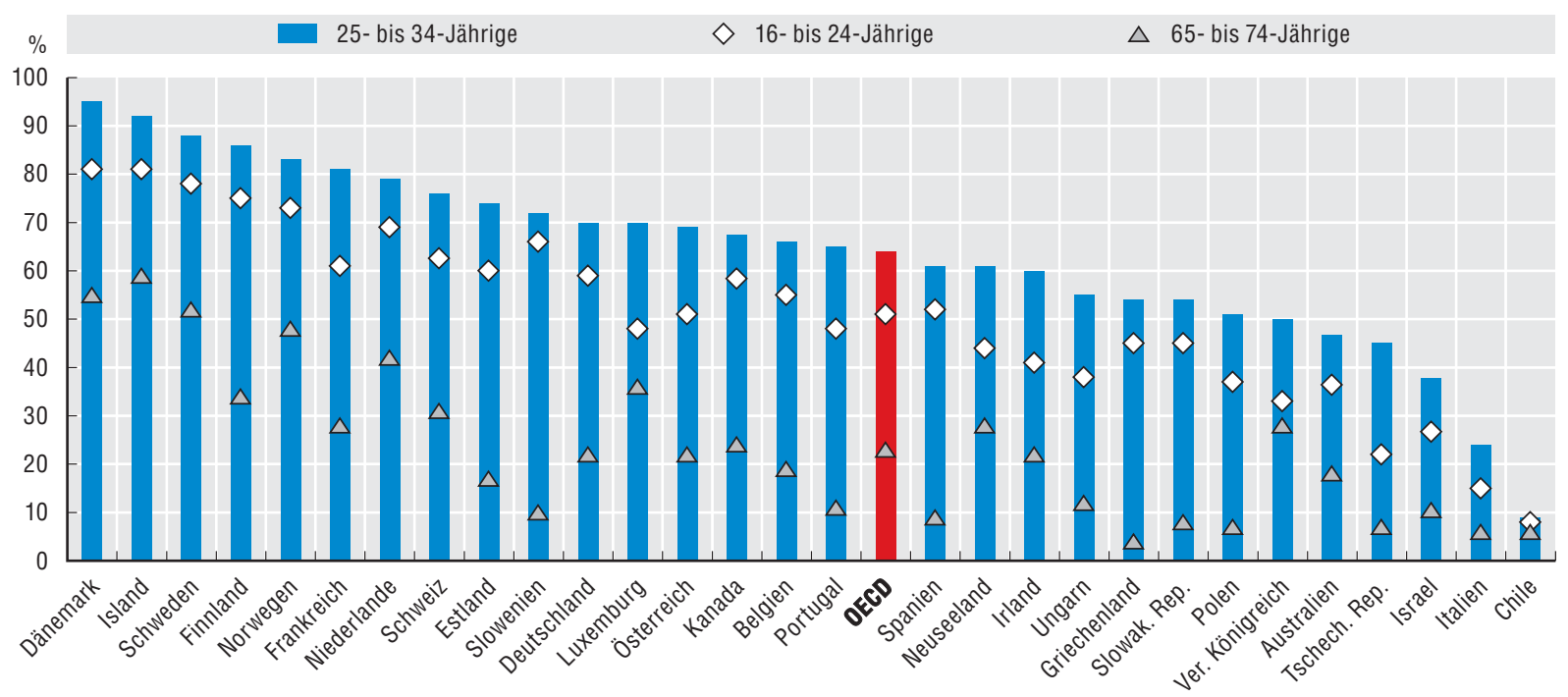

Quelle: OECD, ICT Database; Eurostat, Information Society Statistics (Datenbank). 
Responsive öffentliche Güter und Dienstleistungen zeichnen sich dadurch aus, dass sie die Bedürfnisheterogenität der Bürger explizit anerkennen und sich darauf einstellen. Anstatt lediglich Dienstleistungen in „Einheitsgröße“ anzubieten, setzen responsive Dienstleister Strategien zur Segmentierung ihrer Kundenbasis ein und nutzen Mechanismen, um proaktiv Feedback bzw. Beschwerden von Bürgern einzuholen und zu berücksichtigen. Darüber hinaus sind responsive öffentliche Güter und Dienstleistungen durch das Bestreben um eine bedarfsorientierte Anpassung, möglichst schnelle Reaktionszeiten und die Minimierung von Verzögerungen gekennzeichnet. Dementsprechend ist die Zeitgerechtigkeit der Leistungserbringung ein wichtiger Responsivitätsindikator mit besonders starken Auswirkungen auf das Vertrauen der Bürger darauf, dass die öffentlichen Dienstleistungen ihren Bedürfnissen gerecht werden können.

\section{Gesundheitswesen}

Die Wartezeit ist eine Messgröße für die Zeitgerechtigkeit der Leistungserbringung. Zu lange Wartezeiten können nicht nur die Wahrnehmung der Qualität einer Dienstleistung, sondern auch die erwartete Wirkung der Dienstleistung beeinträchtigen. Beispielsweise kann die Verzögerung einer medizinischen Behandlung bisweilen negative gesundheitliche Auswirkungen haben und in einem akuten Stadium eine vermeidbare Hospitalisierung verursachen. Sie kann darüber hinaus die ArztPatienten-Beziehung und das Vertrauen der Bürger in das Gesundheitssystem beeinträchtigen. Andererseits können Wartezeiten auch dadurch bedingt sein, dass bei Abwesenheit anderer Zuteilungskriterien, wenn die Dienstleistungen völlig kostenlos erbracht werden, der Zeitfaktor bei eingeschränkter Verfügbarkeit der Dienstleistung zu einer Korrekturvariablen werden kann. In den OECD-Mitgliedsländern, für die Daten zur Verfügung stehen, gaben $2010 \mathrm{im}$ Schnitt fast 40\% der an einen Facharzt überwiesenen Bürger an, dass sie mehr als vier Wochen auf den Facharzttermin warten mussten (Abbildung 9.8). Im Ländervergleich bestehen jedoch erhebliche Unterschiede. Der Anteil der Bürger, die mehr als vier Wochen auf einen Termin warten mussten, betrug in Kanada fast $60 \%$ und in Deutschland weniger als $20 \%$.

Der Anteil der Bevölkerung, der mehr als vier Monate auf elektive chirurgische Eingriffe warten musste, war im Schnitt niedriger (rd. 10\%). In Kanada, Schweden, Norwegen und dem Vereinigten Königreich gaben ungefähr 20\% der Bevölkerung an, von langen Wartezeiten betroffen zu sein. In Deutschland musste niemand länger als vier Monate warten. Personalmangel, schlechte Organisation und ein Mangel an Krankenhausbetten zählen zu den Faktoren, die zu langen Wartezeiten für chirurgische Eingriffe beitragen können.

\section{Steuerverwaltung}

Eine Reihe von OECD-Mitgliedsländern zählt das Kriterium der Zeitgerechtigkeit zu den zentralen Leistungsstandards für bestimmte öffentliche Dienstleistungen, insbesondere die Steuerverwaltung. In den Ländern, die Standards für die Bearbeitungsdauer von Steuererklärungen festgelegt haben, betrug die Bearbeitungszeit im Schnitt nicht mehr als 40 Tage für papiergebundene Steuererklärungen und nicht mehr als 35 Tage für elektronische Steuererklärungen (Abbildung 9.9). In zwei Ländern die Niederlande und Polen - wurden Steuererklärungen innerhalb von drei Monaten bearbeitet. In allen anderen Ländern lagen die Zeitvorgaben für die Bearbeitung papiergebundener Steuererklärungen zwischen maximal 10 Tagen in Irland und 42 Tagen in Australien, Dänemark und Japan. In den meisten Ländern war die elektronische Steuererklärung mit keiner wesentlichen Verkürzung der vorgegebenen Standards für die Bearbeitungszeit verbunden; eine Ausnahme stellten lediglich Australien, Irland und Kanada dar. In diesen Ländern profitierten die Bürgerinnen und Bürger von einer drei- bis viermal so schnellen Bearbeitung, wenn sie ihre Steuererklärung nicht in Papierform, sondern elektronisch einreichten.

\section{Justizwesen}

Zeitgerechtigkeit kann auch bei der Beurteilung der Qualität von Justizsystemen eine wichtige Rolle spielen. Verzögerungen können die Fähigkeit von Justizsystemen, die Rechtsstaatlichkeit aufrechtzuerhalten sowie leistungsfähige und gleiche Rahmenbedingungen für die Klärung wirtschaftlicher Streitigkeiten zu schaffen, in schlechtem Licht erscheinen lassen und dadurch das Vertrauen in die Institutionen der Justiz untergraben. Verzögerungen können auch zusätzliche Kosten verursachen, da Rechtssachen länger anhängig sind und die wirtschaftliche Situation ungeklärt bleibt, was sich negativ auf die Investitionsaussichten auswirkt. Allzu kurze Bearbeitungszeiten hingegen können zu Lasten des Erfordernisses eines ordnungsgemäßen Gerichtsverfahrens gehen.

Die Verfahrensdauer ist ein häufig verwendeter Indikator für die Zeitgerechtigkeit im Justizwesen. In den 31 OECD-Mitgliedsländern, für die Daten zur Verfügung stehen, lag die durchschnittliche Dauer für den Abbau des Bestands anhängiger Zivilsachen in erster Instanz zwischen ungefähr 100 Tagen in Japan und mehr als 550 Tagen in Italien, bei einem OECDDurchschnitt von rd. 242 Tagen (Abbildung 9.10). Länder, die dem französischen Rechtssystem folgen, weisen die längste Dauer für den Abbau des Bestands anhängiger Rechtssachen aus. Neben den Unterschieden zwischen einzelnen Rechtssystemen in der Ausgestaltung des formellen und materiellen Rechts kann sich jedoch auch die Organisation des Justizsystems - z.B. hinsichtlich der Personalgewinnung und -führung, der IT-Nutzung und der Kapazitäten zur Bewältigung der Fallzahlen - auf die Zeitdauer auswirken, die für den Abschluss eines Gerichtsverfahrens erforderlich ist. 


\section{Methodik und Definitionen}

Die Daten zu den Wartezeiten für Facharzttermine und elektive chirurgische Eingriffe stammen aus OECD Health Data 2011. Die Wartezeit für Facharzttermine und elektive chirurgische Eingriffe bezeichnet die Zeit, die zwischen der Überweisung des Patienten und dem entsprechenden Facharzt- oder Operationstermin vergeht. Nur Befragte, die Termine bei Fachärzten oder elektive chirurgische Eingriffe in Anspruch genommen hatten, wurden gebeten, ihre Wartezeiten anzugeben.

Die Daten zur Bearbeitungsdauer von Einkommensteuererklärungen stammen aus Tax Administration 2013: Comparative Information on OECD and other Advanced and Emerging Economies. Die Bearbeitungsdauer bezieht sich auf die Zeit zwischen dem Einreichen der Einkommensteuererklärung und der Entscheidung der Steuerbehörde über eine Steuerrückzahlung. Steuererklärungen sind die Formulare, auf denen die Bürger den zuständigen Behörden ihr steuerpflichtiges Einkommen melden. Steuerrückzahlungen beziehen sich auf die Rückerstattung, die die Bürger erhalten, wenn der von ihnen entrichtete Betrag höher ist als ihre Steuerschuld.

Die Justizdaten zur durchschnittlichen Verfahrenslänge erstinstanzlicher Zivilsachen stammen aus dem OECD-Bericht „Judicial Performance and its Determinants: A Cross-Country Perspective“. Die Verfahrenslänge wird mit einer häufig in der Literatur verwendeten Formel geschätzt: [(anhängig ( $-1_{1}+$ anhängig $\left._{t}\right) /\left(n e u_{t}\right.$ + abgeschlossen $\left._{t}\right]^{*} 365$. In Fällen, in denen keine Informationen zur Zahl der anhängigen Rechtssachen zur Verfügung standen, das betreffende Land aber Angaben zur tatsächlichen Verfahrenslänge machen konnte, wurden letztere verwendet (England und Wales, Mexiko, Neuseeland und die Niederlande). Für Länder, für die weder die geschätzte noch die tatsächliche Verfahrenslänge verfügbar war, wurde die Verfahrenslänge durch Imputation des erwarteten Werts aus der Regression der geschätzten Verfahrenslänge gemäß der Weltbank-Datenbank Doing Business berechnet.

\section{Weitere Informationen}

OECD (2013a), Tax Administration 2013: Comparative Information on OECD and other Advanced and Emerging Economies, OECD Publishing, Paris, http://dx.doi.org/10.1787/9789264200814-en.

OECD (2013b, erscheint demnächst), Health at a Glance 2013: OECD Indicators, OECD Publishing, Paris.

Palumbo, G. et al. (2013), „Judicial Performance and its Determinants: A Cross-Country Perspective“, OECD Economic Policy Papers, No. 5, OECD Publishing, Paris, http://dx.doi.org/10.1787/ 5k44x00md5g8-en.

\section{Anmerkungen zu den Abbildungen}

9.9: In der Abbildung sind nur Länder aufgeführt, in denen Leistungsstandards in der Verwaltungspraxis eingesetzt werden. Für die Niederlande konnte die Anzahl der Tage nicht mit hinreichender Näherung geschätzt werden und wurde daher nicht berücksichtigt. Daten für Österreich: Für papiergebundene und elektronische Steuererklärungen gelten die gleichen Standards. Daten für Chile: Für Steuererklärungen, die zwischen 1. und 19. April eingereicht werden, sind Steuerrückzahlungen per Überweisung bis 10. Mai und per Scheck bis 30. Mai fällig; für Steuererklärungen, die zwischen 20. und 27. April eingereicht werden, läuft die Frist für Steuerrückzahlungen per Überweisung bis 17. Mai und per Scheck bis 30. Mai; für Steuererklärungen, die zwischen 28. April und 9. Mai eingereicht werden, ist eine Steuerrückzahlung per Überweisung bis 26. Mai und per Scheck bis 30. Mai zu erwarten. Die Daten für Ungarn beziehen sich auf die von den Steuerbehörden vorgegebenen Standards und nicht die tatsächliche Bearbeitungszeit. Daten für die Vereinigten Staaten: Die angegebenen Standards gelten lediglich für papiergebundene Einkommensteuererklärungen. Es gelten keine separaten Standards für elektronische Steuererklärungen. Für elektronische Steuererklärungen wird angestrebt, Steuerrückzahlungen innerhalb von 5 bis 21 Tagen zu veranlassen. Diese Zielvorgabe wird von der Steuerbehörde der Vereinigten Staaten (Internal Revenue Service - IRS) bei den meisten elektronischen Steuererklärungen erreicht. Die Daten zur papiergebundenen Steuererklärung sind für Estland und Portugal nicht anwendbar.

9.10: Die Daten für das Vereinigte Königreich beziehen sich auf England und Wales.

Hinweis zu den Daten für Israel: http://dx.doi.org/10.1787/888932315602. 


\section{SONDERBEITRAG - IM DIENSTE DER BÜRGER: ZUGÄNGLICHKEIT UND QUALITÄT}

\section{Bürgerorientierung öffentlicher Dienstleistungen: Zeitgerechtigkeit}

\subsection{Wartezeiten für Facharzttermine und elektive chirurgische Eingriffe (2010)}

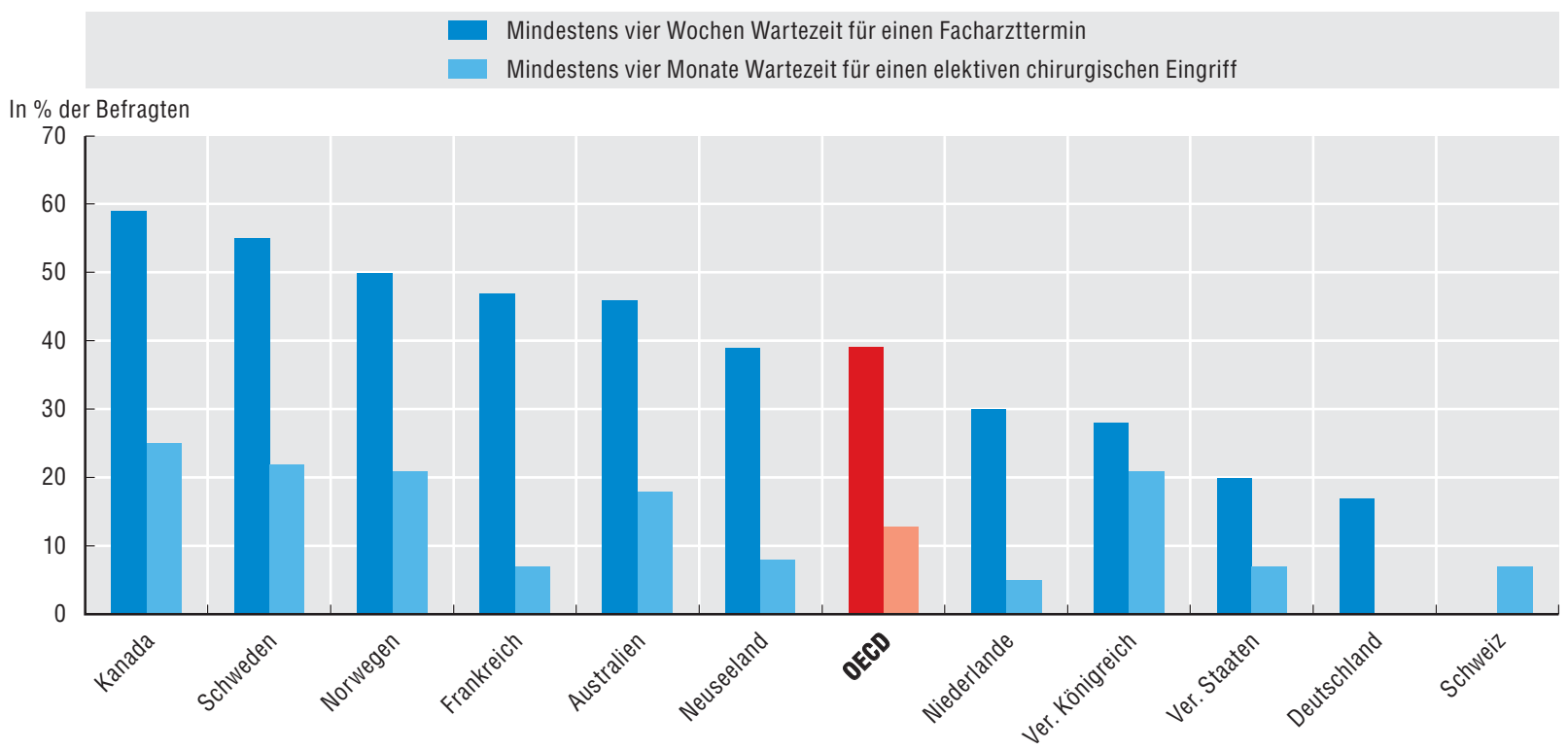

Quelle: Commonwealth Fund International Health Policy Survey 2010.

9.9. Bearbeitungszeit für Einkommensteuererklärungen, bei denen eine Steuerrückzahlung zu erwarten ist (2011) Durchschnittliche Dauer in Tagen für mindestens $80 \%$ der Steuererklärungen

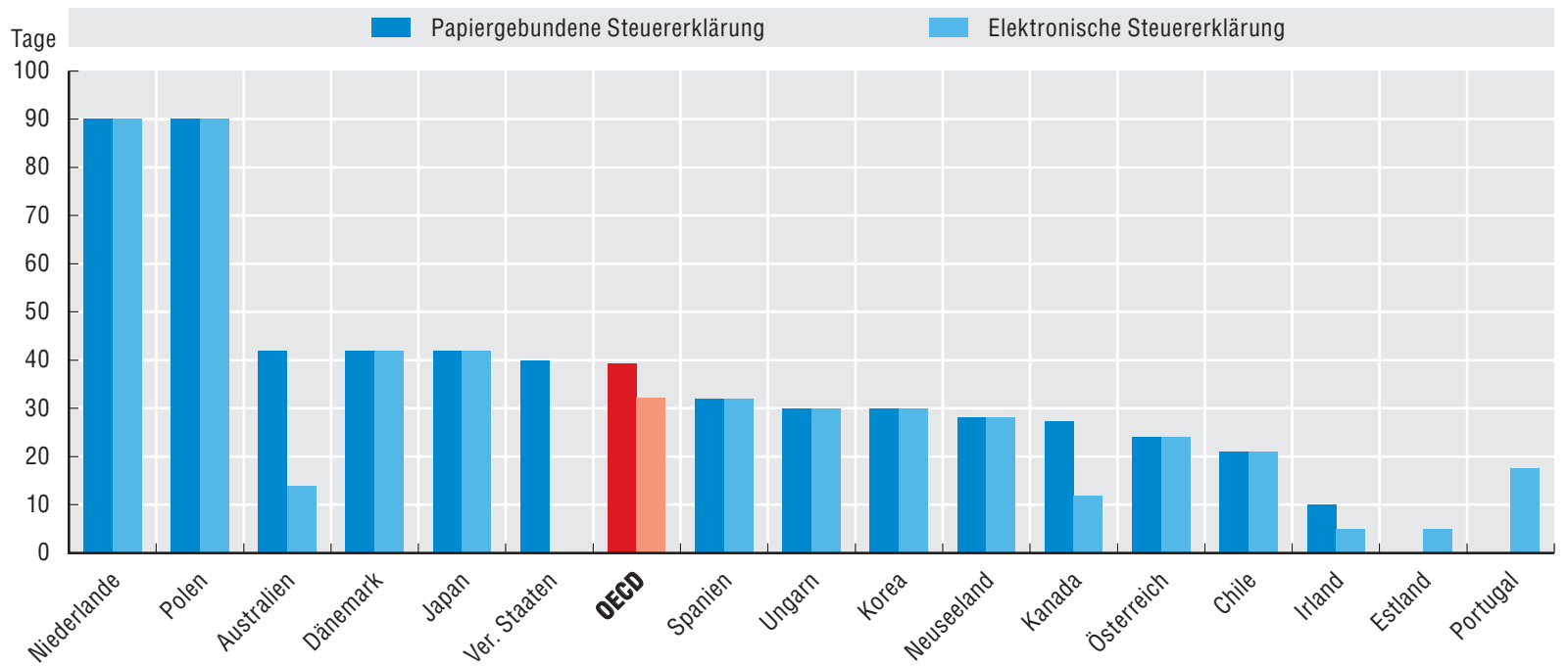

Quelle: OECD (2013), Tax Administration 2013: Comparative Information on OECD and other Advanced and Emerging Economies, OECD Publishing, Paris, http://dx.doi.org/10.1787/9789264200814-en. 


\section{SONDERBEITRAG - IM DIENSTE DER BÜRGER: ZUGÄNGLICHKEIT UND QUALITÄT}

Bürgerorientierung öffentlicher Dienstleistungen: Zeitgerechtigkeit

9.10. Verfahrensdauer erstinstanzlicher Rechtssachen in Tagen (2012)

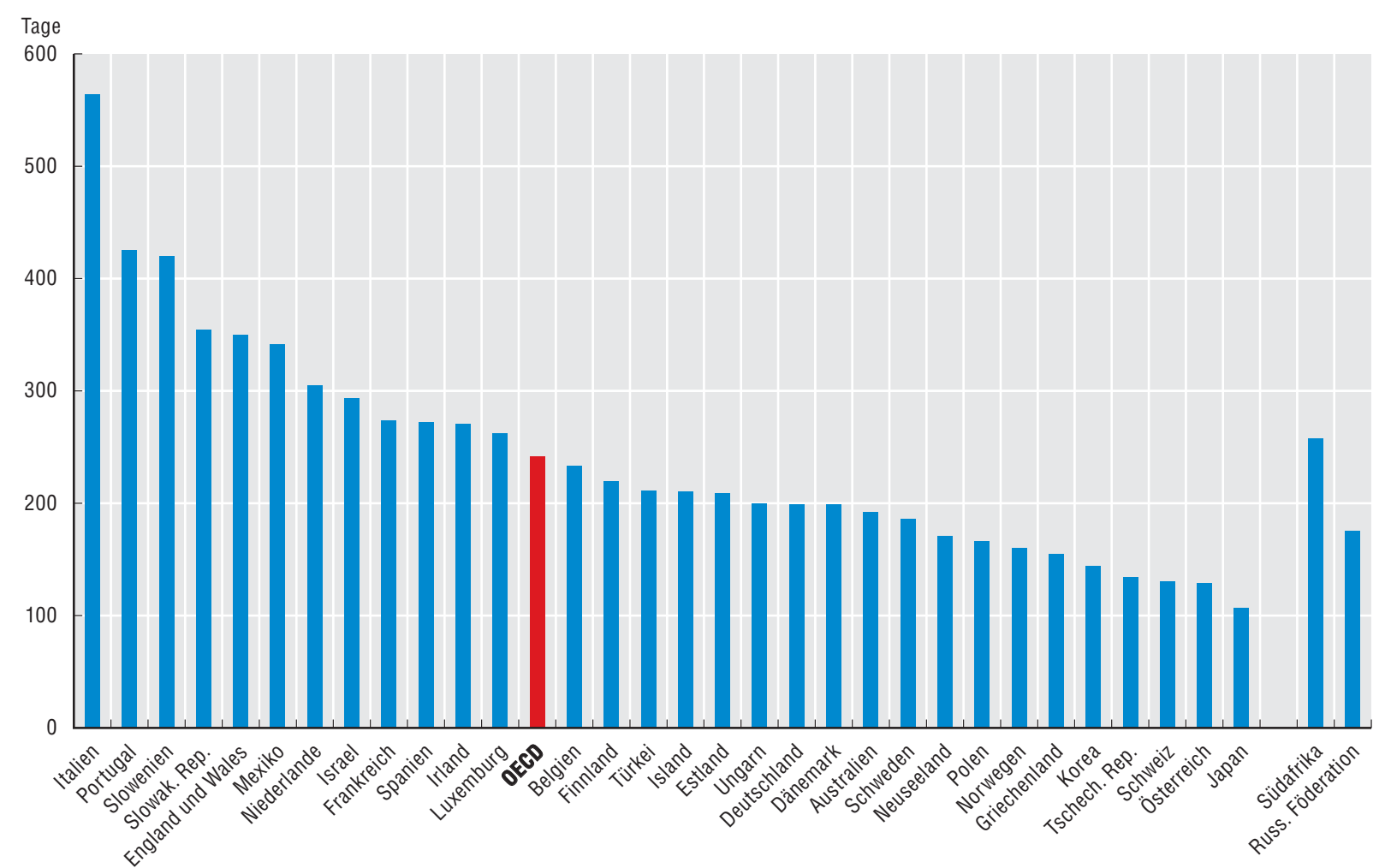

Quelle: OECD, CEPEJ, Weltbank sowie Palumbo, G. et al. (2013), „Judicial Performance and its Determinants: A Cross-Country Perspective“, OECD Economic Policy Papers, No. 5, OECD Publishing, Paris, http://dx.doi.org/10.1787/5k44x00md5g8-en. 
In jeder demokratischen Gesellschaftsordnung haben die Bürgerinnen und Bürger eine Reihe grundlegender Rechte und Verpflichtungen gegenüber dem Staat und seinen Organen. Die Messung der Nutzerzufriedenheit mit Gütern und Dienstleistungen des öffentlichen Sektors gilt als wichtiger Indikator für die Evaluierung der Leistungserbringung in den OECD-Ländern. Allerdings gibt es bisher wenig konsensuell entwickelte Messgrößen, mit denen ermittelt werden kann, inwiefern der institutionelle Rahmen der einzelnen Staaten darauf ausgelegt ist, den Rechten der Bürger Gehör und Anerkennung zu verschaffen. Ein solcher Rahmen trägt dazu bei, die Verlässlichkeit öffentlicher Dienstleistungen zu gewährleisten, indem die Bürgerinnen und Bürger über ihre Rechte informiert werden und Kanäle für Rechtsbehelfe und Qualitätssicherung zur Verfügung haben. In einer Charta, in der die Rechte der Bürger beschrieben sind, könnten auch grundlegende Dienstleistungs- und Verfahrensstandards definiert werden, wie es beispielsweise bei der Citizen's Charter im Vereinigten Königreich der Fall war.

Angesichts des schwindenden Vertrauens in Staat und Regierung ist es mittlerweile noch wichtiger geworden, die Anerkennung der Rechte von Bürgerinnen und Bürgern zu unter suchen. Nur wenige Länder verfügen über eine allgemeine Definition der Rechte von Patienten oder Steuerzahlern, ganz zu schweigen von einem standardisierten Regelungsrahmen für Beschwerdeverfahren.

\section{Steuerverwaltung}

Im Bereich der Steuerverwaltung schaffen die verschiedenen nationalen Steuerpolitiken unterschiedliche Rahmenbedingungen für die Tätigkeit der Finanzbehörden der einzelnen Länder. Dementsprechend sind von Land zu Land gewisse Unterschiede bei der detaillierten Ausgestaltung der Rechte der Steuerzahler festzustellen. Dennoch lassen sich mehrere gemeinsame Trends erkennen und analysieren. Ein zu Beginn des vergangenen Jahrzehnts veröffentlichtes Dokument der Arbeitsgruppe Nr. 8 des OECD-Ausschusses für Steuerfragen zeigte, dass zwar die meisten Länder zu diesem Zeitpunkt nicht über eine explizite „Steuerzahlercharta“ verfügten, dass aber dennoch die folgenden grundlegenden Rechte der Steuerzahler in allen Systemen verankert waren: „das Recht auf Information, Unterstützung und Anhörung; das Recht auf Einspruch; das Recht, nicht mehr als den ordnungsgemäßen Steuerbetrag zu zahlen; das Recht auf Verlässlichkeit; das Recht auf Schutz der Privatsphäre; sowie das Recht auf Vertraulichkeit und Wahrung des Steuergeheimnisses".

In vielen OECD-Mitgliedsländern wurde in den letzten Jahren eine Steuerzahler- oder Dienstleistungscharta erarbeitet, um diese grundlegenden Rechte zu formalisieren. Unter einer Steuerzahler- oder Dienstleistungscharta sind Dokumente zu verstehen, die Aussagen dazu enthalten, welches Verhalten von Staatsbediensteten einerseits und Steuerzahlern andererseits erwartet wird. Die genaue Beschaffenheit solcher Chartas kann in den einzelnen OECD-Ländern unterschiedliche Formen annehmen. In einigen Fällen werden regulatorische Maßnahmen, die zum Schutz der Steuerzahler ergriffen wurden, zu einer „Steuerzahlercharta“ zusammengefasst, die später allgemein veröffentlicht wird, es sind jedoch auch andere Szenarien möglich. Daher sollte bei internationalen Vergleichen berücksichtigt werden, dass Länder ohne Steuerzahlercharta den Rechten der Steuerzahler möglicherweise ebenso große Bedeutung beimessen wie Länder mit formeller Steuerzahlercharta.
Wie aus Tabelle $9.12 \mathrm{zu}$ ersehen, verfügten 2012 fast alle Finanzbehörden mit Zuständigkeit für Steuerangelegenheiten über eine formalisierte Dokumentation der Rechte der Steuerzahler in Form von gesetzlichen und/oder administrativen Regelungen. Von den untersuchten 33 OECD-Mitgliedsländern haben 30 diese Rechte (teilweise oder vollständig) im Steuergesetz oder anderen Gesetzen kodifiziert; die einzigen Ausnahmen bilden Irland, Neuseeland und die Türkei. 26 Finanzbehörden arbeiten mit einem Katalog von Rechten und Verpflichtungen, die in administrativen Dokumenten (manchmal als „Steuerzahler-“ oder „Dienstleistungscharta“ bezeichnet) beschrieben sind. Neben kulturellen und rechtlichen Fragestellungen scheinen verschiedene weitere Faktoren die Entscheidung der Länder für einen kodifizierten oder einen administrativen Ansatz zu beeinflussen. Für einen administrativen Ansatz werden u.a. folgende Gründe angeführt: schneller Umsetzungsprozess, „leserfreundlichere“ Sprache, breiteres Anwendungsspektrum (darunter beispielsweise umfassendere Rechte, die in einem Gesetzgebungsprozess nicht berücksichtigt werden könnten), größere Flexibilität und einfache Rechtsbehelfsmöglichkeiten. Die Vorteile eines kodifizierten Ansatzes beruhen dagegen auf folgenden Erwägungen: positiverer Eindruck auf die Steuerzahler auf Grund der stärkeren Verpflichtungen, die für die Finanzbehörden damit verbunden sind; rasche Einhaltung der neuen Regelungen durch die Bediensteten; Beständigkeit des Dokuments gegenüber Veränderungen auf Grund unterschiedlicher politischer Interessen (Langlebigkeit) sowie etablierte Rechtsbehelfs- und Anfechtungsmechanismen.

\section{Gesundheitswesen}

Im Gesundheitssektor gelten die Einflussmöglichkeiten von Patienten und Patientenrechte - wie z.B. Klagemöglichkeiten als grundlegende Standards, an denen die Nutzerorientierung bei der Erbringung öffentlicher Dienstleistungen gemessen wird. Bei der Analyse wurden fünf Aspekte untersucht: Existiert eine formelle Definition der Patientenrechte bzw. Patientencharta auf nationaler Ebene? Können Patienten bei Behandlungsfehlern gerichtliche Rechtsbehelfe in Anspruch nehmen? Sind Krankenhäuser verpflichtet, eine Beschwerdestelle für die Entgegennahme und Klärung von Patientenbeschwerden zu unterhalten? Existiert eine Ombudsstelle, die für die Untersuchung und Klärung von Beschwerden über Gesundheitsdienstleistungen zuständig ist? Sind Sammelklagen gegen Gesundheitsdienstleister und Pharmakonzerne zulässig?

Die überwiegende Mehrheit der OECD-Länder gab an, dass eine formelle Definition der Patientenrechte auf nationaler Ebene existiert (26 von 33 Ländern). Lediglich Israel, Kanada, Luxemburg, Schweden und die Schweiz gaben an, nicht über derartige Regelungen zu verfügen. In allen Ländern außer Finnland, Island, Neuseeland und der Slowakischen Republik können Patienten bei Behandlungsfehlern gerichtliche Rechtsbehelfe in Anspruch nehmen. In einer Mehrzahl der Länder (19) sind Krankenhäuser verpflichtet, eine Beschwerdestelle zur Entgegennahme von Patientenbeschwerden zu haben. Ein Großteil der OECD-Länder gab an, über Ombudsstellen zu verfügen, die für die Untersuchung und Klärung von Patientenbeschwerden über Gesundheitsdienstleistungen zuständig sind. Lediglich Dänemark, Japan, Korea, die Niederlande und die Türkei haben keine derartigen Mediationsbeauftragten. 


\section{Methodik und Definitionen}

Die Daten zu den Rechten der Steuerzahler stammen aus Tax Administration 2013: Comparative Information on OECD and other Advanced and Emerging Economies (OECD, 2013). Die Informationen wurden durch zwei Erhebungen erfasst, die in den OECD-Mitgliedsländern und anderen bedeutenden Volkswirtschaften durchgeführt wurden: der IBFD Tax Survey und der CIS Survey. Unter einer Steuerzahler- oder Dienstleistungscharta sind Dokumente zu verstehen, die beschreiben, welches Verhalten von Staatsbediensteten einerseits und von Steuerzahlern andererseits erwartet wird.

Die Daten zu den Patientenrechten stammen aus „Health Systems Institutional Characteristics: A Survey of 29 OECD Countries" (Paris et al., 2010). Die Informationen wurden durch den OECD Survey on Health System Characteristics (2008-09 und 2012) erhoben. Dem Survey aus dem Jahr 2012 wurden folgende Informationen entnommen: Frage 90: „Existiert eine formelle Definition der Patientenrechte auf nationaler Ebene (z.B. durch ein Gesetz oder eine Charta)?" Aus dem Survey 2008-09 wurden folgende Fragen verwendet: Frage 76: „Sind Krankenhäuser verpflichtet, eine Beschwerdestelle zu unterhalten, die Patientenbeschwerden entgegennimmt und klärt?" Frage 78: „Was für eine Art von Schadenersatzsystem gilt in dem Land?" Frage 80: „Sind Sammelklagen gegen Gesundheitsdienstleister, Pharmakonzerne usw. zulässig?“ Frage 81: „Gibt es Ombudsleute, die dafür zuständig sind, Patientenbeschwerden über Gesundheitsdienstleistungen zu untersuchen und zu klären?" Eine Sammelklage kann als Klage definiert werden, die von einer oder mehreren Personen für sie selbst und eine wesentlich größere Gruppe von Personen, die alle denselben Klagegrund haben, eingereicht wird.

\section{Weitere Informationen}

OECD (2013), Tax Administration 2013: Comparative Information on OECD and other Advanced and Emerging Economies, OECD Publishing, Paris, http://dx.doi.org/10.1787/9789264200814-en.

Paris, V., M. Devaux und L. Wei (2010), „Health Systems Institutional Characteristics: A Survey of 29 OECD Countries", OECD Health Working Papers, No. 50, OECD Publishing, Paris, http:// dx.doi.org/10.1787/5kmfxfq9qbnr-en.

\section{Anmerkungen zu den Abbildungen}

9.12: Kanada: Die Taxpayer Bill of Rights beschreibt 15 Rechte, die sich aus gesetzlich verankerten Rechten einerseits und Leistungsansprüchen andererseits zusammensetzen. Gesetzlich verankerte Rechte sind in der Steuergesetzgebung kodifiziert und beinhalten im Allgemeinen das Recht auf Inanspruchnahme von Rechtsbehelfen. Die Leistungsansprüche regeln das Verhältnis zwischen den Finanzbehörden und den Steuerzahlern und umfassen einen behördlichen Rechtsbehelfsprozess. Als erster Schritt in einem solchen Beschwerdeprozess wird zunächst versucht, die Angelegenheit mit dem für den Fall zuständigen Bediensteten der Behörde zu klären; wenn der Steuerzahler damit nicht zufrieden ist, kann er über das Beschwerdeverfahren (Service Complaints Program) eine formelle Beschwerde einreichen; wenn die Angelegenheit auch dadurch nicht zu seiner Zufriedenheit geregelt wird, kann er eine Beschwerde an die zuständige Ombudsstelle (Taxpayers' Ombudsman) richten, die eine unparteiische und unabhängige Überprüfung vornimmt. Chile: Im Februar 2010 wurde das Steuerrecht durch einen neuen Paragrafen ergänzt, der den Mindestumfang der gesetzlich anerkannten und garantierten Rechte der Steuerzahler festlegt und beschreibt. Irland: Es gibt zwar ein internes Verfahren zur Abwicklung von Beschwerden, die Steuerzahler können jedoch auch Rechtsbehelfe nach gesetzlichen Bestimmungen über verschiedene Schiedsstellen (Appeals Commissioners, Ombudsman's Office, Equality Tribunal) in Anspruch nehmen. Luxemburg: Die in administrativen Dokumenten formell definierten Rechte der Steuerzahler betreffen nur direkte Steuern.

Hinweis zu den Daten für Israel: http://dx.doi.org/10.1787/888932315602. 
9. SONDERBEITRAG - IM DIENSTE DER BÜRGER: ZUGÄNGLICHKEIT UND QUALITÄT

Verlässlichkeit öffentlicher Dienstleistungen: Sicherung der Rechte der Bürger

9.11. Rechte der Bürger in den nationalen Gesundheitssystemen (2009-2012)

\begin{tabular}{|c|c|c|c|c|c|}
\hline & $\begin{array}{c}\text { Formelle Definition } \\
\text { oder Charta } \\
\text { der Patientenrechte } \\
\text { auf nationaler Ebene (2012) }\end{array}$ & $\begin{array}{c}\text { Patienten können } \\
\text { bei Behandlungsfehlern } \\
\text { gerichtliche Rechtsbehelfe } \\
\text { in Anspruch nehmen (2009) }\end{array}$ & $\begin{array}{l}\text { Krankenhäuser müssen } \\
\text { eine Beschwerdestelle } \\
\text { zur Entgegennahme } \\
\text { und Klärung von } \\
\text { Patientenbeschwerden } \\
\text { haben (2009) }\end{array}$ & $\begin{array}{l}\text { Ombudsstelle mit } \\
\text { Zuständigkeit für die } \\
\text { Untersuchung und Klärung } \\
\text { von Beschwerden über } \\
\text { Gesundheitsdienstleistungen } \\
\text { (2009) }\end{array}$ & $\begin{array}{l}\text { Sammelklagen gegen } \\
\text { Gesundheitsdienstleister } \\
\text { und Pharmakonzerne } \\
\text { zulässig (2009) }\end{array}$ \\
\hline Australien & - & ○ & $\bullet$ & $\bullet$ & $\bullet$ \\
\hline Österreich & $\bullet$ & $\bullet$ & $\bigcirc$ & ○ & ○ \\
\hline Belgien & $\bullet$ & ○ & 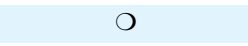 & $\bullet$ & $\bullet$ \\
\hline Kanada & O & - & $\bigcirc$ & - & - \\
\hline Chile & $\bullet$ &.. & .. & .. & .. \\
\hline Tschech. Rep. & - & • & • & .. & - \\
\hline Dänemark & - & - & $\bullet$ & O & ○ \\
\hline Finnland & $\bullet$ & O & $\bullet$ & - & O \\
\hline Frankreich & - & - & - & 0 & O \\
\hline Deutschland & - & - & $\bigcirc$ & - & .. \\
\hline Griechenland & $\bullet$ & $\bullet$ & .. & $\bullet$ & .. \\
\hline Ungarn & - & - & - & - & .. \\
\hline Island & ○ & O & $\bullet$ & $\bullet$ & O \\
\hline Irland & - & - & - & - & O \\
\hline Israel & O & .. & .. & .. & .. \\
\hline Italien & - & - & - & .. & O \\
\hline Japan & $\bullet$ & $\bullet$ & $\bullet$ & O & $\bullet$ \\
\hline Korea & ○ & - & - & O & O \\
\hline Luxemburg & O & ○ & $\bullet$ & • & O \\
\hline Mexiko & - & 0 & - & $\bullet$ & O \\
\hline Niederlande & - & $\bullet$ & $\bullet$ & O & .. \\
\hline Neuseeland & - & O & - & $\bullet$ & O \\
\hline Norwegen & $\bullet$ & $\bullet$ & $\bigcirc$ & $\bullet$ & $\bullet$ \\
\hline Polen & $\bullet$ & 0 & $\mathrm{O}$ & $\bullet$ & $\bullet$ \\
\hline Portugal & - & $\bullet$ & $\bullet$ & $\bullet$ & $\bullet$ \\
\hline Slowak. Rep. & .. & O & .. & .. & .. \\
\hline Slowenien & $\bullet$ & .. & .. & .. & .. \\
\hline Spanien & $\bullet$ & 0 & $\bullet$ & $\bullet$ & $\bullet$ \\
\hline Schweden & O & $\bullet$ & O & $\bullet$ & - \\
\hline Schweiz & O & ○ & $\bigcirc$ & $\bullet$ & O \\
\hline Türkei & .. & $\bullet$ & 0 & O & $\bullet$ \\
\hline Ver. Königreich & 0 & $\bullet$ & $\bullet$ & $\bullet$ & $\bullet$ \\
\hline Ver. Staaten & $\bullet$ & .. & .. & .. & .. \\
\hline OECD insgesamt & 26 & 25 & 19 & 21 & 14 \\
\hline
\end{tabular}

\section{$\mathrm{Ja}$}

Nein

Quelle: Paris, V., M. Devaux und L. Wei (2010), „Health Systems Institutional Characteristics: A Survey of 29 OECD Countries“, OECD Health Working Papers, No. 50, OECD Publishing, Paris, http://dx.doi.org/10.1787/5kmfxfq9qbnr-en; 
9. SONDERBEITRAG - IM DIENSTE DER BÜRGER: ZUGÄNGLICHKEIT UND QUALITÄT

Verlässlichkeit öffentlicher Dienstleistungen: Sicherung der Rechte der Bürger

9.12. Rechte der Bürger in der Steuerverwaltung (2013)

\begin{tabular}{|c|c|c|}
\hline & $\begin{array}{c}\text { Rechte der Steuerzahler sind formell } \\
\text { in der Steuergesetzgebung oder anderen } \\
\text { Gesetzen definiert }\end{array}$ & $\begin{array}{l}\text { Rechte der Steuerzahler sind formell } \\
\text { in administrativen Dokumenten definiert }\end{array}$ \\
\hline Australien & $\bullet$ & $\bullet$ \\
\hline Österreich & - & - \\
\hline Belgien & ○ & $\bullet$ \\
\hline Kanada & - & - \\
\hline Chile & - & - \\
\hline Tschech. Rep. & ○ & $O$ \\
\hline Dänemark & 0 & - \\
\hline Finnland & $\bullet$ & - \\
\hline Frankreich & $\bullet$ & $\bullet$ \\
\hline Deutschland & - & O \\
\hline Griechenland & $\bullet$ & - \\
\hline Ungarn & - & - \\
\hline Island & - & 0 \\
\hline Irland & $\bigcirc$ & - \\
\hline Israel & $\bullet$ & $\bullet$ \\
\hline Italien & - & - \\
\hline Japan & $\bullet$ & $O$ \\
\hline Korea & $\bullet$ & $\bullet$ \\
\hline Luxemburg & $\bullet$ & $\bullet$ \\
\hline Mexiko & $\bullet$ & $\bullet$ \\
\hline Niederlande & $\bullet$ & $\bullet$ \\
\hline Neuseeland & $\bigcirc$ & - \\
\hline Norwegen & $\bullet$ & - \\
\hline Polen & - & $\bigcirc$ \\
\hline Portugal & $\bullet$ & $\bullet$ \\
\hline Slowak. Rep. & $\bullet$ & $\bigcirc$ \\
\hline Slowenien & - & - \\
\hline Spanien & $\bullet$ & - \\
\hline Schweden & $\bullet$ & $\bullet$ \\
\hline Schweiz & - & $\mathrm{O}$ \\
\hline Türkei & O & $\bullet$ \\
\hline Ver. Königreich & $\bullet$ & - \\
\hline Ver. Staaten & ○ & $\bullet$ \\
\hline OECD insgesamt & 30 & 26 \\
\hline
\end{tabular}

$\mathrm{Ja}$

Nein

Quelle: OECD (2013), Tax Administration 2013: Comparative Information on OECD and other Advanced and Emerging Economies, OECD Publishing, Paris, http://dx.doi.org/10.1787/9789264200814-en. 
Die Messung der Nutzerzufriedenheit mit öffentlichen Gütern und Dienstleistungen ist das Kernstück eines bürgerorientierten Ansatzes bei der Leistungserbringung und eine wichtige Komponente organisatorischer Erfolgsstrategien zur kontinuierlichen Verbesserung. Üblicherweise werden Daten aus Meinungsumfragen herangezogen, um die Erfahrungen der Bürger beim Umgang mit staatlichen Stellen zu evaluieren und ihre Meinung zu den erhaltenen Leistungen einzuholen. Diese Informationen liefern Verwaltungsmanagern Erkenntnisse darüber, welche Elemente der Leistungserbringung die Zufriedenheit steigern, und helfen ihnen dabei, die Auswirkungen von Reformen auf die Endnutzer zu überwachen. Außerdem wird es den politischen Entscheidungsträgern und Verwaltungsmanagern durch die Messung der Bürgerzufriedenheit ermöglicht, die Nutzer der öffentlichen Dienstleistungen besser zu verstehen und Untergruppen von Nutzern sowie Bedürfnisse oder Zugänglichkeitsdefizite zu identifizieren. Darüber hinaus kann die Zufriedenheit der Bürger ein wichtiger Outcome-Indikator für die Gesamtleistung des Staats sein.

Immer mehr staatliche Stellen sind bestrebt, proaktiv Feedback von Bürgern einzuholen und darauf zu reagieren, um so die Bürgerorientierung und Qualität öffentlicher Dienstleistungen zu verbessern. Das in Kanada genutzte „gemeinsame Messinstrument" sowie die in Italien und Frankreich verwendeten "Qualitätsbarometer" beispielsweise erfassen Meinungsdaten von Bürgern und sind dafür konzipiert, verschiedenen staatlichen Stellen die Messung und Überwachung der Qualität öffentlicher Dienstleistungen im Zeitverlauf zu ermöglichen. Frankreich hat ein Befragungspanel eingerichtet, das kontinuierliches Feedback zu den erbrachten Dienstleistungen, insbesondere im Zusammenhang mit wichtigen Lebensereignissen, liefert.

Im Allgemeinen erfreuen sich die öffentlichen Dienstleistungen in den OECD-Mitgliedsländern bei der Mehrheit der Bevölkerung einer hohen Wertschätzung. Beispielsweise gaben 2012 durchschnittlich $72 \%$ der Bürger in den OECD-Mitgliedsländern an, Vertrauen in die örtliche Polizei zu haben. Ein fast ebenso hoher Prozentsatz äußerte Zufriedenheit mit der Verfügbarkeit guter Gesundheitsversorgung, während sich $66 \%$ mit dem Bildungssystem und den Schulen in ihrer Stadt oder Region zufrieden zeigten.

Im Schnitt blieben die Zufriedenheitswerte während und unmittelbar nach der wirtschaftlichen und sozialen Krise relativ konstant. Zwischen 2007 und 2012 steigerte sich die Zufriedenheit der Bürger in allen drei hier analysierten Bereichen nur geringfügig - um weniger als 2 Prozentpunkte. Dabei sind jedoch große Unterschiede zwischen den einzelnen Ländern festzustellen. Das Vertrauen in die örtliche Polizei stieg in weniger OECDLändern (15) als die Zufriedenheit mit dem Bildungs- (19) und dem Gesundheitswesen (19 Länder). Am stärksten nahm das Vertrauen in die örtliche Polizei in Estland (+14 Prozentpunkte) und in der Slowakischen Republik (+13 Prozentpunkte) zu, während es in Mexiko (-15 Prozentpunkte) und Ungarn (-7 Prozentpunkte) deutlich zurückging. Die Zufriedenheit mit dem Bildungssystem verzeichnete in Israel und im Vereinigten Königreich den größten Anstieg (jeweils um 9 Prozentpunkte), während Chile und Mexiko den stärksten Rückgang (um 17 bzw. 11 Prozentpunkte) auswiesen. Bei der Zufriedenheit mit der Gesundheitsversorgung bewegten sich die Veränderungen in allen außer neun Ländern innerhalb einer Spanne von 5 Prozentpunkten. Die deutlichste Steigerung wurde in Ungarn (10 Prozentpunkte) und im Vereinigten Königreich (8 Prozentpunkte), der stärkste Rückgang in Griechenland (23 Prozentpunkte) und Japan (10 Prozentpunkte) festgestellt.
Insgesamt gesehen ist die Zufriedenheit der Bürgerinnen und Bürger mit öffentlichen Dienstleistungen größer als ihr Vertrauen in die Regierung. Im OECD-Durchschnitt waren 2012 sowohl das Vertrauen in die örtliche Polizei als auch die Zufriedenheit mit der Gesundheitsversorgung um 30 Prozentpunkte höher als das Vertrauen in die Regierung, während die Zufriedenheit mit dem Bildungssystem um 26 Prozentpunkte höher war.

\section{Methodik und Definitionen}

Die Daten stammen aus der Gallup-Umfrage (Gallup World Poll), die in rd. 140 Ländern weltweit auf der Basis eines einheitlichen, in die wichtigsten Sprachen jedes Landes übersetzten Fragebogens durchgeführt wird. Von wenigen Ausnahmen abgesehen, sind alle Stichproben zufallsbasiert und national repräsentativ für die Wohnbevölkerung des gesamten Landes (einschließlich ländlicher Gebiete) ab 15 Jahren. Die Ergebnisse können jedoch durch Stichprobenfehler und sonstige Fehler beeinflusst werden. Der Stichprobenumfang liegt bei mindestens 1000 Einzelpersonen pro Land. Vgl. Kapitel 1 wegen einer umfassenderen Erläuterung zur Messung des Vertrauens in den Staat.

Die Daten zum Vertrauen in die örtliche Polizei beziehen sich auf den Prozentsatz der Befragten, die mit "Ja“ auf die folgende Frage antworteten: „Haben Sie Vertrauen in die Polizei Ihres Ortes/Ihrer Umgebung?“

Die Daten zur Zufriedenheit mit dem Bildungssystem und den Schulen beziehen sich auf den Prozentsatz der Befragten, die mit „Zufrieden“ auf die folgende Frage antworteten: „In der Stadt oder der Region, in der Sie leben, sind Sie zufrieden oder nicht zufrieden mit dem Bildungssystem oder den Schulen?"

Die Daten zur Zufriedenheit mit der Verfügbarkeit von guter Gesundheitsversorgung beziehen sich auf den Prozentsatz der Befragten, die mit „Zufrieden“ auf die folgende Frage antworteten: „In der Stadt oder der Region, in der Sie leben, sind Sie zufrieden oder nicht zufrieden mit der Verfügbarkeit von guter Gesundheitsversorgung?“

Abbildung 9.14, „Levels of satisfaction and confidence across selected public services compared to confidence in national government (2012)" ist online verfügbar unter http://dx.doi.org/10.1787/888932943153.

\section{Weitere Informationen}

OECD (2012), Measuring Regulatory Performance: A Practitioner's Guide to Perception Surveys, OECD Publishing, Paris, http:// dx.doi.org/10.1787/9789264167179-en.

\section{Anmerkungen zu den Abbildungen}

Die Daten für Finnland, Frankreich, Irland, Österreich, Portugal, die Schweiz, die Slowakische Republik und Slowenien beziehen sich auf 2006 anstelle von 2007. Die Daten für Chile, Deutschland und das Vereinigte Königreich beziehen sich auf 2011 anstelle von 2012. Die Daten für Japan, Korea und Mexiko zum Vertrauen in die örtliche Polizei und zur Zufriedenheit mit der Gesundheitsversorgung beziehen sich auf 2011 anstelle von 2012.

Hinweis zu den Daten für Israel: $h t t p: / / d x . d o i . o r g / 10.1787 / 888932315602$. 


\section{SONDERBEITRAG - IM DIENSTE DER BÜRGER: ZUGÄNGLICHKEIT UND QUALITÄT}

Zufriedenheit der Bürger mit öffentlichen Dienstleistungen

9.13. Zufriedenheits- bzw. Vertrauenswerte für verschiedene öffentliche Dienstleistungen (2007 und 2012)

2012

$\diamond 2007$
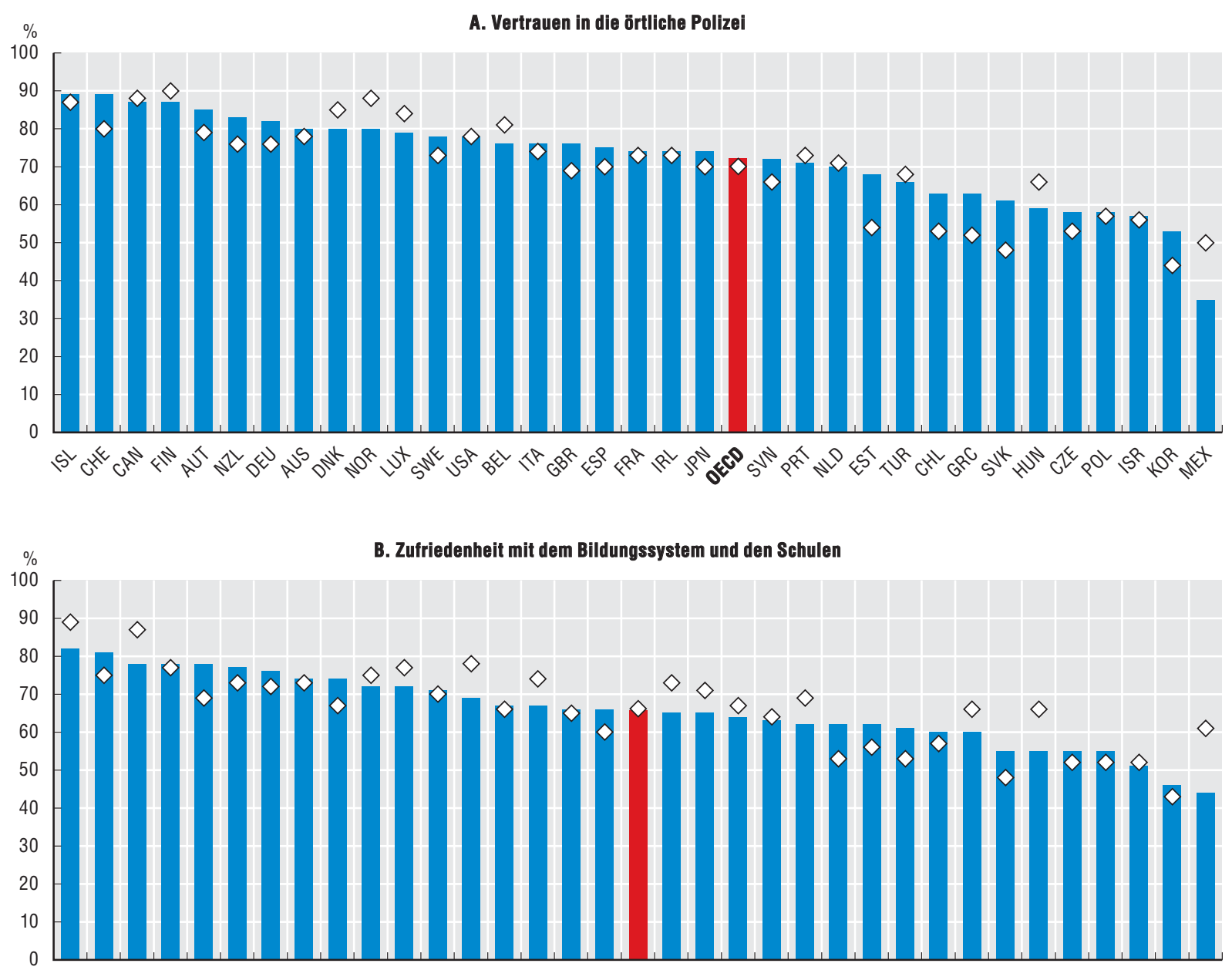

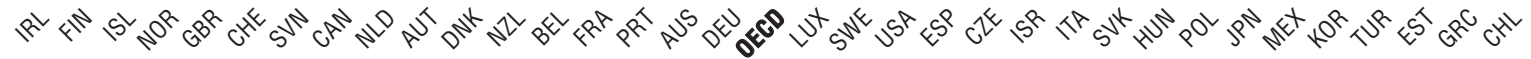

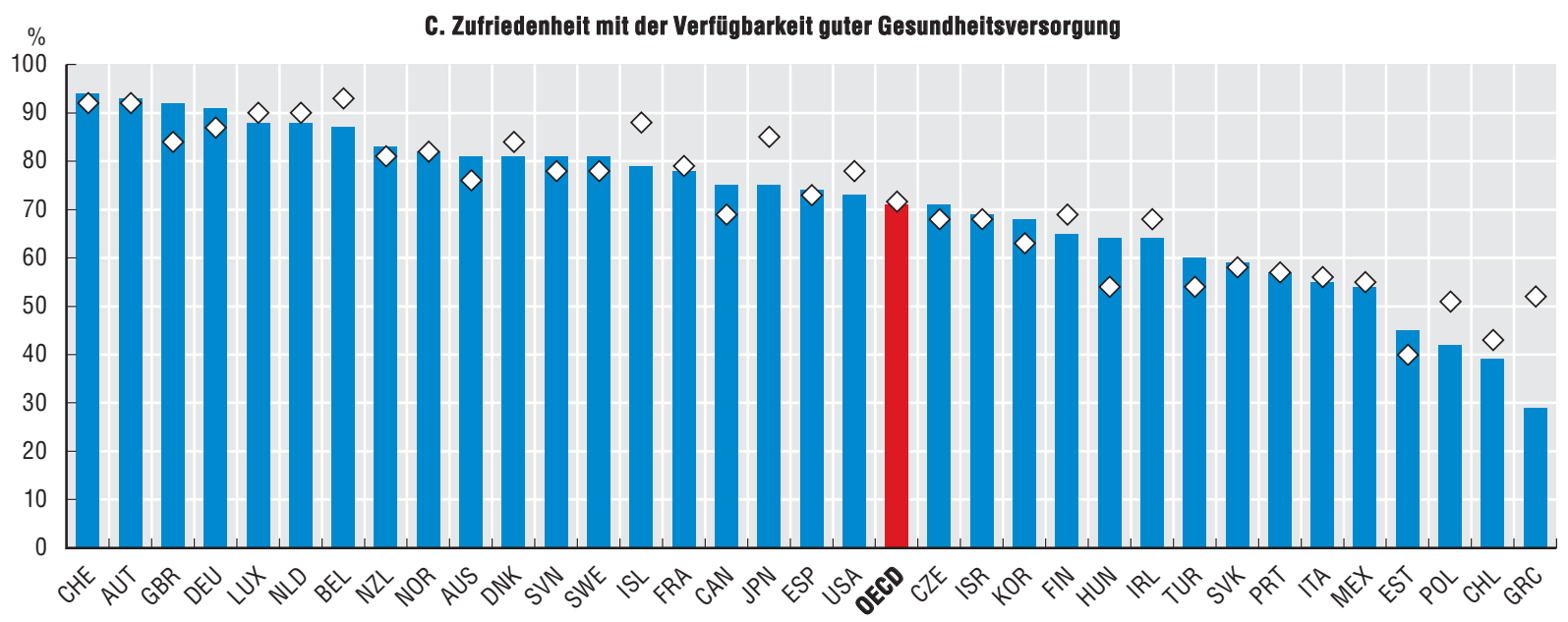

Quelle: Gallup World Poll. 

ANHANG A

\section{Methodik bezüglich der Einnahmeaggregate}

Die nachstehende Tabelle enthält detaillierte Informationen über den Aufbau der in Kapitel 3 „Öffentliche Finanzen und wirtschaftliches Handeln des Staates“ verwendeten Aggregate Steuern, Sozialversicherungsbeiträge, Zuwendungen und sonstige Einkünfte auf der Basis der OECD National Accounts Data.

Tabelle A.1. Einnahmeaggregate

\begin{tabular}{|c|c|c|}
\hline $\begin{array}{l}\text { Bezeichnung in } \\
\text { Regierung und Verwaltung } \\
\text { auf einen Blick }\end{array}$ & $\begin{array}{l}\text { Bezeichnung im } \\
\text { System der Volkswirtschaftlichen Gesamtrechnungen }\end{array}$ & $\begin{array}{c}\text { Code in den } \\
\text { OECD National Accounts Data } \\
\text { (Wichtigste Aggregate } \\
\text { des Sektors Staat) }\end{array}$ \\
\hline \multicolumn{3}{|l|}{ Steuern } \\
\hline Indirekte Steuern & Empfangene Produktions- und Importabgaben & GD2R \\
\hline Direkte Steuern & Empfangene direkte Einkommen- und Vermögensteuern & GD5R \\
\hline Vermögenswirksame Steuern & Vermögenswirksame Steuern & GD91R \\
\hline Sozialversicherungsbeiträge & Sozialversicherungsbeiträge & GD61R \\
\hline \multicolumn{3}{|l|}{ Zuwendungen und sonstige Einkünfte } \\
\hline \multirow[t]{2}{*}{ Laufende Transfers und Zuwendungen } & Empfangene sonstige Vermögenstransfers & GD7R \\
\hline & $\begin{array}{l}\text { Empfangene sonstige Vermögenstransfers und } \\
\text { Investitionszuschüsse }\end{array}$ & GD92R_D99R \\
\hline \multirow[t]{2}{*}{ Umsätze und Gebühren } & $\begin{array}{l}\text { Marktbestimmte Produktion und Nichtmarktproduktion für die } \\
\text { Eigenverwendung }\end{array}$ & GP11_P12R \\
\hline & Zahlungen für sonstige Nichtmarktproduktion & GP131R \\
\hline Vermögenseinkommen & Empfangenes Vermögenseinkommen & GD4R \\
\hline Subventionen & Empfangene sonstige Produktionssubventionen & GD39R \\
\hline Gesamteinkünfte & Gesamteinkünfte & GTR \\
\hline
\end{tabular}




\section{ANHANG B \\ Klassifikation der Aufgabenbereiche des Staats (COFOG)}

Die von der OECD konzipierte Klassifikation der Aufgabenbereiche des Staats (COFOG) erfasst die Daten zu den Ausgaben des Staats aus dem System der Volkswirtschaftlichen Gesamtrechnungen nach Aufgabenbereichen. Wie aus Tabelle B.1 hervorgeht, werden die Ausgaben auf der ersten COFOG-Stufe in zehn „funktionale“ Abteilungen bzw. Ausgabenbereiche untergliedert (wie Verteidigung, Bildungswesen, soziale Sicherung), und auf der zweiten COFOG-Stufe wird jede Abteilung (Gruppe der ersten Stufe) weiter in bis zu neun Untergruppen unterteilt. Während für 32 der 34 Länder COFOG-Daten der ersten Stufe zur Verfügung stehen, sind COFOG-Daten der zweiten Stufe derzeit nur für 21 europäische OECD-Mitgliedsländer und Japan verfügbar.*

Tabelle B.1. Erste und zweite COFOG-Stufe

\begin{tabular}{ll}
\hline Erste Stufe & Zweite Stufe \\
\hline Allgemeine öffentliche Verwaltung & Exekutiv- und Legislativorgane, Finanz- und Steuerwesen, auswärtige Angelegenheiten \\
& - Wirtschaftshilfe für das Ausland \\
- Allgemeine Verwaltung & Grundlagenforschung \\
- Angewandte Forschung und experimentelle Entwicklung im Bereich allgemeine öffentliche & Verwaltung \\
- Allgemeine öffentliche Verwaltung, a.n.g. \\
- Staatsschuldentransaktionen \\
- Transfers allgemeiner Art zwischen verschiedenen staatlichen Ebenen \\
- Militärische Verteidigung \\
- Zivile Verteidigung \\
- Militärhilfe für das Ausland \\
- Angewandte Forschung und experimentelle Entwicklung im Bereich Verteidigung \\
Verteidigung, a.n.g. \\
\hline
\end{tabular}

* Für Chile und Mexiko stehen keine COFOG-Daten der ersten Stufe zur Verfügung. Bis vor kurzem waren COFOG-Daten der zweiten Stufe in einigen nationalen statistischen Ämtern verfügbar, wurden von internationalen Organisationen aber nicht erfasst. Zudem waren die COFOG-Daten der zweiten Stufe unter den Ländern nicht vergleichbar, da das SNA-Handbuch und das Handbuch des Internationalen Währungsfonds zu den Statistiken über die Staatsfinanzen (International Monetary Fund Manual on Government Finance Statistics) keine wesentlichen praktischen Informationen zur Anwendung der COFOG-Konzepte enthalten. Jedoch richtete Eurostat im Jahr 2005 eine Task Force ein, die ein Handbuch zur Anwendung des COFOG-Konzepts bei Ausgabendaten der Volkswirtschaftlichen Gesamtrechnung erstellen und die Erfassung von COFOG-Daten der zweiten Stufe für europäische Länder erörtern sollte. Für die Schweiz und alle nichteuropäischen OECD-Mitgliedsländer (außer Japan), d.h. Australien, Chile, Israel, Kanada, Korea, Mexiko, Neuseeland und die Vereinigten Staaten, stehen keine COFOG-Daten der zweiten Stufe zur Verfügung. Darüber hinaus sind diese Daten in einigen EU-Mitgliedsländern nur für ausgewählte COFOG-Abteilungen verfügbar. Es wird derzeit mit diesen Ländern an einer Einigung zur Übermittlung dieser Daten an die OECD gearbeitet. 
Tabelle B.1. Erste und zweite COFOG-Stufe (Forts.)

\begin{tabular}{|c|c|}
\hline Erste Stufe & Zweite Stufe \\
\hline Öffentliche Ordnung und Sicherheit & $\begin{array}{l}\text { - Polizei } \\
\text { - Brandschutz } \\
\text { - Gerichte } \\
\text { - Justizvollzug } \\
\text { - Angewandte Forschung und experimentelle Entwicklung im Bereich öffentliche Ordnung } \\
\text { - und Sicherheit } \\
\text { - Offentliche Ordnung und Sicherheit, a.n.g }\end{array}$ \\
\hline Wirtschaftliche Angelegenheiten & $\begin{array}{l}\text { - Allgemeine Angelegenheiten der Wirtschaft und des Arbeitsmarkts } \\
\text { - Land- und Forstwirtschaft, Fischerei und Jagd } \\
\text { - Brennstoffe und Energie } \\
\text { - Bergbau, Herstellung von Waren und Bauwesen } \\
\text { - Verkehrswesen } \\
\text { - Kommunikation } \\
\text { - Andere Wirtschaftsbereiche } \\
\text { - Angewandte Forschung und experimentelle Entwicklung im Bereich wirtschaftliche Angelegenheiten } \\
\text { - Wirtschaftliche Angelegenheiten, a.n.g. }\end{array}$ \\
\hline Umweltschutz & $\begin{array}{l}\text { - Abfallwirtschaft } \\
\text { - Abwasserwirtschaft } \\
\text { - Vermeidung und Beseitigung von Umweltverunreinigungen } \\
\text { - Arten- und Landschaftsschutz } \\
\text { - Angewandte Forschung und experimentelle Entwicklung im Bereich Umweltschutz } \\
\text { - Umweltschutz, a.n.g. }\end{array}$ \\
\hline $\begin{array}{l}\text { Wohnungswesen und kommunale } \\
\text { Einrichtungen }\end{array}$ & $\begin{array}{l}\text { - Wohnungswesen } \\
\text { - Raumplanung } \\
\text { - Wasserversorgung } \\
\text { - Straßenbeleuchtung } \\
\text { - Angewandte Forschung und experimentelle Entwicklung im Bereich Wohnungswesen } \\
\text { und kommunale Einrichtungen } \\
\text { - Wohnungswesen und kommunale Einrichtungen, a.n.g. }\end{array}$ \\
\hline Gesundheitswesen & $\begin{array}{l}\text { - Medizinische Erzeugnisse, Geräte und Ausrüstungen } \\
\text { - Ambulante Behandlung } \\
\text { - Stationäre Behandlung } \\
\text { - Öffentlicher Gesundheitsdienst } \\
\text { - Angewandte Forschung und experimentelle Entwicklung im Bereich Gesundheitswesen } \\
\text { - Gesundheitswesen, a.n.g. }\end{array}$ \\
\hline $\begin{array}{l}\text { Freizeitgestaltung, Sport, Kultur und } \\
\text { Religion }\end{array}$ & $\begin{array}{l}\text { - Freizeitgestaltung und Sport } \\
\text { - Kultur } \\
\text { - Rundfunk- und Verlagswesen } \\
\text { - Religiöse und andere Gemeinschaftsangelegenheiten } \\
\text { - Angewandte Forschung und experimentelle Entwicklung im Bereich Freizeitgestaltung, Sport, } \\
\text { Kultur und Religion } \\
\text { - Freizeitgestaltung, Sport, Kultur und Religion, a.n.g. }\end{array}$ \\
\hline Bildungswesen & $\begin{array}{l}\text { - Vorschul- und Primarbereich } \\
\text { - Sekundarbereich } \\
\text { - Postsekundärer nichttertiärer Bereich } \\
\text { - Tertiärbildung } \\
\text { - Nichtzuordenbares Bildungswesen } \\
\text { - Hilfsdienstleistungen für das Bildungswesen } \\
\text { - Angewandte Forschung und experimentelle Entwicklung im Bereich Bildungswesen } \\
\text { - Bildungswesen, a.n.g. }\end{array}$ \\
\hline Soziale Sicherung & $\begin{array}{l}\text { - Krankheit und Erwerbsunfähigkeit } \\
\text { - Alter } \\
\text { - Hinterbliebene } \\
\text { - Familien und Kinder } \\
\text { - Arbeitslosigkeit } \\
\text { - Wohnverhältnisse } \\
\text { - Soziale Ausgrenzung, a.n.g. } \\
\text { - Angewandte Forschung und experimentelle Entwicklung im Bereich soziale Sicherung } \\
\text { - Soziale Sicherung, a.n.g. }\end{array}$ \\
\hline
\end{tabular}

a.n.g.: Anderweitig nicht genannt. 


\section{ANHANG C}

\section{Zusammengesetzte Indizes zur Haushaltspraxis}

Diese Ausgabe von Regierung auf einen Blick enthält zwei zusammengesetzte Indizes zur Haushaltspraxis, die den Einsatz einer mittelfristigen Perspektive im Haushaltsprozess und die Anwendung eines Verfahrens der ergebnisorientierten Budgetierung betreffen. Die für die Erstellung der zusammengesetzten Indizes verwendeten Daten stammen aus dem OECD Survey on Budgeting Practices and Procedures von 2012 und dem OECD Survey on Performance Budgeting von 2011. Die Befragten waren vornehmlich hochrangige Haushaltsverantwortliche aus dem Finanzministerium.

Anhand dieser in Regierung und Verwaltung auf einen Blick dargelegten eng definierten Gesamtindikatoren lassen sich diskrete, qualitative Informationen zu wichtigen Aspekten der Haushaltspraxis, wie mittelfristige Ausgabenrahmen und ergebnisorientierte Budgetierung, am besten zusammenfassen. „Die Interpretation von Gesamtindikatoren ist sehr viel einfacher als der Versuch, bei vielen getrennten Indikatoren einen gemeinsamen Trend zu finden“ (Nardo et al., 2004). Ihre Erstellung und Verwendung können jedoch zu Kontroversen Anlass geben. Diese Indikatoren werden von den Nutzern leicht und häufig fehlinterpretiert, was auf einen Mangel an Transparenz im Hinblick auf ihre Zusammensetzung und die sich daraus ergebenden Schwierigkeiten zurückzuführen ist, genau zu erfassen, was sie tatsächlich messen.

Die OECD hat mehrere Maßnahmen ergriffen, um die geläufigen Probleme im Umgang mit Gesamtindikatoren zu vermeiden oder zu lösen. Die in dieser Publikation dargelegten Gesamtindikatoren werden den Grundsätzen gerecht, die im Handbook on Constructing Composite Indicators (Nardo et al., 2008) als notwendige Voraussetzung für die zweckmäßige Erstellung von Gesamt- bzw. synthetischen Indikatoren ermittelt wurden.

Jeder Gesamtindikator basiert auf einem theoretischen Rahmen, der in dem von ihm abgedeckten spezifischen Bereich ein vereinbartes Konzept widerspiegelt. Die Variablen, aus denen sich die Indikatoren zusammensetzen, wurden von einer Expertengruppe innerhalb der OECD und nach Konsultation von Ländervertretern in den jeweiligen Arbeitsgruppen anhand ihrer Relevanz für das Konzept ausgewählt.

- Es kamen verschiedene statistische Instrumente zum Einsatz - darunter eine Faktoranalyse - um sicherzustellen, dass die verschiedenen Variablen, aus denen sich die einzelnen Indikatoren zusammensetzen, konsistent sind und auf demselben Konzept fußen.

- Es wurden verschiedene Methoden untersucht, um fehlende Werte zu ersetzen.

- Alle Teilindikatoren und Variablen wurden zu Vergleichszwecken standardisiert. 
- Zum Aufbau der Gesamtindikatoren wurden alle Teilindikatoren linear aggregiert, was die allgemein übliche Methode ist.

- Unter Einsatz der Monte-Carlo-Simulation wurde eine Sensitivitätsanalyse durchgeführt, um die Robustheit der Indikatoren gegenüber verschiedenen Gewichtungsmethoden (Gleichgewichtung, Gewichtung nach Faktoren, Expertengewichtung) zu bestimmen. Die Expertengewichtung hat sich als die geeignetste Gewichtungsmethode herausgestellt.

Die Indikatoren sollen nicht die Gesamtqualität der Haushaltssysteme messen. Hierzu wären eine wesentlich solidere konzeptuelle Grundlage und normative Hypothesen vonnöten. Vielmehr sind die in Regierung und Verwaltung auf einen Blick dargelegten Gesamtindikatoren deskriptiver Natur und wurden entsprechend benannt, um dies zum Ausdruck zu bringen. Zur Erstellung der Indikatoren wurden in den Erhebungen in allen Ländern dieselben Fragen gestellt, um die Vergleichbarkeit der Indikatoren zu gewährleisten.

Während die zusammengesetzten Indizes in Zusammenarbeit mit den Mitgliedsländern erstellt wurden und auf den besten Verfahrensweisen und/oder Theorien fußen, stehen sowohl die Variablen, aus denen sich die Gesamtindikatoren zusammensetzen als auch ihre Gewichtungen zur Diskussion offen und können sich folglich im Zeitverlauf noch ändern. Die OECD nimmt derzeit eine Neudefinition der besten Verfahrensweisen zur Erhöhung der Haushaltstransparenz vor und überarbeitet das Konzept der Haushaltsflexibilität, weshalb in dieser Ausgabe keine Gesamtindikatoren zu diesen Themenkomplexen enthalten sind. Die in der Ausgabe 2013 von Regierung und Verwaltung auf einen Blick dargelegten Gesamtindikatoren sind nicht mit denen der Ausgabe 2009 vergleichbar, da sie auf neuen Versionen der Umfragen beruhen, die zusätzliche bzw. anders formulierte Fragen sowie andere Gewichtungen enthalten.

Die Gesamtindikatoren wurden anhand der folgenden Methodik erstellt: Jeder Themenbereich wurde in große Kategorien unterteilt, die die theoretisch relevanten Aspekte für beide Elemente umfassen (mittelfristige Ausgabenrahmen und ergebnisorientierte Budgetierung). Jeder großen Kategorie wurde eine Gewichtung zugeordnet. Innerhalb jeder großen Kategorie wurden die relevanten Fragen identifiziert, jeder Frage wurde eine Untergewichtung zugeordnet und jeder Antwort auf die Fragen jeweils eine Punktzahl verliehen. Die Länderpunktzahl für jede Frage setzt sich zusammen aus dem Produkt der Gewichtung für die große Kategorie und der Untergewichtung für die Frage, multipliziert mit der von jedem Land gegebenen Antwort (1 oder 0); der Gesamtindikator entspricht der Summe dieser Punktwerte für jedes Land. Beide Gesamtindikatoren schwanken zwischen 0 und 1; ein Ergebnis von 1 spricht für den Einsatz solider Praktiken in Bezug auf eine gegebene Thematik. 


\section{Variablen, Gewichtungen und Punktzahlen im mittelfristigen Ausgabenrahmen (MTEF)}

Bei der Erstellung des MTEF-Gesamtindikators sind folgende Kriterien und Gewichtungen berücksichtigt worden.

\section{Abbildung C.1. Im MTEF-Index verwendete Variablen und Gewichtungen}

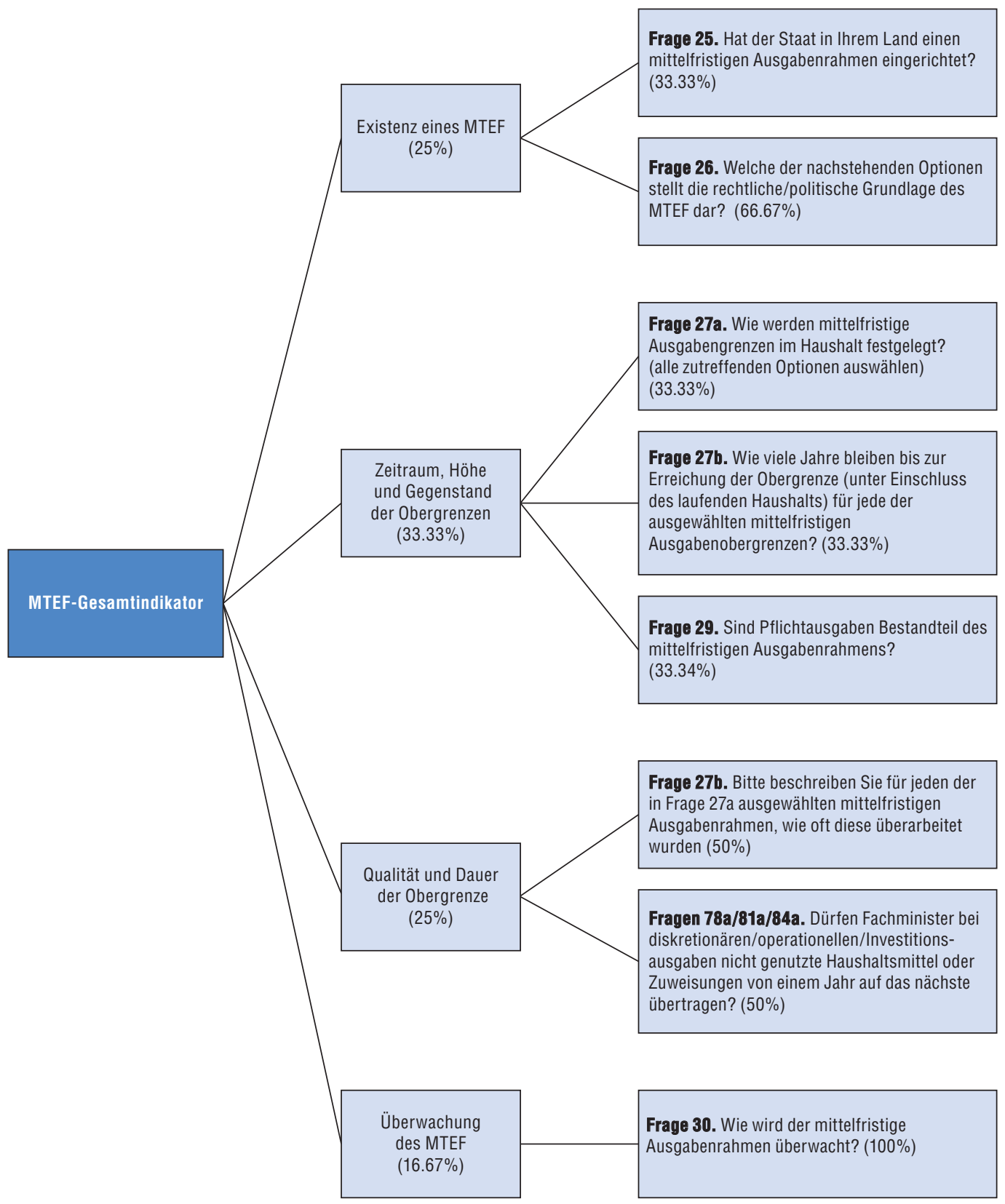

Anmerkung: Nähere Einzelheiten zum theoretischen Rahmen, zur Erstellung und Gewichtung der einzelnen Bestandteile finden sich unter: www.oecd.org/gov/govataglance.htm. 


\section{Verwendung eines Verfahrens der ergebnisorientierten Budgetierung auf Ebene der Zentralregierung, Gewichtungen und Punktzahlen}

Bei der Erstellung des MTEF-Gesamtindikators sind folgende Kriterien und Gewichtungen berücksichtigt worden.

\section{Abbildung C.2. Im Index der ergebnisorientierten Budgetierung verwendete Variablen und Gewichtungen}
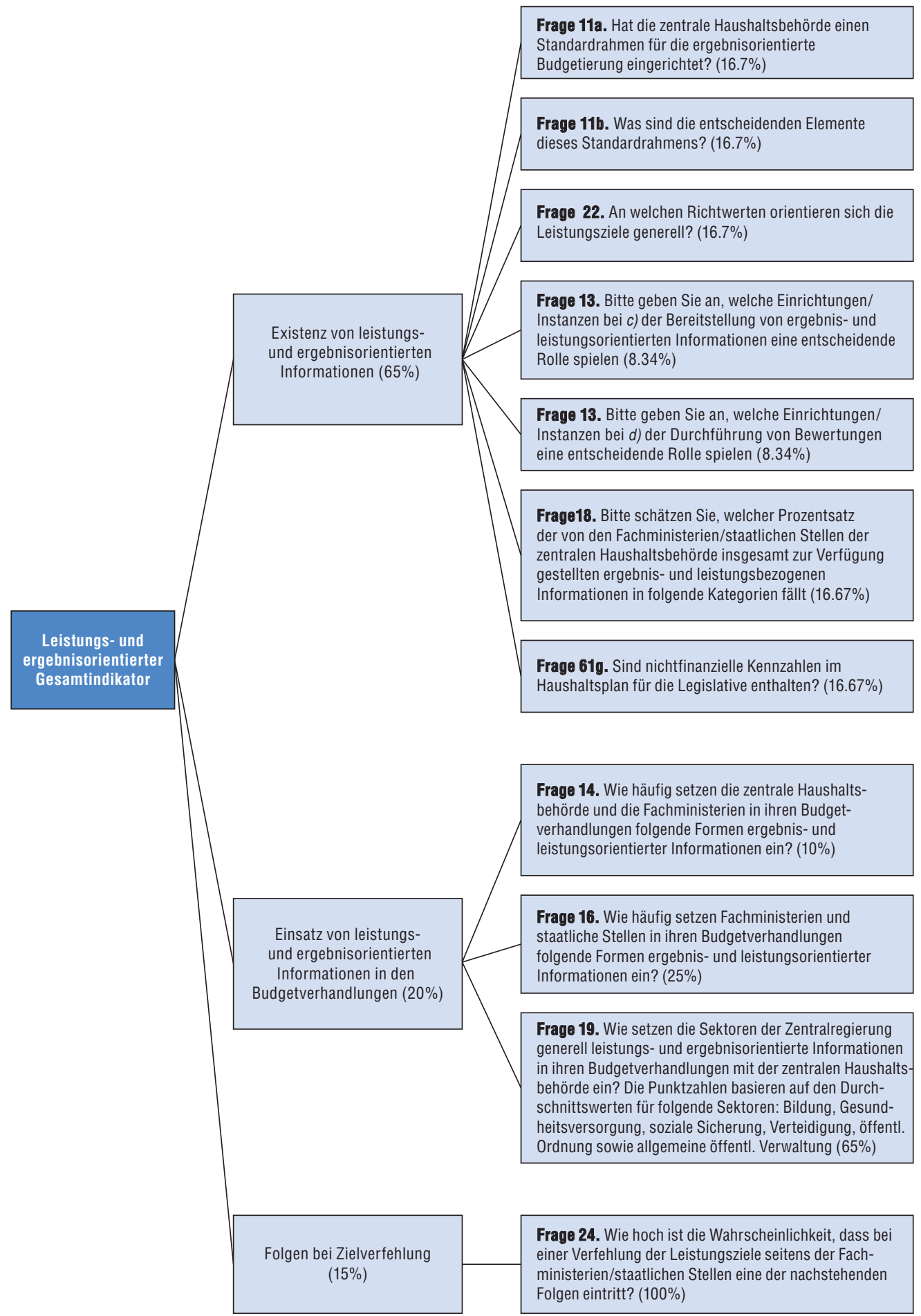

Anmerkung: Nähere Einzelheiten zum theoretischen Rahmen, zur Erstellung und Gewichtung der einzelnen Bestandteile finden sich unter: www.oecd.org/gov/govataglance.htm. 


\section{ANHANG D \\ Methodik und weitere Anmerkungen zur Vergütung der Beschäftigten im öffentlichen Dienst}

Die Vergütung spielt eine wichtige Rolle, wenn es gilt, qualifizierte Arbeitskräfte für den öffentlichen Dienst anzuwerben und zu motivieren. Im Rahmen der fiskalischen Konsolidierungsanstrengungen in vielen OECD-Ländern wird die Besoldung der öffentlich Bediensteten in Frage gestellt, was in einigen Fällen Einbußen bei den Gehältern und/oder Leistungen bzw. einen Lohnstopp zur Folge hat. Im Jahr 2010 erstellte die OECD eine Datenbank zum Besoldungsniveau typischer Berufe auf Ebene der Zentralregierung in den wichtigsten Ministerien, die zu einem besseren Verständnis der Gehaltsstrukturen und Lohnniveaus im öffentlichen Dienst beiträgt und 2012 aktualisiert wurde. Da es keine allgemeingültige Definition der Führungspositionen gibt und die Zahl der Führungsebenen in den Ländern und Ministerien variiert, bietet diese Vergütungserhebung eine gemeinsame Typologie für bestimmte Berufe auf Ebene der Zentralregierung. Ein Vergleich der Durchschnittsbesoldung im öffentlichen Dienst kann irreführend sein, da der öffentliche Sektor in verschiedenen Ländern unterschiedliche und heterogene Berufe umfasst. Diese Erhebung stellt aber Vergütungsdaten zu vergleichbaren Berufen zur Verfügung, so dass unsere Kenntnisse über den öffentlichen Sektor verbessert werden.

Der Vergleich der Besoldungsniveaus für leitende Führungskräfte, mittlere Führungskräfte, akademische Fachkräfte und Sekretariatskräfte zeigt ihre relative Gesamtvergütung in den OECD-Ländern, die nicht nur die Löhne und Gehälter, sondern auch die Beiträge zu den Gesundheits- und Rentenleistungen umfasst. Eine Gegenüberstellung der Vergütungsniveaus ergibt also ein mehr oder minder vollständiges Bild der Gesamtlohnkosten, das kohärente Vergleiche zwischen den Ländern zulässt.

Die Vergütungsdaten werden auch innerhalb der Länder mit dem Durchschnittsgehalt verglichen, das Arbeitskräfte mit Tertiärabschluss beziehen, was die relative Attraktivität dieser Berufe im Vergleich zu anderen zum Ausdruck bringt, die ein ähnliches Bildungsniveau voraussetzen. Beim Vergleich muss auch den unterschiedlichen wirtschaftlichen Entwicklungsstadien in den Ländern Rechnung getragen werden, daher die Korrektur um das Pro-Kopf-BIP. Allerdings ist beim Ländervergleich auf Grund der unterschiedlichen Lage an den Arbeitsmärkten, des divergierenden kulturellen und

Die statistischen Daten für Israel wurden von den zuständigen israelischen Stellen bereitgestellt, die für sie verantwortlich zeichnen. Die Verwendung dieser Daten durch die OECD erfolgt unbeschadet des völkerrechtlichen Status der Golanhöhen, von Ost-Jerusalem und der israelischen Siedlungen im Westjordanland. 
politischen Konsenses sowie möglicher Unterschiede zwischen den Ländern bei den lohndefinierenden Merkmalen selbst innerhalb derselben Berufsgruppen Vorsicht geboten, da diese in der vorliegenden Analyse unbereinigt bleiben.

\section{Berufe}

Der OECD Survey on Compensation of Employees in Central/Federal Government aktualisiert die 2010 durchgeführte Erhebung. Die im Verlauf dieser Erhebung gesammelten Daten werden die Durchführung vergleichender Analysen und weiterer Arbeiten auf dem Gebiet der Vergütungspolitik und -praxis ermöglichen. Ziel dieser Erhebung ist die Sammlung von Informationen zur Jahresvergütung von Arbeitskräften für eine Stichprobe ausgewählter Berufe auf Ebene der Zentralregierung/Bundesregierung/ nationalen Regierung. Es soll eine Datenbank zum Vergütungsniveau typischer Berufe auf Ebene der Zentralregierung eingerichtet werden, die zu einem besseren Verständnis der Gehaltsstrukturen und Lohnniveaus im öffentlichen Dienst beiträgt.

Die Erhebung konzentriert sich auf die Ebene der Zentral-/Bundesregierung; die Ebene der Bundesstaaten, nachgeordneten Gebietskörperschaften und Kommunen sowie Sozialversicherungseinrichtungen bleibt unberücksichtigt. Alle öffentlichen und quasiöffentlichen Unternehmen auf allen Verwaltungsebenen bleiben in der Erhebung unberücksichtigt. Sie erstreckt sich nicht auf die nachgeordneten Ämter/Organisationen der Ministerien auf Ebene der Zentralregierung, die oft als „Behörden“ bezeichnet werden. Im Mittelpunkt der Erhebung stehen ferner Vollzeitarbeitskräfte, Consultants und Zeitarbeitskräfte sind ausgenommen.

Im Fragebogen werden Daten zu vier typischen Berufsgruppen auf Ebene der Zentralregierung/Bundesregierung gesammelt: leitende Führungskräfte, mittlere Führungskräfte, akademische Fachkräfte und Sekretariatskräfte. Diese Berufe gelten als relativ repräsentativ und zwischen den einzelnen Ländern vergleichbar. Die Informationen zu diesen Berufen - mit Ausnahme der Dienstleistungsberufe - entstammen drei Schlüsselministerien (Innenministerium, Finanzministerium und Justizministerium) und drei Fachministerien (Bildung, Gesundheit und Umwelt). Kasten D.1 beschreibt die typischen Aufgaben der in dieser Erhebung betrachteten Ministerien.

Kasten D.2 enthält die Klassifizierung und Definitionen der in dieser Erhebung betrachteten Berufe, die in jedem Staat als einigermaßen typisch gelten. Das Hauptaugenmerk liegt auf der Kategorie der Führungskräfte im Allgemeinen, da die Kriterien, die eine Führungskraft im öffentlichen Dienst von anderen Kräften unterscheidet, darin bestehen, dass sie die Arbeit von mindestens 3 Personen beaufsichtigt und leitet. Da es extrem schwierig ist, eine detailliertere Beschreibung der Aufgaben und Zuständigkeiten zur Verfügung zu stellen, die auf den verschiedenen Führungsebenen eine Differenzierung ermöglichen, wurde beschlossen, den Schwerpunkt eher auf eine hierarchische Differenzierung zu legen als eine detailliertere Beschreibung der Funktionen vorzunehmen.

Die Klassifizierung und Definition der Berufe wurde anhand der Internationalen Standardklassifikation der Berufe (ISCO-08) der Internationalen Arbeitsorganisation (ILO) adaptiert. Das ist darauf zurückzuführen, dass nur wenige Länder ihre Berufe in der staatlichen Verwaltung anhand des ISCO-Modells klassifizieren. 


\section{Kasten D.1. Typische Aufgaben der in dieser Erhebung betrachteten Ministerien}

Die nachstehende Beschreibung der Tätigkeiten oder Funktionen der in dieser Erhebung betrachteten Ministerien dient nur Orientierungszwecken. In einigen Ländern tragen die Ministerien möglicherweise einen anderen Namen oder werden sogar als Department oder Secretariat bezeichnet.

\section{Innenministerium/Ministerium für innere Angelegenheiten}

- Vertritt den Staat auf dem gesamten Hoheitsgebiet.

- Stellt die Achtung der Bürgerrechte durch das allgemeine Wahlrecht sicher.

- Garantiert die Achtung der Kompetenzen nachgeordneter Körperschaften im Rahmen der Dezentralisierung.

- Definiert die Zuwanderungspolitik.

- Stellt die Politik der nationalen Sicherheit auf und koordiniert sie.

- Gewährleistet das Fortbestehen einer friedlichen und sicheren Gesellschaft.

- Garantiert die Wahrung der inneren Sicherheit und den Schutz der verfassungsmäßigen Ordnung.

\section{Finanzministerium}

- Plant und erarbeitet den Staatshaushalt.

- Analysiert und konzipiert die Steuerpolitik.

- Entwickelt Bestimmungen für Finanzinstitute und sorgt für deren Durchsetzung.

- Verfolgt wirtschaftliche und finanzielle Entwicklungen.

- Verwaltet den Mitteltransfer von der Ebene der nationalen/Zentral-/Bundesregierung zu nachgeordneten Gebietskörperschaften.

\section{Justizministerium}

- Gewährleistet das gute Funktionieren des Justizsystems.

- Erarbeitet die Gesetzestexte und Bestimmungen für einige spezifische Fachgebiete.

- Definiert die Hauptorientierungen der staatlichen Justizpolitik und sorgt für ihre Durchsetzung.

- Leistet Verbrechensopfern Unterstützung.

- Sorgt für eine gerechte, konsistente und effektive Durchsetzung von Strafen und sonstigen Sanktionen.

\section{Bildungsministerium}

- Regelt, koordiniert und organisiert das nationale Bildungssystem, im Allgemeinen von der Grundschule bis zur Sekundarschule.

- Gewährleistet gleichberechtigten Zugang zur staatlichen Bildung.

- Kontrolliert und beurteilt private und öffentliche Schulen und Hochschuleinrichtungen.

- Stellt eine effektives Management der Lehrerschaft und Verwaltungskräfte sicher.

\section{Gesundheitsministerium}

- Konzipiert und implementiert die öffentliche Gesundheitspolitik (Vorsorge, Organisation des Gesundheitswesens und Ausbildung von Fachkräften).

- Definiert die Sportpolitik und Maßnahmen zur Bekämpfung der Drogensucht.

- In Zusammenarbeit mit anderen Ministerien definiert es die Arbeitsschutz- und Sozialversicherungs bestimmungen.

\section{Umweltministerium}

- Definiert und verwaltet den Gesetzes- und Regulierungsrahmen für den Umweltschutz und den effizienten Ressourceneinsatz.

- Zertifiziert die Einrichtungen, Landnutzungen und Industriebranchen im Land, um sicherzustellen, dass die potenziellen Risiken für Umwelt und menschliche Gesundheit, Sicherheit sowie Grund und Boden auf ein Mindestmaß reduziert sind. 


\section{Kasten D.2. Klassifizierung und Definition der Berufe}

\section{Leitende Führungskräfte}

Führungskräfte der Stufe D1 (Teil von ISCO-08 1112) sind Spitzenbeamte direkt unterhalb des Ministers oder Staatssekretärs. Sie können dem höheren Dienst angehören und/oder von der Regierung oder dem Regierungschef ernannt werden. Sie beraten die Regierung in Politikfragen, überwachen die Interpretation und Umsetzung der staatlichen Politik, und sie haben in einigen Ländern auch exekutive Befugnisse. Führungskräfte der Stufe D1 sind u.U. berechtigt, an bestimmten Kabinettssitzungen/Ministerräten teilzunehmen, gehören dem Kabinett/Ministerrat aber nicht an. Ihnen obliegt die Gesamtleitung und das Management eines spezifischen Verwaltungsbereichs für den Minister/Staatssekretär, wie dies in Ländern mit einem System unabhängiger Einrichtungen, dezentralisierten Machtbefugnissen, einer flacheren Organisationsstruktur und mit entsprechenden Befugnissen ausgestatteten Führungskräften der Fall ist. Die genaue Berufsbezeichnung kann in den einzelnen Ländern unterschiedlich lauten.

Führungskräfte der Stufe D2 (Teil von ISCO-O8 11 und 112) sind direkt unter den D1-Kräften angesiedelt. Sie formulieren und überprüfen die ergriffenen Maßnahmen und planen, steuern, koordinieren und evaluieren die Gesamttätigkeiten des Ministeriums oder der spezifischen Abteilung/Verwaltungseinheit mit der Unterstützung anderer Führungskräfte. Sie können dem höheren Dienst angehören. Sie liefern Orientierungen bei der Koordinierung und Verwaltung des Arbeitsprogramms und leiten Teams akademischer Fachkräfte in verschiedenen Politikbereichen. Sie stellen die Ziele, Strategien und Programme für die spezifische Verwaltungseinheit/Abteilung auf, die unter ihrer Aufsicht steht.

\section{Mittlere Führungskräfte (haben Führungsverantwortung für mindestens 3 Mitarbeiter)}

Führungskräfte der Stufe D3 (Teil von ISCO-O8 12) sind direkt unter den D2-Kräften angesiedelt. Sie planen, lenken und koordinieren die allgemeinen Arbeitsabläufe einer spezifischen Abteilung/Verwaltungseinheit innerhalb des Ministeriums mit der Unterstützung anderer Führungskräfte, gewöhnlich im Rahmen der Leitlinien, die von einem Aufsichtsgremium oder Verwaltungsrat aufgestellt worden sind. Sie übernehmen die Leitung und das Management von Teams akademischer Fachkräfte auf ihrem spezifischen Fachgebiet. Diese Beamten erstellen das Arbeitsprogramm und übernehmen das Personalmanagement für Verwaltungseinheiten, Abteilungen oder Politikbereiche. Sie stellen die Haushalte auf und verwalten sie, kontrollieren die Ausgaben und stellen den effizienten Einsatz der Ressourcen sicher. Sie kontrollieren und evaluieren die Leistungen der verschiedenen Teams akademischer Fachkräfte.

Führungskräfte der Stufe D4 (Teil von ISCO-O8 121) sind direkt unter den D3-Kräften angesiedelt. Sie formulieren und leisten Politikberatung sowie strategische und Finanzplanung. Sie erstellen und steuern Betriebs- und Verwaltungsabläufe und beraten leitende Führungskräfte. Sie kontrollieren Auswahl, Ausbildung und Leistungsniveau der Mitarbeiter, erstellen die Haushaltsentwürfe und überwachen finanzielle Transaktionen, kontrollieren die Ausgaben und sorgen für einen effizienten Ressourceneinsatz. Spezifische Teams akademischer Fachkräfte innerhalb einer Verwaltungseinheit stehen unter ihrer Leitung.

\section{Akademische Fachkräfte}

Leitende Volkswirte/Fachkräfte im Bereich Politikanalyse (Teil von ISCO-08 242 und 2422) nehmen (über die Führung von höchstens drei Mitarbeitern hinaus) keine Führungsaufgaben wahr und sind rangmäßig über den Nachwuchskräften im Bereich Politikanalyse sowie Verwaltungs-/Sekretariatskräften angesiedelt. Sie müssen gewöhnlich einen Hochschulabschluss haben. Sie haben gewisse Führungsverantwortlichkeiten in einem Arbeitsbereich oder bei verschiedenen Projekten, entwickeln und analysieren Politikmaßnahmen, die für die Gestaltung, Umsetzung und Änderung staatlicher Aktionen und Programme maßgeblich sind. Diese akademischen Fachkräfte überprüfen die bestehenden Maßnahmen und die Gesetzgebung, um Anomalien und veraltete Vorschriften zu identifizieren. Sie analysieren und formulieren Politikoptionen, verfassen Themenpapiere und Empfehlungen für Politikveränderungen. Ferner beurteilen sie die Auswirkungen, die finanziellen Folgen und die Machbarkeit staatlicher Maßnahmen in politischer und verwaltungstechnischer Hinsicht. Mitarbeiter in dieser Kategorie haben die Möglichkeit, im Rahmen ihrer beruflichen Karriere zur Führungskraft aufzusteigen. Sie können in unterschiedlichen Fachgebieten tätig sein, die von Rechtswissenschaften, Wirtschaft, Politik, öffentliche Verwaltung, internationalen Beziehungen bis hin zu Ingenieurwissenschaften, Umwelt, Pädagogik, Gesundheitswirtschaft usw. reichen. Leitende Politikexperten/Volkswirte haben mindestens fünf Jahre Berufserfahrung. 


\section{Kasten D.2. Klassifizierung und Definition der Berufe (Forts.)}

Nachwuchsvolkswirte/-fachkräfte im Bereich Politikanalyse (Teil von ISCO-08 242 und 2422) sind rangmäßig über den Verwaltungs-/Sekretariatskräften angesiedelt. Sie müssen gewöhnlich einen Hochschulabschluss haben. Sie haben keine Führungsverantwortung. Sie konzipieren und analysieren Politikmaßnahmen, die für die Gestaltung, Durchsetzung und Veränderung staatlicher Aktionen und Programme maßgeblich sind. Diese Fachkräfte überprüfen die bestehenden Maßnahmen und die Gesetzgebung, um Anomalien und veraltete Vorschriften zu identifizieren. Sie analysieren und formulieren Politikoptionen, verfassen Themenpapiere und Empfehlungen für Politikveränderungen. Ferner beurteilen sie die Auswirkungen, die finanziellen Folgen und die Machbarkeit staatlicher Maßnahmen in politischer und verwaltungstechnischer Hinsicht. Sie können in unterschiedlichen Fachgebieten tätig sein, die von Rechtswissenschaften, Wirtschaft, Politik, öffentliche Verwaltung, internationalen Beziehungen bis hin zu Ingenieurwissenschaften, Umwelt, Pädagogik, Gesundheitswirtschaft usw. reichen. Nachwuchsfachkräfte im Bereich Politikanalyse/-volkswirte haben weniger als fünf Jahre Berufserfahrung.

\section{Sekretariatskräfte}

Bei Sekretariatskräften (allgemeinen Bürokräften) (Teil von ISCO-08 411 und 4110) wird generell kein Hochschulabschluss vorausgesetzt, wenngleich viele über eine solche Qualifikation verfügen. Sie erledigen ein breites Spektrum an Sekretariats- und Verwaltungsaufgaben in Verbindung mit Geldoperationen, Reisebuchungen, Kundenanfragen und Terminabwicklung. Sie erfassen, erstellen, sortieren, klassifizieren und erteilen Informationen; sie sortieren, öffnen und verschicken Postsendungen; sie bereiten Berichte vor und erledigen die Korrespondenz; sie verwalten das Büromaterial für die Mitarbeiter und nehmen Telefonate entgegen, beantworten elektronische Anfragen oder leiten diese an geeignete Personen weiter; sie überprüfen Zahlen, bereiten Rechnungen vor und erfassen erledigte Finanztransaktionen, übertragen Informationen auf den PC, lesen Korrektur und arbeiten Korrekturen ein. Einige sind an der Aufstellung des Abteilungsbudgets, der Ausgabenkontrolle, Aufsetzung von Verträgen sowie Auftragsverwaltung beteiligt. Die erfahrensten Mitarbeiter, die die Arbeit der Sekretariatskräfte überwachen, gehören nicht zu dieser Kategorie.

Da es keine allgemeingültige Definition der Positionen auf Führungsebene gibt und die Zahl der Führungsebenen in den Ländern und Ministerien variiert, entspricht D1 in diesem Bericht der obersten Führungsebene direkt unterhalb der (vom Präsidenten oder Regierungschef bestimmten) Minister/Staatssekretäre, die wiederum vom jeweiligen Minister ernannt (und in manchen Fällen vom Präsidenten/Regierungschef bestimmt) werden. In dieser Erhebung werden die Führungsebenen bis D4 berücksichtigt, wobei es sich bei den Ebenen D1 und D2 um leitende Führungspositionen und bei den Ebenen D3 und D4 um mittlere Führungspositionen handelt.

Die Kategorie der „akademischen Fachkräfte“ ist in Nachwuchskräfte und leitende Kräfte unterteilt, da diese Kategorie Mitarbeiter mit ganz unterschiedlichem Erfahrungshorizont umfasst.

Ferner wurden in der Erhebung 2012 Informationen zu einigen Berufen zusammengetragen, in denen Dienstleistungen an vorderster Front erbracht werden (Kriminaleamte/ Polizeikommissare, Polizisten, Grenzbeamte, Zollbeamte und Steuerprüfer). Diese Funktionen werden auf Ebene der Zentralregierung eingerichtet und sie können auf die Ministerien oder Behörden übertragen werden. An dieser Stelle sei darauf hingewiesen, dass Funktionen wie Grenzbeamte in einigen Ländern nicht existieren, da diese Tätigkeiten von der Polizei ausgeübt werden. In anderen Ländern werden einige der oben erwähnten Funktionen von den Bundesländern oder nachgeordneten Gebietskörperschaften übernommen. 


\section{Kasten D.3. Dienstleistungserbringer - verschiedene Berufsbeschreibungen}

Polizeikommissare und Kriminalbeamte (Teil von ISCO-08 3355) untersuchen Fakten und Umstände im Zusammenhang mit begangenen Straftaten, um Tatverdächtige zu identifizieren. Sie suchen auch nach Informationen, die nicht direkt zugänglich oder offensichtlich sind und Einrichtungen oder Umstände und das Verhalten von Personen betreffen, primär zur Verhinderung von Straftaten. Zu ihrem Aufgabenbereich zählt u.a. auch die Suche nach Kontakten und Informationsquellen zu geplanten oder begangenen Straftaten mit dem Ziel, Straftaten zu verhindern bzw. Tatverdächtige zu identifizieren, die Sicherung, Überprüfung und Analyse von Beweismitteln, um Straftaten aufzuklären, die Vornahme von Verhaftungen sowie die Aussage als Zeuge vor Gericht. Sie tragen gewöhnlich Führungsverantwortung. Polizeikommissare und Kriminalbeamte benötigen gewöhnlich einen Hochschulabschluss und/oder werden nach einer bestimmten Anzahl von Jahren der Berufserfahrung als Polizisten (generell mehr als 5 Jahre) in diese höheren Funktionen befördert.

Polizisten (Teil von ISCO-08 5412) sorgen für die Sicherung von Recht und Ordnung, übernehmen Streifendienste bzw. Revierkontrollen von öffentlichen Flächen, setzen Gesetze und Bestimmungen durch und nehmen Tatverdächtige fest. $\mathrm{Zu}$ den weiteren Aufgaben zählen u.a. die Verkehrsregelung und Aufklärung bei Unfällen, Leistung von Nothilfe für Opfer von Unfällen, Straftaten und Naturkatastrophen. Polizisten haben gewöhnlich höchstens drei Mitarbeiter unter sich. Für Polizisten wird in der Regel kein Hochschulabschluss vorausgesetzt.

Grenzbeamte (Teil von ISCO-08 3351) kontrollieren Personen, die nationale Grenzen überqueren, um die einschlägigen Vorschriften und Bestimmungen anzuwenden und durchzusetzen. Zu ihren Aufgaben gehören u.a. Streifengänge bzw. -fahrten entlang der nationalen Grenzen und Küstengewässer, um Personen vom illegalen Zutritt oder Verlassen des Landes abzuhalten, das Überprüfen von Reisedokumenten, um sicherzustellen, dass die betreffenden Personen über die notwendigen Genehmigungen und Dokumente verfügen, die Koordinierung und Kooperation mit anderen an Rechtsvollzug, Ausweisung und Strafverfolgung beteiligten Stellen. Grenzbeamte haben, wenn überhaupt, nicht mehr als drei Personen unter sich.

Fachkräfte im Zolldienst (Teil von ISCO-08 3351) kontrollieren Fahrzeuge, die nationale Grenzen überqueren, um die einschlägigen Vorschriften und Bestimmungen anzuwenden und durchzusetzen. Zu ihren Aufgaben gehört die Inspektion des Gepäcks von Personen, die nationale Grenzen überqueren, um sicherzustellen, dass dieses mit den staatlichen Vorschriften und Bestimmungen hinsichtlich der Ein- und Ausfuhr von Waren und Währungen übereinstimmt, die Prüfung von Transportdokumenten und Fahrzeugladungen, die nationale Grenzen überqueren, um ihre Konformität mit den staatlichen Vorschriften und Bestimmungen zu gewährleisten, die Festnahme von Personen und Beschlagnahme von verbotenen und nicht deklarierten Waren, die u.a. gegen Einwanderungs- bzw. Zollgesetze verstoßen. Zollbeamte haben, wenn überhaupt, generell nicht mehr als drei Personen unter sich.

Steuerprüfer (Teil von ISCO-08 3352) untersuchen Steuererklärungen, Rechnungen und andere Dokumente, um die Art und Höhe der Steuern, Abgaben und Gebühren zu bestimmen, die von Einzelpersonen oder Unternehmen zu entrichten sind, wobei außergewöhnliche oder besonders wichtige Fälle an Buchsachverständige oder hochrangige Beamte überwiesen werden. Sie beraten Organisationen, Unternehmen und Bürger in Bezug auf die staatlichen Gesetze, Regeln und Bestimmungen hinsichtlich der Ermittlung und Zahlung von Steuern, Abgaben und Gebühren sowie die Rechte und Pflichten der Bürger; sie untersuchen Steuererklärungen, Rechnungsbelege und andere relevante Dokumente, sie prüfen eingereichte Steuererklärungen und Buchhaltungsaufzeichnungen und -systeme sowie interne Kontrollen von Unternehmen, um u.a. die Einhaltung der Steuergesetze und -bestimmungen zu gewährleisten. Steuerprüfer haben, wenn überhaupt, höchstens 3 Personen unter sich. 


\section{Vergütung}

Die Erhebung legt den Schwerpunkt auf die Gesamtvergütung, die aus zwei Hauptkomponenten besteht: 1) den Bruttolöhnen und -gehältern und 2) den Arbeitgeberbeiträgen zur Sozialversicherung. Gefragt wurde nach Angaben zum Gehaltsniveau von Vollzeitarbeitskräften:

1. Die Bruttolöhne und -gehälter enthalten die Sozialversicherungsbeiträge, Einkommensteuern usw., die vom Arbeitnehmer zu entrichten sind, selbst wenn sie, um den bürokratischen Aufwand zu reduzieren oder aus sonstigen Gründen, de facto vom Arbeitgeber zurückgehalten und für den Arbeitnehmer direkt an die Sozialversicherungssysteme, Steuerbehörden usw. abgeführt werden. Die Arbeitgeberbeiträge zur Sozialversicherung sind in den Bruttolöhnen und -gehältern nicht enthalten. Sachleistungen bleiben in der Erhebung unberücksichtigt. Die Bruttolöhne und -gehälter umfassen:

- Grundlöhne und -gehälter, bei denen es sich um die regulären Jahresgehälter handelt, die den Bediensteten für die von ihnen geleisteten Arbeitsstunden und für den Staat erbrachten Dienstleistungen gezahlt werden. Wenngleich die Löhne und Gehälter in regelmäßigen wöchentlichen, monatlichen oder sonstigen Intervallen gezahlt werden, sollte für die Zwecke dieser Erhebung das Jahresgehalt angegeben werden. Zahlungen für Überstunden sind aus den Daten ausgeklammert.

- Zusätzliche Zahlungen - wobei diese auf Grund der Schwierigkeiten, lückenlose Daten zu bekommen und die Vergleichbarkeit zwischen den Ländern zu garantieren, auf die wichtigsten Kategorien begrenzt wurden, darunter:

* Vergütungen für Zeiten, in denen nicht gearbeitet wurde, die sich aber nur auf den bezahlten Jahresurlaub und gesetzliche Feiertage beziehen.

* Unter regelmäßig gezahlte Sondervergütungen und Zuwendungen fallen das 13. Monatsgehalt und saisonale Bonuszahlungen, Gewinnbeteiligungen sowie über das normale Urlaubsgeld hinausgehende ergänzende urlaubsbedingte Zahlungen sowie sonstige Sondervergütungen und Zuwendungen.

* Nicht regelmäßig gezahlte Sondervergütungen und Zuwendungen (leistungsbezogene Vergütung) beziehen sich auf Ad-hoc-Prämien oder sonstige außergewöhnliche Zahlungen in Verbindung mit der Gesamtleistung des anspruchsberechtigten Arbeitnehmers.

2. Arbeitgeberbeiträge zur Sozialversicherung sind Sozialversicherungsbeiträge, die der Arbeitgeber an die Sozialversicherung oder andere beschäftigungsbezogene Sozialversicherungssysteme abzuführen hat, um die Erbringung von Sozialleistungen (Krankenversicherung, Rentenversicherung) für seine Angestellten sicherzustellen:

- Arbeitgeberbeiträge zu gesetzlichen oder privaten Sozialversicherungssystemen für die Alters- und Krankheitsvorsorge. Bei den Arbeitgeberbeiträgen zur Sozialversicherung handelt es sich um Sozialversicherungsbeiträge, die der Arbeitgeber an die Sozialversicherungen oder andere beschäftigungsbezogene Sozial-versicherungssysteme abzuführen hat, um den Bezug von Sozialleistungen (Krankenversicherung, Rentenversicherung) für seine Beschäftigten sicherzustellen. In einigen Ländern fließen diese Sozialversicherungsbeiträge in staatliche, in anderen indessen in private Versicherungssysteme. Die Arbeitgeberbeiträge zur Sozialversicherung umfassen manchmal auch Mittel für Sonderfonds, die beispielsweise in Tarifvereinbarungen eingerichtet wurden. In der Erhebung von 2011 beschränkten sich die über die 
Arbeitgeberbeiträge zur Sozialversicherung gesammelten Daten auf Zahlungen an die Kranken- und Rentenversicherung, die den Großteil der Sozialversicherungsbeiträge ausmachen.

- Die vom Arbeitgeber für den Arbeitnehmer entrichteten nicht kapitalgedeckten Sozialleistungen beschränken sich auf Leistungen der Kranken- und Rentenversicherung. Der Begriff „nicht kapitalgedeckt“ bezieht sich auf Sozialleistungen, die nicht bei einem Sozialversicherungsträger finanziell gedeckt sind und bei denen über die Entrichtung der Sozialversicherungsbeiträge nicht offiziell Buch geführt wird. Nicht kapitalgedeckte Renten- oder Krankenversicherungssysteme existieren in vielen Ländern: In diesem Fall werden die Renten- und Krankenversicherungsleistungen der Beamten aus dem gesamtstaatlichen Haushalt finanziert. In einer Reihe von Ländern decken die Arbeitnehmer- und Arbeitgeberbeiträge nicht die Gesamtheit der Kosten ab, die mit den Sozialleistungen für öffentlich Bedienstete einhergehen. In diesen Fällen enthält der Haushalt häufig spezielle Posten, die für die Finanzierung dieses nicht kapitalgedeckten Teils der Sozialleistungen bestimmt sind.

Nicht alle Länder waren in der Lage, das Element der Sozialversicherungsbeiträge in ihre Survey-Antworten einzubeziehen (was hauptsächlich den nicht kapitalgedeckten Rentenversicherungssystemen zuzuschreiben ist). Folglich war es notwendig, diese Komponente unter Verwendung anderer Datenquellen für diese Länder zu schätzen. Im System der Volkswirtschaftlichen Gesamtrechnungen werden die fiktiven Buchungen für nicht kapitalgedeckte Rentenversicherungen vom Konzept her in allen Ländern einheitlich vorgenommen. Daher war es unter Verwendung der Daten der Volkswirtschaftlichen Gesamtrechnungen möglich, den Gesamtanteil der Arbeitgeberbeiträge zur Sozialversicherung zu schätzen, der in den verschiedenen bestehenden Datenbanken zur Vergütung öffentlich Bediensteter angegeben war. Der in den Daten für diese Publikation zur Berechnung der Personalvergütungen zu Grunde gelegte Anteil ist nach einer genauen Analyse und Diskussion mit den Ländern festgelegt worden. Bestimmt wurde dieser Anteil anhand der Daten der Volkswirtschaftlichen Gesamtrechnungen aus folgenden Ländern: Deutschland, Finnland, Griechenland, Japan und Norwegen. Zudem wurde dieser Anteil für Belgien anhand einer Kombination von Informationen aus der Vergütungserhebung und Daten der Volkswirtschaftlichen Gesamtrechnungen geschätzt.

An dieser Stelle sei darauf hingewiesen, dass anders als in der Vergütungserhebung, wo sich die Arbeitgeberbeiträge auf das Gesundheits- und Rentensystem beschränken, die Daten im Rahmen der Volkswirtschaftlichen Gesamtrechnungen die Gesamtheit der arbeitgeberseitigen Sozialversicherungsbeiträge berücksichtigen. Infolgedessen war der sich ergebende Anteil unter Zugrundelegung dieser Quelle bis zu einem gewissen Grad zu hoch ausgewiesen. Ferner beziehen sich die Anteile der Arbeitgeberbeiträge zur Sozialversicherung aus den Daten der Volkswirtschaftlichen Gesamtrechnungen auf die Gesamtheit der öffentlich Bediensteten. Bei Verwendung dieser Anteile wird etwaigen Unterschieden, die beispielsweise zwischen den Berufen bei den Prozentsätzen der Sozialversicherungsbeiträge bestehen können, nicht Rechnung getragen. Für die Länder, die in der Erhebung Daten zu den Arbeitgeberbeiträgen zur Sozialversicherung zur Verfügung gestellt haben, wurden für die Sozialversicherungsbeiträge die exakten Daten verwendet (die in den einzelnen Berufen abweichen können). 
Die Höhe der Sozialversicherungsbeiträge ist nur eine Ersatzvariable. Quantität und Qualität der Leistungen, die die Arbeitnehmer durch die Arbeitgeber- und Arbeitnehmerbeiträge zur Sozialversicherung beziehen, hängen von vielen Variablen ab, darunter u.a. auch der Qualität und Effizienz der Mittel- und Leistungsverwaltung in jedem Land.

\section{Einsatz von Vergleichsgrößen}

Es sind Berechnungen durchgeführt worden, in denen die Vergütungsdaten unter Einsatz der KKP-Methode in US-\$ umgewandelt wurden. Hiermit wurden Unterschiede bei den Wechselkursen und relativen Preisniveaus ausgeglichen. Die KKP trägt den verhältnismäßig unterschiedlichen Lebenshaltungskosten in den (Landes-)Hauptstädten in den Ländern und im Ländervergleich nicht Rechnung. In vielen Ländern ist der Großteil der öffentlich Bediensteten auf Ebene der Zentralregierung in der Hauptstadt beschäftigt. Die Löhne und Gehälter können helfen, die bei den Lebenshaltungskosten in den Großstädten bestehenden Unterschiede etwas auszugleichen.

Die OECD hat auch Ländervergleiche anhand von Daten vorgenommen, die mit dem Pro-Kopf-BIP normalisiert wurden, das durch die OECD National Accounts Statistics (Datenbank) zur Verfügung stand. Mit dieser Normalisierung lassen sich Unterschiede bei den durchschnittlichen Vermögensverhältnissen innerhalb eines Landes beseitigen.

Die OECD hat auch das Verhältnis zwischen der durchschnittlichen Gesamtvergütung und der durchschnittlichen Vergütung von Personen mit Tertiärabschluss berechnet (Bruttogehalt zuzüglich Arbeitgeberbeiträge zur Sozialversicherung). Da die in dieser Erhebung betrachteten Berufe der öffentlich Bediensteten auf Ebene der Zentralregierung generell ein hohes Bildungsniveau voraussetzen, wirft das Verhältnis die Frage der Vergleichbarkeit der Vergütung im öffentlichen Sektor mit der der Gesamtwirtschaft auf. Die Angaben zu den Gehältern von Personen mit Tertiärabschluss basieren auf einer Kombination von Quellen, wie der Publikation OECD Bildung auf einen Blick (2013 oder frühere Ausgaben) zu entnehmen ist. Aber selbst bereinigt um das Bildungsniveau, bleiben andere Merkmale der Personen in diesen Berufen unberücksichtigt, wie beispielsweise das Dienstalter, Alter, Geschlecht usw.

Das Verhältnis zwischen der Vergütung der öffentlich Bediensteten in Bezug auf das Pro-Kopf-BIP und dem Arbeitsentgelt von Personen mit Tertiärabschluss ist nicht um die Arbeitszeit bereinigt worden. Dieser Ansatz wurde gewählt, um zwischen den drei verglichenen Messgrößen Konsistenz zu wahren.

\section{Arbeitszeitbereinigungen}

Die Unterschiede zwischen der Zeit, in der Menschen tatsächlich arbeiten, und der jahresdurchschnittlichen Vergütung (jahresdurchschnittlicher Bruttolohn zuzüglich Arbeitgeberbeiträge zur Sozialversicherung) wird berechnet, um eine bereinigte jahresdurchschnittliche Vergütung zu erhalten. Um die Vergütung der betrachteten Arbeitnehmer auf eine länderübergreifend vergleichbare Grundlage zu stellen, werden die Unterschiede in der Arbeitszeit (Zahl der im öffentlichen Dienst geleisteten wöchentlichen Arbeitsstunden, der gesetzliche oder durchschnittliche Urlaubsanspruch sowie die Anzahl der den öffentlichen Dienst betreffenden Feiertage) für die Berechnung der bereinigten jahresdurchschnittlichen Vergütung verwendet.

Für alle Führungskräfte (in D1-, D2-, D3- und D4-Position) wurden die Angaben nur um die Urlaubsansprüche bereinigt, da die wöchentliche Arbeitszeit in dieser Beschäftigtenkategorie sehr uneinheitlich ist. 
Die Bereinigungen um die Arbeitszeit sind in Tabelle D.1 wiedergegeben.

Die jahresdurchschnittlichen Vergleichsgehälter lauten:

$$
\mathrm{W}_{\mathrm{co}}^{a^{*}}=\frac{\left(\frac{\mathrm{W}_{\mathrm{co}}^{a}}{\mathrm{P}_{\mathrm{c}}}\right)}{\mathrm{H}_{\mathrm{c}}^{a^{*}}}
$$

mit:

$\mathrm{W}_{c 0}^{a^{*}}=$ Jahresdurchschnittliche Vergütung der Arbeitnehmer in Land c innerhalb der Beschäftigtengruppe $o$, in KKP zwecks Arbeitszeitbereinigung.

$\mathrm{W}_{\mathrm{co}}^{a}=$ Jahresdurchschnittliche Vergütung in Landeswährung in Land c innerhalb der Beschäftigtengruppe $o$ in Landeswährung.

$P_{c}=$ Kaufkraftparität von Land c.

$H_{c}^{a^{*}}=$ Durchschnittliche Arbeitszeit in Land c. Diese entspricht den jahresdurchschnittlichen Arbeitsstunden in Land c (gemäß den Erhebungsdaten), dividiert durch 2 088. Die Zahl 2088 entspricht den theoretischen Arbeitsstunden pro Jahr bei einer 40-Stunden-Woche, ohne Urlaub und Fehlzeiten jeglicher Art. Hieraus ergeben sich ferner im Durchschnitt 261 Arbeitstage pro Jahr mit jeweils 8 Arbeitsstunden. 
Tabelle D.1. Arbeitszeitbereinigungen

\begin{tabular}{|c|c|c|c|c|c|c|c|c|}
\hline & $\begin{array}{l}\text { Vertraglich } \\
\text { vereinbarte } \\
\text { wöchentliche } \\
\text { Arbeitszeit }\end{array}$ & $\begin{array}{c}\text { Durchschnittliche } \\
\text { Anzahl der } \\
\text { Urlaubstage }\end{array}$ & $\begin{array}{l}\text { Durchschnittliche } \\
\text { Anzahl der } \\
\text { Feiertage für } \\
\text { den öffentlichen } \\
\text { Dienst }\end{array}$ & $\begin{array}{l}\text { Anzahl der } \\
\text { Arbeitstage im } \\
\text { Jahresdurch- } \\
\text { schnitt in Land }\end{array}$ & $\begin{array}{l}\text { Anzahl der } \\
\text { Arbeitsstunden } \\
\text { im Jahresdurch- } \\
\text { schnitt in Land }\end{array}$ & $\begin{array}{l}\text { Koeffizient } \\
\text { der Arbeits- } \\
\text { zeitbereinigung, } \\
\text { Wochenstunden } \\
\text { und Urlaub }\end{array}$ & $\begin{array}{c}\text { Koeffizient } \\
\text { der Arbeits- } \\
\text { zeitbereinigung, } \\
\text { Urlaub }\end{array}$ & $\begin{array}{c}\text { Koeffizient } \\
\text { der Arbeits- } \\
\text { zeitbereinigung, } \\
\text { keine Korrektur }\end{array}$ \\
\hline Australien & 37.5 & 20 & 10 & 231 & 1730 & 0.830 & 0.885 & 1.000 \\
\hline Österreich & 40 & 25 & 10 & 226 & 1806 & 0.866 & 0.866 & 1.000 \\
\hline Belgien & 38 & 26 & 10 & 225 & 1708 & 0.819 & 0.862 & 1.000 \\
\hline Chile & 44 & 15 & 9 & 237 & 2083 & 0.999 & 0.908 & 1.000 \\
\hline Dänemark & 37 & 30 & 9 & 222 & 1641 & 0.787 & 0.850 & 1.000 \\
\hline Estland & 40 & 34 & 5 & 222 & 1774 & 0.850 & 0.850 & 1.000 \\
\hline Finnland & 36.3 & 32 & 7 & 222 & 1607 & 0.771 & 0.850 & 1.000 \\
\hline Frankreich & 35.5 & 25 & 7.5 & 228 & 1620 & 0.777 & 0.875 & 1.000 \\
\hline Deutschland & 41 & 29.5 & 10 & 221 & 1814 & 0.870 & 0.848 & 1.000 \\
\hline Griechenland & 40 & 25 & 12 & 224 & 1790 & 0.858 & 0.858 & 1.000 \\
\hline Island & 40 & 30 & 14 & 217 & 1734 & 0.831 & 0.831 & 1.000 \\
\hline Israel & 42.5 & 24 & 9 & 228 & 1936 & 0.928 & 0.873 & 1.000 \\
\hline Italien & 36 & 32.0 & 8 & 221 & 1589 & 0.762 & 0.847 & 1.000 \\
\hline Japan & 38.8 & 20 & 17 & 224 & 1734 & 0.831 & 0.858 & 1.000 \\
\hline Korea & 40 & 20 & 14 & 227 & 1814 & 0.870 & 0.870 & 1.000 \\
\hline Niederlande & 36 & 23 & 8 & 230 & 1654 & 0.793 & 0.881 & 1.000 \\
\hline Neuseeland & 40 & 20 & 11 & 230 & 1838 & 0.881 & 0.881 & 1.000 \\
\hline Norwegen & 37.5 & 25 & 10 & 226 & 1693 & 0.812 & 0.866 & 1.000 \\
\hline Polen & 40 & 26 & 11 & 224 & 1790 & 0.858 & 0.858 & 1.000 \\
\hline Portugal & 35 & 25 & 11 & 225 & 1573 & 0.754 & 0.862 & 1.000 \\
\hline Slowak. Rep. & 37.5 & 25 & 15 & 221 & 1655 & 0.794 & 0.847 & 1.000 \\
\hline Slowenien & 40 & 29.9 & 15 & 216 & 1727 & 0.828 & 0.828 & 1.000 \\
\hline Spanien & 37.5 & 22 & 14 & 225 & 1685 & 0.808 & 0.862 & 1.000 \\
\hline Schweden & 39.8 & 33 & 9 & 219 & 1739 & 0.834 & 0.839 & 1.000 \\
\hline Ver. Königreich & 37 & 25 & 8 & 228 & 1685 & 0.808 & 0.873 & 1.000 \\
\hline Ver. Staaten & 40 & 20 & 10 & 231 & 1846 & 0.885 & 0.885 & 1.000 \\
\hline
\end{tabular}

Anmerkungen: Die Zahlen in der Tabelle wurden gerundet.

Höchstzahl an Arbeitstagen pro Jahr bei einer Fünftagewoche: 261. Höchstzahl an Arbeitsstunden pro Jahr bei einem Achtstundentag: 2088. Österreich: Seit dem 1. Januar 2011 hängt die Zahl der bezahlten Urlaubstage vom Alter ab: Eine Vollzeitkraft hat Anspruch auf 240 Stunden (30 Tage/6 Wochen) ab dem Jahr, in dem ihr 43. Geburtstag vor dem 1. Juli liegt. Liegt der 43. Geburtstag nach dem 30. Juni, hat sie im darauffolgenden Jahr Anspruch auf die 240 Stunden.

Estland: Die Dauer des bezahlten Jahresurlaubs hängt von der Beschäftigungsdauer ab. 35 Kalendertage $=28$ Arbeitstage + Beamte, deren Dienstalter 3 Jahre übersteigt, erhalten einen weiteren bezahlten Urlaubstag je Dienstjahr, maximal 10 Tage.

Finnland: Der bezahlte Jahresurlaub beträgt 30 oder 38 Tage, je nach Dienstalter.

Deutschland: Für Angestellte im öffentlichen Dienst und Beamte gelten unterschiedliche Arbeitszeitregelungen.

Italien: Die Zahl der gesetzlichen Urlaubstage variiert. 30 Tage in den ersten 3 Arbeitsjahren, 32 ab dem 4 . Arbeitsjahr. In der Polizei gibt es zwei Korridore, je nach Anzahl der Dienstjahre: 36 Tage bei 15-25 Dienstjahren, 45 Tage bei über 25 Dienstjahren.

Korea: Beamte haben Anspruch auf 3-21 Tage bezahlten Jahresurlaub, je nach Dienstalter.

Slowakische Republik: Die vertraglich vereinbarte Arbeitszeit liegt bei 40 Wochenstunden bzw. 37,5 Wochenstunden, je nach Arbeitsvertrag. Die Zahl der bezahlten Urlaubstage hängt vom Alter ab: 25 Tage (für unter 33-Jährige) oder 30 Tage (bei über 33-Jährigen).

Slowenien: Bei der Durchschnittszahl der bezahlten Urlaubstage pro Jahr handelt es sich um eine Schätzung. Eine Arbeitskraft hat Anspruch auf bezahlten Jahresurlaub, der vier Wochen nicht unterschreiten darf. Zudem hat die Arbeitskraft für jedes Kind unter 15 Jahren Anspruch auf einen weiteren Tag Jahresurlaub. Leistungsabhängig hat ein Beamter Anspruch auf maximal 3 weitere Tage bezahlten Jahresurlaub. Der Jahresurlaub kann im Fall erschwerender Arbeitsbedingungen (Lärm, Hitze, usw), einer schlechten gesundheitlichen Verfassung oder für die Leitung einer Verwaltungseinheit um bis zu drei Tage verlängert werden.

Schweden: Die Zahl der Arbeitstage variiert laut zentralem Tarifvertrag auf Ebene der Zentralregierung mit dem Alter. Beschäftigte unter 30 Jahren haben 28 Tage bezahlten Jahresurlaub, Bedienstete zwischen 30 und 39 Jahren 31 Tage und Beschäftigte im Alter von über 40 Jahren 35 Tage.

Vereinigtes Königreich: Die Zahl der wöchentlichen Arbeitsstunden liegt bei 37 außerhalb von London, wo die meisten Beamten tätig sind, und bei 36 Stunden in der Londoner Innenstadt. Die Zahl der durchschnittlichen Arbeitstage je Arbeitsjahr mit Urlaubsanspruch variiert je nach Ministerium.

Quelle: 2011 OECD Survey on Compensation of Employees in Central/Federal Government. 


\section{ANHANG E \\ Detaillierte Daten über die Offenlegung von Interessenkonflikten}

Dieser Anhang enthält für jedes Land, das an der Erhebung teilnahm, Daten über die Arten privater Interessen, zu deren Offenlegung Entscheidungsträger der Zentralregierung verpflichtet sind, sowie über den Transparenzgrad dieser Offenlegung. Die Daten bilden die Grundlage für die in Abbildung 8.4 aufgeführten summarischen Daten.

\section{Anmerkung zu Tabelle E.1}

Die Daten beziehen sich auf die Verfahrensweisen in den Mitgliedsländern. Bei der Berechnung eines Aggregats aus den länderspezifischen Daten über die Offenlegung privater Interessen in den drei Bereichen der staatlichen Gewaltenteilung und den Zugang der Öffentlichkeit zu diesen Daten wurden alle Privatinteressen und alle Positionen als gleichermaßen wichtig betrachtet und deshalb gleich gewichtet. Bei der Umrechnung der Antworten der Länder in ein Punktesystem wurde den Kategorien „Verboten“ und „Die Information wird offengelegt und der Öffentlichkeit online oder im Druckformat zur Verfügung gestellt“ mit 100 Punkten die höchstmögliche Punktzahl zugewiesen. „Die Information wird offengelegt und der Öffentlichkeit auf Antrag zur Verfügung gestellt“ erhielt mit 67 Punkten die zweithöchste Punktzahl und „Die Information wird offengelegt und der Öffentlichkeit nicht zur Verfügung gestellt" erhielt mit 33 Punkten die dritthöchste Punktzahl. Die Kategorie „Offenlegung ist nicht vorgeschrieben“ erhielt keine Punkte. Alle untersuchten Privatinteressen wurden gleich gewichtet.

Die Daten zu Brasilien, der Tschechischen Republik, Griechenland, Israel und der Russischen Föderation beziehen sich auf 2010. Die Daten zur Legislative und Judikative von Spanien beziehen sich ebenfalls auf 2010.

Die statistischen Daten für Israel wurden von den zuständigen israelischen Stellen bereitgestellt, die für sie verantwortlich zeichnen. Die Verwendung dieser Daten durch die OECD erfolgt unbeschadet des völkerrechtlichen Status der Golanhöhen, von Ost-Jerusalem und der israelischen Siedlungen im Westjordanland. 
In Luxemburg gibt es keine Auflagen in Bezug auf die Offenlegung von Privatinteressen. In den Vereinigten Staaten ist die Staatsanwaltschaft ein Organ der Exekutive. Hohe Beamte im Justizministerium sind wie alle anderen Beschäftigten der Exekutivorgane verpflichtet, ihre Finanzlage in einem der Öffentlichkeit zugänglichen Bericht offenzulegen. Die Bundesstaatsanwälte der unteren Ebene haben ein separates, nicht öffentliches Berichtssystem zur Offenlegung von Interessenkonflikten. In Norwegen beziehen sich die Daten zu den Richtern nicht auf Laienrichter und Richter in Schlichtungsstellen. In Neuseeland sind Richter und Staatsanwälte nicht verpflichtet, Interessenkonflikte offenzulegen. Es gibt jedoch einen Verhaltenskodex mit dem Titel "Guidelines for Judicial Conduct" (verfügbar auf der Webseite Courts of New Zealand: www.courtsofnz.gout.nz/business/guidelines/guidelines-for-judicial-conduct). Darüber hinaus sind Staatsanwälte häufig Rechtsanwälte, die einer Berufsordnung unterliegen (und bestraft werden sowie je nach Sachlage wegen Pflichtverletzung sogar ihre Berufszulassung verlieren können). In Brasilien traten im Juli 2013 ein neues Gesetz über Interessenkonflikte für öffentliche Amtsträger in den Exekutivorganen und Verbote für die Zeit nach dem Ausscheiden aus dem Dienst in Kraft (Gesetz 12.813).

Bezahlte außerdienstliche Nebentätigkeiten: In Österreich und Belgien sind alle Beamten an die Entscheidung der Verwaltung gebunden, falls eine bezahlte außerdienstliche Nebentätigkeit zu einem Interessenkonflikt führen kann, in Island und der Schweiz gilt diese Regelung nur für Richter. In Dänemark dürfen Richter nur dann eine außerdienstliche Nebenbeschäftigung ausüben (die offengelegt werden muss), wenn diese Tätigkeit Richtern gesetzlich vorbehalten ist oder von einem Sondergremium genehmigt wird. In Estland ist es dem Premierminister sowie den Ministern, Richtern und Staatsanwälten gesetzlich untersagt, eine bezahlte außerdienstliche Nebenbeschäftigung auszuüben, wobei für Forschungs- und Lehrtätigkeiten, die offengelegt werden sollten, Ausnahmeregelungen gelten. In Japan dürfen Richter keine bezahlte außerdienstliche Nebentätigkeit ausüben, ohne im Verlauf ihrer Amtszeit die Erlaubnis des Obersten Gerichtshofs eingeholt zu haben. In ähnlicher Weise müssen Richter ebenfalls grundsätzlich die Zustimmung des Obersten Gerichtshofs oder ihrer Vorgesetzten einholen, um eine unbezahlte außerdienstliche Nebentätigkeit auszuüben. Es gibt jedoch auch Fälle, in denen Richter vor der Aufnahme einer unbezahlten außerdienstlichen Nebenbeschäftigung, wie z.B. der Vorsitz in einer Eltern-Lehrer-Vereinigung, keine Erlaubnis des Obersten Gerichtshofs oder ihrer Vorgesetzten eingeholt haben. Falls Informationen über außerdienstliche Nebenbeschäftigungen von Richtern verlangt werden, werden personenbezogene Daten von Richtern u.U. nicht veröffentlicht. In Polen dürfen der Premierminister und die Minister bestimmte Tätigkeiten, die zu einem Interessenkonflikt führen können, nicht ausüben. Sie sind darüber hinaus verpflichtet, ihre Mitgliedschaft in Gremien von Stiftungen, Handelsgesellschaften und Genossenschaften zu melden, selbst wenn diese Tätigkeit unentgeltlich ausgeübt wird.

Vorherige Beschäftigungsverhältnisse: In Estland sind die Mitglieder der Exekutive und der Legislative gesetzlich nicht verpflichtet, Informationen über vorherige Beschäftigungsverhältnisse offenzulegen, in der Praxis werden diese Informationen aber proaktiv veröffentlicht.

Vermögen, Verbindlichkeiten, Einkommensquellen und -höhe sowie Geschenke: In Island ist der Premierminister nur verpflichtet, Kredite offenzulegen, die abgeschrieben oder zu seinen Gunsten geändert wurden. In Irland werden die Gehälter und Zulagen der Parlamentarier veröffentlicht. Darüber hinaus sind alle Parlamentarier, einschließlich der Amtsinhaber, nach den Ethikgesetzen verpflichtet, ihre persönlichen Interessen in einer 
jährlichen Interessenerklärung offenzulegen. Dies umfasst Einkommen aus anderen Quellen (d.h. bezahlte außerdienstliche Nebentätigkeiten), Aktien, Mandate in Führungsgremien, Grund und Boden, Geschenke, vergünstigte Dienstleistungen oder Reisen, Beratertätigkeit sowie ein direktes Interesse an einem öffentlichen Auftrag. Die Interessen der Abgeordneten sind im Interessenregister des Parlaments öffentlich zugänglich. In Mexiko müssen Geschenke angegeben werden, wenn ihr Wert mindestens dem Zehnfachen des Mindestlohns entspricht. Informationen über Staatsbedienstete werden online veröffentlicht, sofern diese zustimmen. In der Praxis stellen rd. 66\% der Staatsbediensteten der Öffentlichkeit die Informationen zur Verfügung. In Polen sind der Premierminister und die Minister verpflichtet, eine Vermögenserklärung abzugeben, die z.B. das Vermögen, die Verbindlichkeiten sowie Einkommensquelle und -höhe ausweist. Obwohl eine Veröffentlichung der Erklärungen gesetzlich nicht vorgeschrieben ist, erklären sich fast alle Minister zu einer Veröffentlichung im Internet bereit.

In Irland und Italien ergreifen die Behörden nach Einholung der Offenlegungsformulare zwar keinerlei Maßnahmen, der Großteil der offengelegten Informationen wird jedoch veröffentlicht, so dass die Bürger die übermittelten Informationen selbst prüfen können. 


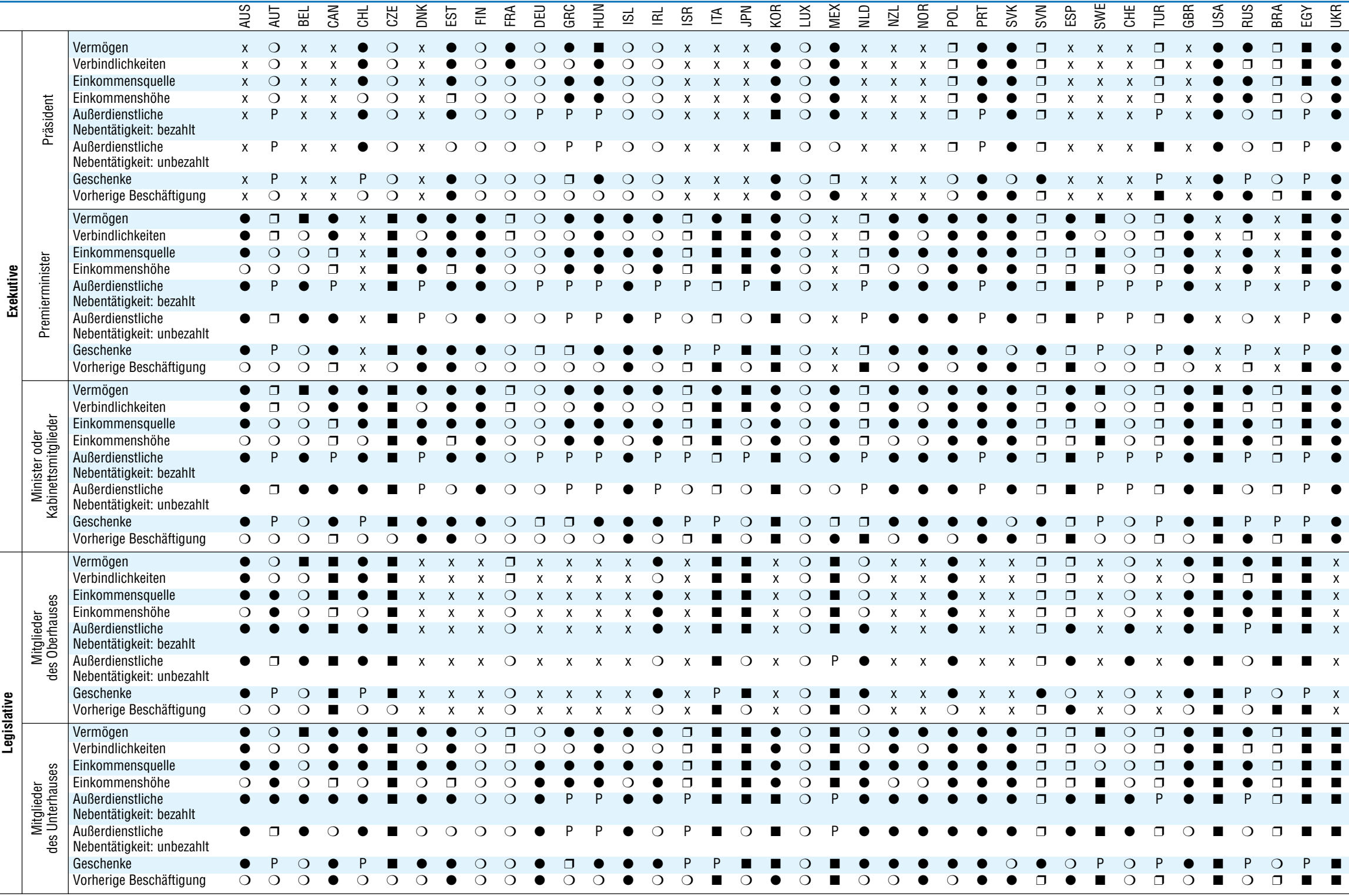


Tabelle E.1. Offenlegung ausgewählter privater Interessen und Zugang der Öffentlichkeit zu den offengelegten Informationen in den drei Staatsgewalten nach Land (2012) (Forts.)

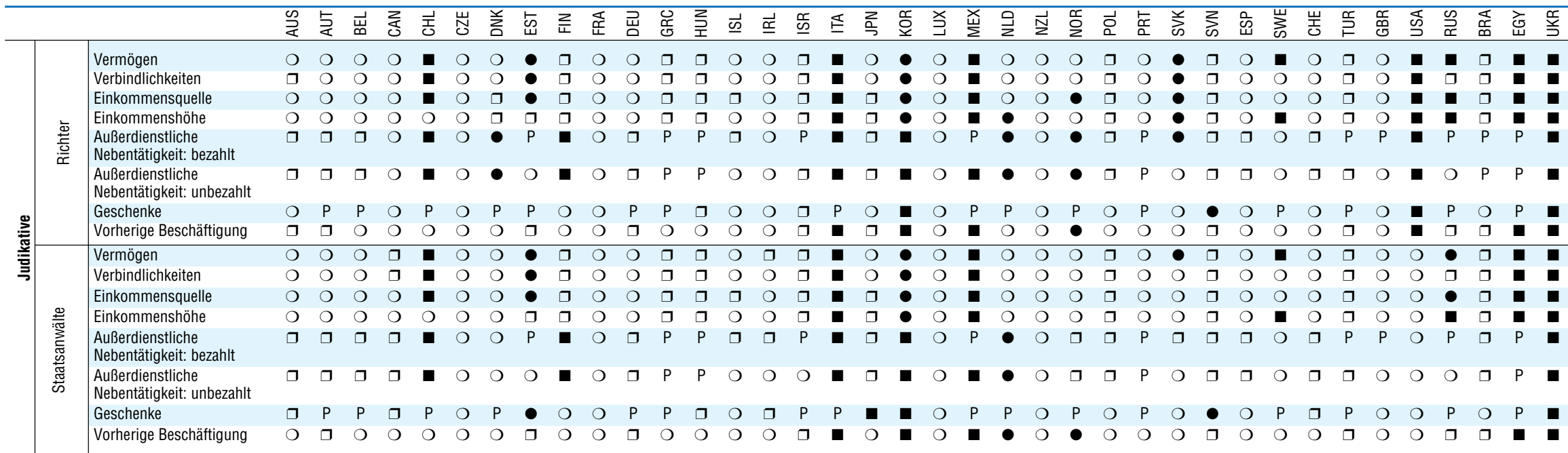

- Die Information wird offengelegt und der Öffentlichkeit online oder im Druckformat zur Verfügung gestellt.

$\square$ Die Information wird offengelegt und der Öffentlichkeit nicht zur Verfügung gestellt.

- Die Information wird offengelegt und der Öffentlichkeit auf Anfrage zur Verfügung gestellt.

O Offenlegung ist nicht vorgeschrieben.

P Verboten.

x Nicht anwendbar (z.B. das Land hat keinen Präsidenten).

Quelle: OECD Survey on Managing Conflict of Interest von 2012. 
ANHANG F

\section{Mitglieder des Lenkungsausschusses}

\begin{tabular}{|c|c|c|c|}
\hline & Name & Titel/Stellung & Ministerium \\
\hline Australien & Kathie Potts & Stv. Beauftragte für den öffentlichen Dienst & Australian Public Service Commission \\
\hline Österreich & Michael Kallinger & Generaldirektor & $\begin{array}{l}\text { Bundeskanzleramt, Öffentlicher Dienst } \\
\text { und Verwaltungsinnovation }\end{array}$ \\
\hline Belgien & Jacques Druart & Leiter Internationale Koordinierung & $\begin{array}{l}\text { Föderaler öffentlicher Dienst Personal } \\
\text { und Organisation }\end{array}$ \\
\hline Kanada & Nicolas Wise & Geschäftsführender Direktor & Treasury Board Secretariat \\
\hline Dänemark & David Fjord Nielson & Sonderberater & Finanzministerium \\
\hline Finnland & Katju Holkeri & Leiterin der Abteilung Regierungspolitik & Finanzministerium \\
\hline Frankreich & Daniel Aunay & $\begin{array}{l}\text { Leiter des Bereichs internationale } \\
\text { Beziehungen }\end{array}$ & $\begin{array}{l}\text { Ministère du Budget, des Comptes publics, } \\
\text { de la Fonction publique et de la Réforme de l'État }\end{array}$ \\
\hline Italien & Dr. Pia Marconi & Generaldirektorin & $\begin{array}{l}\text { Abteilung Öffentliche Verwaltung } \\
\text { des Ministerratspräsidiums }\end{array}$ \\
\hline Japan & Irie Akifumi & Erster Sekretär & Ständige Vertretung Japans bei der OECD \\
\hline Korea & Seong Ju Kang & $\begin{array}{l}\text { Generaldirektor für } \\
\text { Informationssicherheitspolitik }\end{array}$ & $\begin{array}{l}\text { Büro für Informationsstrategie, Ministerium } \\
\text { für öffentliche Verwaltung und Sicherheit }\end{array}$ \\
\hline Niederlande & Peter van Der Gaast & $\begin{array}{l}\text { Leiter der Abteilung für Analyse, } \\
\text { Arbeitsmarkt und makroökonomische } \\
\text { Beratung }\end{array}$ & $\begin{array}{l}\text { Ministerium für Inneres } \\
\text { und Königreichsbeziehungen }\end{array}$ \\
\hline Norwegen & Kleng Bratveit & & $\begin{array}{l}\text { Ministerium für öffentliche Verwaltung und Reform, } \\
\text { Abteilung für IKT-Politik und Reform des öffentlichen } \\
\text { Sektors }\end{array}$ \\
\hline Schweden & Susanne Johansson & Beraterin & Statskontoret \\
\hline Ver. Königreich & Liz McKeown & Stv. Direktorin, Analyseabteilung & Cabinet Office \\
\hline
\end{tabular}




\section{Glossar}

Begriff

Allgemeine Beschäftigungsbedingungen im öffentlichen Dienst

Arbeitszeitanpassung Anpassung der durchschnittlichen jährlichen Vergütung von öffentlich Bediensteten, die die Unterschiede bei der Arbeitszeit ausgleicht, wobei je nach Fall die durchschnittliche Zahl der wöchentlichen Arbeitstage oder die durchschnittliche Zahl der wöchentlichen Arbeitsstunden berücksichtigt wird.

Bruttoinlandsprodukt Standardmessgröße des Werts der von einer Volkswirtschaft in einem (BIP)

Budget

Datensatz

\section{Bedeutung in Regierung und Verwaltung auf einen Blick}

Die allgemeinen Beschäftigungsbedingungen gelten generell für die meisten öffentlich Bediensteten und sicherlich für die meisten externen Mitarbeiter, die dem Gesetz nach als Angestellte behandelt werden. Gelegenheitsarbeiter werden nach dieser Definition von den allgemeinen Beschäftigungsbedingungen für öffentlich Bedienstete nicht erfasst. $\mathrm{Zu}$ beachten ist, dass die allgemeinen Beschäftigungsbedingungen in einigen Ländern von wenigen Ausnahmen abgesehen (gegebenenfalls einige Gelegenheitsarbeiter) für alle Beschäftigten, einschließlich der kurzfristig Beschäftigten, gelten. bestimmten Zeitraum produzierten Waren und Dienstleistungen. Konkret entspricht es der Summe der Bruttowertschöpfung aller gebietsansässigen, an der Produktion beteiligten institutionellen Einheiten (zuzüglich aller Gütersteuern und abzüglich der Gütersubventionen, die im Produktionswert nicht enthalten sind). Alternativ dazu kann es berechnet werden als die Summe der Ausgaben für die Endverwendung von Waren und Dienstleistungen (alle Verwendungen außer Vorleistungen), gemessen in Anschaffungspreisen, abzüglich des Werts der Waren und Dienstleistungsimporte, oder als die Summe der von gebietsansässigen produzierenden Einheiten geleisteten Primäreinkommen (OECD Glossary of Statistical Terms).

Eine umfassende Aufstellung der staatlichen Finanzpläne, die Ausgaben, Einnahmen, Defizit bzw. Überschuss und Verschuldung umfasst. Das Budget ist das wichtigste wirtschaftspolitische Dokument der Regierung, in dem die Regierung darlegt, wie sie die öffentlichen Mittel einsetzen will, um bestimmte Politikziele zu erreichen, und in gewissem Umfang ihre Politikprioritäten verdeutlicht.

Satz von Indikatoren und Variablen zu einem bestimmten Thema (z.B. Regulierungsqualität). 
Effizienz

Effektivität

Einheitsstaat

Ergebnis

Erwerbsbevölkerung

Fiskalregel

Föderalstaat

Gender

Governance Gesamtrechnungen
Erzielung einer maximalen Leistung mit einem gegebenen Niveau an Ressourcen bei der Erledigung einer Tätigkeit (OECD Glossary of Statistical Terms).

Grad der Erreichung der mit einer Tätigkeit verfolgten Ziele (OECD Glossary of Statistical Terms).

Land ohne verfassungsmäßig verankerte Teilung der Staatsgewalt zwischen einer zentralen und mehreren eigenständigen regionalen oder gliedstaatlichen Regierungsebenen. Allerdings können auch Einheitsstaaten administrativ in verschiedene Regierungsebenen wie Gemeinden, Provinzen oder Regionen unterteilt sein.

Bezieht sich auf das, was mit einer Tätigkeit letztlich erreicht wird. Im Ergebnis drücken sich die beabsichtigten oder unbeabsichtigten Folgen staatlichen Handelns aus, wobei andere Faktoren aber ebenfalls eine Rolle spielen können (OECD Glossary of Statistical Terms).

Die Erwerbsbevölkerung oder gegenwärtig ökonomisch aktive Bevölkerung umfasst alle Personen, die die Voraussetzungen für die Einbeziehung in die Kategorie der Erwerbstätigen oder der Erwerbslosen in einem bestimmten, kurzen Referenzzeitraum erfüllen (OECD Glossary of Statistical Terms).

Europäisches System International vergleichbarer Buchungsrahmen, der von den MitgliedsVolkswirtschaftlicher ländern der Europäischen Union für eine systematische und detaillierte Beschreibung einer Gesamtwirtschaft (Region, Land oder Ländergruppe), ihrer Bestandteile und Beziehungen zu anderen Gesamtwirtschaften verwendet wird (OECD Glossary of Statistical Terms). Volle Übereinstimmung mit dem System der Volkswirtschaftlichen Gesamtrechnungen (System of National Accounts - SNA).

Für die Zwecke dieses Dokuments verwendet die OECD eine ähnliche Definition wie die Europäische Kommission. Eine nummerische Fiskalregel ist eine dauerhafte Begrenzung der fiskalpolitischen Aggregate (Vorgaben für das laufende Jahr werden z.B. nicht erfasst).

Land mit einer verfassungsmäßig verankerten Teilung der Staatsgewalt zwischen einer zentralen und mehreren eigenständigen regionalen oder gliedstaatlichen Regierungsebenen.

Sozial vorgegebene und anerzogene Verhaltensweisen und Erwartungen in Bezug auf die weibliche und männliche Geschlechterrolle. Alle Kulturen interpretieren die biologischen Unterschiede zwischen Frauen und Männern und entwickeln soziale Erwartungen in Bezug auf angemessene Verhaltensweisen und Tätigkeiten und die Aufteilung von Rechten, Ressourcen und Macht zwischen Frauen und Männern. Gender ist wie Rasse, ethnische Zugehörigkeit und Klasse eine soziale Kategorie, die die Lebenschancen des Einzelnen maßgeblich bestimmt. Genderfragen prägen die Teilhabe am gesellschaftlichen und wirtschaftlichen Leben.

Ausübung politischer, wirtschaftlicher und administrativer Befugnisse 
Haushaltsdaten für Bürger

Indikator

Individuelle Güter und Dienstleistungen

Input

Kollektive Güter und Dienstleistungen

Leistungs- und ergebnisorientierte Informationen

Öffentliche Dienstleistungen
Ein Haushaltsleitfaden für die Bürger ist hier als leicht verständliche Zusammenfassung der wesentlichen Merkmale des dem Gesetzgeber vorgelegten Jahreshaushalts definiert. Es sollte sich um ein selbsterklärendes Dokument handeln, in dem die Inhalte des Jahreshaushaltsentwurfs sowie die voraussichtlichen Wirkungen erläutert werden. Auch wenn er Links oder Verweise auf ausführlichere Dokumente enthält, sollte ein Leitfaden den Lesern nicht abverlangen, diese zu nutzen bzw. mit ihren Inhalten vertraut zu sein, um den Leitfaden zu verstehen.

Von einer Reihe beobachteter Fakten abgeleitete quantitative oder qualitative Messgröße, die über die relative Stellung (z.B. eines Landes) in einem bestimmten Bereich Aufschluss geben kann. Wird ein Indikator in regelmäßigen Abständen evaluiert, kann aus ihm die Richtung von Veränderungen abgelesen werden, die sich bei unterschiedlichen Einheiten im Zeitverlauf vollziehen (Nardo et al., 2005).

Güter und Dienstleistungen, die hauptsächlich dem Einzelnen zugute kommen. Beispiele hierfür sind Bildungs- und Gesundheitsleistungen oder Sozialversicherungsleistungen.

In der Produktion von Waren und Dienstleistungen zum Einsatz kommende Arbeits-, Kapital-, Waren- und Dienstleistungseinheiten. „Wird der Gesundheitssektor als Beispiel zu Grunde gelegt, umfasst der Input definitionsgemäß die vom medizinischen und nichtmedizinischen Personal geleistete Arbeitszeit, die zum Einsatz kommenden Arzneimittel, den Stromverbrauch und die sonstigen gekauften Vorleistungen sowie die Kapitaldienste der eingesetzten Ausrüstungen und Gebäude“ (Lequiller, 2005).

Güter und Dienstleistungen, die der Allgemeinheit zugute kommen. Beispiele hierfür sind öffentliche Ausgaben für Verteidigung sowie öffentliche Ordnung und Sicherheit.

Leistungs- und ergebnisorientierte Informationen können sowohl von staatlichen als auch von nichtstaatlichen Organisationen erstellt werden und sowohl qualitativer als auch quantitativer Art sein. Sie beziehen sich auf Messgrößen/Indikatoren/allgemeine Informationen zu den Inputs, Verfahren, Outputs und Ergebnissen staatlicher Politik/ Programme/Organisationen und können letztlich zur Bewertung von deren Effektivität, Kostenwirksamkeit und Effizienz genutzt werden. Leistungs- und ergebnisorientierte Informationen finden sich beispielsweise in Statistiken, den Finanz- und/oder operationalen Daten staatlicher Organisationen, den von staatlichen Organisationen erstellten Ergebnisberichten, der Evaluierung von Politiken, Programmen oder Organisationen sowie in Ausgabenprüfungen.

Dienstleistungen, die für die Öffentlichkeit oder öffentliche Einnichtungen erbracht werden. Öffentliche Dienstleistungen werden von staatlichen Stellen für die Bürger erbracht, entweder direkt (durch den öffentlichen Sektor) oder durch die Finanzierung privater Dienstleistungen. Sie basieren auf dem sozialen Konsens, dass bestimmte Dienstleistungen unabhängig vom Einkommen für alle verfügbar sein sollten. Selbst 
Öffentlicher Sektor

Output (Leistung)

Produktivität

Staat

System der Volks-

wirtschaftlichen

Gesamtrechnungen wenn öffentliche Dienstleistungen weder öffentlich bereitgestellt noch öffentlich finanziert werden, unterliegen sie aus sozialen und politischen Gründen generell einer strengeren Regulierung als die meisten anderen Wirtschaftssektoren.

Sektor Staat zuzüglich (quasi-)öffentliche Unternehmen (System der Volkswirtschaftlichen Gesamtrechnungen - SNA 1993).

In der Leistungsbewertung der öffentlichen Verwaltung werden Outputs definiert als Güter und Dienstleistungen, die von staatlichen Stellen erzeugt bzw. erbracht werden (z.B. erteilte Unterrichtsstunden, ermittelte Leistungsansprüche und ausgezahlte Sozialleistungen) (OECD Glossary of Statistical Terms).

Produktivität wird gemeinhin definiert als das Verhältnis zwischen einer Volumenmessgröße der Produktion und einer Volumenmessgröße des Inputeinsatzes (OECD Glossary of Statistical Terms). Ökonomen unterscheiden zwischen der Gesamtproduktivität, d.h. der Gesamtproduktion dividiert durch die Veränderung des (gewichteten) Inputs, und der Grenzproduktivität, mit anderen Worten der Veränderung der Produktion dividiert durch die Veränderung des (gewichteten) Inputs (Coelli et al., 1999).

Der Sektor Staat umfasst: a) alle Organe der zentralen, gliedstaatlichen und lokalen Ebene, b) alle Sozialversicherungsträger der verschiedenen staatlichen Ebenen und c) alle nicht marktorientierten Einrichtungen ohne Erwerbszweck, die staatlicher Kontrolle unterliegen und größtenteils von staatlichen Stellen finanziert werden. Nicht zum Sektor Staat gehören öffentliche Unternehmen, selbst wenn sich das gesamte Eigenkapital dieser Unternehmen im Besitz staatlicher Stellen befindet. Nicht inbegriffen sind ferner QuasiUnternehmen, die sich im Besitz staatlicher Einheiten befinden und staatlicher Kontrolle unterliegen. Demgegenüber sind Unternehmenseinheiten ohne eigene Rechtspersönlichkeit, die sich im Besitz staatlicher Einheiten befinden und keine QuasiUnternehmen sind, fester Bestandteil dieser Einheiten und gehören somit zum Sektor Staat (System der Volkswirtschaftlichen Gesamtrechnungen - SNA 1993).

Das System der Volkswirtschaftlichen Gesamtrechnungen (System of National Accounts - SNA) ist ein zusammenhängendes, konsistentes und integriertes System makroökonomischer Konten, Vermögensbilanzen und Tabellen, die auf einem Katalog international vereinbarter Konzepte, Definitionen, Klassifikationen und Rechnungslegungsregeln beruhen (SNA 1.1).

Das System der Volkswirtschaftlichen Gesamtrechnungen 1993 (SNA 1993) wurde unter der gemeinsamen Verantwortung der Vereinten Nationen, des Internationalen Währungsfonds, der Kommission der Europäischen Gemeinschaften, der OECD und der Weltbank erstellt (OECD Glossary of Statistical Terms).

Das SNA 2008 wurde vor kurzem abgeschlossen und enthält eine Reihe von Änderungen gegenüber dem SNA 1993. Die im SNA aufgeführten Indikatoren basieren bei allen OECD-Ländern mit 
Transferleistungen

Unabhängige

fiskalische Institution

Variable

Verwaltungsablauf im öffentlichen

Sektor

Vollzeitäquivalent (VZÄ)

Zentrale

Haushaltsbehörde
Ausnahme von Australien (sowie Kanada bei der Staatsverschuldung) auf dem SNA 1993. Es ist zu beachten, dass es einige Jahre dauern wird (bei den meisten Ländern bis 2014), bis die Volkswirtschaftlichen Gesamtrechnungen diese Änderungen widerspiegeln, was einen gewissen Einfluss auf einige der in dieser Publikation aufgeführten ausgewählten Indikatoren haben wird.

Anspruchsberechtigten Personen vom Staat gewährte Mittel, die nicht an ein bestimmtes Gut oder eine bestimmte Dienstleistung gebunden sind. Beispiele für Transferleistungen sind Renten, Arbeitslosenunterstützung und Leistungen der Entwicklungszusammenarbeit.

Ein öffentlich finanziertes unabhängiges Gremium, das kraft Gesetz unter der Kontrolle der Exekutive oder der Legislative steht und die Fiskalpolitik und Haushaltsergebnisse objektiv überwacht und analysiert und in einigen Fällen diesbezüglich Empfehlungen abgibt. Unabhängige fiskalische Institutionen führen zukunftsgerichtete Ex-ante-Evaluierungen durch (im Gegensatz zu den öffentlichen Auditeinrichtungen, die eine ebenso unerlässliche Ex-post-Prüfung durchführen).

Merkmal einer beobachteten Einheit, das mehr als einen Wert aus einer Reihe von Werten annehmen kann, wobei es sich um Zahlen oder Klassifizierungskategorien handeln kann (z.B. Einkommen, Alter, Gewicht usw. oder „Beruf“, „Branche“, „Krankheit“ usw.) (OECD Glossary of Statistical Terms).

Strukturen, Verfahren und Verwaltungsbestimmungen, die im öffentlichen Sektor weit verbreitet sind.

Die Zahl der Arbeitsplätze in Vollzeitäquivalenten ist definiert als die Gesamtzahl der geleisteten Arbeitsstunden dividiert durch die Zahl der in einer Vollzeitbeschäftigung jährlich durchschnittlich geleisteten Arbeitsstunden (OECD Glossary of Statistical Terms).

Die zentrale Haushaltsbehörde ist eine öffentliche Stelle oder eine aus mehreren koordinierten Stellen bestehende Einrichtung, die auf der zentralen/nationalen/Bundesebene des Staates angesiedelt ist und für die treuhänderische Kontrolle und Verwaltung des nationalen/ Bundeshaushalts zuständig ist. In vielen Ländern ist sie Teil des Finanzministeriums. Die konkreten Zuständigkeiten unterscheiden sich von Land zu Land, die zentrale Haushaltsbehörde ist jedoch generell zuständig für die Formulierung der Haushaltsentwürfe, die Durchführung der Haushaltsverhandlungen, die Zuteilung und Neuverteilung der Finanzmittel, die Einhaltung der Haushaltsgesetze und die Durchführung von Leistungsbewertungen und/oder Effizienzprüfungen. Die Behörde reguliert den Haushaltsvollzug, ist aber nicht zwangsläufig für die Auszahlung öffentlicher Mittel zuständig. Eine sehr wichtige Aufgabe der zentralen Haushaltsbehörde besteht abschließend darin, die Haushaltsdisziplin auf gesamtstaatlicher/nationaler Ebene zu überwachen und aufrechtzuerhalten und eine effektive Kontrolle der Haushaltsausgaben zu gewährleisten. 
Zusammengesetzter Indikator, der durch die Zusammenfassung einzelner Indikatoren Index Izu einem Gesamtindex nach einem festgelegten Modell gebildet wird (Nardo et al., 2005). 


\section{ORGANISATION FÜR WIRTSCHAFTLICHE ZUSAMMENARBEIT UND ENTWICKLUNG}

Die OECD ist ein in seiner Art einzigartiges Forum, in dem die Regierungen gemeinsam an der Bewältigung von Herausforderungen der Globalisierung im Wirtschafts-, Sozial- und Umweltbereich arbeiten. Die OECD steht auch in vorderster Linie bei den Bemühungen um ein besseres Verständnis der neuen Entwicklungen und durch sie ausgelöster Befürchtungen, indem sie Untersuchungen zu Themen wie Corporate Governance, Informationswirtschaft oder Bevölkerungsalterung durchführt. Die Organisation bietet den Regierungen einen Rahmen, der es ihnen ermöglicht, ihre Politikerfahrungen auszutauschen, nach Lösungsansätzen für gemeinsame Probleme zu suchen, empfehlenswerte Praktiken aufzuzeigen und auf eine Koordinierung nationaler und internationaler Politiken hinzuarbeiten.

Die OECD-Mitgliedstaaten sind: Australien, Belgien, Chile, Dänemark, Deutschland, Estland, Finnland, Frankreich, Griechenland, Irland, Island, Israel, Italien, Japan, Kanada, Korea, Luxemburg, Mexiko, Neuseeland, die Niederlande, Norwegen, Österreich, Polen, Portugal, Schweden, Schweiz, die Slowakische Republik, Slowenien, Spanien, die Tschechische Republik, Türkei, Ungarn, das Vereinigte Königreich und die Vereinigten Staaten. Die Europäische Union nimmt an den Arbeiten der OECD teil.

OECD Publishing sorgt dafür, dass die Ergebnisse der statistischen Analysen und der Untersuchungen der Organisation zu wirtschaftlichen, sozialen und umweltpolitischen Themen sowie die von den Mitgliedstaaten vereinbarten Übereinkommen, Leitlinien und Standards weite Verbreitung finden. 


\section{Regierung und Verwaltung auf einen Blick 2013}

Die Messung der Leistungsfähigkeit des Staates gilt zwar schon seit langem als wichtige Voraussetzung für die Steigerung der Effektivität und Effizienz der öffentlichen Verwaltung, angesichts der Folgen der Wirtschaftskrise und der in vielen Ländern vorgenommenen Straffung der Fiskalpolitik sind die staatlichen Instanzen heute jedoch mehr denn je auf verlässliche Indikatoren angewiesen, um sachkundige Entscheidungen treffen zu können. Regierung und Verwaltung auf einen Blick 2013 nimmt im einführenden Politikkapitel die Frage des Vertrauens in den Staat unter die Lupe und stellt dem Leser anschließend einen umfassenden Katalog wichtiger Indikatoren zu folgenden Themen vor:

- Strategische Governance

- Öffentliche Finanzen und wirtschaftliches Handeln des Staates

- Haushaltspraxis und -verfahren

- Beschäftigung und Bezahlung im öffentlichen Sektor

- Frauen in Regierung und Verwaltung

- Öffentliches Beschaffungswesen

- Offene und inklusive Regierung

- Im Dienste der Bürger: Zugänglichkeit und Qualität öffentlicher Dienstleistungen

Über die StatLinks, die in der gesamten Publikation zu finden sind, kann auf die Excel ${ }^{\mathrm{TM}}$-Arbeitsblätter zugegriffen werden, die zur Erstellung der Tabellen und Abbildungen in Regierung und Verwaltung auf einen Blick verwendet wurden. Kurzdarstellungen zu den einzelnen Ländern und sonstige Online-Ergänzungen sind über die OECD-Website zu Regierung und Verwaltung auf einen Blick verfügbar:

www.oecd.org/gov/govataglance.htm.

Diese Publikation kann online eingesehen werden unter: http://dx.doi.org/10.1787/9789264209541-de.

Diese Studie ist in der OECD iLibrary veröffentlicht, die alle Bücher, periodisch erscheinenden Publikationen und statistischen Datenbanken der OECD enthält.

Weitere Informationen finden Sie unter: www.oecd-ilibrary.org. 